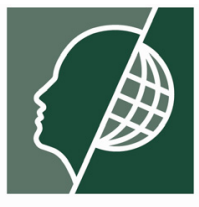

Organization for Human Brain Mapping

\title{
Schedule of Poster Presentations and List of Posters
}

\author{
*Indicates poster will also be presented during an Oral Session. \\ Information listed below appears as author submitted.
}

Schedule of Poster Presentations and List of Posters

Monday, June 16, 2008

11:30 - 12:30 You Yangs Hall (Level 3)

COGNITION \& ATTENTION

Attention (auditory, tactile, motor)
Fractal based method for alertness measurement using EEG, Sridhar Poosapadi Arjunan ${ }^{\text {, }}$ Dinesh Kant Kumar ${ }^{1}$, Tzyy-Ping Jung ${ }^{2},{ }^{1}$ SECE, RMIT University, Melbourne, Australia, ${ }^{2}$ SCCN, University of California, San Diego, USA

Cortically constrained current source density analysis of duration-deviant mismatch negativity in schizophrenia, Ross Fulham ${ }^{1}$, Ulrich Schall ${ }^{1,5}$, Patrica Michie ${ }^{2,5}$, Phillip Ward ${ }^{3,5}$, Matthew Hughes ${ }^{2,5}$, Patrick Johnston ${ }^{4}$, Paul Rasser ${ }^{1,5},{ }^{1}$ Centre for Brain and Mental Health Studies, Newcastle University, Newcastle, Australia, ${ }^{2}$ School of Psychology, Newcastle University, Newcastle, Australia, ${ }^{3}$ University of NSW, Sydney, Australia, ${ }^{4}$ Swinburn University of Technology, Melbourne, Australia, ${ }^{5}$ Schizophrenia Research Institute, NSW, Australia, ${ }^{6}$ Hunter Medical Research Institute, Newcastle, Australia

Attention-dependent modulation of neural activity in primary motor cortex, Annette Milnik, Isabella Nowak, Notger Müller, Cognitive Neurology Unit, Frankfurt, Germany

Chronotype-dependent performance modulation according to time of day : a functional neuroimaging approach, Christina Schmidt ${ }^{l, 2}$, Fabienne Collette ${ }^{1,2}$, Virginie Sterpenich ${ }^{l}$, Gilles Vandewalle , Gilberte Tinguely', Annabelle Darsaud', Steffen Gais ', Manuel Schabus ${ }^{I}$, Martin Deseilles ${ }^{l}$, Thanh DangVu ${ }^{I}$,Eric Salmon ${ }^{I}$, André Luxen ${ }^{I}$, Pierre Maquet ${ }^{l}$, Christian Cajochen ${ }^{3}$, Philippe Peigneux ${ }^{4},{ }^{1}$ Cyclotron Research Center, University of Liège, Liège, Belgium,

${ }^{2}$ Department of Cognitive Science, University of Liège, Liège, Belgium, ${ }^{3}$ Center for Chronobiology Psychiatric University Clinics, Basel, Switzerland, ${ }^{4}$ UR2NF-Neuropsychology and Functional Neuroimaging Research Unit, Brussels, Belgium

The effects of the glutamate antagonist memantine on brain activation to an auditory discrimination task: A pharmacological fMRI study, Heidi van Wageningen ${ }^{l}$, Hugo A. Jørgensen ${ }^{2}$, Tom Eichele ${ }^{1}$, Karsten Specht ${ }^{l}$, Kenneth Hugdahl ${ }^{1,2},{ }^{1}$ Department of Biological and Medical Psychology, University of Bergen, Bergen, Norway, ${ }^{2}$ Division of Psychiatry, Haukeland University Hospital, Bergen, Norway

\section{COGNITION \& ATTENTION Attention (visual)}

Study of Distraction-related EEG Dynamics in Virtual Reality Driving Simulation, Chin-Teng Lin ${ }^{1,2}$, Yu-Chieh Chen ${ }^{1,2}$, Chun-Ling Lin ${ }^{1,2}$, Chih-Feng Chao ${ }^{l}$, Jeng-Ren Duann ${ }^{1,3}$, Tzyy-Ping Jung ${ }^{1,3}{ }^{1}$, 'Brain Research Center, University System of Taiwan, Hsinchu, Taiwan, ${ }^{2}$ Department of Electrical and Control Engineering, National Chiao-Tung University, Hsinchu, Taiwan, ${ }^{3}$ Institute for Neural Computation, University of California, San Diego, USA

Top-down modulation of FFA by semantics associated with ignored and attended faces, Francesco Gentile, Bernadette M. Jansma, Dept. of Cognitive Neuroscience, Faculty of Psychology, University of Maastricht, Maastricht, Netherlands
$1 \mathrm{M}-\mathrm{AM}$

5 M-AM

9 M-AM

13 M-AM

17 M-AM 
Increased activity in human visual cortex during fixation in the absence of foveal visual stimulation, Xiaoqi Huang ${ }^{1,2}$, Paul C. Knox ${ }^{3}$, Su Lv ${ }^{1}$, Hehan Tang ${ }^{l}$, Qiyong Gong ${ }^{l},{ }^{1}$ Huaxi Magnetic Resonance Research Center, Department of Radiology, West China hospital of Sichuan University, Chengdu, China, ${ }^{2}$ Department of Psychiatry, West China hospital of Sichuan University, Chengdu, China, ${ }^{3}$ Division of Orthoptics, School of Health Sciences, University of Liverpool, Liverpool, United Kingdom

Neural multivariate decoding in early visual cortex is not modulated by high attentional demands in an unrelated task., Christian Kaul ${ }^{1,2}$, Nilli Lavie ${ }^{3}$, Geraint Rees ${ }^{1,2}$, ${ }^{1}$ Institute of Cognitive Neuroscience, University College London, London, United Kingdom, ${ }^{2}$ Wellcome Trust Centre for Neuroimaging, University College London, London, United Kingdom, ${ }^{3}$ Department of Psychology, University College London, London, United Kingdom

Activation during Joint Attention is Context Dependent as Measured with Magnetoencephalography (MEG): Substrates of Social Cognition, Renee Lajiness-O'Neill, ${ }^{1,2}$, Nicholas Velissaris ${ }^{I}$, Lesley Pawluk ${ }^{I}$, Susan Bowyer ${ }^{2,3,4}$, 'Eastern Michigan University, Ypsilanti, USA, ${ }^{2}$ Henry Ford Medical Group, Detroit, USA, ${ }^{3}$ Oakland University, Rochester, USA, ${ }^{4}$ Wayne State Universiry, Detroit, USA

An fMRI study of item similarity effects in visual search, Steven Phillips, Yuji Takeda, AIST, Tsukuba, Japan

Brain Substrates Associated with Working Memory among Subjects with Alcohol Use Disorders, Mi-Sook Park ${ }^{1}$, In Kyu YU ${ }^{2}$, Hyunsoo Khang ${ }^{2}$, Sunju Sohn ${ }^{3}$, Jin-Hun Sohn ${ }^{1},{ }^{1}$ Dept. of Psychology, Institute for Brain Research, Chungnam National UniversityDaejeon, Daejeon, South Korea, ${ }^{2}$ Dept. of Radiology, College of Medicine, Eulji University, Daejeon, South Korea, ${ }^{3}$ School of Social Work, University of Texas Austin, Austin, USA

Pathways for visual-spatial attention, Roza Umarova ${ }^{l}$, Dorothee Saur ${ }^{1}$, Susanne Schnell ${ }^{2}$, Björn Kreher $^{2}$, Magnus-Sebastian Vry ${ }^{I}$, Volkmar Glauche ${ }^{I}$, Cornelius Weiller ${ }^{I},{ }^{I}$ Freiburg Brain Imaging, Department of Neurology, University Hospital, Freiburg, Germany, ${ }^{2}$ Medical Physics, Department of Diagnostic Radiology, University Hospital, Freiburg, Germany

\section{COGNITION \& ATTENTION Cognitive Aging}

Automated 3D mapping of caudate atrophy in Parkinson's disease with and without dementia, Liana Apostolova ${ }^{l}$, Mona Beyer ${ }^{2}$, Amity Green ${ }^{1}$, Jonathan Morra ${ }^{1}$, Kristy Hwang ${ }^{1}$, Dag Aarsland ${ }^{2}$, Carmen Janvin ${ }^{2}$, Jan Larsen ${ }^{2}$, Jeffrey Cummings ${ }^{l}$, Paul Thompson ${ }^{I},{ }^{1}$ UCLA, Los Angeles, USA, ${ }^{2}$ Stavanger University, Stavanger, Norway

Establishing Quantitative Linkages of Cognitive Impairments and Leukoaraiosis by CT Imaging, Wei-Shih Huang ${ }^{1}$,Shu-Wen Huang ${ }^{2}$, Chon-Haw Tsai ${ }^{1}$, Chung-Ta Lu' ${ }^{1}$, Chih-Chien Yang ${ }^{2,3},{ }^{1}$ Department of Neurology, China Medical University Hospital, Taichung, Taiwan, ${ }^{2}$ Graduate School of Educational Measurement \& Statistics, National Taichung University, Taichung, Taiwan, ${ }^{3}$ Cognitive NeuroMertics Laboratory, National Taichung University, Taichung, Taiwan

Brain Localization of Cognitive Domains with Diffusion MRI, Efrat Sasson ${ }^{1}$, Glen Doniger ${ }^{2}$, Ofer Pasternak ${ }^{3}$, Yaniv Assaf ${ }^{1,4},{ }^{I}$ Department of Neurobiochemistry, Faculty of Life Sciences, Tel Aviv University, Tel Aviv, Israel, ${ }^{2}$ Department of Clinical Science, NeuroTrax Corporation, Newark, USA, ${ }^{3}$ School of Computer Science, Tel Aviv University, Tel Aviv, Israel, ${ }^{4}$ Functional brain imaging unit, Tel Aviv Sourasky Medical Center, Tel Aviv, Israel

\section{COGNITION \& ATTENTION Cognitive Development}

Neural correlates of successful and partial inhibitions in children: An ERP study of go/no-go performance, Lucy Cragg ${ }^{1,3}$, Allison Fox ${ }^{2}$, Kate Nation ${ }^{3}$, Corinne Reid ${ }^{4}$, Mike Anderson ${ }^{2},{ }^{I}$ Brain and Body Centre, University of Nottingham, Nottingham, United Kingdom, ${ }^{2}$ School of Psychology, University of Western Australia, Perth, Australia, ${ }^{3}$ Dept. of Experimental Psychology, University of Oxford, Oxford, United Kingdom, ${ }^{4}$ School of Psychology, Murdoch University, Perth, Australia 
Abnormal Structural Integrity of the Ventral Frontostriatal pathway: A Diffusion Tensor Tractography Study of Young Male children with Fragile X Syndrome, Brian W. Haas ${ }^{l}$, Naama Barnea-Goraly ${ }^{l}$, Amy Lightbody ${ }^{I}$, Sweta Patnaik', Fumiko Hoeft ${ }^{l}$, Joseph Piven ${ }^{2}$, Reiss Allan ${ }^{l},{ }^{l}$ Center for Interdisplinary Brain Sciences Research, Stanford University Medical Center, $69 \mathrm{M}-\mathrm{AM}$ Stanford, USA, ${ }^{2}$ Neurodevelopmental disorder Research Center, University of North Carolina, Chapel Hill, USA

Effects of Donepezil on neural network reorganization in patients with post-stroke cognitive impairment: a preliminary study, Yun-Hee Kim ${ }^{l}$, Yun H. Park ${ }^{l}$, Suk Hoon Ohn ${ }^{l}$, Duk Ryul Na ${ }^{2}$, Sung Tae Kim ${ }^{3}$, Chang-hyun Park ${ }^{1,4}$, Woo-Kyoung Yoo ${ }^{1}$, Peter K.W. Lee ${ }^{1},{ }^{1}$ Department of Physical Medicine and Rehabilitation, Samsung Medical Center, Sungkyunkwan University School of Medicine, Seoul, Korea, ${ }^{2}$ Department of Neurology, Samsung Medical Center, Sungkyunkwan University School of Medicine, Seoul, Korea, ${ }^{3}$ Department of Radiology, Samsung Medical Center, Sungkyunkwan University School of Medicine, Seoul, Korea, ${ }^{4}$ Department of Physics, Korea Advanced Institute of Science and Technology, Daejeon, Korea

Response inhibition is associated with right inferior frontal gyrus and right preSMA white matter microstructure in children, Kathrine Skak Madsen ${ }^{1,2}$, Martin Vestergaard Hansen ${ }^{1}$, William F. Baaré, ${ }^{1,2}$, Lisser Rye Ejersbo ${ }^{4}$, Christian Gerlach ${ }^{4}$, Olaf B. Paulson ${ }^{1,2}$, Terry L. Jernigan ${ }^{1,2,3},{ }^{I}$ Danish Research Centre for MR, Copenhagen University Hospital, Hvidovre, Denmark, ${ }^{2}$ Center for Integrated Molecular Brain Imaging, Copehagen, Denmark, ${ }^{3}$ Laboratory of Cognitive Imaging, University of California, San Diego, USA, ${ }^{4}$ Learning Lab Denmark, Danish School of Education, University of Aarhus, Copenhagen, Denmark

Relation between the cerebral organization of arithmetic and language correlates: perspective from a large scale database of healthy subjects, philippe pinel $^{1,2,3}$, alex lopez Rolon ${ }^{4}$, stanislas dehaene ${ }^{1,2,3,5},{ }^{1}$ inserm, saclay, France, ${ }^{2}$ cea, saclay, France, ${ }^{3}$ Université ParisSud, orsay, France, ${ }^{4}$ Medizinische Universität Innsbruck, Innsbruck, Austria, ${ }^{5}$ college de France, paris, France

\section{COGNITION \& ATTENTION}

Perception, Imagery, Awareness

Visual awareness during binocular rivalry: Structural connectivity and a truly nonrivalrous comparison condition, Juliane C. Wilcke ${ }^{1,2}$, Robert P. O'Shea ${ }^{3}$, Richard Watts ${ }^{1,4}$, ${ }^{1}$ Department of Physics and Astronomy, University of Canterbury, Christchurch, New Zealand, ${ }^{2}$ Department of Psychology, University of Canterbury, Christchurch, New Zealand, ${ }^{3}$ Department of Psychology, University of Otago, Dunedin, New Zealand, ${ }^{4}$ Van der Veer Institute for Parkinson's and Brain Research, Christchurch, New Zealand

Implication of two distinct neuronal networks in the awareness of environment and of self, Audrey Vanhaudenhuyse ${ }^{I}$, Athena Demertzi ${ }^{1}$, Manuel Schabus ${ }^{2}$, Christophe Phillips ${ }^{1}$, Serge Bredart ${ }^{3}$, Steven Laureys ${ }^{1,4}$, Melanie Boly ${ }^{1,4},{ }^{1}$ Coma Science Group, Cyclotron Research Center, University of Liège, Liège, Belgium, ${ }^{2}$ Department of Psychology, University of Salzburg, Salzburg, Belgium, ${ }^{3}$ Department of Cognitive Science, University of Liège, Liège, Austria, ${ }^{4}$ Neurology Department, CHU Sart Tilman, University of Liège, Liège, Belgium

Differential Neuromagnetic Activity Associated with Time Perception of Short and Long Tones, Frederick Carver ${ }^{l}$, Brita Elvevaag ${ }^{l}$, Tom Holroyd ${ }^{l}$, Terry Goldberg ${ }^{2}$, Richard Coppola ${ }^{l}$, ${ }^{I}$ NIMH, Bethesda, USA, ${ }^{2}$ Albert Einstein CoM, Glenn Oaks, USA

When the brain takes BOLD 'steps': Controlling differential brain activation levels via realtime fMRI-based neurofeedback training, Brigitte Dahmen ${ }^{1,2}$, Bettina Sorger ${ }^{1,2}$, Charlotte Sinke ${ }^{1,2}$, Rainer Goebel ${ }^{1,2}$, IDepartment of Cognitive Neuroscience, Maastricht University, Maastricht, Netherlands, ${ }^{2}$ Maastricht Brain Imaging Center (M-BIC), Maastricht, Netherlands

Neural Correlates of Perception in Chess, Merim Bilalic, Michael Erb, Wolfgang Grodd, Section Exp. MR of the CNS, Department of Neuroradiology, University of Tübingen, Tuebinge, Germany

Does Mental Rotation of Hands and Feet Involve Somatotopically Organized Brain Regions?, Takashi Hanakawa, Chihiro Hosoda, Manabu Honda, National Center of Neurology and Psychiatry, Kodaira, Japan 
The Comparison of Buddhist Meditation with Different Phrases by Using fMRI, Chao-Hsien Hsieh $^{l}$, Chien-Hui Liou ${ }^{l}$, Chang-Wei Hsieh ${ }^{1}$, Chi-Hong Wang ${ }^{2}$, Li-Kang Ho ${ }^{3}$, Jyh-Horng Chen ${ }^{l}$, ${ }^{l}$ Interdisciplinary MRI/MRS Lab, Department of Electrical Engineering, National Taiwan University, Taipei, Taiwan, ${ }^{2}$ Department of Neurology, Cardinal Tien Hospital Yung Ho Branch, Taipei, Taiwan, ${ }^{3}$ Department and Institute of Pharmacology, National Yang-Ming University, Taipei, Taiwan

An fMRI Investigations of Temporal Discrimination: The Relationship Between Right Prefrontal Cortex and Interval Duration., Kelly Jantzen ${ }^{1}$, Steve Sedita ${ }^{2}$, J.A. Scott Kelso ${ }^{2}$, ${ }^{I}$ Western Washington University, Bellingham, USA, ${ }^{2}$ Florida Atlantic University, Boca Raton, USA

\section{COGNITION \& ATTENTION Reasoning \& Problem Solving}

Dynamics of conditional inference and top down effects : A MEG study, Mathilde Bonnefond ${ }^{l}$, Jean-Baptiste Van Der Henst ${ }^{l}$, Anne Cheylus ${ }^{l}$, Olivier Bertrand ${ }^{2}$, Ira Noveck ${ }^{l}$, ${ }^{1}$ CNRS-LAboratoire sur le langage, le cerveau et la cognition, France, France, ${ }^{2}$ INSERM-U821 Dynamique Cérébrale et Cognition, France, France

Sex differences in cortical activation patterns during mental rotation task in schizophrenia patients., Jose Jimenez ${ }^{1,2}$, Adham Mancini-Marie ${ }^{1,2}$, Melissa Rinaldi ${ }^{1,2}$, Emmanuel Stip ${ }^{1,2}$, Marc Lavoie $^{1,2}$, Francois Guillem ${ }^{1,2}$, Adrianna Mendrek ${ }^{1,2},{ }^{1}$ Department of Psychiatry, Fernand-Seguin Research Center, Louis-H Lafontaine Hospital, University of Montreal, Montreal, Canada, ${ }^{2}$ Department of Psychiatry, Biomedical Sciences Program, Faculty of Medicine, University of Montreal, Montreal, Canada

The Effect of Chicken Essence on Cognitive Processing in the Brain Revealed by fMRI Using the Tower of London Task, Jin-Hun Sohn', Ji-Eun Park', Jin-Sup Eom ${ }^{l}$, Chia Chew $\mathrm{Sern}^{2}$, Daniel Tsi ${ }^{2}$, Hajime Nagai ${ }^{2},{ }^{l}$ Dept. of Psychology, Institute for Brain Research, Chungnam Nat'l University, Daejeon, South Korea, ${ }^{2}$ BRAND'S Health Science Center, Cerebos Pacific Limited, China square central, Singapore

\section{COGNITION \& ATTENTION}

Space, Time, \& Number Coding

Numerical Specialisation: Within and Between Dimensions, Roi Cohen Kadosh, Bahador Bahrami, Vincent Walsh, Brian Butterworth, Cathy Price, University College London, London, United Kingdom

Effective Connectivity of Frontal and Parietal Cortex in Quantifier Comprehension, Vanessa Troiani, Jonathan Peelle, Murray Grossman, University of Pennsylvania, Philadelphia, USA

\section{DISORDERS OF THE NERVOUS SYSTEM Alzheimer \& Dementia}

Diffusion tensor analysis of optic radiation changes after optic neuritis, Clare Bajraszewski $^{1}$, Scott Kolbe ${ }^{1,2}$, Caron Chapman ${ }^{2}$, Peter Mitchell ${ }^{3}$, Helmut Butzkueven ${ }^{1,2,3}$ Trevor Kilpatrick ${ }^{1,2,3}$, Gary Egan ${ }^{1,2},{ }^{1}$ Howard Florey Institute, Florey Neuroscience Institutes, Australia, ${ }^{2}$ Centre for Neuroscience, University of Melbourne, Australia, ${ }^{3}$ Royal Melbourne Hospital, Australia

Regional brain changes in Mild Alzheimer's Disease: A Combination of Voxel-based Morphometry and Diffusion Tensor Imaging, Qin Chen ${ }^{1,2}$, Ling Zou ${ }^{2}$, Zheng-Yan $\mathrm{Li}^{2}$, Luo Ou-Yang ${ }^{3}$, Wei-Wei Zhang ${ }^{2}$, Li-Jun Jiang ${ }^{4}$, Dong Zhou', Qi-Yong Gong ${ }^{2,5}$, Qiang Yuan ${ }^{1}$, ${ }^{l}$ Department of Neurology, West China hospital of Sichuan University, Chengdu, China, ${ }^{2}$ Huaxi MR Research Center (HMRRC), Department of Radiology, West China Hospital of Sichuan University, Chengdu, China, ${ }^{3}$ Department of Psychology, Southwest University, Chongqin, China, ${ }^{4}$ Psychiatric Center, West China Hospital of Sichuan University, Chengdu, China, ${ }^{5}$ Division of Medical Imaging, University of Liverpool, Liverpool, United Kingdom 
Evidence for cortical reorganisation in cognitive domains in Multiple Sclerosis from functional MRI, Christian Enzinger ${ }^{1,3}$, Marisa Loitfelder ${ }^{1,2}$, Stefan Ropele ${ }^{l}$, Christa Neuper ${ }^{2}$, Katja Petrovic ${ }^{1}$, Faton Gorani ${ }^{1}$, Siegrid Fuchs ${ }^{I}$, Franz Fazekas ${ }^{1},{ }^{I}$ Dept. of Neurology, Medical University Graz, Graz, Austria, ${ }^{2}$ Institute of Psychology, Karl Franzens University Graz, Graz, Austria, ${ }^{3}$ Section of Neuroradiology, Dept. of Radiology, Medical University Graz, Graz, Austria

Physical Fitness is Associated with Preservation of Hippocampal Volume in Alzheimer's Disease, Robyn Honea ${ }^{1}$, George Thomas ${ }^{1}$, Amith Harsha ${ }^{1}$, Benjamin Cronk ${ }^{l}$, Joseph Donnelly ${ }^{2}$, William Brooks ${ }^{I}$, Jeffrey M. Burns ${ }^{I},{ }^{I}$ Departments of Neurology, University of Kansas Medical Center, Kansas City, USA, ${ }^{2}$ Energy Balance Laboratory and Center for Physical Acitivity, Nutrition, and Weight, Kansas City, USA

Diffusion tensor imaging in clinically isolated syndrome and relapsing-remitting multiple sclerosis, Yaou Liu ${ }^{1,3}$, Chunshui Yu ${ }^{l}$, Kuncheng Li ${ }^{l}$, Yunyun Duan ${ }^{l}$, Wen Qin ${ }^{l}$, Fuchun Lin ${ }^{2}$, Gary Egan ${ }^{3},{ }^{I}$ Department of radiology, Xuanwu Hospital, Capital University of Medical Sciences, Beijing, China, ${ }^{2}$ Institute of Physics and Mathematics, Chinese Academic of Science, Wuhan, China, ${ }^{3}$ Howard Florey Institute, Melbourne, Australia

Stability of fMRI Hippocampal Activation in Normal Older Subjects Over Two Years, Jacqueline O'Brien ${ }^{1}$, Peter LaViolette ${ }^{2}$, Kelly O'Keefe ${ }^{I}$, Amy DeLuca ${ }^{1}$, Keith Johnson ${ }^{2}$, Reisa Sperling I, 'Brigham and Women's Hospital, Boston, USA, ${ }^{2}$ Massachusetts General Hospital, Boston, USA

Cortical neurodegeneration syndromes target human structural-functional covariance networks, William Seeley ${ }^{l}$, Richard Crawford ${ }^{l}$, Bruce Miller ${ }^{l}$, Michael Greicius ${ }^{2},{ }^{1}$ Memory \& Aging Center, University of California, San Francisco, San Francisco, USA, ${ }^{2}$ Stanford University, Palo Alto, USA

How Treatment of donepezil influence the brain structures in Alzheimer's: A Diffusion Tensor Imaging Study at 3T, Ling Zou ${ }^{1}$, Qin Chen ${ }^{2}$, Qiang Yuan ${ }^{2}$, Zhengyan Li ${ }^{1}$, Weiwei Zhang ${ }^{l}$, Yi Wei ${ }^{l}$, Xiaoling Wen ${ }^{1}$, Qiyong Gong ${ }^{1}{ }^{1}$ Huaxi MR Research Center(HMRRC), Huaxi Hospital, Sichuan University, chengdu, China, ${ }^{2}$ Department of Neurology, Huaxi Hospital, Sichuan University, chengdu, China

\section{DISORDERS OF THE NERVOUS SYSTEM Mood \& Anxiety Disorders}

Decreased Amygdala Anisotropy by DTI in Early Onset MDD: An Epidemiologic Twin Study, Kelly Botteron ${ }^{1}$, Tomoyuki Nishino ${ }^{1}$, Melissa Munn ${ }^{2}$, Dimitrios Alexopolous ${ }^{1}$, Babb Casey ${ }^{l}$, McKinstry Robert ${ }^{l},{ }^{I}$ Washington University School of Medicine, St Louis, USA, ${ }^{2}$ University of Colorado, Boulder, USA

Thinner Prefrontal Cortex in Veterans with Posttraumatic Stress Disorder, Elbert Geuze $e^{1,2}$, Eric Vermetten ${ }^{1,2}$, Rainier Goebel ${ }^{3}$,Herman Westenberg ${ }^{2},{ }^{1}$ Research Centre-Military Mental Healthcare, Utrecht, Netherlands, ${ }^{2}$ Utrecht University Medical Centre, Utrecht, Netherlands, ${ }^{3}$ Maastricht University, Maastricht, Netherlands

Increased Amygdala Activation in Subjects with Bulimia Nervosa, Timo Lukkarinen ${ }^{1,2,3}$, Ilkka Nissilä ${ }^{1,3}$, Aila Rissanen ${ }^{2}$, Jaakko Kaprio ${ }^{4,5}$, Anna Keski-Rahkonen ${ }^{2,4}$, Elina Sihvola ${ }^{4,6}$, Leila Karhunen $^{7}$, Salla Kaurijoki ${ }^{7}$, Oili Salonen ${ }^{3}$, Milla Linna ${ }^{1,2}$, Synnöve Carlson ${ }^{1,8},{ }^{1}$ Neuroscience Unit, Institute of Biomedicine/physiology, University of Helsinki, Helsinki, Finland, ${ }^{2}$ Obesity Research Unit, Department of Psychiatry, University of Helsinki, Helsinki, Finland, ${ }^{3}$ Functional Brain Imaging Unit, HBRC, Medical Imaging Center, University of Helsinki, Helsinki, Finland, ${ }^{4}$ Department of Public Health, University of Helsinki, Helsinki, Finland, ${ }^{5}$ Department of Mental Health and Alcohol Research, National Public Health Institute, Helsinki, Finland, ${ }^{6} 6$ HUS Department of Psychiatry, Helsinki University Central Hospital, Helsinki, Finland, ${ }^{7}$ Department of Clinical Nutrition, University of Kuopio, Kuopio, Finland, ${ }^{8}$ Medical School, University of Tampere, Tampere, Finland

Hippocampo-amygdaloid structure predicts HPA axis dysregulation in the acute phase of major depression (MD), Philipp Sämann, David Höhn, Stefan Kloiber, Natalya Chechko, Susanne Lucae, Michael Czisch, Max Planck Institute of Psychiatry, Munich, Germany 
Aberrant functional connectivity of dorsolateral prefrontal and cingulate networks in patients with major depression during working memory processing, Nenad Vasic ${ }^{l}$, Henrik Walter $^{2}$, Fabio Sambataro ${ }^{3}$, Robert Christian Wolf ${ }^{1},{ }^{1}$ University CLinic of Ulm, Department of Psychiatry III, Ulm, Germany, ${ }^{2}$ Department of Psychiatry, Division of Medical Psychology, University of Bonn, Bonn, Germany, ${ }^{3}$ Clinical Brain Disorders Branch, Genes Cognition and Psychosis Program, National Institute of Mental Health, National Institutes of Health, Bethesda, Washington, USA

\section{DISORDERS OF THE NERVOUS SYSTEM Parkinson's Disease \& Other Basal Ganglia}

White matter degeneration in early Huntington's disease; a Diffusion Tensor Imaging and Tract-Based Spatial Statistics study, India Bohanna ${ }^{l}$, Gary Egan ${ }^{l}$, Anusha Sritharan ${ }^{2}$, Leigh Johnston $^{l, 3}$, Hamed Asadi $^{1}$, Ross Cunnington ${ }^{4}$, Andrew Churchyard ${ }^{5}$, Nellie Georgiou-Karistianis ${ }^{2}$, ${ }^{1}$ Howard Florey Institute, Florey Neuroscience Institutes, Melbourne, Australia, ${ }^{2}$ School of Psychology, Psychiatry and Psychological Medicine, Monash University, Melbourne, Australia, ${ }^{3}$ Department of Electrical and Electronic Engineering, University of Melbourne \& NICTA Victorian Research Laboratory, Melbourne, Australia, ${ }^{4}$ Queensland Brain Institute, University of Queensland, Brisbane, Australia, ${ }^{5}$ Department of Neurology, Monash Medical Centre, Melbourne, Australia

A Joint Conditional-Independence, FDR-Controlled Method for Functional Connectivity Insights into L-Dopa Effectiveness in Parkinson's Disease, Martin McKeown ${ }^{1,2,3}$, Junning Li ${ }^{4}$, Samantha Palmer ${ }^{2}$, Jane Wang ${ }^{4},{ }^{I}$ Pacific Parkinson's Research Center, Vancouver, Canada, ${ }^{2}$ Brain Research Center, Vancouver, Canada, ${ }^{3}$ Dept. of Medicine (Neurology), Vancouver, Canada, ${ }^{4}$ Dept. of Electrical and Computer Engineering, Vancouver, Canada

Spatial mapping of coherence and phase shift between electromyographic activities and local field potentials in the subthalamic nucleus in patients with Parkinson's disease and resting tremor, Christiane Reck ${ }^{1,2}$, Esther Florin ${ }^{1,4}$, Lars Wojtecki ${ }^{2}$, Holger Krause ${ }^{2}$, Stefan Groiss ${ }^{2}$, Jürgen Voges ${ }^{3}$, Mohammad Maarouf ${ }^{3}$, Volker Sturm ${ }^{3}$, Alfons Schnitzler ${ }^{2}$, Lars Timmermann ${ }^{1}$, ${ }^{I}$ Department of Neurology, Cologne, Germany, ${ }^{2}$ Department of Neurology, Düsseldorf, Germany, ${ }^{3}$ Department of Stereotactic Neurosurgery, Cologne, Germany, ${ }^{4}$ Institute of Neuroscience and Biophysics-Medicine, Jülich, Germany

\section{DISORDERS OF THE NERVOUS SYSTEM Schizophrenia}

Prefrontal cortical activation in people at ultra-high risk of psychosis: An fMRI study of voluntary eye movements., Elizabeth Bowman ${ }^{1,2}$, Larry Abel ${ }^{1}$, Cali Bartholomeusz ${ }^{2,3}$, Barnaby Nelson $^{3}$, Alison Yung ${ }^{3}$, Murat Yucel $^{2}$, Christos Pantelis ${ }^{2}$, Beatriz Luna ${ }^{4}$, Katerina Velanova ${ }^{4}$, Patrick McGorry, Stephen Wood ${ }^{2}$, IDepartment of Optometry and Vision Sciences, The University of Melbourne, Melbourne, Australia, ${ }^{2}$ Melbourne Neuropsychiatry Centre, Department of Psychiatry, The University of Melbourne, Melbourne, Australia, ${ }^{3}$ ORYGEN Youth Health, Melbourne, Australia, ${ }^{4}$ Department of Psychiatry, The University of Pittsburgh, Pittsburgh, USA

On the difference between auditory verbal hallucinations and inner speech; a group-wise analysis of fMRI scans in 24 psychotic patients, Iris Sommer ${ }^{l}$, Kelly Diederen ${ }^{l}$, Jan Dirk Blom ${ }^{2}$, Leila Kushan ${ }^{1}$, Karin Slotema ${ }^{2}$, Marco Boks ${ }^{1}$, Kirstin Daalman ${ }^{l}$, Wijbrand Hoek ${ }^{2}$, Bas Neggers ${ }^{1}$, Rene Kahn ${ }^{l},{ }^{l}$ University Medical Centre, Utrecht, Netherlands, ${ }^{2}$ Parnassia Psycho-Medical centre, The Hague, Netherlands

Mapping grey matter reductions in schizophrenia: an ALE meta-analysis of voxel-based morphometry studies, Alex Fornito ${ }^{l}$, Yücel Murat ${ }^{1,2}$, Jessica Patti ${ }^{3}$, Stephen Wood ${ }^{l}$, Christos Pantelis ${ }^{l},{ }^{I}$ Melbourne Neuropsychiatry Centre, The University of Melbourne, Melbourne, Australia, ${ }^{2}$ ORYGEN Research Centre, The University of Melbourne, Melbourne, Australia, ${ }^{3}$ Department of Psychology, The University of Melbourne, Melbourne, Australia

Functional and anatomical connectivity abnormalities of left inferior frontal gyrus in schizophrenia, Bum Seok Jeong ${ }^{1,2,3}$, R.W. McCarley ${ }^{2}$, M.E. Shenton ${ }^{3}$, C.G. Wible, M. Kubicki ${ }^{3}$, R.H. Hashimoto ${ }^{2},{ }^{1}$ Dept. of Psychiatry, Eulji University, Daejeon, South Korea, ${ }^{2}$ Clinical Neuroscience Division, Laboratory of Neuroscience, Boston VA Healthcare System, Dept. of Psychiatry,

(193 M-AM

.

Harvard Medical School, Boston, USA, ${ }^{3}$ Psychiatry Neuroimaging Laboratory, Department of Psychiatry, Brigham and Women's Hospital and Harvard Medical School, Boston, USA 
EEG alpha activity reflects hypofrontality in schizophrenia, Maria G. Knyazeva ${ }^{1,2}$, Mahdi Jalili $^{3}$, Reto Meuli ${ }^{2}$, Martin Hasler ${ }^{3}$, Oscar De Feo ${ }^{4}$, Kim Q. Do ${ }^{5},{ }^{1}$ Dept of Neurology, Centre Hospitalier Universitaire Vaudois and University of Lausanne, Lausanne, Switzerland, ${ }^{2}$ Dept of Radiology, Centre Hospitalier Universitaire Vaudois and University of Lausanne, Lausanne, Switzerland, ${ }^{3}$ École Polytechnique Fédérale de Lausanne (EPFL), School of Computer and Communication Sciences, IC-LANOS, Lausanne, Switzerland, ${ }^{4} 4$ Department of Microelectronic Engineering, University College Cork, Cork City, Ireland, ${ }^{5}$ Center for Psychiatric Neuroscience, Dept of Psychiatry, Centre Hospitalier Universitaire, Lausanne, Switzerland

Morphological abnormalities of the cerebral cortical thickness in schizophrenia, Tao $\mathrm{Liu}^{l}$ Feng Shi', Yuan Zhou ${ }^{2}$, Wanlin Zhu', Lei Lin ${ }^{2}$, Jesse Jin ${ }^{l}$, Tianzi Jiang ${ }^{2}$, Suhuai Luo ${ }^{1}$, Mira Park ${ }^{1}$, Paul Rasser, Ulrich Schall, ${ }^{3}$ 'School of Design, Communication \& I.T, The University of Newcastle, Callaghan NSW, Australia, ${ }^{2}$ National Laboratory of Pattern Recognition, Institute of Automation, Chinese Academy of Sciences, Beijing, China, ${ }^{3}$ Schizophrenia Research Institute, Sydney, Australia, Priority Centre for Brain \& Mental Health Research, University of Newcastle, Newcastle, Australia, Hunter Medical Research Institute, Newcastle, Australia, ${ }^{4}$ Neuropsychiatric Institute, Prince of Wales Hospital, Randwick NSW, Australia

\section{LINKING CEREBRAL GREY MATTER AND MISMATCH NEGATIVITY (MMN) IN} SCHIZOPHRENIA, Paul E. Rasser ${ }^{1,2}$, Juanita Todd ${ }^{1,2}$, Paul M. Thompson ${ }^{3}$, Patricia T. Michie $^{1,2}$, Philip B. Ward ${ }^{4}$, Patrick Johnston ${ }^{5}$, Katrin Helmbold ${ }^{2,6}$, Vanessa Case ${ }^{2}$, Paul A. Tooney ${ }^{l, 2}$, Ulrich Schall ${ }^{1,2},{ }^{1}$ Schizophrenia Research Institute, Sydney, Australia, ${ }^{2}$ Priority Centre for Brain \& Mental Health Research, University of Newcastle, Newcastle, Australia, ${ }^{3}$ Laboratory of Neuro Imaging, University of California Los Angeles, Los Angeles, USA, ${ }^{4}$ Schizophrenia Research Unit, Liverpool Hospital, University of New South Wales, Sydney, Australia, ${ }^{5}$ Brain Sciences Institute, Swinburne University of Technology, Melbourne, Australia, ${ }^{6}$ Department of Psychology, University of Konstanz, Konstanz, Germany

3D Pattern of Brain Abnormalities in Chronic Schizophrenia Visualized Using Tensor-Based Morphometry: a Multi-Site Structural Imaging Study, Theo G.M. van Erp ${ }^{I}$, Ming-Chang Chiang $^{2}$, Daqiang Sun ${ }^{1}$, Molly E. Hardt ${ }^{l}$, Jeremy H. Bockholt ${ }^{3}$, Jessica A Turner ${ }^{4}$, Vince D. Calhoun, $^{3,5,6}$, Hans J. Johnson ${ }^{7}$, Doug N, Greve ${ }^{8}$, Greg G. Brown', Judith M. Ford ${ }^{10}$, Steven G. Potkin $^{4}$, Tyrone D. Cannon ${ }^{11}$, Paul M. Thompson ${ }^{2}$, Arthur W. Toga ${ }^{2}, F$. BIRN ${ }^{l}$, I Department of Psychology, University of California Los Angeles, Los Angeles, USA, ${ }^{2}$ Lab of Neuroimaging and Department of Neurology, University of California Los Angeles, Los Angeles, USA, ${ }^{3}$ The Mind Research Network, Albuquerque, USA, ${ }^{4}$ Department of Psychiatry and Human Behavior, University of California Irvine, Irvine, USA, ${ }^{5}$ Department of Electrical and Computer Engineering, University of New Mexico, Albuquerque, USA, ${ }^{6}$ Department of Psychiatry, Yale University, New Haven, USA, ${ }^{7}$ Iowa Mental Health Clinical Research Center, The University of Iowa Hospitals and Clinics, Iowa City, USA, ${ }^{8}$ Department of Radiology, Massachusetts General Hospital, Boston, USA, ${ }^{9}$ Psychology Services, Veterans Administration San Diego Healthcare System, and Psychiatry Department, University of San Diego, San Diego, USA, ${ }^{10}$ Department of Psychiatry, Yale University School of Medicine, West Haven, USA, ${ }^{11}$ Departments of Psychology and Psychiatry and Biobehavioral Sciences, University of California Los Angles, Los Angeles, USA

\section{EMOTION \& MOTIVATION}

Reward

Anterior Cingulate and Vulnerability to Depression; Blunted Response to Incongrous Feedback in a Novel Reward-Related Task., Darragh Downey ${ }^{1}$, Shane McKie ${ }^{2}$, JFW Deakin ${ }^{2}$, Ian Anderson ${ }^{2}$, Rebecca Elliott ${ }^{2},{ }^{1}$ Imaging Science and Biomedical Engineering, University of Manchester, Manchester, United Kingdom, ${ }^{2}$ Neuroscience and Psychiatry Unit, University of Manchester, Manchester, United Kingdom

The roles of expectation and dopamine release in the mechanism of the placebo effect in Parkinson's disease: A high-resolution PET study with [11C] raclopride, Sarah Lidstone ${ }^{l}$, Katherine Dinelle ${ }^{2}$, Stephan Blinder ${ }^{1}$, Tom Ruth ${ }^{3}$, Vesna Sossi ${ }^{2}$, Jon Stoessl ${ }^{\text {, }}$, Pacific Parkinson's Research Centre, Vancouver, Canada, ${ }^{2}$ Department of Physics \& Astronomy, Vancouver, Canada, ${ }^{3}$ TRIUMF, Vancouver, Canada

Neural Activity in a Delay Discounting Task Correlates with Interindividual Differences in Impulsivity and Self-Control, Lioba Schmitz, Corinna Nuesser, Susanne Erk, Dina Schardt, Henrik Walter, Dept. of Psychiatry, Div. of Medical Psychology, University of Bonn, Bonn, 
Detachment effectuates suspension of reward magnitude and prediction error coding in ventral striatum, Markus Staudinger ${ }^{1}$, Susanne Erk ${ }^{2}$, Birgit Abler ${ }^{3}$, Henrik Walter ${ }^{4},{ }^{1}$ University of Bonn, Bonn, Germany, ${ }^{2}$ University of Bonn, Bonn, Germany, ${ }^{3}$ University of Ulm, Ulm, Germany, $245 \mathrm{M}-\mathrm{AM}$

${ }^{4}$ University of Bonn, Bonn, Germany

Neural encoding of object valence using parametric modulation and multivariate pattern classification, Anita Tusche ${ }^{l}$, John-Dylan Haynes ${ }^{1,2}$, ${ }^{I}$ Max Planck Institute for Cognitive and Brain Sciences, Leipzig, Germany, ${ }^{2}$ Bernstein Center for Computational Neuroscience Berlin, Charité - Universitätsmedizin, Berlin, Germany

\section{EMOTION \& MOTIVATION}

Sexual Behavior

The resting frontal alpha asymmetry across the menstrual cycle: a magnetoencephalographic study, Ren-Jen Hwang ${ }^{l}$, Li-Fen Chen ${ }^{2,3,4}$, Tzu-Chen Yeh ${ }^{2,3,4}$, Pei-Chi Tu ${ }^{l}$, Chung-Haow Tu', Jen-Chuen Hsieh ${ }^{1,2,3,4},{ }^{1}$ Insitute of Neuroscience, National Yang-Ming University, Taipei, Taiwan, ${ }^{2}$ Institute of Brain of Brain Science, National Yang-Ming University, Taipei, Taiwan, ${ }^{3}$ Dept. Medical Research \& Education, Taipei Veterans General Hospital, Taipei, Taiwan, ${ }^{4}$ Brain Research Center, National Yang-Ming University, Taipei, Taiwan

\section{EMOTION \& MOTIVATION Social Behavior}

Learning to like: social observation influences prefrontal activation for viewing others, Jeffrey C. Cooper, Tamar Kreps, Brian Knutson, Department of Psychology, Stanford University, Stanford, USA

Investigation of Brain Activity under Social Pressure using the Asch Paradigm: An fNIRS study, Takashi X. FUJISAWA, Toyoharu HOSOKAWA, Noriko NAGATA, Haruhiro KATAYOSE, Kwansei Gakuin University, Hyogo, Japan

Investigating Neural Correlates of Frustration with fMRI, Johan Lambregs ${ }^{1}$, Johan Ormel ${ }^{2}$, André Aleman ${ }^{I},{ }^{1}$ University Medical Center Groningen, BCN-NIC, Groningen, Netherlands,

${ }^{2}$ University Medical Center Groningen, Dept. Psychiatry, Groningen, Netherlands

Functional Imaging of "Development of Parenting Brain" in Adolescents, Akio NAKAI', Ayako SASAKI ${ }^{2}$, Hirotaka KOSAKA ${ }^{3}$, Ken-ichi MATSUKI ${ }^{4}$, Michiko TANABE ${ }^{2},{ }^{1}$ Department of Pediatrics, Faculty of Medical Sciences, University of Fukui, Fukui, Japan, ${ }^{2}$ Department of Maternity, Child Health Nursing, and Midwifery, Faculty of Medical Sciences, University of Fukui, Fukui, Japan, ${ }^{3}$ Department of Neuropsychiatry, Faculty of Medical Sciences, University of Fukui, Fukui, Japan,

${ }^{4}$ Department of Developmental Sciences, Faculty of Education and Regional Studies, Fukui, Japan

\section{GENETICS}

Catechol-o-methyltransferase val ${ }^{158}$ met genotype influences neural incentive processing, Katharina Schmack', Florian Schlagenhauf ${ }^{I}$, Philipp Sterzer ${ }^{I}$, Jana Wrase ${ }^{I}$, Anne Beck ${ }^{I}$, Theresa Dembler ${ }^{l}$, Peter Kalus ${ }^{I}$, Imke Puls ${ }^{l}$, Thomas Sander ${ }^{2}$, Jürgen Gallinat ${ }^{l}$, Andreas Heinz ${ }^{l},{ }^{\prime}$ Dept.of Psychiatry, Charité University Medical Center, Berlin, Germany, ${ }^{2}$ Max-Delbrück Center for Molecular Medicine, Berlin, Germany

The impact of gene-environment interactions on neural pathways in risk for syndromal depression and anxiety, Justine M. Gatt ${ }^{1,2}$, Charles B. Nemeroff ${ }^{3}$, Carol Dobson-Stone ${ }^{4}$, Stacey A. Kuan ${ }^{1,2}$, Robert H. Paul ${ }^{5}$, Richard A. Bryant ${ }^{1,6}$, Peter R. Schofield ${ }^{4}$, Evian Gordon ${ }^{1,2,7}$, Leanne M. Williams ${ }^{1,2},{ }^{1}$ The Brain Dynamics Centre, Westmead Millennium Institute, Westmead Hospital and Western Clinical School, University of Sydney, Sydney, Australia, ${ }^{2}$ Psychological Medicine, Western Clinical School, University of Sydney, Sydney, Australia, ${ }^{3}$ Department of Psychiatry and Behavioral Sciences, Emory University School of Medicine, Atlanta, USA, ${ }^{4}$ Prince of Wales Medical Research Institute, University of New South Wales, and Garvan Institute of Medical Research, Sydney, Australia, ${ }^{5}$ Department of Psychiatry, Behavioral Neuroscience, University of Missouri, St. Louis, USA, ${ }^{6}$ School of Psychology, University of New South Wales, Sydney, Australia, ${ }^{7}$ The Brain Resource International Database and the Brain Resource Company, and Faculty of Medicine, University of Sydney, Sydney, Australia 
Genetics of cerebral sulcation: Does genetics offer a new way of sulcal classification?, Peter Kochunov ${ }^{I}$, David Glahn ${ }^{1}$, Peter Fox ${ }^{1}$, Oliver Coulon ${ }^{2}$, Karl Zilles ${ }^{3}$, Wendy Shelledy ${ }^{4}$, Jack Lancaster ${ }^{1}$, John Blangero ${ }^{4}$, Jeff Rogers ${ }^{4},{ }^{1}$ Research Imaging Center, University of Texas Health Science Center at San Antonio, san antonio, USA, ${ }^{2}$ Laboratoire des Sciences de l'Information et des Systèmes, Marseille, France, ${ }^{3}$ Institut für Medizin (IME), Jülich, Germany, ${ }^{4}$ Southwest Research Foundation, San Antonio, USA

Brain-Derived Neurotrophic Factor and Volumes of Hippocampus and Amygdala in Adolescents, Tomas Paus ${ }^{1,2}$, Marie Chupin ${ }^{6}$, Line Garnero ${ }^{6}$, Gabriel Leonard ${ }^{2}$, Michel Perron ${ }^{3,4}$, Bruce Pike $^{2}$, Alain Pitiot ${ }^{1}$, Louis Richer ${ }^{5}$, Roberto Toro ${ }^{l}$, Suzanne Veillette ${ }^{3,4}$, Zdenka Pausova, ${ }^{1,3}$, ${ }^{1}$ University of Nottingham, Nottingham, United Kingdom, ${ }^{2}$ McGill University, Montreal, Canada, ${ }^{3}$ University of Montreal, Montreal, Canada, ${ }^{4}$ CEGEP Jonquiere, Jonquiere, Canada, ${ }^{5}$ University of Quebec, Chicoutimi, Canada, ${ }^{6} \mathrm{CNRS}$, Paris, France

Neuroimaging endophenotypes for emotion perception? Variation with COMT Val ${ }^{108 / 158}$ Met genotypes, level of awareness and sex differences, Leanne (Lea) Williams ${ }^{1,2}$, Stacey Kuan ${ }^{1,2}$, Justine Gatt ${ }^{1,2}$, Dobson-Stone Carol ${ }^{3}$, Schofield Peter ${ }^{3}$, Gordon Evian ${ }^{1,2,4}$, ' Brain Dynamics Centre, Westmead Millennium Institute, Sydney, Australia, ${ }^{2}$ University of Sydney, Sydney, Australia, ${ }^{3}$ Prince of Wales Medical Research Institute, Sydney, Australia, ${ }^{4}$ Brain Resource, Sydney, Australia

\section{IMAGING TECHNIQUES \& CONTRAST MECHANISM EEG}

Modulation of Resting EEG Nonlinear Topography by NMDA Receptor Antagonist Nitrous Oxide, Brett Foster, Mathew Dafilis, Peter Cadusch, David Liley, Brain Dynamics Research Unit, Brain Sciences Institute, Swinburne University of Technology, Melbourne, Australia

Tracking inter-hemispheric transfer with high-density event-related brain potentials, Ryan $D^{\prime}$ Arcy $^{1,2,3}$, Erin Mazerolle ${ }^{1,2}$, Nicole Pelot ${ }^{1},{ }^{1}$ Institute for Biodiagnostics (Atlantic), National Research Council, Halifax, Canada, ${ }^{2}$ Department of Psychology/Neuroscience, Dalhousie

University, Halifax, Canada, ${ }^{3}$ Department of Radiology, Dalhousie University, Halifax, Canada

\section{IMAGING TECHNIQUES \& CONTRAST MECHANISM} Functional MRI

Neuroimaging Analysis and Visualization Tools For Remote Collaboration, Michael Andric, Uri Hasson, Steven Small, The University of Chicago, Chicago, USA

Oxygen Calibrated Functional MRI, Daniel Bulte, Peter Jezzard, University of Oxford, Oxford, United Kingdom

Functional Changes in Cerebral Blood Flow and Venous Blood Volume: what is the SteadyState Relationship?, J. Jean Chen, G. Bruce Pike, McConnell Brain Imaging Centre, Montreal Neurological Institute, Montreal, Canada

Test-Retest Reliability of Functional Activation in Schizophrenia and Unaffected Individuals During Working Memory Tasks: Differences, Implications, and the Effects of Denoising, Kristen Haut ${ }^{1}$, Maria Prom ${ }^{2}$, Angus MacDonald III $^{I}$, 'Iniversity of Minnesota, Minneapolis, USA, ${ }^{2}$ Carleton College, Northfield, USA

Effects of current timing and local shimming in neuronal current imaging: experiment and simulation, Ivana Drobnjakl, Gaby Pell ${ }^{2}$, Mark Jenkinson ${ }^{I},{ }^{I}$ FMRIB Centre, University of Oxford, Oxford, United Kingdom, ${ }^{2}$ Brain Research Institute, Melbourne, Australia

Faster response of diffusion-weighted fMRI signal compared to BOLD and NIRS signals in the human brain, Satoru Kohno ${ }^{l, 2}$, Nobukatsu Sawamoto , Shin-ichi Urayamal, Toshihiko Aso ${ }^{1,4}$, Akitoshi Seiyama ${ }^{3}$, Denis Le Bihan ${ }^{4}$, Hidenao Fukuyamal ${ }^{1}{ }^{1}$ Human Brain Research Center, Kyoto University Graduate School of Medicine, Kyoto, Japan, ${ }^{2} R \& D$ Department, Medical Systems Division, Shimadzu Corporation, Kyoto, Japan, ${ }^{3}$ Human Health Science, Kyoto University Graduate School of Medicine, Kyoto, Japan, ${ }^{4}$ CEA, NeuroSpin, Saclay, France
281 M-AM

285 M-AM*

289 M-AM

293 M-AM

297 M-AM

301 M-AM

305 M-AM

309 M-AM

313 M-AM

317 M-AM

321 M-AM 
Searching the reference image for selecting default network components in fMRI, $S$ - $J \mathrm{Lin}^{l}$, T-C Yeh ${ }^{1,2}, C-M$ Cheng $^{2}, J-C H$ sieh $^{1,2}, L-T H^{2}{ }^{2},{ }^{l}$ Institute of Brain science, National Yang-Ming University, Taipei, Taiwan, ${ }^{2}$ Department of Medical Research and Education, Taipei Veterans General Hospital, Taipei, Taiwan

Resting State Sensorimotor Functional Connectivity in Multiple Sclerosis Correlates with Transcallosal Motor Pathway Transverse Diffusivity, Mark Lowe ${ }^{l}$, Erik Beall ${ }^{l}$, Ken Sakaie ${ }^{l}$, Katherine Koenig ${ }^{I}$, Lael Stone ${ }^{I}$, RuthAnn Marrie ${ }^{2}$, Micheal Phillips ${ }^{I},{ }^{I}$ Cleveland Clinic,

Cleveland, USA, ${ }^{2}$ University of Manitoba, Winnipeg, Canada

Exploring the Neuro-Cognitive Significance of the Negative BOLD Response: Attenuation of the BOLD Response Appears to Inhibit Hippocampal-Dependent Memory, Sinéad Mullally, Shane O'Mara, Trinity College Institute of Neuroscience, Dublin, Ireland

Impact of COMT val ${ }^{158}$ met polymorphism on processing speed in healthy volunteers, Devon C. Nixon ${ }^{l}$, Bart Rypma ${ }^{2}$, Rachel G. Higier ${ }^{l}$, Steven Sust ${ }^{l}$, Morgan J. Prust ${ }^{l}$, Hao Yang Tan ${ }^{l}$, Brad Zoltickl $^{l}$, Jennifer K. Brooke ${ }^{l}$, Venkata S. Mattay ${ }^{I}$, Daniel R. Weinberger ${ }^{I}$, Joseph H. Callicott ${ }^{l}$, ${ }^{l} \mathrm{CBDB} / \mathrm{GCAP} / \mathrm{NIMH} / \mathrm{NIH}$, Bethesda, USA, ${ }^{2}$ University of Texas at Dallas, University of Texas Southwestern, Dallas, USA

Measuring Brain Connectivity using Diffusion Tensor Imaging and Resting State Temporal Correlations, Pawel Skudlarski ${ }^{1,2}$, Kanchana Jagannathan ${ }^{l}$, Vince Calhoune ${ }^{3}$, Beata Skudlarska ${ }^{4}$ Godfrey Pearlson ${ }^{1,2},{ }^{1}$ Olin Neuropsychiatry Research Center,, Hartford, USA, ${ }^{2}$ Department of Psychiatry Yale University School of Medicine, New Haven, USA, ${ }^{3}$ The Mind Institute, Albuquerue, NM, University of New Mexico, Albuquerque, USA, ${ }^{4}$ Center on Geriatrics, bridgeport Hospital, bridgeport, USA

Is T2* always the optimum Echo Time in BOLD fMRI? Challenging a classic concept with a new functional Contrast to Noise Ratio model, Pierre-Francois Van de Moortele ${ }^{l}$, Eddie Auerbach ${ }^{1}$,Kamil Ugurbil ${ }^{1}$, Stephane Lehericy ${ }^{1,2},{ }^{l}$ CMRR-University of Minnesota, Minneapolis, USA, ${ }^{2}$ Université Pierre et Marie Curie,Hôpital Pitié-Salpêtrière, Paris, France

Direct measurement of neuronal magnetic field changes evoked by median nerve stimulation using MRI: TE dependence, Yiqun Xue, ${ }^{1,2}$, Thomas Grabowski ${ }^{3}$, Jinhu Xiong ${ }^{2},{ }^{1}$ Biomedical Engineering, University of Iowa, Iowa city, USA, ${ }^{2}$ Radiology, University of Iowa, Iowa city, USA, ${ }^{3}$ Neurology, University of Iowa, Iowa city, USA

High-Resolution fMRI at 7T using Generalized Series Parallel Imaging Technique, Sungdae Yun ${ }^{l}$, Jun-Young Chung ${ }^{2}$, Sung Suk Oh ${ }^{1}$, Hyo Woon Yoon ${ }^{2}$, Zang-Hee Cho ${ }^{2,3}$, HyunWook Park ${ }^{l}$, ${ }^{I}$ Department of Electrical Engineering, Korea Advanced Institute of Science and Technology, Daejeon, Korea, ${ }^{2}$ Neuroscience Research Institute, Gachon University of Medicine and Science, Incheon, Korea, ${ }^{3}$ Department of Radiological Sciences, University of California, Irvine, USA

\section{IMAGING TECHNIQUES \& CONTRAST MECHANISM MEG}

Simultaneous MEG source imaging and depth recordings in Humans, Florence GOMBERT ${ }^{l}$, Claude ADAM ${ }^{1,2}$, Guido NOLTE ${ }^{3}$, Line GARNERO ${ }^{l}$, Sylvain BAILLET ${ }^{l},{ }^{l}$ Coginitive Neuroscience \& Brain Imaging Laboratory LENA, CNRS, MEG-EEG center, UPMC University-Paris 6, Paris, France, ${ }^{2}$ Epilepsy Unit, La Salpêtrière Hospital, Paris, France, ${ }^{3}$ Fraunhofer FIRST, Berlin, France

\section{LANGUAGE}

Language Acquisition

Error-related Responses Supporting Grammatical Plasticity, Douglas Davidson ${ }^{1,2}$, Peter Indefrey ${ }^{I, 2},{ }^{I}$ F. C. Donders Centre for Cognitive Neuroimaging, Nijmegen, Netherlands, ${ }^{2}$ Max Planck Institute for Psycholinguistics, Nijmegen, Netherlands

Neural correlates of foreign language sound imitation, Hiroshi Hashizume ${ }^{1}$, Hyeonjeong Jeong ${ }^{1,2}$, Naho Ikuta ${ }^{l}$, Motoaki Sugiura ${ }^{1,3}$, Ryuta Kawashima ${ }^{l},{ }^{1}$ Department of Functional Brain Imaging, IDAC, Tohoku University, Sendai, Japan, ${ }^{2}$ Japan Society for the Promotion of Science,

349 M-AM 
fMRI shows that language lateralisation is affected in BECTS, Leasha Lillywhite ${ }^{1,2}$, Simon Harvey ${ }^{3}$, Michael Saling ${ }^{4}$, David Abbott ${ }^{1,2}$, John Archer $^{1,2}$, Danya Vears ${ }^{2}$, Ingrid Scheffer ${ }^{2}$, Graeme Jackson ${ }^{1,2},{ }^{1}$ Brain Research Institute, Melbourne, Australia, ${ }^{2}$ Department of Medicine, The University of Melbourne, Melbourne, Australia, ${ }^{3}$ Royal Children's Hospital, Melbourne, Australia, ${ }^{4}$ Department of Psychology, The University of Melbourne, Melbourne, Australia

Functional Neuroimaging of Novel Word Learning, Amy Clements-Stephens ${ }^{1,2}$, April Materek $^{1,4}$, Pooja Gaur ${ }^{1}$, Laurie Cutting ${ }^{1,2,3},{ }^{1}$ Kennedy Krieger Institute, Baltimore, USA, ${ }^{2}$ Johns Hopkins University, Baltimore, USA, ${ }^{3}$ Johns Hopkins Medical Institute, Baltimore, USA, ${ }^{4}$ Loyola University, Baltimore, USA

\section{LANGUAGE Production}

Bold response changes with ageing evidenced during a semantic fluency task., Christophe Destrieux ${ }^{1,2,3,4}$, Florence Domengie ${ }^{1,3}$, Giovanni de Marco ${ }^{5}$, Jean-Philippe Cottier ${ }^{1,2,3,4}$, Caroline Hommet ${ }^{1,2,3,4},{ }^{1}$ CHRU, Tours, France, ${ }^{2}$ INSERM, U619, Tours, France, ${ }^{3}$ Université François Rabelais, Tours, France, ${ }^{4}$ IFR135, Tours, France, ${ }^{5}$ Université de Picardie Jules Verne, Amiens, France

Semantic context and visual feature effects on verbal self-monitoring measured with Arterial Spin Labelling, Julia Hocking, Katie McMahon, Matthew Meredith, Greig de Zubicaray, fMRI Laboratory, University of Queensland, Brisbane, Australia

Second Language Communication: Effects of Interview Types and Oral Proficiency Levels on Brain Activation, Hyeonjeong Jeong ${ }^{1,2}$, Hiroshi Hashizume ${ }^{2}$, Yuko Sassa ${ }^{2}$, Satoru Yokoyama ${ }^{2}$ Motoaki Sugiura ${ }^{2,3}$, Kensaku Ishimaki ${ }^{4}$, Ryuta Kawashima ${ }^{2},{ }^{1}$ Japan Society for the Promotion of Science, Tokyo, Japan, ${ }^{2}$ Department of Functional Brain Imaging, IDAC, Tohoku University, Sendai, Japan, ${ }^{3}$ National Institute for Physiological Science, Okazaki, Japan, ${ }^{4}$ The Society for testing English Proficiency, Tokyo, Japan

Intraoperative cortical stimulation mapping and presurgical fMRI - complement or contradiction?, Janpeter Nickel', Michael C. Sabel ${ }^{2}$, Walter Stummer ${ }^{2}$, Hans-Jakob Steiger ${ }^{2}$, Rüdiger J. Seitz ${ }^{l},{ }^{I}$ Department of Neurology, University Hospital Düsseldorf, Düsseldorf, Germany, ${ }^{2}$ Department of Neursurgery, University Hospital Düsseldorf, Düsseldorf, Germany

Examining cortical representational overlap for singing with lyrics and propositional language, Sarah Wilson ${ }^{1,2}$, David Abbott ${ }^{2,3}$, Anthony Waites ${ }^{2,3}$, Regula Briellmann ${ }^{2}$, Dean Lusher ${ }^{1}$, Gaby Pell 2,3, Jenni Ogden ${ }^{4}$, Michael Saling ${ }^{1,2}$, Graeme Jackson ${ }^{2,3},{ }^{1}$ School of Behavioural Science, The University of Melbourne, Victoria, Australia, ${ }^{2}$ Brain Research Institute, Austin Health, Melbourne, Victoria, Australia, ${ }^{3}$ Department of Medicine, The University of Melbourne, Victoria, Australia, ${ }^{4}$ Department of Psychology, The University of Auckland, Auckland, New Zealand

\section{MEMORY \& LEARNING \\ Plasticity (normal \& following pathology)}

Do Baseline Neurocognitive Deficits In Hypothyroid Patients Indicate A Difference In BOLD Activity?, Gillian Cooke ${ }^{1}$, Sinead Mullally ${ }^{I}$, Neuman Correia ${ }^{2}$, Maria Fitzgibbon ${ }^{3}$, James Gibney', Shane O'Mara ${ }^{I},{ }^{1}$ Trinity College Institute of Neuroscience \& School of Psychology, Trinity College Dublin, Dublin, Ireland, ${ }^{2}$ Adelaide \& Meath Hospital, incorporating National Children's Hospital, Dublin, Ireland, ${ }^{3}$ University College Hospital Galway, Newcastle Road, Galway, Ireland

Gender differences in navigation and neural plasticity: Does training matter?, Petra Neumann, Georg Grön, University Ulm, Ulm, Germany

The structural and functional basis of variability in normal motor skill learning, Valentina Tomassini, Saad Jbabdi, Tamas Kincses, Rose Bosnell, Paul M Matthews, Heidi Johansen-Berg, FMRIB Centre, University of Oxford, Oxford, United Kingdom

\section{( 01 M-AM}




\section{MEMORY \& LEARNING \\ Working Memory}

Tracking the cerebro-cerebellar verbal working memory circuitry using functional MRI and Diffusion Spectrum Imaging, Jing-Syun $\mathrm{Yu}^{l}$, Wen-Yang Chiang ${ }^{2}$, Yumie Ono ${ }^{3}$, Wen-Yih Isaac Tseng ${ }^{2}$, SH Annabel Chen ${ }^{1}$, 'Department of Psychology, National Taiwan University, Taipei, Taiwan, ${ }^{2}$ Department of Radiology, National Taiwan University College of Medicine, Taipei, Taiwan, ${ }^{3}$ Physiology and Neuroscience, Kanagawa Dental College, Kanagawa, Japan

Regional variability in the BOLD HRF assessed using concurrent TMS-fMRI, Eva Feredoes ${ }^{I}$, Tom Johnstone ${ }^{2}$, Giulio Tononi ${ }^{3}$, Bradley R Postle ${ }^{1,3}$, ${ }^{I}$ Dept. of Psychology, University of Wisconsin-Madison, Madison, USA, ${ }^{2}$ School of Psychology and CLS, University of Reading, Reading, United Kingdom, ${ }^{3}$ Dept. of Psychiatry, University of Wisconsin-Madison, Madison, USA

Nonlinear and factorial brain responses during associative working memory with increasing implicit task load, Nicole Kochan ${ }^{I}$, Perminder Sachdev ${ }^{I}$, Melissa Slavin ${ }^{I}$, Michael Valenzuela ${ }^{l}$, Michael Breakspear ${ }^{2},{ }^{1}$ School of Psychiatry, University of New South Wales, Neuropsychiatric Institute, Prince of Wales Hospital, Sydney, Australia, ${ }^{2}$ School of Psychiatry, University of New South Wales, Black Dog Institute, Prince of Wales Hospital, Sydney, Australia

Fronto-parietal Dysfunction during Spatial Working Memory Task in Subjects at Ultra-High-Risk for Schizophrenia, Ji-Young Park', Do-Hyung Kang ${ }^{2}$, Jung-Suk Choi ${ }^{2}$, Myeong-Hoon Jung ${ }^{2}$, Wi-Hoon Jung ${ }^{3}$, Na-Young Shin ${ }^{1}$, Chi-Hoon Choi ${ }^{4}$, Jong-Min Lee ${ }^{5}$, Jun Soo Kwon ${ }^{l-3}$, Interdisciplinary Program in Cognitive Science, Seoul National University, Seoul,

Korea, ${ }^{2}$ Department of Psychiatry, Seoul National University Hospital, Seoul, Korea,

${ }^{3}$ Interdisciplinary Program in Brain Science, Seoul National University, Seoul, Korea,

${ }^{4}$ Department of Radiology, National Medical Center, Seoul, Korea, ${ }^{5}$ Department of Biomedical Engineering, Hanyang University, Seoul, Korea

A cortico-hippocampal network emerging with subsequent memory dependent theta oscillation, Naoyuki Sato ${ }^{1}$, Takashi Ozaki ${ }^{1}$, Yoshiaki Someya ${ }^{2}$, Kimitaka Anami ${ }^{2}$, Seiji Ogawa ${ }^{2}$, Hiroaki Mizuhara ${ }^{1,3}$, Yoko Yamaguchi ${ }^{1},{ }^{1}$ RIKEN Brain Science Institute, Saitama, Japan, ${ }^{2}$ Hamano Life Sci Res Foundation, Tokyo, Japan, ${ }^{3}$ Kyoto Univ, Kyoto, Japan

\section{MODELING \& ANALYSIS \\ Bayesian Modeling}

A predictive coding account of the mismatch negativity, Marta I Garrido, James M Kilner, Stefan J Kiebel, Karl J Friston, Wellcome Trust Centre for Neuroimaging, UCL, London, United Kingdom

Stratification and Complexity of Brain Connectivity, Gloria Haro ${ }^{1}$, Christophe Lenglet ${ }^{2}$, Guillermo Sapiro ${ }^{3}$, Paul Thompson ${ }^{4},{ }^{1}$ Universitat Politecnica de Catalunya, Barcelona, Spain, ${ }^{2}$ Siemens Corporate Research, Princeton, USA, ${ }^{3}$ University of Minnesota, Minneapolis, USA,

${ }^{4}$ UCLA Medical School, Los Angeles, USA

Regularisation of High Angular Resolution Diffusion Imaging Data, Leigh Johnston ${ }^{1,2}$, Scott Kolbe $^{2,3}$, Iven Mareels ${ }^{1}$, Gary Egan ${ }^{2,3},{ }^{1}$ Department of Electrical and Electronic Engineering, University of Melbourne \& NICTA Victorian Research Laboratory, Melbourne, Australia, ${ }^{2}$ Howard Florey Institute, Florey Neuroscience Institutes, Melbourne, Australia, ${ }^{3}$ Centre for Neuroscience, University of Melbourne, Melbourne, Australia

Evaluation of Probabilistic Tractography on Bayesian Tensor Estimation, Dae-Jin Kim, Hae-Jeong Park, Department of Diagnostic Radiology, Yonsei University, College of Medicine, 134 Shinchon-dong, Seodaemun-gu, Seoul, South Korea

Multimodal Fusion: A generative model for EEG and fMRI, Maria Joao Rosa ${ }^{I}$, James Kilner ${ }^{I}$, Felix Blankenburg ${ }^{2}$, Oliver Josephs ${ }^{I}$, Will Penny ${ }^{l},{ }^{l}$ Wellcome Trust Centre for Neuroimaging, University College London, London, United Kingdom, ${ }^{2}$ Department of Neurology, Charité, 
11:30 - 12:30 Corryong Hall (Level 2)

\author{
MODELING \& ANALYSIS \\ Classification \& Predictive Modeling
}

Classification of Resting State fMRI Scans using Temporal Network Associations in Schizophrenic and Normal Patients, Ariana Anderson ${ }^{1}$, Mark Cohen ${ }^{2}$, Ivo Dinov ${ }^{1,3}$, Javier Quintana ${ }^{4}$, Jon Sherin ${ }^{4}$, Alan Yuille ${ }^{I},{ }^{1}$ Department of Statistics, University of California, Los Angeles, Los Angeles, USA, ${ }^{2}$ Psychiatry \& Behavioral Sciences, University of California, Los $453 \mathrm{M}-\mathrm{AM}$ Angeles, Los Angeles, USA, ${ }^{3}$ Center for Computation Biology, Los Angeles, USA, ${ }^{4}$ West Los Angeles Veterans Administration, UCLA, Los Angeles, USA

Gaussian smoothing and brain area activation relationship - determination of optimal filter size through ROC curves, Lilian Contin ${ }^{l}$, João Sato ${ }^{l}$, Griselda Garrido ${ }^{2},{ }^{1}$ NIF/LIM44 Institute of Radiology - University of São Paulo, São Paulo, Brazil, ${ }^{2}$ Instituto Israelita de Ensino e Pesquisa, $461 \mathrm{M}-\mathrm{AM}$ São Paulo, Brazil

An investigation of the visual coding of faces using kernel canonical correlation analysis,

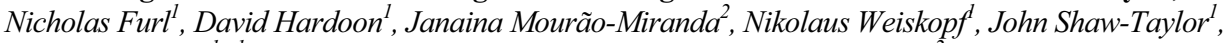
Raymond Dolan ${ }^{1},{ }^{1}$ University College London, London, United Kingdom, ${ }^{2}$ Kings College London, London, United Kingdom

Feature Analysis of Event-related Brain Potentials by Statistical Classification: Application of Naive Bayes Method and Principal Component Analysis to Predicting Auditory Stimuli, Yasuyuki Inoue ${ }^{1}$, Akitoshi Ogawa ${ }^{2}$, Kota Arai $^{3}$, Hidehiko Matsumoto ${ }^{3}$, Atsuhito Toyomaki ${ }^{4}$, Hiroshige Takeichi ${ }^{2}$, Takashi Omori ${ }^{4}$, Sachiko Koyama ${ }^{4}$, Takashi Morotomi ${ }^{3}$, Michiteru Kitazaki ${ }^{1},{ }^{I}$ Toyohashi University of Technology, Toyohashi-shi, Japan, ${ }^{2}$ RIKEN, Wako-shi, Japan, ${ }^{3}$ Sakushin Gakuin University, Utsunomiya-shi, Japan, ${ }^{4}$ Hokkaido University, Sapporo-shi, Japan

Profiling brain function for source imaging in EEG and MEG: A similarity ranking method for evaluating individual activation, Yannick Marchand ${ }^{l, 2,3,4}$, Ryan $D^{\prime}$ Arcy $^{1,2,5}$, Vanessa Versteeg ${ }^{1,2}$, Erin Mazerolle ${ }^{1,2},{ }^{1}$ Institute for Biodiagnostics (Atlantic), National Research Council Canada, Halifax, Canada, ${ }^{2}$ Department of Psychology, Dalhousie University, Halifax, Canada, ${ }^{3}$ Faculty of Computer Science, Dalhousie University, Halifax, Canada, ${ }^{4}$ School of Human Communication Disorders, Dalhousie University, Halifax, Canada, ${ }^{5}$ Department of Radiology, Dalhousie University, Halifax, Canada

Neuroimaging Platform for Neuroinfomatics: NIMG-PF, Ryouji Suzuki ${ }^{1}$, Kazuhisa Niki ${ }^{2}$, Norio Fujimaki ${ }^{3}$, Shinobu Masaki ${ }^{4}$, Kazuhisa Ichikawa ${ }^{l}$, Shiro Usui ${ }^{5},{ }^{1}$ Kanazawa Institute of Technology, Kanazawa, Japan, ${ }^{2}$ Neuroscience Research Institute, National Institute of Advanced Industrial Science and Technology, Tsukuba, Japan, ${ }^{3}$ National Institute of Information and Communications Technology, Kobe, Japan, ${ }^{4}$ Brain Activity Imaging Center, ATR-Promotions, Kyoto, Japan,

${ }^{5}$ RIKEN Brain Science Institute,, Wako, Japan

Classifying Cortical Surface Folding: An Adaptive Filter based on Spatial and Frequency Curvature Properties, Rudolph Pienaar ${ }^{1,2}$, Bruce Fischl ${ }^{1,2}$, Nasser Al Dossary, Nikos Makris ${ }^{1,2}$, P Ellen Grant ${ }^{1,2},{ }^{1}$ Harvard Medical School, Boston, USA, ${ }^{2}$ Massachusetts General Hospital, Boston, USA, ${ }^{3}$ King Faisal Specialist Hospital and Research Centre, Riyadh, Saudi Arabia

Quantitative Multiscale Brain Modeling: Toward a Large-Scale "Working Brain" Model, Peter Robinson, Andrew Phillips, Parry Chen, Anthony Krensel, Peter Drysdale, Christopher Rennie, University of Sydney, Sydney, Australia

Sex Differences in Short Brain Waves: Where and When., Akaysha Tang ${ }^{1,2}$, Peng Sun ${ }^{3}$, Zhen Yang ${ }^{l}$, Amy Korzekwal, Matthew Sutherland ${ }^{l},{ }^{l}$ Department of Psychology, Albuquerque, USA, ${ }^{2}$ Department of Neurosciences, Albuquerque, USA, ${ }^{3}$ Department of Electrical and Computer Engineering, Albuquerque, USA

Decoding unconscious determinants of human decisions in real-time, Martin Weygandt ${ }^{l}$, Chun Siong Soon ${ }^{1,2}$, John-Dylan Haynes ${ }^{1,2},{ }^{1}$ Bernstein Center for Computational Neurosciences Berlin, Berlin, Germany, ${ }^{2}$ Max Planck Institute for Cognitive and Brain Sciences, Leipzig, Germany 


\section{MODELING \& ANALYSIS \\ Motion Correction/Spatial Normalization, Atlas Construction}

FreeSurfer-Initiated Fully-Automated Subcortical Brain Segmentation in MRI Using Large Deformation Diffeomorphic Metric Mapping, Ali Khan ${ }^{l}$, Lei Wang ${ }^{2}$, Mirza Faisal Beg,

${ }^{I}$ Medical Image Analysis Laboratory, Simon Fraser University, Burnaby, Canada, ${ }^{2}$ Washington 497 M-AM University, St. Louis, USA

False Sense of EPI-to-Structural Alignment with Common Cross-Modality Registration Methods, Robert Cox ${ }^{l}$, Ziad Saad ${ }^{1}$, Daniel Glen ${ }^{l}$, Michael Beauchamp ${ }^{2}$, Rutvik Desai ${ }^{3},{ }^{I}$ NIMH, Bethesda, USA, ${ }^{2}$ UT Health Science Center, Houston, USA, ${ }^{3}$ Medical College of Wisconsin, Milwaukee, USA

Reducing Erroneous Influence from Neighboring Structures by Diffeomorphic Registration of fMRI Data, Behrang Nosrat-Makouei ${ }^{1}$, Lei Wang ${ }^{2}$, Deanna M. Barch ${ }^{2}$, Mirza Faisal Beg ${ }^{I}$, ${ }^{I}$ Medical Image Analysis Lab, Simon Fraser University, Burnaby, Canada, ${ }^{2}$ Washington University, St Louis, USA

Employing the general linear model for creating customized pediatric templates, Marko Wilke $^{1,2}$, Scott Holland ${ }^{3,4}$, Mekibib Altaye ${ }^{4}$, Christian Gaser ${ }^{5},{ }^{1}$ Department of Pediatric Neurology and Developmental Medicine, University Children's Hospital, Tuebingen, Germany, ${ }^{2}$ Section for Experimental MR of the CNS, Dept. of Neuroradiology, Tuebingen, Germany, ${ }^{3}$ Department of Pediatrics, University of Cincinnati, Cincinnati, USA, ${ }^{4}$ Imaging Research Center, Cincinnati Children's Hospital Medical Center, Cincinnati, USA, ${ }^{5}$ Department of Psychiatry, Jena, Germany

\section{MODELING \& ANALYSIS}

Univariate Modeling, Linear, \& Nonlinear

Detection of single-trial events in BOLD fMRI without prior stimulus information, Cesar Caballero $^{1}$, Natalia Petridou ${ }^{2}$, Susan Francis ${ }^{2}$, Ian Dryden ${ }^{3}$, Li Bai ${ }^{I}$, Penny Gowland ${ }^{2},{ }^{1}$ School of Computer Science, University of Nottingham, Nottingham, United Kingdom, ${ }^{2}$ Sir Peter Mansfield Magnetic Resonance Centre, University of Nottingham, Nottingham, United Kingdom, ${ }^{3}$ School of Mathematical Sciences, University of Nottingham, Nottingham, United Kingdom

Comparison of Spherical Deconvolution Methods Based on the Spherical Harmonic Basis, Maxime Descoteaux ${ }^{l}$, Alfred Anwander ${ }^{2}$, Rachid Deriche ${ }^{l},{ }^{I}$ INRIA Sophia Antipolis - Mediterranee, Sophia Antipolis, France, ${ }^{2}$ Max Planck Institute, Leipzig, Germany

Transient neuroenergetics: Towards dynamic calibrated fMRI, Basavaraju G. Sanganahalli, Peter Herman ${ }^{l}$, Fahmeed Hyder ${ }^{1,2},{ }^{I}$ Diagnostic Radiology, Yale University, New Haven, USA, ${ }^{2}$ Biomedical Engineering, Yale University, New Haven, USA

Validation of resampling methods for fMRI data, Mingwu Jin, Dietmar Cordes, Unviersity of Colorado Denver, Denver, USA

\section{$513 \mathrm{M}-\mathrm{AM}$}

Bayesian Deconvolution of FMRI data using Bilinear Dynamical Systems, Salima Makni ${ }^{1}$, Mark Woolrich ${ }^{1}$, Steve Smith ${ }^{1}$, Christian Beckmann ${ }^{1,2},{ }^{1}$ FMRIB, Oxford, United Kingdom, ${ }^{2} I C L$, London, United Kingdom

Real-time EEG Mapping System, Jan Muzik, Karel Hana, Czech Technical University, Prague, Czech Republic

MEG predicts stimulus-rate dependence of BOLD responses in human SI, Cathy Nangini ${ }^{l}$, Yevhen Hlushchuk ${ }^{1,2}$, Riitta Hari ${ }^{1,2},{ }^{1}$ Brain Research Unit, Low Temperature Physics Laboratory, Helsinki University of Technology, Espoo, Finland, ${ }^{2}$ Advanced Magnetic Imaging Centre, Helsinki University of Technology, Espoo, Finland

Disconnection's Renaissance takes shape: formal incorporation in group-level lesion studies, David Rudrauf, Sonya Mehta, Thomas Grabowski, University of Iowa, Department of Neurology, Iowa City, USA

CamBA (CAMbridge Brain Analysis): multi-level nonparametric analysis of neuroimaging studies using permutation tests, Alle Meije Wink ${ }^{1,2}$, Cinly Oo $^{2}$, Sanja Abbott ${ }^{2}$, Anna Barnes ${ }^{2}$, 
Manfred Kitzbichler ${ }^{2}$, Levent Sendur ${ }^{2}$, Ed Bullmore ${ }^{2}$, John Suckling ${ }^{2},{ }^{1}$ Imaging Sciences Department, Imperial College, MRC Clinical Sciences Centre, Hammersmith Campus, London, United Kingdom, ${ }^{2}$ Brain Mapping Unit, Department of Pyschiatry, Addenbrooke's Hospital, Hills Road, Cambridge, United Kingdom

Genetic analysis of cortical thickness in 8-year-old twins, Uicheul Yoon ${ }^{l}$, Cherine Fahim ${ }^{1,2}$, Daniel Perusse ${ }^{2}$, Alan Evans ${ }^{I},{ }^{1}$ McConnell Brain Imaging Centre, Montreal Neurological Institute, Montreal, Canada, ${ }^{2}$ The Research Centre at the Sainte Justine Hospital, Montreal, Canada

\section{MOTOR BEHAVIOR \\ Hand Movements}

Time-frequency analysis of brain activity during polyrhythmic motor performance, Tjeerd Boonstra $^{1,2}$, Michael Breakspear ${ }^{1}$, Andreas Daffertshofer ${ }^{2}$, Peter Beek ${ }^{2},{ }^{1}$ University of New South Wales, Randwick, Australia, ${ }^{2}$ VU University, Amsterdam, Netherlands

The execution and the observation of grasping movements elicit overlapping activations. Luca Turella ${ }^{1}$, Wolfgang Grodd ${ }^{2}$, Umberto Castiello ${ }^{1,3}$, ' Departement of General Psychology, University of Padova, Italy, Padova, Italy, ${ }^{2}$ Section on Experimental MR of the CNS, Department of Neuroradiology, University of Tuebingen, Germany, Tübingen, Germany, ${ }^{3}$ Department of Psychology, Royal Holloway, University of London, United Kingdom, London, United Kingdom

How does my finger jointly act with yours?, Idil Kokal, Valeria Gazzola, Christian Keysers, BCN Neuroimaging Center, University Medical Center, Groningen, Netherlands

Neural substrates involved in the recognition and imitation of a point-light biological motion representation of the human hand, Aidan Roche ${ }^{1,2}$, Zarinah Agnew ${ }^{2,3}$, Anil Bharath ${ }^{1}$, Anthony Bull ${ }^{1}$, Basant Puri ${ }^{2},{ }^{I}$ Department of Bioengineering, Imperial College London, London, United Kingdom, ${ }^{2}$ ISD, MRC CSC \& Imperial College London, London, United Kingdom, ${ }^{3}$ Cognitive Neuroscience Group, MRC CSC \& Imperial College London, London, United Kingdom

Laterality of the Neural Mechanisms for Gesture Imitation, Thomas Zeffiro ${ }^{l}$, Christos Vasios $^{2}$, Fa-Hsuan Lin' ${ }^{2}$, Gary Strangman ${ }^{1}$, Christina Supelanal, John Belliveau', ' Neural Systems Group, Massachusetts General Hospital, Charlestown, USA, ${ }^{2}$ Athinoula A. Martinos Center for

Biomedical Imaging, Massachusetts General Hospital, Charlestown, USA

\section{MOTOR BEHAVIOR \\ Motor-Premotor Cortex/Motor Cortical Functions}

The organization of cognitive control within lateral prefrontal cortex in schizophrenia, Guillaume Barbalat $^{1,2}$, Valerian Chambon ${ }^{1}$, Nicolas Franck ${ }^{1,2}$, Etienne Koechlin ${ }^{3}$, Chloe Farrer ${ }^{1}$, ${ }^{1}$ Institut des Sciences Cognitives, CNRS, Lyon, France, ${ }^{2}$ Centre Hospitalier le Vinatier, Lyon, France, ${ }^{3}$ Université Pierre et Marie Curie and INSERM, Paris, France

Modality differences in rhythmic sequence production, Anke Karabanov, Örjan Blom, Lea Forsman, Fredrik Ullen, Karolinska Institutet, Stockholm, Sweden

Changes of the hemodynamic response after administration of ethanol in different cerebral regions, Michael Luchtmann ${ }^{1}$, Tobias Moench ${ }^{l}$, Maurice Hollmann ${ }^{1}$, Katja Jachau ${ }^{2}$, Johannes Bernardingl, I'Otto-von-Guericke University, Medical Faculty, Institute for Biometry and Medical Informatics, Magdeburg, Germany, ${ }^{2}$ Otto-von-Guericke University, Medical Faculty, Institute for Forensic Medicine, Magdeburg, Germany

Local and Remote Changes in Resting Cerebral Blood Flow Following a Single Session of 5Hz rTMS Applied to the Primary Motor Cortex, Shalini Narayana ${ }^{l}$, Wei Zhang ${ }^{l}$, Crystal Franklin $^{I}$, Joseph Panzarella ${ }^{I}$, Peter Fox ${ }^{I, 2}$, ${ }^{I}$ Research Imaging Center, UT Health Science Center, San Antonio, USA, ${ }^{2}$ South Texas Veterans Health Care Center, San Antonio, USA

Mechanisms underlying functional changes in the primary motor cortex ipsilateral to an active hand, Monica A. Perez, Leonardo G. Cohen, Human Cortical Physiology Section NINDS, NIH, Bethesda, USA 
fMRI of Violent Video Gaming and Fiber-Optic Joystick Evaluation, Joseph Santos ${ }^{I}$, Javier Gonzalez-Castillo $^{1}$, Jeffrey Jackson ${ }^{2,3}$, Olumide Olalude ${ }^{2}$, John Ulmer $^{4}$, Thomas Talavage ${ }^{1,2}$, ${ }^{I}$ Weldon School of Biomedical Engineering, West Lafayette, USA, ${ }^{2}$ School of Electrical and Computer Engineering, West Lafayette, USA, ${ }^{3}$ Red Leaf Designworks, LLC, Lafayette, USA, ${ }^{4}$ Department of Radiology, Medical College of Wisconsin, Milwaukee, USA

Motor Cortex Somatotopy in Congenital Paraplegic Patients, Christoph Stippich ${ }^{l}$, Michael Akbar $^{2}$, Javier Leon Alonso ${ }^{1}$, Katharina Riffel ${ }^{l}$, Alfred Aschoff ${ }^{3},{ }^{l}$ Division of Neuroradiology, University of Heidelberg, Medical Center, Heidelberg, Germany, ${ }^{2}$ Department of Orthopedic Surgery, University of Heidelberg, Heidelberg, Germany, ${ }^{3}$ Department of Neurosurgery, University of Heidelberg, Medical Center, Heidelberg, Germany

Time course of corticospinal excitability and the direction of evoked movements during motor preparation, Gijs van Elswijk ${ }^{1,2}$, Willemijn Schot ${ }^{3}$, Dick Stegeman ${ }^{2,3}$, Sebastiaan Overeem ${ }^{2}{ }^{1}$ F.C. Donders Centre for Cognitive Neuroimaging, Nijmegen, Netherlands, ${ }^{2}$ Department of Clinical Neurophysiology, Radboud University Nijmegen Medical Centre, Nijmegen, Netherlands, ${ }^{3}$ Faculty of Human Movement Sciences, VU University, Amsterdam, Netherlands

\section{NEUROANATOMY Anatomical Studies}

Relationships between age, neuropsychological scores and structural brain measures in 236 healthy aged adults of Chinese origin, Michael Chee, ${ }^{l, 2}$,Hui Zheng ${ }^{I}$, Maria Schuchinsky ${ }^{l}$, Samuel Sim ${ }^{l}$, Karren Chen ${ }^{I}$, Karen Chan ${ }^{2}$, Lisa Chuah ${ }^{l},{ }^{\prime}$ Duke NUS Graduate Medical School, Singapore, Singapore, ${ }^{2}$ Singapore Health Services, Singapore, Singapore

The postcentral sulcus: depth profiles in sulci grouped by cluster analysis, Matthew Cykowski ${ }^{1}$, Olivier Coulon ${ }^{2}$, Peter Kochunov ${ }^{1}$, Jack Lancaster ${ }^{1}$, Peter Fox ${ }^{1,3},{ }^{1}$ 1Research Imaging Center, University of Texas Health Science Center at San Antonio, San Antonio, USA, ${ }^{2}$ Laboratoire des Sciences de l'Information et des Systèmes, Marseille, France, ${ }^{3}$ VA Medical Center, San Antonio, USA

Efferents of Area 25 in the non-human primate brain, Stephen Frey, Veronika Zlatkina, Vladimir V. Rymar, Abbas F. Sadikot, Michael Petrides, Montreal Neurological Institute, Montreal, Canada

Fluid flow deformation analysis of postnatal rhesus macaque brain, Julia Hamstra, Evan Fletcher, Charles DeCarli, David Amaral, Univ. of California, Davis, Davis, USA

Probabilistic Anatomic Mapping of Cerebral Blood Flow Distribution of the Middle Cerebral Artery, Seong-Jang Kim ${ }^{1,2}$, In-Ju Kim ${ }^{1,2}$, Yong-Ki Kim ${ }^{1,2}$, Tae-Hong Lee ${ }^{2,3}$, Jung Sub Lee $^{2,4}$, Sungmin Jun ${ }^{1}$, Hyun-Yeol Nam ${ }^{1}$, Jae Sung Lee, Yu Kyeong Kim ${ }^{5}$, Dong Soo Lee ${ }^{5}$, 'Nuclear Medicine, Pusan National University Hospital, Busan, Korea, ${ }^{2}$ Nuclear Medicine, Pusan National University Hospital, Busan, Korea, ${ }^{3}$ Nuclear Medicine, Pusan National University Hospital, Busan, Korea, ${ }^{4}$ Radiology, Busan, Korea, ${ }^{5}$ Orthopaedic Surgery, Busan, Korea, ${ }^{6}$ Nuclear Medicine, Pusan National University Hospital, Busan, Korea, ${ }^{7}$ Nuclear Medicine, Pusan National University Hospital, Busan, Korea, ${ }^{8}$ Departement of Nuclear Medicine, Seoul National University, Seoul, Korea, ${ }^{9}$ Departement of Nuclear Medicine, Seoul National University, Seoul, Korea,

${ }^{10}$ Departement of Nuclear Medicine, Seoul National University, Seoul, Korea

Structure-Function Relationship of the Human Motor Thalamus, Susan Kouloyan-Ilic ${ }^{1,2}$, Hamed Akhlaghi ${ }^{1}$, Gary Egan ${ }^{1}$, Peter Brotchie ${ }^{3},{ }^{1}$ Howard Florey Institute, Melbourne, Australia, ${ }^{2}$ The Alfred, Melbourne, Australia, ${ }^{3}$ Barwon Health, Melbourne, Australia

Polymorphism in the Fibroblast Growth Factor-20 gene modulates grey matter volume in the medial temporal lobe, Herve Lemaitre ${ }^{l}$, Vankata Mattay ${ }^{l}$, Fabio Sambataro ${ }^{I}$, Beth Verchinski ${ }^{I}$, Richard Straub ${ }^{l}$, Joseph Callicott ${ }^{l}$, Ronald McKay ${ }^{2}$, Daniel Weinberger ${ }^{l},{ }^{I}$ CBDB, NIMH, Bethesda, USA, ${ }^{2} L M B$, NINDS, Bethesda, USA

Superior temporal gyrus subvolumes in healthy individuals and in treatment resistant schizophrenia with auditory hallucinations, Paul Fitzgerald ${ }^{l}$, Jerome Maller ${ }^{l}$, Justin Yuen ${ }^{1}$, Zafiris Daskalakis ${ }^{2},{ }^{\prime}$ Alfred Psychiatry Research Centre, Monash University, Melbourne, 
Australia, ${ }^{2}$ Alfred Psychiatry Research Centre, Monash University, Melbourne, Australia, ${ }^{3}$ Alfred Psychiatry Research Centre, Monash University, Melbourne, Australia, ${ }^{4}$ Centre for Addiction and Mental Health, Toronto, Canada

Accelerated aging in type 1 diabetes demonstrated with voxel-based analyses of volume and T2 images, Gaby Pell ${ }^{1}$, Ashleigh Lin $^{2}$, Mark Wellard ${ }^{3}$, Debbie Rankins ${ }^{2}$, George Werther ${ }^{4}$, Fergus Cameron ${ }^{4}$, Graeme Jackson ${ }^{1}$ Elisabeth Northam ${ }^{2},{ }^{1}$ Brain Research Institute, Melbourne, Australia, ${ }^{2}$ Murdoch Childrens Research Institute, Melbourne, Australia, ${ }^{3}$ Queensland University of Technology, Brisbane, Australia, ${ }^{4}$ Royal Children's Hospital, Melbourne, Australia

Age-related thinning of cortical grey matter, Rolf Kötter ${ }^{l, 2}$, Andrew Reid ${ }^{l, 2}$, Anouk van Norden ${ }^{3}$, Karlijn de Laat ${ }^{3}$, Lucas van Oudheusden ${ }^{3}$, Alan Evans ${ }^{4}$, Frank-Erik de Leeuw ${ }^{3},{ }^{1}$ UMC Radboud Nijmegen Department of Cognitive Neuroscience, Nijmegen, Netherlands, ${ }^{2} \mathrm{C} \& \mathrm{O}$ Vogt Institute for Brain Research, University Clinics Düsseldorf, Düsseldorf, Germany, ${ }^{3}$ UMC Radboud Nijmegen Department of Neurology, Nijmegen, Netherlands, ${ }^{4}$ McConnell Brain Imaging Center, Montreal Neurological Institute, McGill University, Montreal, Canada

BrainVisa Plugin for Automated Measurements of Sulcal Length and Depth, Bill Rogers ${ }^{I}$ Peter Kochunov ${ }^{I}$, David Glahn ${ }^{I}$, Jeff Rogers ${ }^{2}$, Peter Fox ${ }^{l},{ }^{\prime}$ University of Texas Health Science Center, San Antonio, USA, ${ }^{2}$ Southwest Foundation for Biomedical Research, San Antonio, USA

Gender differences in the neuroanatomical correlates of the affective startle reflex, Sarah Whittle $^{1,3}$, Jonathan Kettle ${ }^{1,3}$, Laurie O'Brien-Simpson ${ }^{3}$, Murat Yucel ${ }^{1,2}$, Julian Simmons ${ }^{1}$, Nicholas Allen ${ }^{1,3},{ }^{l}$ ORYGEN Research Centre, University of Melbourne, Melbourne, Australia, ${ }^{2}$ Melbourne Neuropsychiatry Centre, University of Melbourne, Melbourne, Australia, ${ }^{3}$ Department of Psychology, University of Melbourne, Melbourne, Australia

\section{PHYSIOLOGY, METABOLISM, \& NEUROTRANSMISSION}

Effects of aging on blood flow, oxygen metabolism and blood oxygenation level dependent (BOLD) responses to visual stimulation, Beau Ances, Christine Liang, Oleg Leontiev, Joanna Perthen, Adam Fleisher, Amy Lansing, Richard Buxton, University of California San Diego, La Jolla, USA

Effects of Levodopa on the neural mechanisms of meaning suppression: A 4T fMRI study, David Copland ', Greig De Zubicaray ${ }^{2}$, Katie McMahon', ' ${ }^{I}$ School of Health and Rehabilitation Sciences, The University of Queensland, Brisbane, Australia, ${ }^{2}$ Centre for Magnetic Resonance, The University of Queensland, Australia

Neural integration of baroreflex and cognitive/sensory processing shapes central regulation of beat-to-beat blood pressure, Marcus Gray ${ }^{1}$, Karin Rylander ${ }^{2}$, Neil Harrison ${ }^{3}$, Mikael Elam ${ }^{2}$, B. Gunnar Wallin', Hugo Critchley ${ }^{1,3},{ }^{I}$ CISC, Brighton Sussex Medical School, The University of Sussex, Brighton, United Kingdom, ${ }^{2}$ Institute of Clinical Neurosciences, Unit of Clinical Neurophysiology Sahlgren University Hospital, Goteborg, Sweden, ${ }^{3}$ Institute of Cognitive Neuroscience, Alexandra House, University College London, London, United Kingdom

Imaging of Glucose Metabolic Response in Human Brain Induced by Stimulation of Acupoint ST 36: A FDG PET Study, Xianglan Jin ${ }^{1}$, Yilong $\mathrm{Ma}^{2}$, Jintao Zhang, ${ }^{3}$ Yigen $\mathrm{Wu}^{4}$, Baoci Shan ${ }^{5}$, Dayi Yin ${ }^{3}$, Jinping Sun ${ }^{3}$, Xian Shi ${ }^{3}$, Jiahe Tian ${ }^{3}$, Shulin Yao ${ }^{3}$, Bo Yu ${ }^{l}$, Ling Yin ${ }^{3},{ }^{1}$ Neurology Department, 202nd Hospital of PLA, Shenyang, China, ${ }^{2}$ Department of Neurology, New York University School of Medicine, New York, USA, ${ }^{3} 1$ Neuroinformatics Center, PLA General Hospital, Beijing, China, ${ }^{4}$ Wuhan Institute of Physics and Mathematics, Chinese Academy of Sciences, Wuhan, China, ${ }^{5}$ Institute of High Energy Physics, Chinese Academy of Sciences, Beijing, China

Imaging oxygen consumption with Near-Infrared Spectroscopy and fMRI simultaneously, Rickson Mesquita ${ }^{1,2}$, Harsha Radhakrishnan ${ }^{2}$, Joseph Mandeville ${ }^{2}$, Theodore Huppert ${ }^{3}$, Maria Franceschini ${ }^{2}$, Roberto Covolan ${ }^{1}$, David Boas ${ }^{2},{ }^{1}$ Universidade Estadual de Campinas, Campinas, Brazil, ${ }^{2}$ Massachusetts General Hospital, Charlestown, USA, ${ }^{3}$ University of Pittsburgh, Pittsburgh, USA

Understanding your inhibitions: neuropharmacological perturbations of GABAergic systems, metabolic outcomes and network correlations., Caroline Rae ${ }^{1,2}$, Fatima Nasrallah ${ }^{1,2}$, Julian Griffin ${ }^{3}$, Vladimir Balcar ${ }^{4},{ }^{1}$ Prince of Wales Medical Research Institute, Randwick, 
Australia, ${ }^{2}$ The University of New South Wales, Sydney, Australia, ${ }^{3}$ The University of Cambridge, Cambridge, United Kingdom, ${ }^{4}$ The University of Sydney, Sydney, Australia

Resting State Networks - Neither Low Frequency Nor Anticorrelated?, Stephen Smith ${ }^{l}$, Rami Niazy $^{2}$, Christian Beckmann ${ }^{3,1}$, Karla Miller ${ }^{I},{ }^{I} F M R I B$, Oxford University, Oxford, United Kingdom, ${ }^{2}$ CUBRIC, Cardiff University, Cardiff, United Kingdom, ${ }^{3}$ Imperial College London, London, United Kingdom

Caffeine is not a universal BOLD contrast booster, Lucie Yang, Merideth Addicott, Ann Peiffer, Robert Kraft, Joseph Maldjian, Jonathan Burdette, Luke Burnett, Michael Chen, Paul Laurienti, Wake Forest University School of Medicine, Winston-Salem, USA

Functional Connectivity within the Human Thalamocortical System, Dongyang Zhang, Abraham Snyder, Michael Fox, Mark Sansbury, Joshua Shimony, Marcus Raichle, Washington University, Saint Louis, USA

\author{
SENSORY SYSTEMS \\ Multisensory \& Crossmodal
}

Cross-modal temporal processing in dyslexia assessed with Biological Parametric Mapping, W. David Hairston ${ }^{1}$, Ramon Casanoval, Jonathan Burdette ${ }^{l}$, Frank Wood ${ }^{2}$, Joseph Maldjian ${ }^{l}$, ${ }^{I}$ ANSIR Lab, Dept of Radiology, Wake Forest University School of Medicine, Winston-salem, USA, ${ }^{2}$ Section of Neuropsychology, Wake Forest University School of Medicine, Winston-salem, USA

Audiovisual interactions during access to speech meaning in cochlear implantees: $\mathrm{A}_{\mathbf{2}}{ }^{15} \mathrm{O}$ PET study, Hyo-Jeong Lee ${ }^{1,2}$, Michael Gaebler ${ }^{1}$, Eric Truy ${ }^{3,4}$, Anne-Lise Giraud ${ }^{l},{ }^{1}$ Inserm U742, Laboratoire de Neurosciences Cognitives, Département d'Etudes Cognitives, ENS, Paris, France, ${ }^{2}$ Department of Otolaryngology, Hallym University College of Medicine, Anyang, South Korea, ${ }^{3}$ Département d'ORL, de Chirurgie Cervico-Maxillo-Faciale et d'Audiophonologie, Hôpital Edouard Herriot, Lyon, France, ${ }^{4}$ CNRS UMR 5020, Université Claude Bernard Lyon1, Lyon, France

\title{
SENSORY SYSTEMS
}

\section{Pain \& Autonomic Function}

Differentiating Pain Encoding in Neuropathic Pain Patients, Lino Becerra ${ }^{1,2}$, Gautam Pendse ${ }^{l}$, David Borsook ${ }^{1,2},{ }^{1}$ P.A.I.N. Group McLean Hospital, Belmont, USA, ${ }^{2}$ Harvard Medical School, Boston, USA

High-Resolution fMRI of heat pain perception at 7 T in Humans, Li Min Chen, Christopher Gatenby, Elzabeth Stringer, Robert Friedman, Feng Wang, John Gore, Vanderbilt University, Nashville, USA

Activation of the Trigeminal Principal Sensory Nucleus by Orofacial Muscle Pain, Paul $\mathrm{Nash}^{l}$, Vaughan Macefield ${ }^{2}$, Iven Klineberg ${ }^{3}$, Greg Murray ${ }^{3}$, Luke Henderson ${ }^{l},{ }^{1}$ Dept Anatomy and Histology, University of Sydney, Sydney, Australia, ${ }^{2}$ School of Medicine, University of Western Sydney, Sydney, Australia, ${ }^{3}$ Jaw Function and Orofacial Pain Research Unit, Faculty of Dentistry, The University of Sydney, Sydney, Australia

No evidence for central hypersensitivity in post-operative pain: a serial fMRI study, Ron Kupers ${ }^{1,2}$ Fabien Schneider ${ }^{3}$, Rune Christensen ${ }^{1}$, Henrik Kehlet ${ }^{2},{ }^{1}$ PET Unit, Copenhagen, Denmark, ${ }^{2}$ Dept. Surgical Pathophysiology, Copenhagen, Denmark, ${ }^{3}$ Dept. Radiology, Saint-Etienne, France

Supraspinal response of mechanically induced osteoarthritic knee pain, Albert Leung ${ }^{1,3}$, Dan Muhtar ${ }^{1}$, Jeng-Ren Duann ${ }^{2}$, Artour Torossi ${ }^{4}$, Tony Yaksh ${ }^{l},{ }^{\prime}$ The University of California, San Diego, School of Medicine, La Jolla, USA, ${ }^{2}$ The University of California, San Diego, Institutue for Neurocomputation, La Jolla, USA, ${ }^{3}$ VA San Diego Healthcare System, La Jolla, USA, ${ }^{4}$ The University of California, San Diego, La Jolla, USA

Lateralization of Pain Matrix Areas related or unrelated to the side of stimulation, Kai $\mathrm{Lutz}^{l}$, Michael Meier ${ }^{l}$, Mike Bruegger ${ }^{I}$, Thierry Keller ${ }^{2}$, Ashley Barlow ${ }^{3}$, Roger Luechinger ${ }^{4}$, Lutz 
Zürich, Zürich, Switzerland, ${ }^{2}$ Automatic Control Laboratory, Swiss Federal Institute of Technology, Zürich, Switzerland, ${ }^{3}$ GlaxoSmithKline, Consumer Healthcare, Weybridge, United Kingdom, ${ }^{4}$ Institute of Biomedical Engineering, Swiss Federal Institute of Technology and the University of Zürich, Zürich, Switzerland, ${ }^{5}$ Center for Dental and Oral Medicine and Craniomaxillofacial Surgery, Clinic for Removable Prosthodontics, Masticatory Disorders and Special Care Dentistry, University of Zürich, Zürich, Switzerland

Cerebral response to acute pain correlates with degree of diabetic neuropathy, Iain Wilkinson, Rajiv Gandhi, Dinesh Selvarajah, Mike Hunter, Ceila Emery, Paul Griffiths, Solomon Tesfaye, University of Sheffield, Sheffield, United Kingdom

COGNITION \& ATTENTION

Attention (auditory, tactile, motor)

Keeping track of emerging rules: The neural circuitry of dynamic auditory change detection, Alexandra Bendixen ${ }^{l}$, Urte Roeber ${ }^{l}$, Nelson J. Trujillo-Barreto ${ }^{2}$, Erich Schröger ${ }^{l},{ }^{\prime}$ University of Leipzig, Leipzig, Germany, ${ }^{2}$ Cuban Neuroscience Center, Havana, Cuba

Simultaneous ERP and fMRI in an oddball paradigm with standard and deviant conceptual pairs, Ilan Laufer, Michiro Negishi, Nallakkandi Rajeevan, Cheryl Lacadie, R. Todd Constable, Yale University School of Medicine, Department of Diagnostic Radiology, New Haven, USA

Characteristics and EEG spectral dynamics of behavioural microsleeps in a Mock-MRI scanner, Govinda Poudel ${ }^{1,2}$, Richard Jones ${ }^{1,2,3,4}$, Carrie Innes ${ }^{1,3}$, Philip Bones ${ }^{1,4}$, ${ }^{1}$ Van der Veer Institute for Parkinson's and Brain Research, Christchurch, New Zealand, ${ }^{2}$ Medicine, University of Otago, Christchurch, New Zealand, ${ }^{3}$ Medical Physics and Bioengineering, Christchurch Hospital, Christchurch, New Zealand, ${ }^{4}$ Electrical and Computer Engineering, University of Canterbury, Christchurch, New Zealand

Prominent dysfunction of neural network associated with sustained attention in the patients with schizophrenia compared to the patients with major depression and the healthy controls, Jeong-Ho Seok', Jae-Jin Kim², Jong-Doo Lee ${ }^{3}$, Jeonghun Ku ${ }^{4}$, Hae-Jeong Park ${ }^{3}$, Sang Joon Son ${ }^{2}$, Hyeongrae Lee ${ }^{4}$, Hye-Sun Kim ${ }^{2}$, Maeng-Keun $\mathrm{Oh}^{3},{ }^{1}$ Department of Psychiatry, Hallym University Sacred Heart Hospital, Anyang, Korea, ${ }^{2}$ Department of Psychiatry, Yonsei University, College of Medicine, Seoul, Korea, ${ }^{3}$ Department of Diagnostic Radiology, Yonsei University, College of Medicine, Seoul, Korea, ${ }^{4}$ Department of Biomedical Engineering, Hanyang University, Seoul, Korea

Neural substrates of warning effect: a functional MRI study, Hisakazu T. Yanaka ${ }^{1,2,3}$, Daisuke N. Saito ${ }^{I}$, Norihiro Sadato ${ }^{1,2,3,4},{ }^{I}$ Division of Cerebral Integration, Department of Cerebral Research, National Institute for Physiological Sciences, Okazaki, Japan, ${ }^{2}$ Department of Physiological Sciences, The Graduate University for Advanced Studies (Sokendai, Okazaki, Japan, ${ }^{3}$ Research Institute of Science and Technology for Society (RISTEX), Japan Science and Technology Agency (JST), Tokyo, Japan, ${ }^{4}$ Department of Functional Neuroimaging, Faculty of Medical Sciences, University of Fukui, Fukui, Japan

\section{COGNITION \& ATTENTION Attention (visual)}

Attentional effect on emotional Chinese word processing in the human brain: An eventrelated fMRI study, Hsin-Mei Chen ${ }^{l}$, Su-Ling Yeh ${ }^{l}$, Kuan-Ming Chen ${ }^{l}$, Jyh-Horng Chen ${ }^{2}$, ${ }^{I}$ Department of Psychology, National Taiwan University, Taipei, Taiwan, ${ }^{2}$ Interdisciplinary MR lab, Department of Electrical Engineering, National Taiwan University, Taipei, Taiwan

Modulation of FFA activation by attention and processing demands, Daniel Grupe ${ }^{2}$, Robert Schultz ${ }^{l}$, Elinora Hunyadi ${ }^{l},{ }^{l}$ Center for Autism Research, Children's Hospital of Philadelphia, Philadelphia, USA, ${ }^{2}$ Yale University Child Study Center, New Haven, USA

The Different Roles of Posterior Parietal Cortex and Frontal Eye Field in Control of Visual Selection: Effects of Repetitive Transcranial Magnetic Stimulation on Partial Report Analyzed
$2 \mathrm{M}-\mathrm{PM}$

$6 \mathrm{M}-\mathrm{PM}$

$10 \mathrm{M}-\mathrm{PM}$

14 M-PM

18 M-PM

$22 \mathrm{M}-\mathrm{PM}$

$26 \mathrm{M}-\mathrm{PM}$

$30 \mathrm{M}-\mathrm{PM}$ 
by Bundesen's Theory of Visual Attention, James J. Hung ${ }^{l}$, Jon Driver ${ }^{2}$, Vincent Walsh ${ }^{2}$, ${ }^{I}$ Department of Neurology, Chang Gung Memorial Hospital, Chang Gung University College of Medicine, Taipei, Taiwan, ${ }^{2}$ Institute of Cognitive Neuroscience \& Department of Psychology, University College London, London, United Kingdom

Differences in activation latencies during an attention task as measured by rapid event-related fMRI, Thilo Kellermann ${ }^{l}$, Martina Reske ${ }^{I}, N$. Jon Shah ${ }^{2,3,4}$, Frank Schneider ${ }^{1,3}$, Ute Habel ${ }^{l},{ }^{I}$ RWTH Aachen University, Aachen, Germany, ${ }^{2}$ Research Centre Jülich, Jülich, Germany, ${ }^{3}$ Brain Imaging Centre West, Jülich, Germany, ${ }^{4}$ University of Dortmund, Dortmund, Germany

Competitive Interactions in Human Extrastriate Cortex are Modulated by Collinear Alignment, Stephanie McMains ${ }^{1,2}$, Sabine Kastner ${ }^{1,2},{ }^{l}$ CSBMB, Princeton, USA, ${ }^{2}$ Psychology Dept, Princeton, USA

Context-dependent influences of fronto-parietal areas on visual cortex: Direct confirmation with concurrent TMS-fMRI, Christian Ruff, $f^{1,2}$,Felix Blankenburg ${ }^{1,2}$, Sven Bestmann ${ }^{2}$, Otto Bjoertomt ${ }^{1}$, Nikolaus Weiskopf ${ }^{1,2}$, Jon Driver ${ }^{1,2},{ }^{1}$ UCL Institute of Cognitive Neuroscience, London, United Kingdom, ${ }^{2}$ Wellcome Trust Centre for Neuroimaging at UCL, London, United Kingdom

Content-Specific Top-Down Control from Prefrontal to Visual Cortex during Mental Imagery, Mark Stokes ${ }^{1,2}$, Russell Thompson ${ }^{1}$, Rhodri Cusack ${ }^{1}$, John Duncan ${ }^{1},{ }^{1}$ MRC-CBU, Cambridge, United Kingdom, ${ }^{2}$ Oxford Uniersity, Oxford, United Kingdom

Single pulse TMS on frontal eyefields and intraparietal sulcus enhances coupling of visuospatial attention and saccadic eye movements, Helene Veenstra ${ }^{l, 2}$, Bas Neggers ${ }^{I},{ }^{I}$ Department of psychiatrics, UMC, Utrecht, Netherlands, ${ }^{2}$ Department of Experimental Psychology and Psychopharmacology, Utrecht University, Utrecht, Netherlands

\section{COGNITION \& ATTENTION} Cognitive Aging

Effects of age on EEG activity during driving, Kui-Ming Chen ${ }^{1}$, Tong-Ping Su ${ }^{2}$, Chia-Min Huang $^{2}$, Chin-Teng Lin ${ }^{3}$, Li-Wei Ko ${ }^{3}$, I-Fang Chung ${ }^{1}$, Tzyy-Ping Jung ${ }^{4}$, Institute of Biomedical Informatics, National Yang-Ming University, Taipei, Taiwan, ${ }^{2}$ Psychiatry Department, Taipei Veterans General Hospital, Taipei, Taiwan, ${ }^{3}$ Brain Research Center, National Chiao-Tung University, Hsinchu, Taiwan, ${ }^{4}$ Swartz Center for Computational Neuroscience, Institute for Neural Computation, University of California, San Diego, USA

Nature of cognitive demands determines a distinct relationship between corpus callosum size and interhemispheric efficiency, Jennyfer Ansado ${ }^{1,2}$, Sven Joubert ${ }^{1,3}$, Yves Joanette ${ }^{1,2}$, Sylvane Faure ${ }^{2},{ }^{1}$ Centre de Recherche, IUGM \& Faculté de médecine, Université de Montréal, Montréal, Canada, ${ }^{2}$ Département de psychologie et CERNEC, Montréal, Canada, ${ }^{3}$ Laboratoire de Psychologie Expérimentale et Quantitative, Université Nice-Sophia Antipolis, Nice, France

Diffusion Tensor Imaging of Memory Decline, Efrat Sasson ${ }^{1}$, Glen Doniger ${ }^{2}$, Ofer Pasternak ${ }^{3}$, Yaniv Assaf ${ }^{d, 4},{ }^{\prime}$ Department of Neurobiochemistry, Tel Aviv University, Tel Aviv, Israel, ${ }^{2}$ Department of Clinical Science, NeuroTrax Corporation, Newark, USA, ${ }^{3}$ School of Computer Science, Tel Aviv University, Tel Aviv, Israel, ${ }^{4}$ Functional brain imaging unit, Tel Aviv Sourasky Medical Center, Tel Aviv, Israel

\section{COGNITION \& ATTENTION} Cognitive Development

Developmental changes in inter-regional correlations in cortical thickness during adolescence: The influence of working memory ability and IQ, Lucy Cragg ${ }^{l}$, Gabriel Leonard ${ }^{2}$, Michel Perron $^{3,4}$, Bruce Pike ${ }^{2}$, Louis Richer ${ }^{5}$, Roberto Toro ${ }^{1}$, Suzanne Veillette ${ }^{3,4}$, Zdenka Pausova ${ }^{1,3}$, Tomas Paus ${ }^{1,2},{ }^{1}$ Brain and Body Centre, University of Nottingham, Nottingham, United Kingdom, ${ }^{2}$ Montreal Neurological Institute, McGill University, Montreal, Canada, ${ }^{3}$ Universite de Montreal, Montreal, Canada, ${ }^{4}$ Groupe ECOBES, CEJEP Jonquiere, Jonquiere, Canada, ${ }^{5}$ Department of Psychology, University of Quebec in Chicoutimi, Chicoutimi, Canada 
DTI parameters in the superior longitudinal fasciculus associated with spatial working memory performance in children, Martin Vestergaard Hansen ${ }^{1}$, Kathrine Skak Madsen ${ }^{1,2}$, Lisser Rye Ejersbo ${ }^{4}$, Christian Gerlach ${ }^{4}$, Thomas Z. Ramsøy ${ }^{1}$, Olaf B. Paulson ${ }^{1,2}$, Terry L. Jernigan ${ }^{1,2,3}$, IDanish Research Centre for MR, Copenhagen University Hospital, Hvidovre, $70 \mathrm{M}-\mathrm{PM}$ Denmark, ${ }^{2}$ Center for Integrated Molecular Brain Imaging, Copenhagen, Denmark, ${ }^{3}$ Laboratory of Cognitive Imaging, University of California, San Diego, USA, ${ }^{4}$ Learning Lab Denmark, Danish School of Education, University of Aarhus, Copenhagen, Denmark

Age-related changes in face induced gamma oscillations, Natasa Kovacevic ${ }^{1}$, Roxane Itier ${ }^{1}$, Anthony McIntosh ${ }^{1,2},{ }^{1}$ Rotman Research Institute, Toronto, Canada, ${ }^{2}$ Department of Psychology, University of Toronto, Toronto, Canada

Effects of bilingualism on numerical neurocognition in a paediatric population. An fMRI investigation, Katrien Mondt ${ }^{l}$, Esli Struys ${ }^{l}$, Danielle Balériaux ${ }^{2}$, Piet Van de Craen ${ }^{l},{ }^{1}$ Department of Linguistics, Vrije Universiteit Brussel, Brussels, Belgium, ${ }^{2}$ MR Unit, Université Libre de Bruxelles, Brussels, Belgium

Music and the infant brain: a fMRI study in newborns, Maria Cristina Saccuman ${ }^{l}$, Paola Scifo $^{2,3}$, Guido Andreolli ${ }^{l}$, Danilo Spada ${ }^{5}$, Federica Navarra ${ }^{2,3}$, Cristina Baldoli ${ }^{3,4}$, Stefan Koelsch ${ }^{6}$, Daniela Perani ${ }^{1,2,3},{ }^{1}$ Vita-Salute San Raffaele University, Milan, Italy, ${ }^{2}$ Department of Nuclear Medicine, Scientific Institute San Raffaele, Milan, Italy, ${ }^{3}$ CERMAC San Raffaele Scientific Institute, Milan, Italy, ${ }^{4}$ Department of Neuroradiology, Scientific Institute San Raffaele, Milan, Italy, ${ }^{5}$ Psychology Institue, School of Medicine, Universita' degli Studi, Milan, Italy, ${ }^{6}$ Max-Planck-Institute for Neuropsychology, Leipzig, Germany

\section{COGNITION \& ATTENTION Executive Function}

Unconscious formation of free intentions: functional dissociation between regions in prefrontal cortex, Chun Siong Soon ${ }^{1}$, Anna He ${ }^{l}$, John-Dylan Haynes ${ }^{1,2}$, ${ }^{1}$ Max Planck Institute for Cognitive and Brain Sciences, Leipzig, Germany, ${ }^{2}$ Bernstein Center for Computational Neuroscience Berlin, Berlin, Germany

\section{COGNITION \& ATTENTION \\ Perception, Imagery, Awareness}

Motor familiarity modulates mirror neurons system activity during auditory action recognition in sighted and congenitally blind individuals, Daniela Bonino ${ }^{1,2}$, Emiliano Ricciardi $^{1,3}$, Lorenzo Sani ${ }^{1}$, Tomaso Vecchi ${ }^{2}$, Mario Guazzelli ${ }^{4}$, James Haxby ${ }^{5}$, Luciano Fadiga ${ }^{6}$, Pietro Pietrini ${ }^{1},{ }^{1}$ Laboratory of Clinical Biochemistry and Molecular Biology, University of Pisa, Pisa, Italy, ${ }^{2}$ Department of Psychology, University of Pavia, Pavia, Italy, ${ }^{3}$ MRI Lab, Institute of Clinical Physiology, C.N.R. Research Area, Pisa, Italy, ${ }^{4}$ Psychology Chair, University of Pisa, Pisa, Italy, ${ }^{5}$ Department of Psychology, Princeton University, Princeton, USA, ${ }^{6}$ Department of Biomedical Sciences and Advanced Therapy - Physiology Section, University of Ferrara, Ferrara, Italy

Specificity of neural responses to observed and executed actions revealed with fMR-adaptation, Trevor Chong ${ }^{1,2}$, Ross Cunnington ${ }^{2}$, Mark Williams ${ }^{1}$, Nancy Kanwisher ${ }^{3}$, Jason Mattingley ${ }^{2}$, ${ }^{1}$ Macquarie University, Sydney, Australia, ${ }^{2}$ The University of Queensland, St Lucia, Australia,

${ }^{3}$ Massachusetts Institute of Technology, Cambridge, USA

Hearing moves seeing: converging psychophysical and fMRI evidence of auditory-driven visual apparent motion, Elliot Freeman ${ }^{1}$, Su Watkins ${ }^{2}$, Jon Driver ${ }^{2}$, Geraint Rees ${ }^{2},{ }^{1}$ Brunel University, Uxbridge, United Kingdom, ${ }^{2}$ University College London, London, United Kingdom

Restless Minds - A relation between rest and the self in the brain - A deep-TMS study, Michal Gruberger ${ }^{1,2,3}$, Talma Hendler ${ }^{1,3,4}$, Eran-Vadim Harel ${ }^{2,4}$, Hagai Harari ${ }^{2,4}$, Levkovitz Yechiel ${ }^{2,4}$, Abraham Zangen ${ }^{5},{ }^{1}$ Department of Psychology, Tel-Aviv University, Tel-Aviv, Israel, ${ }^{2}$ The Emotion-Cognition Research Center, Shalvata Mental Health Center, Hod-Hasharon, Israel, ${ }^{3}$ Functional Brain Center, Tel-Aviv Sourasky Medical Center, Tel-Aviv, Israel, ${ }^{4}$ Sackler Faculty of Medicine, Tel-Aviv University, Tel-Aviv, Israel, ${ }^{5}$ Department of Neurobiology, The Weizmann Institute of Science, Rehovot, Israel 
Consistency and functional specialization in the default mode brain network, Ben Harrison ${ }^{1,2}$, Jesus Pujol ${ }^{l}$, Marina López-Solà ${ }^{l}$, Rosa Hernández-Ribas ${ }^{l}$, Joan Deus ${ }^{l}$, Hector Ortiz ${ }^{l}$, Carles Soriano-Mas ${ }^{1}$, Murat Yücel ${ }^{2}$, Christos Pantelis ${ }^{2}$, Narcís Cardoner ${ }^{l},{ }^{1}$ Institut d'Alta Tecnologia-PRBB, Barcelona, Spain, ${ }^{2}$ Melbourne Neuropsychiatry Centre, Department of Psychiatry, The University of Melbourne, Melbourne, Australia

Modulations of induced gamma power and synchrony during gaze processing, Roxane Itier ${ }^{l}$, Natasa Kovacevic ${ }^{l}$, Anthony McIntosh ${ }^{1,2},{ }^{1}$ Rotman Research Institute, Toronto, Canada,

${ }^{2}$ University of Toronto, Toronto, Canada

High frequency gamma rhythm in parietal cortex during imagined hand movements, Blake Johnson, Macquarie Centre for Cognitive Science, Sydney, Australia

\section{COGNITION \& ATTENTION Reasoning \& Problem Solving}

MEG-EEG correlates of mentalistic and mechanical reasoning in healthy subjects. An $118 \mathrm{M}-\mathrm{PM}$ analysis of the effects of incongruity, Eric Brunet-Gouet, Damien Vistoli, Emilie Bobin, Christine Passerieux, Inserm ERI15 / UVSQ EA 4047, Versailles, France

Creative Achievement and Cortical Thickness in a Large Healthy Cohort, Rex E. Jung ${ }^{1,2,3}$, H. Jeremy Bockholt ${ }^{l}$, Judith Segall ${ }^{l}$, Arvind Caprihan ${ }^{l}$, Robert Chavez ${ }^{l}$, Shirley Smith ${ }^{l}$, M. Layne Kalbfleisch ${ }^{4},{ }^{1}$ MIND Research Network, Albuquerque, USA, ${ }^{2}$ Department of Neurology, University of New Mexico, Albuquerque, USA, ${ }^{3}$ Department of Psychology, University of New Mexico, Albuquerque, USA, ${ }^{4}$ Krasnow Institute, George Mason University, Fairfax, USA

The neural basis of autistic performance on Raven's Progressive Matrices, Isabelle Soulieres $^{1,2}$, Thomas Zeffiro ${ }^{1}$, Fabienne Samson ${ }^{2,3}$, Elise Barbeau ${ }^{2,3}$, Cherif Sahyoun ${ }^{4}$, Michelle Dawson ${ }^{2}$, Laurent Mottron ${ }^{2,3},{ }^{1}$ Neural Systems Group, Psychiatry Department, Massachusetts General Hospital, Boston, USA, ${ }^{2}$ Clinique spécialisée de l'autisme, Hôpital Rivière-des-Prairies, Montreal, Canada, ${ }^{3}$ Psychiatry Department, Université de Montréal, Montreal, Canada, ${ }^{4}$ Harvard-MIT Division of Health Sciences and Technology, Boston, USA

\section{COGNITION \& ATTENTION \\ Space, Time, \& Number Coding}

Effect of sex and menstrual cycle phase on brain activation for 3D mental rotation, Christine Corbly, Linah Al-Alem, Xun Liu, Thomas Kelly, Thomas Curry, Jane Joseph, University of Kentucky, Lexington, USA

Brain indices of complexity in arithmetic expressions, Naiyi Wang ${ }^{I}$, Burkhard Maess ${ }^{1}$, Yuejia Luo $^{2}$, Angela D Friederici ${ }^{l},{ }^{1}$ Max Planck Institute for Human Cognitive and Brain Sciences, Leipzig, Germany, ${ }^{2}$ State Key Laboratory of Cognitive Neuroscience and Learning, Beijing, China

\section{DISORDERS OF THE NERVOUS SYSTEM} Alzheimer \& Dementia

Neuroanatomical correlates of neuropsychiatric symptoms in mild Alzheimer's disease, Peita D Bruen ${ }^{1,2}$, William J McGeown ${ }^{1}$, Michael F Shanks ${ }^{1}$, Annalena Venneri ${ }^{1,3},{ }^{1}$ Clinical Neuroscience Centre, University of Hull, Hull, United Kingdom, ${ }^{2}$ Department of Neuroscience University of Parma, Parma, Italy, ${ }^{3}$ Department of Neuroscience, University of Modena and Reggio Emilia, Modena, Italy

\section{MICROSTRUCTURAL ALTERATIONS AND NEUROGENESIS-RELATED BRAIN} REGIONS IN ALZHEIMER'S DISEASE, Andrea Cherubini ${ }^{1,2}$, Patrice Péran ${ }^{2}$, Margherita Di Paola $^{l}$, Giacomo Luccichenti ${ }^{2}$, Umberto Sabatini ${ }^{2}$, Gianfranco Spalletta ${ }^{I},{ }^{I}$ Department of Clinical and Behavioral Neurology, Santa Lucia Foundation, Rome, Italy, ${ }^{2}$ Department of Radiology, Santa Lucia Foundation, Rome, Italy

\section{MAPPING DEMYELINATION OF THE SUBCORTICAL WHITE MATTER IN EARLY}

ALZHEIMER'S DISEASE, Eleonora Fornari ${ }^{l}$, Maria G. Knyazeva ${ }^{l, 2}$, Reto Meuli ${ }^{l}$, Joseph Ghika $^{2}$, Andrea Brioschi ${ }^{2}$, Isabelle Bourquin ${ }^{2}$, Philippe Maeder ${ }^{l},{ }^{1}$ Radiology Dept, University Hospital and University of Lausanne, Lausanne, Switzerland, ${ }^{2}$ Neurology Dept, University 
3D Mapping of Brain Atrophy in Alzheimer's Disease and Mild Cognitive Impairment with Tensor-Based Morphometry, Xue Hua ${ }^{l}$, Alex Leow ${ }^{l}$, Suh Lee ${ }^{l}$, Neelroop Parikshak ${ }^{l}$, Andrea Klunder ${ }^{I}$, Arthur Toga ${ }^{l}$, Natasha Lepore ${ }^{I}, Y i-Y u$ Chou $^{l}$, Caroline Brun ${ }^{l}$, Ming-Chang Chiang ${ }^{l}$, Marina Barysheval, Clifford Jack $\mathrm{Jr}^{2}$, Michael Weiner ${ }^{3}$, Paul Thompson ${ }^{l},{ }^{1}$ Laboratory of Neuro Imaging, Dept. of Neurology, UCLA School of Medicine, Los Angeles, USA, ${ }^{2}$ Mayo Clinic College of Medicine, Rochester, USA, ${ }^{3}$ Dept. Radiology, Medicine and Psychiatry, UC San Francisco, San Francisco, USA

Use of MetaROIs and Minimal Deformation Templates to identify FDG PET Biomarker Regions in Alzheimer's Disease, Cindee Madison ${ }^{I}$, Susan Landau ${ }^{I}$, Rayhan Lal ${ }^{I}$, Connie Cheung ${ }^{I}$, Norman Foster ${ }^{3}$,Eric Reiman ${ }^{4}$, Robert Koeppe ${ }^{5}$, Michael Weiner ${ }^{2}$, Willam Jagust ${ }^{1},{ }^{1}$ UC Berkeley, Berkeley, USA, ${ }^{2}$ UC San Francisco, San Francisco, USA, ${ }^{3}$ University of Utah, Salt Lake City, USA, ${ }^{4}$ Banner Alzheimer's Institute, Salt Lake City, USA, ${ }^{5}$ University of Michigan, Ann Arbor, USA

Cognitive decline associated with loss of hippocampal activation on longitudinal fMRI in non-demented older subjects, Kelly O'Keefe ${ }^{I}$, Jacqueline O'Brien ${ }^{l}$, Amy DeLuca ${ }^{l}$, Peter LaViolette ${ }^{2}$, Bradford Dickerson ${ }^{2}$, Ali Atri ${ }^{2}$, Deborah Blacker ${ }^{2}$, Maija Pihlajamaki ${ }^{1}$, Keith Johnson ${ }^{2}$, Reisa Sperling ${ }^{1,2},{ }^{1}$ Brigham and Women's Hospital, Boston, USA, ${ }^{2}$ Massachusetts General Hospital, Boston, USA

Brain functional activity predicts subsequent structural atrophy in Alzheimer's disease but not normal aging, Benjamin Shannon ${ }^{1}$, Abraham Snyder ${ }^{1,2}$, Cindy Lustig ${ }^{3}$, Randy Buckner ${ }^{4,5}$, Marcus Raichle ${ }^{1,2},{ }^{1}$ Department of Radiology, Washington University, Saint Louis, USA,

${ }^{2}$ Department of Neurology, Washington University, Saint Louis, USA, ${ }^{3}$ Department of Psychology, University of Michigan, Ann Arbor, USA, ${ }^{4}$ Department of Psychology, Harvard University,

Cambridge, USA, ${ }^{5}$ Howard Hughes Medical Institute, Chevy Chase, USA

\section{DISORDERS OF THE NERVOUS SYSTEM Mood \& Anxiety Disorders}

State or Trait? The effect of stressful-experience on brain activation correlates with neuroticism, Roee Admon ${ }^{1,2}$, Orit Stern ${ }^{1}$, Keren Rosenberg ${ }^{1,2}$, Gadi Lubin ${ }^{4}$, Talma Hendler ${ }^{1,2,3}$, ${ }^{I}$ Functional Brain Center, Wohl Institute for Advanced Imaging, Tel Aviv Sourasky Medical Center, Tel Aviv, Israel, ${ }^{2}$ Physiology Dept, Tel Aviv University, Tel Aviv, Israel, ${ }^{3}$ Psychology Dept, Tel Aviv University, Tel Aviv, Israel, ${ }^{4}$ Mental Health Section, Israeli Defense Forces, Israel

Reduced cortico-limbic connectivity in remitted recurrent depression, Naranjargal Dashdorj ${ }^{\text {, }}$ Neil Nixon ${ }^{2}$, Graham Worwood ${ }^{2}$, Mario Liotti ${ }^{2}$, Elena Georgiadi ${ }^{2}$, Dorothee Auer ${ }^{1}$, Peter Liddle ${ }^{2}$, ${ }^{l}$ Academic Radiology, School of Medical and Surgical Sciences, University of Nottingham, Nottingham, United Kingdom, ${ }^{2}$ Division of Psychiatry, School of Medical and Surgical Sciences, University of Nottingham, Nottingham, United Kingdom

Cortical thickness and depression in a population-based sample of adolescents, Marije Jansen', Gabriel Leonard', Michel Perron ${ }^{3,4}$, Bruce Pike ${ }^{2}$, Louis Richer ${ }^{5}$, Roberto Toro ${ }^{1}$, Suzanne Veillette ${ }^{3,4}$, Zdenka Pausova ${ }^{1,3}$, Tomas Paus ${ }^{1,2},{ }^{1}$ Brain \& Body Centre, University of Nottingham, Nottingham, United Kingdom, ${ }^{2}$ Montreal Neurological Institute, McGill University, Montreal, Canada, ${ }^{3}$ Université de Montréal, Montreal, Canada, ${ }^{4}$ Groupe ECOBES, CEJEP Jonquiere, Jonquiere, Canada, ${ }^{5}$ Department of Psychology, University of Quebec in Chicoutimi, Chicoutimi, Canada

SELF-REPORTED RUMINATION AS TRAIT MARKER FOR DEPRESSION: EVIDENCE FROM FUNCTIONAL NEUROIMAGING, Danilo Arnone, Emma Pegg, Shane McKie, Darragh Downey, Rebecca Elliott, Bill Deakin, Ian Anderson, Neuroscience and Psychiatry Unit, University of Manchester, Manchester, United Kingdom

Longitudinal assessment of brain structural alterations in major depressive disorder, Carles Soriano-Mas ${ }^{1}$, Rosa Hernández-Ribas ${ }^{1,2}$, Narcís Cardoner ${ }^{1,2}$, Mikel Urretavizcaya ${ }^{2}$, Joan Deus ${ }^{1,3}$, Héctor Ortiz ${ }^{1,4}$, Marina López-Solà ${ }^{1,5}$, Ben J. Harrison ${ }^{1,6}$, José M. Menchón $^{2}$, Julio Vallejo ${ }^{2}$, Jesús Pujol ${ }^{1},{ }^{1}$ Institut d'Alta Tecnologia-PRBB, CRC Corporació Sanitària, Barcelona, Spain, ${ }^{2}$ Department of Psychiatry, Hospital Universitari Bellvitge, Barcelona, Spain, ${ }^{3}$ Department of Clinical and Health Psychology, Universitat Autònoma de Barcelona, Barcelona, Spain,

${ }^{4}$ Electronic Engineering Department, Technical University of Catalonia (UPC), Barcelona, Spain,

${ }^{5}$ Clinical Sciences Departament, Faculty of Medicine, University of Barcelona, Barcelona, Spain,

${ }^{6}$ Melbourne Neuropsychiatry Centre, Department of Psychiatry, The University of Melbourne,

Melbourne, Australia

$166 \mathrm{M}-\mathrm{PM}^{*}$

170 M-PM

174 M-PM

178 M-PM 
Reduced orbitofrontal-amygdala resting-state connectivity in anxiety disorder patients, Christian Windischberger ${ }^{1,2}$, Andreas Weissenbacher ${ }^{1,2}$, Florian Gerstl ${ }^{1,2}$, Ewald Moser ${ }^{1,2}$, Rupert Lanzenberger ${ }^{3},{ }^{1}$ MR Center of Excellence, Medical University, Vienna, Austria, ${ }^{2}$ Center for Biomedical Engineering and Physics, Medical University, Vienna, Austria, ${ }^{3}$ Department of Psychiatry and Psychotherapy, Medical University, Vienna, Austria

\section{DISORDERS OF THE NERVOUS SYSTEM Parkinson's Disease \& Other Basal Ganglia}

On the cerebral effects of L-DOPA in Morbus Parkinson - a study using fMRI, Christian Enzinger $^{1,3}$, Petra Schwingenschuh ${ }^{1}$, Petra Katschnig ${ }^{1}$, Stefan Ropele ${ }^{1}$, Faton Gorani ${ }^{1}$, Marisa Loitfelder ${ }^{l, 2}$, Erwin Ott ${ }^{l}$, Franz Fazekas ${ }^{I},{ }^{I}$ Dept. of Neurology, Medical University Graz, Graz, Austria, ${ }^{2}$ Institute of Psychology, Karl Franzens University Graz, Graz, Austria, ${ }^{3}$ Section of Neuroradiology, Dept. of Radiology, Medical University Graz, Graz, Austria

Effect of L-Dopa therapy on the fronto-striatal activity observed in patients with Parkinson's disease during set-shifting., Thomas Jubault ${ }^{1,5}$, Laura Monetta ${ }^{1,5}$, Antonio P. Strafella ${ }^{2,6}$, AnneLouise Lafontaine ${ }^{3}$, Michel Panisset ${ }^{4}$, Alain Ptito ${ }^{3}$, Claudine Gauthier ${ }^{1,5}$, Oury Monchi ${ }^{1,5}$, ${ }^{I}$ Functional Neuroimaging Unit, Montreal Geriatric's Institute, Montreal, Canada, ${ }^{2}$ Toronto Western Hospital, Toronto, Canada, ${ }^{3}$ Montreal Neurological Institute and Hospital, Montreal, Canada, ${ }^{4}$ Andre Barbeau's Movement Disorders Unit, University of Montreal Hospital Centre, Montreal, Canada, ${ }^{5}$ University of Mon treal, Montreal, Canada, ${ }^{6}$ Centre for Addiction and Mental Health, Toronto, Canada

Effects of deep brain stimulation on somatosensory evoked magnetic fields in Parkinsonian patients, Jyrki Mäkelä ${ }^{1}$,Juha Pohjola ${ }^{2}$, Samu Taulu ${ }^{3}$, Antti Ahonen ${ }^{3}$,Eero Pekkonen ${ }^{4},{ }^{1}$ BioMag Laboratory, Helsinki University Central Hospital, Helsinki, Finland, ${ }^{2}$ Department of Neurosurgery, Helsinki University Central Hospital, Helsinki, Finland, ${ }^{3}$ Elekta Neuromag Oy, Helsinki, Finland, ${ }^{4}$ Department of Neurology, Helsinki University Central Hospital, Helsinki, Finland

\section{DISORDERS OF THE NERVOUS SYSTEM Schizophrenia}

Working memory network activation and functional relationships: MEG studies in patients, unaffected siblings, and normal volunteers, Richard Coppola ${ }^{l, 2}$, Sreenivasan Rajamoni ${ }^{l}$, Fred Carver $^{l}$, Tom Holroyd ${ }^{l}$, Stefano Marenco ${ }^{2}$, Daniel Weinberger ${ }^{2},{ }^{1} M E G$ Core Facility, Bethesda, USA, ${ }^{2} C B D B, N I M H$, Bethesda, USA

Cortical activation preceding the perception of auditory verbal hallucinations: an fMRI study, Kelly Diederen ${ }^{1}$, Iris Sommer ${ }^{1}$, Jan Dirk Blom ${ }^{2}$, Rutger Goekoop ${ }^{2}$, Kirstin Daalman ${ }^{l}$, Marco Boks ${ }^{l}$, Bas Neggers ${ }^{l}$, Rene Kahn ${ }^{1},{ }^{1}$ University Medical Centre, Utrecht, Netherlands, ${ }^{2}$ Parnassia Psycho-Medical centre, The Hague, Netherlands

Functional imaging of emotional self concept in schizophrenia, Ute Habel, Katharina Pauly, Frank Schneider, Tilo Kircher, Department of Psychiatry and Psychotherapy, RWTH Aachen University, Aachen, Germany

Different neural correlates of ambivalence in schizophrenia and depression: $\mathbf{a ~}_{\mathbf{2}}{ }^{15} \mathrm{O}$ PET study, Jae-Jin Kim ${ }^{1,2,3}$, Young-Chul Jung ${ }^{1,2}$, Il Ho Park ${ }^{1,2}$, Ji-Won Chun ${ }^{l}$, Hye Sun Kim ${ }^{1}$, Jeong Ho Seok ${ }^{4}$, Joon Suk Lim², Maeng-Gun Oh', Hae-Jeong Park ${ }^{3}$, Jong Doo Lee ${ }^{3},{ }^{3}$ Institute of Behavioral Science in Medicine, Severance Mental Health Hospital, Yonsei University College of Medicine, Gwangju-si, South Korea, ${ }^{2}$ Department of Psychiatry, Yonsei University College of Medicine, Seoul, South Korea, ${ }^{3}$ Department of Diagnostic Radiology, Yonsei University College of Medicine, Seoul, South Korea, ${ }^{4}$ Department of Psychiatry, Hallym University Sacred Heart Hospital, Anyang, South Korea

A combined fMRI and ${ }^{1} \mathrm{H}-\mathrm{MRS}$ study of the ACC and the hippocampus in patients with schizophrenia, Luke Stoeckel ${ }^{1,2}$, Meredith Reid ${ }^{1,3}$, Jan Den Hollander ${ }^{4}$, Shastry Akella ${ }^{3}$, Kathy Avsar ${ }^{1,2}$, Adrienne C. Lahti ${ }^{l},{ }^{I}$ Neuroimaging and Translational Research Lab, Department of Psychiatry and Behavioral Neurobiology, University of Alabama at Birmingham (UAB), Engineering, UAB, Birmingham, USA, ${ }^{4}$ Department of Medicine, UAB, Birmingham, USA 
Cerebral Asymmetry and Functional Laterality in Psychosis, Clare Mackay ${ }^{I}$, Neil Roberts ${ }^{2}$, Roozbeh Rezaie ${ }^{2}$, Tom Barrick ${ }^{3}$, Digby Quested ${ }^{l}$, Guy Goodwin ${ }^{1}$, Julie Connell ${ }^{1}$, Manaan Kar Ray ${ }^{I}$, Tim Crow ${ }^{I},{ }^{I}$ Department of Psychiatry, University of Oxford, Oxford, United Kingdom, ${ }^{2}$ University of Liverpool, Liverpool, United Kingdom, ${ }^{3}$ St Georges Hospital Medical School, London, United Kingdom

Abnormal white matter microstructure in schizophrenia: a voxelwise analysis of axial and radial diffusivity., Marc Seal ${ }^{l}$, Murat Yücel ${ }^{1,2}$, Alex Fornito ${ }^{l}$, Stephen Wood ${ }^{l}$, Ben Harrison ${ }^{1,3}$, Mark Walterfang ${ }^{I}$, Gaby Pell ${ }^{1,4}$, Christos Pantelis ${ }^{l},{ }^{l}$ Melbourne Neuropsychiatry Centre, The University of Melbourne, Victoria, Australia, ${ }^{2}$ ORYGEN Research Centre, Victoria, Australia, ${ }^{3}$ Institut d'Alta Tecnologia-PRBB, CRC Corporació Sanitària, Barcelona, Spain, ${ }^{4}$ Brain Research Institute, Austin Health, Victoria, Australia

\section{EMOTION \& MOTIVATION}

Reward

Brain responses to hunger and its satiation: an arterial spin labeling study, Michael Farrell ${ }^{1,2}$, John Dixon ${ }^{3}$, Julie Playfair ${ }^{3}$, Maureen Dixon ${ }^{3}$, Maria Gavrilescu ${ }^{I}$, Michael McKinley ${ }^{l}$, Melissa Hayden ${ }^{3}$, Derek Denton ${ }^{4}$, Paul O'Brien ${ }^{3}$, Gary Egan ${ }^{1,2},{ }^{1}$ Howard Florey Institute, University of Melbourne, Parkville, Australia, ${ }^{2}$ Centre for Neuroscience, University of Melbourne, Parkville, Australia, ${ }^{3}$ Centre for Obesity Research and Education, Monash University, Prahran, Australia, ${ }^{4}$ Baker Heart Research Institute, Alfred Hospital, Prahran, Australia

Common and distinct brain regions involved in processing different nature of positive and negative reinforcements during uncertain situations, Elise METEREAU, Jean-Claude DREHER, 'Reward and decison making' team, Centre de Neuroscience Cognitive, CNRS Université de Lyon 1, Lyon, France

The medial orbitofrontal cortices and the nucleus accumbens contribute to reward processing under passive situations for monetary gain and loss, Atsushi Sekiguchi ${ }^{1,2}$, Motoaki Sugiura ${ }^{3,1}$, Naho Ikuta ${ }^{1}$, Shigeru Sato ${ }^{4}$, Kaoru Horie ${ }^{4}$, Ryuta Kawashima ${ }^{1},{ }^{1}$ Department of Functional Brain Imaging, IDAC, Tohoku University, Sendai, Japan, ${ }^{2}$ Department of Psychosomatic Medicine, Kyushu University, Fukuoka, Japan, ${ }^{3}$ Department of Cerebral Research, NIPS, Okazaki, Japan, ${ }^{4}$ Graduate School of International Cultural Studies, Tohoku University, Sendai, Japan

Effects of visual cues related to the beginning and the end of smoking on the brains of deprived and non-deprived smokers, Bastian Stippekohl, Rudolf Stark, Dieter Vaitl, Bender Institute of Neuroimaging, Giessen, Germany

An fMRI study of normal-weight restrained versus unrestrained eaters, Jason van Steenburgh $^{1}$, Maria Colettal, Deborah Green ${ }^{1}$, Feroze Mohamed $^{3}$, Steve Platek ${ }^{2}$, Schweta Moonat ${ }^{3}$, Michael Lowe ${ }^{I},{ }^{I}$ Drexel University, Philadelphia, USA, ${ }^{2}$ University of Liverpool, Liverpool, United Kingdom, ${ }^{3}$ Temple University, Philadelphia, USA

\section{EMOTION \& MOTIVATION Social Behavior}

Empathy for Pain is Modulated by the Social Context: An Event-related fMRI Investigation, Yuko Akitsuki ${ }^{1,2}$, Jean Decety ${ }^{l},{ }^{I}$ Departments of Psychology and Psychiatry and Center for Cognitive and Social Neuroscience, The University of Chicago, Chicago, USA, ${ }^{2}$ Department of Functional Brain Imaging, Institute of Development, Aging and Cancer (IDAC), Tohoku University, Sendai, Japan

Facial emotion recognition and amygdala activation across the menstrual cycle, Birgit Derntl $^{1,2,3}$, Christian Windischberger ${ }^{1,4}$, Simon Robinson ${ }^{5}$, Elisabeth Lamplmayr ${ }^{2}$, Ilse Kryspin-Exner ${ }^{2}$, Ruben Gur ${ }^{6}$, Ewald Moser ${ }^{1,4,6}$, Ute Habel ${ }^{3},{ }^{1}$ MR Centre of Excellence, Medical University of Vienna, Vienna, Austria, ${ }^{2}$ Institute for Clinical, Biological and Differential Psychology, Faculty of Psychology, University of Vienna, Vienna, Austria, ${ }^{3}$ Department of Psychiatry and Psychotherapy, University of Aachen RWTH, Aachen, Germany, ${ }^{4}$ Centre for Biomedical Engineering and Physics, Medical University of Vienna, Vienna, Austria, ${ }^{5}$ Center of Mind/Brain Studies, University of Trento, Matarello, Italy, ${ }^{6}$ Department of Psychiatry, University of Pennsylvania, Philadelphia, USA

234 M-PM 
Monoamines regulate emotional impact in inferomedial prefrontal cortex, Albert Gjedde ${ }^{l, 2}$, Jacob Geday ${ }^{1,3},{ }^{I}$ PET Center, Aarhus University Hospitals, Aarhus, Denmark, ${ }^{2}$ Center of Functionally Integrative Neuroscience, Aarhus University, Aarhus, Denmark, ${ }^{3}$ Dept of Neurology, Aarhus University Hospitals, Aarhus, Denmark

Dissociable neural pathways are involved in the perception of someone else's congruent and incongruent emotional facial response, Evelyne Lepron, Jean-François Démonet, Inserm, Toulouse, France

Neural Basis of Social Cooperation with Reputations, Kazuhisa Niki ${ }^{\text {, }}$, Shinsuke Suzuki ${ }^{2}$, Syoken Fujisaki ${ }^{2}$,Eizo Akiyama ${ }^{2},{ }^{1}$ Neuroscience Research Institute, National Institute of Advanced Industrial Science and Technology, Tsukuba, Japan, ${ }^{2}$ Graduate School of Systems \& Information engineering, University of Tsukuba, Tsukuba, Japan

\section{GENETICS}

Human vs Computer Algorithm Choices in Identifying Identical Twin Pairs Based on Cortical Shape Characteristics - Who's Better?, Kelly Botteron ${ }^{1,2}$, Donna Dierker ${ }^{3}$, Richard Todd $^{l}$, Jim Alexopolous ${ }^{1}$, Daniel Seung Kyun Han ${ }^{1}$, Tomoyuki Nishino ${ }^{1}$, Erin Reid ${ }^{3}$, Alex Todorov ${ }^{1}$, David Van Essen ${ }^{3},{ }^{1}$ Washington University School of Medicine, Dept Psychiatry, St. Louis, USA, ${ }^{2}$ Washington University School of Medicine, Mallinckrodt Institute of Radiology, St Louis, USA, ${ }^{3}$ Washington University School of Medicine Anatomy and Neurobiology, St Louis, USA

Neurodevelopmental Candidate Gene Variation and MRI-defined Brain Structural Differences in Healthy Controls and Major Depressive Disorder Patients, Becky Inkster ${ }^{\text {, }}$ Thomas Nichols ${ }^{1}$, Pierandrea Muglia ${ }^{2}$, Paul Matthews ${ }^{l},{ }^{1}$ Clinical Imaging Centre, Hammersmith Hospital, London, Clinical Pharmacology and Discovery Medicine, GlaxoSmithKline, London, United Kingdom, ${ }^{2}$ Medical Genetics, Verona, Clinical Pharmacology and Discovery Medicine, GlaxoSmithKline, Verona, Italy

Allelic variation in NOS1AP is associated with altered prefrontal cortex function and functional connectivity during working memory, Laura A. Libby, Kristin K. Nicodemus, Rachel G. Higier, Morgan J. Prust, Hao Yang Tan, Joshua W. Buckholtz, Bhaskar Kolachana, Richard E. Straub, Daniel R. Weinberger, Joseph H. Callicott, CBDB, GCAP, NIMH IRP, NIH, DHHS, Bethesda, USA

Epistasis of BDNF and SLC6A4 in Depression, Lukas Pezawas ${ }^{1,2}$, Andreas Meyer-Lindenberg ${ }^{1,5}$, Aaron Goldman ${ }^{1}$, Beth Verchinski ${ }^{1}$, Gang Chen ${ }^{3}$, Bhaskar Kolachana ${ }^{1}$, Michael Egan ${ }^{1}$, Venkata Mattay ${ }^{I}$, Ahmad Hariri ${ }^{4}$, Daniel Weinberger ${ }^{I},{ }^{1}$ Genes, Cognition and Psychosis Program, National Institute of Mental Health, National Institutes of Health, Bethesda, USA, ${ }^{2}$ Division of Biological Psychiatry, Medical University of Vienna, Vienna, Austria, ${ }^{3}$ Scientific and Statistical Computing Core, National Institute of Mental Health, National Institutes of Health, Bethesda, USA, ${ }^{4}$ Department of Psychiatry, University of Pittsburgh School of Medicine, Western Psychiatric Institute and Clinic, Pittsburgh, USA, ${ }^{5}$ Central Institute of Mental Health, Mannheim, Germany

\section{IMAGING TECHNIQUES \& CONTRAST MECHANISM EEG}

A Mobile Wearable Wireless Brain Computer Interface Platform for Operational

Neuroscience, Chin-Teng Lin ${ }^{l}$, Tzyy-Ping Jung ${ }^{2}$, Jin-Chern Chiou ${ }^{l}$, Li-Wei Ko ${ }^{l}$, Chih-Feng Chao ${ }^{I}$, Sheng-Fu Liang ${ }^{3},{ }^{1}$ Natl. Chiao-Tung Univ, Hsinchu, Taiwan, ${ }^{2}$ UCSD, La Jolla, San Diego, USA, ${ }^{3}$ Natl. Cheng Kung Univ, Tainan, Taiwan

Rejection of the ballistocardiographic artefact using a cICA based algorithm, Yves Leclercq, Pierre Maquet, Christophe Phillips, Cyclotron Research Center, Liège, Belgium

Functional localization in EEG+MEG using EM estimation on a state-space model with spatial and time smoothness constraint, Antonio Molins ${ }^{3,2}$, Matti Hämäläinen ${ }^{2,4,3}$, Emery Brown ${ }^{3,2,5,1}$, ${ }^{1}$ Brain and Cognitive Sciences, MIT, Cambridge, USA, ${ }^{2}$ MGH-MIT-HMS Athinoula A. 
Martinos Ctr. for Biomed. Imaging, Charlestown, USA, ${ }^{3}$ Harvard-MIT division for Hlth. Sci. and Technology, Cambridge, USA, ${ }^{4}$ Radiology, MGH, Boston, USA, ${ }^{5}$ Anesthesiology, MGH, Boston, USA

\section{IMAGING TECHNIQUES \& CONTRAST MECHANISM Functional MRI}

fMRI with TX SENSE at High Field: The importance of the Reception Field, Fernando Boada $^{l}$, Tamer Ibrahim ${ }^{l}$, Victor Stenger ${ }^{2},{ }^{1}$ University of Pittsburgh, Pittsburgh, USA, ${ }^{2}$ University of Hawaii, Honolulu, USA

Mapping current waveforms with Multiple Alternating Balanced Steady States, Giedrius Buracas ${ }^{1}$, Jongho Lee ${ }^{2}$, Richard Buxton ${ }^{1}$, Eric Wong ${ }^{I}$, Thomas Liu ${ }^{I},{ }^{1}$ UCSD, Dept. Radiology, La Jolla, USA, ${ }^{2}$ Advanced MRI, LFMI, NINDS, NIH, Bethesda, USA

EEG Default Mode Network: Fast vs. Slow Music of Movie Episodes, Weijia Feng, Andrew CN Chen*, Center for Higher Brain Functions, Capital Medical University,, Beijing, China

High Resolution Mapping of $\mathbf{V} 5$ at 7 Tesla, Robin Heidemann ${ }^{l}$, Robert Trampel ${ }^{l}$, Enrico Reimer ${ }^{1}$, Joeran Lepsien ${ }^{1}$, Markus Raabe ${ }^{2}$, Fabrizio Fasano ${ }^{3}$, Robert Turner ${ }^{l},{ }^{1}$ Max Planck Institute for Human Cognitive and Brain Sciences, Leipzig, Germany, ${ }^{2}$ Institute for Experimental Psychology, University of Regensburg, Regensburg, Germany, ${ }^{3}$ Neuroimaging Laboratory, Fondazione Santa Lucia, Rome, Italy

Transit Time and Cerebrovascular Reactivity, Michael Jurkiewicz, Julien Poublanc, Adrian Crawley, David Mikulis, Department of Medical Imaging, The Toronto Western Hospital of the University Health Network, Toronto, Canada

SSFP fMRI at 7 Tesla, Jongho Lee, Masaki Fukunaga, Jeff Duyn, Advanced MRI, LFMI, NINDS, National Institute of Health, Bethesda, USA

Volumetric Magnetic Resonance Inverse Imaging improves the sensitivity of fMRI by reducing physiological noise, Fa-Hsuan Lin ${ }^{1,2}$, Thomas Witzel ${ }^{3}$, Polly Dhond ${ }^{l}$, Thomas Zeffiro ${ }^{4}$, Lawrence Wald', Graham Wiggins ${ }^{I}$, John Belliveau, , 'Athinoula A. Martinos Center for Biomedical Imaging, Massachusetts General Hospital, Charlestown, USA, ${ }^{2}$ Institute of Biomedical Engineering, National Taiwan University, Taipei, Taiwan, ${ }^{3}$ Harvard-MIT Division of Health Sciences and Technology, Cambridge, USA, ${ }^{4}$ Neural Systems Group, Massachusetts General Hospital, Charlestown, USA

Computer-controlled hypercapnic vasodilation for accurate and reproducible BOLD calibration, C.I. Mark, G.B. Pike, McConnell Brain Imaging Center, Montreal Neurological Institute, McGill University, Montreal, Canada

Upper Bound Estimation of Neuronal Current-Induced Magnetic Field Changes in Humans, Kevin Murphy ${ }^{l}$, Jerzy Bodurka ${ }^{2}$, Peter A. Bandettini ${ }^{1,2}$, ' Section on Functional Imaging Methods, National Institute of Mental Health, Bethesda, USA, ${ }^{2}$ Functional MRI Facility, National Institute of Mental Health, Bethesda, USA

Superresolution Parallel Functional MRI, Ricardo Otazo ${ }^{1}$, Fa-Hsuan Lin ${ }^{2,3}$, Stefan Posse I, $^{1,5}$, ${ }^{I}$ Electrical and Computer Engineering Department, University of New Mexico, Albuquerque, USA, ${ }^{2}$ MGH-HMS-MIT Athinoula A. Martinos Center for Biomedical Imaging, Charlestown, USA, ${ }^{3}$ Department of Radiology, Massachusetts General Hospital, Boston, USA, ${ }^{4}$ Department of Psychiatry, University of New Mexico, Albuquerque, USA, ${ }^{5}$ Department of Physics and Astronomy, University of New Mexico, Albuquerque, USA

An easily used method to detect brain regions associated with individual differences using spontaneous functional connectivity, Ming Song, Tianzi Jiang, national key laboratory of pattern recognition, beijing, China

Estimation of vascular contribution to DfMRI (Diffusion weighted fMRI) signal., Shin-ichi Urayama $^{I}$, Kenji Aso ${ }^{I}$, Toshihiko Aso ${ }^{2,1}$, Satoru Kohno ${ }^{I}$, Nobukatsu Sawamotol, Hidenao Fukuyama ${ }^{1}$, Denis Le Bihan ${ }^{2,1},{ }^{1}$ Human Brain Research Center, Graduate School of Medicine, 
Inline Distortion Correction for Echo-Planar fMRI, Markus Vogler ${ }^{1,2}$, Sheeba Arnold ${ }^{3}$, Oliver Hinds $^{3}$, Susan Whitfield-Gabrieli ${ }^{4}$, Josef Pfeuffer ${ }^{1}$, Christina Triantafyllou ${ }^{3,5}$, ' Siemens Medical Solutions, Applications Development, Erlangen, Germany, ${ }^{2}$ University of Applied Sciences, Hof, Germany, ${ }^{3}$ McGovern Institute for Brain Research, MIT, Cambridge, USA, ${ }^{4}$ Department of Brain and Cognitive Sciences, MIT, Cambridge, USA, ${ }^{5}$ Athinoula A. Martinos Center, Department of Radiology, MGH, Harvard Medical School, Cambridge, USA

Changes in Tissue Volume Fraction and T1 during Brain Activation, Wanyong Shin, Hong Gu, Yihong Yang, Neouimaging Research Branch, National Insititute on Drug Abuse, NIH, Baltimore, USA

\section{IMAGING TECHNIQUES \& CONTRAST MECHANISM MEG}

EEG Default Mode Network: Gender Consistency and Difference, Andrew CN Chen*, Huixuan Zhao, Weijia Feng, Center for Higher Brain Functions, Capital Medical University, Beijing, China

Large-Scale Parameter Estimation and Dynamic Source Localization for the Magnetoencephalography (MEG) Inverse Problem., Camilo Lamus ${ }^{1,2}$, Simona Temereanca ${ }^{1,3}$, Chris J. Long ${ }^{1,3}$, Matti S. Hämäläinen ${ }^{3,4}$, Emery N. Brown ${ }^{1,2,4}$, Patrick L. Purdon ${ }^{1,2},{ }^{1}$ Department of Brain and Cognitive Sciences, Massachusetts Institute of Technology, Cambridge, USA,

${ }^{2}$ Department of Anaethesia and Critical Care, Massachusetts General Hospital, Boston, USA,

${ }^{3}$ MGH/MIT/HMS Martinos Center for Biomedical Imaging, Charlestown, USA, ${ }^{4}$ Harvard-MIT

Division of Health Science and Technology, Cambridge, USA

\section{LANGUAGE \\ Language Acquisition}

Phoneme categorization elicits reversed response in the left premotor cortex in control versus dyslexic readers: a support to the 'allophonic' hypothesis of dyslexia, Olivier Dufor ${ }^{1}$, Willy Serniclaes ${ }^{2}$, Liliane Sprenger-Charolles ${ }^{2}$, Jean-Francois Démonet ${ }^{1},{ }_{1}^{1}$ Inserm UMR S825, Toulouse, France, ${ }^{2}$ CNRS Laboratoire Psychologie de la Perception, Paris, France

The Bilingual Semantic System in the Late Korean-English Bilinguals : An fMRI Study, Minjung Kim, Woorim Jeong, Seungbok Lee, Department of Psychology, Chungbuk National University, Cheongju, South Korea

Neural bases of word and non-word reading in trained children with developpemental dyslexia., Rodolphe Nenert ${ }^{l, 3}$, Christophe Levêque ${ }^{2}$, Marie-thérèse LeNormand ${ }^{4}$, Philippe Evrard ${ }^{4}$, Scania De Schonen ${ }^{3,4},{ }^{1}$ Inserm U825, Hopital Purpan, Toulouse, France, ${ }^{2}$ Department of Radiology, Hôpital d'Instruction des Armées du Val-de-Grâce, Paris, France, ${ }^{3}$ LPP, Université Descartes-CNRS, Paris, France, ${ }^{4}$ Laboratory of Developmental Physiology Hopital Robert Debré, Paris, France

Signal processing for whole-head MEG data from awake infants, Toshiaki Imada ${ }^{l}$, Alexis Bosseler ${ }^{1}$, Samu Taulu ${ }^{2}$, Elina Pihko ${ }^{3}$, Jyrki Mäkelä ${ }^{3}$, Antti Ahonen ${ }^{2}$, Patricia Kuhl ${ }^{1}$, Institute for Learning and Brain Sciences, University of Washington, Seattle, USA, ${ }^{2}$ Elekta Neuromag Oy, Helsinki, Finland, ${ }^{3}$ BioMag Laboratory, Helsinki University Central Hospital, Helsinki, Finland

\section{LANGUAGE Production}

Language Functioning after Lesions to the Arcuate Fasciculus, Nina Dronkers ${ }^{1,2,3}$, And Turken ${ }^{l}$, Robert Knight ${ }^{4}$, Juliana Baldo ${ }^{I},{ }^{1}$ VA North. California Health Care System, Martinez, USA, ${ }^{2}$ University of California, Davis, USA, ${ }^{3}$ University of California, San Diego, USA,

${ }^{4}$ University of California, Berkeley, USA

Substrates of Switching of Phonology between the First and Second Languages, Chihiro Hosoda ${ }^{1,2}$, Takashi Hanakawa ${ }^{1}$, Tadashi Nariai ${ }^{2}$, Kikuo Ohno $^{2}$, Manabu Honda ${ }^{l},{ }^{l}$ Department of Cortical Functional Disorders, National Center of Neurology and Psychiatry, Kodaira, Japan,

$350 \mathrm{M}-\mathrm{PM}$

354 M-PM

358 M-PM

362 M-PM

370 M-PM

374 M-PM

378 M-PM

382 M-PM*

${ }^{2}$ Department of Neurosurgery, Tokyo Medical and Dental University, Tokyo, Japan 
Dynamic brain activation during language processing in temporal lobe epilepsy: longitudinal fMRI analysis, Jae-Hun Kim ${ }^{l}$, Jong-Min Lee , Hang Joon Jo ${ }^{1}$, June Sic Kim², Chi Heon Kim ${ }^{2}$, Chun Kee Chung ${ }^{2}$,Eunjoo Kang ${ }^{3}$, Sun I. Kim ${ }^{l},{ }^{l}$ Department of Biomedical Engineering, Hanyang University, Seoul, Korea, ${ }^{2}$ MEG Center, Department of Neurosurgery, Seoul National University College of Medicine, Seoul, Korea, ${ }^{3}$ Department of Psychology, Kangwon National University, Kangwon, Korea

Generation of action verbs in Parkinson's disease: a fMRI study, Patrice Péran ${ }^{1,2}$, Dominique Cardebat $^{2}$, Andrea Cherubini ${ }^{1}$, Fabrizio Piras ${ }^{3,4}$, Giacomo Luccichenti ${ }^{1}$, Antonella Peppe ${ }^{5}$, Carlo Cartagirone $^{5,6}$, Olivier Rascol ${ }^{2}$, Jean-François Démonet ${ }^{2}$, Umberto Sabatini ${ }^{1}$, 'Department of Radology, IRCCS. Foundation Santa Lucia, Rome, Italy, ${ }^{2}$ INSERM U825, Toulouse, France, ${ }^{3}$ Center for Research in Language, University of California, San Diego, USA, ${ }^{4}$ Neuroimaging laboratory, Rome, Italy, ${ }^{5}$ Laboratory of Clinical and Behavioural Neurology, IRCCS Santa Lucia Foundation, Rome, Italy, ${ }^{6}$ Neurological Clinic, Department of Neurosciences, Tor Vergata University of Rome, Rome, Italy

\section{MEMORY \& LEARNING \\ Plasticity (normal \& following pathology)}

Task-induced changes in short-range and long-range synchronization during subsequent sleep, Ysbrand Van Der Werf ${ }^{1,2}$, Cornelis Stam ${ }^{2}$, Eus Van Someren ${ }^{1,2},{ }^{I}$ Dept. Sleep and Cognition, Netherlands Institute for Neuroscience, an Institute of the Royal Netherlands Academy of Arts and Sciences, Amsterdam, Netherlands, ${ }^{2}$ Dept. Clinical Neurophysiology, VU University medical center, Amsterdam, Netherlands

Striatal Contribution to Sleep-dependent Consolidation of Motor Sequence Learning, Karen Debas $^{1}$, Julie Carrier ${ }^{2,3}$, Pierre Orban ${ }^{I}$, Marc Barakat ${ }^{l}$, Gilles Vandewalle ${ }^{I}$, Abdallah Hadj Tahar ${ }^{I}$, Avi Karni $^{4}$, Leslie Ungerleider ${ }^{5}$, Habib Benali ${ }^{2,6}$, Julien Doyon ${ }^{1,3,5,6},{ }^{1}$ Functional Neuroimaging Unit, Department of Psychology, University of Montreal, Montreal, Canada, ${ }^{2}$ Centre d'étude du sommeil et des rythmes biologiques, Hôpital du Sacré-Cour de Montréal, Montreal, Canada, ${ }^{3}$ Centre de recherche en neuropsychologie et en cognition, Department of Psychology, University of Montreal, Montreal, Canada, ${ }^{4}$ Laboratory for Functional Brain Imaging and Learning Research, The Brain-Behavior Center, Haifa, Israel, ${ }^{5}$ Laboratory of Brain and Cognition, NIMH, NIH, Bethesda, USA, ${ }^{6}$ Unité Mixte de Recherche-S 678, Institut National de la Santé et de la Recherche Médicale/University of Paris 6, Centre Hospitalier Universitaire Pitié-Salpêtriere, Paris, France

Localization of Cognitive Function in Rats- MRI Study, Tamar Blumenfeld-Katzir, Ofer Pasternak, Yaniv Assaf, Tel Aviv University, Tel Aviv, Israel

\section{MEMORY \& LEARNING Working Memory}

Working memory in women: fMRI comparison of face processing vs. mental rotation in n-back format, Bonnie Alexander ${ }^{1}$, Sheila Crewther ${ }^{1}$, David Crewther ${ }^{2},{ }^{1}$ La Trobe University, Bundoora, Australia, ${ }^{2}$ Brain Sciences Institute, Swinburne University, Hawthorn, Australia

Variations in task difficulty dissociate activity in prefrontal cortex and medial temporal lobe during working memory encoding, Wesley Clapp, Jonas Karlsson, Michael Rubens, Theodore Zanto, Adam Gazzaley, University of California San Francisco (UCSF), San Francisco, USA

Automatic Coding of Old-New Effect during Working Memory: Evidence from Multimodal Imaging, Chunyan Guo ${ }^{1,2}$, Jessica Clark ${ }^{2,3}$, Adam Lawson' ${ }^{2}$, Yang Jiang ${ }^{2},{ }^{1}$ Department of Psychology, Capital Normal University, Beijing, China, ${ }^{2}$ Behavioral Science Department, University of Kentucky College of Medicine, Lexington, USA, ${ }^{3}$ Psychology Department, University of Kentucky, Lexington, USA

Age-associated changes in the neural correlates of episodic and working memory, Helen Macpherson ${ }^{1}$, Andrew Pipingas ${ }^{2}$, Richard Silberstein ${ }^{3},{ }^{1}$ Swinburne University, Melbourne, Australia, ${ }^{2}$ Swinburne University, Melbourne, Australia, ${ }^{3}$ Swinburne University, Melbourne, Australia

Frontal and Parietal Activation During Working Memory Differentiates Dyslexia from Controls as Revealed by Magnetoencephalography (MEG), Nicholas Velissaris ${ }^{l}$, Lesley 
Pawluk ${ }^{l}$, Laszlo Erdodi ${ }^{l}$, Renee Lajiness-O'Neill ${ }^{1,2}$, Susan Bowyer ${ }^{2,3,4},{ }^{1}$ Eastern Michigan University, Ypsilanti, USA, ${ }^{2}$ Henry Ford Hospital, Detroit, USA, ${ }^{3}$ Oakland University, Rochester, USA, ${ }^{4}$ Wayne State University, Detroit, USA

Efficiency \& trial-to-trial variance of spatial working memory performance is manifest across overlapping load-dependent networks, Michael Valenzuela ${ }^{1,2}$, Nicole Kochan ${ }^{1,2}$, Melissa Slavin $^{1}$, Julian Trollor ${ }^{1,2}$, Perminder Sachdev ${ }^{I, 2}$, Anthony McIntosh $^{3}$, Michael Breakspear ${ }^{1,4}$, ${ }^{I}$ School of Psychiatry, University of NSW, Sydney, Australia, ${ }^{2}$ Neuropsychiatric Institute, Prince of 430 M-PM Wales Hospital, Sydney, Australia, ${ }^{3}$ Rotman Research Institute, Baycrest Centre, Toronto, Canada, ${ }^{4}$ Black Dog Institute, Sydney, Australia

\section{MODELING \& ANALYSIS Bayesian Modeling}

Bayesian Brain Source Imaging based on combined MEG/EEG and fMRI using MCMC, Sung C. Jun ${ }^{1,2}$, John S. George ${ }^{1}$, Juliana Par'e-Blagoev ${ }^{3}$, Sergey Plis ${ }^{4}$, Doug M. Ranken ${ }^{1}$, David M. Schmidt ${ }^{1},{ }^{1}$ Applied Modern Physics Group, MS-D454, Los Alamos, USA, ${ }^{2}$ Department of Information and Communications, Gwangju Institute of Science and, Gwangju, South Korea, ${ }^{3}$ The MIND Institute, Albuquerque, USA, ${ }^{4}$ Department of Computer Science, University of New Mexico, Albuquerque, USA

Graph partitioned spatial priors for imaging, Lee Harrison, William Penny, Guillaume Flandin, Karl Friston, Wellcome Trust Centre for Neuroimaging, London, United Kingdom $J U N^{l}$, SERGEY PLIS ${ }^{2},{ }^{I}$ Gwangju Inst. of Science \& Technology, Gwangju, Korea, ${ }^{2}$ University of New Mexico, Albuquerque, USA

Combining ICA and GLM for FMRI data analysis, Salima Makni ${ }^{l}$, Christian Beckmann ${ }^{1,2}$, Steve Smith ${ }^{l}$, Mark Woolrich ${ }^{l},{ }^{l}$ FMRIB, Oxford, United Kingdom, ${ }^{2}$ Department of Clinical Neurosciences, ICL, London, United Kingdom

Neuroimaging of human face processing by Bayesian MCMC method, Gokcen Yildiz ${ }^{1,2}$, A. Deniz Duru', Ahmet Ademoglu ${ }^{3},{ }^{1}$ Katholieke Universiteit Leuven, Leuven, Belgium,

${ }^{2}$ Galatasaray University, Istanbul, Turkey, ${ }^{3}$ Bogazici University, Istanbul, Turkey

\section{MODELING \& ANALYSIS \\ Classification \& Predictive Modeling}

Phase effect of spontaneous alpha rhythm on the visual evoked potential, Robert Becker ${ }^{\text {, }}$ Petra Ritter ${ }^{l}$, Robert Schmidt ${ }^{2}$, Richard Kempter ${ }^{2}$, Arno Villringer ${ }^{l, 3},{ }^{1}$ Berlin NeuroImaging Center, Dept. of Neurology, Charité, Universitätsmedizin, Berlin, Germany, ${ }^{2}$ Theoretical Neuroscience Lab, ITB, Humboldt-University, Berlin, Germany, ${ }^{3}$ Max-Planck-Institute for Human Cognitive and Brain Science, Leipzig, Germany

Beyond Prediction: More Robust Sparse fMRI Models Reveal Distributed Clusters of Local Activity, Melissa Carroll ${ }^{1}$, Guillermo Cecchi ${ }^{2}$, Irina Rish ${ }^{2}$, Rahul Garg ${ }^{2}$, Ravi Rao ${ }^{2},{ }^{\prime}$ Princeton University Computer Science Department, Princeton, USA, ${ }^{2} I B M$ TJ Watson Research Center, Yorktown Heights, USA

Predicting EEG power oscillations using fMRI, Federico De Martino, Giancarlo Valente, Rainer Goebel, Elia Formisano, Department of Neurocognition, University of Maastricht, Maastricht, Netherlands

Total variation approach for high temporal resolution event detection in fMRI, Mostafa Ghannad Rezaie, Luis Hernandez-Garcia, University of Michigan, Ann Arbor, USA

An Informatics System for the Management of Distributed Neuroimaging Research Data, Neil Killeen ${ }^{l}$, Jason Lohrey ${ }^{2}$, Wee Siong Soh ${ }^{3}$, Wilson Liu ${ }^{1,3}$, Steve Melnikoff ${ }^{4}$, Gary Egan ${ }^{1,3}$, ${ }^{I}$ Centre for Neuroscience, the University of Melbourne, Melbourne, Australia, ${ }^{2}$ Arcitecta, 
Melbourne, Australia, ${ }^{3}$ Howard Florey Institute, the Florey Neuroscience Institutes, Melbourne, Australia, ${ }^{4}$ Victorian E-Research Strategic Initiative, Melbourne, Australia

Probabilistic classification models for Brain Computer Interfaces, Jérémie Mattout ${ }^{1,2}$, Guillaume Gibert ${ }^{1,2}$, Virginie Attina ${ }^{1,2}$, Emmanuel Maby ${ }^{1,2}$, Olivier Bertrand ${ }^{1,2},{ }^{1}$ Brain Dynamics and Cognition, U821 INSERM, Lyon, France, ${ }^{2}$ Lyon 1 - Université Claude Bernard, Lyon, France

Comparison of small clinical samples with Voxel-Based Morphometry: a quantitative approach to the analysis of outliers effects by means of virtual phantoms, Federico Nocchi $^{1,2}$, Tiziana Franchin ${ }^{1,3}$, Elisabetta Genovese ${ }^{4}$, Daniela Longo ${ }^{5}$, Giuseppe Fariello ${ }^{5}$, Vittorio Cannatà ${ }^{4},{ }^{1}$ Clinical Engineering Service, Bambino Gesù Children's Hospital, Rome, Vatican City, ${ }^{2}$ Philips Medical Systems, Monza, Italy, ${ }^{3}$ Department of Bioengineering, Polytechnic of Milan, Milan, Italy, ${ }^{4}$ Health Physics, Bambino Gesù Children's Hospital, Rome, Vatican City, 'Department of Paediatric Radiology, Bambino Gesù Children's Hospital, Rome, Vatican City

Contribution of cortical thickness measurement to the prediction of fast conversion from Mild Cognitive Impairment to Alzheimer's Disease, Olivier QUERBES ${ }^{1,2}$, Jean-Albert LOTTERIE $^{1,2}$, Jérémie PARIENTE ${ }^{1,2}$, Isabelle BERRY $Y^{1,2}$, Jean-Claude FORT ${ }^{2,3}$, Florent AUBRY $Y^{1,2}$, Pierre CELSIS ${ }^{1,2}$, INSERM U825, Toulouse, France, ${ }^{2}$ University Toulouse III Paul Sabatier, Toulouse, France, ${ }^{3}$ Laboratoire de Statistiques et probabilités, Toulouse, France

Automatic Classification of Human Brain Constituents including Crossing Fibres using HARDI and a Support Vector Machine, Susanne Schnell ${ }^{l}$, Björn Kreher ${ }^{I}$, Jürgen Hennig ${ }^{I}$, Hans Burkhardt ${ }^{2}$,Valerij Kiselev ${ }^{I},{ }^{I}$ Medical Physics, Dept. of Diagn. Radiology, University Hospital Freiburg, Freiburg, Germany, ${ }^{2}$ Chair in Pattern Recognition and Image Processing, Institute of Computer Science, University of Freiburg, Freiburg, Germany

\section{A NEW TRIANGULATION METHOD TO LOCALIZE FUNCTIONAL ACTIVITY ON} THE CORTICAL SURFACE, Alan Tucholka ${ }^{1,2}$, Bertrand Thirion ${ }^{1,2}$, Philippe Pinel ${ }^{3}$, Jean-Baptiste Poline ${ }^{I}$, Jean-François Mangin' ${ }^{1}$, 'CEA Saclay, Neurospin/LNAO, Bat 145, 91191, Gif-suf-Yvette, France, ${ }^{2}$ INFIA Futurs, Parietal, Paris, France, ${ }^{3}$ INSERM UNICOG, Neurospin, Paris, France

Estimating Structural Complexity Changes in Alzheimer's Disease and Frontotemporal Dementia, Karl Young ${ }^{1,3}$, An-Tao Du ${ }^{3}$, Joel Kramer ${ }^{2}$,Howard Rosen ${ }^{2}$, Bruce Miller ${ }^{2}$, Michael Weiner ${ }^{1,3}$, Norbert Schuff ${ }^{1,3},{ }^{1}$ Department of Radiology, University of California San Francisco, San Francisco, USA, ${ }^{2}$ Department of Neurology, University of California San Francisco, San Francisco, USA, ${ }^{3}$ Center For Imaging of Neurodegenerative Diseases, Department of Veterans Affairs Medical Center, San Francisco, USA

\section{MODELING \& ANALYSIS \\ Motion Correction/Spatial Normalization, Atlas Construction}

Affine and nonlinear spatial normalization techniques using derivatives of brain magnetic resonance images, Jia-Xiu Liu ${ }^{l}$, Yong-Sheng Chen ${ }^{l}$, Li-Fen Chen ${ }^{2,3}$, ' Department of Computer Science, National Chiao Tung University, Hsinchu, Taiwan, ${ }^{2}$ Institute of Brain Science, National Yang-Ming University, Taipei, Taiwan, ${ }^{3}$ Integrated Brain Research Laboratory, Taipei Veterans General Hospital, Taipei, Taiwan

SLICE TIMING CORRECTION IN BOLD FMRI DATA, Rute Martins ${ }^{l}$, Alexandre Andrade ${ }^{2}$, Patricia Figueiredo ${ }^{1}$, 'School of Engineering, Technical University of Lisbon, Lisbon, Portugal, ${ }^{2}$ Institute of Biophysics and Biomedical Engineering University of Lisbon, Lisbon, Portugal

Structural differences can affect functional interpretation: Differences between modulated and unmodulated fMRI in healthy aging, Jonathan Peelle, Murray Grossman, Department of Neurology, University of Pennsylvania, Philadelphia, USA

Comparison of Registration Techniques on Inter-subject Variation of Diffusion Tensor Imaging, Xiujuan Geng ${ }^{l}$, Hong Gu ${ }^{l}$, Thomas Ross ${ }^{l}$, Gary Christensen ${ }^{2}$, Yihong Yang ${ }^{l}$,

${ }^{I}$ Neuroimaging Research Branch, National Institute on Drug Abuse, NIH, Baltimore, USA,

${ }^{2}$ University of Iowa, Iowa City, USA 


\section{MODELING \& ANALYSIS \\ Univariate Modeling, Linear, \& Nonlinear}

Increased Frontal Delta Synchronization of Bipolar Patients: a MEG Study, Shyan-Shiou Chen ${ }^{1}$, Li-Fen Chen ${ }^{2,3}$, Pei-Chi Tu ${ }^{4}$, Tung-Ping Su ${ }^{5,6}$, Jen-Chuen Hsieh ${ }^{2,3}$, Ying-Chia Lin ${ }^{3}$, ${ }^{I}$ Department of Mathematics, National Taiwan Normal University, Taipei, Taiwan, ${ }^{2}$ Institute of Brain Science, School of Medicine, National Yang-Ming University, Taipei, Taiwan, ${ }^{3}$ Integrated 514 M-PM Brain Research Laboratory, Department of Medical Research and Education, Taipei Veterans General Hospital, Taipei, Taiwan, ${ }^{4}$ Division of Neuroscience, School of Life Sciences, National Yang-Ming University, Taipei, Taiwan, ${ }^{5}$ Division of Psychiatry, School of Medicine, National Yang-Ming University, Taipei, Taiwan, ${ }^{6}$ Psychiatric Department, Taipei Veterans General Hospital, Taipei, Taiwan

The Global Mean Should be Abandoned as Default Normalization Reference in PET Perfusion and Metabolism Studies, Per Borghammer ${ }^{1,2}$, Albert Gjedde ${ }^{1,2}$, ${ }^{1}$ PET Center, Aarhus University Hospitals, Aarhus, Denmark, ${ }^{2}$ Center of Functionally Integrative Neuroscience, Aarhus University, Aarhus, Denmark

The relationship between brain size and cortical structure in the adult human brain, Kiho $\mathrm{Im}^{l}$, Jong-Min Lee ${ }^{I}$, Oliver Lyttelton ${ }^{2}$, Sun Hyung Kim ${ }^{1}$, Alan Evans ${ }^{2}$, Sun I. Kim ${ }^{l},{ }^{1}$ Department of Biomedical Engineering, Hanyang University, Seoul, South Korea, ${ }^{2}$ McConnell Brain Imaging Centre, Montreal Neurological Institute, McGill University, Montreal, Canada

Advances in False Discovery Rate control applied to neuroimaging analyses, Glenn Lawyer ${ }^{1}$, Egil Ferkingstad ${ }^{2}$, Ragnar Nesvåg ${ }^{3}$, Katarina Varnäs ${ }^{4}$, Arnoldo Frigessi $^{2}$, Erik G. Jönsson ${ }^{4}$, Ingrid Agartz ${ }^{1,3,4}$, ${ }^{1}$ Department of Psychiatry, University of Oslo, Oslo, Norway, ${ }^{2}$ Department of Biostatistics, University of Oslo, Oslo, Norway, ${ }^{3}$ Department of Psychiatric Research, Diakonhjemmet Hospital, Oslo, Norway, ${ }^{4}$ Department of Clinical Neuroscience, Psychiatry Section, Karolinska Institutet, Stockholm, Sweden

Spatio-temporal dynamics of P300-related neuronal activation: an EEG/fMRI study, Dante Mantini $^{1,2}$, Laura Marzetti ${ }^{1,2}$, Armando Tartaro ${ }^{1,2}$, Gian Luca Romani ${ }^{1,2}$, Cosimo Del Gratta ${ }^{1,2}$, ${ }^{I}$ Institute for Advanced Biomedical Technologies, University Foundation " G. D'Annunzio", Chieti, Italy, ${ }^{2}$ Department of Clinical Sciences and Bio-imaging, University "G. D'Annunzio”, Chieti, Italy

An Application of Dynamic Analysis of t-Statistics to Clinical fMRI - Initial Evaluation of Brain Tumor Cases, Toshiharu Nakai ${ }^{1}$, Epifanio Bagarinao ${ }^{2}$, Satoshi Nakao ${ }^{3}$, Tomohisa Okada ${ }^{4}$, Chikako Nakai , Kayako Matsuo ${ }^{1}$, 'Functional Brain Imaging Lab, National Center for Geriatrics and Gerontology, Ohbu, Japan, ${ }^{2}$ Grid Technology Research Center, National Institute of Advanced Industrial Science and Technology, Tsukuba, Japan, ${ }^{3}$ Nakao Clinic, Kobe, Japan, ${ }^{4}$ Institute of Biomedical Research and Innovation, Kobe, Japan, ${ }^{5}$ Faculty of Business and Informatics, Toyohashi Sozo University, Toyohashi, Japan

GLM Permutation - Nonparametric Inference for Arbitrary General Linear Models, Thomas Nichols ${ }^{1,3}$, Gerard Ridgway ${ }^{2}$, Matthew Webster ${ }^{3}$, Stephen Smith ${ }^{3},{ }^{1}$ GlaxoSmithKline Clinical Imaging Centre, London, United Kingdom, ${ }^{2}$ Centre for Medical Image Computing, University College London, London, United Kingdom, ${ }^{3}$ FMRIB Centre, Oxford University, Oxford, United Kingdom

Age-related nonlinear properties of EEG variation in post-photic stimulation: A multiscale entropy analysis, Tetsuya Takahashi ${ }^{1}$, Tetsuhito Murata ${ }^{1}$, Tomoyuki Mizuno ${ }^{1}$, Mitsuru Kikuchi $^{2}$, Kimiko Mizukami ${ }^{3}$, Kosuke Narita ${ }^{4}$, Hirotaka Kosaka $^{1}$, Koichi Takahashi, Yuji Wada $^{\boldsymbol{l}}$, 'Department of Neuropsychiatry, Faculty of Medical Sciences, University of Fukui, Fukui, Japan, ${ }^{2}$ Department of Psychiatry and Neurobiology, Graduate School of Medical Science, Kanazawa University, Kanazawa, Japan, ${ }^{3}$ Department of Psychology, Faculty of Human studies, Jin-ai University, Fukui, Japan, ${ }^{4}$ Department of Psychiatry and Human Behavior, Gunma University Graduate School of Medicine, Gunma, Japan, ${ }^{5}$ Department of Informatics, Faculty of Science and Engineering, Kinki University, Osaka, Japan

On Non-normality, Non-parametric Tests and Pooling Permutations Over Space for Voxel Based Morphometry, Anderson M. Winkler ${ }^{1}$, Thomas E. Nichols ${ }^{2,3}$, David C. Glahn ${ }^{l}$,

${ }^{I}$ Department of Psychiatry, University of Texas Health Science Center at San Antonio, San Antonio, USA, ${ }^{2}$ FMRIB Centre, Oxford University,, United Kingdom, ${ }^{3}$ GSK Clinical Imaging Centre, United Kingdom 
Optimizing Kernel Size for the Smoothed Variance t-test, Hui Zhang ${ }^{1}$, Timothy Johnson ${ }^{1}$, Jeffery Fessler ${ }^{1}$, Kent Kiehl ${ }^{4}$, Thomas Nichols ${ }^{2,3,1}$, IUniversity of Michigan, Ann Arbor, USA, ${ }^{2}$ GlaxoSmithKline Clinical Imaging Centre, London, United Kingdom, ${ }^{3}$ FMRIB Centre, Oxford,

United Kingdom, ${ }^{4}$ University of New Mexico, Logan, USA

\title{
MOTOR BEHAVIOR \\ Hand Movements
}

Somatosensory areas $3 \mathrm{a}$ and $4 \mathrm{p}$ are activate during motor imagery in patients with hemiparetic stroke, Andrew Butler ${ }^{1}$, Linda Confalonieri ${ }^{2}$, Giuseppe Pagnoni ${ }^{3}$, Lawrence Barsalou ${ }^{4},{ }^{1}$ Department of Rehabilitation Medicine, Emory University, Atlanta, USA, ${ }^{2}$ CESCOM, University of Milan Bicocca, Milan, Italy, ${ }^{3}$ Department of Psychiatry and Behavioral Sciences, Emory University, Atlanta, USA, ${ }^{4}$ Department of Psychology, Emory University, Atlanta, USA

Gender and Handedness Effects on Corticospinal and Spinothalamic Tracts: A structural asymmetry study using diffusion spectrum imaging, Su-Chun Huang ${ }^{l}$, Fang-Chen Yeh ${ }^{l}$, Hsiao-Lan Wang ${ }^{3}$, V. J. Wedeen ${ }^{4}$, Wen-Yih Isaac Tseng ${ }^{1,2},{ }^{1}$ Center for Optoelectronic Biomedicine, National Taiwan University College of Medicine, Taipei, Taiwan, ${ }^{2}$ Department of Medical Imaging, National Taiwan University Hospital, Taipei, Taiwan, ${ }^{3}$ Faculty of Education, Centre for Neuroscience in Education, University of Cambridge, Cambridge, United Kingdom, ${ }^{4}$ MGH Martinos Center for Biomedical Imaging, Harvard Medical School, Charlestown, USA

Planning to grasp haptically experienced objects reactivates the lateral occipital complex, Gregory Kroliczak ${ }^{1}$, Scott H. Frey ${ }^{1,2}$, 'Department of Psychology, University of Oregon, Eugene, USA, ${ }^{2}$ Lewis Center for Neuroimaging, University of Oregon, Eugene, USA

Touch typing performance correlates with white matter integrity in specific regions of the motor system, Jan Scholz, Heidi Johansen-Berg, FMRIB Centre, Oxford, United Kingdom

\section{MOTOR BEHAVIOR}

\author{
Locomotion
}

Functional potential demonstrated by Diffusion Tensor Tractography in hemiplegic patients with cerebral palsy, Su Min Son ${ }^{l}$, Sung Ho Jang ${ }^{2}$, Ho Lee ${ }^{l}$, In Kyu Yu ${ }^{3}$, Seung Yeon Kim ${ }^{4}$, Han $K u M o o n{ }^{5},{ }^{1}$ Dept of $P M \& R$, college of medicine, Eulji university, Daejeon, Korea, ${ }^{2}$ Dept of PM\&R, college of Medicine, Yeungnam univeristy, Daegu, Korea, ${ }^{3}$ Dept of diagnostic radiology, college of medicine, Eulji university, Daejeon, Korea, ${ }^{4}$ Dept of Pediatrics, college of medicine, Eulji university, Daegeon, Korea, ${ }^{5}$ Dept of Pediatrics, college of medicine, Yeungnam university, Daegu, Korea

\section{MOTOR BEHAVIOR \\ Motor-Premotor Cortex/Motor Cortical Functions}

Physiological Correlates of Motion Sickness Induced by Dynamic Virtual Reality Environment, Chin-Teng Lin ${ }^{1,2}$, Yu-Chieh Chen ${ }^{1,2}$, Chun-Ling Lin ${ }^{1,2}$, Chih-Feng Chao ${ }^{1}$, Jeng-Ren Duann ${ }^{1,3}$, Tzyy-Ping Jung ${ }^{1,3},{ }^{1}$ Brain Research Center, University System of Taiwan, Hsinchu, Taiwan, ${ }^{2}$ Department of Electrical and Control Engineering, National Chiao-Tung University, HsinChu, Taiwan, ${ }^{3}$ Institute for Neural Computation, University of California, San Diego, USA

Resting-state connectivity of the motor network in acute stroke patients, Woo-Kyoung Yoo ${ }^{l}$, Chang-hyun Park ${ }^{1,2}$, Suk Hoon Ohn ${ }^{l}$, Sung H. Yoo ${ }^{3}$, Myoung-Hwan Ko ${ }^{4}$, Sung Tae Kim ${ }^{5}$, Kwang Ho Lee 6 , Yun-Hee Kim ${ }^{1},{ }^{1}$ Department of Physical Medicine and Rehabilitation, Samsung Medical Center, Sungkyunkwan University School of Medicine, Seoul, Korea, ${ }^{2}$ Department of Physics, Korea Advanced Institute of Science and Technology, Daejeon, Korea, ${ }^{3}$ Department of Physical Therapy, Yonsei University, Wonju, Korea, ${ }^{4}$ Department of Physical Medicine and Rehabilitation, Chonbuk National University Medical School, Jeonju, Korea, ${ }^{5}$ Department of Radiology, Samsung Medical Center, Sungkyunkwan University School of Medicine, Seoul, Korea, ${ }^{6}$ Department of Neurology, Samsung Medical Center, Sungkyunkwan University School of Medicine, Seoul, Korea 
Neuropsychology, Institute for Psychology, University Zürich, Zürich, Switzerland, ${ }^{2}$ Exercise Physiology, Institute for Human Movement Sciences, Swiss Federal Institute of Technology and Institute of Physiology, University of Zurich, Zürich, Switzerland, ${ }^{3}$ Institute of Biomedical Engineering, Swiss Federal Institute of Technology and the University of Zurich, Zürich, Switzerland

Local and Remote Changes in Cerebral Blood Flow During Motor Task Following a Single Session of $5 \mathrm{~Hz}$ rTMS Applied to the Primary Motor Cortex, Shalini Narayana ${ }^{l}$, Wei Zhang ${ }^{l}$, Crystal Franklin ${ }^{l}$, Joseph Panzarella ${ }^{l}$, Peter Fox ${ }^{1,2},{ }^{1}$ Research Imaging Center, UT Health Science Center, San Antonio, USA, ${ }^{2}$ South Texas Veterans Health Care Center, San Antonio, USA

586 M-PM

A gradient for neuroplastic capacity within the primary motor cortex: Indications from a case report post hemispherectomy, Jakob Rath ${ }^{1}$, Robert Schmidhammer ${ }^{2}$, Thomas Steinkellner ${ }^{1}$, Nicolaus Klinger ${ }^{I}$, Alexander Geißler ${ }^{1}$, Roland Beisteiner ${ }^{l}$, 'Study Group Clinical fMRI, MR Center of Excellence, Department of Neurology, Medical University of Vienna, A-1090 Vienna, Austria, ${ }^{2}$ Ludwig Boltzmann Institute for Experimental and Clinical Traumatology, Research Center for Traumatology, A-1200 Vienna, Austria

Probing ipsilateral premotor-to-motor connectivity during movement selection, Sergiu Groppa $^{1}$, Boris Schlaag ${ }^{1}$, Oliver Granert ${ }^{l}$, Bart van Nuenen ${ }^{1,2}$, Gesa Hartwigsen ${ }^{1}$, Thomas Weyh ${ }^{3}$, Hartwig Siebner ${ }^{l},{ }^{1}$ Department of Neurology, Christian-Albrechts-University, Kiel, Germany, ${ }^{2}$ Department of Neurology, Radboud University Nijmegen Medical Centre, Nijmegen, Netherlands, ${ }^{3}$ Institute for Medical Electronics, University of Technology Munich, Munich, Germany

I did this! brain response to visually presented hand actions reveals recently perfomed acts, Alon Talmor ${ }^{1}$, Hezy Yeshurun ${ }^{1}$, Talma Hendler ${ }^{2,3},{ }^{1}$ School of Computer Science, Sackler Faculty of Exact Sciences, Tel Aviv University, Israel, Tel Aviv, Israel, ${ }^{2} 2$ Functional Brain Center, Wohl Institute for Advanced Imaging, Tel Aviv Sourasky Medical Center, Israel, Tel Aviv, Israel, ${ }^{3}$ Psychology Department, Sackler Faculty of Medicine, Tel Aviv University, Israel, Tel Aviv, Israel

The changes of both gray matter density and white matter integrity in pianist's brain: a combined structural and diffusion MRI study, Ying $\mathrm{Han}^{1}$, Hong Yang ${ }^{2}$, Ya-Ting $\mathrm{Lv}^{3}$, Chao-Zhe

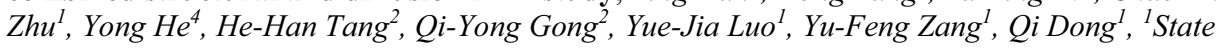
Key Laboratory of Cognitive Neuroscience and Learning, Beijing Normal University, Beijing, China, ${ }^{2}$ Huaxi MR Research Center (HMRRC), Department of Radiology, West China Hospital of Sichuan University, Chengdu, China, ${ }^{3}$ National Laboratory of Pattern Recognition, Institute of Automation, Chinese Academy of Sciences, Beijing, China, ${ }^{4}$ McConnell Brain Imaging Center, Montreal Neurological Institute, McGill University, Montreal, Canada

\section{NEUROANATOMY \\ Anatomical Studies}

Effects of hypertension on grey matter volumes over 4 years in healthy adults aged 60-64: a voxel based morphometry study, Xiaohua Chen ${ }^{1.2}$, Wei Wen ${ }^{1,2}$, Perminder Sachdev ${ }^{1,2}$, Kaarin Anstey, ${ }^{3}$ School of Psychiatry, University of New South Wales, Sydney, Australia,

${ }^{2}$ Neuropsychiatric Institute, Prince of Wales Hospital, Sydney, Australia, ${ }^{3}$ Centre for Mental Health Research, Australian National University, Canberra, Australia

Optimized high-resolution mapping of magnetisation transfer at 3 Tesla reveals substructures in the human thalamus in clinically feasible measurement time, Peter Dechent ${ }^{l}$, Tabea Gringel ${ }^{1,2}$, Erck Elolf ${ }^{2}$, Walter Schulz-Schaeffer ${ }^{3}$, Gunther Helms ${ }^{1},{ }^{1}$ MR-Research in Neurology and Psychiatry, University Medical Center, Göttingen, Germany, ${ }^{2}$ Department of Neuroradiology, University Medical Center, Göttingen, Germany, ${ }^{3}$ Department of Neuropathology, University Medical Center, Göttingen, Germany

Transmitter receptor mapping reveals hierarchy and input specificity in human primary somatosensory cortex, Valentina Garibotto ${ }^{1,2}$, Simon B. Eickhoff ${ }^{l}$, Nicola Palomero-Gallagher ${ }^{1}$, Karl Zilles ${ }^{1,3},{ }^{1}$ Institute of Neuroscience and Biophysics, INB-3 Medicine, Research Centre, Jülich, Germany, ${ }^{2}$ San Raffaele Scientific Institute, Milan, Italy, ${ }^{3}$ C.\&O. Vogt-Institute of Brain Research, University, Düsseldorf, Germany 
Observer-Independent Cytoarchitectonic Mapping of the Human Medial Orbitofrontal Cortex, Anton Henssen ${ }^{l}$, Simon B. Eickhoff ${ }^{1,2,3}$, Karl Zilles ${ }^{1,2,3}$, Axel Schleicher ${ }^{1}$, Hartmut Mohlberg ${ }^{2,3}$, Katrin Amunts, ${ }^{2,3},{ }^{1}$ C\&O. Vogt Institut für Hirnforschung, Düsseldorf, Germany, ${ }^{2}$ Institut für Medizin, Forschungszentrum Jülich, Jülich, Germany, ${ }^{3}$ Brain Imaging Center West $618 \mathrm{M}-\mathrm{PM}$ (BICW), Jülich, Germany, ${ }^{4}$ Klinik für Psychiatrie und Psychotherapie, Universitätsklinikum Aachen, RWTH Aachen, Aachen, Germany

Gender and Age Associated Differences of Cerebral Glucose Metabolism in Normal Healthy Populations: Statistical Parametric Mapping Analysis of F-18 FDG Brain Positron Emission Tomography, Seong-Jang Kim ${ }^{1}$, Sang Heon Song ${ }^{2}$, Tae-Hong Lee ${ }^{3},{ }^{\prime}$ Department of Nuclear Medicine, PNUH, Busan, Korea, ${ }^{2}$ Department of Internal Medicine, PNUH, Busan, Korea, ${ }^{3}$ Department of Radiology, PNUH, Busan, Korea

Functional differentiation of the human insula revealed by ALE meta-analysis, Florian Kurth $^{l}$, Simon B. Eickhoff ${ }^{d, 2,3}$, Katrin Amunts ${ }^{2,3,4}$, Karl Zilles ${ }^{1,2,3},{ }^{l}$ C. \& O. Vogt Institute of Brain Research, University Düsseldorf, Düsseldorf, Germany, ${ }^{2}$ Institute of Neuroscience and Biophysics, INB-3 Medicine, Research Centre Jülich, Jülich, Germany, ${ }^{3}$ Brain Imaging Center West (BICW), Jülich, Germany, ${ }^{4}$ Department of Psychiatry and Psychotherapy, RWTH Aachen University, Aachen, Germany

Common cortical fold variants explored using PALS and CIVET surface registration techniques, Oliver Lyttelton ${ }^{1}$, Donna Dierker ${ }^{2}$, David Van Essen ${ }^{2}$, Alan Evans ${ }^{1},{ }^{1}$ McConnell Brain Imaging Center, McGill University, Montreal, Canada, ${ }^{2}$ Department of Anatomy \& Neurobiology, School of Medicine, Washington University, St Louis, USA

Reversed sexual dimorphism in hippocampal Grey Matter density in women and men with schizophrenia compared to matched healthy controls using 3 Tesla MRI, Adham Mancini-Marïe ${ }^{1,2}$, José Jimenez ${ }^{1,2}$, Cheryl Corcoran ${ }^{3}$, Emmanuel Stip ${ }^{1,2}$, Melissa Rinaldi ${ }^{1}$, Tania Pampoulova $^{1,2}$, Adrianna Mendrek ${ }^{1,2},{ }^{1}$ Department of Psychiatry, Centre de Recherche Fernand Seguin, L-H Lafontaine Hospital, University of Montreal, Montreal, Canada, ${ }^{2}$ Department of Psychiatry, Faculty of Medicine, University of Montreal, Montreal, Canada, ${ }^{3}$ Center of Prevention and Evaluation, New York State Psychiatric Institute, Columbia University, New York, USA

Brain structure and the female menstrual cycle, Jennifer Perrin, Pierre-Yves Herve, Alain Pitiot, John Totman, Tomas Paus, Brain \& Body Centre, University of Nottingham, Nottingham, United Kingdom

Amygdala Structural Deficits in Psychopathy, Yaling Yang Rofman ${ }^{1}$, Adrian Raine ${ }^{2}$, Katherine Narr $^{3}$, Patrick Colletti ${ }^{4}$, Arthur Toga ${ }^{3},{ }^{1}$ Department of Psychology, University of Southern California, Los Angeles, USA, ${ }^{2}$ Department of Criminology, Psychiatry, and Psychology, University of Pennsylvania, Pennsylvania, USA, ${ }^{3}$ Laboratory of Neuro Imaging, Department of Neurology, David Geffen School of Medicine at UCLA, Los Angeles, USA, ${ }^{4}$ Department of Radiology, U.S.C. School of Medicine, Los Angeles, USA

Relationship between body mass index and gray matter volumes in healthy individuals: Cross-sectional and longitudinal analyses, Yasuyuki Taki ${ }^{l}$, Shigeo Kinomura ${ }^{l}$, Kazunori Sato ${ }^{l}$, Ryoi Gotol, Ryuta Kawashima ${ }^{2}$, Hiroshi Fukudal ${ }^{I}{ }^{I}$ Department of Nuclear Medicine \& Radiology, Institute of Development, Aging and Cancer (IDAC), Tohoku University, Sendai, Japan,

${ }^{2}$ Department of Functional Brain Imaging, IDAC, Tohoku University, Sendai, Japan

Tissue Orientation - and thus Structure - Affects T2* Contrast in Ultra High Field MRI., Christopher Wiggins, Valdis Gudmundsdottir, Denis Le Bihan, Vincent Lebon, Myriam Chaumeil, CEA/NeuroSpin, Saclay, France

\section{PHYSIOLOGY, METABOLISM, \& NEUROTRANSMISSION}

Gender Differences in Age-related Decline of Regional Cerebral Glucose Metabolism, Seong Ae Bang ${ }^{1,2}$, Sang Soo Cho ${ }^{1,2}$, Eun Jin Yoon ${ }^{1,2}$, Eun Ju Lee ${ }^{1,2}$, Yu Kyeong Kim ${ }^{1,2}$, Sang Eun Kim ${ }^{1,2}$, ${ }^{1}$ Seoul National University College of Medicine, Seoul, South Korea, ${ }^{2}$ Seoul National University Bundang Hospital, Seoul, South Korea

The post-stimulation undershoot in BOLD fMRI of human brain is not caused by elevated cerebral blood volume, Peter Dechent ${ }^{1}$, Jürgen Baudewig ${ }^{1}$, Kai Kallenberg ${ }^{1,2}$, Andreas Kastrup ${ }^{3}$, 
K. Dietmar Merboldt ${ }^{4}$, Jens Frahm ${ }^{4},{ }^{1}$ MR-Research in Neurology and Psychiatry, University Medical Center, Göttingen, Germany, ${ }^{2}$ Department of Neuroradiology, University Medical Center, Göttingen, Germany, ${ }^{3}$ Department of Neurology, University Medical Center, Göttingen, Germany, ${ }^{4}$ Biomedizinische NMR Forschungs GmbH am Max-Planck-Institut für biophysikalische Chemie, Göttingen, Germany

Electrophysiological correlates of the brain's intrinsic large-scale functional architecture, Biyu He, Abraham Snyder, John Zempel, Matthew Smyth, Marcus Raichle, Washington University School of Medicine, St. Louis, USA

Deactivation of the Pregenual Anterior Cingulate Cortex May Predict Increased Hypothalamic Pituitary Adrenal Activation., Najmeh Khalili-Mahani, Jens C. Pruessner, McGill University, Montreal, Canada

Correlations between regional 5-HTT and 5-HT1A receptor availability in healthy subjects, Allison Nugent ${ }^{1}$, Dara Cannon ${ }^{2}$, Paul Carlson ${ }^{l}$, Rebecca Davis ${ }^{1}$, Wayne Drevets ${ }^{l},{ }^{I}$ Section on Neuroimaging in Mood and Anxiety Disorders, NIMH, Bethesda, USA, ${ }^{2}$ Department of Psychiatry, National University of Ireland, Galway, Ireland

Exogenous cortisol administration results in medial temporal hypoactivation in the human brain, Jennifer Robinson ${ }^{1}$, William Lovallo ${ }^{2}$, Sibel Cakir ${ }^{3}$, Jennifer Barrett ${ }^{1}$, Peter Fox ${ }^{4}$, David Glahn ${ }^{1,4}$, ' Department of Psychiatry, University of Texas Health Science Center, San Antonio,

USA, ${ }^{2}$ Behavioral Sciences Laboratories, Veterans Affairs Medical Center, Oklahoma City, USA, ${ }^{3}$ Department of Psychiatry, Istanbul University, Istanbul, Turkey, ${ }^{4}$ Research Imaging Center, University of Texas Health Science Center, San Antonio, USA

Pharmacological modulation during fMRI: muscarinic and nicotinic proportions of the attention network according to Posner, Renate Thienel ${ }^{1,2}$, Bianca Voss ${ }^{2}$, Martina Reske ${ }^{2,3}$, Thilo Kellermann ${ }^{2}$, Sarah Halfter ${ }^{2}$, Abigail Sheldrick ${ }^{2}$, Katrin Radenbach ${ }^{4}$, Ute Habel ${ }^{2}$, Frank Schneider ${ }^{2}$, NJ Shah ${ }^{5}$, Tilo Kircher ${ }^{2},{ }^{1}$ Centre for Rural \& Remote Mental Health, University of Newcastle, Orange, Australia, ${ }^{2}$ Dept. of Psychiatry \& Psychotherapy, University Clinics, Aachen, Germany, ${ }^{3}$ Dept. of Psychiatry, University of California, San Diego, USA, ${ }^{4}$ Dept. of Psychiatry, Georg-August-University, Goettingen, Germany, ${ }^{5}$ Research Centre Juelich, Helmholtzgesellschaft, Juelich, Germany

Normative Blood Flow Values in Adults in the Posterior Fossa Using MR Perfusion, Ali Shaibani ${ }^{l}$, Amir H. Yassari ${ }^{l}$, Jessy Mouannes ${ }^{2}$, Aaron Skolnik ${ }^{l}$, Shahram Rahimi ${ }^{l}$, Aghaei Anahita ${ }^{l}$, Timothy J Carrol ${ }^{2}$, Bernard R Bendok ${ }^{1}$, Matthew T Walker ${ }^{l},{ }^{1}$ Northwestern University, Feinberg School of Medecine, Chicago, USA, ${ }^{2}$ Northwestern University, Evanston, USA

\section{SENSORY SYSTEMS \\ Multisensory \& Crossmodal}

A hierarchy of visual predictions on auditory speech processing, Luc Arnal, Benjamin Morillon, Anne-Lise Giraud, Inserm U742. Université Pierre et Marie Curie-Paris 6, Paris, France

$686 \mathrm{M}-\mathrm{PM}$

Flash VEP is reduced in children when preceded by an audio-visual stimulus., Hamish InnesBrown, Ayla Barutchu, Mohit Shivdasani, Antonio Paolini, Auditory Clinical Neuroscience Unit, Bionic Ear Institute, Melbourne, Australia

Cortical processing of human vs. non-human categories of action sounds, Lauren Engel, Aina Puce, James Lewis, Center for Advanced Imaging, West Virginia University, Morgantown, WV USA, Morgantown, USA

\section{SENSORY SYSTEMS}

Pain \& Autonomic Function

Perfusion based functional MRI study of thirst and satiation using an arterial spin labeling method, Tharushini Bowala ${ }^{1}$, Michael Farrell ${ }^{1}$, Michael McKinley ${ }^{l}$, Michael Mathai ${ }^{l}$, Robin McAllen ${ }^{1}$, Paddy Phillips ${ }^{2}$, Derek Denton ${ }^{3,4}$, Gary Egan ${ }^{1},{ }^{1}$ Howard Florey Institute, Florey Neurosciences Institutes, University of Melbourne, Parkville, Australia, ${ }^{2}$ Flinders University, Southern Adelaide Health Service and Repatriation General Hospital, Bedford Park, Australia, ${ }^{3}$ Office of the Dean, Faculty of Medicine, Dentistry and Health Sciences, University of Melbourne, Parkville, Australia, ${ }^{4}$ Baker Heart Research Institute, Alfred Hospital, Prahran, Australia 
EVOKED MAGNETIC BRAIN RESPONSES IN TRAUMATIC PERIPHERAL

NEUROPATHIC PAIN (before and after a local block), P.J. Theuvenet ${ }^{l}, B . W$. van Dijk ${ }^{l}$, Maria J. Peters ${ }^{I}$, F.L. Lopes da Silval ${ }^{1}$, J.M. van Ree ${ }^{I}$, Andrew C.N. Chen ${ }^{2},{ }^{I}$ Dept. of

Anesthesiology, Alkmaar Medical Center, Alkmaar, Netherlands, ${ }^{2}$ Center for Higher Brain

Functions, Capital Medical University, Beijing, China

Cortical Activation during the Urge to Cough in Healthy Volunteers, Lisan Ho ${ }^{l}$, Kevin McGuinness ${ }^{2}$, Douglas R Corfield ${ }^{3}$, Graham Kemp ${ }^{1}$, Sandy Jack ${ }^{4}$, John Earis ${ }^{4}$, Peter Calverley ${ }^{5}$, Neil Roberts ${ }^{1}$, Ashley Woodcock ${ }^{2}$,Jacky Smith ${ }^{2},{ }^{1}$ Magnetic Resonance \& Image Analysis Research Centre (MARIARC), University of Liverpool, Pembroke Place, Liverpool, United Kingdom, ${ }^{2}$ Respiratory Research Group, University of Manchester, Manchester, United Kingdom,

${ }^{3}$ Institute of Science \& Technology in Medicine, Keele University, Keele, United Kingdom,

${ }^{4}$ University Hospital Aintree, Liverpool, United Kingdom, ${ }^{5}$ School of Infection and Immunity,

University of Liverpool, Liverpool, United Kingdom

Augmented cerebral activation by lumbar mechanical stimulus in chronic low back pain patients - an fMRI study, Jiro Kurata ${ }^{1}$, Yoshitaka Kobayashi ${ }^{2}$, Mika Kokubun $^{3}$, Takashi Akaishizawa ${ }^{3}$, Miho Sekiguchi ${ }^{2}$, Shin-ichi Konno ${ }^{2}$, Shin-ichi Kikuchi ${ }^{2},{ }^{1}$ Department of Anesthesia, Teikyo University School of Medicine, Itabashi, Japan, ${ }^{2}$ Department of Orthopaedic Surgery, Fukushima Medical University School of Medicine, Fukushima, Japan, ${ }^{3}$ Department of Radiology, Southern Tohoku General Hospital, Koriyama, Japan

Mapping brain response to pain in fibromyalgia patients using temporal analysis of fMRI, Marina López-Solà ${ }^{I}$, Jesús Pujol ${ }^{1,2}$, Hector Ortiz ${ }^{1,3}$, Joan Carles Vilanova ${ }^{4}$, Benjamin Harrison ${ }^{1,5}$, Murat Yücel ${ }^{5}$, Carles Soriano-Mas ${ }^{1}$, Narcís Cardoner ${ }^{1,6}$, Carme Busquets ${ }^{7}$, Rosa HernándezRibas $^{1,6}$, Joan Deus ${ }^{8},{ }^{1}$ Institut d'Alta Tecnologia-PRBB, CRC Corporació Sanitària, Barcelona, Spain, ${ }^{2}$ Clinical Sciences Departament. Faculty of Medicine. University of Barcelona, Barcelona, Spain, ${ }^{3}$ Department of Electronic Engineering, Technical University of Catalonia, Barcelona, Spain, ${ }^{4}$ Magnetic Ressonance, Girona Clinic, Girona, Spain, ${ }^{5}$ Melbourne Neuropsychiatry Centre, Department of Psychiatry, Melbourne, Australia, ${ }^{6}$ Department of Psychiatry, Bellvitge University Hospital, Barcelona, Spain, ${ }^{7}$ Pain Unit, Girona Universitary Hospital Doctor Josep Trueta, Girona, Spain, ${ }^{8}$ Department of Clinical and Health Psychology, Autonomous University of Barcelona, Barcelona, Spain

Perceptual and activation differences between experimental muscle and cutaneous pain, Heather Cameron ${ }^{l}$, Arshad Zaman ${ }^{1}$, Neil Roberts ${ }^{I}$, Turo Nurmikko ${ }^{1,2}$, I University of Liverpool, Liverpool, United Kingdom, ${ }^{2}$ The Walton Centre for Neurology and Neurosurgery NHS Trust, Liverpool, United Kingdom

\section{EMOTION \& MOTIVATION Emotional Perception}

Regulation of Vagal Tone by Medial Prefrontal Cortex Varies By Emotional Valence, Richard D. Lane ${ }^{l}$, Kateri McCrae ${ }^{l, 2}$, Eric M. Reiman ${ }^{1,3,4}$, Carolyn L. Fort ${ }^{1}$, Julian F. Thayer ${ }^{5},{ }^{1}$ Department of Psychiatry, University of Arizona, Tucson, USA, ${ }^{2}$ Department of Psychology, Stanford University, Palo Alto, USA, ${ }^{3}$ Translational Genomics Research Institute, Phoenix, USA, ${ }^{4}$ Banner Alzheimer Institute, Banner Positron Emission Tomography Center, Banner Good Samaritan Medical Center, Phoenix, USA, ${ }^{5}$ Department of Psychology, Ohio State University, Columbus, USA 
11:30 - 12:30 You Yangs Hall (Level 3)

COGNITION \& ATTENTION

Attention (auditory, tactile, motor)
Neural mechanisms underlying error correction and spatial realignment during adaptation to optical wedge prisms, Heidi Chapman ${ }^{1}$, Ranmalee Eramudugolla ${ }^{2}$, Mark Strudwick ${ }^{3}$, Andrea Loftus ${ }^{4}$, Ross Cunnington ${ }^{2}$, Jason Mattingley ${ }^{2},{ }^{1}$ Department of Psychology, University of Melbourne, Melbourne, Australia, ${ }^{2}$ Queensland Brain Institute, University of Queensland, Brisbane, Australia, ${ }^{3}$ Centre for Magnetic Resonance, University of Queensland, Brisbane, Australia, ${ }^{4}$ Department of Psychology, University of Western Australia, Perth, Australia

An exploration of the cortical sources of the P3a, P3b and Novelty P3 sub-components of the ERP., Jacqueline Rushby ${ }^{1,2}$, Robert Barry ${ }^{I}$, Thomas Weickert ${ }^{2},{ }^{I}$ Brain \& Behaviour Research Institute and School of Psychology, University of Wollongong, Wollongong, Australia, ${ }^{2}$ Prince of Wales Medical Research Institute and School of Psychiatry, University of NSW, Randwick, Australia

Dance floor in the brain, Karsten Specht ${ }^{1,2}$, Berge Osnes ${ }^{1}$, Kenneth Hugdahl ${ }^{1,3}$, ${ }^{I}$ Department of Biological and Medical Psychology, University of Bergen, Bergen, Norway, ${ }^{2}$ Clinical Engineering Department, Haukeland University Hospital, Bergen, Norway, ${ }^{3}$ Division of Psychiatry and Bergen Mental Health Center, Haukeland University Hospital, Bergen, Norway

\section{COGNITION \& ATTENTION Attention (visual)}

Effect of endogenous attention on the human brain response to illusory line motion, Tomoaki Ayabe $^{1,2,4}$, Tomohiro Ishizu ${ }^{3}$, Tomokazu Urakawa ${ }^{l}$, Yoshiki Kaneoke ${ }^{l}$, Ryusuke Kakigi ${ }^{I},{ }^{1}$ National Institute for Physiological Science, Okazaki, Japan, ${ }^{2}$ The Graduate University for Advanced Studies, Okazaki, Japan, ${ }^{3}$ Keio University, Tokyo, Japan, ${ }^{4}$ Japan Sciety for Promotion of Science, Tokyo, Japan

The neurocognitive effects of donepezil on visual short-term memory capacity following $\mathbf{2 4} \mathbf{h}$ of sleep deprivation, Lisa Chuah ${ }^{1}$, Annette Chen ${ }^{1}$, Delise Chong ${ }^{l}$, Rekshan William ${ }^{1}$, Jiat-Chow Tan $^{l}$, Martin Pan ${ }^{2}$, Robert Lai ${ }^{2}$, Vincenzo Libri ${ }^{2}$, Michael Chee ${ }^{I},{ }^{1}$ Cognitive Neuroscience Lab, Duke-NUS Graduate Medical School, Singapore, Singapore, ${ }^{2}$ Neurology Centre of Excellence of Drug Discovery, GlaxoSmithKline, Harlow, United Kingdom

Neuroanatomical correlates of performance enhancement by nicotine under conditions of selective attention, divided attention and stimulus detection, Britta Hahn ${ }^{l}$, Thomas J. Ross ${ }^{l}$, Frank A. Wolkenberg ${ }^{1}$, Diaa M. Shakleya ${ }^{2}$, Marilyn A. Huestis ${ }^{2}$, Elliot A. Stein ${ }^{I},{ }^{\text {INational }}$ Institute on Drug Abuse, Neuroimaging Research Branch, Baltimore, USA, ${ }^{2}$ National Institute on Drug Abuse, Chemistry and Drug Metabolism Section, Baltimore, USA

Early attention: local modulations and network changes, Andreas A. Ioannides ${ }^{l}$, Vahe Poghosyan ${ }^{2}$, Marotesa Voultsidou, ${ }^{\prime}{ }^{I}$ RIKEN, Brain Science Institute (BSI), Laboratory for Human Brain Dynamics, Wako-shi, Japan, ${ }^{2}$ AAI Scientific and Cultural Services Limited, Laboratory for Human Brain Dynamics, Nicosia, Cyprus

Change of ERP features with respect to the task difficulty of visual oddball task, Kyung Hwan Kim, Ja Hyun Kim, Jin Yun, Department of Biomedical Engineering Yonsei University, Wonju, South Korea

Activation in V1 reflects the local saliency of pop-out stimuli, Lucia Melloni ${ }^{1,2}$, Sara van Leeuwen $^{1,2}$, Arjen Alink ${ }^{2,3}$, Notger Müller ${ }^{1,2},{ }^{l}$ Cognitive Neurology Unit, Johann Wolfgang Goethe-University, Frankfurt am Main, Germany, ${ }^{2}$ ) Brain Imaging Center, Frankfurt am Main, Germany, ${ }^{3}$ ) Department of Neurophysiology, Max Planck Institute for Brain Research, Frankfurt am Main, Germany

Conflict resolution in a focused visual attentional task. A MEG study, Carmen Santisteban ${ }^{1,2}$, Jesus M. Alvarado ${ }^{1,2}$, Manuel Cortijo ${ }^{1,3}$, 'Instituto de Estudios Biofuncionales, Madrid, Spain,

${ }^{2}$ Facultad de Psicologia, Madrid, Spain, ${ }^{3}$ Facultad de Farmacia, Madrid, Spain 
Selectivity of visual attention is relatively preserved following $24 \mathrm{~h}$ of sleep deprivation, Jiat Chow Tan, Delise Chong, William Rekshan, Michele Veldsman, Annette Chen, Michael Chee, Cognitive Neuroscience Laboratory, Duke-NUS Graduate Medical School, Singapore, Singapore

\section{EMOTION \& MOTIVATION \\ Emotional Learning}

The influence of contingency awareness on neural responses, valence ratings and skin conductance responses in a picture-picture conditioning paradigm, Tim Klucken, Rudolf Stark, 51 T-AM Dieter Vaitl, Bender Institute of Neuroimaging, Giessen, Germany

\section{COGNITION \& ATTENTION Cognitive Aging}

Age-related slowing of task switching is associated with decreased integrity of frontoparietal white matter, Brian Gold ${ }^{1}$, David Powell ${ }^{2}$, Liang Xuan ${ }^{2}$, Greg Jicha ${ }^{3}$, Charles Smith ${ }^{2,3},{ }^{1}$ Anatomy and Neurobiology, Lexington, USA, ${ }^{2}$ Magnetic Resonance Imaging and Spectroscopy Center, Lexington, USA, ${ }^{3}$ Neurology, Lexington, USA

Functional and structural changes in the ageing human brain: how EEG and (f)MRI measures complement each other to further our understanding of cognitive aging, $M . M$. Lorist $^{1,2}$, N.M. Maurits ${ }^{2,3},{ }^{1}$ Department of Experimental and Work Psychology, University of Groningen, Groningen, Netherlands, ${ }^{2} 2 B C N$-NeuroImaging Center, University Medical Center Groningen, University of Groningen, Groningen, Netherlands

Normal Aging: an Executive Function FMRI Study, David Zhu ${ }^{l, 2}$, Rose Zacks ${ }^{l}$, Jill Slade ${ }^{2}$, ${ }^{I}$ Department of Psychology, Michigan State University, East Lansing, USA, ${ }^{2}$ Department of Radiology, Michigan State University, East Lansing, USA

\section{COGNITION \& ATTENTION Cognitive Development}

DEVELOPMENTAL MATURATION OF NEURAL SYSTEMS SUBSERVING

CALCULATION AND ITS ALTERATION IN A CASE OF MATH LEARNING

DISABILITY, Paul Eslinger, Clancy Blair, David Baker, Jianli Wang, Jennifer Realmuto, Qing Yang, Penn State University, Hershey, USA

Differences in Interhemispheric Communication Due to Handedness: a Structural and Functional Study, Sarina Iwabuchi, Ian Kirk, Research Centre for Cognitive Neuroscience, Department of Psychology, University of Auckland, Auckland, New Zealand

Where in the brain is intelligence: a diffusion tensor imaging study on mental retardation

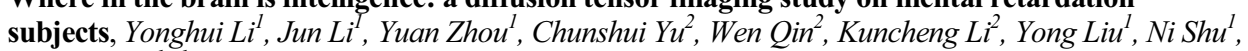
Tianzi Jiang ${ }^{I},{ }^{I}$ National Laboratory of Pattern Recognition, Institute of Automation, Chinese Academy of Sciences, Beijing, China, ${ }^{2}$ Department of Radiology, Xuanwu Hospital of Capital Medical University, Beijing, China

Developmental Changes in Verbal Working Memory Load-Dependency: An fMRI Investigation, Elizabeth $O^{\prime}$ Hare $^{1,2}$, Lisa Lu ${ }^{1,4}$, Suzanne Houston ${ }^{l}$, Sarah McCourt ${ }^{1}$, Susan Bookheimer ${ }^{2,3}$, Elizabeth Sowell ${ }^{1,2},{ }^{1}$ UCLA Laboratory of Neuro Imaging, Los Angeles, USA, ${ }^{2}$ UCLA Interdepartmental Neuroscience Program, Los Angeles, USA, ${ }^{3}$ UCLA Department of Psychiatry and Biobehavioral Sciences, Los Angeles, USA, ${ }^{4}$ Roosevelt University, Dept. of Psychology, Chicago, USA

\section{COGNITION \& ATTENTION \\ Perception, Imagery, Awareness}

Neural correlates of human body perception, Rosanne Aleong ${ }^{l}$, Tomas Paus ${ }^{1,2},{ }^{l}$ Cognitive Neurosciences Unit, Montreal Neurological Institute, Montreal, Canada, ${ }^{2}$ Brain \& Body Centre, University of Nottingham, Nottingham, United Kingdom

Fragmentation of fMRI resting state networks (RSN) in deep non Rapid Eye Movement (REM) sleep as compared to wakefulness as revealed by a group probabilistic ICA analysis in healthy volunteers, Melanie Boly ${ }^{1,2}$, Vincent Perlbarg ${ }^{3}$, Guillaume Marrelec ${ }^{3}$, Thanh Dang-Vu ${ }^{2,4}$, 
Manuel Schabus ${ }^{5}$, Melanie Pelegrini ${ }^{3}$, Audrey Vanhaudenhuyse ${ }^{1}$, Genevieve Albouy ${ }^{4}$, Evelyne Balteau $^{4}$, Christophe Phillips ${ }^{4}$, Virginie Sterpenich ${ }^{4}$, Gilles Vandewalle ${ }^{4}$, Andre Luxen ${ }^{4}$, Steven Laureys ${ }^{1,2}$, Habib Benali ${ }^{3}$, Pierre Maquet ${ }^{2,4},{ }^{1}$ Coma Science Group, Cyclotron Research Center, University of Liège, Liège, Belgium, ${ }^{2}$ Neurology Department, CHU Sart Tilman Hospital, University of Liège, Liège, Belgium, ${ }^{3}$ Inserm, U678 and Pierre et Marie Curie University, Faculty of medicine Pitie-Salpetriere, Paris, France, ${ }^{4}$ Cyclotron Research Center, University of Liège, Liège, Belgium, ${ }^{5}$ Department of Psychology, University of Salzburg, Salzburg, Austria

Neuronal ensemble dynamics during a fast visual recognition task: application of Segmental analysis in an event-related design, Sergey Borisov ${ }^{1,2,3}$, Sergey Shishkin ${ }^{2}$, Andrei Medvedev ${ }^{I}$, Alexander Kaplan ${ }^{2}$, John VanMeter ${ }^{I},{ }^{l}$ Center for Functional and Molecular Imaging, Georgetown University Medical Center, Washigton, USA, ${ }^{2}$ Dept. of human physiology, Biological faculty, M.V.Lomonosov Moscow State University, Moscow, Russia, ${ }^{3}$ Brain Image Center and Dept. of Neurology, Johann Wolfgang Goethe University Clinic, Frankfurt am Main, Germany

The processing of different face dimensions depends on attention, but not only: an fMRadaptation study, Kathrin Cohen Kadosh ${ }^{1}$, Richard N. A. Henson ${ }^{2}$, Roi Cohen Kadosh ${ }^{3}$, Mark H. Johnson ${ }^{l}$, Frederic Dick ${ }^{1,4}$, 'Centre for Brain and Cognitive Development, School of Psychology, Birkbeck College, London, United Kingdom, ${ }^{2}$ MRC Cognition \& Brain Sciences Unit, Cambridge University, Cambridge, United Kingdom, ${ }^{3}$ Institute of Cognitive Neuroscience \& Department of Psychology, University College London, London, United Kingdom, ${ }^{4}$ Center for Research in Language, University of California, San Diego, USA

Social Modulation of Touch Representation - an fMRI study, valeria gazzola ${ }^{l}$, michael spezio $^{2}$, fulvia castelli ${ }^{2}$, ralph adolphs ${ }^{2}$, christian keysers ${ }^{1,2}$, ISocial Brain Lab, BCNNeuroImaging Center, University Medical Center Groningen, University of Groningen, groningen, Netherlands, ${ }^{2}$ Division of Humanities and Social Sciences, Caltech, Pasadena, USA

Neural correlates of bodily self-awareness, Pär Halje, Bigna Lenggenhager, Olaf Blanke, Laboratory of Cognitive Neuroscience, Brain Mind Institute, Ecole Polytechnique Federale de Lausanne, Lausanne, Switzerland

Local ongoing BOLD fluctuations in hMT+ bias the perception of visual motion, Guido Hesselmann ${ }^{l}$, Christian Kell ${ }^{2}$, Evelyn Eger ${ }^{I}$, Andreas Kleinschmidt ${ }^{I},{ }^{l}$ CEA Neurospin, INSERM U562, Gif-sur-Yvette, France, ${ }^{2}$ University of Frankfurt, Dep. of Neurology, Frankfurt, Germany

Dynamic neural responses during 3-D object structure perception from motion, Sunao Iwaki ${ }^{1,2}$, Giorgio Bonmassar ${ }^{2}$, John W. Belliveau ${ }^{2},{ }^{1}$ Natl Inst Adv Indust Sci \& Tech, Ikeda, Japan, ${ }^{2}$ Mass General Hospital, Boston, USA

\section{COGNITION \& ATTENTION Reasoning \& Problem Solving}

Voxel-based Lesion Symptom Mapping and White Matter Tractography: Analysis of Regions Mediating Non-verbal Reasoning, Juliana Baldo ${ }^{1}$, Nina Dronkers ${ }^{1,2,3}$, And Turken ${ }^{1},{ }^{1}$ VA 115 T-AM Northern California Health Care System, Martinez, USA, ${ }^{2}$ University of California, Davis, USA,

${ }^{3}$ University of California, San Diego, USA

The Effects of Choice on Discourse Processing: An fMRI Study, Eunsoo Cho, Sun-Hee Back, Yoonkyung Chung, Sung-il Kim, Korea University, Seoul, Korea

General Intelligence ( $g$ ) and Intelligence in General (FSIQ) as Manifested in the Brain, Rex E. Jung ${ }^{1,2,3}$, H. Jeremy Bockholt ${ }^{I}$, Judith Segall ${ }^{l}$, Arvind Caprihan ${ }^{l}$, Shirley Smith ${ }^{l}$, Robert Chavez', Ronald A. Yeo ${ }^{3}$, Richard J. Haier ${ }^{4},{ }^{1}$ MIND Research Network, Albuquerque, USA, ${ }^{2}$ Department of Neurology, University of New Mexico, Albuquerque, USA, ${ }^{3}$ Department of Psychology, Albuquerque, USA, ${ }^{4}$ Department of Medicine, University of California, Irvine, USA

Integrating information in conditional reasoning: an EEG study, Jean-Baptiste Van der Henst, Mathilde Bonnefond, CNRS, Laboratoire sur le Langage le Cerveau et la Cognition, Bron, France 
COGNITION \& ATTENTION

Space, Time, \& Number Coding

Navigation in a virtual office landscape; effects of landmarks and obstacles, Carl S Pintzka, Hallvard Evensmoe, Jian Xu, Hanne Lehn, Asta Håberg, Department of Circulation and Medical Imaging, Norwegian University for Science and Technology (NTNU), Trondheim, Norway

Parietal areas involved in format-independent representation of mathematical functions, Anna Wilson ${ }^{1}$, Mike Thomas ${ }^{2}$, Vanessa Lim ${ }^{1}$, Michael Corballis ${ }^{1},{ }^{I}$ Department of Psychology, The University of Auckland, Auckland, New Zealand, ${ }^{2}$ Department of Mathematics, The University of Auckland, Auckland, New Zealand

\section{DISORDERS OF THE NERVOUS SYSTEM Alzheimer \& Dementia}

Patterns of Brain Activation in Persons at Genetic Risk for Alzheimer's disease: An fMRI Follow-Up, Alison Burggren ${ }^{l}$, Kenji Ogura ${ }^{I}$, Jesse Brown ${ }^{l, 2}$, Gary Small ${ }^{l}$, Susan Bookheimer ${ }^{I}$, ${ }^{1}$ UCLA Department of Psychiatry and Biobehavioral Sciences, Los Angeles, USA, ${ }^{2}$ UCLA Neuroscience IDP, Los Angeles, USA

The Substantia Innominata in Mild Cognitive Impairment: Implications as a Potential Biomarker, Terence Chua ${ }^{l, 2}$, Wei Wen ${ }^{1,2}$, Xiaohua Chen ${ }^{1,2}$, Perminder Sachdev ${ }^{1,2}$,

${ }^{I}$ Neuropsychiatric Institute, Euroa Centre, Prince of Wales Hospital, Randwick, NSW 2031, Sydney, Australia, ${ }^{2}$ School of Psychiatry, University of New South Wales, NSW 2052, Sydney, Australia

Reduced precuneus deactivation during object naming in dementia, Lars Frings ${ }^{1,2,6}$, Katharina Dressel ${ }^{1,5}$, Stefanie Abel ${ }^{1,5}$, Dorothee Saur ${ }^{1,3}$, Dorothee Kümmerer ${ }^{1,3}$, Hansjörg Mast ${ }^{1,4}$, Cornelius Weiller ${ }^{1,3}$, Michael Hüll, ${ }^{2,6}$, IFreiburg Brain Imaging, University of Freiburg, Freiburg, Germany, ${ }^{2}$ Gerontopsychiatry \& Neuropsychology Section, Department of Psychiatry \& Psychotherapy, University Medical Center, Freiburg, Freiburg, Germany, ${ }^{3}$ Department of Neurology, University Medical Center, Freiburg, Freiburg, Germany, ${ }^{4}$ Department of Neuroradiology, University Medical Center, Freiburg, Freiburg, Germany, ${ }^{5}$ Neurolinguistics Section, RWTH Aachen University, Aachen, Germany, ${ }^{6}$ Centre of Geriatrics and Gerontology, Freiburg, Germany

Reverse association between corpus callosum size and interhemispheric efficency in normal aging and Alzheimer's disease, Jennyfer Ansado ${ }^{1,2}$, Sven Joubert ${ }^{1,3}$, Sylvane Faure ${ }^{2}$, Yves Joanette ${ }^{I},{ }^{I}$ Centre de Recherche, IUGM \& Faculté de médecine, Université de Montréal, Montréal, Canada, ${ }^{2}$ Laboratoire de Psychologie Expérimentale et Quantitative, Université Nice-Sophia Antipolis, Nice, France, ${ }^{3}$ Département de psychologie et CERNEC, Montréal, Canada

Volumetric reduction of anterior medial temporal lobe structures precedes amnestic mild cognitive impairment, Sarah Martin ${ }^{1}$, Charles Smith ${ }^{2,4,5}$, Fred Schmitt ${ }^{2,3}$, Brian Gold ${ }^{1,5}$, ${ }^{I}$ Department of Anatomy and Neurobiology, Lexington, USA, ${ }^{2}$ Department of Neurology, Lexington, USA, ${ }^{3}$ Department of Psychiatry, Lexington, USA, ${ }^{4}$ Alzheimer's Disease Center and Sanders-Brown Center on Aging, Lexington, USA, ${ }^{5}$ Magnetic Resonance Imaging and Spectroscopy Center, Lexington, USA

Changes in serotonin transporter density in Alzheimer's disease, Yasuomi Ouchi ${ }^{l}$, Etsuji Yoshikawa ${ }^{2}$, Masami Futatsubashi ${ }^{2}$, Toshihiko Kanno ${ }^{3}$, Genichi Sugihara ${ }^{4}$, Kazuhiko Nakamura ${ }^{4}$, Yasuhiro Magata ${ }^{l},{ }^{1}$ Molecular Imaging Frontier Res Ctr, Hamamatsu University School of Medicine, Hamamatsu, Japan, ${ }^{2}$ Hamamatsu Photonics KK, Hamamatsu, Japan, ${ }^{3}$ Hamamatsu Medical Ctr, Hamamatsu, Japan, ${ }^{4}$ Psychiatry, Hamamatsu University School of Medicine, Hamamatsu, Japan

Cortical Thickness Mediates Relationships between Lesion Area and Verbal Working Memory Performance in Multiple Sclerosis, Lawrence Sweet ${ }^{1,2}$, Denise Cote ${ }^{2}$, Stephen Rao ${ }^{3}$, Emily Trittschuh ${ }^{4}$, Beth Jerskey ${ }^{1}$, Richard Mulligan ${ }^{2}$, James Paskavitz ${ }^{5},{ }^{1}$ Warren Alpert Medical School of Brown University, Providence, USA, ${ }^{2}$ Butler Hospital, Providence, USA, ${ }^{3}$ Cleveland Clinic, Cleveland, USA, ${ }^{4}$ Northwestern University, Chicago, USA, ${ }^{5}$ Perceptive Informatics, Waltham, USA 


\section{DISORDERS OF THE NERVOUS SYSTEM Mood \& Anxiety Disorders}

Turboprop-DTI Reveals White Matter Abnormalities in Social Anxiety Disorder., Anton Orlichenko ${ }^{1}$, K. Luan Phan ${ }^{2}$, Huiling Peng ${ }^{3}$, Emil F. Coccaro ${ }^{4}$, Konstantinos Arfanakis ${ }^{3}$, ${ }^{I}$ Department of Electrical and Computer Engineering, Illinois Institute of Technology, Chicago, USA, ${ }^{2}$ Department of Psychiatry, University of Michigan, Ann Arbor, USA, ${ }^{3}$ Department of Biomedical Engineering, Illinois Institute of Technology, Chicago, USA, ${ }^{4}$ Department of Psychiatry, University of Chicago, Chicago, USA

Magnetic resonance volumetric analysis of brain regions in body dysmorphic disorder, Jamie Feusner ${ }^{l}$, Jennifer Townsend ${ }^{2}$, Alexander Bystritsky ${ }^{I}$, Malin McKinley ${ }^{I}$, Susan Bookheimer ${ }^{2}$, ${ }^{1}$ UCLA Semel Institute for Neuroscience and Human Behavior, Los Angeles, USA, ${ }^{2}$ UCLA Center for Cognitive Neuroscience, Los Angeles, USA

Evidence of dysfunctional pain inhibition in Fibromyalgia reflected in rACC during provoked pain, Karin Jensen ${ }^{l}$, Eva Kosek ${ }^{1}$, Frank Petzke ${ }^{2}$, Peter Fransson ${ }^{l}$, Hanke Marcus ${ }^{2}$, Steven C R Williams ${ }^{3}$, Serena Carville ${ }^{3}$,Ernest Choy ${ }^{3}$, Yves Mainguy ${ }^{4}$, Richard Gracely ${ }^{5}$, Martin Ingvar ${ }^{I},{ }^{1}$ Karolinska Institute, Stockholm, Sweden, ${ }^{2}$ University hospital of Cologne, Cologne, Germany, ${ }^{3}$ Kings College, London, United Kingdom, ${ }^{4}$ Pierre Fabre Médicament, Labège, France, ${ }^{5}$ University of Michigan, Ann Arbour, USA

Response to task failure is modulated by past depression and rumination, Emma Pegg, Shane McKie, Bill Deakin, Ian Anderson, Rebecca Elliott, Neuroscience and Psychiatry Unit, University of Manchester, Manchester, United Kingdom

Aberrant intrinsic functional organization in medication-naïve patients with first depressive episode revealed by resting-state fMRI, Yuan Zhou ${ }^{1,2}$, Chuishui $\mathrm{Yu}^{3}$, Yong Liu ${ }^{2}$, Ming Song ${ }^{2}$, Kuncheng $\mathrm{Li}^{3}$, Tianzi Jiang ${ }^{2},{ }^{1}$ Center for Social and Economic Behavior, Institute of Psychology, Chinese Academy of Sciences, Beijing 100101, P. R. China, Beijing, China, ${ }^{2}$ National Laboratory of Pattern Recognition, Institute of Automation, Chinese Academy of Sciences, Beijing 100080, P. R. China, Beijing, China, ${ }^{3}$ Department of Radiology, Xuanwu Hospital of Capital University of Medical Science, Beijing 100053, P. R. China, Beijing, China

\section{DISORDERS OF THE NERVOUS SYSTEM Parkinson's Disease \& Other Basal Ganglia}

SPM Analysis of F-18 FDG PET in Parkinson's Syndrome Patients with Urinary Dysfunction, Kyung Hoon Hwang ${ }^{l}$, Nam-Bum Kim ${ }^{2}$, Min-Kyung Lee ${ }^{l}$, Wonsick Choe ${ }^{l},{ }^{l}$ Gachon Univ Gil Hospital, Incheon, South Korea, ${ }^{2}$ Gachon Univ Neuroscience Research Institute, Incheon, South Korea

Effects of Subthalamic Nucleus Deep Brain Stimulation on Parkinsonian Resting Tremor : An MEG Study, Hame Park ${ }^{1,4}$, June Sic Kim ${ }^{1,2}$, Sun Ha Paik2, Beom Seok Jeon ${ }^{3,4}$, Chun Kee Chung ${ }^{1,2,4}$, Jee-Young Lee ${ }^{3},{ }^{1}$ MEG Center, Seoul National University Hospital, Seoul, Korea, ${ }^{2}$ Department of Neurosurgery, Seoul National University College of Medicine, Seoul, Korea, ${ }^{3}$ Department of Neurology, Seoul National University College of Medicine, Seoul, Korea,

${ }^{4}$ Interdisciplinary Program in Cognitive Science, Seoul National University, Seoul, Korea

Evidence for cortical and subcortical alterations in Restless Legs Syndrome: the pathoanatomy of RLS revisited, Alexander Unrath ${ }^{1}$, Hans-Peter Mueller ${ }^{1}$, Freimuth Juengling ${ }^{2}$, Jan Kassubek ${ }^{l},{ }^{1}$ University of Ulm, Department of Neurology, Ulm, Germany, ${ }^{2}$ St. Clara Spital, Department of Nuclear Medicine, Basel, Switzerland

\section{DISORDERS OF THE NERVOUS SYSTEM Schizophrenia}

GREY MATTER VOLUME INCREASE AFTER EARLY ANTIPSYCHOTIC TREATMENT IN DRUG NAÏVE, FIRST EPISODE SCHIZOPHRENIC COHORT, $Y i$ Deng $^{l}$, Gráinne McAlonan ${ }^{l}$, Hasan Merali ${ }^{2}$, Charlton Cheung ${ }^{l}$, Vinci Cheung ${ }^{l}$, Eric Chen ${ }^{l}$, Siew Chual, ' Department of Psychiatry, The University of Hong Kong, Hong Kong, Hong Kong,

${ }^{2}$ Harvard Medical School, Harvard University, Boston, USA

171 T-AM 
Trial-by-trial analysis of combined EEG and fMRI shows dynamic of cognitive function in healthy controls and patients with schizophrenia, Ana Diukova, Pavan Malikarjun, Dorothee Auer, Peter Liddle, Institute of Neuroscience, University of Nottingham, Nottingham, United Kingdom

Multimodal neuroimaging of executive-emotional processing in adolescents at genetic risk for schizophrenia, Sarah Hart ${ }^{1,3}$, Guido Gerig ${ }^{4}$, Diana Perkins ${ }^{2}$, Joseph Blocher $^{2}$, Joshua Bizzell, ${ }^{2,}$, Justin Woodlief ${ }^{3}$, Hongbin Gu ${ }^{2}$, Aysenil Belger ${ }^{1,2,3},{ }^{1}$ Neurodevelopmental Disorders Research Center, University of North Carolina at Chapel Hill, Chapel Hill, USA, ${ }^{2}$ Department of Psychiatry, University of North Carolina at Chapel Hill School of Medicine, Chapel Hill, USA, ${ }^{3}$ Duke-UNC Brain Imaging and Analysis Center, Duke University Medical Center, Durham, USA, ${ }^{4}$ Scientific Computing and Imaging Institute, University of Utah, Salt Lake City, USA

Dysfunctional modulation of emotional interference in the medial prefrontal cortex in schizophrenia, Il Ho Park ${ }^{l, 2}$, Hae-Jeong Park ${ }^{3}$, Ji-Won Chun ${ }^{2}$,Eung Yeop Kim ${ }^{3}$, Jae-Jin Kim $^{1,2,3}{ }^{1}{ }^{1}$ Department of Psychiatry, Yonsei University College of Medicine, Seoul, South Korea, ${ }^{2}$ Institute of Behavioral Science in Medicine, Yonsei University College of Medicine, Seoul, South Korea, ${ }^{3}$ Department of Diagnostic Radiology, Yonsei University College of Medicine, Seoul, South Korea

\section{EFFECT OF EARLY ANTIPSYCHOTIC TREATMENT ON CAUDATE AND AMYGDALA VOLUME IN NEUROLEPTIC NAÏVE, NEWLY DIAGNOSED} SCHIZOPHRENIA, Meikei Leung ${ }^{I}$, Siew Chua ${ }^{l}$, Hasan Merali ${ }^{2}$, Yi Deng ${ }^{l}$, Charlton Cheung ${ }^{I}$, Vinci Cheungl, Eric Chen ${ }^{l}$, Gráinne McAlonan ${ }^{l},{ }^{l}$ Department of Psychiatry, The University of Hong Kong, Hong Kong, Hong Kong, ${ }^{2}$ Harvard Medical School, Harvard University, Boston, USA

Cortical and Subcortical Reward Prediction Error Learning in Psychosis, Graham Murray $^{1,2,3}$, Phil Corlett ${ }^{1,3}$, Luke Clark ${ }^{1,3}$, Mathias Pessiglione ${ }^{4}$, Ed Bullmore ${ }^{1,2,3}$, Peter Jones ${ }^{1,2,3}$, Garry Honey, Andy Blackwell ${ }^{1}$, Trevor Robbins ${ }^{3}$, Paul Flethcer ${ }^{1,2,3},{ }^{1}$ Brain Mapping Unit, Department of Psychiatry, University of Cambridge, Cambridge, United Kingdom, ${ }^{2}$ CAMEO Early Psychosis Service, Cambridge, United Kingdom, ${ }^{3}$ Behavioural and Clinical Neuroscience Institute, Cambridge, United Kingdom, ${ }^{4}$ Pitié-Salpêtrière Hospital, Paris, France

Cerebellar grey and white matter changes associated with cannabis use in schizophrenia and in healthy controls., Nadia Solowij ${ }^{1,2}$, Colleen Respondek ${ }^{l}$, Sarah Whittle ${ }^{3}$, Evelyn Lindsay ${ }^{3}$, Dan Lubman ${ }^{3,4}$, Murat Yücel ${ }^{3,4},{ }^{1}$ School of Psychology and Illawarra Institute for Mental Health, University of Wollongong, Wollongong, NSW, Australia, ${ }^{2}$ Schizophrenia Research Institute, Sydney, NSW, Australia, ${ }^{3}$ Melbourne Neuropsychiatry Centre, Department of Psychiatry, University of Melbourne and Melbourne Health, Melbourne, VIC, Australia, ${ }^{4}$ ORYGEN Research Centre, Melbourne, VIC, Australia

$\mathbf{T}_{2}$ relaxometry detects temporal lobe pathology in people at ultra-high risk for psychosis, Damien Kennedy ${ }^{1,2}$, Lisa Phillips ${ }^{2}$, Pat McGorry ${ }^{3}$, Alison Yung ${ }^{3}$, Marc Seal ${ }^{1}$, Dennis Velakoulis ${ }^{1}$, Christos Pantelis ${ }^{1}$, Stephen Wood ${ }^{l},{ }^{l}$ Melbourne Neuropsychiatry Centre, University of Melbourne, Melbourne, Australia, ${ }^{2}$ Department of Psychology, University of Melbourne, Melbourne, Australia, ${ }^{3}$ ORYGEN Research Centre, University of Melbourne, Melbourne, Australia

\section{EMOTION \& MOTIVATION} Reward

Cue reactivity in abstinent problem gamblers and nicotine dependent persons: an fMRI study, Anna Goudriaan ${ }^{1}$, Michiel De Ruiter ${ }^{2,4}$, Dick Veltman ${ }^{1,2}$, Jaap Oosterlaan ${ }^{3}$, Wim van den Brink ${ }^{1},{ }^{1}$ University of Amsterdam, Department of Psychiatry, Academic Medical Center, Amsterdam, Netherlands, ${ }^{2}$ Department of Psychiatry, VUmc, Vrije Universiteit Amsterdam, Amsterdam, Netherlands, ${ }^{3}$ Department of Clinical Neuropsychology, Vrije Universiteit Amsterdam, Amsterdam,

Netherlands, ${ }^{4}$ Netherlands Cancer Institute, NKI, Amsterdam, Netherlands

Incentive-induced performance decrements in a reward pursuit task, Dean Mobbs ${ }^{1,2}$, Demis Hassabis $^{l}$, Ben Seymour ${ }^{l}$, Jennifer Marchant ${ }^{l}$, Nikolaus Weiskopf ${ }^{\prime}$, Ray Dolan ${ }^{I}$, Chris Frith ${ }^{1,3}$,

${ }^{I}$ Wellcome Trust Centre for Neuroimaging, University College London, London, United Kingdom,

${ }^{2}$ MRC-Cognition and Brain Sciences Unit, Cambridge, United Kingdom, ${ }^{3}$ Niels Bohr project

"Interacting Minds", CFIN, University of Aarhus, Aarhus, Denmark 
Modulation of the Orbitofrontal Cortex as a Function of Expertise, Martin Skov ${ }^{l}$, Ulrich Kirk ${ }^{2}$, Mark S. Christensen ${ }^{I, 3}$, Niels Nygaard ${ }^{4},{ }^{\prime}$ Danish Research Centre for Magnetic Resonance, Copenhagen University Hospital Hvidovre, Copenhagen, Denmark, ${ }^{2}$ Anatomy Department, Wellcome Department of Imaging Neuroscience, University, London, United Kingdom, ${ }^{3}$ Institute for Physical Exercise and Sport Science, University of Copenhagen, Copenhagen, Denmark, ${ }^{4}$ Institute for Architecture and Aesthetics, Aarhus School of Architecture, Aarhus, Denmark

Aberrant reward network connectivity in obese women in response to high- and low-calorie food images, Luke Stoeckel ${ }^{1}$, Jieun Kim ${ }^{2}$, Rosalyn Weller ${ }^{1}$, James Cox ${ }^{1}$, Barry Horwitz ${ }^{2}$,

${ }^{I}$ Department of Psychology, University of Alabama at Birmingham (UAB), Birmingham, USA,

${ }^{2}$ Brain Imaging and Modeling Section, NIDCD, NIH, Bethesda, USA

Cue-reactivity and subjective craving in abstinent opiate-dependent males: an fMRI study., Fleur Zijlstra ${ }^{1,2,3}$, Dick Veltman ${ }^{2}$, Jan Booij ${ }^{3}$, Wim van den Brink ${ }^{2}$, Ingmar H.A. Franken ${ }^{4}$,

${ }^{1}$ Amsterdam Institute for Addiction Research, Amsterdam, Netherlands, ${ }^{2}$ Department of

Psychiatry, Academic Medical Centre, Amsterdam, Netherlands, ${ }^{3}$ Department of Nuclear Medicine, Academic Medical Centre, Amsterdam, Netherlands, ${ }^{4}$ Institute of Psychology, Erasmus University Rotterdam, Rotterdam, Netherlands

\section{EMOTION \& MOTIVATION Social Behavior}

In the blink of an eye: Similar N170 but different late ERPs while viewing blinks vs. meaningful eye movements., Julie Brefczynski-Lewis, Michael Berrebi, Marie McNeely, Aina Puce, Center for Advanced Imaging, West Virginia University, Morgantown, USA

Gender differences in the neural correlates of empathic behavior, Birgit Derntl, Andreas Finkelmeyer, Thilo Kellermann, Timur Toygar, Frank Schneider, Irina Falkenberg, Ute Habel, Department of Psychiatry and Psychotherapy, University Aachen RWTH, Aachen, Germany

Moral emotion to usual behaviors - a NIRS study -, Hirotoshi HIRAISHI, Primate Research Institute, Kyoto Univ, INuyama, Aichi, Japan

Differences between physical and social cognition: An ERP study, Yue-Jia Luo ${ }^{1,2}$, Junfeng $G u o^{2},{ }^{l}$ State Key Laboratory of Cognitive Neuroscience and Learning $\square$ Beijing Normal University, Beijing, China, ${ }^{2}$ Key Laboratory of Mental Health, Institute of Psychology, The Chinese Academy of Sciences, Beijing, China

Effects of Gonadectomy on Social Behavior, Cognition, and Amygdala Volume in the Rhesus Macaque, A. Brent Richards ${ }^{l}$, Sarah Ward ${ }^{l}$, Debora Rothmond ${ }^{2}$, Stephanie Schmitz ${ }^{3}$, Rhoshel Lenroot ${ }^{4}$, Jay Giedd ${ }^{4}$, Pam Noble ${ }^{3}$, Ruth Woodward ${ }^{5}$, James Winslow ${ }^{3}$, Cynthia Shannon Weickert ${ }^{2}$, ${ }^{I}$ MiNDS Unit, National Institute of Mental Health, Bethesda, USA, ${ }^{2}$ Neuroscience Institute of Schizophrenia and Allied Disorders, University of New South Wales, Randwick, Australia, ${ }^{3}$ Nonhuman Primate Core Facility, National Institute of Mental Health, Poolesville, USA, ${ }^{4}$ Child Psychiatry Branch, National Institute of Mental Health, Bethesda, USA, ${ }^{5}$ Research Animal Management Branch, National Institute of Child Health and Human Development, Bethesda, USA

\section{GENETICS}

\section{VOLUMETRIC DIFFERENCES IN BRAIN STRUCTURE IN IDENTICAL AND FRATERNAL TWINS COMPUTED USING RIEMANNIAN TENSOR-BASED} MORPHOMETRY, Caroline Brun ${ }^{1}$, Natasha Lepore ${ }^{l}$, Xavier Pennec ${ }^{2}, Y$-Y Y Chou ${ }^{l}$, Agatha D. Lee $^{I}$, Marina Barysheval, Katie McMahon ${ }^{3}$, Greig de Zubicaray ${ }^{3}$, Margie Wright ${ }^{4}$, Arthur W. Toga ${ }^{I}$, Paul M. Thompson ${ }^{I},{ }^{1}$ Laboratory of Neuro Imaging, UCLA, Los Angeles, USA, ${ }^{2}$ Asclepios Research Project, INRIA, Sophia-Antipolis, France, ${ }^{3}$ Centre for Magnetic Resonance, University of Queensland, Brisbane, Australia, ${ }^{4}$ Genetic Epidemiology Lab, Queensland Institute of Medical Research, Brisbane, Australia

BDNF impacts on brain structure of patients with schizophrenia, Kempf Lucas ${ }^{1}$, Robyn Honea $^{2}$, Bhaskar Kolachana ${ }^{l}$, Kolachana Mattay ${ }^{l}$, Andreas Meyer-Lindenberg ${ }^{3}$, Daniel Weinberger ${ }^{I},{ }^{I}$ GCAP/NIMH/NIH, Bethesda, USA, ${ }^{2}$ KUMC, Kansas City, USA, ${ }^{3}$ Central Institue of Mental Health, Manneheim, Germany 
BDNF val66met polymorphism and short-term, experience-dependent plasticity in motor cortex of elderly human subjects, Stephanie McHughen ${ }^{l}$, Kristin Pearson Fuhrhop ${ }^{l}$, Jeffrey Kleim ${ }^{4}$,Erin Kleim ${ }^{4}$, Vincent Procaccio ${ }^{3}$, Steven Cramer ${ }^{1,2},{ }^{1}$ Dept. of Anatomy \& Neurobiology, University of California, Irvine, Irvine, USA, ${ }^{2}$ Dept. of Neurology, University of California, Irvine, 283 T-AM Irvine, USA, ${ }^{3}$ Dept. of Pediatrics, Center for Molecular and Mitochondrial Medicine and Genetics, University of California, Irvine, Irvine, USA, ${ }^{4}$ Dept. of Neuroscience, University of Florida, Gainesville, USA

Gene Expression Mapping in Adult Human Cortex: An Open Access Resource, Elaine Shen ${ }^{1}$, Chinh Dang ${ }^{1}$, Ed Lein ${ }^{1}$, Michael Hawrylycz ${ }^{1}$, John Hohmann ${ }^{1}$, Thomas Hyde ${ }^{2}$, Andreas Jeromin ${ }^{1}$, Susan Sunkin', Paul Wohnoutka', Hongkui Zeng ${ }^{1}$, Joel Kleinman', Allan Jones ${ }^{l},{ }^{1}$ Allen Institute for Brain Science, Seattle, USA, ${ }^{2}$ Section on Neuropathology, Clinical Brain Disorders Branch $(C B D B)$, Genes Cognition and Psychosis Program (GCAP), Intramural Research Program (IRP), NIMH, NIH, Bethesda, USA

\section{IMAGING TECHNIQUES \& CONTRAST MECHANISM EEG}

EEG Default Mode Network: Spectral Field Power Mapping, Andrew CN Chen*, Weijia Feng, Huixuan Zhao, Yanlin Yin, Peipei Wang, Center for Higher Brain Functions, Capital Medical University, Beijing, China

\section{MACROSCOPIC STATE TRANSITIONS IN ELECTROENCEPHALOGRAPHIC}

DYNAMICS, David Liley, Mathew Dafilis, Brett Foster, Peter Cadusch, Brain Dynamics Group, Brain Sciences Instutute, Swinburne University of Technology, Hawthorn, Victoria 3122, Australia

Solving the EEG problems without the individual's MRI using a database of images, Pedro Valdés-Hernández ${ }^{1}$, Nicolás von-Ellenrieder ${ }^{2}$, Alejandro Ojeda-Gonzalez ${ }^{1}$, Yasser Alemán-Gómez ${ }^{1}$, Silvia Kotchen ${ }^{3}$, Carlos Muravchik ${ }^{2}$, Pedro Valdés-Sosa ${ }^{I},{ }^{I}$ Cuban Neuroscience Center, Havana, Cuba, ${ }^{2}$ University of La Plata, La Plata, Argentina, ${ }^{3}$ University of Buenos Aires, Buenos Aires, Argentina

\section{IMAGING TECHNIQUES \& CONTRAST MECHANISM Functional MRI}

Real Time Software for Monitoring MRI Scanner Operation., Jerzy Bodurka ${ }^{l}$, Peter Bandettini ${ }^{l, 2},{ }^{I}$ Functional MRI Facilility, national Institute of Mental Health, NIH, Bethesda, USA, ${ }^{2}$ Section on Functional Imaging Method, National Institute of Mental Health, NIH, Bethesda, USA

Comparison of the phase encoded and the multifocal mapping of the primary visual cortex, Bordier Cecile ${ }^{1}$, Dojat Michel ${ }^{2,3}$, Vasseur Flor ${ }^{2,3}$, James Andrew ${ }^{I},{ }^{I}$ ARC Centre of Excellence in Vision Science and Research School of Biological Sciences, Australian National University, Canberra, Australia, ${ }^{2}$ INSERM, U836, Grenoble, F-38043, Grenoble, France, ${ }^{3}$ Joseph Fourier University, Institute of Neurosciences, Grenoble, France

Optimizing the detection of fMRI activation in white matter using asymmetric spin echo spiral, Jodie Gawryluk ${ }^{1,2}$, Kimberly Brewer ${ }^{1,2}$, Steven Beyea ${ }^{l, 2}$, Ryan D'Arcy ${ }^{1,2}$, ${ }^{1}$ National Research Council, Institute for Biodiagnostics, Halifax, Canada, ${ }^{2}$ Dalhousie University, Halifax, Canada

Quantitative comparison of online and offline motion compensation methods, Oliver Hinds ${ }^{l}$, Susan Gabrieli ${ }^{1,2}$, Julie Yoo ${ }^{1}$, John Gabrieli ${ }^{1,2}$, Christina Triantafyllou ${ }^{l, 3},{ }^{l}$ McGovern Institute for Brain Research, Massachusetts Institute of Technology, Cambridge, USA, ${ }^{2}$ Department of Brain and Cognitive Sciences, Massachusetts Institute of Technology, Cambridge, USA, ${ }^{3}$ Athinoula A. Martinos Center, Department of Radiology, MGH, Harvard Medical School, Charlestown, USA

The Neuroimaging Informatics Tools and Resources Clearinghouse (NITRC), Robert Buccigrossi $^{1}$, Mark Ellisman ${ }^{2}$, Jeff Grethe ${ }^{2}$, Christian Haselgrove ${ }^{3}$, David Kennedy ${ }^{4}$, Maryann Martone ${ }^{2}$, Nina Preuss ${ }^{1}$, Maureen Sullivan ${ }^{1}$, Keith Wagner ${ }^{1},{ }^{1}$ Turner Consulting Group, Inc, Washington, USA, ${ }^{2}$ University of California, San Diego, USA, ${ }^{3}$ Neuromorphometrics, Inc, 
Distortion-Free High-Resolution Functional MRI for Neurosciences Using Passband Balanced-SSFP at 3T, Jin Hyung Lee, Serge Dumoulin, Gary Glover, Brian Wandell, Dwight Nishimura, John Pauly, Stanford University, Stanford, USA

BOLD signal dropout in EPI: recovery, Guoxiang Liu ${ }^{1,2}$, Seiji Ogawa ${ }^{2},{ }^{1}$ National Institute of Information and Communications Technology, Kobe, Japan, ${ }^{2}$ Ogawa Laboratories for Brain Function Research, Tokyo, Japan

Detecting fMRI activation in white matter: Interhemispheric transfer of functionally lateralized stimuli across the corpus callosum, Erin Mazerolle ${ }^{1,2}$, Ryan D'Arcy ${ }^{1,2,3}$, Xiaowei Song ${ }^{1,4}$, Steven Beyea ${ }^{1,3,5}$, Institute for Biodiagnostics (Atlantic), National Research Council, Halifax, Canada, ${ }^{2}$ Department of Psychology/Neuroscience, Dalhousie University, Halifax, Canada, ${ }^{3}$ Department of Radiology, Dalhousie University, Halifax, Canada, ${ }^{4}$ Department of Medicine, Dalhousie University, Halifax, Canada, ${ }^{5}$ Department of Physics, Dalhousie University, Halifax, Canada

Fast whole brain fMRI acquisition above heart-rate nyquist frequency: applications of very fast imaging using PRESTO-2DSENSE, Sebastiaan F.W. Neggers ${ }^{l}$, Martijn P. van den Heuvel ${ }^{1}$, René C.W. Mandl ${ }^{1}$,Erno J. Hermans ${ }^{2,3}$, Christian F. Beckmann ${ }^{4}$, Hilleke E. Hulshoff Pol ${ }^{l}$, ${ }^{l}$ Dept. of Psychiatry, Rudolf Magnus Institute for Neuroscience, University Medical Center, Utrecht, Netherlands, ${ }^{2}$ F.C. Donders Centre at the Radboud University Nijmegen, Nijmegen, Netherlands, ${ }^{3}$ Department of Neurology at the Radboud University Nijmegen Medical Centre, Nijmegen, Netherlands, ${ }^{4}$ Clinical Neuroscience Department, Division of Neuroscience and Mental Health, Imperial College London, London, United Kingdom

Optimized EPI for fMRI studies using a Common Gradient Template to compensate local Susceptibility-Induced Signal Loss: A pilot Study, Jochen Rick ${ }^{1}$, Simon Maier ${ }^{2}$, Oliver Tüscher ${ }^{3}$, Maxim Zaitsev ${ }^{1}$, Oliver Speck ${ }^{4},{ }^{1}$ Dept. of Diagnostic Radiology, Medical Physics, University Hospital Freiburg, Freiburg, Germany, ${ }^{2}$ Dept. of Psychiatry and Psychotherapy, University Hospital Freiburg, Freiburg, Germany, ${ }^{3}$ Dept. of Neurology, University Hospital Freiburg, Freiburg, Germany, ${ }^{4}$ Dept. of Biomedical Magnetic Resonance, Otto-von-Guericke University, Magdeburg, Germany

Improvements of prospective motion compensation using real-time shim correction, Daniel Splitthoff, Juergen Hennig, Maxim Zaitsev, Dept. of Diagnostic Ragiology, Medical Physics, University Hospital Freiburg, Freiburg, Germany

Feasibility of k-t BLAST for functional fMRI at (ultra-) high magnetic field strengths, Jane Utting $^{1,3}$, Sebastian Kozerke ${ }^{2}$, René Vohn ${ }^{1}$, Ralph Schnitker ${ }^{1}$, Roger Luechinger ${ }^{2}$, Thoralf Niendorf ${ }^{\text {, }}$ IIZKF-BIOMAT, Medical Faculty, RWTH-Aachen, Aachen, Germany, ${ }^{2}$ Institute for Biomedical Engineering, University and ETH, Zuerich, Switzerland, ${ }^{3}$ Experimental MRI, Radiology, Medical Faculty, RWTH-Aachen, Aachen, Germany

Integration of motion correction into the GLM for fMRI analysis of moving subjects, Alle Meije Wink ${ }^{1,2}$, Shuzhou Jiang ${ }^{1,2}$, Jo Hajnal ${ }^{1,2},{ }^{1}$ Imaging Sciences Department, Imperial College, Robert Steiner MR Unit, Hammersmith Hospital, London, United Kingdom, ${ }^{2}$ MRC Clinical Sciences Centre, Hammersmith Campus, London, United Kingdom

Nonlinear Registration across Subjects in Functional Connectivity Analysis at a Group Level, Hong Gu, Xiujuan Geng, Elliot Stein, Yihong Yang, Neuroimaging Research Branch, National Institute on Drug Abuse, NIH, Baltimore, USA

\section{IMAGING TECHNIQUES \& CONTRAST MECHANISM} MEG

Fast retinotopic mapping of visual fields using MEG, Benoit Cottereau ${ }^{1,2}$, Alexandre Gramfort ${ }^{3}$, Jean Lorenceau ${ }^{1}$, Bertrand Thirion ${ }^{4}$, Maureen Clerc ${ }^{3}$, Sylvain Baillet ${ }^{1},{ }^{1}$ CNRS UPR 640, Paris, France, ${ }^{2}$ ESME-Sudria, Ivry, France, ${ }^{3}$ Odyssée Laboratory-ENPC/ENS/INRIA, Sophia-Antipolis, France, ${ }^{4}$ INRIA Futur,Neurospin, Saclay, France

Comparing MEG source localization algorithms with fMRI statistical maps and neuroanatomy, Johanna Zumer ${ }^{1,2}$, Elizabeth Disbrow ${ }^{2,3}$, Hagai Attias ${ }^{4}$, Matt Brookes ${ }^{1}$, Peter Morris ${ }^{1}$, Srikantan Nagarajan ${ }^{2},{ }^{1}$ University of Nottingham, Nottingham, United Kingdom, ${ }^{2}$ University of California, San Francisco, San Francisco, USA, ${ }^{3}$ University of California, Davis, Davis, USA, ${ }^{4}$ Golden Metallic, Inc, San Francisco, USA 


\section{LANGUAGE}

Language Acquisition

Fast learning of action words evidenced by MEG, Stefanie Enriquez-Geppert ${ }^{1}$, Pienie Zwitserlood ${ }^{2}$, Markus Junghöfer ${ }^{l}$, Christo Pantev ${ }^{l}$, Christian Dobel ${ }^{l}$, 'Institute for Biomagnetism and Biosignalanalysis, University of Münster, Münster, Germany, ${ }^{2}$ Departement of Psychology, University of Münster, Münster, Germany

Dissociate Effects of Age-of-Acquisition from Word Frequency Effects in Picture Naming Using Functional MRI, WEN-JUI KUO, DAISY HUNG, OVID TZENG, Institute of Neuroscience, National Yang-Ming University, Taipei, Taiwan

EEG signatures of the BOLD-defined language network in resting state, Marcel CM Bastiaansen ${ }^{1,2}$, Tom Eichele ${ }^{3}$, René Scheeringa ${ }^{l},{ }^{1}$ F.C. Donders Centre for Cognitive Neuroimaging, Radboud University Nijmegen, Nijmegen, Netherlands, ${ }^{2}$ Max Planck Institute for Psycholinguistics, Nijmegen, Netherlands, ${ }^{3}$ Department of Biological and Medical Psychology, University of Bergen, Bergen, Norway

\section{LANGUAGE \\ Production}

Correct and erroneous naming responses in healthy subjects, Stefanie Abel ${ }^{l}$, Katharina Dressel ${ }^{l}$, Ralph Schnitker ${ }^{2}$, Dorothee Kümmerer ${ }^{3}$, Dorothee Saur ${ }^{3}$, Cornelius Weiller ${ }^{3}$, Walter Huber ${ }^{1}$,

${ }^{I}$ Neurolinguistics at the Department of Neurology, RWTH Aachen University, Aachen, Germany,

${ }^{2}$ Interdisciplinary Center for Clinical Research - Neurofunctional Imaging Lab, RWTH Aachen

University, Aachen, Germany, ${ }^{3}$ Department of Neurology, Neurocenter, University of Freiburg,

Freiburg, Germany

The involvement of cytoarchitectonic BA 44 and BA 45 in different types of verbal fluency, Stefan Heim ${ }^{1,2}$, Simon B. Eickhoff ${ }^{1,2}$, Katrin Amunts ${ }^{1,2,3}$, ${ }^{1}$ Institute of Neuroscience and Biophysics, INB-3 Medicine, Research Centre Jülich, Jülich, Germany, ${ }^{2} 3$ Brain Imaging Center West (BICW), Jülich, Germany, ${ }^{3}$ Dept. of Psychiatry and Psychotherapy, RWTH Aachen, Aachen, Germany

Regional cerebral blood flow intercorrelations during speech production by adults who stutter, Roger Ingham ${ }^{1,2}$, Janis Ingham ${ }^{1,2}$, Frank Zamarripa ${ }^{2}$, Peter Fox ${ }^{2},{ }^{1}$ UC Santa Barbara, Santa Barbara, USA, ${ }^{2}$ UT Health Science Center in San Antonio, San Antonio, USA

Neural correlates of lexical semantic recovery after treatment in aphasia, Swathi Kiran ${ }^{\text {, }}$, Rajani Sebastian ${ }^{1}$, Padmadevan Chettiar ${ }^{1}$, Micheal Devous ${ }^{2},{ }^{1}$ University of Texas at Austin, Austin, USA, ${ }^{2}$ UT Southwestern, Dallas, USA

White matter correlates of lexical retrieval in elderly adults, Elena Rykhlevskaia ${ }^{l}$, Manuella Clark-Cotton ${ }^{2,3}$, Avron Spiro III ${ }^{4,5}$, Loraine Obler ${ }^{2,3,6}$, Martin Albert ${ }^{2,3},{ }^{1}$ Stanford Cognitive and Systems Neuroscience Laboratory, Stanford, CA, ${ }^{2}$ Medical Research Service, VA Boston Healthcare System, Boston, MA, ${ }^{3}$ Department of Neurology, Boston University School of Medicine, Boston, MA, ${ }^{4}$ Normative Aging Study and MAVERIC, VA Boston Healthcare System, Boston, MA, ${ }^{5}$ Department of Epidemiology, Boston University School of Public Health, ${ }^{6}$ Program in Speech-Language-Hearing Sciences, CUNY Graduate Center, New York, NY,

\section{MEMORY \& LEARNING Plasticity (normal \& following pathology)}

Cerebellum and Cognition: Plasticity during the automatisation of rule-based information processing, Joshua Balsters, Narender Ramnani, Dept Psychology, Royal Holloway University of London, LONDON, United Kingdom

Dependence of hemispheric dominance on fMRI normalization and region of interest procedures, Alexander Geißler, Thomas Steinkellner, Jakob Rath, Nicolaus Klinger, Roland Beisteiner, Study Group Clinical fMRI, MR Center of excellence, Department of Neurology, Medical University of Vienna, Vienna, Austria

Learning rules changes connectivity between the prefrontal cortex and cerebellum, Yuri Saalmann ${ }^{I}$, Joshua Balsters ${ }^{I}$, Michael Wright ${ }^{2}$, Narender Ramnani ${ }^{l},{ }^{\prime}$ Department of Psychology, Royal Holloway, University of London, Egham, United Kingdom, ${ }^{2}$ Department of Psychology, Brunel University, Uxbridge, United Kingdom 


\section{MEMORY \& LEARNING Working Memory}

Perceptual memory representations studied in delayed discrimination of spatial frequency behavioral and fMRI evidence for high-fidelity visual stores in early visual cortex, Oliver Baumann $^{1,2,3}$, Tor Endestad 3 , Svein Magnussen ${ }^{3}$, Mark Greenlee ${ }^{2},{ }^{1}$ University of Queensland, Brisbane, Australia, ${ }^{2}$ University of Regensburg, Regensburg, Germany, ${ }^{3}$ University of Oslo, Oslo, Norway

Inefficient recruitment of working memory updating networks in post-traumatic stress disorder, Richard Clark ${ }^{l}$, Kathryn Moores ${ }^{I}$, Alexander McFarlane ${ }^{2}{ }^{I}$ Flinders University, Adelaide, Australia, ${ }^{2}$ Adelaide University, Adelaide, Australia

Increase of Alpha Coherence in a Working Memory Network: An MEG Study, Hyojin Park $^{1,2}$, June Sic Kim ${ }^{4}$, Chun-Kee Chung ${ }^{1,4}$, Dong Soo Lee ${ }^{1,2}$, Eunjoo Kang ${ }^{3},{ }^{1}$ Interdisciplinary Program in Cognitive Science, Seoul National University, Seoul, South Korea, ${ }^{2}$ Department of Nuclear Medicine, Seoul National University Hospital, Seoul, South Korea, ${ }^{3}$ Department of Psychology, Kangwon National University, Chuncheon, South Korea, ${ }^{4}$ MEG Center, Department of Neurosurgery, Seoul National University Hospital, Seoul, South Korea

Prefrontal cortex and basal ganglia control access to working memory, Fiona McNab, Torkel Klingberg, Stockholm Brain Institute, Karolinska Institutet, Stockholm, Sweden

A TMS “ping” during fMRI reveals physiological consequences of functional connectivity and dissociates multivariate from univariate maps of working memory storage, Bradley Postle $^{1,2}$, Eva Feredoes ${ }^{1,3}$, Todd Woodward ${ }^{3}$, Giulio Tononi ${ }^{2},{ }^{1}$ Univ. of Wisconsin Psychology, Madison, USA, ${ }^{2}$ Univ. of Wisconsin Psychiatry, Madison, USA, ${ }^{3}$ Univ. of British Columbia Psychiatry, Vancouver, Canada

\section{MODELING \& ANALYSIS Bayesian Modeling}

Observing the Observer : a nested Bayesian approach to studies of learning and decision making, Jean Daunizeau ${ }^{l}$, Mathias Pessiglione ${ }^{2}$, Klaas Stephan ${ }^{l}$, Hanneke Den Ouden ${ }^{l}$, Karl Friston ${ }^{1},{ }^{1}$ Welcome Trust Centre for Neuroimaging, London, United Kingdom, ${ }^{2}$ INSERM U610, Paris, France

Combined spatial and non-spatial Gaussian process prior for fMRI analysis, Adrian Groves, Mark Woolrich, FMRIB Centre, Oxford, United Kingdom

The choice of forward model in MEG localisation, Richard Henson ${ }^{l}$, Jeremie Mattout ${ }^{2}$, Karl $^{2}$ Friston ${ }^{3},{ }^{1}$ MRC CBU, Cambridge, United Kingdom, ${ }^{2}$ Brain Dynamics and Cognition, U821 INSERM, Lyon, France, ${ }^{3}$ FIL, London, United Kingdom

How Should Anatomical Connectivity Be Defined?, Enrico Kaden, Alfred Anwander, Thomas R. Knösche, Max Planck Institute for Human Cognitive and Brain Sciences, Leipzig, Germany

A Unified Bayesian Framework for MEG/EEG Source Imaging, David Wipf ${ }^{\text {, Hagai Attias }}{ }^{2}$, Kensuke Sekhara ${ }^{3}$, Srikantan Nagarajan ${ }^{1},{ }^{1}$ UCSF, San Francisco, USA, ${ }^{2}$ Golden Metallic, San Francisco, USA, ${ }^{3}$ Tokyo Metropolitan University, Tokyo, Japan

\section{MODELING \& ANALYSIS}

\section{Classification \& Predictive Modeling}

A multivariate approach to fMRI activation detection using pattern recognition and information entropy on tactile data, Malin C.B. Aberg, Line Löken, Johan Wessberg, Department of neuroscience and physiology, Göteborg University, Göteborg, Sweden

Exploiting EEG inverse problem in an asynchronous BCI experiment, Michel Besserve, Jacques Martinerie, Line Garnero, Laboratoire de Neurosciences Cognitives et Imagerie Cérébrale, CNRS UPR 640 LENA \& UPMC Univ Paris 06, Paris, France 
Classification of Brain Magnetic Resonance Images for Bipolar Disorders Based on Voxel-based Morphometry and Bayesian Theorem, Yong-Sheng Chen ${ }^{1}$, Li-Fen Chen ${ }^{2,3}$, Ya-Ting Chang ', Yung-Tien Huang', Jen-Chuen Hsieh ${ }^{2,3}$, Tzu-Chen Yeh ${ }^{3.2}$, I Department of Computer Science, National Chiao Tung University, Hsinchu, Taiwan, ${ }^{2}$ Institute of Brain Science, National Yang-Ming University, Taipei, Taiwan, ${ }^{3}$ Integrated Brain Research Laboratory, Taipei Veterans General Hospital, Taipei, Taiwan

A porous elastic BOLD hemodynamic model with spatiotemporal response, Peter Drysdale ${ }^{1,2}$, Jacqueline Huber ${ }^{1,3}$, Peter Robinson ${ }^{I, 2,3}$, ' School of Physics, University of Sydney, Sydney, Australia, ${ }^{2}$ Brain Dynamics Center, Westmead Millenium Institute, Westmead Hospital and Western Clinicial School of University of Sydney, Westmead, Australia, ${ }^{3}$ Faculty of Medicine, University of Sydney, Sydney, Australia

Classifying brain states based on regional homogeneity of fMRI data, Bin $\mathrm{Lv}^{l}$, Huiguang $H e^{l}$, Zhiqiang Zhang ${ }^{2}$, Wei Huang ${ }^{2}$, Meng Li ${ }^{l}$, Guangming Lu ${ }^{2}$, ${ }^{1}$ Institute of Automation, Chinese Academy of Sciences, Beijing, China, ${ }^{2}$ Department of Medical Imaging, Nanjing Jinling Hospital, Nanjing, China

Classification and control strategies of epileptic seizures via bifurcation analysis, Jong Won Kim $^{1,2}$, James Roberts ${ }^{1,2}$, Peter Robinson ${ }^{1,2,3}$, ' School of Physics, The University of Sydney, Sydney, Australia, ${ }^{2}$ Brain Dynamics Center, Westmead Hospital, Westmead, Australia, ${ }^{3}$ Faculty of Medicine, The University of Sydney, Sydney, Australia

Mutual Information-Based Feature Selection enhances fMRI-based brain activity classification, Vincent Michel ${ }^{l}$, Cécilia Damon ${ }^{l}$, Alan Tucholka ${ }^{2}$, Merlin Keller ${ }^{1}$, Bertrand Thirion ${ }^{l},{ }^{1}$ Inria Saclay, Saclay, France, ${ }^{2}$ CEA-Neurospin, Gif sur Yvette, France

Mapping Neuronal Fibers Through Partial Volume Voxels, Ofer Pasternak ${ }^{l}$, Nir Sochen ${ }^{l}$, Nathan Intrator ${ }^{l}$, Yaniv Assaf ${ }^{d, 2},{ }^{I}$ Tel Aviv university, Tel Aviv, Israel, ${ }^{2}$ Functional Brain Imaging Unit, Tel Aviv Sourasky Medical Center, Tel Aviv, Israel

A model of realistic conducting volume including or excluding brain lesional area, Pauletto Giada , Valiante Gabriele ${ }^{I}$, Skrap Miran ${ }^{2}$, Budai Riccardo ${ }^{1},{ }^{l}$ Department of Neurology and DPMSCAzienda Ospedaliero-Universitaria, S. Maria della Misericordia, Udine, Italy, ${ }^{2}$ Department of Neurosurgery - Azienda Ospedaliero-Universitaria, S. Maria della Misericordia, Udine, Italy

Co-Clustering Approach to Neural Representation of Objects, Svetlana Shinkareva, Julie Conder, University of South Carolina, Columbia, USA

A Comparison of Feature Selection Strategies for Classification of fMRI Activation Patterns, Giancarlo Valente, Federico De Martino, Rainer Goebel, Elia Formisano, University of Maastricht, Department of Cognitive Neuroscience, Maastricht, Netherlands

MODELING \& ANALYSIS

Motion Correction/Spatial Normalization, Atlas Construction

EVALUATION OF DTI IMAGE ANALYSIS USING NONLINEAR SPATIAL NORMALIZATION AND TISSUE-SPECIFIC, SMOOTHING-COMPENSATED VOXEL BASED ANALYSIS: APPLICATION IN AUTISM, Andrew Alexander ${ }^{l}$, Jee Eun Lee ${ }^{l}$, Babak Ardekani ${ }^{2}$, Moo Chung ${ }^{1}$, Erin Bigler ${ }^{3}$, Janet Lainhart ${ }^{4},{ }^{1}$ University of Wisconsin, Madison, USA, ${ }^{2}$ Nathan Kline Institute, Orangeburg, USA, ${ }^{3}$ Brigham Young University, Provo, USA, ${ }^{4}$ University of Utah, Salt Lake City, USA

Improving voxel-based morphometry with diffeomorphic non-linear registration by DARTEL toolbox: conventional SPM normalization vs DARTEL Normalization, Carlton CHU, Geoffrey Tan, John Ashburner, Wellcome Trust Centre for Neuroimaging(FIL), London, United Kingdom

499 T-AM

The Structural-Functional Correspondence Project, Martin Frost, Rainer Goebel, Dept. Cognitive Neuroscience, Maastricht University, Maastricht, Netherlands

503 T-AM

Subcortical Structure Template Generation with its Applications in Shape Analysis, Anqi Qiu $^{l}$, Timothy Brown ${ }^{2}$, Bruce Fischl ${ }^{3,4}$, Anthony Kolasny', Jun $\mathrm{Ma}^{2}$, Randy Buckner ${ }^{3,5}$, Michael Miller ${ }^{2},{ }^{1}$ Division of Bioengineering, National University of Singapore, Singapore, Singapore, ${ }^{2}$ Center for Imaging Science, Johns Hopkins University, Baltimore, USA, ${ }^{3}$ Athinoula A Martinos 
Center for Biomedical Imaging at MGH, Boston, USA, ${ }^{4}$ Computer Science and Arti ${ }^{-}$cial Intelligence Laboratory, Massachusetts Institute of Technology, Boston, USA, ${ }^{5}$ Department of Psychology, Center for Brain Science, Harvard University, Boston, USA

\section{MODELING \& ANALYSIS}

\section{Univariate Modeling, Linear, \& Nonlinear}

An improved method for voxel-based T2-weighted MRI analysis, David $F$ Abbott ${ }^{1,2,3}$, Gaby $S$ Pell $^{1,2,3}$, Heath Pardoe ${ }^{1,2,3}$, Graeme Jackson ${ }^{1,2,3}{ }^{1}$ Brain Research Institute, Melbourne, Australia, ${ }^{2}$ The University of Melbourne, Melbourne, Australia, ${ }^{3}$ Florey Neuroscience Institutes, Melbourne, Australia

Detection of Local Cortical Asymmetry via Discriminant Power Analysis, Moo K. Chung, Daniel J. Kelley, Kim M. Dalton, Richard J. Davidson, Waisman Laboratory for Brain Imaging and Behavior, University of Wisconsin, Madison, USA

Phase Modeling in Arterial Spin Labeling FMRI, Luis Hernandez-Garcia ${ }^{1}$, Daniel Rowe ${ }^{2}$,

${ }^{1}$ University of Michigan, Ann Arbor, USA, ${ }^{2}$ Medical College of Wisconsin, Milwaukee, USA

Stimulus interaction effects in parietal and limbic system in an executive task: practical use of a simple rapid event related fMRI method to measure main and interaction effects, $J$. Martijn Jansma, Allison Nugent, Rebecca Davis, Wayne Drevets, NIH/NIMH/SNMAP, Bethesda, USA

Noninvasive Quantifying the Regional CBV Using FWE Model Based on VASO Functional MRI Technique, Chia-Wei Li, Chang-Wei Wu, Jyh-Horng Chen, Interdisciplinary MRI/MRS Laboratory, Department of Electrical Engineering, National Taiwan University, Taipei, Taiwan

Advanced simulations of fMRI data sets, Radek Marecek ${ }^{l}$, Michal Mikl ${ }^{1,2}$, Petr Hlustik ${ }^{3},{ }^{1} 1 \mathrm{st}$ Department of Neurology, St. Anne's University Hospital and Masaryk University, Brno, Czech Republic, ${ }^{2}$ Department of Biomedical Engineering, FEEC, Brno University of Technology, Brno, Czech Republic, ${ }^{3}$ Department of Neurology and Radiology, School of Medicine, Palacky University and University Hospital, Olomouc, Czech Republic

The Effect of Task Switching on the t-Statistics Correlation to Explore the Neuronal Basis of Motor Execution - An Approach Using Dynamic fMRI, Toshiharu Nakai ${ }^{1}$, Epifanio Bagarinao $^{2}$, Yoshio Tanaka ${ }^{2}$, Chikako Nakai ${ }^{3}$, Kayako Matsuo ${ }^{l},{ }^{I}$ Functional Brain Imaging Lab, National Center for Geriatrics and Gerontology, Ohbu, Japan, ${ }^{2}$ Grid Technology Research Center, National Institute of Advanced Industrial Science and Technology, Tsukuba, Japan, ${ }^{3}$ Faculty of Business and Informatics, Toyohashi Sozo University, Toyohashi, Japan

Predicting the BOLD time courses from simultaneously recorded LFPs, Christopher Tyler ${ }^{\text {, }}$ Nikos Logothetis ${ }^{2},{ }^{1}$ Smith-Kettlewell Institute, San Francisco, USA, ${ }^{2}$ MPI for Biological Cybernetics, Tuebingen, Germany

Robust Group Analysis Using Outlier Modelling, Mark Woolrich, FMRIB Centre, Dept. of Clinical Neurology, University of Oxford, Oxford, United Kingdom

\section{MOTOR BEHAVIOR}

Brain-machine Interface

Reading the Mind: Identification and Prediction of the Intended Targets of Reaching Movements Using Magnetoencephalography. Applications for an Implicit Brain Computer Interface, Claudia Bonin ${ }^{1}$, Kory Johnson ${ }^{2}$, Mark Hallett ${ }^{I},{ }^{1}$ Human Motor Control Section, National Institute of Neurological Disorders and Strokes, National Institutes of Health, Bethesda, USA, ${ }^{2}$ Bioinformatics Neuroscience Group, Information Technology Program, National Institute of Neurological Disorders and Stroke, National Institutes of Health, Bethesda, USA

\section{MOTOR BEHAVIOR \\ Hand Movements}

Effects of timing and task uncertainty on the fronto-parietal motor circuits: An fMRI study, Oliver Jakobs ${ }^{1}$, Ling Wang ${ }^{2,3}$, Christian Grefkes ${ }^{1,4}$, Anton Henssen ${ }^{1}$, Manuel Dafotakis ${ }^{2,3}$, Karl Zilles $^{1,2,3}$, Simon B. Eickhoff ${ }^{2},{ }^{I} \mathrm{C} \&$ O. Vogt Institute of Brain Research, University of Düsseldorf, 
Düsseldorf, Germany, ${ }^{2}$ Institute of Neuroscience and Biophysics, INB-3 Medicine, Research Centre Jülich, Jülich, Germany, ${ }^{3}$ Brain Imaging Center West (BICW), Jülich, Germany,

${ }^{4}$ Max-Planck-Institut for Neurological Research, Cologne, Germany

fMRI in patients with writer's cramp treated by repetitive transcranial magnetic stimulation (rTMS) of the primary somatosensory cortex, Robert Jech ${ }^{l}$, Petra Havrankova ${ }^{l}$, Nolan Walker ${ }^{1}$, Jana Tauchmanova ${ }^{2}$, Josef Vymazal ${ }^{3}$, Evzen Ruzicka ${ }^{1},{ }^{\prime}$ Department of Neurology, 1 st Medical Faculty, Charles University, Prague, Czech Republic, ${ }^{2}$ Department of Control Engineering, Faculty of Electrical Engineering, Czech Technical University, Prague, Czech Republic, ${ }^{3}$ Na Homolce Hospital, Prague, Czech Republic

Dissociating networks of delayed imitation by independent component analysis, Mareike $M$. Menz, Kathrin Reetz, Adam McNamara, Ferdinand Binkofski, Department of Neurology and NeuroImage Nord, University of Luebeck, Luebeck, Germany

Neural correlates of improved visuomotor functions following stimulation of the noradrenergic system in humans, Ling E. Wang ${ }^{1,2}$, Gereon R. Fink ${ }^{1,3}$, Manuel Dafotakis ${ }^{1}$, Christian Grefkes ${ }^{1,3,4}$, ' Cognitive Neurology Section, Institute of Neuroscience and BiophysicsMedicine, Research Centre Juelich, Juelich, Germany, ${ }^{2}$ International Graduate School of Neuroscience, Ruhr University Bochum, Bochum, Germany, ${ }^{3}$ Department of Neurology, University of Cologne, Cologne, Germany, ${ }^{4}$ Neuromodulation \& Neurorehabilitation Section, Max-Planck-Institute of Neurological Research, Cologne, Germany

\section{MOTOR BEHAVIOR \\ Motor-Premotor Cortex/Motor Cortical Functions}

Neural correlates of action prediction in sports: How important is expertise?, Ana Maria Abreu $^{1,2}$, Emiliano Macaluso ${ }^{1}$, Paola Cesari ${ }^{3}$, Cosimo Urgesi ${ }^{4}$, Salvatore Maria Aglioti ${ }^{2}$, ${ }^{I}$ Neuroimaging Laboratory, Santa Lucia Foundation, Rome, Italy, ${ }^{2}$ Department of Psychology, University of Rome 'La Sapienza', Rome, Italy, ${ }^{3}$ Department of Neurological and Visual Sciences, University of Verona, Verona, Italy, ${ }^{4}$ Scientific Institute Eugenio Medea, San Vito al Tagliamento, Pordenone, Italy

Premotor mirror neuron activation in schizophrenia, Peter Enticott ${ }^{1}$, Kate Hoy ${ }^{1}$, Sally Herring ${ }^{1}$, Patrick Johnston ${ }^{2}$, Paul Fitzgerald ${ }^{l},{ }^{1}$ Alfred Psychiatry Research Centre, Monash University, Melbourne, Australia, ${ }^{2}$ Brain Sciences Institute, Swinburne University of Technology, Melbourne, Australia

The Enhancement of Cortical Excitability by Transcranial Direct Current Stimulation in Human Brain, Yong Hyun Kwon ${ }^{1}$, Sung Ho Jang ${ }^{2}$, Sang Ho Ahn ${ }^{2},{ }^{1}$ Department of Physical Therapy, Yeungnam College of Science \& Technology, Daegu, South Korea, ${ }^{2}$ Department of Physical Medicine and Rehabilitation, School of Medicine, Yeungnam University, Daegu, South Korea

Making EMG recordings during fMRI work: experiences from fundamental and applied studies of the motor system, N.M. Maurits ${ }^{1,2}$, R.J. Renken ${ }^{2}$, J.H. van der Hoeven ${ }^{l}$, A.F. van Rootselaar, , ${ }^{3}$ Department of Neurology, University Medical Center Groningen, Groningen, Netherlands, ${ }^{2} B C N$-NeuroImaging Center, University Medical Center Groningen, University of Groningen, Groningen, Netherlands, ${ }^{3}$ Department of Neurology and Clinical Neurophysiology, Academic Medical Center Amsterdam, Amsterdam, Netherlands

Normal variation in representation area of thenar and tibial muscles in healthy motor cortex: navigated transcranial magnetic stimulation study, Eini Niskanen ${ }^{1,2}$, Laura Säisänen ${ }^{1}$, Petro Julkunen ${ }^{1}$, Ritva Vanninen ${ }^{3}$, Mervi Könönen ${ }^{1,3},{ }^{l}$ Department of Clinical Neurophysiology, Kuopio University Hospital, Kuopio, Finland, ${ }^{2}$ Department of Physics, University of Kuopio, Kuopio, Finland, ${ }^{3}$ Department of Radiology, Kuopio University Hospital, Kuopio, Finland

An Image-Guided, Robotic, Transcranial Magnetic Stimulation (irTMS) Virtual Lesion Study of Speech, Donald Robin ${ }^{l, 2}$, Frank Guenther ${ }^{3}$, Shalini Narayana ${ }^{1}$, Adam Jacks ${ }^{1}$, Jason Tourville ${ }^{3}$, Amy Ramage ${ }^{1}$, Jack Lancaster ${ }^{l}$, Crystal Franklin ${ }^{l}$, Peter Fox ${ }^{2}$, ' Research Imaging Center, University of Texas Health Science Center at San Antonio, San Antonio, USA, ${ }^{2}$ Honor's College, University of Texas, San Antonio, San Antonio, USA, ${ }^{3}$ Center for Neurocomputation, Boston University, Boston, USA 
Observing multiple people acting: Separability of cortical processing streams associated with each person's actions, Jeremy I. Skipper ${ }^{1}$, Ekaterina Dobryakova ${ }^{2}$, Natalie Sebanz ${ }^{3}$, ${ }^{1}$ Sackler Institute for Developmental Psychobiology, Weill-Cornell Medical College, New York, USA, ${ }^{2}$ Rutgers University, Newark, USA, ${ }^{3}$ University of Birmingham, Birmingham, United Kingdom

EEG spectrum power and EEG -EMG coherence mapping during voluntary movement in children aged 7 to 10 years with different attention and impulsivity, Alexander Trembach ${ }^{l}$, Yanina Bugaez ${ }^{2}$, Maxim Beljaev ${ }^{3}$, Katrin Vitko ${ }^{l}$, Eduard Moskalev ${ }^{I},{ }^{I}$ Department of Adaptive Training and Physical Rehabilitation, Kuban State University of Physical Education, Sport and Tourism, Krasnodar, Russia, ${ }^{2}$ Department of Physiology, Kuban University of Physical Education, Sport and Tourism, Krasnodar, Russia, ${ }^{3}$ Department of Biomechanics, Kuban State University of Physical Education, Sport and Tourism, Krasnodar, Russia, ${ }^{4}$ Department of Adaptive Training and Physical Rehabilitation, Kuban State University of Physical Education, Sport and Tourism, Krasnodar, Russia, ${ }^{5}$ Department of Adaptive Training and Physical Rehabilitation, Kuban State University of Physical Education, Sport and Tourism, Krasnodar, Russia

\section{NEUROANATOMY \\ Anatomical Studies}

Nerve Fiber Mapping in Histological Sections of the Human Brain by Means of Polarized Light, Markus Axer ${ }^{l}$, Jürgen Dammers ${ }^{1}$, David Gräßel ${ }^{l}$, Katrin Amunts ${ }^{1,2}$, Uwe Pietrzyk ${ }^{1,3}$, Karl Zilles ${ }^{1,4},{ }^{1}$ Institute of Neurosciences and Biophysics 3 - Medicine, Research Center Jülich, Jülich, Germany, ${ }^{2}$ Department for Psychiatry and Psychotherapy, RWTH Aachen University, Aachen, Germany, ${ }^{3}$ Department of Physics, University of Wuppertal, Wuppertal, Germany, ${ }^{4}$ C. and O. Vogt Institute of Brain Research, University of Düsseldorf, Düsseldorf, Germany

A comparison of manual tracing and automated measure of hippocampal volume in a large community-based sample, Nicolas Cherbuin ${ }^{I}$, Kaarin J. Anstey ${ }^{I}$, Chantal Meslin ${ }^{I}$, Perminder S. Sachdev ${ }^{2},{ }^{I}$ Centre for Mental Health Research, Australian National University, Canberra, Australia, ${ }^{2}$ School of Psychiatry, University of New South Wales, Sydney, Australia

Delineation of the subthalamic nucleus (STN) on high-resolution maps of R2*, Peter Dechent ${ }^{1}$,Erck Elolf ${ }^{2}$, Tabea Gringel ${ }^{1,2}$, Michael Knauth ${ }^{2}$, Gunther Helms ${ }^{I},{ }^{1}$ MR-Research in Neurology and Psychiatry, University Medical Center, Göttingen, Germany, ${ }^{2}$ Department of Neuroradiology, University Medical Center, Göttingen, Germany

Congestive heart failure is associated with changes in grey matter volume that cannot be entirely explained by cardiovascular disease, Griselda Garrido ${ }^{1}$, Leon Flicker ${ }^{2}$, Christopher Beer $^{2}$, Nicola Lautenschlager ${ }^{2}$, Leonard Arnolda ${ }^{3}$, Andrew Campbell ${ }^{4}$, Nat Lenzo ${ }^{5}$, Osvaldo Almeida ${ }^{2},{ }^{\prime}$ Serviço de Informática Médica, Instituto do Coração, Hospital das Clínicas da Faculdade de Medicina da Universidade de São Paulo, São Paulo, Brazil, ${ }^{2}$ Western Australia Centre for Health \& Ageing, University of Western Australia, Perth, Australia, ${ }^{3}$ School of Medicine and Pharmacology, University of Western Australia, Perth, Australia, ${ }^{4}$ Department of Medical Engineering and Physics, Royal Perth Hospital, Perth, Australia, ${ }^{5}$ Department of Nuclear Medicine, Royal Perth Hospital, Perth, Australia

New structural brain findings in maltreated children with PTSD using deformation tensor morphometry: a preliminary report, Andrea Jackowski ${ }^{1,2}$, Colin Studholme ${ }^{3}$, Heather DouglasPalumberi ${ }^{2}$, Joan Kaufman ${ }^{2,4},{ }^{1}$ LiNC, Universidade Federal de Sao Paulo, Sao Paulo, Brazil, ${ }^{2}$ Child Study Center, Yale University, New Haven, USA, ${ }^{3}$ Radiology, University of California, San Francisco, USA, ${ }^{4}$ Psychiatry, Yale University, New Haven, USA

Can regional structural MRI measurement of cerebral health explain age-related cognitive change?, Peter Kochunov ${ }^{1,2}$, Donald Robin ${ }^{1}$, Anita Schlosser ${ }^{1}$, Valeria Kochunov ${ }^{1}$, Jack Lancaster ${ }^{1,2}$, Peter Fox ${ }^{1,2}$, ${ }^{1}$ Research Imaging Center, University of Texas Health Science Center at San Antonio, san antonio, USA, ${ }^{2}$ International Consortium for Brain Mapping (ICBM), USA

Laterality Differences in Klinefelter's Syndrome: A voxel-based morphometry study, Francois Lalonde ${ }^{1}$, Gregory Ihrie ${ }^{2}$, Gregory Wallace ${ }^{3}$, Liv Clasen ${ }^{1}$, Jay Giedd ${ }^{l},{ }^{l}$ Child Psychiatry Branch, NIMH, NIH, Bethesda, USA, ${ }^{2}$ University of Maryland, College Park, USA, ${ }^{3}$ Laboratory of Brain and Cognition, NIMH, NIH, Bethesda, USA 
Characterization of cortical pathology heterogeneity in multiple sclerosis using 7T MRI, Caterina Mainero $^{l}$, Andre van der Kouwe ${ }^{l}$, Thomas Benner ${ }^{l}$, Graham Wiggins ${ }^{l}$, R Phillip Kinkel ${ }^{2}$, Bruce R Rosen ${ }^{l},{ }^{I}$ Martinos Center for Biomedical Imaging, Massachusetts General Hospital,

Charlestown, USA, ${ }^{2}$ Neurology, Beth Israel Deaconess Medical Center, Boston, USA

Patterns of cortical thickness in obsessive compulsive disorder (OCD), Veena M. Narayan ${ }^{l}$, Owen R. Phillips ${ }^{I}$, Katherine L. Narr ${ }^{l}$, Paul M. Thompson ${ }^{I}$, Arthur W. Toga ${ }^{l}$, Philip R. Szeszko ${ }^{2}$, ${ }^{I}$ Laboratory of Neuro Imaging, Dept. of Neurology, UCLA, Los Angeles, USA, ${ }^{2}$ Department of Psychiatry Research, The Zucker Hillside Hospital, North-Shore Long Island Jewish Health Systems, Glen Oaks, USA

The APOE e4 allele is associated with greater hippocampal atrophy in the subicular and CA1 areas in Alzheimer's disease: an in vivo MR study, Michela Pievani ${ }^{l}$, Francesca Sabattoli $^{1}$, Cristina Testa ${ }^{1,2}$, Matteo Bonetti ${ }^{3}$, Rebecca Dutton ${ }^{4}$, Agatha Lee $^{4}$, Paul Thompson ${ }^{4}$ Giovanni Frisoni ${ }^{1,5,6},{ }^{1}$ LENITEM Laboratory of Epidemiology, Neuroimaging and TelemedicineIRCCS Centro S. Giovanni di Dio - FBF, Brescia, Italy, ${ }^{2}$ Machine Vision Laboratory, Department of Mathematics and Computer Science, University of Udine, Udine, Italy, ${ }^{3}$ Service of Neuroradiology, Istituto Clinico Città di Brescia, Brescia, Italy, ${ }^{4}$ Laboratory of Neuroimaging, Department of Neurology, UCLA School of Medicine, Los Angeles, USA, ${ }^{5}$ Psychogeriatric WardIRCCS Centro San Giovanni di Dio - FBF, Brescia, Italy, ${ }^{6}$ A.Fa.R. Associazione Fatebenefratelli per la Ricerca, Rome, Italy

BrainVISA Plugin for Cortical Thickness Measurement Using Surface Normals with Curvature Thresholding, Bill Rogers ${ }^{l}$, Peter Kochunov ${ }^{1}$, David Glahn ${ }^{l}$, Jeff Rogers ${ }^{2}$, Peter Fox ${ }^{l}$, ${ }^{1}$ University of Texas Health Science Center, San Antonio, USA, ${ }^{2}$ Southwest Foundation for Biomedical Research, San Antonio, USA

Unbiased High Resolution T1 Weighted Brain Images at High Field with a New Interleaved 3D-MPRAGE/Proton Density GE sequence, Pierre-Francois Van de Moortele, Eddie Auerbach, Cheryl Olman, Essa Yacoub, Kamil Ugurbil, Steen Moeller, CMRR-University of Minnesota, Minneapolis, USA

A surface-based fractal information dimension method for cortical complexity analysis, Yuanchao zhang ${ }^{1,3}$, Jiefeng Jiang ${ }^{l}$, Lei Lin ${ }^{l, 3}$, Feng Shi ${ }^{1}$, Chunshui Y ${ }^{2}$, Tianzi Jiang ${ }^{l},{ }^{l}$ National Laboratory of Pattern Recognition, Institute of Automation, Chinese Academy of Sciences, Beijing, China, ${ }^{2}$ Department of Radiology, Xuanwu Hospital of Capital Medical University, Beijing,

China, ${ }^{3}$ Department of Mathematics, Zhejiang University, Hangzhou, China

\section{PHYSIOLOGY, METABOLISM, \& NEUROTRANSMISSION}

Striatal dopamine release induced by repetitive transcranial magnetic stimulation over dorsolateral prefrontal cortex: Effect of aging, Seong Ae Bang ${ }^{1,2}$, Sang Soo Cho ${ }^{1,2}$, Eun Jin Yoon $^{1,2}$, Ji Sun Kim ${ }^{1,2}$, Byung Chul Lee ${ }^{1,2}$, Yu Kyeong Kim ${ }^{1,2}$, Sang Eun Kim ${ }^{1,2}$, ' Seoul National University College of Medicine, Seoul, South Korea, ${ }^{2}$ Seoul National University Bundang Hospital, Seoul, South Korea

Laminar distribution and co-distribution of neurotransmitter receptors in early human visual cortex, Claudia Rottschy ${ }^{1,2,3}$, Simon B. Eickhoff ${ }^{2}$, Karl Zilles ${ }^{1,2,4},{ }^{I} C \& O$. Vogt Institute of Brain Research, University of Düsseldorf, Düsseldorf, Germany, ${ }^{2}$ Institute of Neuroscience and Biophysics, INB-3 Medicine, Research Centre Jülich, Jülich, Germany, ${ }^{3}$ Dept. of Neurology, RWTH Aachen, Aachen, Germany, ${ }^{4}$ Brain Imaging Center West (BICW), Jülich, Germany

fMRI correlates of EEG slow oscillations during sleep in humans, Silvina Horovitz ${ }^{l}$, Masaki Fukunaga $^{1}$, Dante Picchioni ${ }^{2}$, Walter Carr ${ }^{3}$, Jacco de Zwart ${ }^{1}$, Peter van Gelderen ${ }^{1}$, Thomas Balkin', Allen Braun ${ }^{4}$, Jeff Duyn ${ }^{l},{ }^{1}$ NINDS - National Institutes of Health, Bethesda, USA, ${ }^{2}$ Walter Reed Army Institute of Research, Silver Spring, USA, ${ }^{3}$ Naval Medical Research Center, Silver Spring, USA, ${ }^{4}$ NIDCD -National Institutes of Health, Bethesda, USA

Brain activation involved in appetite change in schizophrenia patients treated with atypical antipsychotic, Emmanuel Stip ${ }^{1,2,3,4}$, Adham Mancini-Marie ${ }^{1,2}$, Karyne Anselmo ${ }^{1}$, Genevieve Létourneau $^{1,2}$, Pascal Dellamillieure ${ }^{3,4}$, Adrianna Mendrek ${ }^{1,2}$, Lahcen Ait Bentaleb ${ }^{1,2}$, Olivier Lipp $^{1,2}$, Marie-Claude Delisle ${ }^{1,2}$, Pierre Léouffre ${ }^{1,2}$, Tania Pampoulova ${ }^{1}$, Pierre Lalonde ${ }^{1,2}$, Sonia Dollfus ${ }^{3,4},{ }^{1}$ Department of Psychiatry, Centre de Recherche Fernand Seguin, L-H Lafontaine 
Hospital, University of Montreal, Montreal, Canada, ${ }^{2}$ Department of Psychiatry, Faculty of Medicine, University of Montreal, Montreal, Canada, ${ }^{3}$ Centre Esquirol, Université de Basse Normandie, CHU Cote de Nacre, Caen, France, ${ }^{4}$ Centre Cyceron, Caen, France

Use of FDG-PET to Evaluate the Limbic-Pituitary-Adrenal Axis During Estrogen Challenge: A Preliminary Analysis, William Ottowitz ${ }^{1}$, Martin Lindquist ${ }^{2}$, Darin Dougherty ${ }^{3}$, Alan Fischman , Janet Hall ${ }^{5},{ }^{1}$ GSAS, Columbia University, New York, USA, ${ }^{2}$ Dept Statistics, Columbia University, New York, USA, ${ }^{3}$ MGH Psychiatric Neuroscience Program, Boston, USA, ${ }^{4}$ MGH Dept of Nuclear Medicine, Boston, USA, ${ }^{5}$ Reproductive Endocrinology, Boston, $U S A$

Prospective Neurochemical Characterization of Child Offspring of Parents with Bipolar Disorder, Manpreet Singh ${ }^{1}$, Kiki Chang ${ }^{l}$, Daniel Spielman ${ }^{2},{ }^{1}$ Stanford University School of Medicine, Stanford, USA, ${ }^{2}$ Richard Lucas Center for Magnetic Resonance Spectroscopy and Imaging, Stanford, USA

Regional distribution of aerobic glycolysis in the resting human brain, S. Neil Vaishnavi, Andrei Vlassenko, Melissa Rundle, Abraham Snyder, Mark Mintun, Marcus Raichle, Dept. Radiology, Washington Univ. School of Medicine, St. Louis, USA

BOLD Response in Lateral Geniculate Nucleus (LGN) at Very Short Stimulus Durations, Barış̧ Yeşilyurt ${ }^{1}$, Kamil Uğurbil ${ }^{1,2}$, Kamil Uludağ ${ }^{1},{ }^{1}$ Max-Planck-Institute for Biological Cybernetics, High-Field Magnetic Resonance Center, Tübingen, Germany, ${ }^{2}$ Center for Magnetic Resonance Research, Department of Radiology, University of Minnesota Medical School, Minneapolis, USA

\section{SENSORY SYSTEMS Multisensory \& Crossmodal}

An electrophysiological study of the development of multisensory facilitation in children., Ayla Barutchu ${ }^{l}$, Hamish Innes-Brown ${ }^{l}$, Mohit N. Shivdasani ${ }^{l}$, Sheila Crewther ${ }^{2}$, Tony G. Paolini ${ }^{l}{ }^{2}$, ${ }^{I}$ Auditory Clinical Neuroscience Unit, The Bionicear Institute, Melbourne, Australia, ${ }^{2}$ School of Psychological Sciences, Melbourne, Australia

Sound-induced illusory flashes: issues for a psychophysiological investigation., Hamish InnesBrown $^{l, 2}$, David Crewther ${ }^{2},{ }^{I}$ Bionic Ear Institute, Melbourne, Australia, ${ }^{2}$ Brain Sciences Institute, Swinburne University, Melbourne, Australia

Neural correlates of sensory feedback loops in reaching, Alexandra Reichenbach ${ }^{1,2}$, Jean-Pierre Bresciani ${ }^{2}$, Angelika Peer ${ }^{3}$, Kamil Uludag ${ }^{I}$, Heinrich Bülthoff ${ }^{2}$, Axel Thielscher ${ }^{1}$, ${ }^{I}$ Max-Planck Institute for Biological Cybernetics, High-Field Magnetic Resonance Center, Tübingen, Germany, ${ }^{2}$ Max-Planck Institute for Biological Cybernetics, Dept. for Cognitive and Computational Psychophysics, Tübingen, Germany, ${ }^{3}$ Technische Universität München, Institute of Automatic Control Engineering, Munich, Germany

SEGREGATED VISUO-HAPTIC PROCESSING OF TEXTURE AND LOCATION, Gregory Gibson ${ }^{1,2}$, Randall Stilla ${ }^{2}$, Krish Sathian ${ }^{1,2},{ }^{1}$ Rehabilitation R\&D Center of Excellence, Atlanta VAMC, Decatur, USA, ${ }^{2}$ Department of Neurology, Emory University, Atlanta, USA

\section{SENSORY SYSTEMS}

Pain \& Autonomic Function

Cola-bottle Tonic Pain Test (C-TPT) on EEG Default Mode Spectral Field Power Mapping, Andrew CN Chen*, Liping Song, Li Du, Yanling Luo, Center for Higher Brain Functions, Capital Medical University, Beijing, China

Enhanced functional connectivity of the dorsolateral prefrontal cortex during intermittent pain in patients with Alzheimer's disease, Leonie Cole ${ }^{1,2,3}$, Maria Gavrilescu', Stephen Gibson $^{3,4,5}$, Michael Farrell ${ }^{1,2,3}$, Gary Egan ${ }^{1,2},{ }^{1}$ Howard Florey Institute, Florey Neurosciences Institute, Parkville, Australia, ${ }^{2}$ Centre for Neuroscience, University of Melbourne, Parkville, Australia, ${ }^{3}$ National Ageing Research Institute, Parkville, Australia, ${ }^{4}$ Department of Medicine, University of Melbourne, Parkville, Australia, ${ }^{5}$ Caulfield Pain Management and Research Centre, Caulfield, Australia 
Illness Behaviour in Chronic Low Back Pain Patients is Associated with Reduced Insular Cortex Volume, Sioban Kelly, ${ }^{1,2}$, Donna Lloyd ${ }^{3}$, Gordon Findlay ${ }^{4}$, John Downes ${ }^{2}$, Turo Nurmikkol, Neil Roberts ${ }^{5},{ }^{1}$ Pain Research Institute, Liverpool, United Kingdom, ${ }^{2}$ School of Psychology, University of Liverpool, Liverpool, United Kingdom, ${ }^{3}$ School of Psychology, University of Manchester, Manchester, United Kingdom, ${ }^{4}$ The Walton Centre for Neurology and Neurosurgery, Liverpool, United Kingdom, ${ }^{5}$ Magnetic Resonance Image Analysis Research Centre, University of Liverpool, Liverpool, United Kingdom

Brain responses to visceral pain - influence of central serotonin signaling, Jennifer Labus ${ }^{l}$, Michiel van Nieuwenhoven ${ }^{3}$, Shin Fukudo ${ }^{2}$, Emeran Mayer ${ }^{I},{ }^{\prime}$ Center for Neurobiology of Stress, Brain Research Institute, Depts of Psychiatry and Biobehavioral Sciences and Medicine at the University of California, Los Angeles, Los Angeles, USA, ${ }^{2}$ Behavioral Medicine, Tohoku University Graduate School of Medicine, Sendai, Miyagi, Japan, Sendai, Miyagi, Japan, ${ }^{3}$ Gastroenterology, University Hospital Maastricht, The Netherlands, Maastricht, Netherlands

NMDA-antagonist and morphine reduce pain and fMRI-activation of pain areas in CRPS, Anja Schwarz ${ }^{1}$, Sylvia Gustin ${ }^{1}$, Niels Birbaumer ${ }^{1}$, Nektarius Sinis ${ }^{2}$, Ralf Veit ${ }^{1}$, Wolfgang Larbig ${ }^{1}$, Herta Flor ${ }^{3}$, Martin Lotze ${ }^{4},{ }^{1}$ Institute of Medical Psychology and Behavioral Neurobiology, Tuebingen, Germany, ${ }^{2}$ Traumatology Hospital of the University of Tuebingen, Tuebingen, Germany, ${ }^{3}$ Department of Clinical and Cognitive Neuroscience at the University of Heidelberg, Central Institute of Mental Health, Mannheim, Germany, ${ }^{4}$ Functional Imaging Institute for Diagnostic Radiology and Neuroradiology, University of Greifswald, Greifswald, Germany

Acupuncture Mediated Brain Activity Demonstrated with fMRI at 4 Tesla, Mark Strudwick ${ }^{l}$, Katie McMahon ${ }^{l}$, Stephen Wilson ${ }^{2}$, Greig DeZubicaray ${ }^{l},{ }^{l}$ Centre for Magnetic Resonance, University of Queensland, Brisbane, Australia, ${ }^{2}$ School of ITEE, University of Queensland, Brisbane, Australia

\section{COGNITION \& ATTENTION}

Attention (auditory, tactile, motor)

The "VP1": an early voice-preferential electrophysiological response, Ian Charest ${ }^{l}$, Cyril Pernet $^{2}$, Guillaume Rousselet ${ }^{1}$, Sarah Fillion-Bilodeau ${ }^{3}$, Pascal Belin ${ }^{1,4},{ }^{1}$ Centre for Cognitive Neuroimaging, Department of Psychology, University of Glasgow, Glasgow, United Kingdom, ${ }^{2}$ SFC Brain Imaging Research Center, Department of Clinical Neurosciences, University of Edinburgh, Edinburgh, United Kingdom, ${ }^{3}$ Département de Psychologie, Université de Montréal, Montréal, Canada, ${ }^{4}$ International Laboratory for Brain, Music and Sound Research, Université de Montréal and McGill University, Montréal, Canada

Examining the Pharmacology of Mismatch Negativity: Electrophysiological Investigations in Healthy Subjects, Sumie Leung ${ }^{1}$, Rodney Croft ${ }^{1}$, Torsten Baldeweg ${ }^{2}$, Barry O'Neill', Pradeep Nathan ${ }^{3},{ }^{1}$ Biological Psychiatry Research Unit, Brain Sciences Institute, Faculty of Life and Social Sciences, Swinburne University of Technology, Melbourne, Australia, ${ }^{2}$ Institute of Child Health (University College London) and Great Ormond Street Hospital for Children NHS Trust, London, United Kingdom, ${ }^{3}$ Department of Psychiatry, University of Cambridge, Cambridge, United Kingdom

Top-down and bottom-up control of auditory attention: A combined fMRI and probabilistic tractography study, Juha Salmi ${ }^{1,2,3}$, Teemu Rinne ${ }^{1}$, Sonja Koistinen ${ }^{1,3}$, Tuomas Neuvonen ${ }^{2,3}$, Synnöve Carlson ${ }^{2,4,5}$, Oili Salonen ${ }^{6}$, Kimmo Alho ${ }^{1},{ }^{1}$ Department of Psychology, University of Helsinki, Finland, ${ }^{2}$ Neuroscience Unit, Institute of Biomedicine/physiology, University of Helsinki, Finland, ${ }^{3}$ Advanced Magnetic Imaging Centre, Helsinki University of Technology, Finland, ${ }^{4}$ Medical School, University of Tampere, Finland, ${ }^{5}$ Brain Research Unit, Helsinki University of Technology, Finland, ${ }^{6}$ Helsinki Medical Imaging Center, Helsinki University Central Hospital, Finland

Schizophrenia-associated deficits of mismatch negativity reflect stimulus presentation and auditory feature with special focus on emotional prosody, Heike Thoennessen ${ }^{l}$, Mikhail Zvyagintsev ${ }^{1}$, Frank Boers ${ }^{2}$, Juergen Dammers ${ }^{2}$, Christine Norra ${ }^{3}$, Klaus Mathiak ${ }^{1,4},{ }^{1}$ Dept. of Psychiatry and Psychotherapy, RWTH Aachen University, Aachen, Germany, ${ }^{2}$ Institute of Medicine, 
Research Center Jülich, Juelich, Germany, ${ }^{3}$ Max-Planck-Institute of Experimental Medicine, Goettingen, Germany, ${ }^{4}$ Institute of Psychiatry King's Colllege London, London, United Kingdom

\section{COGNITION \& ATTENTION}

Attention (visual)

Right Parietal Cortex and Top-Down Visuospatial Attention: Combined on-line rTMS and fMRI, Felix Blankenburg ${ }^{1}$, Christian Ruff, ${ }^{2}$, Sven Bestmann ${ }^{2}$, Oliver Josephs ${ }^{2}$, Ralf Deichmann ${ }^{4}$, Otto Bjoertomt ${ }^{2,3}$, Jon Driver ${ }^{2,3},{ }^{1}$ Bernstein Center for Computational Neuroscience, Charite, Berlin, Germany, ${ }^{2}$ Institute of Cognitive Neuroscience, University College London, London, United Kingdom, ${ }^{3}$ Wellcome Trust Centre for Neuroimaging at UCL, Institute of Neurology, London, United Kingdom, ${ }^{4}$ University Hospital, Brain Imaging Center, Frankfurt, Germany

Attentional Modulation of Multisensory Audiovisual Integration during Speech Perception, Scott Fairhall, Emiliano Macaluso, Santa Lucia Foundation, Rome, Italy

Probing the link between sources and targets of attentional control: a concurrent TMS-fMRI study of visuospatial selection, Klaartje Heinen ${ }^{1}$, Christian Ruff ${ }^{1}$, Sven Bestmann ${ }^{2}$, Bertram Schenkluhn ${ }^{1}$, Felix Blankenburg ${ }^{3}$, Otto Bjoertomt ${ }^{1}$, Vincent Walsh ${ }^{1}$, Jon Driver ${ }^{1}$, Chris Chambers ${ }^{1}$, ${ }^{I}$ Institute of Cognitive Neuroscience, UCL, London, United Kingdom, ${ }^{2}$ Wellcome Trust Centre for Neuroimaging, London, United Kingdom, ${ }^{3}$ Department of Neurology and Neuroscience Research Center, Charité, Berlin, Germany

Brain responses to direct gaze: An optical topography study, Yuko Isogayal, Akiko Obata ${ }^{2}$, Hiroki Sato ${ }^{2}$, Atsushi Maki ${ }^{2}$, Takao Sato ${ }^{1}$, Norito Kawakami ${ }^{1}$, 'The University of Tokyo, Tokyo, Japan, ${ }^{2}$ Advanced Research Laboratory, Hitachi, Ltd, Saitama, Japan

\section{EFFECTS OF TRANSCRANIAL ANODAL DIRECT CURRENT STIMULATION OVER THE RIGHT PARIETAL CORTEX ON UNILATERAL NEGLECT IN STROKE}

PATIENTS, Myoung-Hwan Ko ${ }^{l}$, Sang-Hyoung Han ${ }^{l}$, Jeong-Hwan Seo ${ }^{l}$, Yun-Hee Kim ${ }^{2}$, ${ }^{1}$ Chonbuk National University Medical School \& Hospital, Jeonju, South Korea, ${ }^{2}$ Sungkyunkwan

University School of Medicine, Samsung Medical Center, Seoul, South Korea

Selective guidance of attention by items in working memory: converging fMRI and ERP results, Judith Peters ${ }^{1,2}$, Pieter Roelfsema ${ }^{3,4}$, Rainer Goebel ${ }^{1,2},{ }^{i}$ Cognitive Neuroscience Dept, Faculty of Psychology, Maastricht University, Maastricht, Netherlands, ${ }^{2}$ Brain Imaging Center (M-BIC), Maastricht University, Maastricht, Netherlands, ${ }^{3}$ Department of Vision and Cognition, Netherlands Institute for Neuroscience, an institute of the Royal Netherlands Academy of Arts and Sciences (KNAW), Amsterdam, Netherlands, ${ }^{4}$ Department of Experimental Neurophysiology, Center for Neurogenomics and Cognitive Research, Vrije Universiteit, Amsterdam, Netherlands

Working Memory Consolidation Delays Perceptual Processing in Visual Cortex: A TimeResolved fMRI Study, Paige Scalf ${ }^{\prime}$, Paul Dux ${ }^{2}$, Rene' Marois ${ }^{2},{ }^{I}$ Beckman Institute, University of Illinois at Urbana-Champaign, Urbana, USA, ${ }^{2}$ Department of Psychology, Vanderbilt Vision Research Center, Center for Integrative and Cognitive Neuroscience, Vanderbilt University, Nashville, USA

Lapses in attention during sleep deprivation: more than meets the eye, Michael WL Chee ${ }^{I}$, Jiat Chow Tan ${ }^{l}$, Hui Zheng ${ }^{1}$, Parimal Sarayu ${ }^{l}$, Weismann H Daniel ${ }^{2}$, Zagorodnov Vitali ${ }^{3}$, David F Dinges ${ }^{4},{ }^{1}$ Cognitive Neuroscience Laboratory, Duke-NUS Graduate Medical School, Singapore, Singapore, ${ }^{2}$ Department of Psychology, University of Michigan, Michigan, USA, ${ }^{3}$ School of Computer Engineering, Nanyang Technological University, Singapore, Singapore, ${ }^{4}$ Unit for Experimental Psychiatry, niversity of Pennsylvania School of Medicine, Pennsylvania, USA

\section{COGNITION \& ATTENTION Cognitive Aging}

Structural consequences of chronic insomnia: a voxel-based morphometric study, Ellemarije Altena $^{1,2}$, Hugo Vrenken ${ }^{2}$, Ysbrand Van der Werf ${ }^{1,2}$, Eus Van Someren ${ }^{1,2}$, ${ }^{1}$ Netherlands Institute for Neuroscience, Amsterdam, Netherlands, ${ }^{2}$ VU University Medical Center, Amsterdam, Netherlands

Age-Related Neural Inefficiency and Compensation Across Multiple Cognitive Domains, Cheryl Grady, Andrea Protzner, Magda Wojtowicz, Darryl Bannon, Randy McIntosh, Rotman Research Institute, Toronto, Canada 
Cognitive training impacts functional brain activity and cerebral blood flow of healthy older adults in a randomized controlled trial, Jennifer Mozolic ${ }^{1,2}$, Ashley Morgan ${ }^{l}$, Paul Laurienti ${ }^{l}$, ${ }^{I}$ Department of Radiology, Wake Forest University School of Medicine, Winston-Salem, USA,

${ }^{2}$ Graduate Program in Neuroscience, Wake Forest University School of Medicine, Winston-Salem, USA

\section{COGNITION \& ATTENTION \\ Cognitive Development}

The development of white matter tracts and response inhibition examined using diffusion tensor imaging, Jessica Cohen ${ }^{1}$, Fred Sabb ${ }^{2}$, Robert Bilder ${ }^{1,2}$, Susan Bookheimer ${ }^{2,3,4,5}$, Barbara Knowlton ${ }^{1,3,4}$, Robert Asarnow ${ }^{2}$, Russell Poldrack ${ }^{1,3,4},{ }^{1}$ UCLA Department of Psychology, Los Angeles, USA, ${ }^{2}$ UCLA Department of Psychiatry, Los Angeles, USA, ${ }^{3}$ UCLA Brain Research Institute, Los Angeles, USA, ${ }^{4}$ UCLA Interdepartmental Neuroscience Program, Los Angeles, USA, ${ }^{5}$ UCLA Brain Mapping Center, Los Angeles, USA

Early Development of Cortical Brain Responses to Rapidly Presented Auditory Stimulation: a Magnetoencephalographic Study, Carolin Sheridan ${ }^{l, 2}$, Rossitza Draganova ${ }^{3}$, Hubert Preissl ${ }^{1,2}$, Eric Siegel ${ }^{1}$, Rathinaswamy Govindan ${ }^{I}$, Hari Eswaran ${ }^{l}$, Curtis Lowery ${ }^{I},{ }^{I}$ University of Arkansas for Medical Sciences, Little Rock, USA, ${ }^{2}$ University of Tuebingen, Tuebingen, Germany,

${ }^{3}$ University of Muenster, Muenster, Germany

Development of Default Mode and Task Positive Network Integrity and Interactions from Childhood to Young Adulthood, AM Clare Kelly', Lucina Uddin', Zarrar Shezad ${ }^{l}$, Dylan Gee ${ }^{l}$, Daniel Margulies ${ }^{1,2}$, Adriana Di Martino ${ }^{I}$, F Xavier Castellanos ${ }^{l}$, Michael Milham ${ }^{l},{ }^{I}$ Phyllis Green and Randolph Cowen Institute for Pediatric Neuroscience, NYU Child Study Center, New York, USA, ${ }^{2}$ Berlin School of Mind and Brain, Humboldt-Universität zu Berlin, Berlin, Germany

Basal Perfusion in Adolescents at Risk for Alcohol Use Disorders, Ai-Ling Lin ${ }^{l}$, David Glahn ${ }^{1}$, Rene Olvera ${ }^{2}$, Peter Fox ${ }^{1}$, Ahmad Hariri ${ }^{3}$, Douglas Williamson ${ }^{4},{ }^{1}$ Research Imaging Center, University of Texas Health Science Center, San Antonio, USA, ${ }^{2}$ Department of Psychiatry, University of Texas Health Science Center, San Antonio, USA, ${ }^{3}$ Department of Psychiatry, University of Pittsburgh, Pittsburgh, USA, ${ }^{4}$ Department of Psychiatry, Epidemiology and Biostatistics, University of Texas Health Science Center, San Antonio, USA

Emotion, cognition and its interaction in adolescent-onset schizophrenia: an fMRI study, Katharina Pauly ${ }^{1}$, Nina Seiferth ${ }^{1}$, Thilo Kellermann ${ }^{1}$, Timo Vloet ${ }^{2,3,4}$, N. Jon Shah ${ }^{3,4,5}$, Frank Schneider ${ }^{1,3,4}$, Ute Habel ${ }^{l}$, Tilo Kircher ${ }^{1,3,4}$, ${ }^{l}$ Department of Psychiatry and Psychotherapy, RWTH Aachen University, Aachen, Germany, ${ }^{2}$ Department of Child and Adolescent Psychiatry and Psychotherapy, RWTH Aachen University, Aachen, Germany, ${ }^{3}$ Brain Imaging Center West, Juelich, Germany, ${ }^{4}$ Institute of Neuroscience and Biophysics - Medicine, Research Center Juelich, Juelich, Germany, ${ }^{5}$ Institute of Physics, University of Dortmund, Dortmund, Germany

\section{COGNITION \& ATTENTION}

Perception, Imagery, Awareness

Components in Continuous Meditation. An fMRI Investigation, Klaus B. Bcerentsen ${ }^{1,2}$, Bo Sommerlund ${ }^{l}$, Johannes Damsgaard-Madsen ${ }^{1,2}$, Mark Fosnoes ${ }^{l}$, Pernille Bruhn ${ }^{1,2}$, Hans StødkildeJørgensen ${ }^{2},{ }^{l}$ Department of Psychology, University of Aarhus, Aarhus, Denmark, ${ }^{2} M R$ ResearchCenter, Aarhus University Hospital, Aarhus, Denmark

Resting state connectivity integrity in the default network reflects the level of consciousness impairment in brain-injured patients. An fMRI study in brain death, coma, vegetative state, minimally conscious state and locked-in syndrome, Melanie Boly ${ }^{1,2}$, Audrey Vanhaudenhuyse ${ }^{l}$, Luaba Tshibanda ${ }^{3}$, Marie-Aurelie Bruno ${ }^{1}$, Pierre Boveroux ${ }^{1,4}$, Quentin Noirhomme ${ }^{l}$, Caroline Schnakers ${ }^{l}$, Athena Demertzi ${ }^{l}$, Didier Ledoux ${ }^{1,4}$, Bernard Lambermont ${ }^{5}$, Gustave Moonen ${ }^{2}$, Robert-Ferninand Dondelinger ${ }^{3}$, Christophe Phillips ${ }^{1}$, Pierre Maquet ${ }^{1,2}$, Steven Laureys ${ }^{1,2},{ }^{1}$ Coma 88 T-PM* Science Group, Cyclotron Research Center, University of Liège, Liège, Belgium, ${ }^{2}$ Neurology Department, CHU Sart Tilman Hospital, University of Liège, Liège, Belgium, ${ }^{3}$ Radiology Department, CHU Sart Tilman Hospital, University of Liège, Liège, Belgium, ${ }^{4}$ Anesthesiology, Department, CHU Sart Tilman Hospital, University of Liège, Liège, ${ }^{5}$ Internal Medicine Department, CHU Sart Tilman Hospital, University of Liège, Liège, 
Segregating parietal areas related to number processing and response times, Marinella Cappelletti ${ }^{1}$, Hwee-Ling Lee ${ }^{2}$, Elliot Freeman ${ }^{1}$, Cathy Price ${ }^{2},{ }^{1}$ Institute of Cognitive Neuroscience, 92 T-PM London, United Kingdom, ${ }^{2}$ Wellcome Trust Centre for Neuroimaging, London, United Kingdom

Neuromagnetic correlates of mental rotation of hands, Lincoln J. Colling, Blake Johnson, Macquarie Centre for Cognitive Science, Macquarie University, Sydney, Australia

Different cues to the beat during auditory sequence perception modulate motor area activity: an fMRI investigation of musicians and non-musicians, Jessica Grahn, James Rowe, Medical Research Council, Cognition and Brain Sciences Unit, Cambridge, United Kingdom

A Repetition Suppression Study of the Visual Processing of Gait and Configuration from Biological Motion, Ashley Hamlin, James Thompson, George Mason University, Fairfax, USA

Simultaneous recording of fNIRS and SCR improves lie detection accuracy, Toyoharu Hosokawa ${ }^{l}$, Koji Kazai ${ }^{I}$, Akihiro Yagi ${ }^{2}$, Haruhiro Katayose ${ }^{1},{ }^{1}$ Kwansei Gakuin University, Sanda, Japan, ${ }^{2}$ Kwansei Gakuin University, Nishinomiya, Japan

Complexity-dependent changes of the spontaneous brain activities in the parietal cortices during mental arithmetic, Sunao Iwaki, Hiroko Kou-Shimazaki, Natl. Inst. Adv. Indust. Sci. \& Tech (AIST), Ikeda, Japan

\section{COGNITION \& ATTENTION \\ Reasoning \& Problem Solving}

\section{REGIONAL DOPAMINE D2 RECEPTOR DENSITY AND INDIVIDUAL DIFFERENCES IN PSYCHOMETRIC CREATIVITY, Örjan Blom ${ }^{1,3}$, Simon Červenka ${ }^{2,3}$, Anke Karabanov ${ }^{1,3}$,} Hans Forssberg ${ }^{1,3}$, Lars Farde ${ }^{2,3}$, Fredrik Ullén ${ }^{1,3},{ }^{1}$ Department of Woman and Child Health, Division for Neuropediatrics, Karolinska Institutet, Karolinska University Hospital, Stockholm, Sweden, ${ }^{2}$ Department of Clinical Neuroscience, Psychiatry Section, Karolinska Institutet, Karolinska University Hospital, Stockholm, Sweden, ${ }^{3}$ Stockholm Brain Institute, Stockholm, Sweden

Dissociable contributions of ventrolateral prefrontal and frontopolar cortex sub-regions during analogical reasoning, Adam Hampshire, John Duncan, Adrian Owen, MRC Cognition \& Brain Sciences Unit, Cambridge, United Kingdom

Parietal deactivation in major depressive disorder during cognitive performance: a functional magnetic resonance imaging study, Adham Mancini-Marie ${ }^{1,2}$, Emmanuel Stip ${ }^{1,2}$, Stephane Potvin ${ }^{1,2}$, Boualem Mensour ${ }^{4}$, Jean-Maxime Leroux ${ }^{4}$, Gilles Beaudouin ${ }^{4}$, Cherine Fahim $^{1,2,3}$, Mario Beauregard ${ }^{4,5}$, 'Department of Psychiatry, Centre de Recherche Fernand Seguin, L-H Lafontaine Hospital, University of Montreal, Montreal, Canada, ${ }^{2}$ Department of Psychiatry, Faculty of Medicine, University of Montreal, Montreal, Canada, ${ }^{3}$ Department of Neurology and Neurosurgery, McConnell Brain Imaging Centre, Montreal Neurological Institute, Mcgill University, Montreal, Canada, ${ }^{4}$ Department of Radiology, Centre Hospitalier de l'Université de Montréal (CHUM), Notre Dame Hospital, University of Montreal, Montreal, Canada, ${ }^{5}$ Department of Psychology, University of Montreal, Montreal, Canada

\section{COGNITION \& ATTENTION Space, Time, \& Number Coding}

Conceptual but not perceptual number processing is affected by TMS to the parietallobe, Marinella Cappelletti, Neil Muggleton, Vincent Walsh, University college London, London, United 128 T-PM Kingdom

Orienting Attention to Numbers: Involvement of Frontal Lobes, Elena Rusconi ${ }^{l}$, Domenica Bueti $^{2}$, Marianna Riello ${ }^{3}$, Vincent Walsh ${ }^{4}$, Brian Butterworth ${ }^{5},{ }^{1}$ CIMEC Center of Mind Brain Science, Italy, ${ }^{2} I C N$, United Kingdom, ${ }^{3}$ CIMEC, Italy, ${ }^{4}$ ICN, United Kingdom, ${ }^{5}$ ICN, United Kingdom

\section{DISORDERS OF THE NERVOUS SYSTEM Alzheimer \& Dementia}

Volumetric and functional brain changes in Huntington's disease: a two year longitudinal study, Hamed Asadi ${ }^{1}$, Nellie Georgiou-Karistian ${ }^{2}$, Maree Farrow ${ }^{3}$, Anusha Sritharan ${ }^{2}$, Ross 
Cunnington ${ }^{4}$, Gary Egan ${ }^{1},{ }^{1}$ Howard Florey Institute, University of Melbourne, Melbourne, Australia, ${ }^{2}$ Experimental Neuropsychology Research Unit, School of Psychology, Psychiatry and Psychological Medicine, Monash University, Melbourne, Australia, ${ }^{3}$ Alzheimer's Australia, Melbourne, Australia, ${ }^{4}$ Cognitive Neuroscience Laboratory, The Queensland Brain Institute, Brisbane, Australia

Amyloid deposition related to cortical thinning, J. Alex Becker ${ }^{l}$, Jeremy Carmasin ${ }^{l}$, Bruce Fischl $^{l}$, Doug Greve ${ }^{l}$, Amy DeLuca ${ }^{I}$, Pete LaViolette ${ }^{l}$, Jacqueline O'Brien ${ }^{l}$, Kelly O'Keefe ${ }^{l}$, Alan Fischman ${ }^{l}$, Dorene Rentz ${ }^{2}$, Reisa Sperling ${ }^{l, 2}$, Keith Johnson ${ }^{l, 2}{ }^{\prime}{ }^{I}$ Massachusetts General Hospital, Boston, USA, ${ }^{2}$ Brigham and Women's Hospital, Boston, USA

Reduced resting state activity in dorsal visual-spatial attention system in Alzheimer's disease, Jessica Damoiseauxl, Christian Beckmann ${ }^{2}$, Ernesto Sanz Arigita ${ }^{1}$, Cornelis Stam ${ }^{1}$, Frederik Barkhof ${ }^{1}$, Stephen Smith ${ }^{2}$, Philip Scheltens ${ }^{1}$, Serge Rombouts ${ }^{3},{ }^{1}$ VU University Medical Center, Amsterdam, Netherlands, ${ }^{2}$ Oxford Centre for Functional Magnetic Resonance Imaging of the Brain, Oxford, United Kingdom, ${ }^{3}$ Leiden Institute for Brain and Cognition (LIBC), Leiden University Medical Center, Institute for Psychological Research, Leiden University, Leiden, Netherlands

Cholinergic dysfunction in subcortical ischemic vascular dementia: a transcranial magnetic stimulation study, Stefan Golaszewski ${ }^{1,3}$, Raffaele Nardone ${ }^{2}$, Juergen Bergmann ${ }^{1,7}$, Christian Siedentop $f^{3,4}$, Florian Koppelstaetter ${ }^{3,4}$, Eugen Gallasch ${ }^{6}$, Anja Ischebeck, Gunther Ladurner ${ }^{1}$, ${ }^{I}$ Department of Neurology, Paracelsus Medical University Salzburg, Salzburg, Austria, ${ }^{2}$ Department of Neurology, F. Tappeiner Hospital Meran, Meran, Italy, ${ }^{3}$ fMRI Lab, Department of Psychiatry, Medical University Innsbruck, Innsbruck, Austria, ${ }^{4}$ Department of Radiology, Medical University Innsbruck, Innsbruck, Austria, ${ }^{5}$ Department of Neurology, Medical University Innsbruck, Innsbruck, Austria, ${ }^{6}$ Institute of Physiology, Medical University Graz, Graz, Austria, ${ }^{7}$ Institute of Psychology, University of Salzburg, Salzburg, Austria

Correlation between "Ala score" and CBF in Alzheimer's disease -A SPECT study, Takashi Kawachi $^{1}$, Hiroyasu Kusakabe ${ }^{3}$, Haruhiko Oda ${ }^{2}$, Yasuji Yamamoto ${ }^{2}$, Toshio Kawamata ${ }^{2}$, Kiyoshi Maeda ${ }^{2},{ }^{1} I B R I$, Kobe, Japan, ${ }^{2}$ Kobe university, Kobe, Japan, ${ }^{3}$ Ohara Hospital, Kobe, Japan

Correlation between findings of $\mathrm{rCBF}$ and ${ }^{1} \mathrm{H}-\mathrm{MRS}$ in posterior cingulate gyrus for the patients with memory impairment, Takashi Nihashi ${ }^{1}$, Kazumasa Hayasaka ${ }^{2}$, Yutaka Arahata ${ }^{3}$, Katsushige Iwai ${ }^{3}$, Akinori Takeda ${ }^{3}$, Yoshiko Yamaoka ${ }^{3}$,Youko Konagaya ${ }^{3}$, Yukihiko Washimi ${ }^{3}$, Kenji Yoshiyama ${ }^{4}$, Hideyuki Hattori ${ }^{4}$, Shousuke Satake ${ }^{5}$, Hisayuki Miura ${ }^{5}$, Hidetoshi Endo ${ }^{5}$, Hiroshi Yatsuya 6 , Shinji Naganawa ${ }^{1},{ }^{1}$ Department of Radiology, Nagoya University Graduate School of Medicine, Nagoya, Japan, ${ }^{2}$ Department of Radiology, National Hospital for Geriatric Medicine, Obu, Japan, ${ }^{3}$ Department of Neurology, National Hospital for Geriatric Medicine, Obu, Japan, ${ }^{4}$ Department of Psychiatry, National Hospital for Geriatric Medicine, Obu, Japan, ${ }^{5}$ Department of General Outpatient services, National Hospital for Geriatric Medicine, Obu, Japan, ${ }^{6}$ Department of Pubic Health, Nagoya University School of Medicine, Nagoya, Japan

Deconstructing Frontotemporal Lobar Degenerations, Matthias Schroeter ${ }^{1,2}$, Karolina Raczka $^{3}$, Jane Neumann ${ }^{l}$, D. Yves von Cramon ${ }^{1,2}$, ${ }^{l}$ Max-Planck-Institute for Human Cognitive and Brain Sciences, Leipzig, Germany, ${ }^{2}$ Day Clinic, Leipzig, Germany, ${ }^{3}$ Institute for Systems Neuroscience, University Medical Center Hamburg Eppendorf, Hamburg, Germany

Regional thalamic degeneration in Alzheimer's disease characterised by structural vertexand diffusion tractography-based analyses, Brian Patenaude ${ }^{l}$, Mark Jenkinson ${ }^{l}$, Jeske Damoiseaux ${ }^{2}$, Steve Smith ${ }^{1}$, Paul Matthews ${ }^{1}$, Frederik Barkhof ${ }^{2}$, Serge Rombouts ${ }^{3}$, Ernesto Sanz-Arigita ${ }^{2}$, Mojtaba Zarei ${ }^{l},{ }^{1}$ Oxford Centre for Functional Magnetic Resonance Imaging of the Brain, Oxford, United Kingdom, ${ }^{2} V U$ University Medical Center, Amsterdam, Netherlands, ${ }^{3}$ Leiden Institute for Brain and Cognition, Leiden, Netherlands

\section{DISORDERS OF THE NERVOUS SYSTEM Mood \& Anxiety Disorders}

Correlation between fractional anisotropy and cerebral measurements of gray and white matter substances in late-life depression, Diana M Bezerra, ${ }^{I}$, Marco A A Moscoso ${ }^{I}$, Salma R I Ribeiz ${ }^{l}$, Renata Ávila ${ }^{l}$, Fábio L S Duran ${ }^{2}$, Geraldo F Busatto ${ }^{2}$, Rodrigo Batistelo ${ }^{3}$, Marcel P Jackowski ${ }^{3}$ Cássio M C Bottino I, ' I'Old Age Research Group (PROTER), Department and Institute 
of Psychiatry, Faculty of Medicine, University of Sao Paulo, Sao Paulo, Brazil, ${ }^{2}$ Neuroimaging Laboratory, Department and Institute of Psychiatry, Faculty of Medicine, University of Sao Paulo, Sao Paulo, Brazil, ${ }^{3}$ Computer Science Department and Institute of Mathematics and Statistics, University of Sao Paulo, Sao Paulo, Brazil

Effects of Cholinergic Inhibition in Major Depressive Disorder on Interactions between Attention and Emotional Processing in the Amygdala, Maura Furey, Julie frost-Bellgowen, Ashish Khanna, Mark Opal, Wayne Drevets, Mood and Anxiety Disorders Program, NIMH, NIH, Bethesda, USA

Widely Spread Cortical Morphology Abnormalities in Major Depressive Disorder, Lei Lin ${ }^{1,3}$, Chunshui Yu ${ }^{2}$, Yuan Zhou ${ }^{I}$, Feng Shi ${ }^{1}$, Kuncheng Li ${ }^{2}$, Tianzi Jiang ${ }^{I},{ }^{1}$ National Laboratory of Pattern Recognition, Institute of Automation, Chinese Academy of Sciences, Beijing, China, ${ }^{2}$ Department of Radiology, Xuanwu Hospital of Capital Medical University, Beijing, China,

${ }^{3}$ Department of Mathematics, Zhejiang University, Hangzhou, China

Differences between bipolar disorder patients and control subjects using DTI and trackbased spatial statistics, Jadwiga Rogowska ${ }^{I}$, Tomasz Soltysinski ${ }^{l, 2}$, Deborah Yurgelun-Todd ${ }^{l}$, ${ }^{l}$ Cognitive Neuroimaging Laboratory, Brain Imaging Center, McLean Hospital \& Harvard Medical School, Belmont, USA, ${ }^{2}$ Institute for Precision and Biomedical Engineering, Warsaw University of Technology, Warsaw, Poland

Differential activation of fronto-striato-limbic circuitry in panic disorder and posttraumatic stress disorder, Oliver Tuescher ${ }^{1,3,9}$, Xenia Protopopescu ${ }^{1,2,9}$, Hong Pan ${ }^{1}$, Marylene Cloitre ${ }^{4}$, Tracy Butler ${ }^{1}$, Martin Goldstein ${ }^{1,5}$, Almut Engelien ${ }^{1,6}$, Daniella Furman ${ }^{1}$, Michael Silverman ${ }^{1,5}$, Yihong Yang ${ }^{1}$, Elizabeth Phelps ${ }^{7}$, Jack Gorman ${ }^{5}$, Joseph LeDoux ${ }^{8}$, David Silbersweig ${ }^{1}$, Emily Stern', IFunctional Neuroimaging Laboratory, Weill Medical College of Cornell University, New York, USA, ${ }^{2}$ The Rockefeller University Laboratory of Neuroendocrinology, New York, USA, ${ }^{3}$ epartment of Neurology, Albert-Ludwigs-University, Freiburg, Germany, ${ }^{4}$ NYU Child Studies Center, New York University School of Medicine, New York, USA, ${ }^{5}$ Mount Sinai School of Medicine, New York, USA, ${ }^{6}$ Department of Psychiatry, Münster, Germany, ${ }^{7}$ Deptartment of Psychology, New York University, New York, USA, ${ }^{8}$ Center for Neural Science, New York University, New York, USA, ${ }^{9}$ both authors contributed equally to this work

\section{DISORDERS OF THE NERVOUS SYSTEM Parkinson's Disease \& Other Basal Ganglia}

Changes in Tissue Intensity Associated with Disease Severity in Huntington's Disease, Elizabeth Aylward ${ }^{l}$, Jennifer Dines ${ }^{l}$, Katherine Field ${ }^{l}$, Olivia Liang ${ }^{l}$, Reading Sarah ${ }^{2}$, Ross Christopher ${ }^{2},{ }^{1}$ University of Washington, Seattle, USA, ${ }^{2}$ Johns Hopkins University, Baltimore, USA

Diffusion tensor imaging in the analysis of white matter alterations in idiopathic restless legs syndrome, Jan Kassubek, Hans-Peter Müller, Anne-Dorte Sperfeld, Alexander Unrath, Dept. of Neurology, University of Ulm, Ulm, Germany

Patterns of fractional anisotropy changes in white matter of cerebellar peduncles sensitive for distinguishing cerebellar diseases, Neal Prakash ${ }^{1,2}$, Nathan Hageman ${ }^{2}$, Xue Hua ${ }^{2}$, Arthur Toga ${ }^{2}$, Susan Perlman ${ }^{2}$, Noriko Salamon ${ }^{3},{ }^{1}$ Kaiser Hawaii, Honolulu, USA, ${ }^{2}$ UCLA, Neurology, Los Angeles, USA, ${ }^{3}$ UCLA, Radiology, Los Angeles, USA

Mean-Field Modelling of Parkinsonian Tremor, Sacha van Albada ${ }^{1,2}$, Peter Robinson ${ }^{1,2,3}$, ${ }^{I}$ School of Physics, University of Sydney, Sydney, Australia, ${ }^{2}$ The Brain Dynamics Centre, Westmead Millennium Institute, Westmead Hospital and Western Clinical School of the University of Sydney, Westmead, Australia, ${ }^{3}$ Faculty of Medicine, University of Sydney, Sydney, Australia

\section{DISORDERS OF THE NERVOUS SYSTEM Schizophrenia}

Dopamine-induced changes in neural network patterns supporting aversive conditioning, Andreea Diaconescul, Mahesh Menon ${ }^{2}$, Shitij Kapur ${ }^{2}$, Anthony McIntosh ${ }^{1},{ }^{1}$ Rotman Research Institute, Toronto, Canada, ${ }^{2}$ Centre for Addiction and Mental Health, Toronto, Canada 
Jenkinson ${ }^{1}$, Paul Matthews ${ }^{2}$, Anthony James ${ }^{3},{ }^{1}$ FMRIB Centre, Oxford University, Oxford, United Kingdom, ${ }^{2}$ CIC, GSK, London, United Kingdom, ${ }^{3}$ Warneford Hospital, Oxford, United Kingdom

Mechanism of Nicotinic Enhancement of Visual Attention in Schizophrenia, L. Elliot Hong ${ }^{l}$, Thomas Ross ${ }^{2}$, Betty Jo Salmeron ${ }^{2}$, Gunvant Thaker ${ }^{1}$, Elliot Stein ${ }^{2},{ }^{1}$ Maryland Psychiatric Research Center, Department of Psychiatry, University of Maryland School of Medicine, Baltimore, USA,

${ }^{2}$ Neuroimaging Research Branch, National Institute on Drug Abuse, NIH, Baltimore, USA

Brain regions associated with presence in the virtual environment: Comparison between patients with schizophrenia and healthy controls, Soo Hee Choi ${ }^{1}$, Jae-Jin Kim ${ }^{1,2}$, Jeonghun $\mathrm{Ku}^{2,3}$, So Young Kim ${ }^{2}$, Hyeong Rae Lee ${ }^{3}$, Il Ho Park ${ }^{1,2}$, Kang-Jun Yoon ${ }^{4}$, Sun I. Kim ${ }^{3},{ }^{1}$ Department of Psychiatry, Yonsei University College of Medicine, Seoul, South Korea, ${ }^{2}$ Institute of Behavioral Science in Medicine, Yonsei University College of Medicine, Gwangju, South Korea, ${ }^{3}$ Department of Biomedical Engineering, Hanyang University, Seoul, South Korea

Decreased Information Transmission Efficiency in Schizophrenia, Yong Liu ${ }^{l}$, Yuan Zhou, Ming Song , Yihui Hao ${ }^{2}$, Haihong Liu', Zhening Liu ${ }^{2}$, Tianzi Jiang ${ }^{l},{ }^{\prime}$ National Laboratory of Pattern Recognition, Institute of Automation, Chinese Academy of Sciences, Beijing, China,

${ }^{2}$ Institute of Mental Health, Second Xiangya Hospital, Central South University, Changsha, China

Dysbindin is Associated with Imaging Phenotypes in Schizophrenia, Katherine L Narr ${ }^{l}$, Philip R Szeszko ${ }^{2}$, Todd Lencz ${ }^{2}$, Roger P Woods ${ }^{1}$, Liberty S Hamilton ${ }^{l}$, Owen Phillips ${ }^{1}$, Delbert G Robinson ${ }^{2}$, Katherine E Burdick, Pamela DeRosse ${ }^{2}$, Raju Kucherlapati, Paul M Thompson', Arthur W Toga ${ }^{2}$, Anil K Malhotra ${ }^{2}$, Robert M Bilder ${ }^{I},{ }^{I}$ Departments of Neurology and Psychiatry, David Geffen School of Medicine, University of California at Los Angeles, USA, ${ }^{2}$ Division of Psychiatry Research, The Zucker Hillside Hospital, North Shore-Long Island Jewish Health System, USA, ${ }^{3}$ Harvard Medical School-Partners Healthcare Center for Genetics and Genomics, Cambridge, USA

fMRI study of a matched-performance visual discrimination task in individuals with schizophrenia and first-degree relatives, Luke Stoeckel ${ }^{1,2}$, Kathy Avsar ${ }^{2}$, Martin Weiler $^{3}$, Adrienne Lahti ${ }^{l},{ }^{l}$ Neuroimaging and Translational Research Lab, Department of Psychiatry and Behavioral Neurobiology, University of Alabama at Birmingham (UAB), Birmingham, USA, ${ }^{2}$ Department of Psychology, UAB, Birmingham, USA, ${ }^{3}$ Maryland Psychiatric Research Center, University of Maryland at Baltimore, Baltimore, USA

\section{EMOTION \& MOTIVATION Reward}

Comparison of Cerebral Activation during Verbal and Monetary Reward: Individual Differences in Achievement Goal, Eunsoo Cho, Yoonkyung Chung, Eun Mo Yeon, Hun Jeon, Soonkoo Kwon, Sung-il Kim, Korea University, Seoul, Korea

Functional MRI study of reward anticipation and outcomes in the patients with obsessivecompulsive disorder, Wi Hoon Jung ${ }^{l}$, Ji Yeon Han ${ }^{l}$, Do-Hyung Kang ${ }^{2}$, Ji Young Park ${ }^{1}$, Jung-Seok Choi ${ }^{2}$, Myung-Hoon Jung, ${ }^{2}$ Chi-Hoon Choi ${ }^{3}$, Jong-Min Lee ${ }^{3}$, Jun Soo Kwon ${ }^{1,2}$, ${ }^{I}$ Interdisciplinary Program in Brain Science and in Cognitive Science, Seoul National University, Seoul, South Korea, ${ }^{2}$ Department of Psychiatry, Seoul National University College of Medicine, Seoul, South Korea, ${ }^{3}$ Department of Biomedical Engineering, Hanyang University, Seoul, South Korea

Delay Discounting during Different Reward Episodes and its Genetic Correlates, Corinna Nuesser ${ }^{l}$, Dina Schardt ${ }^{l}$, Susanne Erk ${ }^{l}$, Markus Noethen ${ }^{3,4}$, Marcella Rietschel ${ }^{5}$, Per Hoffmann ${ }^{3,4}$, Markus Skowronek, Sven Cichon ${ }^{3,4}$, Kerstin Ludwig ${ }^{3,4}$, Thomas Goschke ${ }^{2}$, Henrik Walter ${ }^{1}$, ${ }^{1}$ Division of Medical Psychology, Department of Psychiatry, University of Bonn, Bonn, Germany, ${ }^{2}$ Institute of Psychology II, Technische Universität Dresden, Dresden, Germany, ${ }^{3}$ Department of Genomics, Life \& Brain Center, University of Bonn, Bonn, Germany, ${ }^{4}$ Institute of Human Genetics, University of Bonn, Bonn, Germany, ${ }^{5}$ Central Institute for Mental Health, Div. Genetic Epidemiology in Psychiatry, Mannheim, Germany

Smoking or eating? Neuronal mechanisms underlying nicotine's effect on eating behavior, Michael N. Smolka', Lena Krebs ${ }^{2}$, Oliver Grimm ${ }^{2}$, Andrea Kobiella ${ }^{2}$, Sabine Klein ${ }^{2},{ }^{1}$ Section of Systems Neuroscience, Department of Psychiatry and Psychotherapy, Technische Universität 
Temporal dynamics of reward probability coding: a Magnetoencephalographic study in humans, Julie Thomas, Giovanna Vanni-Mercier, Jean-Claude Dreher, 'Reward and decison making' team, Centre de Neuroscience Cognitive, CNRS - Université Lyon1, Bron, France

\section{EMOTION \& MOTIVATION Sexual Behavior}

The feasibility of PULSAR arterial spin labeling in the investigation of the male sexual response, Janniko Georgiadis ${ }^{1}$, Michael Farrell ${ }^{2,3}$, Ruud Boessen ${ }^{2,4}$, Derek Denton ${ }^{5,6}$, Maria Gavrilescu ${ }^{2}$, Rudie Kortekaas ${ }^{1}$, Remco Renken ${ }^{7}$, Hans Hoogduin ${ }^{4,7}$, Gary Egan ${ }^{2,3}$, ${ }^{1}$ Dept. Neuroscience, University Medical Center Groningen, University of Groningen, Groningen, Netherlands, ${ }^{2}$ Howard Florey Institute, Florey Neuroscience Institutes, University of Melbourne, Melbourne, Australia, ${ }^{3}$ Centre for Neuroscience, University of Melbourne, Melbourne, Australia, ${ }^{4}$ Rudolf Magnus Institute for Neurosciences, University Medical Center Utrecht, Utrecht, Netherlands, ${ }^{5}$ Office of the Dean, Faculty of Medicine, Dentistry and Health Sciences, University of Melbourne, Melbourne, Australia, ${ }^{6}$ Baker Heart Research Institute, Alfred Hospital, Prahran, Australia, ${ }^{7} B C N$ NeuroImaging Center, University Medical Center Groningen, University of Groningen, Groningen, Netherlands

\section{EMOTION \& MOTIVATION Social Behavior}

Neural correlates of message tailoring and self-relatedness in smoking cessation programming, Hannah Faye Chua, Israel Liberzon, Robert Welsh, Victor Strecher, University of Michigan, Ann Arbor, USA

Neuroanatomical Correlates of Human Personality Characteristics: Introversion/Extraversion, Matthew A. Howard, Sarah L. Gregory, Steven C. R. Williams, Centre for Neuroimaging Sciences, Institute of Psychiatry, King's College London, London, United Kingdom

Adult Attachment Security Predicts Maternal Brain Responses using Functional MRI, Lane Strathearn ${ }^{l}$, Peter Fonagy ${ }^{l, 2}$, Read Montague ${ }^{I},{ }^{I}$ Baylor College of Medicine, Houston, USA,

${ }^{2}$ University College London, London, United Kingdom

\section{GENETICS}

MAPPING GENETIC INFLUENCES ON THE LATERAL VENTRICLES USING MULTI-ATLAS FLUID IMAGE ALIGNMENT IN TWINS, Yi-Yu Chou ${ }^{l}$, Natasha Lepore ${ }^{l}$, Marina Barysheval, Ming-Chang Chiang', Katie McMahon ${ }^{2}$, Greig de Zubicaray ${ }^{2}$, Matthew Meredith ${ }^{2}$, Margaret Wright ${ }^{3}$, Arthur Togal, Paul Thompson ${ }^{1},{ }^{1}$ Laboratory of Neuro Imagimg, Department of Neurology, UCLA, Los Angeles, USA, ${ }^{2}$ Centre for Magnetic Resonance, University of Queensland, Brisbane, Australia, ${ }^{3}$ Genetic Epidemiology Laboratory, Queensland Institute of Medical Research, Brisbane, Australia

Genetic influences over cortical gyrification. An across species comparison of heritability of gyrification index in extended pedigrees of baboons and humans, Peter Kochunov ${ }^{1}$, David Glahn $^{1}$, Peter Fox ${ }^{1}$, Karl Zilles ${ }^{2}$, Wendy Shelledy ${ }^{3}$, Jack Lancaster ${ }^{1}$, John Blangero ${ }^{3}$, Jeff Rogers ${ }^{3}$, ${ }^{I}$ The University of Texas Health Science Center at San Antonio, san antonio, USA, ${ }^{2}$ Institut für Medizin (IME), Jülich, USA, ${ }^{3}$ Southwest Foundation for Biological Research and Education (SFBR), San Antonio, TX, san antonio, Germany

Building Confidence in Single-Cohort Imaging Genetics Results, Thomas Nichols ${ }^{1,2}$, Becky Inkster ${ }^{l}$, Pierandrea Muglia ${ }^{3}$, Paul Matthews ${ }^{1},{ }^{1}$ GlaxoSmithKline, London, United Kingdom, ${ }^{2}$ FMRIB Centre, Oxford, United Kingdom, ${ }^{3}$ GlaxoSmithKline, Verona, Italy

Multiple influences of the androgen receptor polyglutamine polymorphism on the healthy human brain, Geoffrey CY Tan ${ }^{1,2}$, Weiguang Christopher Ho ${ }^{3}$, Ese E Mudanohwo ${ }^{4}$, Chia-Yeh Carlton Chu', John Ashburner ${ }^{1}$, Nina Somal ${ }^{5}$, Henrietta Gordon ${ }^{6}$, Mary Davis ${ }^{4}$, Nicholas $W$ Wood $^{2,4}$, Richard SJ Frackowiak ${ }^{1,7},{ }^{1}$ Wellcome Trust Centre for Neuroimaging, Institute of Neurology, UCL, London, United Kingdom, ${ }^{2}$ Dept of Molecular Neuroscience, Institute of Neurology, UCL, London, United Kingdom, ${ }^{3}$ Imperial College Medical School, London, United Kingdom, ${ }^{4}$ Neurogenetic Laboratory, Institute of Neurology, UCL, London, United Kingdom, ${ }^{5}$ Psychology Department, Goldsmiths College, London, United Kingdom, ${ }^{6}$ Dept of Anatomy, London, United Kingdom, ${ }^{7}$ Ecole Normale Superieure, Paris, United Kingdom 


\section{IMAGING TECHNIQUES \& CONTRAST MECHANISM} EEG

EEG Default Mode Network: 3D Spectral Coherence Topology, Andrew CN Chen*, Huixuan Zhao, Center for Higher Brain Functions, Capital Medical University, Beijing, China

PHYSIOLOGICALLY CAUSAL ANALYSIS OF THE HUMAN ELECTROENCEPHALOGRAM USING FIXED ORDER AUTOREGRESSIVE MOVING AVERAGE MODELING, Nicholas Sinclair ${ }^{1,2}$, Bugler Susan ${ }^{3}$, Delacretaz Louis ${ }^{2}$, Leslie Kate ${ }^{3}$, Liley David ${ }^{1,2},{ }^{1}$ Brain Dyanmics Group, Brain Sciences Instutute, Swinburne University of Technology, Hawthorn, Victoria 3122, Australia, ${ }^{2}$ Cortical Dynamics Pty Ltd, Scoresby, Victoria, Australia, ${ }^{3}$ Department of Anaesthesia and Pain Management, Royal Melbourne Hospital, Melbourne, Australia

\section{IMAGING TECHNIQUES \& CONTRAST MECHANISM} Functional MRI

Neural Origin of Low Frequency Synchrony in BOLD fcMRI, Jeffrey Anderson, University of Utah, Salt Lake City, USA

SENSE Optimized Sixteen Element Receive Array for Cervical Spinal Cord Imaging at 3T., Jerzy Bodurka ${ }^{l}$, Patrick Ledden ${ }^{2}$, Peter Bandettini ${ }^{1,3}$, ${ }^{1}$ Functional MRI Facilility, national Institute of Mental Health, NIH, Bethesda, USA, ${ }^{2}$ Nova Medical Inc, Wilmington, USA, ${ }^{3}$ Section on Functional Imaging Method, National Institute of Mental Health, NIH, Bethesda, USA

High Resolution FMRI of the Medial Temporal Lobe - Is SSFP a Viable Option?, Michael Chappell $^{1}$, Anders Kristoffersen ${ }^{2}$, Pål Erik Goa ${ }^{2}$, Hanne Lehn $^{1}$, Olav Haraldseth ${ }^{1,3}$, Asta Håberg ${ }^{1}$, ${ }^{I}$ Department of Circulation and Medical Imaging, Norwegian University of Science and Technology, Trondheim, Norway, ${ }^{2}$ St Olavs Hospital, Trondheim, Norway, ${ }^{3}$ Department of Circulation and Medical Imaging, St Olavs Hospital, Trondheim, Norway

DEPRESSION VULNERABILITY IS REFLECTED IN SUBGENUAL CINGULATE FUNCTION, Beate Hartinger ${ }^{l}$, Sharon Russo-Schwarzbaum ${ }^{l}$, Christian Kasess ${ }^{2}$, Barbara Kandler $^{I}$, Christian Scharinger ${ }^{1}$, Gerald Pail ${ }^{1}$, Andreas Erfurth ${ }^{1}$, Harald Esterbauer ${ }^{3}$, Christian Windischberger ${ }^{2}$, Siegfried Kasper ${ }^{1}$, Ewald Moser ${ }^{2}$, Lukas Pezawas ${ }^{I},{ }^{I}$ Division of Biological Psychiatry, Department of Psychiatry and Psychotherapy, Medical University of Vienna, Vienna, Austria, ${ }^{2}$ MR Center of Excellence, Center for Biomedical Engineering and Physics, Medical University of Vienna, Vienna, Austria, ${ }^{3}$ Clinical Institute of Medical and Chemical Laboratory Diagnostics, Medical University of Vienna, Vienna, Austria

ROI Based Analysis of fMRI Data to Investigate the Neuronal Pathway after Acupuncture Stimulation, Geon-Ho Jahng ${ }^{l}$, Kyung Hwan Ryu', Sun Hee Lee ${ }^{l}$, Young Jin Kim ${ }^{2}$, Chang Woo Ryu ${ }^{l}$, Sabina Lim ${ }^{2},{ }^{1}$ Department of Radiology, East-West Neo Medical Center, Kyung-Hee University, Seoul, South Korea, ${ }^{2}$ Dept. Applied Korean Medicine, Kyung-Hee University, Seoul, South Korea

Temporal Response and Spatial Specificity in Passband SSFP fMRI, Taek S. Kim ${ }^{l}$, Jongho Lee $^{2}$, Gary H. Glover ${ }^{3}$, John M. Pauly ${ }^{1},{ }^{\prime}$ Electrical Engineering, Stanford University, Stanford, USA, ${ }^{2}$ Advanced MRI/LFMI/NINDS, National Institute of Health, Bethesda, USA, ${ }^{3}$ Radiology, Stanford University, Stanford, USA

Imaging of autonomic activity in forebrain white matter, C. Leith ${ }^{l}$, J. Rosengarten ${ }^{2,3}$, M. Rosengarten ${ }^{2}$, S. Ouyang ${ }^{4}$, H. Sun ${ }^{I},{ }^{I}$ Neurodynamics Research Institute, Chicago, USA, ${ }^{2}$ Global Medical Imaging, Libertyville, USA, ${ }^{3}$ Rosalind Franklin University School of Medicine, North Chicago, USA, ${ }^{4}$ University of California, Los Angeles, USA

Task-Free Pre-Surgical Mapping Using fMRI Intrinsic Activity, Hesheng Liu ${ }^{l}$, Randy Buckner ${ }^{1,2,3}$, Tanveer Talukdar ${ }^{1}$, Naoro Tanakal, Joseph Madsen ${ }^{4}$, Steven Stufflebeam ${ }^{l}, I^{1}$ Athinoula A. Martinos Center for Biomedical Imaging, Massachusetts General Hospital, Boston, USA, ${ }^{2}$ Harvard University Department of Psychology, Center for Brain Science, Boston, USA, ${ }^{3}$ Howard Hughes Medical Institute, Chevy Chase, USA, ${ }^{4}$ Children's Hospital Boston, Boston, USA

Pharmacological fMRI study in Over Active Bladder (OAB) patients, Feroze Mohamed, Shweta Moonat, Steve Lebovitch, Brett Lebed, Scott Faro, Michael Pontari, Temple University, Philadelphia, USA

300 T-PM

304 T-PM 308 T-PM

312 T-PM

316 T-PM 320 T-PM 324 T-PM 328 T-PM 
FMRI results differ between display devices in visual oddball task, Eini Niskanen ${ }^{1,2,3}$, Perttu Ranta-aho ${ }^{l}$, Mika Tarvainen ${ }^{I}$, Mervi Könönen ${ }^{2,4}$, Pasi Karjalainen ${ }^{l},{ }^{\prime}$ Department of Physics, University of Kuopio, Kuopio, Finland, ${ }^{2}$ Department of Clinical Neurophysiology, Kuopio University Hospital, Kuopio, Finland, ${ }^{3}$ Department of Neurology, Kuopio University Hospital, Kuopio, Finland, ${ }^{4}$ Department of Radiology, Kuopio University Hospital, Kuopio, Finland

fMRI in Patients with Lumbar Radiculopathy, Harish Sharma ${ }^{1}$, Raj Gupta ${ }^{2}$, Bill Olivero ${ }^{3}$, ${ }^{1}$ University of Illinois at Urbana-Champaign, Urbana, USA, ${ }^{2}$ University of Illinois college of Medicine, Urbana, USA, ${ }^{3}$ Carle foundation Hospital, Urbana, USA

Reducing variability due to subject positioning in longitudinal structural and functional MRI studies, Adam Thomas ${ }^{l}$, Sandeep Gupta ${ }^{2}$, Peter Bandettini ${ }^{l}$, Sean Marrett ${ }^{l},{ }^{1}$ Function MRI Facility, Bethesda, USA, ${ }^{2}$ GE Global Research Center, Niskayuna, USA

Improved Event-Related Experimental Design when Stimuli have Undefined Event Types, Andrew Vahabzadeh-Hagh ${ }^{1}$, Julie Yoo ${ }^{2}$, Oliver Hinds ${ }^{2}$, John Gabrieli ${ }^{1,2,3},{ }^{1}$ Harvard-MIT Division of Health Sciences and Technology, Cambridge, USA, ${ }^{2}$ McGovern Institute for Brain Research, Massachusetts Institute of Technology, Cambridge, USA, ${ }^{3}$ Department of Brain and Cognitive Sciences, Massachusetts Institute of Technology, Cambridge, USA

Direct measurement of neuronal magnetic field changes evoked by median nerve stimulation using MRI: Magnitude or Phase?, Yiqun Xue ${ }^{1,2}$, Thomas Grabowski ${ }^{3}$, Jinhu Xiong ${ }^{2},{ }^{1}$ Biomedical Engineering, University of Iowa, Iowa city, USA, ${ }^{2}$ Radiology, University of Iowa, Iowa city, USA, ${ }^{3}$ Neurology, Univerisity, Iowa city, USA

Gender Difference in Default Networks Detected by BOLD-based fMRI at 3T, Tzu-Chen Yeh $^{1,2}$, Sue-Jin Lin ${ }^{2}$, Wen-Jui Kuo ${ }^{1,2}$, Chou-Ming Cheng ${ }^{1}$, Jen-Chuen Hsieh ${ }^{1,3}$, Low-Ton Ho ${ }^{1}$, ${ }^{l}$ Department of Medical Research and Education, Taipei Veterans General Hospital, Taipei, Taiwan, ${ }^{2}$ Institute of Neuroscience, National Yang-Ming University, Taipei, Taiwan, ${ }^{3}$ Center for Neuroscience, National Yang-Ming University, Taipei, Taiwan

\section{IMAGING TECHNIQUES \& CONTRAST MECHANISM MEG}

Experimental calculation of magnetic lead fields using MEG simultaneously acquired with intracranial EEG, Sarang Dalal ${ }^{1}$, Karim Jerbi ${ }^{l, 2}$, Olivier Bertrand ${ }^{l}$, Line Garnero ${ }^{2}$, Sylvain Baillet $^{2}$, Jacques Martinerie ${ }^{2}$, Jean-Philippe Lachaux ${ }^{1},{ }^{1}$ INSERM U821, Lyon, France, ${ }^{2}$ CNRS UPR640-LENA, Paris, France

\section{LANGUAGE \\ Language Acquisition}

Functional Development and Structural Maturation of Language Areas in the Human Brain, Jens Brauer, Alfred Anwander, Angela Friederici, Max Planck Institute for Human Cognitive and Brain Sciences, Leipzig, Germany

FRONTOSTRIATAL CIRCUITRY IN ARTIFICIAL SYNTACTIC CLASSIFICATION: AN FMRI INVESTIGATION IN HUNTINGTON'S DISEASE, Christian Forkstam ${ }^{1,2}$, Marieke Dekkers $^{2,3}$, Nicol Voermans ${ }^{2,3}$, Berry Kremer ${ }^{3}$, Guillen Fernández ${ }^{2,3}$, Karl Magnus Petersson ${ }^{1,2,4}$, I Cognitive Neurophysiology Research Group, Stockholm Brain Institute, Department of Clinical Neuroscience, Karolinska Institutet, Stockholm, Sweden, ${ }^{2}$ F. C. Donders Centre for Cognitive Neuroimaging, Radboud University Nijmegen, Nijmegen, Netherlands, ${ }^{3}$ Department of Neurology, University Medical Center Nijmegen, Nijmegen, Netherlands, ${ }^{4}$ Centre for Intelligent Systems, University of Algarve, Faro, Portugal

Extensive metabolic connectivity predicts the ability of speech language acquisition after cochlear implantation in prelingual deaf children, Hyejin Kang ${ }^{1,2}$, Heejung Kim ${ }^{2}$, Eunjoo $\mathrm{Kang}^{4}$, Jae Sung Lee ${ }^{2}$, Hyo-Jeong Lee ${ }^{3}$, Seung-Ha Oh ${ }^{3}$, Dong Soo Lee ${ }^{2},{ }^{1}$ Brain and Neuroscience Major, Seoul, Korea, ${ }^{2}$ Department of Nuclear Medicine, Seoul, Korea, ${ }^{3}$ Department of Otolaryngology, Seoul, Korea, ${ }^{4}$ Department of Psychology, Chuncheon, Korea

Using fMRI to study lateralization of cortical language areas in patients with medically intractable temporal lobe epilepsy, Tanu Sharma, Salah Baz, Seyed Mirsattari, Frank Bihari, Andrea Dencev, Brent Hayman-Abello, London Health Sciences Center, London, Canada 


\section{LANGUAGE \\ Production}

Phonological processing in reading Japanese kanji: Does reading heterophonic-homographic characters make any difference?, Chiao-Yi $\mathrm{Wu}^{1}$, Kayako Matsuo ${ }^{2}$, Epifanio Bagarinao ${ }^{3}$, Wen-Yih Issac Tseng ${ }^{4}$, Toshiharu Nakai ${ }^{2}$, S.H. Annabel Chen ${ }^{1},{ }^{1}$ Department of Psychology, National Taiwan University, Taipei, Taiwan, ${ }^{2}$ Functional Brain Imaging Laboratory, Department of Gerontechnology, National Center for Geriatrics and Gerontology, Aichi, Japan, ${ }^{3}$ Grid Technology Research Center, National Institute of Advanced Industrial Science and Technology, Ibaraki, Japan, ${ }^{4}$ Center for Optoelectronic Biomedicine, National Taiwan University College of Medicine, Taipei, Taiwan

Language Laterality Determined from High Anisotropy Arcuate Fasciculus Tracts, Timothy Ellmore ${ }^{I}$, Michael Beauchamp ${ }^{2}$, Jeremy Slater ${ }^{3}$, Joshua Breier ${ }^{4}$, Thomas O'Neill $^{1}$, Nitin Tandon ${ }^{I},{ }^{1}$ Dept of Neurosurgery, UT Medical School at Houston, Houston, USA, ${ }^{2}$ Dept of Neurobiology \& Anatomy, UT Medical School at Houston, Houston, USA, ${ }^{3}$ Dept of Neurology, UT Medical School at Houston, Houston, USA, ${ }^{4}$ Dept of Pediatrics, UT Medical School at Houston, Houston, USA

Neuroanatomical correlates of age-related change in verbal abilities, Adam Jacks ${ }^{1}$, Peter Kochunov ${ }^{1}$, Valeria Kochunov ${ }^{1}$, Donald Robin ${ }^{1}$, Anita Schlosser ${ }^{2}$, Peter Fox ${ }^{1},{ }^{1}$ The University of Texas Health Science Center, Research Imaging Center, San Antonio, USA, ${ }^{2}$ Department of Neurology, Sykehuset Østfold Fredrikstad, Fredrikstad, Norway

The neural correlates of Semantic Feature Analysis in a Primary Progressive Aphasia patient: an event-related fMRI study, Karine Marcotte ${ }^{1,2}$, Ana Inés Ansaldo ${ }^{1,3},{ }^{9}$ CRIUGM-UNF, Montreal, Canada, ${ }^{2}$ Faculty of Medicine, University of Montreal, Montreal, Canada, ${ }^{3}$ Speech-Communication Sciences Department, University of Montreal, Montreal, Canada

Sex differences in handedness, asymmetry of the Planum Temporale and functional language lateralization, Iris Sommer ${ }^{l}$, Andre Aleman ${ }^{2}$, Marco Boks ${ }^{l}$, Metten Somers ${ }^{l}$, Rene Kahn ${ }^{l}$, ${ }^{I}$ Universitary Medical Center Utrecht, Utrecht, Netherlands, ${ }^{2} B C N$ Neuroimaging Centre, University Medical Centre Groningen, Groningen, Netherlands

\section{MEMORY \& LEARNING Plasticity (normal \& following pathology)}

Hippocampal correlates of memory dysfunction 10 years after childhood TBI, Miriam Beauchamp ${ }^{1,2,4}$, Jerome Maller ${ }^{4,5}$, Cathy Catroppa ${ }^{1,2,4}$, Celia Godfrey ${ }^{1,4}$, Michael Ditchfield ${ }^{1,3}$, Vicki Anderson ${ }^{1,2,3,4},{ }^{1}$ Murdoch Childrens Research Institute, Melbourne, Australia, ${ }^{2}$ University of Melbourne, Melbourne, Australia, ${ }^{3}$ Royal Children's Hospital, Melbourne, Australia, ${ }^{4}$ Australian Center for Child Neuropsychological Studies, Melbourne, Australia, ${ }^{5} 5$ Alfred Psychiatry Research Centre, Melbourne, Australia

Non-monotonic changes in the cerebellar cortex during the acquisition of skilled cognitive operations, A. L. Hayter, D. W. Langdon, N. Ramnani, Royal Holloway, University of London, London, United Kingdom

Neurophysiological Correlates of Strategic Verbal Learning in Traumatic Brain Injury, Gary Strangman $^{1,2}$, Therese O'Neil-Pirozzi $^{2,3}$, Richard Goldstein ${ }^{2}$, Christina Supelana ${ }^{l}$, Kalika Kelkar $^{2}$, David Burke ${ }^{4}$, Douglas Katz ${ }^{5}$, Scott Rauch ${ }^{6}$, Cary Savage ${ }^{7}$, Mel Glenn ${ }^{2},{ }^{1}$ Massachusetts General Hospital, Harvard Medical School, Boston, USA, ${ }^{2}$ Spaulding Rehabilitation Hospital, Harvard Medical School, Boston, USA, ${ }^{3}$ Northeastern University, Boston, USA, ${ }^{4}$ Emory University, Atlanta, USA, ${ }^{5}$ Boston University, Boston, USA, ${ }^{6}$ McLean Hospital, Belmont, USA, ${ }^{7}$ Kansas University Medical Center, Kansas City, USA

\section{MEMORY \& LEARNING Working Memory}

Gender differences in functional activity for working memory, Suz-Chieh Sung ${ }^{l}$, Jing-Syun $\mathrm{Yu}^{l}$, Wen-Yih Isaac Tseng ${ }^{2}$, S.H. Annabel Chen ${ }^{l},{ }^{1}$ Department of Psychology, National Taiwan University, Taipei, Taiwan, ${ }^{2}$ Department of Radiology, National Taiwan University College of

380 T-PM 
Effect of sex and menstrual cycle phase on brain activation for verbal working memory, Jane Joseph, Christine Corbly, Linah Al-Alem, Garretson Epperly, Xun Liu, Thomas Curry, Thomas Kelly, University of Kentucky, Lexington, USA

Effects of transcranial direct current stimulation on verbal working memory in patients with stroke, Yun-Hee Kim ${ }^{l}$, Jung Mi Jo ${ }^{l}$, Suk Hoon Ohn ${ }^{l}$, Myoung-Hwan Ko ${ }^{2}$, Gyoung Moon $\mathrm{Kim}^{3}$, Woo-Kyoung Yoo ${ }^{1}$, Peter K.W. Lee ${ }^{1},{ }^{1}$ Department of Physical Medicine and Rehabilitation, Samsung Medical Center, Sungkyunkwan University School of Medicine, Seoul, Korea, ${ }^{2}$ Department of Physical Medicine and Rehabilitation, Chonbuk National University Medical School, Jeonju, Korea, ${ }^{3}$ Department of Neurology, Samsung Medical Center, Sungkyunkwan University School of Medicine, Seoul, Korea

Examining Working Memory Component Processes, Michael Motes ${ }^{1,2,3}$, Bart Rypma $^{1,2,3}$, ${ }^{l}$ Center for BrainHealth, Dallas, USA, ${ }^{2}$ School of Behavioral \& Brain Sciences, Dallas, USA,

${ }^{3}$ University of Texas Southwestern Medical Center, Dallas, USA

Functional connectivity of updating in working memory and refreshing information, Jennifer Roth $^{I}$, Marcia Johnson ${ }^{2}$, R. Todd Constable ${ }^{I},{ }^{I}$ Department of Diagnostic Radiology, Magnetic Resonance Research Center, Yale University, New Haven, USA, ${ }^{2}$ Department of Psychology, Yale University, New Haven, USA

\title{
MODELING \& ANALYSIS \\ Bayesian Modeling
}

Empirical Markov Chain Monte Carlo Bayesian analysis of fMRI data, Francesco de Pasquale $^{1}$, Cosimo Del Gratta ${ }^{1,2}$, Gian Luca Romani ${ }^{1,2},{ }^{1}$ ITAB, Institute for Advanced Biomedical Technologies, University G. D'Annunzio, Chieti, Chieti, Italy, ${ }^{2}$ Department of Clinical Sciences and Biomedical Imaging, University of Chieti, Chieti, Italy

Fast Bayesian nonlinear model fitting for analysis of simultaneous BOLD \& ASL data, Adrian Groves, Mark Woolrich, FMRIB Centre, Oxford, United Kingdom

Multiple-subjects connectivity-based parcellation using hierarchical infinite mixture models, Saad Jbabdi ${ }^{l}$, Mark Woolrich ${ }^{l}$, Timothy Behrens ${ }^{1,2},{ }^{I}$ FMRIB Centre, Oxford, United Kingdom, ${ }^{2}$ Depratment of Experimental Psychology, Oxford, United Kingdom

\section{MEG SOURCE CHARACTERIZATION THROUGH CURRENT MULTIPOLE}

MOMENTS, Sheraz Khan ${ }^{1,2,6}$, Benoît Cottereau ${ }^{1,3}$, Richard M. Leahy ${ }^{4}$, John C. Mosher ${ }^{5}$, Habib Ammari ${ }^{6}$, Sylvain Baillet ${ }^{1,2},{ }^{l}$ Cognitive Neuroscience \& Brain Imaging Laboratory, CNRS Hopital de la Salpetriere, Paris, France, ${ }^{2}$ University Pierre \& Marie CURIE, Paris 6, Paris, France, ${ }^{3}$ ESME-Sudria College of Engineering, Ivry, France, ${ }^{4}$ University of Southern California, Los Angeles, USA, ${ }^{5}$ Los Alamos National Laboratory, Los Alamos, USA, ${ }^{6}$ Laboratoire Ondes et Acoustique, CNRS \& ESPCI, Paris, France

MEG source modeling by Bayesian tracking: Validation of the particle filter approach, Lauri Parkkonen $^{1,5}$, Alberto Sorrentino ${ }^{2}$, Cristina Campi ${ }^{3}$, Annalisa Pascarella ${ }^{4}$, Michele Piana ${ }^{2,4}$, ${ }^{l}$ Brain Research Unit, Low Temperature Lab, Helsinki Univ. of Technology, Espoo, Finland, ${ }^{2}$ INFM - CNR Lamia, Genova, Italy, ${ }^{3}$ Dipartimento di Matematica, Universita di Genova, Genova, Italy, ${ }^{4}$ Dipartimento di Informatica, Universita di Verona, Verona, Italy, ${ }^{5}$ Elekta Neuromag Oy, Helsinki, Finland

13:45 - 14:45 Corryong Hall (Level 2)

\section{MODELING \& ANALYSIS \\ Classification \& Predictive Modeling}

\begin{abstract}
Assessment of placebo-controlled benzodiazepine sedation by means of indices derived from fMRI auditory responses, Charilaos Alexakis ${ }^{I}$, Ana Diukova ${ }^{1,3}$, Quazi Siddiqui ${ }^{2}$, Carolyn Steward ', Jaroslav Hlinkal, Paul Morgan', Jonathan Hardman', Dorothee Auerl, , 'Division of Academic Radiology, University of Nottingham, Nottingham, United Kingdom, ${ }^{2}$ Division of Anaesthesia, University of Nottingham, Nottingham, United Kingdom, ${ }^{3}$ Division of Psychiatry, University of Nottingham, Nottingham, United Kingdom
\end{abstract}


A Neural Predictor of Schizophrenia Based on Striatal [18F] Fluorodopa Uptake Measured with PET, Subrata Bose ${ }^{l}$, Federico Turkheimer ${ }^{1,2}$, Oliver Howes ${ }^{3}$, Mitul Mehta ${ }^{3}$, Rhian Cunliffe ${ }^{l}$, Paul Stokes ${ }^{I}$, Paul Grasby ${ }^{1,2},{ }^{I}$ MRC-Clinical Sciences Centre, Imperial College London, London, United Kingdom, ${ }^{2}$ Division of Neuroscience \& Mental Health, Imperial College London, London, United Kingdom, ${ }^{3}$ Institute of Psychiatry, King's College London, London, United Kingdom

Multiclass classification of fMRI pattern by relevance vector regression, Carlton $\mathrm{CHU}^{l}$, Janainan Mourão-Miranda ${ }^{2}$,John Ashburner ${ }^{l},{ }^{1}$ Wellcome Trust Centre for Neuroimaging, Institute of Neurology, UCL, London, United Kingdom, ${ }^{2}$ Brain Image Analysis Unit, Biostatics Department, Centre for Neuroimaging Sciences (PO 89), Institute of Psychiatry, London, United Kingdom

Combining top-down and bottom-up methods for ERP pattern classification, Gwen Frishkoff, ${ }^{1,}$, Robert Frank ${ }^{2}$, Jiawei Rong ${ }^{2}$, Dejing Dou ${ }^{2},{ }^{1}$ University of Pittsburgh, Pittsburgh, USA, ${ }^{2}$ University of Oregon, Eugene, USA

Towards shorter scan times using subject-dependent processing pipelines for clinical tasks in fMRI, Wayne Lee ${ }^{1,2}$, Richard Mraz ${ }^{3}$, Fred Tam ${ }^{1}$, Simon Graham ${ }^{1,2}$, Stephen Strother ${ }^{1,2}$, ${ }^{1}$ Rotman Research Institute, Toronto, Canada, ${ }^{2}$ University or Toronto, Toronto, Canada, ${ }^{3}$ Sunnybrook Health Sciences Centre, Toronto, Canada

Innovation approach to detect the respiratory related neuronal activity in the brainstem based on optical imaging data, Fumikazu Miwakeichi ${ }^{1}$, Yoshitaka Oku ${ }^{2}$, Yasumasa Okada ${ }^{3}$, Shigeharu Kawai ${ }^{4}$, Yoshiyasu Tamura ${ }^{5}$, Makio Ishiguro ${ }^{6},{ }^{1}$ Medical System Course, Graduate School of Engineering, Chiba University, Chiba, Japan, ${ }^{2}$ Department of Physiology, Hyogo College of Medicine, Hyogo, Japan, ${ }^{3}$ Department of Medicine, Tsukigase Rehabilitation Center, Keio University, Shizuoka, Japan, ${ }^{4}$ Department of Statistical Science, The Graduate University for Advanced Studies, Tokyo, Japan, ${ }^{5}$ Department of Data Science, The Institute of Statistical Mathematics, Tokyo, Japan, ${ }^{6}$ Department of Statistical Modeling, The Institute of Statistical Mathematics, Tokyo, Japan

Multivriate Bayes regression of CRS-R score from FDG-PET images, Christophe Phillips ${ }^{l}$, Mélanie Boly ${ }^{1,2}$, Pierre Maquet ${ }^{1,2}$, Caroline Schnakers ${ }^{l}$, Marie-Aurelie Bruno ${ }^{l}$, Audrey Vanhaudenhuyse ${ }^{1}$, Roland Hustinx ${ }^{3}$, Gustave Moonen ${ }^{2}$, Steven Laureys ${ }^{1,2},{ }^{1}$ Cyclotron Research Centre, University of Liège, Liège, Belgium, ${ }^{2}$ Neurology Department, CHU Hospital, Universiy of Liège, Liège, Belgium, ${ }^{3}$ Nuclear Medecine Department, CHU Hospital, Universiy of Liège, Liège, Belgium

Classification analysis of rapid event-related fMRI studies, Angela Rizk-Jackson, Jeanette Mumford, Russell Poldrack, UCLA Dept. of Psychology, Los Angeles, USA

Threshold-Free Cluster Enhancement - Practical Examples, Stephen Smith ${ }^{1}$, Gwenaelle Douaud , Thomas Nichols ${ }^{2,1},{ }^{I} F M R I B$, Oxford University, Oxford, United Kingdom, ${ }^{2}$ GSK CIC, London, United Kingdom

Image Intensity Correction for Detecting White Matter Hyperintensity (WMH) Progression in Longitudinal Fluid Attenuation Inversion Recovery (FLAIR) Whole Brain Scans, Wanlin Zhu', Wei Wen ${ }^{l}$, Aihua Xia ${ }^{2}$, Perminder Sachdev ${ }^{I},{ }^{I}$ School of Psychiatry, University of NSW, Sydney, Australia, ${ }^{2}$ Department of Mathematics and Statistics, Melbourne University, Melbourne, Australia

\section{MODELING \& ANALYSIS}

Motion Correction/Spatial Normalization, Atlas Construction

FNIRT - FMRIB's Non-linear Image Registration Tool, Jesper Andersson, Steve Smith, Mark Jenkinson, FMRIB-Centre, Oxford, United Kingdom

Inter-subject Functional Connectivity Alignment, Bryan Conroy ${ }^{l}$, Benjamin Singer ${ }^{2}$, Peter Ramadge ${ }^{1}$, James Haxby ${ }^{3},{ }^{1}$ Department of Electrical Engineering, Princeton University, Princeton, USA, ${ }^{2}$ Center for the Study of Brain, Mind, and Behavior, Princeton University, Princeton, USA, ${ }^{3}$ Department of Psychology, Princeton University, Princeton, USA

Agreement of independent structural and functional methods for locating the human V1 boundary, Oliver Hinds ${ }^{1}$, Jonathan Polimeni ${ }^{2}$, Mukund Balasubramanian ${ }^{3}$, Bruce Fischl ${ }^{2,4}$, Eric Schwartz ${ }^{3,5,6}$, Christina Triantafyllou ${ }^{1,2},{ }^{1}$ McGovern Institute for Brain Research, Massachusetts 
Hospital, Harvard Medical School, Charlestown, USA, ${ }^{3}$ Department of Cognitive and Neural Systems, Boston University, Boston, USA, ${ }^{4}$ Computer Science and Artificial Intelligence Lab, Massachusetts Institute of Technology, Cambrdige, USA, ${ }^{5}$ Department of Electrical and Computer Engineering, Boston University, Boston, USA, ${ }^{6}$ Department of Anatomy and Neurobiology, Boston University Medical School, Boston, USA

Comparison of Talairach and MNI coordinates in functional neuroimaging data: Validation of the icbm2tal transform, Jennifer Robinson ${ }^{1}$, Angela Laird ${ }^{2}$, Kathryn McMillan ${ }^{3}$, Diana Tordesillas-Gutiérre ${ }^{1}$, Sarah Thelen ${ }^{2}$, Kimberly Ray ${ }^{4}$, David Glahn ${ }^{1,2}$, Peter Fox ${ }^{2}$, Jack Lancaster ${ }^{2}$, ${ }^{I}$ Department of Psychiatry, University of Texas Health Science Center, San Antonio, USA, ${ }^{2}$ Research Imaging Center, University of Texas Health Science Center, San Antonio, USA, Department of Radiology, Vanderbilt University, Nashville, USA, ${ }^{4}$ Department of Physics, Texas Lutheran University, Seguin, USA

\section{MODELING \& ANALYSIS}

Univariate Modeling, Linear, \& Nonlinear

Novel suppression method of spatially correlated noise improves detection of fMRI responses to ultra-short stimuli at 7T, Marta Bianciardi, Masaki Fukunaga, Jeff H. Duyn, Peter van Gelderen, Jacco A. de Zwart, Advanced MRI Section, LFMI, NINDS, NIH, Bethesda, USA

New Validation Technique for Cortical Data Smoothing, Moo K. Chung, Department of Biostatistics and Medical Informatics, University of Wisconsin, Madison, USA

508 T-PM

Single volume estimates of neural activation computed in real-time, Oliver Hinds ${ }^{l}$, Todd Thompson ${ }^{2}$, Susan Gabrieli, ${ }^{1,2}$, John Gabrieli, ${ }^{1,2}$, Christina Triantafyllou ${ }^{2,3},{ }^{1}$ McGovern Institute for Brain Research, Massachusetts Institute of Technology, Cambridge, USA, ${ }^{2}$ Department of Brain and Cognitive Sciences, Massachusetts Institute of Technology, Cambridge, USA, ${ }^{3}$ Athinoula A. Martinos Center, Department of Radiology, MGH, Harvard Medical School, Charlestown, USA

Exploring the effectiveness of spatial smoothing in fMRI, Mingwu Jin, Dietmar Cordes, University of Colorado Denver, Denver, USA

524 T-PM

Estimating distributions of onset times and durations from multi-subject fMRI studies, $L u c y$ Robinson, Tor Wager, Martin Lindquist, Columbia University, New York, USA

Sample Size Recalculation Using Internal Pilot Studies For Group fMRI, Jeanette Mumford, Department of Psychology, University of California, Los Angeles, Los Angeles, USA

A Bayesian approach to fMRI data analysis using Stochastic Search Variable Selection, Rajesh Nandy, Brad Mcevoy, University of California, Los Angeles, USA

536 T-PM

The Mann-Whitney-Wilcoxon random field, with applications to brain mapping, Farzan Rohani ${ }^{1}$, Masoud Asgharian ${ }^{1}$, Keith Worsley ${ }^{1,2},{ }^{1}$ Department of Mathematics and Statistics, McGill University, Montreal, Canada, ${ }^{2}$ Montreal Neurological Institute, McGill University, Montreal, Canada

Change in fractal properties of resting fMRI time series after different tasks, Alle Meije Wink $^{1,2}$, Anna Barnes ${ }^{2}$, Ulrich Muller ${ }^{2}$, Ed Bullmore ${ }^{2}$, John Suckling ${ }^{2},{ }_{1}^{1}$ Imaging Sciences Department, Imperial College, MRC Clinical Sciences Centre, Hammersmith Campus, London, United Kingdom, ${ }^{2}$ Brain Mapping Unit, Department of Psychiatry, University of Cambridge, Addenbrookes Hospital, Hills Road, Cambridge, United Kingdom

Non-negative least-squares random field theory, Keith Worsley ${ }^{I}$, Jonathan Taylor ${ }^{2},{ }^{1}$ McGill University, Montreal, Canada, ${ }^{2}$ Stanford University, Palo Alto, USA

\section{MOTOR BEHAVIOR}

Brain-machine Interface

Improved Gazed-Dependent Brain Computer Interface by using Onset and Offset Flash Visual Evoked Potential, Chi-Hsun $W u^{l}$, Po-Lei Lee ${ }^{1,2,3}$, ' Department of Electrical Engineering, National Central University, Taoyuan, Taiwan, ${ }^{2}$ Department of Medical Research and Education, Taipei General Veterans Hospital, Taipei, Taiwan, ${ }^{3}$ Institute of Brain Science, National YangMing University, Taipei, Taiwan 


\section{MOTOR BEHAVIOR \\ Hand Movements}

Can low frequency repetitive transcranial magnetic stimulation to the non-lesioned hemisphere improve paretic arm reach-to-grasp performance after stroke?, Beth Fisher ${ }^{\text {, }}$ Jool Tretriluxana ${ }^{2}$, Shailesh Kantak ${ }^{l}$, Allan $\mathrm{Wu}^{3}{ }^{1},{ }^{1}$ Division of Biokinesiology and Physical Therapy, University of Southern California, Los Angeles, USA, ${ }^{2}$ Physical Therapy and Applied Movement Science, Mahidol University, Bankok, Thailand, ${ }^{3}$ Department of Neurology, University of California Los Angeles, Los Angeles, USA

Speed-dependent change of intercerebellar coupling during finger movement, Chang-hyun Park $^{1,2}$, Woo-Kyoung Yoo ${ }^{1}$, Suk Hoon Ohn ${ }^{1}$, Sung H. You ${ }^{3}$, Bo Hyun Lee ${ }^{l}$, Sung Tae Kim ${ }^{4}$, Yun-Hee Kim ${ }^{I},{ }^{I}$ Department of Physical Medicine and Rehabilitation, Samsung Medical Center, Sungkyunkwan University School of Medicine, Seoul, Korea, ${ }^{2}$ Department of Physics, Korea Advanced Institute of Science and Technology, Daejeon, Korea, ${ }^{3}$ Department of Physical Therapy, Yonsei University, Wonju, Korea, ${ }^{4}$ Department of Radiology, Samsung Medical Center, Sungkyunkwan University School of Medicine, Seoul, Korea

Brain activity during voluntary movement and exercise imagery using Near-infrared spectroscopy (NIRS), Noriyuki Oka ${ }^{1}$, Kayoko Yoshino ${ }^{2}$, Syun Ishizaki ${ }^{3}$, Toshinori Kato ${ }^{4},{ }^{1}$ Fujita Health University Health Department Rehabilitation Science Major occupational therapy The 4 th grade, Toyoake, Japan, ${ }^{2}$ Graduate school of Media and Governance, Keio University, Kanagawa, Japan, ${ }^{3}$ Department of Media and Governance, Keio University, Kanagawa, Japan, ${ }^{4}$ Department of Brain Environmental Research, KATOBRAIN Co, Ltd,, Tokyo, Japan

Longitudinal Evaluation of fMRI Motor Activation Pattern in Multiple Sclerosis using Surface-based Analysis - a 6-month follow-up case study, Jun Wang ${ }^{I}$, Daniel Hier ${ }^{2}$, ${ }^{I}$ State Key lab of Cognitive and Learning, Beijing Normal University, P.R.China, 100875, Beijing, China, ${ }^{2}$ Department of Neurology and Rehabilitation, University of Illinois at Chicago, Chicago, IL 60612, USA, Chicago, USA

\section{MOTOR BEHAVIOR}

Motor-Premotor Cortex/Motor Cortical Functions

Effects of timing and sequencing on pre-movement brain activity, Marta Bortoletto ${ }^{I}$, Ross Cunnington ${ }^{2},{ }^{1}$ Cognitive Neuroscience Unit, IRCCS Centro S. Giovanni di Dio Fatebenefratelli, Brescia, Italy, ${ }^{2}$ Queensland Brain Institute and School of Psychology, University of Queensland, Brisbane, Australia

Changes in MRS Response Following Activation of Motor Cortex, Mick Hunter ${ }^{l, 2}$, Neva Bull $^{1,3}$, Peter Stanwell ${ }^{4},{ }^{1}$ Hunter Medical Research Institute, Newcastle, Australia, ${ }^{2}$ University of Newcastle, Newcastle, Australia, ${ }^{3}$ John Hunter Hospital, Newcastle, Australia, ${ }^{4}$ Brigham and Women's Hospital, Boston, USA

The enhancement of cortical activation of the hand motor representation induced by a briefloudly sound, Mi Young Lee ${ }^{l}$, Yong Hyun Kwon ${ }^{2}$, Ji Won Park ${ }^{3}$, Sang Ho Ahn ${ }^{4}$, Sung Ho Jang ${ }^{4}$, ${ }^{I}$ Department of Rehabilitation Science, Graduate School, Daegu University, Daegu, South Korea, ${ }^{2}$ Department of Physical Therapy, Yeungnam College of Science \& Technology, Daegu, South Korea, ${ }^{3}$ Dept. of Physical Therapy, College of Health and Medical Scinece, Catholic University of Daegu, Daegu, South Korea, ${ }^{4}$ Department of Physical Medicine \& Rehabilitation, Yeungnam University College of Medicine, Daegu, South Korea

Direct recording of mirror neurons in human frontal and temporal lobes, Roy Mukamel ${ }^{1,2}$, Arne Ekstrom ${ }^{2,3}$, Jonas Kaplan ${ }^{1,2}$, Marco Iacoboni ${ }^{1,2,6}$, Itzhak Fried ${ }^{4,5,6}$, ' UCLA AhmansonLovelace Brain Mapping Center, David Geffen School of Medicine, Los Angeles, USA, ${ }^{2}$ UCLA Department of Psychiatry and Biobehavioral Sciences, Semel Institute for Neuroscience and Human Behavior, David Geffen School of Medicine, Los Angeles, USA, ${ }^{3}$ UCLA Center for Cognitive Neuroscience, Semel Institute for Neuroscience and Human Behavior, David Geffen School of Medicine, Los Angeles, USA, ${ }^{4}$ UCLA Division of Neurosurgery, David Geffen School of Medicine, Los Angeles, USA, ${ }^{5}$ Functional Neurosurgery Unit, Tel Aviv Medical Center and Sackler School of Medicine, Tel Aviv University, Tel Aviv, Israel, ${ }^{6}$ UCLA Brain Research Institute, David Geffen School of Medicine, Los Angeles, USA 
Cortical Adaptations in Patients with Clinically Isolated Syndrome; perspectives for predicting MS, Mohammad Ali Oghabian" ${ }^{\prime}$, Mohammad Hosain Harirchian ${ }^{2}$, Ali Reza Rezvanizade ${ }^{1}$, Mohammad Fakhri ${ }^{1},{ }^{1}$ Research Center for Sciences and Technology in Medicine, Tehran University/Medical Sciences, Tehran, Iran, ${ }^{2}$ Neurology Research Center, Emam Hospital, Tehran University/Medical Sciences, Tehran, Iran

The Neural Representation of Praxis: The Slicing Gesture, Donald Robin ${ }^{1,2}$, Howard Poizner ${ }^{3}$, Shalini Narayana ${ }^{1}$, Jack Lancaster ${ }^{1}$, Crystal Franklin ${ }^{1}$, Wayne Hening ${ }^{4}$, Peter Fox ${ }^{1,2},{ }^{1}$ Research Imaging Center, University of Texas Health Science Center at San Antonio, San Antonio, USA, ${ }^{2}$ Honor's 592 T-PM College, University of Texas, San Antonio, San Antonio, USA, Institute for Neural Computation, University of California, San Diego,, USA, ${ }^{4}$ Robert Wood Johnson Medical School, Piscataway, USA

Neural Correlates of Motor Sequence Learning, Christopher J. Steele, Virginia B. Penhune, Concordia University, Montreal, Canada

Neural Abnormalities of Synchronized Tapping in Adult ADHD, Eve Valera ${ }^{l}$, Joseph Biederman ${ }^{2}$, Thomas Zeffiro ${ }^{3}$, Ainat Rogel ${ }^{3}$, Megha Patel ${ }^{4}$, Rebecca Spencer ${ }^{5}$, Nikos Makris ${ }^{6}$, Thomas Spencer ${ }^{2}$, Stephen Faraone ${ }^{7}$, Larry Seidman ${ }^{8},{ }^{1}$ Neuroimaging Program, Clinical and Research Programs in Pediatric Psychopharmacology and Adult ADHD, Psychiatry, Harvard Medical School/Massachusetts General Hospital, Charlestown, USA, ${ }^{2}$ Clinical and Research Programs in Pediatric Psychopharmacology and Adult ADHD, Psychiatry, Harvard Medical School/Massachusetts General Hospital, Boston, USA, ${ }^{3}$ Psychiatry, Massachusetts General Hospital, Charlestown, USA, ${ }^{4}$ Psychology, Brandeis University, Waltham, USA, ${ }^{5}$ Psychology, University of California at Berkeley, Berkeley, USA, ${ }^{6}$ Neurology and Radiology, Harvard Medical School/Massachusetts General Hospital, Boston, USA, ${ }^{7}$ Psychiatry and Neuroscience and Physiology, SUNY Upstate Medical University, Syracuse, USA, ${ }^{8}$ Neuroimaging Program, Clinical and Research Programs in Pediatric Psychopharmacology and Adult ADHD, Psychiatry, Harvard Medical School/Beth Israel Deaconess Medical Center, Boston, USA

\section{NEUROANATOMY \\ Anatomical Studies}

Evolution of the Cerebellar Cortex: Selective expansion of prefrontal-projecting lobules, Joshua Balsters ${ }^{1}$, Emma Cussans ${ }^{1}$, Joern Diedrichsen ${ }^{2}$, Kimberley Phillips ${ }^{3}$, Todd Preuss ${ }^{4}$, James Rilling, ${ }^{5}$ Narender Ramnani ${ }^{1},{ }^{1}$ Dept Psychology, Royal Holloway University of London, London, United Kingdom, ${ }^{2}$ Wolfson Centre for Cognitive Neuroscience, School of Psychology, Bangor University, United Kingdom, ${ }^{3}$ Dept Psychology Hiram College, Hiram, USA, ${ }^{4}$ Div Neuroscience, Yerkes Natl. Primate Research Ctr, Emory University, Atlanta, USA, ${ }^{5}$ Dept Anthropology and Dept of Psychiatry and Behavioral Sciences, Emory University, Atlanta, USA

Insular volume Reduction in Williams Syndrome Using Real-Space Morphometry, Jeremy Cohen ${ }^{1}$, Ursula Bellugi ${ }^{2}$, Asya Karchemskiy ${ }^{l}$,Brian Haas ${ }^{1}$, Allan Reiss ${ }^{l},{ }^{\prime}$ Neuroimaging Laboratory, Department of Psychiatry and Behavioral Sciences, Stanford University School of Medicine, Stanford, USA, ${ }^{2}$ The Salk Institute for Biological Studies, Laboratory for Cognitive Neuroscience, La Jolla, USA

Cerebral change in patients with amnesic Mild Cognitive Impairment single domain and amnesic Mild Cognitive Impairment multiple-domain, Margherita Di Paola ${ }^{1,2}$, Serena Mosti ${ }^{3}$, Augusto Carlesimo ${ }^{3,4}$, Lucia Fadda ${ }^{3,4}$, Monica Ferraccioli ${ }^{5}$, Guido Gainotti ${ }^{5}$, Camillo Marra, Roberta Perri ${ }^{3}$, Carlo Caltagirone ${ }^{3,4}$, ' Neuroimaging Laboratory, IRCCS Santa Lucia Foundation, Rome, Italy, ${ }^{2}$ Department of Internal Medicine and Public Health, University of L'Aquila, L'Aquila, Italy, ${ }^{3}$ Clinical and Behavioural Neurology Laboratory, IRCCS Santa Lucia Foundation, Rome, Italy, ${ }^{4}$ Department of Neurological Sciences, University of Rome "Tor Vergata", Rome, Italy, ${ }^{5}$ Neuropsychology Service - Department of Neurology, Catholic University of Rome, Rome, Italy

Structural brain abnormalities in nonhuman primates exposed to early-life stressors, Andrea Jackowski $^{1,2}$, Griselda Garrido ${ }^{3}$, Andrew Dwork ${ }^{4}$, Tarique Perera ${ }^{5}$, Jeremy Coplan $^{6}$, Joan Kaufman ${ }^{2,7},{ }^{1}$ LiNC, Universidade Federal de Sao Paulo, São Paulo, Brazil, ${ }^{2}$ Child Study, Yale University, New Haven, USA, ${ }^{3}$ Serviço de Informática, Instituto do Coração, Hospital das Clínicas da Faculdade de Medicina da Universidade de São Paulo, São Paulo, Brazil, ${ }^{4}$ Department of Neuroscience, New York State Psychiatric Institute, New York, USA, ${ }^{5}$ Department of Biological Psychiatry, New York State Psychiatric Institute, New York, USA, ${ }^{6}$ Psychiatry, SUNY-Downstate Medical Center, Brooklin, USA, ${ }^{7}$ Psychiatry, Yale University, New Haven, USA 
Left hemisphere language activation depends on the size of the anterior and posterior corpus callosum., Goulven Josse, Ferath Kherif, Mohamed Seghier, Cathy Price, Wellcome Trust Center for Neuroimaging, UCL, London, United Kingdom

620 T-PM

Does smoking affect brain volume change in schizophrenia and healthy controls?, Cédric Koolschijn, Neeltje van Haren, Wiepke Cahn, Hugo Schnack, Hilleke Hulshoff Pol, René Kahn, Rudolf Magnus Institute of Neuroscience, Utrecht, Netherlands

Automated cortical projection of EEG sensors : Anatomical correlation via the international 10-10 system, Laurent KOESSLER ${ }^{1,2}$, Louis MAILLARD ${ }^{2}$, Adnane BENHADID ${ }^{I}$, Jean-Pierre

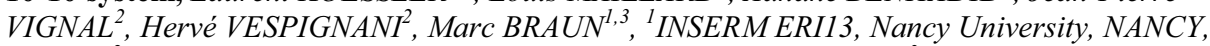
France, ${ }^{2}$ Neurology Department, University Hospital, NANCY, France, ${ }^{3}$ Neuroradiology Department, University Hospital, NANCY, France

Hippocampal volumetrics in treatment-resistant schizophrenia and depression: the importance of the tail, Jerome Maller ${ }^{l}$, Zafiris Daskalakis ${ }^{2}$, Paul Fitzgerald ${ }^{l},{ }^{l}$ Alfred Psychiatry Research Centre, Monash University, Melbourne, Australia, ${ }^{2}$ University of Toronto, Toronto, Canada

Brodmann Areas defined in MNI space using a new Tracing Tool in BioImage Suite, Cheryl M. Lacadie ${ }^{I}$, Robert K. Fulbright ${ }^{I}$, Jagriti Arora ${ }^{I}$, R. Todd Constable ${ }^{1,2,3}$, Xenophon Papademetris ${ }^{1,3},{ }^{1}$ Dept of Diagnostic Radiology, Yale School of Medicine, New Haven, USA, ${ }^{2}$ Dept of Neurosurgery, Yale School of Medicine, New Haven, USA, ${ }^{3}$ Dept. of Biomedical Engineering, New Haven, USA

Differences in Corpus Callosum Area and Shape in Advanced Aging and Alzheimer's Disease, Jidan Zhong ${ }^{1}$, Randy Buckner ${ }^{3,5}$, Bruce Fischl ${ }^{3,4}$, Michael Miller ${ }^{2}$, Anqi Qiu ${ }^{1}$, 'Division of Bioengineering, National University of Singapore, Singapore, Singapore, ${ }^{2}$ Center for Imaging Science, Johns Hopkins University, Baltimore, USA, ${ }^{3}$ Athinoula A Martinos Center for Biomedical Imaging at MGH, Boston, USA, ${ }^{4}$ Computer Science and Artificial Intelligence Laboratory, Massachusetts Institute of Technology, Boston, USA, ${ }^{5}$ Department of Psychology, Center for Brain Science, Harvard University, Boston, USA

Automated Method to Measure Cortical 3D Gyrification Index Implemented as a BrainVISA Plugin, Bill Rogers ${ }^{l}$, Peter Kochunov ${ }^{I}$, Jeff Rogers ${ }^{2}$, David Glahn ${ }^{I}$, Peter Fox ${ }^{1}$, ${ }^{1}$ University of Texas Health Science Center, San Antonio, USA, ${ }^{2}$ Southwest Foundation for Biomedical Research, San Antonio, USA

Characterizing Regional Gray Matter Thickness Trends in Normal Aging, Jing Wan $^{1}$, Aaron Carass $^{l}$, Susan Resnick ${ }^{2}$, Jerry Prince ${ }^{l},{ }^{1}$ Image Analysis and Communications Laboratory, Electrical and Computer Engineering, the Johns Hopkins University, Baltimore, USA, ${ }^{2}$ National Institute on Aging, National Institutes of Health, Baltimore, USA

\section{PHYSIOLOGY, METABOLISM, \& NEUROTRANSMISSION}

The effects of chronic caffeine use on the temporal dynamics of the BOLD signal, Merideth Addicott, Yang Lucie, Casanova Ramon, Peiffer Ann, Maldjian Joseph, Burdette Jonathan, Burnett Luke, Laurienti Paul, Department of Radiology, Wake Forest University School of Medicine, Winston Salem, USA

Measuring Hemodynamic Contributions to the BOLD Post-Stimulus Undershoot, J. Jean Chen, G. Bruce Pike, McConnell Brain Imaging Centre, Montreal Neurological Institute, Montreal, Canada

Mismatched cerebral blood flow and metabolic rate of oxygen in healthy aging: A PET study, Joel Aanerud ${ }^{l, 2}$, Per Borghammer ${ }^{l, 2}$, Manoucher Vafaee ${ }^{2}$, Peter Iversen ${ }^{1}$, Peter Johannsen ${ }^{3}$, Mahmoud Askanian', Albert Gjedde, ${ }^{1,2}$, ${ }^{2}$ PET Center, Aarhus University Hospitals, Aarhus, Denmark, ${ }^{2}$ Center of Functionally Integrative Neuroscience, Aarhus University, Aarhus, Denmark, ${ }^{3}$ Dept of Neurology, Rigshospitalet, Copenhagen, Denmark

Are gamma band power increases in the human brain systematically associated with alpha and beta power suppressions ?, Karim Jerbi ${ }^{1,2}$, Sarang Dalal ${ }^{2}$, Nathan Weisz ${ }^{2}$, Aurélie BidetCaulet $^{2}$, Julien Jung ${ }^{2}$, Lorella Minotti ${ }^{3}$, Phlippe Kahane ${ }^{3}$, Alain Berthoz ${ }^{1}$, Olivier Bertrand ${ }^{2}$, Jean-Philippe Lachaux ${ }^{2},{ }^{1}$ Physiology of Perception and Action Lab, CNRS, Collège de France, Paris, France, ${ }^{2}$ INSERM, U821, Brain Dynamics and Cognition and University Lyon 1, Lyon, France, ${ }^{3}$ Department of Neurology and INSERM U704, Grenoble Hospital, Grenoble, France 
Changes in functional connectivity induced by sevoflurane in the human brain, Roberto Martuzzi $^{l}$, Maolin Qiu ${ }^{l}$, Nallakkandi Rajeevan ${ }^{1}$, Ramachandran Ramani ${ }^{2}$, R. Todd Constable ${ }^{1,3,4}$, ${ }^{l}$ Department of Diagnostic Radiology, Yale University School of Medicine, New Haven, USA,

${ }^{2}$ Department of Anesthesiology, Yale University School of Medicine, New Haven, USA,

668 T-PM

${ }^{3}$ Department of Biomedical Engineering, Yale University School of Medicine, New Haven, USA,

${ }^{4}$ Department of Neurosurgery, Yale University School of Medicine, New Haven, USA

Gender differences of interregional metabolic connectivity in the resting brain explained by less inter-hemispheric transfer in males, Hyojin Park ${ }^{1,2}$, Hyejin Kang ${ }^{1,3}$, Eunjoo Kang ${ }^{4}$, Jungsu S. Oh ${ }^{1,5}$, Jae Sung Lee ${ }^{1}$, Dong Soo Lee ${ }^{1,2}$, I Department of Nuclear Medicine, Seoul National University College of Medicine, Seoul, South Korea, ${ }^{2}$ Interdisciplinary Program in Cognitive Science, Seoul National University, Seoul, South Korea, ${ }^{3}$ Programs in Brain and Neuroscience, Seoul National University, Seoul, South Korea, ${ }^{4}$ Department of Psychology, Kangwon National University, Chuncheon, South Korea, ${ }^{5}$ Psychiatry Neuroimaging Laboratory, Brigham and Women's Hospital, Harvard Medical School, Boston, USA

Changes of neuronal activity after transcranial direct current and photic stimulation are associated with glutamatergic neurotransmission as revealed by functional ${ }^{1}$ H-MR-spectroscopy, Michael Siniatchkin ${ }^{I}$, Friederike Moeller ${ }^{1}$, Mascha Sendacki ${ }^{1}$, Stephan Wolff', Ulrich Stephani ${ }^{I},{ }^{I}$ Pediatric Neurology, Kiel, Germany, ${ }^{2}$ Neuroradiology, Kiel, Germany

Opioids modulate the brain activity associated with breath-holding: an FMRI study, K.T. Pattinson ${ }^{I}$, R.J Governo ${ }^{2}$, E.C. Russell ${ }^{1}$, B.J. Macintosh ${ }^{2}$, I. Ahmad $^{l}$, S.D. Mayhew ${ }^{I}$, D.R. Corfield $^{3}$, I. Tracey ${ }^{2}, R$. G. Wise ${ }^{4}$, ${ }^{1}$ Nuffield Department of Anaesthetics, Oxford University, Oxford, United Kingdom, ${ }^{2}$ Oxford Centre for Functional Magnetic Resonance Imaging of the Brain (FMRIB), Department of Clinical Neurology, Oxford University, Oxford, United Kingdom, ${ }^{3}$ Institute of Science and Technology in Medicine, Keele University, Keele, United Kingdom, ${ }^{4}$ Cardiff University Brain Research Imaging Centre (CUBRIC), School of Psychology, Cardiff University, Cardiff, United Kingdom

Midbrain dopamine autoreceptor availability is inversely associated with novelty seeking traits in humans, David Zald, Ronald Cowan, Patritzia Riccardi, Ronald Baldwin, Ansari M Sib, Rui Li, Evan Shelby, Clarence smith, Robert Kessler, Vanderbilt University, Nashville, USA

\section{SENSORY SYSTEMS \\ Multisensory \& Crossmodal}

Cross-modal plastic changes of effective connectivity in blind subjects: An fMRI study, Takeshi Fujii ${ }^{1,2}$, Hiroki Tanabe ${ }^{1,2}$, Norihiro Sadato ${ }^{1,2,3,4}$, ${ }^{1}$ Division of Cerebral Integration, Department of Cerebral Research, National Institute for Physiological Sciences, Okazaki, Japan, ${ }^{2}$ Department of Physiological Sciences, The Graduate University for Advanced Studies (Sokendai), Kanagawa, Japan, ${ }^{3}$ Research Institute of Science and Technology for Society (RISTEX), Japan Science and Technology Agency (JST), Tokyo, Japan, ${ }^{4}$ Department of Functional Neuroimaging, Faculty of Medical Sciences, University of Fukui, Fukui, Japan

One sound, two percepts: Predicting future speech perception from brain activation during audiovisual exposure, Niclas Kilian-Hütten ${ }^{l}$, Jean Vroomen ${ }^{2}$, Elia Formisano ${ }^{l},{ }^{I}$ Dept of Cognitive Neuroscience, Faculty of Psychology, Maastricht University, Maastricht, Netherlands, ${ }^{2}$ Dept of Psychology, Tilburg, Netherlands

Functional development of the mirror neuron system does not require visual experience: an fMRI study in sighted and congenitally blind individuals, Emiliano Ricciardi ${ }^{1,2}$, Daniela Bonino $^{1,3}$, Lorenzo Sani ${ }^{1,2}$, Tomaso E. Vecchi ${ }^{3}$, Mario Guazzelli ${ }^{4}$, James V. Haxby ${ }^{5}$, Luciano Fadiga ${ }^{6}$ Pietro Pietrini ${ }^{l},{ }^{1}$ Laboratory of Clinical Biochemistry and Molecular Biology, University of Pisa, Pisa, Italy, ${ }^{2}$ MRI Lab, Institute of Clinical Physiology, C.N.R. Research Area, Pisa, Italy, ${ }^{3}$ Department of Psychology, University of Pavia, Pavia, Italy, ${ }^{4}$ Psychology Chair, Universityof Pisa, Pisa, Italy, ${ }^{5}$ Department of Psychology, Princeton University, Princeton, USA, ${ }^{6}$ Department of Biomedical Sciences and Advanced Therapy - Physiology Section, University of Ferrara, Ferrara, Italy

Brain activity in colored-hearing synesthetes when listening to music: An fMRI study, Riuma Takahashi ${ }^{l}$, Mayuka Nishimoto ${ }^{l}$, Takashi X. Fujisawa ${ }^{l}$, Noriko Nagata ${ }^{l}$, Takeshi Sugio ${ }^{2}$, Seiji Inokuchi ${ }^{3},{ }^{1}$ Graduate School of Science and Technology, Kwansei Gakuin University, Hyogo, 
Japan, ${ }^{2}$ Faculty of Culture and Information Science, Doshisha University, Kyoto, Japan, ${ }^{3}$ Faculty of Media Contents, Takarazuka University of Art and Design, Hyogo, Japan

\section{SENSORY SYSTEMS}

Pain \& Autonomic Function

3D TOPOGRAPHIC MAPPING OF MAGNETIC BRAIN RESPONSES IN TRAUMATIC PERIPHERAL NEUROPATHIC PAIN, P.J. Theuvenet ${ }^{1}$, B.W. van Dijk $k^{l}$, Maria J. Peters ${ }^{1}$, F.L. Lopes da Silval, J.M. van Ree ${ }^{I}$, Andrew C.N. Chen ${ }^{2},{ }^{1}$ Dept. of Anesthesiology, Alkmaar Medical Center, Alkmaar, Netherlands, ${ }^{2}$ Center for Higher Brain Functions, Capital Medical University, Beijing, China

Chronic Pain Remodels the Brain's Salience Network: A Resting-State fMRI Study, Michael Greicius ${ }^{I}$, Meredith Barad', Takefumi Ueno ${ }^{2}$, Sean Mackey ${ }^{I},{ }^{I}$ Stanford University Medical Center, Stanford, USA, ${ }^{2}$ Kyushu University, Fukuoka, Japan

A PET study of wind-up pain in patients with postherniotomy pain, Rune Christensen ${ }^{l}$, Eske Aasvang ${ }^{2}$, Henrik Kehlet ${ }^{2}$, Ron Kupers ${ }^{1,2},{ }^{1}$ PET Unit, Copenhagen, Denmark, ${ }^{2}$ Dept. Surgical Pathophysiology, Copenhagen, Denmark

Partial least squares analysis of brain responses to experimentally induced rectal discomfort: Greater engagement of an insula-related network in female Irritable Bowel Syndrome (IBS) patients, Jennifer Labus ${ }^{l}$, Lisa Kilpatrick ${ }^{l}$, Bruce Naliboff ${ }^{1,2}$, Steve Berman ${ }^{l}$, Brandall Suyenobu ${ }^{l}$, Emeran Mayer ${ }^{1},{ }^{l}$ Center for Neurobiology of Stress, Brain Research Institute, Depts of Psychiatry and Biobehavioral Science, University of California, Los Angeles, USA, ${ }^{2}$ VA Greater Los Angeles Healthcare System, Los Angeles, USA

Brain correlates of conditioned placebo analgesia, Luana Colloca ${ }^{l}$, Fausta Lui ${ }^{2}$, Davide Duzzi ${ }^{2}$, Luca Nocetti ${ }^{3}$, Davide Anchisi ${ }^{2}$, Francesca Benuzzi ${ }^{4}$, Patrizia Baraldi ${ }^{2}$, Fabrizio Benedetti ${ }^{2}$, Carlo Adolfo Porro ${ }^{1},{ }^{1}$ Dip. Neuroscienze, Univ. Torino, Torino, Italy, ${ }^{2}$ Dip. Scienze Biomediche, Univ. Modena e Reggio Emilia, Modena, Italy, ${ }^{3}$ Fisica Sanitaria, Policlinico, Modena, Italy, ${ }^{4}$ Dip. Neuroscienze, Univ. Modena e Reggio Emilia, Modena, Italy

Central Representation of Menstrual Cramping Pain in Primary Dysmenorrhea: a PET Study, Cheng-Hao Tu ${ }^{1,4}$, David Niddam ${ }^{2,3,4}$, Ren-Shyan Liu ${ }^{5}$, Hsiang-Tai Cho ${ }^{6}$, Ren-Jen Hwang ${ }^{1,4}$, Jen-Chuen Hsieh ${ }^{1,2,3,4}$, ${ }^{1}$ Institute of Neuroscience, National Yang-Ming University, Taipei, Taiwan, ${ }^{2}$ Institute of Brain of Brain Science, National Yang-Ming University, Taipei, Taiwan, ${ }^{3}$ Brain Research Center, National Yang-Ming University, Taipei, Taiwan, ${ }^{4}$ Laboratory of Integrated Brain Research, Taipei Veteran General Hospital, Taipei, Taiwan, ${ }^{5}$ Department of Nuclear Medicine, Taipei Veteran General Hospital, Taipei, Taiwan, ${ }^{6}$ Department of Obstetrics and Gynecology, Taipei Veteran General Hospital, Taipei, Taiwan 
11:30 - 12:30 You Yangs Hall (Level 3)

\section{COGNITION \& ATTENTION \\ Executive Function}

MEG analysis of inhibitory process during Go/NoGo task in normal children, Eun Young Kim ${ }^{l}$, Yeni Kim², June Sic Kim ${ }^{l}$, Jae-Won Kim², Jun Won Hwang ${ }^{2}$, Boong-Nyun Kim², Soo Churl Cho', Chun Kee Chungl, ' Department of Neurosurgery, Seoul National University College of Medicine, MEG Center, Seoul National University Hospital, Seoul, South Korea, ${ }^{2}$ Department of Neuropsychiatry, Seoul National University College of Medicine, Seoul, South Korea

Functional MRI Deactivations During Working Memory Distinguishes Multiple Sclerosis Patients from Controls, James Paskavitz ${ }^{1}$, Lawrence Sweet ${ }^{2}$, Jesse Samuel ${ }^{I}$, ${ }^{1}$ Perceptive Informatics, Waltham, USA, ${ }^{2}$ Brown University, Providence, USA

Errors and the violation of intention: The functional role of the left ventrolateral prefrontal cortex within a neural system for error processing, Zrinka Sosic ${ }^{1}$, Martin Ruchsow ${ }^{2}$, Georg Grön ${ }^{1}$, ${ }^{I}$ Department of Psychiatry and Psychotherapy III, University of Ulm, Ulm, Germany, ${ }^{2}$ Clinic for Psychiatry and Psychotherapy, Christophsbad, Göppingen, Germany

Neural basis of MPH-induced improvement in working memory differs by DAT genotype in childhood ADHD, Chandan Vaidya ${ }^{l}$, Devon Shook ${ }^{l}$, Jennifer Foss-Feig ${ }^{l}$, Laura Kenealy ${ }^{2}$, Edwin Cook ${ }^{3}$, Mark Stein ${ }^{3},{ }^{1}$ Georgetown University, Washington, USA, ${ }^{2}$ Childrens National Medical Center, Washington, USA, ${ }^{3}$ University of Illinois, Chicago, USA

Paired pulse transcranial magnetic stimulation to investigate cortical inhibition., Paul Fitzgerald ${ }^{l}$, Jerome Maller ${ }^{l}$, Kate Hoy ${ }^{l}$, Faranak Farzan ${ }^{2}$, Zafiris Daskalakis ${ }^{2},{ }^{l}$ Alfred Psychiatry Research Centre, Monash University, Melbourne, Australia, ${ }^{2}$ Alfred Psychiatry Research Centre, Monash University, Melbourne, Australia, ${ }^{3}$ Alfred Psychiatry Research Centre, Monash University, Melbourne, Australia, ${ }^{4}$ Centre for Addiction and Mental Health, Toronto, Canada, ${ }^{5}$ Centre for Addiction and Mental Health, Toronto, Canada

Expertise leads to a more efficient brain utilization: an fMRI study in professional and naïve car drivers during attention and visual-spatial tasks, Lorenzo Sani ${ }^{1,2,3}$, Emiliano Ricciardi ${ }^{1,2,3}$, Alessandra Papasogli $^{4}$, Riccardo Ceccarelli ${ }^{4}$, Ferdinando Franzoni $^{5}$, Gino Santoro ${ }^{5}$, Rainer Goebel ${ }^{6}$, Pietro Pietrini ${ }^{1,3}$, ${ }^{1}$ Laboratory of Clinical Biochemistry and Molecular Biology, University of Pisa, Italy,, ${ }^{2}$ NMR Lab, CREAS-CNR, Pisa, Italy, ${ }^{3}$ Department of Laboratory Medicine and Molecular Diagnostics, AUO Pisa, Italy,, ${ }^{4}$ Formula Medicine, Viareggio, Italy,, ${ }^{5}$ Department of Internal Medicine, University of Pisa, Italy, ${ }^{6}$ Department of Cognitive Neuroscience, Faculty of Psychology, Universiteit Maastricht, The Netherlands,

Parkinson's disease patients fail to deactivate the default mode brain areas., Thilo van Eimeren ${ }^{l}$, Oury Monchi ${ }^{2}$, Benedicte Ballanger ${ }^{I}$, Antonio P. Strafella ${ }^{I},{ }^{1}$ UNH-Toronto Western Hospital, Brain Imaging \& Behaviour Systems Toronto Western Research Institute, CAMH-PET Imaging Centre, University of Toronto, Ontario, Canada, Toronto, Canada, ${ }^{2}$ Centre de Recherche de l'Institut Universitaire de Gériatrie, Université de Montréal, Québec, Canada, Montreal, Canada

Increased activation in prefrontal and striatal areas during planning as a function of depression severity in a representative medication-free sample of Major Depressive Disorder in the general population: Preliminary results from the NESDA-neuroimaging study., Marie-José van Tol ${ }^{l}$, Nic van der Wee ${ }^{1}$, Marjan Nielen ${ }^{2}$, Andre Aleman ${ }^{4}$, Ramona Demenescu ${ }^{4}$, Remco Renken ${ }^{4}$, Mark van Buchem ${ }^{3}$, Frans Zitman ${ }^{1}$, Dick Veltman ${ }^{2},{ }^{1}$ Leiden University Medical Center, Department of Psychiatry, Leiden, Netherlands, ${ }^{2}$ VU medicial Center, Amsterdam, Netherlands, ${ }^{3}$ Leiden University Medical Center, Department of Radiology, Leiden, Netherlands, ${ }^{4}$ University Medical Center Groningen, Groningen, Netherlands

IMAGEN Stop-Signal Task: Validation and Comparison of Brain Networks Subserving Fixed and Adaptive Stop Trials using 3T fMRI, Mira Buehler ${ }^{1,3}$, Mischa de Rover ${ }^{l}$, Sanja Abbott ${ }^{I}$, Luke Clark, Hugh Garavan ${ }^{2}$, Katya Rubia', Gunter Schumann ${ }^{3}$, Laurence Reed ${ }^{3}$, Trevor W. Robbins ${ }^{1}$, IBehavioural and Clinical Neuroscience Institute, Department of Experimental Psychology, University of Cambridge, 
Cambridge, United Kingdom, ${ }^{2}$ School of Psychology and Trinity College Institute of Neuroscience, Trinity College Dublin, Dublin, Ireland, ${ }^{3}$ Institute of Psychiatry, King's College London, London, United Kingdom

Reward expectation in Parkinson's disease: anterior cingulate cortical activation in response to reward expectation and actual reward during disease progression. , James Rowe ${ }^{1,2,3}$, Laura Hughes $^{1,2}$, Roger Barker ${ }^{l}$, Caroline Williams-Gray ${ }^{1}$, Sean Fallon ${ }^{2}$, Adrian Owen ${ }^{2,3},{ }^{1}$ Department of Clinical Neurosciences, Cambridge University, Cambridge, United Kingdom, ${ }^{2}$ MRC Cognition and Brain Sciences Unit, Cambridge, United Kingdom, ${ }^{3}$ MRC Behavioural and Clinical Neurosciences Institute, Cambridge, United Kingdom

A temporal hierarchy of brain dynamics, Stefan Kiebel, Jean Daunizeau, Chris Frith, Karl Friston, Wellcome Trust Centre for Neuroimaging, London, United Kingdom

Action Monitoring in Pediatric Obsessive-Compulsive Disorder, Poyu Chen ${ }^{1,2}$, Kate Fitzgerald ${ }^{3}$, Gregory Hanna ${ }^{3}$, William Gehring ${ }^{l},{ }^{l}$ Department of Psychology, University of Michigan, Ann Arbor, USA, ${ }^{2}$ Department of Psychology, National Chung Cheng University, Chiayi, Taiwan, ${ }^{3}$ Department of Psychiatry, University of Michigan, Ann Arbor, USA

Anticorrelations between task-positive and task-negative brain areas increase during cognition, Michelle Hampson ${ }^{l}$, Naomi Driesen ${ }^{l}$, Jennifer Roth ${ }^{l}$, John Gore ${ }^{2}$, Todd Constable ${ }^{l},{ }^{l}$ Yale School of Medicine, New Haven, USA, ${ }^{2}$ Vanderbilt University Institute on Imaging Science, Nashville, USA

Default Mode of brain function in Schizophrenia. An independent component analysis of fMRI data. V Joseph, A Mendrek, PF Liddle (Division of Psychiatry, Queens Medical Centre), Verghese Joseph $^{1}$, Adrianna Mendrek ${ }^{2}$, Peter Liddle ${ }^{3},{ }^{1}$ University of Nottingham, Nottingham, United Kingdom, ${ }^{2}$ University of British Columbia, Vancover, Canada, ${ }^{3}$ University of Nottingham, Nottingham, United Kingdom

\section{COGNITION \& ATTENTION}

Perception, Imagery, Awareness

Electrical Brain Imaging of Mental Own Body Transformations, Lars Schwabe, Bigna Lenggenhager, Olaf Blanke, Brain Mind Institute, Lausanne, Switzerland

Recognition of point-light possible and impossible motion: Mu rhythms and mirror neuron activity, Naznin Virji-Babul ${ }^{1,2}$, Teresa Cheung ${ }^{1,2}$, Urs Ribary ${ }^{1,2}$, Faisal Beg ${ }^{2},{ }^{1}$ Down Syndrome Research Foundation, Burnaby, Canada, ${ }^{2}$ Simon Fraser University, Burnaby, Canada

Transient and linearly-graded deactivation of the human default-mode network by a visual detection task, Krish D. Singh ${ }^{1}$, Ian P. Fawcett ${ }^{2},{ }^{1}$ CUBRIC, School of Psychology, Cardiff University, Cardiff, United Kingdom, ${ }^{2}$ School of Life and Health Sciences, Aston University, Birmingham, United Kingdom

Neural mechanisms underlying action execution and action observation, Alexander Moiseev ${ }^{l}$, Naznin Virji-Babul ${ }^{1,2}$, Teresa Cheung ${ }^{1,2}$, Douglas Cheyne ${ }^{3}$, Daniel Weeks ${ }^{2},{ }^{1}$ Down Syndrome Research Foundation, Burnaby, Canada, ${ }^{2}$ Simon Fraser University, Burnaby, Canada, ${ }^{3}$ Hospital for Sick Children, Toronto, Canada

Neural correlates of 'feeling of telepresence' during watching a movie, Jeonghun $\mathrm{Ku}^{\mathrm{l}}$, Hyeongrae Lee $^{1}$, Jinsick Park ${ }^{1}$, Dan-Bi Choi ${ }^{2}$, Il Ho Park ${ }^{2}$, Kiwan Han ${ }^{1}$, Kang Joon Yoon ${ }^{3}$, Jae-Jin Kim ${ }^{2}$, In Young Kim ${ }^{l}$, Sun I. Kim ${ }^{l},{ }^{l}$ Department of Biomedical Engineering, Hanyang University, Seoul, Korea, ${ }^{2}$ Department of Psychiatry, College of Medicine, Yonsei University, Seoul, Korea, ${ }^{3}$ St. Peter's Hospital, Seoul, Korea

How special is the self? Neural basis of self-reflection: an fMRI study., Gemma Modinos ${ }^{l}$, Hans Ormel $^{2}$, Lisette van der Meer ${ }^{l}$, Andre Aleman ${ }^{l},{ }^{I} B C N$ Neuroimaging Center, UMCG, Groningen, Netherlands, ${ }^{2}$ Universitair Centrum Psychiatrie, UMCG, Groningen, Netherlands

Activation of the insular cortex during anticipation of feedback stimuli about difficult timing performance, Yasunori Kotani ${ }^{1}$, Yoshimi Ohgami ${ }^{1}$, Tatsuya Yoshihiro ${ }^{1}$, Tetsuji Tsukamoto ${ }^{2}$, Junichiro Arai $^{3}$, Yusuke Inoue ${ }^{4}$, Yasutsugu Aihara ${ }^{5},{ }^{1}$ Tokyo Institute of Technology, Tokyo, Japan, ${ }^{2}$ GE-Yokogawa Medical Systems, Tokyo, Japan, ${ }^{3}$ Daikin Industries, Osaka, Japan, ${ }^{4}$ The University of Tokyo, Tokyo, Japan, ${ }^{5}$ Tokyo Metropolitan University, Tokyo, Japan 
F0 independency of auditory evoked N1m latency is vocal sound specific?, Tomomi Mizuochi ${ }^{l, 5}$, Masato Yumoto ${ }^{2}$, Shotaro Karino ${ }^{3}$, Kenji Itoh $^{4}$, Tatsuya Yamasoba ${ }^{l,}{ }^{3},{ }^{1}$ Department of Sensory and Motor Neuroscience, Graduate School of Medicine, University of Tokyo, Tokyo, Japan, ${ }^{2}$ Department of Clinical Laboratory, Graduate School of Medicine, University of Tokyo, Tokyo, Japan, ${ }^{3}$ Department of Otolaryngology-Head and Neck Surgery, Graduate School of Medicine, University of Tokyo, Tokyo, Japan, ${ }^{4}$ Department of Speech and Cognitive Science, Graduate School of Medicine, University of Tokyo, Tokyo, Japan, 5 JSPS Research Fellow, Tokyo, Japan

\section{DISORDERS OF THE NERVOUS SYSTEM Addiction}

Effects of Acute Alcohol Consumption on Complexity and Functional Connectivity of EEGs in Healthy Subjects., Seongkyun Kim ${ }^{1}$, Dai-Jin Kim ${ }^{2}$, Jaeseung Jeong ${ }^{1,3},{ }^{I}$ Department of Bio and Brain Engineering, Korea Advanced Institute of Science and Technology, Daejeon, South Korea, ${ }^{2}$ Department of Psychiatry, College of Medicine, The Catholic University of Korea, Seoul, South Korea, ${ }^{3}$ Department of Psychiatry, Columbia College of Physicians and Surgeons and the New York State Psychiatric Institute, New York, USA

Pharmacological MRI of cigarette and placebo smoking, Kimberly Lindsey ${ }^{1}$, Blaise Frederick ${ }^{2}$, Lisa Nickerson ${ }^{2}$, Robert Ross MacLean ${ }^{I}$, Scott Lukas ${ }^{1},{ }^{1}$ Behav. Psychopharm. Res. Lab. - McLean Hospital, Belmont, USA, ${ }^{2}$ Brain Imaging Center - McLean Hospital, Belmont, USA

\section{DISORDERS OF THE NERVOUS SYSTEM Autism}

BOLD responses to dynamic facial expressions in autism spectrum disorders, Rahko Jukka ${ }^{l}$, Paakki Jyri-Johan ${ }^{2}$, Ebeling Hanna ${ }^{I}$, Jussila Katja ${ }^{l}$, Jansson-Verkasalo Eira ${ }^{3}$, Kuusikko Sanna ${ }^{1}$, Kätsyri Jari ${ }^{4}$, Mattila Marja-Leena ${ }^{1}$, Moilanen Irma ${ }^{1}$, Nikkinen Juha ${ }^{2}$, Remes Jukka ${ }^{2}$, Sams Mikko , Starck Tuomo ${ }^{2}$, Tervonen Osmo ${ }^{2}$, Kiviniemi Vesa ${ }^{2},{ }^{1}$ Department of Child Psychiatry, Oulu University Hospital, Oulu, Finland, ${ }^{2}$ Department of Diagnostic Radiology, Oulu University Hospital, Oulu, Finland, ${ }^{3}$ Faculty of Humanities, Speech and Language Pathology, University of Oulu, Oulu, Finland, ${ }^{4}$ Laboratory of Computational Engineering, Helsinki University of Technology, Helsinki, Finland

fMRI activation to emotional faces is related to social anxiety in autism spectrum disorders, Natalia Kleinhans ${ }^{1,3}$, Todd Richards ${ }^{1,3}$, Leonard Johnson ${ }^{2,3}$, Jessica Greenson ${ }^{3}$, Geraldine Dawson ${ }^{4}$, Elizabeth Aylward ${ }^{1,3},{ }^{1}$ University of Washington Dept. of Radiology, Seattle, USA, ${ }^{2}$ University of Washington, Dept. of Psychosocial and Community Health, Seattle, USA, ${ }^{3}$ Center on Human Development and Disability, Seattle, USA, ${ }^{4}$ University of Washington, Dept. of Psychology, Seattle, USA

89 W-AM

93 W-AM

$97 \mathrm{~W}-\mathrm{AM}^{*}$

$101 \mathrm{~W}-\mathrm{AM}$

$105 \mathrm{~W}-\mathrm{AM}$

\section{DISORDERS OF THE NERVOUS SYSTEM Brain \& Spinal Cord Trauma}

Investigating the long-term effects of preterm birth on brain volume development using voxelbased morphometry of MRI data, Zoltan Nagy ${ }^{1,2}$, John Ashburner ${ }^{2}$, Bogdan Draganski ${ }^{2}$, Hugo Lagercrantz ${ }^{1},{ }^{I}$ Neonatology Unit of the Deparment of Woman and Child Health, Karolinska Institute, Stockholm, Sweden, ${ }^{2}$ Wellcome Trust Centre for Neuroimaging, University College London, London, United Kingdom

Magnetic Resonance Diffusion Tensor Imaging in Acute and Chronic Diffuse Axonal Injury, Johan Ljungqvist, Daniel Nilsson, Thomas Skoglund, Department of Neurosurgery, Sahlgrenska University Hospital, Goteborg, Sweden

\section{DISORDERS OF THE NERVOUS SYSTEM Developmental Disorders}

Tourette: Nucleus Accumbens Volume Reduction and Somatosensory Cortical Thinning., Cherine Fahim $^{1,4,5}$, Oliver Lyttelton ${ }^{2}$, Alan Evans ${ }^{1,2,3},{ }^{1} 1$.Department of Neurology and Neurosury, McGill University, Montreal, Canada, ${ }^{2}$ Department of Biomedical Engineering, McGill University, Montreal, Canada, ${ }^{3}$ Department of Medical Physics, McGill University, Montreal, Canada, ${ }^{4}$ Sainte Justine Hospital Research Centre, Montreal, Canada, ${ }^{5}$ Department of Psychiatry, University of Montreal, Montreal, Canada
109 W-AM*

$113 \mathrm{~W}-\mathrm{AM}$ 
$T_{2}$ Mapping of the Brains of Human Newborns in Control Group and Prenatally Drug-Exposed Groups, Feng Liu, Yunsuo Duan, Zhengchao Dong, Tove Rosen, Ravi Bansal, Dongrong Xu, Satie Shova, Bradley Peterson, Alayar Kangarlu, Columbia University and New York State Psychiatric Institute, New York, USA

Motor activation in developmental stuttering, Amanda Wood ${ }^{1,2}$, Angela Morgan ${ }^{3}$, Sheena Reilly, Vicki Anderson' ${ }^{2}$ David Reutens ${ }^{1},{ }^{1}$ Department of Medicine, Southern Clinical School, Monash University, Melbourne, Australia, ${ }^{2}$ Australian Centre for Child Neuropsychology Studies, Critical Care Neurosciences Theme, Murdoch Childrens Research Institute, Melbourne, Australia, ${ }^{3}$ Language \& Literacy Group, Healthy Development Theme, Murdoch Childrens Research Institute, Melbourne, Australia

Diffusion tensor imaging abnormalities in boys with attention deficit hyperactive disorder with or without comorbid tic disorders, Jee Wook Choil, Bum Seok Jeong ${ }^{2}$, Myung-Ho Lim ${ }^{3}$, Se Hun Shim ${ }^{4}$, Jung Woo Sonn ${ }^{5}$, Jun Kyun Park ${ }^{6}$, Chang hwa Lee ${ }^{2},{ }^{1}$ Dept. of Psychiatry, Daejeong St. Marry, Catholic University, Daejeon, South Korea, ${ }^{2}$ Dept. of Psychiatry, Eulji University, Daejeon, South Korea, ${ }^{3}$ Dept. of Psychiatry, Dankuk University, Cheonan, South Korea, ${ }^{4}$ Dept. of Psychiatry, Suncheonhyang University, Chenonan, South Korea, ${ }^{5}$ Dept. of Psychiatry, Chungju, South Korea, ${ }^{6}$ Dept. of Psychiatry, Konyang, Daejeon, South Korea, ${ }^{7}$ Dept. of Psychiatry, Eulji University, Daejeon, South Korea

\section{DISORDERS OF THE NERVOUS SYSTEM Epilepsy}

Mapping of entorhinal cortex connectivity in temporal lobe epilepsy, Boris Bernhardt, Jason Lerch, Alan Evans, Neda Bernasconi, Andrea Bernasconi, Brain Imaging Center, Montreal Neurological Institute and Hospital, McGill University, Montreal, Canada

Non-invasive presurgical investigation in epileptic patients using simultaneous EEG-NIRS, Anne Gallagher $^{1,2}$, Dang K. Nguyen ${ }^{3}$, Phetsamone Vannasing ${ }^{1}$, Olivia Florea ${ }^{1}$, Julie Tremblay ${ }^{1}$, Danielle Bastien $^{1,2}$, Isabelle Pelletier ${ }^{1,2}$, Christophe Grova ${ }^{2,4}$, Frédéric Lesage ${ }^{5}$, Alain Bouthillier ${ }^{3}$, Lionel Carmant ${ }^{1,6}$, Franco Lepore ${ }^{1,2}$, Renée Béland ${ }^{2}$, Maryse Lassonde ${ }^{1,2},{ }^{1}$ Centre de Recherche de l'Hôpital Sainte-Justine, Hôpital Sainte-Justine, Montréal, Canada, ${ }^{2}$ Centre de Recherche en Neuropsychologie et Cognition, Université de Montréal, Montréal, Canada, ${ }^{3}$ Service de Neurologie, Hôpital Notre-Dame du CHUM, Montréal, Canada, ${ }^{4}$ Montreal Neurological Institute, Montréal, Canada, ${ }^{5}$ École Polytechnique, Université de Montréal, Montréal, Canada, ${ }^{6}$ Service de Neurologie, Hôpital Sainte-Justine, Montréal, Canada

Automated hippocampal volume measurement can quantify atrophy associated with hippocampal sclerosis in temporal lobe epilepsy, Heath Pardoe, Gaby Pell, Graeme Jackson, Brain Research Institute, Melbourne, Australia

The impact of anterior temporal lobectomy on linguistic ability of temporal lobe epilepsy patients., Savio Wong ${ }^{1}$, Seyed Mirsattari ${ }^{2,3}$, Frank Bihari ${ }^{2}$, Donna Bandur ${ }^{4},{ }^{1}$ Brain and Creativity Institute,

University of Southern California, Los Angeles, USA, ${ }^{2}$ Department of Clinical Neurological Sciences,

The University of Western Ontario, London, Canada, ${ }^{3}$ Department of Medical Biophysics, The

University of Western Ontario, London, Canada, ${ }^{4}$ Speech-Language Pathology Services, Psychological

Services, London, Canada

Visualization of Electroencephalographic Activity during Epileptic Seizures, Michelle Chong ${ }^{l}$, Anthony Burkitt ${ }^{1,3}$, David Grayden ${ }^{1,3}$, Iven Mareels ${ }^{1}$, Karen Fuller ${ }^{2}$, Levin Kuhlmann ${ }^{1}$, Mark Cook ${ }^{2,3}$, ${ }^{I}$ Department of Electrical and Electronics Engineering, The University of Melbourne, Melbourne, Australia, ${ }^{2}$ St. Vincent's Hospital, Melbourne, Australia, ${ }^{3}$ The Bionic Ear Institute, Melbourne, Australia

Single Subject Voxel-Based Relaxometry for Clinical Assessment of Temporal Lobe Epilepsy, Robert Kosior ${ }^{1,2}$, Louis Lauzon ${ }^{2,3}$, Richard Frayne ${ }^{2,3}$, Paolo Federico ${ }^{2,3}$, ${ }^{1}$ Electrical and Computer Engineering, University of Calgary, Calgary, Canada, ${ }^{2}$ Seaman Family MR Centre, Foothills Med. Ctr., Calgary Health Region, Calgary, Canada, ${ }^{3}$ Radiology and Clinical Neurosciences, Hotchkiss Brain Institute, University of Calgary, Calgary, Canada

Post temporal lobe epilepsy surgery fMRI language reorganization, Neelan Pillay ${ }^{1,2,3}$, Anthony Waites $^{1,2,3}$, David Abbott ${ }^{1,2.3}$, Graeme Jackson ${ }^{1,2,3}$, ${ }^{1}$ Brain Research Institute, Melbourne, Australia, ${ }^{2}$ University of Melbourne, Melbourne, Australia, ${ }^{3}$ Austin Health, Melbourne, Australia 


\section{DISORDERS OF THE NERVOUS SYSTEM Stroke \& Recovery of Function}

A longitudinal fMRI study of cortical sensorimotor reorganisation in stroke recovery., Timothy Budd $^{l}$, Mark Parsons ${ }^{1,2}$, Isobelle Hubbard ${ }^{1,2}$, Leeanne Carey ${ }^{3}$, Christopher Levi ${ }^{1,2},{ }^{1}$ University of Newcastle, Newcastle, Australia, ${ }^{2}$ John Hunter Hospital, Newcastle, Australia, ${ }^{3}$ La Trobe University, Melbourne, Australia

Development and Utilization of A New Stroke Registry Containing Quantifiable Imaging Data on A Standard Brain Template, Dong-Eog Kim ${ }^{l}$, Geon-Hwan Kwan ${ }^{2}$, Sang-Wook Jeong ${ }^{l}$, Heung-Kook Choi ${ }^{2},{ }^{l}$ MINER (Molecular Imaging and Neurovascular Research) Lab \& Department of Neurology, Dongguk University International Hospital, Goyang, South Korea, ${ }^{2}$ Department of Computer Science, Kimhae, South Korea

Effect of Repetitive Arm Cycling Combined with Botulinum Toxin on Post-Stroke Spasticity: Evidence from Functional Magnetic Resonance Imaging, Rüdiger Seitz ${ }^{1,2}$, Raimund Kleiser ${ }^{1}$, Sandrin Hyde $^{3}$, Nicolas Perret ${ }^{4}$, Dieter Ruegg ${ }^{3}$, Philippe Vuadens ${ }^{5}$, Eleonora Fornari ${ }^{6}$, Francois Vingerhoets ${ }^{7}$, Karin Diserans ${ }^{7,8},{ }^{1}$ Department of Neurology, Heinrich-Heine-University Düsseldorf, Düsseldorf, Germany, ${ }^{2}$ Brain Imaging Center West, Jülich, Germany, ${ }^{3}$ Department of Medicine, University Hospital, Fribourg, Switzerland, ${ }^{4}$ Neurological Center Plein Soleil, Lausanne, Switzerland, ${ }^{5}$ Clinique de Réadaptation Romande, Sion, Switzerland, ${ }^{6}$ Department of Diagnostic Radiology, University Hospital, Lausanne, Switzerland, ${ }^{7}$ Department of Neurology, University Hospital, Lausanne, Switzerland,

${ }^{8}$ Department of Neurorehabilitation and Neuropsychology, University Hospital, Lausanne, Switzerland

A proof-of-concept study on the effects of a robotic-assisted hand rehabilitation programme after stroke on central movement control, Christian Enzinger ${ }^{1,6}$, Christa Pargfrieder ${ }^{1}$, Sandra Pegritz ${ }^{l}$, Walter Wurm ${ }^{1}$, Regina Linderl-Madrutter ${ }^{2}$, Gudrun Reiter ${ }^{1}$, Reinhold Scherer ${ }^{3}$, Alexander Kollreider ${ }^{4}$, David Ram ${ }^{4}$, Stefan Ropele ${ }^{1}$, Marisa Loitfelder ${ }^{1,5}$, Christa Neuper ${ }^{3,5}$, Franz Fazekas ${ }^{1}$, Peter Grieshofer ${ }^{2}$, ${ }^{l}$ Dept. of Neurology, Medical University Graz, Graz, Austria, ${ }^{2}$ Rehabilitation Clinic JudendorfStrassengel, Graz, Austria, ${ }^{3}$ Technical University Graz, Graz, Austria, ${ }^{4}$ Tyromotion GmbH, Graz, Austria, ${ }^{5}$ Institute of Psychology, Karl-Franzens University Graz, Graz, Austria, ${ }^{6}$ Section of Neuroradiology, Dept. of Radiology, Medical University Graz, Graz, Austria

Motion-processing and visuoconstructive deficits in an occipito-temporal stroke patient, Daniela Bernhardt ${ }^{l}$, Markus Raabe ${ }^{I}$, Ralf Lürding ${ }^{2}$, Ingo Kleiter ${ }^{2}$, Ulrich Bogdahn ${ }^{2}$, Mark W. Greenlee ${ }^{I}$, ${ }^{1}$ University of Regensburg, Institute for Experimental Psychology, Regensburg, Germany, ${ }^{2}$ University of Regensburg, Department of Neurology, Regensburg, Germany

\section{EMOTION \& MOTIVATION Decision Making}

Brain activity during self-referential processing about colors. -An fMRI study-, Hiroko Konno ${ }^{l}$, Yuko Sassa ${ }^{2,3}$, Motoaki Sugiura ${ }^{4}$, Ryuta Kawashima ${ }^{2,3},{ }^{1}$ Tohoku University School of Medicine, Sendai, Japan, ${ }^{2}$ RISTEX, Japan science and technology agency, Sendai, Japan, ${ }^{3}$ Department of Functional Brain Imaging, IDAC, Tohoku University, Sendai, Japan, ${ }^{4}$ Department of Cerebral Research, NIPS, Sendai, Japan

Distinguishing action values from chosen values in the human brain during reward-based decision making, Klaus Wunderlich ${ }^{l}$, Antonio Rangel ${ }^{2}$, John P O'Doherty ${ }^{1,2}$, ${ }^{I}$ Computation and Neural Systems Program, Caltech, Pasadena, USA, ${ }^{2}$ Division of Humanities and Social Sciences, Caltech, Pasadena, USA

Cognitive Dissonance in Free Choice: New Insights from fMRI, Tali Sharot, Benedetto De Martino, Ray Dolan, University College London, London, United Kingdom

\section{EMOTION \& MOTIVATION Emotional Learning}

Dissociable roles for the hippocampus and the amygdala in human cued vs. context fear conditioning., Andreas Marschner ${ }^{1}$, Raffael Kalisch ${ }^{1}$, Bram Vervliet ${ }^{3}$, Debora Vansteenwegen ${ }^{2}$, Christian Büchel', ' Department of Systems Neuroscience, University of Hamburg, Hamburg, Germany, ${ }^{2}$ Department of Psychology, Katholieke Universiteit Leuven, Leuven, Netherlands, ${ }^{3}$ Department of Psychology, University of Amsterdam, Amsterdam, Netherlands 
Neural responses in the amygdala and hippocampus relate with extinction of aversive face and voice stimuli., Tetsuya Iidaka ${ }^{1}$, Daisuke Saito ${ }^{2}$, Hidetsugu Komeda ${ }^{2}$,Yoko Mano ${ }^{2}$, Norio Ozaki ${ }^{1}$, Norihiro Sadato ${ }^{2},{ }^{\prime}$ Nagoya University, Nagoya, Japan, ${ }^{2}$ National Institute for Physiological Sciences, $201 \mathrm{~W}-\mathrm{AM}$ Okazaki, Japan

Switching associations between facial identity and emotional expression is more difficult for angry expressions compared to happy expressions: A behavioural and ERP study., Megan Willis ${ }^{1}$, Romina Palermo ${ }^{1}$, Genevieve McArthur ${ }^{1}$, Darren Burke ${ }^{2}$, Carmen Atkinson ${ }^{l},{ }^{\prime}$ Macquarie Centre for Cognitive Science (MACCS), Macquarie University, Sydney, Australia, ${ }^{2}$ Centre for the Integrative Study of Animal Behaviour (CISAB), Macquarie University, Sydney, Australia

\section{EMOTION \& MOTIVATION Emotional Perception}

Neural activation to harsh faces among patients with Intermittent Explosive Disorder, Michael McCloskey ${ }^{I}$, Emil Coccaro ${ }^{I}$, Mike Angstadt ${ }^{2}$, Royce Lee ${ }^{l}$, Mitchell Berman ${ }^{3}$, K. Luan Phan ${ }^{2}$, ${ }^{I}$ The $^{3}$ University of Chicago, Chicago, USA, ${ }^{2}$ University of Michigan, Ann Arbor, USA, ${ }^{3}$ University of Southern Mississippi, Hattiesburg, USA

The inferior fronto-occipital fasciculus mediates recognition of the facial expression of emotions., Carissa Philippi ${ }^{1}$, Sonya Mehta ${ }^{1}$, Thomas Grabowski ${ }^{1,3}$, Ralph Adolphs ${ }^{2}$, David Rudrauf ${ }^{1}$, Laboratory of Computational Neuroimaging, Department of Neurology, Division of Behavioral Neurology and Cognitive Neuroscience, University of Iowa College of Medicine, 200 Hawkins Drive, Iowa City, USA, ${ }^{2}$ Divisions of Humanities and Social Sciences and Biology, California Institute of Technology, Pasadena, USA, ${ }^{3}$ Department of Radiology, University of Iowa College of Medicine, 200 Hawkins Drive, Iowa City, USA

The cerebral blood flow correlates of Emotional Facial Processing in Mild Alzheimer's disease., Roger T. Staff ${ }^{1}$, Trevor S. Ahearn ${ }^{2}$, Louise H. Phillips ${ }^{2}$, Clare Scott ${ }^{2}$, Donald Mowat ${ }^{2}$, Lawrence J. Whalley $^{2}$, Claude M. Wischik ${ }^{2}$, Alison D. Murray ${ }^{2},{ }^{1}$ Aberdeen Royal infirmary, Aberdeen, Scotland,

${ }^{2}$ University of Aberdeen, Aberdeen, Scotland

Spatial representation of non-verbal emotional perception along the superior temporal sulcus fMRI reveals audiovisual integration area between voice- and face-sensitive regions, Benjamin Kreifelts ${ }^{l}$, Thomas Ethofer ${ }^{1,2}$, Wolfgang Grodd ${ }^{2}$, Thomas Shiozawa ${ }^{3}$, Dirk Wildgruber ${ }^{l},{ }^{1}$ Department of Psychiatry, University of Tuebingen, Tuebingen, Germany, ${ }^{2}$ Section of Experimental MR of the CNS, Department of Neuroradiology, University of Tuebingen, Tuebingen, Germany, ${ }^{3}$ Institute of Anatomy, University of Tuebingen, Tuebingen, Germany

Increased amygdala activation during automatic processing of facial emotion in schizophrenia, Astrid Veronika Rauch ${ }^{1,3}$, Maraike Reker ${ }^{1}$, Patricia Ohrmann ${ }^{1}$, Anya Pedersen ${ }^{1}$, Jochen Bauer ${ }^{l}$, Udo Dannlowski ${ }^{l}$, Liv Harding ${ }^{l}$, Katja Kölkebeck ${ }^{l}$, Carsten Konrad ${ }^{l, 3}$, Harald Kugel ${ }^{2}$, Volker Arolt ${ }^{1}$, Walter Heindel $^{2}$, Thomas Suslow ${ }^{1},{ }^{1}$ Department of Psychiatry, Muenster, Germany, ${ }^{2}$ Department of Clinical Radiology, Muenster, Germany, ${ }^{3}$ IZKF-Research Group 4, Muenster, Germany

Human brain represents valence of another's facial expression, Mikko Viinikainen ${ }^{l}$, Iiro Jääskeläinen ${ }^{l}$, Marja Balk ${ }^{l}$, Taina Autti ${ }^{2}$, Mikko Sams ${ }^{l},{ }^{l}$ Department of Biomedical Engineering and Computational Science, Espoo, Finland, ${ }^{2}$ Helsinki University Central Hospital, Helsinki, Finland

Localization Accuracy of Current Functional Neuroimaging of the Human Amygdala: A MetaAnalysis, Tonio Ball ${ }^{1,2,7}$, Johanna Derix ${ }^{1,7}$, Simon Eickhoff ${ }^{3,4}$, Andreas Schulze-Bonhage ${ }^{1,2,7}$, Isabella Mutschler ${ }^{1,5,6,7},{ }^{1}$ Epilepsy Center, Univerty Clinics, Freiburg, Germany, ${ }^{2}$ Bernstein Center for Computational Neuroscience, University Freiburg, Freiburg, Germany, ${ }^{3}$ Institute for Medicine, Research Center Jülich, Jülich, Germany, ${ }^{4} \mathrm{C} \& O$ Institute for Brain Research, University of Düsseldorf, Düsseldorf, Germany, ${ }^{5}$ Department of Psychiatry, University of Basel, Basel, Switzerland, ${ }^{6}$ Department of Psychology, University of Basel, Basel, Switzerland, ${ }^{7}$ Freiburg Brain Imaging, University Clinics Freiburg, Freiburg, Germany

Decreased frontal gamma oscillations for different facial expressions of patients with bipolar disorder and major depression disorder: a MEG study, Tai-Ying Liu ${ }^{1}$, Li-Fen Chen ${ }^{2,3}$, Jen-Chuen Hsieh $^{2,3}$, Tung-Ping Su ${ }^{4,5}$, Institute of Biomedical Informatics, School of Medicine, National Yang-Ming University, Taipei, Taiwan, ${ }^{2}$ Institute of Brain Science, School of Medicine, National Yang-Ming University, Taipei, Taiwan, ${ }^{3}$ Integrated Brain Research Laboratory, Department of Medical Research 
and Education, Taipei Veterans General Hospital, Taipei, Taiwan, ${ }^{4}$ Division of Psychiatry, School of Medicine, National Yang-Ming University, Taipei, Taiwan, ${ }^{5}$ Psychiatric Department, Taipei Veterans General Hospital, Taipei, Taiwan

Relationships between grey-matter volume and functional brain activity to fearful faces in medial prefrontal and limbic regions in Posttraumatic Stress Disorder., Kim Felmingham ${ }^{1,2}$, Erin Falconer ${ }^{1,3}$, Leanne Williams ${ }^{1,2}$, Thomas Whitford ${ }^{l, 2}$, Anthony Peduto ${ }^{1,4}$, Richard Bryant ${ }^{1,3},{ }^{1}$ Brain Dynamics Centre, Westmead Millenium Institute, Westmead Hospital, Sydney, Australia, 2 Department of 245 W-AM Psychological Medicine, University of Sydney, Sydney, Australia, ${ }^{3}$ School of Psychology, University of New South Wales, Sydney, Australia, ${ }^{4}$ MRI Unit, Department of Radiology, Westmead Hospital, Sydney, Australia

Impact of Arousal on Non-conscious Fear Perception in Posttraumatic Stress Disorder: Enhanced Brainstem - Amygdala - Cortical 'Alarm' System in PTSD Patients with Hyperarousal., Andrew Kemp $^{1}$, Kim Felmingham ${ }^{1}$, Belinda Liddell ${ }^{1}$, Erin Falconer ${ }^{2}$, Richard Bryant ${ }^{2}$, Leanne Williams ${ }^{1},{ }^{\prime}$ Brain Dynamics Centre, Westmead Hospital and Western Clinical School University of Sydney, Sydney, Australia, ${ }^{2}$ School of Psychology, University of New South Wales, Sydney, Australia

Neurocognitive basis in experiencing compassion: A gender approach, Roberto E. Mercadillo ${ }^{l}$, José Luis Diaz ${ }^{2}$,Erick H. Pasaye ${ }^{1,3}$, Perla M. Salgado ${ }^{3}$, Fernando A. Barrios ${ }^{1},{ }^{1}$ Universiad Nacional Autónoma de México, Instituto de Neurobiología, Querétaro, Mexico, ${ }^{2}$ Universidad Nacional Autónoma de México, Facultad de Medicina, México DF, Mexico, ${ }^{3}$ Instituto Nacional de Neurología y

Neurocirugía, MVS, México DF, Mexico

EEG Default Mode Network: Olympic Hymn, Andrew CN Chen*,Huixuan Zhao, Peipei Wang, Center for Higher Brain Functions, Capital Medical University, Beijing, China

Amygdala involved in response to unexpected musical chords, Thomas Fritz ${ }^{1}$, Gottfried Schlaug ${ }^{2}$, Robert Turner ${ }^{l}$, Stefan Koelsch ${ }^{1},{ }^{1}$ Max Planck Institute for Cognitive and Brain Science, Leipzig, Germany, ${ }^{2}$ Harvard Medical School, Boston, USA

Attentional disengagement in response to threatening smoking pictures: An event-related brain potential study, Loes Kessels, Sara Moors, Kelly Pauwels, Rob Ruiter, Maastricht University, Maastricht, Netherlands

\section{IMAGING TECHNIQUES \& CONTRAST MECHANISM} Anatomical MRI

Whole-Brain Myelin Imaging Through Multi-Component Analysis of Steady-State Imaging Data, Sean Deoni ${ }^{1}$, Brian Rutt ${ }^{2}$, Tarunya Arun ${ }^{1}$, Carlo Pierpaoli ${ }^{3}$, Derek Jones ${ }^{4},{ }^{I}$ FMRIB, Oxford, United Kingdom, ${ }^{2}$ Robarts Research Institute, University of Western Ontario, London, Canada, ${ }^{3}$ Section of Tissue Biophysics and Biometrics, National Institutes of Health, Bethesda, USA, ${ }^{4}$ Cardiff University Brain Research Imaging Centre (CUBRIC), Cardiff, Wales

3D image reconstruction of depth electrode recording sites in the human Heschl's gyrus, Paul Poon $^{1,3}$, LS Chen ${ }^{2}$, Hiroyuki Oya ${ }^{3}$, Hiroto Kawasaki ${ }^{3}$, Richard Reale ${ }^{3}$, Kirill Nourski ${ }^{3}$, John Brugge ${ }^{3}$, Matthew Howard III , ' ${ }^{1}$ Dept Physiology, NCKU, Tainan, Taiwan, ${ }^{2}$ Dept Electrical Engineering, NCKU, Tainan, Taiwan, ${ }^{3}$ Dept Neurosurgery, Univ of Iowa, Iowa City, USA

USING ADNI CALIBRATION FOR NON-ADNI STUDIES: How to do it, Berkay Kanberoglu, Lina Karam ${ }^{1}$, Josef Debbins ${ }^{2},{ }^{1}$ Arizona State University, Tempe, USA, ${ }^{2}$ St. Joseph's Hospital and Medical Center, Phoenix, USA

A JPEG 2000 Image Compression Tool for the MIPAV Software Package, Dzung Nguyen ${ }^{1}$, Nam Nguyen ${ }^{1}$, Pierre-Louis Bazin' ${ }^{2}$, Trac Tran ${ }^{1}$, Dzung Pham ${ }^{2},{ }^{I}$ Department of Electrical and Computer Engineering, Johns Hopkins University, Baltimore, USA, ${ }^{2}$ Department of Radiology and Radiological Science, Johns Hopkins University, Baltimore, USA

\section{IMAGING TECHNIQUES \& CONTRAST MECHANISM Diffusion MRI}

Reducing distortions in DW-EPI with a dual-echo blip-reversed sequence, Daniel Gallichan ${ }^{l}$, Jesper L Andersson ${ }^{1}$, Mark Jenkinson ${ }^{l}$, Matthew D Robson ${ }^{2}$, Karla L Miller ${ }^{I},{ }^{I}$ FMRIB Centre, University of 285 W-AM Oxford, Oxford, United Kingdom, ${ }^{2}$ OCMR, University of Oxford, Oxford, United Kingdom 
Probablistic Tractograpy Using Steady-State Diffusion Imaging: A Promising Option For Acheiving Higher Spatial and Angular Resolution, Jennifer McNab ${ }^{l}$, Saad Jbabdi ${ }^{l}$, Sean Deoni ${ }^{1,2}$, Gwenaelle Douaud ${ }^{1}$, Tim Behrens ${ }^{1,3}$, Karla Miller ${ }^{1},{ }^{1}$ Department of Clinical Neurology, Oxford University, Oxford, United Kingdom, ${ }^{2}$ Centre for Neuroimaging Sciences, Institute of Psychiatry, King's College, University of London, London, United Kingdom, ${ }^{3}$ Department of Experimental Psychology, Oxford University, Oxford, United Kingdom

How Many Gradients are Sufficient in High-Angular Resolution Diffusion Imaging (HARDI)?, Liang Zhan ${ }^{l}$, Ming-Chang Chiang ${ }^{l}$, Marina Barysheva ${ }^{l}$, Arthur W. Toga ${ }^{l}$, Katie McMahon ${ }^{2}$, Greig de Zubicaray ${ }^{2}$, Matthew Meredith ${ }^{2}$, Margaret Wright ${ }^{3}$, Paul Thompson ${ }^{l},{ }^{1}$ Laboratory of Neuro Imaging, Department of Neurology, UCLA School of Medicine, Los Angeles, USA, ${ }^{2}$ Functional MRI Laboratory, Centre for Magnetic Resonance, University of Queensland, Brisbane, Australia, ${ }^{3}$ Queensland Institute of Medical Research, Brisbane, Australia

In Vivo Study of White Matter Microvasculature Anisotropy Using the IVIM Technique, Dimitrios C. Karampinos ${ }^{1,3}$, Bradley P. Sutton ${ }^{2,3}$, John G. Georgiadis ${ }^{1,3},{ }^{1}$ Mechanical Science and Engineering Department, University of Illinois at Urbana-Champaign, Urbana, USA, ${ }^{2}$ Bioengineering Department, University of Illinois at Urbana-Champaign, Urbana, USA, ${ }^{3}$ Beckman Institute, University of Illinois at Urbana-Champaign, Urbana, USA

Performance of Spatial Normalization in Diffusion Tensor Imaging, Huiling Peng, Konstantinos Arfanakis, Department of Biomedical Engineering, Illinois Institute of Technology, Chicago, USA

\section{IMAGING TECHNIQUES \& CONTRAST MECHANISM Multi-modal Integration}

Real-time Web-scale Image Annotation for Semantic-based Retrieval of Neuropsychiatric Research Images, H Jeremy Bockholt ${ }^{l}$, Josef Ling ${ }^{l}$, Mark Scully ${ }^{l}$, Adam Scott ${ }^{l}$, Susan Lane ${ }^{l}$, Vincent Magnotta $^{2}$, Tonya White ${ }^{3}$, Kelvin Lim ${ }^{3}$, Randy Gollub ${ }^{4}$, Vince Calhoun ${ }^{1,5},{ }^{1}$ The MIND Institute, Albuquerque, USA, ${ }^{2}$ The University of Iowa, Iowa City, USA, ${ }^{3}$ The University of Minnesota, Minneapolis, USA, ${ }^{4}$ Massachusetts General Hospital, Charlestown, USA, ${ }^{5}$ The University of New Mexico, Albuquerque, USA

DataViewer3D - An open-source, cross-platform multi-modal imaging data visualisation tool, Andre' Gouws, William Woods, Mark Hymers, Gary Green, York Neuroimaging Centre, The Biocentre, York Science Park, University of York, York, United Kingdom

Imaging artefact removal using moving window PCA in simultaneous EEG/fMRI, Perttu Ranta-aho ${ }^{1}$, Stefanos Georgiadis ${ }^{1}$,Eini Niskanen ${ }^{1,2,3}$, Mika Tarvainen ${ }^{l}$, Pasi Karjalainen ${ }^{l},{ }^{l}$ Department of Physcs, University of Kuopio, Kuopio, Finland, ${ }^{2}$ Department of clinical neurophysiology, Kuopio University Hospital, Kuopio, Finland, ${ }^{3}$ Department of Neurology, Kuopio University Hospital, Kuopio, Finland

Simultaneous intracranial EEG-fMRI: A preliminary investigation of RF induced heating., David Carmichael $^{1,2}$, John Thornton $^{3}$, Roman Rodionov ${ }^{1,2}$, Rachel Thornton ${ }^{1,2}$, Andrew McEvoy ${ }^{4}$, Philip Allen ${ }^{5}$, Louis Lemieux ${ }^{1,2}$, ' Department of Clinical and Experimental Epilepsy, UCL Institute of Neurology, London, United Kingdom, ${ }^{2}$ MRI Unit, National society for epilepsy, Chalfont St Peter, United Kingdom, ${ }^{3}$ Lysholm Department of Neuroradiology, National Hospital for Neurology and Neurosurgery, London, United Kingdom, ${ }^{4}$ Victor Horsley Department of Neurosurgery, National Hospital for Neurology and Neurosurgery, London, United Kingdom, ${ }^{5}$ Department of Clinical Neurophysiology, National Hospital for Neurology and Neurosurgery, London, United Kingdom

Analysis on micro-structural integrity of the white matter underlying cortical surface, Bang-Bon Koo ${ }^{l}$, Hua Ning ${ }^{2}$, Dae-Shik Kim ${ }^{2,3}$, Jong-Min Lee ${ }^{1},{ }^{1}$ Department of Biomedical Engineering, Hanyang University, Seoul, Korea, ${ }^{2}$ Center for Biomedical Imaging (CBI), Boston University school of Medicine, Boston, USA, ${ }^{3}$ Department of Anatomy and Neurobiology, Boston University school of Medicine, Boston, USA

Simultaneous measurement of fMRI, TMS and EMG with stepping stone sampling method, Hitoshi Shitara $^{1,2}$, Takashi Hanakawa ${ }^{1}$, Tetsuya Shinozaki ${ }^{2}$, Kenji Takagishi ${ }^{2}$, Manabu Honda $^{l}$, ${ }^{I}$ Department of Cortical Function Disorders, National Institute of Neuroscience, National Center of Neurology and Psychiatry, Tokyo, Japan, ${ }^{2}$ Department of Orthopedic Surgery, Gunma Graduate University School of Medicine, Gunma, Japan 


\section{IMAGING TECHNIQUES \& CONTRAST MECHANISM \\ Optical Imaging/NIRS/MRS (magnetic resonance spectroscopy)}

Identification and removal of motion artefact in functional near infrared imaging with the DYNOT system, F.C. Robertson, T.S. Douglas, E.M. Meintjes, Department of Human Biology, University of Cape Town, Cape Town, South Africa

Event-related hemodynamic optical signal during target detection in a Go-NoGo task, Andrei $V$. Medvedev ${ }^{l}$, Jana Kainerstorfer ${ }^{2}$, Sergey V. Borisov ${ }^{I}$, John VanMeter ${ }^{I},{ }^{l}$ Center for Functional and Molecular Imaging, Georgetown University Medical Center, Washington, USA, ${ }^{2}$ Dept. of Physics,

University of Vienna, Vienna, Austria

\section{IMAGING TECHNIQUES \& CONTRAST MECHANISM Perfusion MRI}

Tracking blood oxygenation within the cerebral vasculature with pulsed ASL using single-shot 3D GRASE, Carol Docherty ${ }^{l}$, Robert Trampel ${ }^{l}$, Matthias Guenther ${ }^{2}$, Marcel Weiss ${ }^{l}$, Enrico Reimer ${ }^{l}$, David Feinberg ${ }^{3}$, Robert Turner', ' Max-Planck Institute for Human Cognitive and Brain Sciences, Leipzig, Germany, ${ }^{2}$ mediri GmbH, Heidelberg, Germany, ${ }^{3}$ Advanced MRI Technologies, Sebastopol, USA

Dynamic Pseudo Continuous Arterial Spin Labeling, Wen-Ming Luh ${ }^{l}$, Afonso Silva ${ }^{2}$, Peter Bandettini ${ }^{1}$, ${ }^{I}$ FMRIF/NIMH, National Institutes of Health, Bethesda, USA, ${ }^{2}$ CMU/NINDS, National Institutes of Health, Bethesda, USA

\section{IMAGING TECHNIQUES \& CONTRAST MECHANISM PET/SPECT}

Automated Standardized Uptake Value Ratio of ${ }^{11}$ C-PIB PET analysis in Alzheimer's disease, Parnesh Raniga $^{1,2}$, Jurgen Fripp ${ }^{1}$, Pierrick Bourgeat ${ }^{1}$, Oscar Acosta ${ }^{1}$, Victor Villemagne ${ }^{5}$, Christopher Rowe ${ }^{5}$, Colin Masters ${ }^{4}$, Olivier Salvado', Sebastien Ourselin ${ }^{1,3},{ }^{1}$ BioMedIA Lab, e-Health Research Center, CSIRO, Brisbane, Australia, ${ }^{2}$ Department of Electrical and Information Engineering, University of Sydney, Sydney, Australia, ${ }^{3}$ University College London, London, United Kingdom, ${ }^{4}$ University of Melbourne, Melbourne, Australia, ${ }^{5}$ Department of Nuclear Medicine, center for PET, Austin Hospital, Melbourne, Australia

\section{IMAGING TECHNIQUES \& CONTRAST MECHANISM TMS}

Determining the Cortical Area Targeted by Transcranial Magnetic Stimulation (TMS), Axel Thielscher, Kamil Uludăg, MPI for biological Cybernetics, Tuebingen, Germany

\section{LANGUAGE \\ Comprehension}

Classification of fMRI during discourse processing in adolescents at ultra high risk for psychosis, Fred w. Sabb ${ }^{l}$, Theo van ERP $P^{2}$, Keng $W^{l}$, Angela Rizk-Jackson ${ }^{2}$, Mirella Dapretto ${ }^{l}$, Rochelle Caplan ${ }^{l}$, Molly Hardt ${ }^{2}$, Russell Poldrack ${ }^{1,2}$, Tyrone Cannon ${ }^{1,2}$, Carrie Bearden ${ }^{1,2}$, ${ }^{1}$ Department of Psychiatry, Semel Institute, UCLA, Los Angeles, USA, ${ }^{2}$ Department of Psychology, UCLA, Los Angeles, USA

Tracing the recovery of aphasia with a joint ICA of functional and structural data, Karsten Specht $t^{1}$, Roland Zahn ${ }^{2}$, Klaus Willmes ${ }^{3}$, Bernd J. Krause ${ }^{4}$, Hans Herzog ${ }^{5}$, Walter Huber ${ }^{6},{ }^{1}$ Department of biological and medical Psychology, University of Bergen \& Department of Medical Engineering, Haukeland University Hospital, Bergen, Norway, ${ }^{2}$ Neuroscience\& Aphasia Research Unit (NARU), University of Manchester, Manchester, United Kingdom, ${ }^{3}$ Section Neuropsychology at the Neurological Clinic, University Hospital Aachen, RWTH Aachen University, Aachen, Germany, ${ }^{4}$ Department of Nuclear Medicine, Technische Universität München, Munich, Germany, ${ }^{5}$ Institute of Medicine, Research Center Jülich, Jülich, Germany, ${ }^{6}$ Section Neurolinguistics at the Neurological Clinic, University Hospital Aachen, RWTH Aachen University, Aachen, Germany

Neural Efficiency for Sentence Comprehension and Working Memory, Satoru Yokoyama ${ }^{l}$, Kei Takahashi $^{123}$, Toshimune Kambara ${ }^{12}$, Tadao Miyamoto ${ }^{2}$, Jorge Riera ${ }^{1}$, Kei Yoshimoto ${ }^{2}$, Ryuta Kawashima , 'IDAC, Tohoku University, Sendai, Japan, ${ }^{2}$ GSICS, Tohoku University, Sendai, Japan, 
Hypoglycemia reduces differential BOLD response to voluntary not automatic language processing, Robin J. Schafer, Jagriti Arora, Maolin Qui, Katie Page, Rachna Relwani, Robert Sherwin, R. Todd Constable, Yale University, New Haven, USA

Processing Misspelled Words in Sentence Context: An ERP Study, Lairoe A. Stowe, Joost Rommers, Hanneke Loerts, John C.J. Hoeks, NeuroImaging Center, University Of Groningen, Groningen, Netherlands

Differences of cerebral oxygen exchange (COE) depending on L1 or L2, Kayoko YOSHINO ${ }^{l}$, Shun ISHIZAKI ${ }^{2}$, Toshinori KATO ${ }^{3},{ }^{1}$ Graduate school of Media and Governance, Keio University, Kanagawa, Japan, ${ }^{2}$ Faculty of Environmental Information, Kanagawa, Japan, ${ }^{3}$ Department of Brain Environmental Research, KATOBRAIN Co., Ltd., Tokyo, Japan

Dynamic ERP Mapping Dictating Concept to Percept: Chinese Olympic Sport Symbols, Andrew CN Chen*, Peipei Wang, Center for Higher Brain Functions, Capital Medical University, Beijing, China

The effect of familiarity in metaphor comprehension: An fMRI study, Claudio Gentili, ${ }^{1,3,4}$, Valentina Bambini ${ }^{2}$,Emiliano Ricciardi ${ }^{3,4,5}$, Pietro Pietrini ${ }^{3,5},{ }^{1}$ Chair of Clinical Psychology, University of Pisa, Pisa, Italy, ${ }^{2}$ Laboratory of Linguistics, Scuola Normale Superiore, Pisa, Italy, ${ }^{3}$ Laboratory of Clinical Biochemistry and Molecular Biology, University of Pisa, Pisa, Italy, ${ }^{4} 4$ MRI Lab, Institute of Clinical Physiology, C.N.R. Research Area, Pisa, Italy, ${ }^{5} 5$ Department of Laboratory Medicine and Molecular Diagnostics, AOUP, Pisa, Italy

Differentiating lexical complexity in fronto-temporal language networks, Mirjana Bozic ${ }^{1}$, Lorraine K Tyler ${ }^{2}$, William Marslen-Wilson ${ }^{1},{ }^{1}$ MRC Cognition and Brain Sciences Unit, Cambridge, United Kingdom, ${ }^{2}$ Department of Experimental Psychology, Cambridge, United Kingdom

World knowledge retrieval during text reading: A dynamic causal modelling study, Ho Ming Chow $^{1,2}$, Barbara Kaup ${ }^{3}$, Uwe Friese ${ }^{I}$, Markus Raabe ${ }^{2}$, Mark W. Greenle ${ }^{2},{ }_{1}^{1}$ Institute of Cognitive Science, University of Osnabrück, Osnabrück, Germany, ${ }^{2}$ Department of Experimental Psychology, University of Regensburg, Regensburg, Germany, ${ }^{3}$ Department of Psychology, Technical University of Berlin, Germany, Berlin, Germany

An fMRI Study of syntactic information on word recognition, Toshimune Kambara ${ }^{I}$, Satoru Yokoyama $^{1}$, Kei Takahashi ${ }^{1,2}$, Naoki Miura ${ }^{1,3}$, Tadao Miyamoto ${ }^{2}$, Daiko Takahashi ${ }^{2}$, Shigeru Sato ${ }^{2}$, Ryuta Kawashima ${ }^{I},{ }^{I}$ Department of Functional Brain Imaging, IDAC, Tohoku University, Sendai, Japan, ${ }^{2}$ Graduate School of International Cultural Studies, Tohoku University, Sendai, Japan, ${ }^{3}$ Department of Intelligent Mechanical Systems Engineering, Kochi University of Technology, Kami, Japan

\section{LANGUAGE \\ Reading/Writing}

MEG Applications for Detecting Dyslexia with Real \& Nonsense Word Reading, Susan Bowyer ${ }^{1,2,3}$, Margaret Greenwald ${ }^{2}$, John Moran ${ }^{1}$, Norman Tepley ${ }^{l, 3}$, Renee Lajiness O'Neill ${ }^{4}$, Henry Ford Hospital, Detroit, USA, ${ }^{2}$ Wayne State University, Detroit, USA, ${ }^{3}$ Oakland University, Rochester, USA, ${ }^{4}$ Eastern Michigan University, Ypsilanti, USA

Neural Basis of Resilient Readers in Dyslexia, Joshua Heitzmann, Candy Ho, Fumiko Hoeft, Allan Reiss, Center for Interdisciplinary Brain Sciences Research, Department of Psychiatry and Behavioral Sciences, Stanford University School of Medicine, Palo Alto, USA

Functional connectivity of reading-related regions in adults, Alecia Vogel ${ }^{1}$, Jessica Church ${ }^{1}$, Fran Miezin $^{1,2}$, Bradley Schlaggar ${ }^{1,2,3,4}$, Steven Petersen ${ }^{1,2,3,5},{ }^{l}$ Department of Neurology, Washington University School of Medicine, St. Louis, USA, ${ }^{2}$ Department of Radiology, Washington University School of Medicine, St. Louis, USA, ${ }^{3}$ Department of Anatomy and Neurobiology, Washington University School of Medicine, St. Louis, USA, ${ }^{4}$ Department of Pediatrics, Washington University School of Medicine, St. Louis, USA, ${ }^{5}$ Department of Psychology, Washington University, St. Louis, USA

The Function of Dorsal Visual Pathway in Chinese Character Recognition: a spTMS Study, Yanlin Luo ${ }^{l}$, Andrew CN Chen ${ }^{l}$, Jie Yang ${ }^{2}$, xiujun Li ${ }^{2}$, Danlin Pen ${ }^{2},{ }^{l}$ Center for Higher Brain Functions, Capital Medical University, Beijing, China, ${ }^{2}$ Beijing normal University, Beijing, China

Abnormal brain responses to sounds in children with language and reading impairments, Genevieve McArthur ${ }^{I}$, Carmen Atkinson ${ }^{l}$, Danielle Ellis ${ }^{2},{ }^{l}$ Macquarie Centre for Cognitive Science, Sydney, Australia, ${ }^{2}$ Macquarie University, Sydney, Australia 


\section{MEMORY \& LEARNING \\ Learning (explicit \& implicit)}

The computational values of information from personal and vicarious experiences are processed in parallel in the ACC, Timothy Behrens ${ }^{1,2,3}$, Laurence Hunt ${ }^{2,3}$, Mark Woolrich ${ }^{1}$. Matthew Rushworth ${ }^{1,2}$, ${ }^{1}$ FMRIB Centre, University of Oxford, Oxford, United Kingdom, ${ }^{2}$ Dept. Experimental Psychology,

University of Oxford, Oxford, United Kingdom, ${ }^{3}$ Equal contribution

Transitions of task-related brain activation during acquisition of a novel perceptual-motor mapping, Oliver Hinds ${ }^{1}$, Susan Gabrieli ${ }^{1,2}$, Noa Ofen ${ }^{2}$, Julie Yoo ${ }^{1}$, Satrajit Ghosh ${ }^{3}$, Nupur Lalal, Daniel Willingham $^{4}$, Christina Triantafyllou ${ }^{1.5}$, John Gabrieli ${ }^{1,2},{ }^{1}$ McGovern Institute for Brain Research, Massachusetts Institute of Technology, Cambridge, USA, ${ }^{2}$ Department of Brain and Cognitive Sciences, Massachusetts Institute of Technology, Cambridge, USA, ${ }^{3}$ Research Laboratory of Electronics, Massachusetts Institute of Technology, Cambridge, USA, ${ }^{4}$ Department of Psychology, University of Virginia, Charllotesville, USA, ${ }^{5}$ Athinoula A. Martinos Center, Department of Radiology, MGH, Harvard Medical School, Charlestown, USA

The bright side of Val - An advantage for the COMT Val genotype in reward-based decision making, Lea Krugel ${ }^{1,2}$, Guido Biele ${ }^{l, 2}$, Peter Mohr ${ }^{1,2}$, Shu-Chen Li $i^{I, 2}$, Hauke Heekeren ${ }^{1,2}$, ${ }^{1}$ Max Planck Institute for Human Development, Berlin, Germany, ${ }^{2}$ Berlin NeuroImaging Center, Berlin, Germany

Consolidation of Motor Memories Encoded by Different Practice Schedules, Satoshi Tanaka ${ }^{I}$, Manabu Honda ${ }^{2}$, Takashi Hanakawa ${ }^{2}$, Leonardo G Cohen ${ }^{1},{ }^{I}$ Human Cortical Physiology Section, NINDS, NIH, Bethesda, USA, ${ }^{2}$ Department of Cortical Function Disorders, National Institute of

Neuroscience, Kodaira, Japan

Visuospatial Working Memory in Adolescents with Dysthymic Disorder: A Functional Magnetic Resonance Imaging (fMRI) Study, Jacqueline Yamada ${ }^{I}$, Melissa Casey ${ }^{1}$, Tim Silk ${ }^{2}$, Ross Cunnington ${ }^{2}$, Mark Bellgrove ${ }^{2}$, Alasdair Vance ${ }^{1},{ }^{\prime}$ Academic Child Psychiatry Unit, Royal Children's Hospital, Murdoch Childrens Research Institute, Melbourne, Australia, ${ }^{2}$ Queensland Brain Institute, Brisbane, Australia

Learning with emotional context affects brain activation during retrieval: an fMRI study, Wenting Jial, Satoru Yokoyama ${ }^{2}$, Motoaki Sugiura ${ }^{23}$, Atsushi Sekiguchi ${ }^{2}$, Ai Fukushima ${ }^{2}$, Ryuta Kawashima ${ }^{2},{ }^{1}$ Tohoku University School of Medicine, Sendai, Japan, ${ }^{2}$ Institute of Development, Aging and Cancer, Tohoku University, Sendai, Japan, ${ }^{3}$ National Institute for Physiological Sciences, Okazaki, Japan

Hippocampal activation during a paired associative learning of faces and names, Kayako Matsuo ${ }^{I}$, Tetsuya Iidaka ${ }^{2}$, Epifanio Bagarinao ${ }^{3}$, Chikako Kato ${ }^{4}$, Akinori Takeda ${ }^{5}$, Toshiharu Nakai ${ }^{1}$, ${ }^{1}$ Dept. Gerontechnology, National Center for Geriatrics and Gerontology, Obu, Japan, ${ }^{2}$ Department of Psychiatry, Nagoya University, Nagoya, Japan, ${ }^{3}$ Grid Technology Research Center, National Institute of Advanced Industrial Science and Technology, Tsukuba, Japan, ${ }^{4}$ Department of Life and Career Design, Toyohashi Sozo University, Toyohashi, Japan, ${ }^{5}$ Department of Advanced Medicine, National Center for Geriatrics and Gerontology, Obu, Japan

11:30 - 12:30 Corryong Hall (Level 2)

MEMORY \& LEARNING

Long-term Memory (episodic, semantic, autobiographical)

Episodic Simulation of Specific and Generic Future Events, Donna Rose Addis ${ }^{1,2}$, Theresa Cheng ${ }^{l}$, Daniel L. Schacter ${ }^{1,2},{ }^{1}$ Dept. of Psychology, Harvard University, Cambridge, USA, ${ }^{2}$ Athinoula A. Martinos Center for Biomedical Imaging, Charlestown, USA

Neural correlates of transmission from lexical-semantic to lexical-phonological stages during name recall: An event-related fMRI study, Sho Yagishita ${ }^{l, 2}$, Takamitsu Watanabe ${ }^{l, 2}$, Hiroshi Ito ${ }^{l}$, Hiroo Ikehira ${ }^{l}$, Motoichiro Kato ${ }^{3}$, Iwao Kanno ${ }^{I}$, Tetsuya Suhara ${ }^{l}$, Hideyuki Kikyo ${ }^{l},{ }^{l}$ National Institute of Radiological Sciences, Chiba, Japan, ${ }^{2}$ The University of Tokyo, Tokyo, Japan, ${ }^{3}$ Keio University, Tokyo, Japan

Dissociated networks mediate retrieval operations via free-recall or recognition, Irit ShapiraLichter $^{1,2}$, Tali Siman-Tov ${ }^{1}$, Daphna Paran ${ }^{3,4}$, Dan Caspi ${ }^{3,4}$, Eli Vakil ${ }^{5}$, Talma Hendler ${ }^{1,4}$,

${ }^{I}$ Functional Brain Imaging Unit, Wohl Institute for Advanced Imaging, Tel Aviv Sourasky Medical 
Center, Tel-Aviv, Israel, ${ }^{2}$ Department of Psychology, Tel Aviv University, Tel-Aviv, Israel, ${ }^{3}$ Department of Rheumatology, Tel-Aviv Sourasky Medical Centre, Tel-Aviv, Israel, ${ }^{4}$ Sackler Faculty of Medicine, Tel Aviv University, Tel-Aviv, Israel, ${ }^{5}$ Department of Psychology and Leslie and Susan Gonda (Goldschmied) Multidisciplinary Brain Research Center, Bar-Ilan University, Ramat-Gan, Israel

Hippocampal activation is associated with encoding distinctiveness of study items, Valerie Carr ${ }^{l}$, Stephen Engel ${ }^{2,3}$, Barbara Knowlton ${ }^{1,2}{ }^{1}$ Interdepartmental Program in Neuroscience, UCLA, Los Angeles, USA, ${ }^{2}$ Department of Psychology, UCLA, Los Angeles, USA, ${ }^{3}$ Department of Psychology, University of Minnesota, Minneapolis, USA

Long-term Motor Training Affected Resting State Brain, Liangsuo Ma ${ }^{l}$, Binquan Wang ${ }^{2}$, Jinhu Xiong ${ }^{1},{ }^{1}$ Department of Radiology, University of Iowa, Iowa City, USA, ${ }^{2}$ Research Imaging Center, University of Texas Health Science Center, San Antonio, USA

A Neural Mechanism Underlying Memory Failure in Older Adults, W. Dale Stevens ${ }^{1}$, Lynn Hasher $^{2,4}$, Kimberly S. Chiew, ${ }^{2}$ Cheryl L. Grady ${ }^{2,3,4}$, 'Department of Psychology, Harvard University, Cambridge, USA, ${ }^{2}$ Rotman Research Institute at Baycrest, University of Toronto, Toronto, Canada, ${ }^{3}$ Department of Psychiatry, University of Toronto, Toronto, Canada, ${ }^{4}$ Department of Psychology, University of Toronto, Toronto, Canada

MODELING \& ANALYSIS

Exploratory Methods, Artifact Removal

Asynchrony of BOLD signal across brain regions, Xu Cui, Allan Reiss, Center for Interdisciplinary Brain Sciences Research, Department of Psychiatry, Stanford University, Stanford, USA

479 W-AM

Artificial shifting of fMRI activation detected by surface-based analyses, Hang Joon Jo ${ }^{l}$, Jong-Min Lee ${ }^{I}$, Jae-Hun Kim ${ }^{l}$, Chi-Hoon Choi ${ }^{2}$, Bon-Mi Gu, Do-Hyung Kang ${ }^{4}$, Jun Soo Kwon ${ }^{4}$, Sun I. Kim ${ }^{l}$, ${ }^{I}$ Department of Biomedical Engineering, Hanyang University, Seoul, Korea, ${ }^{2}$ Department of Diagnostic Radiology, National Medical Center, Seoul, Korea, ${ }^{3}$ Interdisciplinary Program in Brain Science, Seoul National University, Seoul, Korea, ${ }^{4}$ Department of Psychiatry, Seoul National University College of Medicine, Seoul, Korea

Tradeoffs between signal detection accuracy and filter kernel size in high resolution cortical imaging, Benjamin Ramsden, Department of Neurobiology and Anatomy, and Sensory Neuroscience Research Center, School of Medicine, West Virginia University, Morgantown, USA

Characterization of physiological and neural fluctuations in sensory-evoked fMRI of the primary visual cortex., Kevin Aquino ${ }^{1,2,3}$, Peter Robinson ${ }^{1,5,6}$, Mark Schira ${ }^{2,4}$, Peter Drysdale ${ }^{1,5}$, Michael Breakspear ${ }^{2,3},{ }^{1}$ School of Physics, University of Sydney, Sydney, Australia, ${ }^{2}$ School of Psychiatry, University of New South Wales, Sydney, Australia, ${ }^{3}$ The Blackdog Institute, Prince of Wales Hospital, Sydney, Australia, ${ }^{4}$ School of Psychology, University of New South Wales, Sydney, Australia, ${ }^{5}$ Brain Dynamics Center, Westmead Millennium Institute, Westmead Hospital and the University of Sydney, Westmead, Sydney, Australia, ${ }^{6}$ Faculty of Medicine, the University of Sydney, Sydney, Australia

Evaluation of Parameters Used for Retrospective Corrections of the Physiological Noise in fMRI, Arsène Ella, Jochen Rick, Jürgen Hennig, Dept. of Diagnostic Radiology, Medical Physics, University Hospital Freiburg, Freiburg, Germany

MRI Compatible Sleeping-Eye Gaze Tracking System Using Infrared Video Analyzed by ANN based Image Processing, Syoji Kobashi ${ }^{1}$, Yuji Yahatal, Shigeyuki Kan ${ }^{2}$, Masaya Misaki ${ }^{2}$, Takahiko $K_{\text {Koike }}^{2}$, Satoru Miyauchi ${ }^{2}$, Yutaka Hatal, ${ }^{l}$ Graduate School of Engineering, University of Hyogo, Himeji, Japan, ${ }^{2}$ CREST - Brain Function Imaging Team, Kobe Advanced ICT Research Center, National Institute of Information and Communications Technology, Kobe, Japan

Removal of speech-related artifacts in MEG, Mordehay Medvedovsky ${ }^{1}$, Samu Taulu ${ }^{2}$, Rozaliya Bikmullina ${ }^{1}$, Ritva Paetau ${ }^{1,3,4}$, Antti Ahonen ${ }^{2},{ }^{\prime}$ BioMag Laboratory, Helsinki University Central Hospital, Helsinki, Finland, ${ }^{2}$ Elekta Neuromag Oy, Helsinki, Finland, ${ }^{3}$ Hospital for Children and Adolescents, Department of Child Neurology, Helsinki University Central Hospital, Helsinki,

Finland, ${ }^{4}$ Departmetn of Clinical Neurophysiology, Helsinki University Central Hospital, Helsinki, Finland 


\section{MODELING \& ANALYSIS \\ Flattening, Segmentation}

Cortical thickness estimation of Alzheimer's disease patients: Application to the Australian Imaging Biomarkers and Lifestyle (AIBL) study., Pierrick Bourgeat ${ }^{l}$, Oscar Acosta ${ }^{l}$, Jurgen Fripp ${ }^{l}$, Colin Masters ${ }^{2}$, Christopher Rowe ${ }^{3}$,Victor Villemagne ${ }^{3}$, Olivier Slavado ${ }^{1}$, Sebastien Ourselin ${ }^{4}$, ${ }^{I}$ BioMedIA Lab, eHealth Research Centre, CSIRO ICT Centre, Brisbane, Australia, ${ }^{2}$ Centre for Neurosciences, University of Melbourne, Melbourne, Australia, ${ }^{3}$ Department of Nuclear Medicine, Centre for PET, Austin Health, Heidelberg, Australia, ${ }^{4} 2$ Centre for Medical Image Computing, University College London, London, United Kingdom

Anisotropic Diffusion Properties Near The Cortical Surface of The Human Brain, Xiaojian Kang ${ }^{1,2}$, Timothy Herron ${ }^{l}$, And Turken ${ }^{l}$, David Woods ${ }^{1,2,3}$, ' Human Cognitive Neurophysiology Lab, VA Research Service, VA-NCHCS, 150 Muir Road, Martinez, USA, ${ }^{2}$ Department of Neurology and Center for Neuroscience, University of California at Davis, 4860 Y St., Suite 3700, Sacramento, USA, ${ }^{3}$ UC Davis Center for Mind and Brain, 267 Cousteau Place, Davis, USA

Multispectral imaging improves performance of BET skull stripping, Vitali Zagorodnov ${ }^{l}$, Suresh A. Sadananthan ${ }^{1}$, Bradley P. Sutton ${ }^{2,3}$, Michael W.L. Chee ${ }^{4},{ }^{1}$ School of Computer Engineering, Nanyang Technological University, Singapore, Singapore, ${ }^{2}$ Bioengineering, University of Illinois at UrbanaChampaign, Urbana, USA, ${ }^{3}$ Beckman Institute, University of Illinois at Urbana-Champaign, Urbana, USA, ${ }^{4}$ Cognitive Neuroscience Laboratory, Duke-NUS Graduate, Singapore, Singapore

Improved Surface Models for FIRST, Brian Patenaude ${ }^{1}$, Stephen Smith ${ }^{1}$, David Kennedy ${ }^{2}$, Mark Jenkinson ${ }^{1},{ }^{I}$ FMRIB Centre, University of Oxford, Oxford, United Kingdom, ${ }^{2}$ Center for Morphometric Analysis, MGH, Boston, USA

Brain Surface Conformal Slit Mapping, Yalin Wang ${ }^{1,2}$, Xianfeng Gu ${ }^{3}$, Tony Chan ${ }^{2}$, Paul Thompson ${ }^{1}$, ${ }^{1}$ Lab. of Neuro Imaging and Brain Research Institute, UCLA School of Medicine, Los Angeles, USA,

${ }^{2}$ Mathematics Department, UCLA, Los Angeles, USA, ${ }^{3}$ Computer Science Department, Stony Brook University, Stony Brook, USA

\section{MODELING \& ANALYSIS}

Functional Connectivity and Structural Equation Modeling

Modular small-world networks and age-related attenuation of a dominant frontal module in human endogenous fMRI, David Meunier ${ }^{I}$, Sophie Achard ${ }^{l, 2}$, Edward Bullmore ${ }^{I},{ }^{I}$ Brain Mapping Unit, Department of Psychiatry, University of Cambridge, Cambridge, United Kingdom, ${ }^{2}$ GIPSA-lab, UMR CNRS 5216, Grenoble, France

Subcortical Network Shape Analysis via Segmentation Denoising and Random Surface Momentum Maps, Anqi Qiu ${ }^{1}$, Michael Miller ${ }^{2},{ }^{I}$ Division of Bioengineering, National University of Singapore, Singapore, Singapore, ${ }^{2}$ Center for Imaging Science, Johns Hopkins University, Baltimore, USA

A Stimulus-Locked VAR Connectivity Model for Slow Event-Related fMRI Designs, Wesley Thompson, Greg Seigle, University of Pittsburgh Department of Psychiatry, Pittsburgh, USA

Variations in Interhemispheric Correlation Across Development: A Resting-State fMRI Approach, Daniel S Margulies ${ }^{1,2}$, AM Clare Kelly ${ }^{l}$, Lucina Q Uddin ${ }^{1}$, Zarrar Shezhad ${ }^{l}$, Phil Reiss ${ }^{l}$, F Xavier Castellanos ${ }^{I}$, Michael P Milham ${ }^{1},{ }^{1}$ NYU Child Study Center, New York, USA, ${ }^{2}$ Berlin School of Mind and Brain, Berlin, Germany

The Impact of Global Signal Regression on Anti-Correlated Networks in Resting State Connectivity Analyses, Kevin Murphy ${ }^{1}$, Rasmus M. Birn ${ }^{l}$, Peter A. Bandettini ${ }^{1,2}$, 'Section on Functional Imaging Methods, National Institute of Mental Health, Bethesda, USA, ${ }^{2}$ Functional MRI Facility, National Institute of Mental Health, Bethesda, USA

Identifying Stimulus-Induced Functional Connectivity using Partial Directed Coherence, Joao Sato $^{1,2}$, Edson Amaro $\mathrm{Jr}^{1}$, Daniel Takahashi ${ }^{1,2}$, Silvia Arcuri ${ }^{3}$, Koichi Sameshima ${ }^{1}$, Pedro Morettin ${ }^{2}$, Luiz $^{2}$ Baccala ${ }^{4},{ }^{1}$ NIF-LIM44, Institute of Radiology - University of Sao Paulo, Sao Paulo, Brazil, ${ }^{2}$ Institute of Mathematics and Statistics - University of Sao Paulo, Sao Paulo, Brazil, ${ }^{3}$ Institute of Psychiatry - Kings 547 W-AM College London, London, United Kingdom, ${ }^{4}$ Department of Eletric Engineering, Escola Politécnica, University of Sao Paulo, Sao Paulo, Brazil 
The Effect of Atlas-based Parcellation on Small-World Brain Functional Networks, Jinhui Wang ${ }^{1}$, Liang Wang ${ }^{1,2}$, Chaozhe Zhu ${ }^{1}$, Yufeng Zang ${ }^{1}$, Hong Yang ${ }^{3}$, Qiyong Gong ${ }^{3}$, Yong He ${ }^{4},{ }^{1}$ State Key Laboratory of Cognitive Neuroscience and Learning, Beijing Normal University, Beijing, China, ${ }^{2}$ School of Information Science and Technology, Beijing Institute of Technology, Beijing, China, ${ }^{3}$ Huaxi MR Research Center, Department of Radiology, West China Hospital of, Chengdu, China, ${ }^{4}$ McConnell Brain Imaging Centre, Montreal Neurological Institute, McGill University, Montreal, Canada

A Bayesian Hierarchical Framework for Spatial Modeling of fMRI Data, Brian Caffo ${ }^{1}$, DuBois Bowman ${ }^{2}$ Susan Bassett ${ }^{1}$, Clinton Kilts ${ }^{2},{ }^{1}$ Johns Hopkins University, Baltimore, USA, ${ }^{2}$ Emory University, Atlanta, USA

Motor task performance produces reductions in the amplitude of low frequency oscillations across the brain, driving reductions in functional connectivity within brain networks., Eugene Duff, , Leigh Johnston ${ }^{1,3}$, Jinhu Xiong ${ }^{4}$, Peter T. Fox ${ }^{5}$, Iven Mareels ${ }^{3}$, Gary F. Egan ${ }^{1},{ }^{1}$ Howard Florey Institute, Centre for Neuroscience, University of Melbourne, Melbourne, Australia, ${ }^{2}$ Department of Mathematics and Statistics, University of Melbourne, Melbourne, Australia, ${ }^{3}$ Department of Electrical and Electronic Engineering, University of Melbourne, Melbourne, Australia, ${ }^{4}$ Department of Radiology, University of Iowa, Iowa City, USA, ${ }^{5}$ Research Imaging Centre, University of Texas Health Sciences Centre, San Antonio, USA

Mutual information based approach for nonlinear functional connectivity, Seung-Hyun Jin, Peter Lin, Mark Hallett, Human Motor Control Section, Medical Neurological Branch, National Institute of Neurological Disorders and Stroke, National Institutes of Health, Bethesda, USA

Phase Synchrony Analysis of Network Dynamics during Visual Task Performance links EEG and BOLD, Nathan Dees ${ }^{l}$, Linda Larson-Prior ${ }^{2}$, Tracy Nolan ${ }^{2}$, David Politte ${ }^{2}$, Fred Prior ${ }^{2}$, Bahar Sonya ${ }^{l}$, ${ }^{I}$ Center for Neurodynamics, University of Missouri, St. Louis, USA, ${ }^{2}$ Mallinckrodt Institute of Radiology, Washington University School of Medicine, St. Louis, USA

Schizophrenia as a Disruption of Functional Connectivity Patterns, B. Thyreau ${ }^{l}$, R. Garg ${ }^{2}$, G.A.

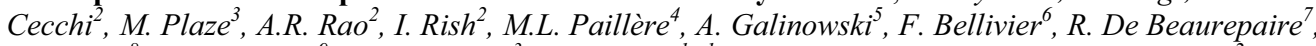
D. Januel ${ }^{8}, C$. Martinelli ${ }^{9}, J-L$. Martinot ${ }^{3}, J-B$. Poline ${ }^{1},{ }^{1}$ Neurospin, CEA, Gif-sur-Yvette, France, ${ }^{2} I B M$ Research, Yorktown Heights, USA, ${ }^{3}$ Unité Inserm-CEA, Neuroimaging and Psychiatry, Orsay, France, ${ }^{4}$ AP-HP Adolescent Medicine Department, Cochin Hospital, Paris, France, ${ }^{5}$ Sainte Anne Hospital, Paris, France, ${ }^{6}$ Henri Mondor Hospital, Créteil, France, ${ }^{7}$ Paul Guiraud Hospital, Villejuif, France, ${ }^{8}$ with CHS Ville-Evrard, Saint-Denis, France, ${ }^{9}$ P. Brousse Hospital, Villejuif, France

Global Signal Regression and Anticorrelations in Resting State fMRI Data, Michael Fox, Abraham Snyder, Marcus Raichle, Washington University in St. Louis, St. Louis, USA

Noise during rest explores the brain's dynamic repertoire, Viktor Jirsa ${ }^{1,3}$, Anandamohan Ghosh ${ }^{l}$, Rolf Kötter ${ }^{2}$, Randy McIntosh ${ }^{4}$, Young-Ah Rho ${ }^{3},{ }^{1}$ Theoretical Neuroscience Group, UMR6152 Institut de Science du Mouvement CNRS, Marseille, France, ${ }^{2}$ University Medical Centre St. Radboud, Nijmegen, Netherlands, ${ }^{3}$ Center for Complex Systems \& Brain Sciences, FAU, Boca Raton, USA, ${ }^{4}$ Rotman Research Institute - Baycrest Centre, Toronto, Canada

The patterns of functional connectivity in pediatric brain at rest: FDG-PET study, Heejung Kim $^{1,2}$, Hyejin Kang ${ }^{1,3}$, Yoon-Kyoung Yim ${ }^{1,2}$, Jae Sung Lee ${ }^{1}$, Dong Soo Lee ${ }^{I},{ }^{1}$ Dept. of Nuclear Medicine, Seoul National University, College of medicine, Seoul, South Korea, ${ }^{2}$ Interdisciplinary program in cognitive science, Seoul National University, Seoul, South Korea, ${ }^{3}$ Brain and neuroscience major, Seoul National University, College of Medicine, Seoul, South Africa

\section{MODELING \& ANALYSIS \\ Multivariate Modeling, PCA, \& ICA}

Modeling the spatial and temporal dependence in fMRI data: An application to a study of inhibitory control in cocaine addiction, F. DuBois Bowman, Gordana Derado, Emory University, Atlanta, USA

Analysis of ictal EEG-fMRI data in focal epilepsy patients using independent component analysis, Pierre LeVan, Louise Tyvaert, Jean Gotman, Montreal Neurological Institute, McGill University, Montreal, Canada

Further Development of the Complex General Linear Model to fMRI - Multiple Input and Output Evoked Response on Single Subject, Daniel Rio ${ }^{l}$, Robert Rawlings ${ }^{l}$, Lawrence Woltz ${ }^{2}$, Jodi Gilman ${ }^{l}$, 
Megan Davis ${ }^{\text {, Daniel Hommer }}$, ' Section of Brain Electrophysiology and Imaging, Laboratory of Clinical Studies, NIH, Bethesda, USA, ${ }^{2}$ Synergy Research Inc., Monrovia, USA

Modulation of the fractal properties of low frequency endogenous brain oscillations in functional MRI by a working memory task., Anna Barnes ${ }^{1}$, Christian Habeck ${ }^{2}$, Garry Honey, ${ }^{1}$ Alle-Meije Wink ${ }^{3}$, Edward Bullmore ${ }^{l}$, John Sucklingl, ${ }^{1}$ Cambridge University, Cambridge, United Kingdom, ${ }^{2}$ Columbia University, New York, USA, ${ }^{3}$ Cambridge University, Cambridge, United Kingdom, ${ }^{4}$ Imperial College, London, United Kingdom, ${ }^{5}$ Cambridge University, Cambridge, United Kingdom, ${ }^{6}$ Cambridge University, Cambridge, United Kingdom

Reliability of multivariate causality measures for neural data, Esther Florin ${ }^{1,2}$, Joachim Gross ${ }^{3}$, Gereon R. Fink ${ }^{1,2}$, Lars Timmermann ${ }^{2},{ }_{1}^{1}$ Institute of Neuroscience and Biophysics - Medicine, Cognitive Neurology, Research Centre Jülich, Juelich, Germany, ${ }^{2}$ b Department of Neurology, University Hospital Cologne, Cologne, Germany, ${ }^{3}$ Centre for Cognitive Neuroimaging (CCNi), Department of Psychology, University of Glasgow, Glasgow, United Kingdom

A frequency domain approach for understanding brain connectivity from EEG data, Laura Marzetti ${ }^{1,2}$, Cosimo Del Gratta ${ }^{1,2}$, Guido Nolte ${ }^{3},{ }^{1}$ Department of Clinical Sciences and Bioimaging, Gabriele D'Annunzio University, Chieti, Italy, ${ }^{2}$ Institute for Advanced Biomedical Technologies, Gabriele D'Annunzio University Foundation, Chieti, Italy, ${ }^{3}$ Fraunhofer FIRST.IDA, Berlin, Germany

Exploring changes in phase of EEG oscillations with tests on complex valued time-frequency representations, Eduardo Martínez-Montes, Pedro A. Valdés-Sosa, Cuban Neuroscience Center, Havana, Cuba

\section{'REAL-TIME' IMAGING OF CARDIOVASCULAR CONTROL IN HUMAN SUBJECTS: CONCURRENT RECORDING OF SPONTANEOUS MUSCLE SYMPATHETIC NERVE ACTIVITY AND SPONTANEOUS FLUCTUATIONS IN BRAINSTEM IMRI SIGNAL} INTENSITY, Vaughan Macefield ${ }^{1,2}$, Luke Henderson ${ }^{3},{ }^{1}$ School of Medicine, University of Western Sydney, Sydney, Australia, ${ }^{2}$ Prince of Wales Medical Research Institute, Sydney, Australia, ${ }^{3}$ Department of Anatomy \& Histology, University of Sydney, Sydney, Australia

\section{MOTOR BEHAVIOR}

Eye Movements/Visuomotor Processing

Cerebral Representations of Space and Time, Martijn Beudel ${ }^{1,2}$, Remko Renken $^{2}$, Klaus Leenders $^{l}$, Bauke de Jong ${ }^{1,2}$, ' dept. Neurology, University Medical Center Groningen, Groningen, Netherlands, ${ }^{2} 2$. BCN Neuroimaging Center, University of Groningen, Groningen, Netherlands

Modulation of BOLD activations of the Smooth Pursuit Eye Movement network as a function of the amount of background dots, Sabine Ohlendorf ${ }^{1,3}$, Andreas Sprenger ${ }^{2}$, Oliver Speck ${ }^{4}$, Volkmar Glauche ${ }^{1}$, Sven Haller ${ }^{5}$, Hubert Kimmig ${ }^{2},{ }^{1}$ Neurologische Universitätsklinik Freiburg, Freiburg, Germany, ${ }^{2}$ Klinik für Neurologie, Universitätsklinikum Schleswig Holstein, Campus Lübeck, Lübeck, Germany, ${ }^{3}$ Abteilung Röntgendiagnostik, Medizin Physik, Universitätsklinikum Freiburg, Freiburg, Germany, ${ }^{4}$ Abteilung Biomedizinische Magnetresonanz, Institut für Experimentelle Physik, Universität Magdeburg, Magdeburg, Germany, ${ }^{5}$ Abteilung für Neuroradiologie, Universitätsspital Basel, Basel, Switzerland

Differential Frontal Controls during Eye Tracking of Visible and Occluded Moving Targets: Simultaneous fMRI and Eye-Movement Recording, Jinhong Ding ${ }^{1,2}$, David Powell ${ }^{3}$, Yang Jiang ${ }^{2}$, ${ }^{1}$ Psychology Dept., Capital Normal University, Beijing, China, ${ }^{2}$ Behavioral Science Dept., University of Kentucky, Lexington, USA, ${ }^{3}$ Magnetic Resonance Imaging and Spectroscopy Center, University of Kentucky, Lexington, USA

\section{NEUROANATOMY}

\section{DTI Studies, Application}

Using multimodal imaging to investigate the structure-function relationship of a sensorimotor cortical U-fiber, Kristi Clark ${ }^{1,2}$, Kenichi Oishi ${ }^{2}$, Roger Woods ${ }^{3}$, Susumu Mori ${ }^{2}$, Arthur Toga ${ }^{1}$, ${ }^{I}$ Laboratory of Neuro Imaging, UCLA, Los Angeles, USA, ${ }^{2}$ Laboratory of Brain Anatomical 
MRI, Johns Hopkins University, Baltimore, USA, ${ }^{3}$ Brain Mapping Center, UCLA, Los Angeles, USA

Remediation-related neuroplasticity of left hemisphere white matter among poor readers: A longitudinal diffusion tensor imaging study, Timothy A. Keller, Ann Meyler, Vladimir L. Cherkassky, Marcel Adam Just, Center for Cognitive Brain Imaging, Department of Psychology, Carrnegie Mellon University, Pittsburgh, USA

Frontal-Limbic White Matter Pathway Differences Associated with Genetic Risk for Major Depressive Disorder, Jennifer Pacheco ${ }^{1,2}$, Christopher Beevers ${ }^{1}$, Cristina Benavides ${ }^{1}$, John McGeary ${ }^{3,4}$, Mithra Sathishkumar ${ }^{2}$, David M. Schnyer ${ }^{1,2}$, ${ }^{I}$ Department of Psychology, The University of Texas at Austin, Austin, USA, ${ }^{2}$ Imaging Research Center, The University of Texas at Austin, Austin, USA, ${ }^{3}$ Research Service, Providence VA Medical Center, Providence, USA, ${ }^{4}$ Center for Alcohol and Addiction Studies, Brown University, Providence, USA

Relating connectional architecture to grey matter function in the human lateral premotor cortex using functional and diffusion imaging, Valentina Tomassini ${ }^{l}$, Saad Jbabdi ${ }^{l}$, Jan Scholz ${ }^{1}$, Tim Behrens ${ }^{1,2}$, Paul M Matthews ${ }^{1}$, Matthew Rushworth ${ }^{1,2}$, Heidi Johansen-Berg ${ }^{l},{ }^{1}$ FMRIB Centre, University of Oxford, Oxford, United Kingdom, ${ }^{2}$ Dept of Experimental Psychology, University of Oxford, Oxford, United Kingdom

Correlation of White Matter Integrity measured by DTI with Intelligence, Personality, and Creativity in Healthy Subjects., Arvind Caprihan, Ranee Barrow, Robert Chavez, H. Jeremy Bockholt, Rex E. Jung, MIND Research Network, Albuquerque, USA

Cortical Connections of Human Inferior Parietal Area PF: Probabilistic Cytoarchitectonic Mapping and Diffusion Tensor Tractography Show a Similar Structural Organization as Compared to Macaques, Stefan Geyer ${ }^{1,3}$, Simon B. Eickhoff, Karl Zilles ${ }^{1,2},{ }^{1}$ C. and O. Vogt Brain Research Institute, Univ. Duesseldorf, Duesseldorf, Germany, ${ }^{2}$ Institute of Neurosciences and Biophysics - Medicine, Research Center, Juelich, Germany, ${ }^{3}$ Max Planck Institute for Human Cognitive and Brain Sciences, Leipzig, Germany

Whole-Brain Analysis of Fractional Anisotropy in Fetal Alcohol Syndrome Using Tract-based Spatial Statistics, Longchuan $\mathrm{Li}^{1}$, Claire Coles ${ }^{2}$, Mary Ellen ${ }^{2}$, Zhihao Li ${ }^{l}$, Mingguo Qiu ${ }^{l}$, Xiaoping $\mathrm{Hu}^{l},{ }^{1}$ Biomedical Imaging Technology Center, Emory University/Georgia Institute of Technology, Atlanta, USA, ${ }^{2}$ Department of Psychiatry and Behavioral Sciences, Emory University, Atlanta, USA

Mapping the Structural Core of Human Cerebral Cortex, Olaf Sporns ${ }^{1}$, Leila Cammoun ${ }^{2}$, Xavier Gigandet ${ }^{2}$, Reto Meuli ${ }^{3}$, Christopher Honey ${ }^{1}$, Patric Hagmann ${ }^{3},{ }^{1}$ Department of Psychological and Brain Sciences, Indiana University, Bloomington, USA, ${ }^{2}$ Signal Processing Institute, Ecole Polytechnique Federale de Lausanne, Lausanne, Switzerland, ${ }^{3}$ Department of Radiology, University Hospital Center and University of Lausanne, Lausanne, Switzerland

Age Related Changes of Human Brains suing Magnetic Resonance Hybrid Diffusion Imaging, $Y u$ Chien Wu $u^{l, 2}$, Frances B. Haeberli, ${ }^{2}$, Yi-Min Huang ${ }^{6}$, Aaron S. Field ${ }^{1,3}$, Andrew L. Alexander ${ }^{2,4,5}$, ${ }^{1}$ Department of Radiology, University of Wisconsin-Madison, Madison, USA, ${ }^{2}$ Waisman Laboratory for Brain Imaging and Behavior, University of Wisconsin-Madison, Madison, USA, ${ }^{3}$ Department of Biomedical Engineering, University of Wisconsin-Madison, Madison, USA, ${ }^{4}$ Department of Medical Physics, University of Wisconsin-Madison, Madison, USA, ${ }^{5}$ Department of Psychiatry, University of Wisconsin-Madison, Madison, USA, ${ }^{6}$ Department of Physics, Madison, USA

\section{SENSORY SYSTEMS \\ Auditory/Vestibular}

Patterns of local gamma activity over the human superior temporal gyrus suggested the presence of FM-selective processing areas, Paul Poon ${ }^{1,2}$, John Brugge ${ }^{2}$, Hiroyuki Oya ${ }^{2}$, Richard Reale ${ }^{2}$, Hiroto Kawasaki ${ }^{2}$, Kirill Nourski ${ }^{2}$, Matthew Howard $\mathrm{III}^{2},{ }^{1}$ Dept Physiology, NCKU, Tainan, Taiwan, ${ }^{2}$ Dept Neurosurgery, Univ of Iowa, Iowa City, USA

Sound-induced activation of vestibular cortex: Electrical neuroimaging during vestibular evoked myogenic potentials, Pär Halje, Christophe Lopez, Olaf Blanke, Laboratory of Cognitive Neuroscience, Brain Mind Institute, Ecole Polytechnique Federale de Lausanne, Lausanne, Switzerland 
Multimodal Functional Imaging of Loss of Consciousness Under Propofol Anesthesia with Simultaneous EEG, fMRI, and 40-Hz ASSR, Patrick Purdon ${ }^{1,2,6}$, Eric Pierce ${ }^{l}$, Giorgio Bonmassar ${ }^{2,3}$, John Walsh ${ }^{l}$, Grace Harrell ${ }^{l}$, Jean Kwo ${ }^{I}$, Daniel Deschler ${ }^{8}$, Catherine Mullaly, Margaret Barlow ${ }^{4}$, Rebecca Merhar ${ }^{1}$, Camilo Lamus ${ }^{6}$, Sharon Maginnis ${ }^{5}$, Debra Skoniecki ${ }^{5}$, Mary Sullivan ${ }^{5}$, Helen-Anne Higgins, ${ }^{5}$ Emery Brown, ${ }^{1,6,7},{ }^{1}$ Mass Gen Hospital Dept Anesthesia \& Critical Care, Boston, USA,

${ }^{2}$ Martinos Ctr. Biomed. Imaging, Charlestown, USA, ${ }^{3}$ Mass Gen Hospital Dept Radiology, Boston, USA,

${ }^{4}$ Mass Gen Hospital Dept Neurology, Boston, USA, ${ }^{5}$ Mass Gen Hospital GCRC, Boston, USA, ${ }^{6}$ MIT Dept Brain Cog Sci, Cambridge, USA, ${ }^{7}$ Harvard/MIT Division Health Sci \& Technology, Cambridge, USA,

${ }^{8}$ Mass Eye Ear Infirmary, Boston, USA

\section{SENSORY SYSTEMS}

Tactile/Somatosensory

Brain white matter differences in lower limb amputees, a Diffusion Tensor Imaging study, Sarael Alcauter $^{1,2}$, Erick H Pasaye ${ }^{2,3}$, Perla M Salgado ${ }^{3}$, Maria del Refugio Pacheco ${ }^{4}$, Maria De Iturbe ${ }^{3}$, Fernando A Barrios ${ }^{5},{ }^{1}$ Instituto Nacional de Psiquiatria INPRF, Mexico DF, Mexico, ${ }^{2}$ Posgrado en Ciencias Biomedicas, UNAM, Mexico DF, Mexico, ${ }^{3}$ Instituto Nacional de Neurologia y Neurocirugía MVS, Mexico DF, Mexico, ${ }^{4}$ Instituto Nacional de Rehabilitacion, Mexico DF, Mexico, ${ }^{5}$ Instituto de Neurobiologia, Universidad Nacional Autónoma de México, Queretaro, Mexico

An fMRI Study of Head Massage Reveals Activity in the Brain's Reward Centres, Lisan Ho ${ }^{l}$, Laura MParkes ${ }^{l}$, Richard L Evans ${ }^{2}$, Neil Roberts ${ }^{1}$, Francis McGlone ${ }^{2},{ }^{I}$ The Magnetic Resonance and Image Analysis Research Centre (MARIARC), University of Liverpool, Liverpool, United Kingdom, ${ }^{2}$ Unilever Research Ltd, Wirral, United Kingdom

A somatotopical relationship between cortical activity and reflexological stimulation: an fMRI study, Tomomi Nakamaru ${ }^{1,2}$, Naoki Miura ${ }^{3,2}$, Ai Fukushima ${ }^{2}$, Ryuta Kawashima ${ }^{2},{ }^{1}$ Tohoku University School of Medicine, 4-1 Seiryo-cho, Aoba-ku, Sendai, Miyagi, Japan, ${ }^{2}$ Department of Functional Brain Imaging, Institute of Development, Aging and Cancer (IDAC), Tohoku University, 4-1 Seiryo-cho, Aoba-ku, Sendai, Miyagi, Japan, ${ }^{3}$ Department of Intelligence Mechanical Systems Engineering, Kochi University of Technology, 185 Miyanokuchi, Kami, Kochi, Japan

Behavioral correlates of negative BOLD signal changes in the primary somatosensory cortex, Jürgen Baudewig ${ }^{1}$, Andreas Kastrup ${ }^{2}$, Sonja Schnaudigel ${ }^{2}$, Lars Becker ${ }^{2}$, Jan Martin Sohns ${ }^{2}$, Peter Dechent ${ }^{l},{ }^{1}$ MR-Research in Neurology and Psychiatry, University Medical Center, Göttingen, Germany, ${ }^{2}$ Department of Neurology, University Medical Center, Göttingen, Germany

Multimodal neuroimaging of somatosensory cortex during somatotopic air-puff stimulation, RueySong Huang ${ }^{1,2}$, Tzyy-Ping Jung ${ }^{I}$, Rey Ramirez ${ }^{I}$, Zeynep Akalin-Acar ${ }^{I}$, Martin Sereno ${ }^{2}$, Scott Makeig ${ }^{I}$, ${ }^{I}$ Swartz Center for Computational Neuroscience, Institute for Neural Computation, University of California, San Diego, La Jolla, USA, ${ }^{2}$ Department of Cognitive Science, University of California, San Diego, La Jolla, USA

Finger representations in areas $3 \mathrm{~b}$ and 1 of human primary somatosensory cortex as revealed by functional MRI of tactile stimulation, Renate Schweizer, Jens Frahm, Biomedizinische NMR Forschungs GmbH am Max-Planck-Institut für biophysikalische Chemie, Goettingen, Germany

\section{SENSORY SYSTEMS}

Vision

Evidence of two alpha rhythm systems in the human brain: a combined EEG/fMRI study, Eti Ben Simon $^{1,2}$, Ilana Podlipsky ${ }^{I}$, Andrey Zhdanov ${ }^{I}$, Talma Hendler ${ }^{1,2,3},{ }^{l}$ Functional Brain Center, Wohl Institute for Advanced Imaging, Tel Aviv Sourasky Medical Center, Tel Aviv, Israel, ${ }^{2} 2$ Sackler Faculty of Medicine, Tel Aviv University, Tel Aviv, Israel, ${ }^{3}$ Psychology department,Tel Aviv University, Tel Aviv, Israel

fMRI of chromatic and achromatic responses in human visual areas: Specializations for spatial $\&$ temporal frequency, Dany V. D'Souza ${ }^{I}$, Barry B. Lee ${ }^{2}$, Jens Frahm ${ }^{l},{ }^{l}$ Biomedizinische NMR Forschungs GmbH am Max-Planck-Institut für biophysikalische Chemie, Goettingen, Germany, ${ }^{2}$ State University of New York, School of Optometry, New York, USA

Cortical Network for Coherent Stereomotion in the Human Brain, Lora Likova, The Smith-Kettlewell Eye Research Institute, San Francisco, USA 
Coincident Visual Retinotopy in simultaneous Slow cortical potentials and fMRI recordings, Hugo Sandoval ${ }^{1,2}$, Stephen Sands ${ }^{1,2}$, J. Andrew Sands ${ }^{1}$, George R. Mangun ${ }^{3}$, Cameron Carter ${ }^{3}$, Joy Geng ${ }^{3}$, ${ }^{l}$ Sands Research, El Paso, USA, ${ }^{2}$ University of Texas, El Paso, USA, ${ }^{3}$ UC Davis, Davis, USA

Volumetric Analysis of the Optic Chiasm in Early-blind Patients., Flemming Andersen ${ }^{l}$, Ron Kupers ${ }^{l}$, Fabien Schneider ${ }^{2}$, Maurice Ptito ${ }^{3},{ }^{1}$ PET Unit, Copenhagen University Hospital, Copenhagen, Denmark, ${ }^{2}$ University of Saint-Etienne, Saint-Etienne, France, ${ }^{3}$ University of Montreal, Montreal, 719 W-AM Canada

ORIENTATION-SPECIFIC CONTEXTUAL MODULATION IN HUMAN VISUAL CORTEX, $J$. Scott McDonald ${ }^{l}$, Kiley Seymour ${ }^{1}$, Mark Schira ${ }^{2}$, Branka Spehar ${ }^{2}$, Colin Clifford ${ }^{l},{ }^{1}$ University of Sydney, Sydney, Australia, ${ }^{2}$ UNSW, Sydney, Australia

Neural correlates of detection and identification of human bodies, Amra Hodzic ${ }^{1,2}$, Amanda Kaas ${ }^{1,3}$, Wolf Singer ', Aglaja Stirn ${ }^{4},{ }^{1}$ Max Planck Institut for Brain Research, Frankfurt am Main, Germany, ${ }^{2}$ Graduate School of Neural and Behavioural Sciences IMPRS, Tübingen, Germany, ${ }^{3}$ Department of Cognitive Neuroscience, University of Maastricht, Maastricht, Netherlands, ${ }^{4}$ Johann Wolfgang Goethe University Clinic, Frankfurt am Main, Germany

Functional decoupling of BOLD and gamma band amplitudes in human visual cortex, Suresh Muthukumaraswamy, Krish Singh, CUBRIC, Cardiff University, Cardiff, United Kingdom

MEG and fMRI studies on the neural basis of global form perception in Glass pattern stimuli., Jennifer B. Swettenham ${ }^{1}$, Stephen J. Anderson ${ }^{2}$, Ngoc J. Thai ${ }^{2},{ }^{l}$ CUBRIC, School of Psychology, Cardiff University, Cardiff, United Kingdom, ${ }^{2}$ The Wellcome Trust Laboratory for MEG Studies, School of Life and Health Sciences, Aston University, Birmingham, United Kingdom

MEMORY \& LEARNING

Long-term Memory (episodic, semantic, autobiographical)

Dissociating Regional Changes in Prefrontal Cortex Structure and Function that Impact Memory Performance during Normal Aging, Luc Valiquette ${ }^{1}$, Rafael Languay ${ }^{1}$, Sidney Pinto ${ }^{1}$, Cheryl Grady ${ }^{2}$, Jens Pruessner ${ }^{l}$, Maria N. Rajah ${ }^{I},{ }^{l}$ McGill University and Douglas Mental Health Univ. Inst., Montreal, QC, Canada ${ }^{2}$ U. of Toronto \& Rotman Research Inst., Toronto, ON, Canada

\section{COGNITION \& ATTENTION Executive Function}

Transiently disrupting right prefrontal cortex interferes with updating of working memory, Neir Eshel, Joseph Luka, Agatha Lenartowicz, Leigh E. Nystrom, Jonathan D. Cohen, Princeton University, Princeton, USA

How positive and negative smells influence cognitive interference processes, Martina Reske ${ }^{1,2}$, Thilo Kellermann $^{2}$, Andreas Finkelmeyer ${ }^{2}$, Thomas Niessen ${ }^{2}$, Michael Schwenzer ${ }^{2}$, Klaus Mathiak ${ }^{2,3}$,

${ }^{1}$ University of California San Diego, Laboratory of Biological Dynamics and Theoretical Medicine, La Jolla, USA, ${ }^{2}$ RWTH Aachen University, Department of Psychiatry, Aachen, Germany, ${ }^{3}$ King's College, Institute of Psychiatry, London, United Kingdom

Modafinil modulates activity in brain regions underlying attentional control in healthy subjects, Beth Stankevich, Roberta Rasetti, Fabio Sambataro, Giuseppe Blasi, Kelsey Skjei, Guilna Alce, Jose Apud, Daniel Weinberger, Venkata Mattay, Clinical Brain Disorders Branch: Genes, Cognition, and Psychosis Program, NIMH, NIH, Bethesda, USA

Comparison of putative default networks in macaque and human cerebral cortex, David Van Essen ${ }^{1}$, Justin Vincent ${ }^{2}$, Avi Snyder ${ }^{1}$, Marcus Raichle ${ }^{I},{ }^{1}$ Washington University, St. Louis, USA, ${ }^{2}$ Harvard University, Cambridge, USA

Activation of self-knowledge reduces conflict during occupational choice: An ERP study., Takashi Nakao, Makoto Miyatani, Akane Okamoto, Kaori Katayama, Mayo Mitsumoto, Yu Watanabe, Hiroshima 18 W-PM Universit, Higashi-Hiroshima, Japan 
CNV resolution does not cause the $\mathbf{N} 2$ and $\mathbf{P} 3$ Go/NoGo effects, Janette Smith ${ }^{1,2}$, Robert Barry ${ }^{2}$, Stuart Johnstone ${ }^{2},{ }^{1}$ University of Newcastle, Newcastle, Australia, ${ }^{2}$ University of Wollongong, Wollongong, Australia

Brain network dynamics during working memory task events in relation to COMT Val(158)Met, Hao-Yang Tan, Qiang Chen, Rachel Higier, Laura Libby, Morgan Prust, Venkata Mattay, Daniel Weinberger, Joseph Callicott, Clinical Brain Disorders Branch, NIMH, NIH, Bethesda, USA

Multi-voxel coding of stimulus-response mapping rules in human frontal and parietal cortex, Alexandra Woolgar, John Duncan, Medical Research Council - Cognition and Brain Sciences Unit, Cambridge, United Kingdom

Transient modulation of intrinsic network connectivity during working memory, Catie Chang ${ }^{l}$, Gary H. Glover ${ }^{1,2,3}$, Moriah E. Thomason ${ }^{3}$, Michael D. Greicius ${ }^{4}$, Vinod Menon ${ }^{5,6},{ }^{1}$ Dept. of Electrical Engineering, Stanford University, Stanford, USA, ${ }^{2}$ Dept. of Radiology, Stanford University, Stanford, USA, ${ }^{3}$ Dept. of Psychology, Stanford University, Stanford, USA, ${ }^{4}$ Dept. of Neurology, Stanford University School of Medicine, Stanford, USA, ${ }^{5}$ Dept. of Psychiatry and Behavioral Sciences, Stanford University School of Medicine, Stanford, USA, ${ }^{6}$ Program in Neuroscience, Stanford, USA

A Longitudinal Investigation of Developmental Changes in Response Inhibition in Early Adolescence, Frances Haeberli, ${ }^{1,2}$, John Ollinger ${ }^{l, 2}$, Dan Kelley ${ }^{l, 2}$, Tom Johnstone ${ }^{3}$, Andrew Alexander ${ }^{1,2},{ }^{1}$ University of Wisconsin - Madison, Madison, USA, ${ }^{2}$ Waisman Center: Lab for Brain Imaging and Behavior, Madison, USA, ${ }^{3}$ Bristol University, Bristol, United Kingdom

Tracing the neural correlates of the $\mathbf{N 2 0 0}$ in a tactile stop-signal task: predominance of the dACC?, Rene Huster ${ }^{1,2,3}$, Rene Westerhausen ${ }^{4}$, Arne Wittling ${ }^{1}$, Werner Wittling ${ }^{1}$, Elisabeth Schweiger ${ }^{1}$, Christo Pantev ${ }^{2},{ }^{I}$ Center for Neuropsychological Research, Trier, Germany, ${ }^{2}$ Institute for Biomagnetism and Biosignalanalysis, Münster, Germany, ${ }^{3}$ Department of Psychiatry and Psychotherapy and Interdisciplinary Center for Clinical Research (IZKF), Münster, Germany, ${ }^{4}$ Cognitive NeuroScience Group, Department of Biological and Medical Psychology, Bergen, Norway

Freely Generating Task Goals and Delayed Intention, Sara L Bengtsson ${ }^{1}$, John-Dylan Haynes ${ }^{2}$, Katsuyuki Sakai ${ }^{3}$, Richard E Passingham ${ }^{4},{ }^{1}$ Wellcome Centre for NeuroImaging, London, United Kingdom, ${ }^{2}$ Max Planck Institute for Human Cognitive and Brain Sciences, Leipzig, Germany, ${ }^{3}$ Department of Cognitive Neuroscience, Univ of Tokyo, Tokyo, Japan, ${ }^{4}$ Department of Experimental Psychology, Oxford, United Kingdom

Changes of EEG Spectra from Alertness to Drowsiness in a Driving Simulator, Sheng-Fu Liang ${ }^{I}$, Chin-Teng Lin ${ }^{2,3}$, Jong-Liang Jeng ${ }^{2}$, Tzai-Wen Chiu ${ }^{2}$, Li-Wei Ko ${ }^{2}$, Ruey-Song Huang ${ }^{2,4}$, Tzyy-Ping Jung ${ }^{2,4}$, Jeng-Ren Duann ${ }^{2,4}$, ${ }^{1}$ Department of Computer Science and Information Engineering, National Cheng-Kung University, Tainan, Taiwan, ${ }^{2}$ Brain Research Center, University System of Taiwan, Hsinchu, Taiwan, ${ }^{3}$ Department of Electrical and Control Engineering, National Chiao-Tung University, Hsinchu, Taiwan, ${ }^{4}$ Institute for Neural Computation, University of California, San Diego, USA

Performance monitoring dysfunction in cannabis users: evidence of anterior cingulate and prefrontal hypoactivity associated with reduced error awareness, Robert Hester ${ }^{\text {, }}$, Liam Nestor ${ }^{2}$, Hugh Garavan ${ }^{2},{ }^{1}$ Queensland Brain Institute and School of Psychology, University of Queensland, St Lucia, Australia, ${ }^{2}$ School of Psychology and Trinity College Institute for Neuroscience, Trinity College Dublin, Dublin, Ireland

Identifying components of task-set reconfiguration using ERP and BESA., Elise Mansfield ${ }^{l}$, Frini Karayanidis ${ }^{1,2,3}$, Kasey Galloway ${ }^{l}$, Janette Smith ${ }^{1,3},{ }^{1}$ Functional Neuroimaging Laboratory, Newcastle, Australia, ${ }^{2}$ Schizophrenia Research Institute, Sydney, Australia, ${ }^{3}$ Hunter Medical Research Institute, Newcastle, Australia

\section{COGNITION \& ATTENTION}

Perception, Imagery, Awareness

Dopaminergic neurotransmission plays a causal role in conscious awareness, Joshua Skewes ${ }^{l}$, Hans

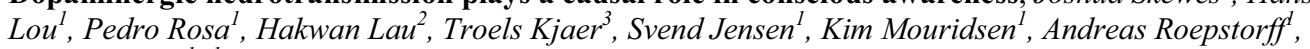
Albert Gjedde ${ }^{I},{ }^{l}$ Center for Functionally Integrative Neuroscience, Aarhus University Hospitals, Aarhus, 62 W-PM Denmark, ${ }^{2}$ Department of Psychology, Columbia University, Manhatten, USA, ${ }^{3}$ Department of Clinical Neurophysiology, Copenhagen University Hospital, Copenhagen, Denmark 
Neural correlates of change detection: how do we tell when a face is different?, Eva Loth ${ }^{l}$, Rik Henson $^{2}$, Andy Calder ${ }^{2}$, Jason Taylor ${ }^{2}$, Sonia Bishop ${ }^{1,2},{ }^{1}$ University of Cambridge, Cambridge, United Kingdom, ${ }^{2}$ MRC CBU, Cambridge, United Kingdom

Local activity patterns in high-level visual cortex reliably encode the category of invisible objects, Philipp Sterzer ${ }^{1,2}$, John-Dylan Haynes ${ }^{3}$, Geraint Rees ${ }^{2},{ }^{I}$ Charité, Dept. of Psychiatry, Berlin, Germany, ${ }^{2}$ University College London, London, United Kingdom, ${ }^{3}$ Bernstein Center for Computational Neuroscience, Berlin, Germany

Location-Invariant Object Information in Foveal Retinotopic Cortex, Mark Williams ${ }^{1,2}$, Chris Baker $^{3}$, Hans Op de Beeck ${ }^{4}$, Sabin Dang ${ }^{1}$, Christina Triantafyllou ${ }^{l}$, Nancy Kanwisher ${ }^{l},{ }^{1}$ MIT, Cambridge, USA, ${ }^{2}$ Macquarie University, Sydney, Australia, ${ }^{3}$ National Institute of Mental Health, Bethesda, USA, ${ }^{4}$ University of Leuven, Leuven, Belgium

Input-specific potentiation in sensory-induced cortical plasticity, Nicolas McNair ${ }^{1}$, Wes Clapp ${ }^{2}$, Jeff Hamm $^{1}$, Tim Teyler ${ }^{3,4}$, Michael Corballis ${ }^{I}$, Ian Kirk ${ }^{l},{ }^{1}$ University of Auckland, Auckland, New Zealand, ${ }^{2}$ University of California, San Francisco, San Francisco, USA, ${ }^{3}$ University of Idaho, Moscow, USA,

${ }^{4}$ Washington State University, Pullman, USA

EEG activity relating to expertise; rapid, knowledge-guided perception of shogi (a Japanese version of chess) piece positions, Hironori Nakatani, Yoko Yamaguchi, Laboratory for Dynamics of Emergent Intelligence, RIKEN Brain Science Institute, Wako, Japan

Different brain activation during perceptual transitions in ambiguous figure associates with perception of binocular rivalry, Chia-Li Liu, National Taiwan University, Taipei, Taiwan

78 W-PM

'Brain Reading' with Real-Time fMRI: Communication via detection of brain states in the absence of motor response, Martin Monti ${ }^{1}$, Martin Coleman ${ }^{2}$, Adrian Owen ${ }^{l},{ }^{1}$ MRC Cognition and Brain Sciences Unit, Cambridge, United Kingdom, ${ }^{2}$ Wolfson Brain Imaging Center, Addenbrookes Hospital, Cambridge, United Kingdom

\section{DISORDERS OF THE NERVOUS SYSTEM Addiction}

Investigating white matter microstructure in opiate addiction, obsessive compulsive disorder and healthy controls, Murat Yücel ${ }^{l, 2}$, Emre Bora ${ }^{l}$, Alex Fornito ${ }^{l}$, Ben Harrison ${ }^{l, 3}$, Marc Seal ${ }^{l}$, Christos Pantelis ${ }^{I}$, Dan Lubman ${ }^{2},{ }^{\prime}$ Melbourne Neuropsychiatry Centre, Department of Psychiatry, University of Melbourne, Melbourne, Australia, ${ }^{2}$ ORYGEN Research Centre, Department of Psychiatry, University of Melbourne, Melbourne, Australia, ${ }^{3}$ Institut d'Alta Tecnologia-PRBB, CRC Corporació Sanitària, Barcelona, Spain

\section{DISORDERS OF THE NERVOUS SYSTEM} Autism

Power Spectral Changes of Resting-State BOLD Signal in Children with Autism Spectrum Disorder, Jukka Remes ${ }^{1,2}$, Tuomo Starck ${ }^{1}$, Jyri-Johan Paakki ${ }^{l}$, Juha Nikkinen ${ }^{1}$, Sanna Kuusikko ${ }^{3}$, Hanna Ebeling $^{3}$, Jukka Rahko ${ }^{3}$, Katja Jussila ${ }^{3}$, Marja-Leena Mattila ${ }^{3}$, Marianne Haapea ${ }^{1}$, Koen van Leemput ${ }^{4,5}$, Irma Moilanen ${ }^{3}$, Osmo Tervonen ${ }^{1}$, Olli Silven ${ }^{2}$, Vesa Kiviniemi ${ }^{I},{ }^{1}$ Department of Diagnostic Radiology, Oulu University Hospital, Oulu, Finland, ${ }^{2}$ Department of Electrical and Information Engineering, University of Oulu, Oulu, Finland, ${ }^{3}$ Department of Child Psychiatry, Oulu University Hospital, Oulu, Finland, ${ }^{4}$ Martinos Center for Biomedical Imaging, Massachusetts General Hospital, Harvard Medical School, Charlestown, USA, ${ }^{5}$ Computer Science and Artificial Intelligence Laboratory, Massachusetts Institute of Technology, Cambridge, USA

\section{DISORDERS OF THE NERVOUS SYSTEM Brain \& Spinal Cord Trauma}

The role of resting state fMRI in Persistent Vegetative State treated with cerebral cortical stimulation, Barbara Massa Micon ${ }^{1,3}$, Franco Cauda $^{2,4}$, Katiuscia Sacco, ${ }^{2,4}$, Elisa Montanaro ${ }^{2}$, Federico D'Agata ${ }^{2,4}$, Sergio Duca $^{4}$, Giuliano Geminiani ${ }^{2,4}$, Antonio Melcarne ${ }^{3}$, Sergio Canavero ${ }^{1},{ }^{\prime}$ Turin Advanced Neuromodulation Group, Torino, Italy, ${ }^{2}$ Department of Psychology, University of Turin, Torino, Italy, ${ }^{3}$ Department of 106 W-PM Neurosurgery, CTO Hospital, Torino, Italy, ${ }^{4}$ CCS MMRI, Koelliker Hospital, Torino, Italy

Diffuse Axonal Injury due to Traumatic Brain Injury Alters Inhibition of Imitative Response Tendencies, Barbara Ettrich ${ }^{I}$, Rainer Scheid ${ }^{l, 2}$, D. Yves von Cramon ${ }^{1,2}$, Matthias Schroeter ${ }^{1,2}$, 
${ }^{1}$ Max-Planck-Institute for Human Cognitive and Brain Sciences, Leipzig, Germany, ${ }^{2}$ Day Clinic of Cognitive Neurology, University of Leipzig, Leipzig, Germany

Impaired Functional Connectivity in Traumatic Brain Injury: An MEG Study, Pratik Mukherjee ${ }^{I}$, Anne Findlay $^{1}$, Hana Lee ${ }^{2}$, Adrian Guggisberg ${ }^{1}$, Susanne Honmal, Michele Meeker ${ }^{2}$, Geoffrey Manley ${ }^{2}$, Srikantan Nagarajan ${ }^{l},{ }^{1}$ Radiology, UCSF, San Francisco, USA, ${ }^{2}$ Neurosurgery, UCSF, San Francisco, USA

\section{DISORDERS OF THE NERVOUS SYSTEM Developmental Disorders}

Connectivity analysis of brain function in control children and children with fetal alcohol spectrum disorder (FASD) during number processing, Robyn Herron ${ }^{l}$, Ernesta Meintjes ${ }^{l}$, Sandra Jacobson $^{2}$, Christopher Molteno ${ }^{3}$, Eric Murphy ${ }^{2}$, Vaibhav Diwadkar ${ }^{2}$, John Gore ${ }^{4}$, Joseph Jacobson ${ }^{2}$, Baxter Rogers ${ }^{4},{ }^{1}$ Department of Human Biology, Faculty of Health Sciences, University of Cape Town, Cape Town, South Africa, ${ }^{2}$ Department of Psychiatry and Behavioural Neurosciences, Wayne State University School of Medicine, Detroit, USA, ${ }^{3}$ Department of Psychiatry, Faculty of Health Sciences, University of Cape Town, Cape Town, South Africa, ${ }^{4}$ Vanderbilt University Institute of Imaging Science, Vanderbilt University, Nashville, USA

Right Inferior Prefrontal Cortex is activated during Response Inhibition in Healthy Controls but not in Children with Fetal Alcohol Spectrum Disorder (FASD), Ernesta Meintjes ${ }^{1}$, Sandra Jacobson ${ }^{2}$, Christopher Molteno ${ }^{1}$, J Christopher Gatenby $^{3}$, Christopher Warton ${ }^{1}$, Christopher Cannistraci ${ }^{3},{ }^{2}$ ohn Gore ${ }^{3}$, Joseph Jacobson ${ }^{2},{ }^{1}$ University of Cape Town, Cape Town, South Africa, ${ }^{2}$ Wayne State University, Detroit, USA, ${ }^{3}$ Vanderbilt University, Nashville, USA

Volumetric reduction of normal appearing cortex in patients with polymicrogyria detected by cortical surface analysis, Pedro P M Oliveira, Claudia C Leite, Edson Amaro, NIF - LIM-44 - InRad Faculdade de Medicina - Universidade de São Paulo, Sao Paulo, Brazil

Functional integrity of malformed cortex: an fMRI study, Florian Koppelstaetter ${ }^{1,2}$, Giorgi Kuchukhidze ${ }^{3}$, Iris Unterberger ${ }^{3}$, Judith Dobesberger ${ }^{3}$, Norbert Embacher ${ }^{3}$, Gerald Walser ${ }^{3}$, Thaddaeus Gotwald $^{l}$, Christian Siedentopf ${ }^{,, 2}$, Stephan Felber ${ }^{4}$, Anja Ischebeck ${ }^{2,3}$, Werner Jaschke ${ }^{l}$, Eugen Trinka ${ }^{3}$, ${ }^{1}$ Department of Radiology, Medical University Innsbruck, Innsbruck, Austria, ${ }^{2}$ fMRI-Lab, Department of Psychiatry, Medical University Innsbruck, Innsbruck, Austria, ${ }^{3}$ Department of Neurology, Medical University Innsbruck, Innsbruck, Austria, ${ }^{4}$ Stiftungsklinikum Mittelrhein St.Martin, Koblenz, Germany

Disruption of right-lateralized fronto-striatal functional circuitry in Fragile $\mathbf{X}$ syndrome, Elizabeth Walter, Fumiko Hoeft, Allan Reiss, Department of Psychiatry, Stanford, USA

\section{DISORDERS OF THE NERVOUS SYSTEM Epilepsy}

How different brain pathologies influence language plasticity in the brain: fMRI study., Massimo Caulo, Carlo Sestieri, Chiara Briganti, Francesco De Pasquale, Armando Tartaro, Gian Luca Romani, University of Chieti-Pescara, Chieti, Italy

Spatiotemporal propagation pattern of ictal scalp EEG in mesial temporal lobe epilepsy associated with hippocampal sclerosis, Ki-Young Jung ${ }^{1}$, Soyoung Kwon ${ }^{1}$, Joong-Koo Kang ${ }^{2}$, Ji Hyun Kim ${ }^{1}$, ${ }^{l}$ Department of Neurology, Korea University Medical Center, Korea University College of Medicine, Seoul, South Korea, ${ }^{2}$ Department of Neurology, Asan Medical Center, Ulsan University College of Medicine, Seoul, South Korea

The Neurodynamics of seizure propagation in focal epilepsy, Andre Peterson ${ }^{1,2,3}$, Anthony Burkitt ${ }^{1,2}$, Iven Mareels ${ }^{1}$, David Grayden ${ }^{1,2}$, Mark Cook ${ }^{3}$, Levin Kuhlmann ${ }^{1},{ }^{\prime}$ Department of Electrical \& Electronic Engineering, Melbourne University, Melbourne, Australia, ${ }^{2}$ Bionic Ear Institute, Melbourne, Australia, ${ }^{3}$ St. Vincents Hospital, Melbourne, Australia

Low Frequency Fluctuation Inhibition in BOLD Deactivation Regions Caused by Ictal Epileptic Discharges, Zhiqiang Zhang ${ }^{1}$, Guangming Lu ${ }^{I}$, Lei Tian ${ }^{2}$, Yijun Liu ${ }^{3},{ }^{1}$ Department of Medical Imaging, Clinical School of Nanjing University, Nanjing, China, ${ }^{2}$ Department of Neurosugeryi, Clinical School of Nanjing University, Nanjing, China, ${ }^{3}$ Department of Psychiatry and Neuroscience, University of Florida, Gainesville, USA 
Reorganization of semantic noun processing in right temporal lobe epilepsy, Elizabeth Jensen ${ }^{1,2}$, Daniel Pittman $^{I}$, Kamal Sahi ${ }^{1}$, Bradley Goodyear ${ }^{1,2,3}$, Paolo Federico ${ }^{1,2,3},{ }^{1}$ Hotchkiss Brain Institute, Calgary, Canada, ${ }^{2}$ Department of Clinical Neurosciences, Calgary, Canada, ${ }^{3}$ Department of Radiology, 154 W-PM Calgary, Canada

Ictal SPECT using Attachable Automatic Injector: Prediction of Ictal Onset Zone, Sang Kun Lee ${ }^{l}$, Jung Ju Lee ${ }^{l}$, Jang-Wuk Choi ${ }^{l}$, Kon Chu ${ }^{I}$, Chun-Kee Chung ${ }^{2}$, Dong Soo Lee ${ }^{3},{ }^{I}$ Neurology, Seoul, Korea, ${ }^{2}$ Neurosurgery, Seoul, Korea, ${ }^{3}$ Nuclear Medicine, Seoul, Korea

Simultaneous EEG-fMRI and Functional Connectivity Analysis for Epilepsy Research and Surgical Planning, Nallakkandi Rajeevan ${ }^{l}$, Michira Negishi ${ }^{l}$, E. Fertig ${ }^{2}$, L. Huh ${ }^{2}$, E. Novotney ${ }^{2}$, H. Blumenfeld ${ }^{2}$, Dennis Spencer', Susan Spencer ${ }^{3}$, Todd Constable ${ }^{1,2}$, 'Diagnostic Radiology, Yale University, New Haven, USA, ${ }^{2}$ Neurosurgery, Yale University, New Haven, USA, ${ }^{3}$ Neurology, Yale University, New Haven, USA

\section{DISORDERS OF THE NERVOUS SYSTEM Stroke \& Recovery of Function}

Post-stroke somatosensory impairment inversely correlates with touch discrimination related BOLD signal in contralesional thalamus., Leeanne Carey ${ }^{1,2}$, David Abbott ${ }^{3}$, Matt Harvey ${ }^{1,3}$, Aina Puce ${ }^{1,4}$, Rudiger Seitz ${ }^{1,5}$, I National Stoke Research Institute, Melbourne, Australia, ${ }^{2}$ LaTrobe University, Melbourne, Australia, ${ }^{3}$ Brain Research Institute, Melbourne, Australia, ${ }^{4}$ Center for Advanced Imaging, Morgantown, USA, ${ }^{5}$ University Hospital, Duesseldorf, Germany

Proprioceptive perception in stroke participants with proprioceptive deficits: an fMRI study, Ettie Ben-Shabat ${ }^{1,2}$, Amy Brodtmann ${ }^{2}$, Thomas A Matyas ${ }^{1,2}$, Leeanne M Carey ${ }^{1,2}$, ${ }^{1}$ La Trobe University, Melbourne, Australia, ${ }^{2}$ National Stroke Research Institute, Melbourne, Australia

Functional MRI in comatose survivors of cardiac arrest demonstrates decreased BOLD signal in patients with unfavourable outcome, Teneille Gofton, Bryan Young, Philippe Choiunard, Andrea Dencev, Frank Bihari, Michael Nicolle, Donald Lee, Michael Sharpe, Seyed Mirsattari, University of Western Ontario, London, Canada

Effects of Blood Pressure, Cholesterol and Glucose Levels on White Matter Tissue Structure: Diffusion Tensor Imaging Tract Based Spatial Statistics (TBSS), David Salat ${ }^{1,4}$, Elizabeth Leritz $^{1,2,3,4}$, Regina McGlinchey ${ }^{2,3,4}$, Caroline Chapman ${ }^{1,2}$, James Rudolph ${ }^{2,3,4}$, William Milberg ${ }^{2,3,4}$, ${ }^{l}$ MGH/MIT/HMS Athinoula A. Martinos Center for Biomedical Imaging, Department of Radiology, Massachusetts General Hospital, Boston, USA, ${ }^{2}$ Geriatric Research, Education and Clinical Center (GRECC), Boston VA Healthcare System, Boston, USA, ${ }^{3}$ Division of Aging, Brigham \& Women's Hospital, Boston, USA, ${ }^{4}$ Harvard Medical School, Boston, USA

\section{EMOTION \& MOTIVATION Decision Making}

Neural Substrates underlying Decision-Making in Adolescents, Uma Rao ${ }^{l}$, Anup Bidesi ${ }^{l}$, Monique Ernst ${ }^{2}$, ${ }^{1}$ UT Southwestern Medical Center, Dallas, USA, ${ }^{2}$ National Insitute of Mental Health, Bethesda, USA

Tracking the unchosen option during stochastic choice in a dynamic world, Erie Boorman ${ }^{1,2}$, Timothy Behrens ${ }^{1,2}$, Mark Woolrich ${ }^{2}$, Matthew Rushworth ${ }^{1,2},{ }^{1}$ Department of Experimental Psychology, University of Oxford, Oxford, United Kingdom, ${ }^{2}$ Centre for Functional MRI of the Brain, University of Oxford, Oxford, United Kingdom

Neural Mechanism of Intertemporal Choice: From Discounting Future Gains to Future Losses, Lijuan Xu ${ }^{l}$, Zhu-Yuan Liang ${ }^{2}$, Kun Wang ${ }^{l}$, Shu Li ${ }^{2}$, Tianzi Jiang ${ }^{l},{ }^{l}$ National Laboratory of Pattern Recognition, Institute of Automation, Chinese Academy of Sciences, Beijing, China, ${ }^{2}$ Center for Social and Economic Behavior, Institute of Psychology, Chinese Academy of Sciences, Beijing, China

\section{EMOTION \& MOTIVATION Emotional Learning}

Recruitment of Frontolimbic Circuitry in Reversal and Extinction Learning, Fatima Soliman ${ }^{l}$, Liat Levita ${ }^{l}$, Alex Millner ${ }^{l}$, Dima Amso ${ }^{1}$, Henning Voss ${ }^{2}$, Gary Glover ${ }^{3}$, BJ Casey ${ }^{l}$, 'Sackler Institute for Developmental Psychobiology, Weill Cornell Medical College, New York, USA, ${ }^{2}$ Citigroup Biomedical Imaging Center, Weill Cornell Medical College, New York, USA, ${ }^{3}$ Lucas Magnetic Resonance Image Center, Stanford University, Palo Alto, USA 
UCS expectancies modulate the diminution of unconditioned fMRI signal responses during Pavlovian fear conditioning, David Knight, Najah Waters, Peter Bandettini, NIMH, Bethesda, USA

\section{EMOTION \& MOTIVATION \\ Emotional Perception}

\section{Association of trait emotional intelligence and individual fMRI-activation patterns during} emotional perception, Benjamin Kreifelts ${ }^{1}$, Thomas Ethofer ${ }^{1,2}$, Wolfgang Grodd ${ }^{2}$, Elisabeth Huberle ${ }^{3}$, Dirk Wildgruber ${ }^{I},{ }^{I}$ Department of Psychiatry, University of Tuebingen, Tuebingen, Germany, ${ }^{2}$ Section of Experimental MR of the CNS, Department of Neuroradiology, University of Tuebingen, Tuebingen, Germany, ${ }^{3}$ Department of Cognitive Neurology, Hertie Institute for Clinical Brain Research, University of Tuebingen, Tuebingen, Germany

The effect of body structure of humanoid robot for emotional empathy: an fMRI study, Naoki MIura $^{1,2}$, Motoaki Sugiura ${ }^{3,2}$, Makoto Takahashi ${ }^{4}$, Tomohisa Moridaira ${ }^{5}$, Atsushi Miyamoto ${ }^{5}$, Yoshihiro Kuroki ${ }^{5}$, Ryuta Kawashima ${ }^{2},{ }^{1}$ Department of Intelligence Mechanical Systems Engineering, Kochi University of Technology, Kami, Japan, ${ }^{2}$ Department of Functional Brain Imaging, Institute of Development, Aging and Cancer (IDAC), Tohoku University, Sendai, Japan, ${ }^{3}$ Department of Cerebral Research, National Institute for Physiological Sciences, Okazaki, Japan, ${ }^{4}$ Graduate School of Engineering, Tohoku University, Sendai, Japan, Information Technologies Laboratories, Sony Corporation, Shinagawa, Japan

Neural correlates of volitional facilitation, Sina Radke ${ }^{l, 2}$, Corinna Nüsser ${ }^{l}$, Susanne Erk ${ }^{l}$, Julius Kuhl ${ }^{2}$, Henrik Walter, ', 'Dept. of Psychiatry, Div. of Medical Psychology, University of Bonn, Bonn, Germany, ${ }^{2}$ Dept. of Differential Psychology and Personality Research, University of Osnabrueck, Osnabrueck, Germany

Neural mechanisms underlying cognition-affect interaction and psychological well-being, Carien M. van Reekum ${ }^{1,2}$, Tom Johnstone ${ }^{1,2}$, Catherine J. Norris ${ }^{I}$, Stacey M. Schaefer ${ }^{I}$, Regina C. Lapate ${ }^{I}$, David Bachhuber', Nicole M. Rute ${ }^{I}$, Richard J. Davidson ${ }^{1},{ }^{1}$ University of Wisconsin-Madison, Madison, USA, ${ }^{2}$ University of Reading, Reading, United Kingdom

Is there a relationship between 5 HT1 a receptor binding and fMRI activation during emotion processing?, Scott Langenecker ${ }^{1,2}$, Susan Kennedy ${ }^{2}$, David Scott ${ }^{2}$, Douglas Noll ${ }^{3}$, Jon-Kar Zubieta ${ }^{1,2}$, ${ }^{1}$ University of Michigan Medical School, Psychiatry Department, Ann Arbor, USA, ${ }^{2}$ University of Michigan, Molecular and Behavioral Neuroscience Institute, Ann Arbor, USA, ${ }^{3}$ University of Michigan, Department of Engineering, Ann Arbor, USA

Skin temperature change in response to threatening stimuli in monkeys, Katsuki Nakamura ${ }^{1,2}$, Koji Kuraoka ${ }^{1,2},{ }^{1}$ National Institute of Neuroscience, NCNP, Kodaira, Japan, ${ }^{2}$ CREST, JST, Kawaguchi, Japan

Do fearful eyes capture attention?, Pia Rothstein ${ }^{1}$, Joy Geng ${ }^{2}$, Glyn Humphreys ${ }^{1},{ }^{1}$ School of Psychology, University of Birmingham, Birmingham, United Kingdom, ${ }^{2}$ Center for Mind and Brain, University California Davis, Davis, USA

The Amygdalar Resting State Network, Christian Windischberger ${ }^{1,2}$, Andreas Weissenbacher ${ }^{1,2}$, Florian Gerstl ${ }^{1,2}$, Rupert Lanzenberger ${ }^{3}$, Ewald Moser ${ }^{1,2},{ }^{I}$ MR Center of Excellence, Medical University, Vienna, Austria, ${ }^{2}$ Center for Biomedical Engineering and Physics, Medical University, Vienna, Austria, ${ }^{3}$ Department of Psychiatry and Psychotherapy, Medical University, Vienna, Austria

Covert visual brand recognition results in a distinct modulation of emotional neuronal networks according to the individual preference: a fMRI study, Silvia Casarotto ${ }^{1,2}$, Emiliano Ricciardi ${ }^{1,2}$, Matteo Corciolani ${ }^{3}$, Simona Romani ${ }^{4}$, Daniele Dalli ${ }^{3}$, Pietro Pietrini ${ }^{1,2},{ }^{1}$ Laboratory of Clinical Biochemistry and Molecular Biology, University of Pisa, Pisa, Italy, ${ }^{2}$ Department of Laboratory Medicine and Molecular Diagnostics, AUO Pisa, Pisa, Italy, ${ }^{3}$ Department of Business Administration, University of Pisa, Pisa, Italy, ${ }^{4}$ Department of Economics, Business, and Regulation, University of Sassari, Sassari, Italy

EEG source localization analysis for empathy of Iconic and Realistic Cartoon Characters, Yeojeong Choi ${ }^{1}$, Takhwan Kim ${ }^{1}$, Jaeseung Jeong ${ }^{2},{ }^{1}$ Graduate School of Culture Technology, Korea Advanced Institute of Science and Technology(KAIST), Daejeon, South Korea, ${ }^{2}$ Department of Bio and Brain Engineering, Korea Advanced Institute of Science and Technology (KAIST), Daejeon, South Korea

Valence - dependent modulation of hypothalamic activity, Martin Fürsatz ${ }^{1,2}$, Christian Windischberger ${ }^{1,2}$, Karl Agir Karlsson ${ }^{3}$, Winfried Mayr ${ }^{2}$, Ewald Moser ${ }^{1,2},{ }^{1}$ MR Centre of Excellence, 
Medical University of Vienna, Vienna, Austria, ${ }^{2}$ Center for Biomedical Engineering and Physics, Medical University of Vienna, Vienna, Austria, ${ }^{3}$ Department of Biomedical Engineering, School of Science and Engineering, Reykjavik University, Reykjavik, Iceland

Inhibiting responses to faces and complex objects: Concurrent empathy, developmental aggression, and neural response, Jessica Kirkland ${ }^{1}$, Marilyn Essex ${ }^{2}$, Jeffrey Armstrong ${ }^{2}$, Richard Davidson ${ }^{l},{ }^{1}$ University of Wisconsin Madison, Psychology Department, Madison, USA, ${ }^{2}$ University of Wisconsin Madison, Department of Psychiatry, Madison, USA

Conscious but not nonconscious perception of social emotions alters the "default mode" brain activity, Franco Cauda ${ }^{2,6}$, Katiuscia Sacco ${ }^{2,6}$, Sergio Duca ${ }^{6}$, Federico D'Agata ${ }^{2,3,6}$, Barbara Massa Micon 1,5 , Giuliano Geminiani ${ }^{2,6}$, Marco Tamietto ${ }^{2,4}$, 'Turin Advanced Neuromodulation Group, Torino, Italy, ${ }^{2}$ Department of Psychology, University of Turin, Tf Psychology, University of Turinorino, Italy, ${ }^{3}$ Department of Neuroscience, Molinette Hospital, Torino, Italy, ${ }^{4}$ Cognitive and Affective Neuroscience Laboratory, Tilburg University, Tilburg, Netherlands, ${ }^{5}$ Department of Neurosurgery, CTO hospital, Torino, Italy, ${ }^{6}$ CCS fMRI, Koelliker Hospital, Torino, Italy

Brain connectivity changes during cognitive-emotional processing in alexithymia, Branislava Ćurčić-B, Marte Swart, André Aleman, Neuroimaging Centrum, University Medical Centre Groningen, Groningen, Netherlands

Fright and Screams: Supra-additive neural responses to perceptually incongruous audio-visual cues of fear, Cindy C. Hagan ${ }^{1}$, Sam Johnson ${ }^{1}$, Will Woods ${ }^{1}$, Andrew J. Calder ${ }^{2}$, Gary R. Green ${ }^{1}$, Andrew W. Young ${ }^{I},{ }^{l}$ Department of Psychology and York Neuroimaging Centre, University of York, York, United 262 W-PM Kingdom, ${ }^{2}$ MRC Cognition and Brain Sciences Unit, Cambridge University, Cambridge, United Kingdom

Nicotine negatively influences the neural processing of visual emotional stimuli in non-smokers, Andrea Kobiella ${ }^{l}$, Dorothea E. Ulshöfer ${ }^{I}$, Christian Vollmert ${ }^{I}$, Sabine Klein ${ }^{I}$, Derik Hermann ${ }^{I}$, Karl Mann ${ }^{1}$, Michael N. Smolka ${ }^{2},{ }^{1}$ Department of Addictive Behavior and Addiction Medicine, Central Institute of Mental Health, Mannheim, Germany, ${ }^{2}$ Section of Systems Neuroscience, Department of Psychiatry and Psychotherapy, Technische Universität Dresden, Dresden, Germany

\section{IMAGING TECHNIQUES \& CONTRAST MECHANISM Anatomical MRI}

Voxel-Guided Morphometry in MS: individual assessment of chronic structural brain tissue changes in MRI - the role of focal lesions for brain atrophy development, Matthias Kraemer ${ }^{1}$, Thorsten Schormann ${ }^{2}$, Andreas Dabringhaus ${ }^{1}$, Jochen Hirsch ${ }^{3}$, Klaus-Martin Stephan ${ }^{1}$, Volker Hoemberg ${ }^{1}$, Achim Gass ${ }^{3},{ }^{1}$ St. Mauritius Therapieklinik, Meerbusch, Germany, ${ }^{2}$ Institut für Anatomie 1, Heinrich-Heine Universität, Düsseldorf, Germany, ${ }^{3}$ Universitätsspital Basel, Neuroradiologie, Basel, Switzerland

A Comparison of Three Different Tractography Software Tools and Their Ease of Application, Brian Snyderl, Jerry Chen ${ }^{2}$, Mojgan Hodaie ${ }^{I},{ }^{I}$ Department of Surgery, Division of Neurosurgery, Toronto Western Hospital, University of Toronto, Toronto, Canada, ${ }^{2}$ University of Toronto, Toronto, Canada

High resolution $\mathbf{R}_{\mathbf{2}}$ * maps reveal laminar structure of human visual cortex in vivo., Masaki Fukunaga, Marta Bianciardi, Peter van Gelderen, Jacco de Zwart, Jeff Duyn, Advanced MRI, LFMI, NINDS, National Institutes of Health, Bethesda, USA

MRI Phase-based Magnetic Susceptibility Mapping of the Human Brain at High Resolution, Karin Shmueli, Peter van Gelderen, Tie-Qiang Li, Jeff Duyn, Advanced MRI Section, Laboratory of Functional and Molecular Imaging, National Institute of Neurological Disorders and Stroke, National Institutes of Health, Bethesda, USA

\section{IMAGING TECHNIQUES \& CONTRAST MECHANISM} Diffusion MRI

Can residual bootstrap reliably estimate uncertainty in fiber orientation obtained by spherical deconvolution from diffusion-weighted MRI?, Ben Jeurissen ${ }^{\prime}$, Alexander Leemans ${ }^{2}$, Jacques-Donald Tournier ${ }^{3,4}$, Jan Sijbers ${ }^{l},{ }^{I}$ Visionlab, Dept. of Physics, University of Antwerp, Antwerp, Belgium, ${ }^{2}$ CUBRIC, School of Psychology, Cardiff University, Cardiff, United Kingdom, ${ }^{3}$ Brain Research Institute, Melbourne, Australia, ${ }^{4}$ Dept. of Medicine, University of Melbourne, Melbourne, Australia 
Employing Bootstrapping Methods to Examine the Need for Pulse Triggering In DiffusionWeighted Imaging, Zoltan Nagy ${ }^{1}$, Chloe Hutton ${ }^{1}$, Daniel Alexander ${ }^{2}$, Ralf Deichmann ${ }^{1,3}$, Nikolaus Weiskopf ${ }^{l},{ }^{1}$ Wellcome Trust Centre for Neuroimaging, University College London, London, United Kingdom, ${ }^{2}$ Centre for Medical Image Computing, Department of Computer Science, University College $290 \mathrm{~W}-\mathrm{PM}$ London, London, United Kingdom, ${ }^{3} 3$ University Hospital Brain Imgaging Centre, University of Frankfurt, Frankfurt, Germany

Structural and Functional Correlations in Subjects with Long-Term Occupational Solvent Exposure: A Combined DTI-fMRI Study, David Carpenter ${ }^{l}$, Cheuk Tang ${ }^{l}$, Gudrun Lang ${ }^{2}$, Eric Leung ${ }^{l}$,Emily Eaves ${ }^{l}$, Nancy Fiedler ${ }^{2},{ }^{1}$ Mount Sinai School of Medicine, New York, USA, ${ }^{2}$ UMDNJRobert Wood Johnson Medical School, New Jersey,

Automated localization of White Matter Hyperintensities (WMH) on DTI white matter tract atlas. Nayoung Lee ${ }^{I}$, Susumu Mori ${ }^{3}$, Kenichi Oishi ${ }^{3}$, Andreia Faria ${ }^{3}$, J. Tilak Ratnanather ${ }^{3}$, Wei Wen ${ }^{2}$, Trollor Julian ${ }^{2}$, Perminder Sachdev ${ }^{2},{ }^{1}$ Center for Imaging Science, Johns Hopkins University, Baltimore, USA, ${ }^{2}$ School of Psychiatry, University of New South Wales, Sydney, Australia, ${ }^{3}$ Department of Radiology, Johns Hopkins University, Baltimore, USA

Sub-millimeter Voxel Diffusion Tensor Imaging of the Optic Chiasm, Joelle Sarlls, Carlo Pierpaoli, National Institutes of Health, Bethesda, USA

\section{IMAGING TECHNIQUES \& CONTRAST MECHANISM Multi-modal Integration}

Registration of a NIRS Functional Time Series Dataset in fMRI Space, Paul Campion ${ }^{1,3}$, Sean Marrett ${ }^{2}$, Eric Wassermann ${ }^{l},{ }^{1}$ Brain Stimulation Section, National Institute of Neurological Disease and Stroke, National Institutes of Health, Bethesda, USA, ${ }^{2}$ Functional MRI Facility, National Institute of Mental Health, National Institutes of Health, Bethesda, USA, ${ }^{3}$ NYU School of Medicine, New York, USA

Development of an fMRI-MEG integrative neuroimaging technique: Improvements of its accuracy by suppression of fMRI-invisible coherent activities, Tetsuo Kobayashi, Yusuke Okada, Kyoto University, Kyoto, Japan

Electrophysiological and hemodynamic correspondence of neuroelectric detection in fMRI data in focal epilepsy, Roman Rodionov ${ }^{1,2}$, Michael Siniatchkin ${ }^{3}$, Christoph Michel ${ }^{4}$, David Carmichael, ${ }^{1,2}$, Rachel Thornton ${ }^{1,2}$, Adam Liston ${ }^{1}$, Louis Lemieux ${ }^{1,2},{ }^{1}$ Department of Clinical and Experimental Epilepsy, Institute of Neurology, London, United Kingdom, ${ }^{2}$ MRI Unit, The National Society for Epilepsy, Chalfont St Peter, Buckinghamshire, United Kingdom, ${ }^{3}$ Christian-Albrechts-University, University Hospital of Pediatric Neurology, Kiel, Germany, ${ }^{4}$ Neurology Clinic, University Hospital, Geneva, Switzerland

Assessing fMRI noise in EEG under simultaneous fMRI-EEG recording: a phantom study, Makoto Miyakoshi ${ }^{1,4}$, Kayako Matsuo ${ }^{2}$, Shigeyuki Kan ${ }^{3}$, Takahiko Koike ${ }^{3}$, Satoru Miyauchi ${ }^{3}$, Toshiharu Nakai ${ }^{2}$, ${ }^{1}$ Graduate School of Environmental Studies, Nagoya University, Nagoya, Japan, ${ }^{2}$ Functional Brain Imaging Laboratory, Department of Gerontechnology, National Center for Geriatrics and Gerontology, Obu, Japan, ${ }^{3}$ Kobe Advanced ICT Research Center, National Institute of Information and

Communications Technology, Kobe, Japan, ${ }^{4}$ Japan Society for the Promotion of Science, Tokyo, Japan

$322 \mathrm{~W}-\mathrm{PM}$

IMAGING TECHNIQUES \& CONTRAST MECHANISM Optical Imaging/NIRS/MRS (magnetic resonance spectroscopy)

Simultaneous measurement of prefrontal hemodynamic changes in multiple subjects by wearable optical topography, Hirokazu Atsumori, Masashi Kiguchi, Akiko Obata, Takusige Katura, Hiroki Sato, Tsukasa Funane, Atsushi Maki, Advanced Research Laboratory, Hitachi, Ltd., Hatoyama, Japan

Blind ICA discrimination of evoked cortical responses in humans with DOT, Joanne Markham ${ }^{l}$,

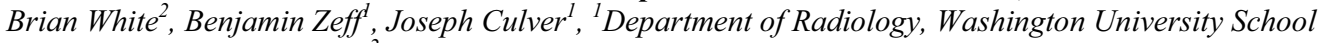
of Medicine, St. Louis, USA, ${ }^{2}$ Department of Physics and School of Medicine, Washington University, St. Louis, USA

Single Trial Analysis of EROS Data with Linear Discriminant Function, Chun-Yu Tse, Monica Fabiani, Gabriele Gratton, Beckman Institute \& Department of Psychology, University of Illinois at Urbana-Champaign, Urbana, USA 


\section{IMAGING TECHNIQUES \& CONTRAST MECHANISM Perfusion MRI}

Comparison of Pulsed Arterial Spin Labeling (PASL) With and Without Parallel Imaging at 3T, Yang Wang, Chen Lin, Andrew Kalnin, Kristine Mosier, John West, Andrew Saykin, IU Center for Neuroimaging, Dept. of Radiology, Indiana University School of Medicine, Indianapolis, USA

Venous outflow effect in arterial spin labeling magnetic resonance imaging: A demonstration in healthy children and children with sickle cell disease, Wen-Chau Wu ${ }^{l}$, Hengyi Rao ${ }^{2}$, Mikolaj Pawlak $^{1}$, Kim Cecil' ${ }^{3}$,John VanMeter ${ }^{4}$, Thomas Zeffiro ${ }^{5}$, John Detre ${ }^{2}$, Elias Melhem ${ }^{1}$, Jiongjiong Wang ${ }^{I},{ }^{I}$ Department of Radiology, University of Pennsylvania, Philadelphia, USA, ${ }^{2}$ Department of Neurology, University of Pennsylvania, Philadelphia, USA, ${ }^{3}$ Department of Radiology, Cincinnati Children's Hospital, Cincinnati, USA, ${ }^{4}$ Department of Neurology, Georgetown University Medical Center, Washington, USA, ${ }^{5}$ Neural system group, Massachusetts General Hospital, Boston, USA

\section{IMAGING TECHNIQUES \& CONTRAST MECHANISM PET/SPECT}

Functional compensation in incipient Alzheimer's disease, Anna Caroli ${ }^{l}$, Cristina Geroldi ${ }^{1,2}$, Flavio Nobili $^{3}$, Leighton R Barnden ${ }^{4}$, Ugo P Guerra ${ }^{5}$, Matteo Bonetti ${ }^{6}$, Giovanni B Frisoni ${ }^{1,2,7},{ }^{1}$ LENITEM Laboratory of Epidemiology, Neuroimaging, \& Telemedicine - IRCCS S. Giovanni di Dio-FBF, Brescia, Italy, ${ }^{2}$ Psychogeriatrics Unit - IRCCS S. Giovanni di Dio-FBF, Brescia, Italy, ${ }^{3}$ Division of Clinical Neurophysiology, Department of Endocrinological and Metabolic Sciences, University of Genoa, Genoa, Italy, ${ }^{4}$ Department of Nuclear Medicine, The Queen Elizabeth Hospital, Adelaide, Australia,

${ }^{5}$ Department of Nuclear Medicine, Ospedali Riuniti, Bergamo, Italy, ${ }^{6}$ Neuroradiology Service, Clinical Institute Città di Brescia, Brescia, Italy, ${ }^{7}$ AFaR Associazione Fatebenefratelli per la Ricerca, Rome, Italy

\section{IMAGING TECHNIQUES \& CONTRAST MECHANISM} TMS

Investigation of the role of S1 and PFC in tactile working memory: a navigated TMS, tractography and EEG study., Tuomas Neuvonen ${ }^{1,2,6}$, Henri Hannula ${ }^{1,2,6}$, Petri Savolainen ${ }^{1}$, Jaana Hiltunen ${ }^{3}$, Oili Salonen ${ }^{4}$, Synnöve Carlson ${ }^{1,5}$, Antti Pertovaara ${ }^{l},{ }^{1}$ Neuroscience Unit, Institute of Biomedicine/Physiology, University of Helsinki, Helsinki, Finland, ${ }^{2}$ Nexstim Ltd, Helsinki, Finland, ${ }^{3}$ Advanced Magnetic Imaging Centre, Helsinki University of Technology, Espoo, Finland, ${ }^{4} H U S$ Helsinki Medical Imaging Centre, Helsinki University of Technology, Helsinki, Finland, ${ }^{5}$ Medical School, University of Tampere, Tampere, Finland, ${ }^{6}$ these authors had an equal contribution to the study

\section{LANGUAGE \\ Comprehension}

The cortical dynamics of intelligible speech, Thomas Schofield, Alex Leff, Klaas Stephan, Jenny Crinion, Karl Friston, Cathy Price, Wellcome Trust Centre for Neuroimaging, 12 Queen Square, University College London, London, United Kingdom

The Stages of Syntactic Processing measured with ERP: Effects of Word Frequency, Laurie A. Stowe, Hanneke Loerts, John C.J. Hoeks, NeuroimagingCenter, University of Groningen, Groningen, Netherlands

Functional networks for semantic and phonological processing assessed with directed partial correlation analysis, Wolfgang Mader ${ }^{1,3}$, David Feess ${ }^{1,3}$, Rüdiger Lange ${ }^{3}$, Cornelius Weiller ${ }^{2,3}$, Jens Timmer ${ }^{1,2}$, Björn Schelter, ${ }^{1,2}$, Dorothee Saur ${ }^{3},{ }^{1}$ FDM, Center for Data Analysis and Modeling, University of Freiburg, Freiburg, Germany, ${ }^{2} B C C N$, Bernstein Center for Computational Neuroscience, University of Freiburg, Freiburg, Germany, ${ }^{3}$ Department of Neurology, University Hospital Freiburg, Freiburg, Germany

Meta-analysis of Neural Representation of First Language and Second Language, Rajani Sebastian ${ }^{l}$, Swathi Kiran ${ }^{1,2},{ }^{1}$ Department of Communication Sciences and Disorders, University of Texas 366 W-PM at Austin, Austin, USA, ${ }^{2}$ Institute of Neuroscience, University of Texas at Austin, Austin, USA 
Neural mechanism of information retrieval unique to sentence processing, Kei Takahashi ${ }^{1,2,3}$, Satoru Yokoyama $^{2}$, Toshimune Kambara ${ }^{2,3}$, Kei Yoshimoto ${ }^{3}$, Ryuta Kawashima ${ }^{2},{ }^{1}$ JSPS, Tokyo, Japan, ${ }^{2}$ IDAC, Tohoku U., Sendai, Japan, ${ }^{3}$ GSICS, Tohoku U., Sendai, Japan

Spatio-temporal patterns of metaphor comprehension: The effect of context, Valentina Bambini ${ }^{l}$, Chiara Bertini ${ }^{1}$, Alessandra Stella ${ }^{2}$, Francesco Di Russo ${ }^{2,3},{ }^{1}$ Laboratory of Linguistics, Scuola Normale Superiore, Pisa, Italy, ${ }^{2}$ Department of Education for Motor Activity and Sport, University Institute of Motor Sciences, Rome, Italy, ${ }^{3}$ Foundation Santa Lucia, Rome, Italy

Dynamic ERP Mapping in Perception of Chinese Pin-Yin Vowels, Andrew CN Chen*, Yanling Yin, Peipei Wang, Weijia Feng, Center for Higher Brain Functions, Capital Medical University, Beijing, China

Language reorganization of patients with auditory deficiencies observed by functional magnetic resonance imaging, Mara Rita Pereira-Jorge ${ }^{1}$, Marcio Sturzbecher ${ }^{2}$, Antonio Carlos Santos ${ }^{3}$, Draulio Barros de Araujo ${ }^{1,2},{ }^{1}$ Universidade de São Paulo, Ribeirão Preto, Brazil, ${ }^{2}$ Universidade de São Paulo, Ribeirão Preto, Brazil, ${ }^{3}$ Universidade de São Paulo, Ribeirão Preto, Brazil, ${ }^{4}$ Universidade de São Paulo, Ribeirão Preto, Brazil

Language dual-tasking: listening to two people makes your brain work twice as hard?, Augusto Buchweitz, Ann Meyler, Marcel Just, Center for Cognitive Brain Imaging, Carnegie Mellon University, Pittsburgh, USA

Sentence Processing and Grammatical Complexity., Anne-Dominique Devauchelle ${ }^{1,2,3}$, Y-Lan Boureau $^{1,2,3}$, Stanislas Dehaene, ${ }^{1,2,4}$, Christophe Pallier ${ }^{1,2,3},{ }^{I}$ INSERM, U562, Cognitive Neuroimaging unit, Gif sur Yvette, France, ${ }^{2} C E A$, DSV/I2BM, NeuroSpin center, Gif sur Yvette, France, ${ }^{3}$ Univ Paris-sud, IFR49, Gif sur Yvette, France, ${ }^{4}$ Collège de France, Paris, France

Cortical networks underlying benefits of audio-visual speech integration, Sungeun $\mathrm{Kim}^{l}$, Thomas M. Talavage ${ }^{1,2,3}$, Rachel Lenhart ${ }^{4}$, Angela Hoffa ${ }^{2}$, Donald Wong ${ }^{5}$, David B. Pisoni ${ }^{6}$, ${ }^{1}$ School of Electrical and Computer Engineering, Purdue University, West Lafayette, USA, ${ }^{2}$ Weldon School of Biomedical Engineering, Purdue University, West Lafayette, USA, ${ }^{3}$ Department of Radiology, Indiana University School of Medicine, Indianapolis, USA, ${ }^{4}$ Department of Mechanical, Aerospace, and Biomedical Engineering, University of Tennessee, Knoxville, USA, ${ }^{5}$ Department of Neuroscience and Clinical Neurology, Indiana University School of Medicine, Indianapolis, USA, ${ }^{6}$ Department of Psychological and Brain Sciences, Indiana University, Bloomington, USA

\section{LANGUAGE Reading/Writing}

Left Posterior Parietal Cortex is Involved in the Spatial Processing of Chinese Character Recognition, Yanlin Luo ${ }^{1}$, Andrew CN Chen ${ }^{1}$, xiujun $\mathrm{Li}^{2}$, Danlin Pen ${ }^{2},{ }^{1}$ Capital Medical University, Beijing, China, ${ }^{2}$ Beijing normal University, Beijing, China

Unbiased classification of developmental dyslexic subtypes using fMRI activation during reading., Ferath kherif, Caroline Ellis, Clare Shakeshaft, Hwee-Ling Lee, Mohamed Seghier, Cathy Price, Wellcome Trust Centre for Neuroimaging, UCL, London, United Kingdom

The Influence of Phonological Transparency on Reading, Atira Bick ${ }^{1}$, Ram Frost ${ }^{2}$, Gadi Goelman ${ }^{3}$, ${ }^{I}$ ICNC, Hebrew University, Jerusalem, Israel, ${ }^{2}$ Psychology Department, Hebrew University, Jerusalem, Israel, ${ }^{3}$ Medical Biophysics, Hadassah Hebrew University Hospital, Jerusalem, Israel

Early Neural Response to Expectancy In Reading Sentences: Convergent ERP and fMRI Findings, Joseph Dien, Aminda O'Hare, University of Kansas, Lawrence, USA

\section{MEMORY \& LEARNING Learning (explicit \& implicit)}

Visuospatial Working Memory in Children with Dysthymic Disorder: A Functional Magnetic Resonance Imaging (fMRI) Study., Karissa Searle ${ }^{I}$, Melissa Casey ${ }^{I}$, Ross Cunnington ${ }^{2}$, Alasdair Vance ${ }^{l},{ }^{\prime}$ Academic Child Psychiatry Unit, Royal Children's Hospital, Murdoch Childrens Research Institute, Melbourne, Australia, ${ }^{2}$ Queensland Brain Institute, Brisbane, Australia

Transfer effects from multiplication to division: An fMRI study on training arithmetic, Anja Ischebeckl, Laura Zamarian ${ }^{l, 2}$, Michael Schocke ${ }^{3}$, Margarete Delazer ${ }^{1},{ }^{1}$ Medical University Innsbruck, 
Dept. of Neurology, Innsbruck, Austria, ${ }^{2}$ University of Trieste, Dept. of Psychology, Trieste, Italy, ${ }^{3}$ Medical University Innsbruck, Dept. of Radiology I, Innsbruck, Austria

An Investigation of Motor Plasticity using Resting State fMRI and Structural Equation Modeling, Liangsuo Ma ${ }^{1}$, Binquan Wang ${ }^{2}$, Donald Robin ${ }^{2}$, Peter Fox ${ }^{2}$, Jinhu Xiong ${ }^{l},{ }^{I}$ Department of Radiology, University of Iowa, Iowa City, USA, ${ }^{2}$ Research Imaging Center, University of Texas Health Science Center, San Antonio, USA

Visuospatial Memory (VSM) in Children with Attention Deficit Hyperactivity Disorder, Combined Type (ADHD-CT): A Functional Magnetic Resonance Imaging (fMRI) Study., Melissa Casey ${ }^{\text {, }}$ Maree Farrow ${ }^{3}$, Ross Cunnington ${ }^{2}$, Alasdair Vance ${ }^{1},{ }^{\prime}$ Academic Child Psychiatry Unit, Royal Children's Hospital, Murdoch Childrens Research Institute, Melbourne, Australia, ${ }^{2}$ Queensland Brain Institute, Brisbane, Australia, ${ }^{3}$ Howard Florey Institute, Melbourne, Australia

Repetition enhancement in perceptual priming: influence of word processing on cortical sharpening, Lebreton Karine ${ }^{l}$, Villain Nicolas ${ }^{l}$, Chételat Gaël ${ }^{1}$,Landeau Brigitte ${ }^{l}$, Seghier Mohammed L ${ }^{2,3}$, Lazeyras François ${ }^{3}$, Eustache Francis ${ }^{1}$, Ibanez Vincent ${ }^{4},{ }^{1}$ Inserm - EPHE - Université de Caen Basse/Normandie, Unité U923, GIP Cyceron, CHU Côte de Nacre, Caen, France, ${ }^{2}$ Department of Radiology, University Hospitals of Geneva, Geneva, Switzerland, ${ }^{3}$ Wellcome Trust Centre for Neuroimaging, Institute of Neurology, UCL, London, United Kingdom, ${ }^{4}$ Psychiatric Neuroimaging Unit, Division of Neuropsychiatry, Department of Psychiatry, University Hospitals of Geneva, Geneva, Switzerland

Functional association of brain and somatic activities accompanying reverse learning, Hideki Ohira $^{l}$, Michio Nomura ${ }^{2}$, Masahiro Matsunaga ${ }^{1,3}$, Tokiko Isowa ${ }^{4}$, Kenta Kimura ${ }^{1}$, Noriaki Kanayama ${ }^{1}$, Hiroki Murakami ${ }^{I}$, Takahiro Osumi ${ }^{1},{ }^{I}$ Nagoya University, Nagoya, Japan, ${ }^{2}$ Tokai Gakuin University, Kakamigahara, Japan, ${ }^{3}$ Aichi Medical University, Nagakute, Japan, ${ }^{4}$ Mie Prefectural College of Nursing, Tsu, Japan

11:30 - 12:30 Corryong Hall (Level 2)

\section{MEMORY \& LEARNING}

Long-term Memory (episodic, semantic, autobiographical)

The neurocognitive benefits of donepezil on episodic memory in young, healthy individuals following $24 \mathbf{h}$ of sleep deprivation, Lisa Chuah ${ }^{I}$, Chong Delise ${ }^{I}$, Jiat-Chow Tan ${ }^{I}$, William Rekshan ${ }^{I}$, Annette Chen ${ }^{l}$, Martin Pan ${ }^{2}$, Robert Lai ${ }^{2}$, Vincenzo Libri ${ }^{2}$, Michael Chee ${ }^{l},{ }^{l}$ Cognitive Neuroscience Lab, Duke-NUS Graduate Medical School, Singapore, Singapore, ${ }^{2}$ Neurology Centre of Excellence of Drug Discovery, GlaxoSmithKline, Harlow, United Kingdom

Autobiographical Retrieval Evokes and Induces Medial Temporal Lobe Theta Oscillatory Activity, Taufik A. Valiante ${ }^{1,2}$, Mary Pat McAndrews ${ }^{1,2},{ }^{1}$ University Health Network, Toronto, Canada, ${ }^{2}$ University of Toronto, Toronto, Canada

Cortical Representations of Famous and Personally-Familiar Places, Motoaki Sugiura ${ }^{1,2}$, Yoko Mano $^{2,1}$, Akihiro Sasaki ${ }^{2,1}$, Norihiro Sadato ${ }^{1,2}$, 'Departmen of Cerebral Research, National Institute for Physiological Sciences, Okazaki, Japan, ${ }^{2}$ Division of Physiological Sciences, Graduate University for Advanced Investigations (SOKENDAI), Okazaki, Japan

Memory performance related to hippocampal activation in non-demented older adults, Amy DeLucal, Peter LaViolette ${ }^{2}$, Kelly O'Keefe ${ }^{I}$, Jacqueline O'Brien ${ }^{l}$, Reisa A. Sperling ${ }^{I},{ }^{\prime}$ Brigham and Women's Hospital, Boston, USA, ${ }^{2}$ Massachusetts General Hospital, Boston, USA

452 W-PM

EEG theta-gamma coupling during explicit memory retention, Hiroaki Mizuhara, ${ }^{1,2}$, Yoko Yamaguchi ${ }^{2},{ }^{I}$ Graduate School of Informatics, Kyoto University, Kyoto, Japan, ${ }^{2}$ Lab. For Dynamics of Emergent Intelligence, RIKEN Brain Science Institute, Wako, Japan

Memory consolidation leads to decreased posterior hippocampal activity during retrieval of face-location associations while anterior hippocampal activity is increased, Atsuko Takashima ${ }^{1}$, Ingrid Nieuwenhuis ${ }^{l}$, Ole Jensen ${ }^{l}$, Lucia Talamini ${ }^{2}$, Mark Rijpkema ${ }^{l}$, Guillen Fernandez ${ }^{1,3}$, ${ }^{1}$ FC Donders Centre, Radboud University Nijmegen, Nijmegen, Netherlands, ${ }^{2}$ Dept. of Psychology, University of Amsterdam, Amsterdam, Netherlands, ${ }^{3}$ Dept. of Neurology, Radboud University Nijmegen, Nijmegen, Netherlands 


\section{MODELING \& ANALYSIS \\ Exploratory Methods, Artifact Removal}

Enabling the Sharing of Functional MRI Datasets with BAXSQL, Epifanio Bagarinao ${ }^{l}$, Yoshio Tanakal, Kayako Matsuo ${ }^{2}$, Toshiharu Nakai ${ }^{2},{ }^{l}$ Grid Technology Research Center, National Institute of Advanced Industrial Science and Technology, Tsukuba City, Japan, ${ }^{2}$ Department of Gerontechnology, National Center for Geriatrics and Gerontology, Ohbu City, Japan

Effect of regressing blink and saccade artefacts out of MEG signals, Pierre Fonlupt ${ }^{1,2}$, Dimitri Bayle ${ }^{1,2}$, Marie-Anne Henaff ${ }^{d, 2},{ }^{1}$ INSERM U821, LYON, France, ${ }^{2}$ Université Lyon1, LYON, France

Intersubjects correlation wavelet analysis: a time-scale data driven analysis., Patricia Lessa ${ }^{1,2}$, João Sato $^{2,3}$, Carlos Griese Neto ${ }^{2,3}$, Elisson Cardoso ${ }^{2}$, Edson Amaro Jr $^{2}$, ' . IIEP - Instituto Israelita de Ensino e Pesquisa Albert Einstein, São Paulo, Brazil, ${ }^{2}$ NIF - Instituto de Radiologia do Hospital das Clínicas Universidade de São Paulo, São Paulo, Brazil, ${ }^{3}$ Instituto de Matemática e Estatística - Universidade de São Paulo, São Paulo, Brazil

Evaluating Latent Functional Cortical Regions Interactions with Structural Equation Modeling, Chih-Chien Yang, Liang-Ting Tsai, Graduate School of Educational Measurement \& Statistics, National Taichung University, Taichung, Taiwan

Unsupervised Clustering Identifies Structured Variability in Single Trial EEG Responses, Andrew Bagshaw ${ }^{1,2}$, Tracy Warbrick ${ }^{1,2},{ }^{1}$ School of Psychology, University of Birmingham, Birmingham, United Kingdom, ${ }^{2}$ Birmingham University Imaging Centre, University of Birmingham, Birmingham, United Kingdom

fMRI noise properties as a function of structure and tissue type: a multi-site study, Douglas Greve ${ }^{l}$, Bryon Mueller ${ }^{2}$, Thomas Liu ${ }^{3}$, Gary Glover ${ }^{4}, F$. BIRN ${ }^{5},{ }^{1}$ MGH Athinoula A. Martinos Center for Biomedical Imaging, Boston, USA, ${ }^{2}$ Center for. Magnetic Resonance Research, Minneapolis, USA, ${ }^{3}$ UCSD Center for Functional MRI, San Diego, USA, ${ }^{4}$ Stanford Radiological Sciences Lab, Stanford, USA, ${ }^{5}$ www.nbirn.net, Irvine, USA

Splines on the Sphere Q-Ball Imaging with Generalized Cross Validated smoothing (GCV-S ${ }^{\mathbf{Q}}$ QBI), Nader Metwalli, ${ }^{l, 2}$, Xiaoping Hu ${ }^{I}$, John Carew ${ }^{3,4}$, IBiomedical Engineering, Georgia Institute of Technology / Emory University, Atlanta, USA, ${ }^{2}$ Biomedical Engineering, Cairo University, Cairo, Egypt, ${ }^{3}$ Radiology and Biostatistics, Emory University, Atlanta, USA, ${ }^{4}$ Bioengineering, Georgia Institute of Technology, Atlanta, USA

HEEG virtual electrodes for synchrony measures, Francois-B. Vialatte ${ }^{l}$, Monique Maurice ${ }^{l}$, Dauwels Justin $^{2,3}$, Andrzej Cichocki ${ }^{1}$, ${ }^{1}$ Riken BSI, Lab. ABSP, Wako-Shi, Japan, ${ }^{2}$ MIT, Boston, USA, ${ }^{3}$ Riken BSI, Amari Research Unit, Wako-Shi, Japan

\section{MODELING \& ANALYSIS \\ Flattening, Segmentation}

Unsupervised Hippocampus Segmentation: Tools, Validation and Clinical Perspectives., Andrea Chincarini $^{1}$, Gianluca Gemme ${ }^{1}$, Piero Calvini ${ }^{1,2}$, Sandro Squarcia ${ }^{1,2}$, Stefania Donadio ${ }^{1,2}$, Luca Rei $^{2}$, Elisabetta Molinaro ${ }^{2}$, Giovanni Frisoni ${ }^{4}$, Flavio Mariano Nobili ${ }^{3}$, Guido Rodriguez ${ }^{3},{ }^{1}$ INFN, sezione di Genova, Genova, Italy, ${ }^{2}$ Laboratorio di Fisica e Statistica Medica, Universita' di Genova, Genova, Italy, ${ }^{3}$ Neurofisiologia Clinica - DTC e DISEM, Azienda Ospedaliera San Martino, Genova, Italy, ${ }^{4}$ RCCS San Giovanni di Dio, Brescia, Italy

MAPS: A Free Medical Image Processing Pipeline, Blake Lucas ${ }^{l}$, Bennett Landman ${ }^{l}$, Jerry Prince ${ }^{I}$, Dzung Pham 2, Image Analysis and Communications Laboratory (IACL), The Johns Hopkins University, Baltimore, USA, ${ }^{2}$ Laboratory of Medical Image Computing (MedIC), The Johns Hopkins University, Baltimore, USA

Comparison of FSL-FIRST with Manual Segmentation of Subcortical Brain Volumes., Janis Breeze $^{1,2}$, Brian Patenaude ${ }^{3}$, Jean Frazier ${ }^{1,2}$, Mark Jenkinson ${ }^{3}$, Stephen Smith ${ }^{3}$, David Kennedy ${ }^{1,2,4}$, ${ }^{1}$ Cambridge Health Alliance, Cambridge, USA, ${ }^{2}$ Harvard Medical School, Boston, USA, ${ }^{3}$ Oxford University, Oxford, United Kingdom, ${ }^{4}$ Massachusetts General Hospital, Boston, USA

Cerebral Surface Extraction with Sub-voxel accuracy from Neonatal MR Images using Thick Rubber Model, Takuma Oshiba ${ }^{1}$, Syoji Kobashi ${ }^{1}$, Kumiko Ando ${ }^{2}$, Masayo Ogawa ${ }^{2}$, Reiichi Ishikura ${ }^{2}$, 
Shozo Hirota 2 , Yutaka Hata ${ }^{l},{ }^{l}$ Graduate School of Engineering, Univerisity of Hyogo, Himeji, Japan,

${ }^{2}$ Hyogo College of Medicine, Nishinomiya, Japan

\section{MODELING \& ANALYSIS}

Functional Connectivity and Structural Equation Modeling

Characterizing the Specific Behavior of Dynamic Causal Modeling Applied to fMRI Signals, Björn Schelter $^{1,2}$, David Feess ${ }^{1,3}$, Wolfgang Mader ${ }^{1,3}$, Rüdiger Lange ${ }^{3}$, Dorothee Saur ${ }^{3}$, Volkmar Glauche ${ }^{3}$, Cornelius Weiller ${ }^{2,3}$, Jens Timmer ${ }^{1,2},{ }^{1}$ FDM, Center for Data Analysis and Modeling, University of Freiburg, Freiburg, Germany, ${ }^{2} B C C N$, Bernstein Center for Computational Neuroscience, University of Freiburg, Freiburg, Germany, ${ }^{3}$ Department of Neurology, University Hospital Freiburg, Freiburg, Germany

Impact of missing responses on fMRI DCM analysis, Michal Mikl ${ }^{1,2}$, Petr Hluštík ${ }^{3}$, Radek Mareček ${ }^{l}$, Martin Havliček ${ }^{2}$, Milan Brázdil ${ }^{l},{ }^{l}$ Department of Neurology, St. Anne's University Hospital and Masaryk Unive, Brno, Czech Republic, ${ }^{2}$ Department of Biomedical Engineering, FEEC, Brno University of Technology, Brno, Czech Republic, ${ }^{3}$ Departments of Neurology and Radiology, School of Medicine, Palacký University and University Hospital, Olomouc, Czech Republic

Modeling the symbiotic relationship between neuronal structure and dynamics., Mika Rubinov ${ }^{I}$, Kelton Temby ${ }^{1}$, Olaf Sporns ${ }^{2}$, Cees van Leeuwen ${ }^{3}$, Michael Breakspear ${ }^{l},{ }^{I}$ University of New South Wales, Sydney, Australia, ${ }^{2}$ Indiana University, Bloomington, USA, ${ }^{3}$ RIKEN Brain Science Institute, Saitama, Japan

Functional pathway discovery using mediation analysis: Approach and application to pain, Tor Wager, Lauren Atlas, Martin Lindquist, Kate Hard, Matthew Davidson, Columbia University, New York, USA

Mutual Information Analysis with Optimized Gaussian Kernel Can Detect Weak Functional Connectivity from MEG Tomographic Estimates, Masaki Maruyama, Andreas Ioannides, RIKEN Brain Science Institute, Wako, Japan

Independent components of EEG signals are associated with widespread networks of simultaneously-measured FMRI activity in the resting state, Rami Niazy, John Evans, Richard Wise, Cardiff University Brain Research Imaging Centre (CUBRIC), School of Psychology, Cardiff University, Cardiff, United Kingdom

Spurious Causality in fMRI, Victor Solo ${ }^{1,2}$, Fa-Hsuan Lin $^{l}$, Mark Vangel ${ }^{1}$, Matti Hamalainen ${ }^{l}$, ${ }^{I}$ MGH/MIT/HMS Athinoula A. Martinos Center for Biomedical Imaging, Harvard Medical School,

Charlestown, USA, ${ }^{2}$ School of Electrical Engineering, University of New South Wales, Sydney, Australia

Each Brain region is organized into both positive and negative connectivity as revealed by restingstate fMRI, Xiang-Yu Long ${ }^{l}$, Xi-Nian Zuo ${ }^{2}$, Qi-Hong Zoul, Chao-Zhe Zhu ${ }^{l}$, Liang Wang ${ }^{l}$, Vesa Kiviniemi ${ }^{3}$, Yong He ${ }^{4}$, Yu-Feng Zang ${ }^{1}$, ${ }^{1}$ State Key Laboratory of Cognitive Neuroscience and Learning, Beijing Normal University, Beijing, China, ${ }^{2}$ National Laboratory of Pattern Recognition, Institute of Automation, Chinese Academy of Sciences, Beijing, China, ${ }^{3}$ Department of Diagnostic Radiology, Oulu University Hospital, Oulu, Finland, ${ }^{4}$ McConnell Brain Imaging Center, Montreal Neurological Institute, McGill University, Montreal, Canada

Resting state functional connectivity estimation in ASL data, Maria Gavrilescu ${ }^{l}$, Michael Farrell ${ }^{l, 2}$, Linda Verhoeven ${ }^{3}$, Derek Denton ${ }^{4,5}$, Gary Egan ${ }^{1,2}$, ${ }^{1}$ Howard Florey Institute, Florey Neuroscience Institutes, Melbourne, Australia, ${ }^{2}$ Centre for Neuroscience, University of Melbourne, Melbourne, Australia, ${ }^{3}$ Biomedical Engineering, Technische Universiteit Eindhoven, Eindhoven, Netherlands, ${ }^{4}$ Office of the Dean, Faculty of Medicine, Dentistry and Health Sciences, University of Melbourne, Melbourne, Australia, ${ }^{5}$ Baker Heart Research Institute, Alfred Hospital, Melbourne, Australia

On the applicability of autoregressive models and Granger causality theory in fMRI analyses, Catherine Davey ${ }^{2}$, David Grayden ${ }^{1}$, Maria Gavrilescu', Michael Farrell ${ }^{2,3}$, Gary Egan ${ }^{2,3}$, Leigh Johnston ${ }^{1,2},{ }^{1}$ Department of Electrical and Electronic Engineering, University of Melbourne \& NICTA Victorian Research Laboratory, Melbourne, Australia, ${ }^{2}$ Howard Florey Institute, Florey Neuroscience Institutes, Melbourne, Australia, ${ }^{3}$ Centre for Neuroscience, University of Melbourne, Melbourne, Australia 
BrainSPANs: An Open Toolbox for Analyzing Brain Spontaneous Activity and Networks, Tianzi Jiang, Yong Liu, Kun Wang, Ming Song, Yuan Zhou, National Laboratory of Pattern Recognition, Institute of Automation, Chinese Academy of Sciences, Beijing, China

A neural code of motor programmes during hand gripping tasks, CC Chen, James Kilner, Nick Ward, Karl Friston, Wellcome Trust Centre for Neuroimaging, london, United Kingdom

568 W-PM

$572 \mathrm{~W}-\mathrm{PM}$

Online Resting Connectivity with Inline Image Reconstruction, Christopher Glielmi ${ }^{l}$, Keith Heberlein ${ }^{2}$, Xiaoping $\mathrm{Hu}^{I},{ }^{l}$ Georgia Institute of Technology / Emory University, Atlanta, USA, ${ }^{2}$ Siemens Medical Solutions, Erlangen, Germany

Possible sources of functional connectivity and under-connectivity in adolescents with autism spectrum disorders., Tyler Jones ${ }^{l}$, Lauren Kenworthy ${ }^{1,2}$, Laura Case ${ }^{I}$, Shawn Milleville ${ }^{l}$, Peter Bandettini ${ }^{l}$, Alex Martin ${ }^{1}$, Rasmus Birn ${ }^{1},{ }^{1}$ Laboratory of Brain and Cognition, Bethesda, USA, ${ }^{2}$ Center for Autism Spectrum Disorders Children's National Medical Center, Washington, USA

\section{MODELING \& ANALYSIS}

Multivariate Modeling, PCA, \& ICA

Improving results from polarized light imaging by means of independent component analysis, Jürgen Dammers ${ }^{l}$, Markus Axer ${ }^{l}$, David Gräßel ${ }^{1}$, Karl Zilles ${ }^{1,2}$, Katrin Amunts ${ }^{1,3}$, Uwe Pietrzyk ${ }^{1,4}$, ${ }^{I}$ Institute of Neuroscience and Biophysics, INB-3 Medicine, Research Center Jülich, Jülich, Germany, ${ }^{2}$ C. and O. Vogt Institute for Brain Research, University of Düsseldorf, Düsseldorf, Germany, ${ }^{3}$ Department of Psychiatry and Psychotherapy, Aachen University Hospital, Aachen, Germany, ${ }^{4}$ Department of Physics, University of Wuppertal, Wuppertal, Germany

Cross-Modal Classification in Human Right Premotor Cortex, Joset Etzel, Valeria Gazzola, Christian Keysers, Unversity Medical Center Groningen/University of Groningen, Groningen, Netherlands

Modulation of ongoing cerebral activity during finger-tapping: A new MEG method for capturing spatio-temporal dynamics, Dante Mantini ${ }^{1,2}$, Stefania Della Penna ${ }^{1,2}$, Laura Marzetti ${ }^{1,2}$, Francesco De Pasquale $^{1,2}$, Paolo Belardinelli, ${ }^{1,2}$, Luca Ciancetta ${ }^{1,2}$, Christofer Lewis ${ }^{1,2,3,4}$, Abraham Z. Snyder ${ }^{3,4}$, Vittorio Pizzella, ${ }^{1,2}$, Gian Luca Romani ${ }^{1,2}$, Maurizio Corbetta ${ }^{1,2,3,4}$, Institute for Advanced Biomedical Technologies, University Foundation "G. D'Annunzio”, Chieti, Italy, ${ }^{2}$ Department of Clinical Sciences and Bio-imaging, University “G. D'Annunzio”, Chieti, Italy, ${ }^{3}$ Department of Neurology, Washington University, St. Louis, USA, ${ }^{4}$ Department of Radiology, Washington University, St. Louis, USA

Multifractal refraction of resting state fMRI time series by age and drug, John Suckling ${ }^{l}$, Alle-Meije Wink ${ }^{2}$, Frederic Bernard ${ }^{3}$, Anna Barnes ${ }^{l}$,Ed Bullmore ${ }^{l}$, 'Brain Mapping Unit, Department of Psychiatry, University of Cambridge, Cambridge, United Kingdom, ${ }^{2}$ 2Imaging Sciences Division, Imperial College, Hammersmith Hospital, London, United Kingdom, ${ }^{3}$ Département d'Etudes Cognitives, Ecole Normale Supérieure, Paris, France

Multivariate Deformation-Based Morphometry Statistics of Cortical Surfaces Reveals Changes in Folding Frequency Correlated with Alzheimer's Disease, Maxime Boucher ${ }^{1,2}$, Oliver Lyttelton ${ }^{l}$, Sue Whitesides ${ }^{2}$, Alan Evans ${ }^{I},{ }^{1}$ Montreal Neurological Institute, Montreal, Canada, ${ }^{2}$ School of Computer Science, McGill University, Montreal, Canada

Event-related Functional Near-infrared Spectroscopy (fNIRS) Analysis by Independent Component Analysis, Yun Jiao ${ }^{1,2}$, Zhenyu Zhou ${ }^{1,2,4}$, Hongyu Yang ${ }^{I}$, Zongcai Ruan ${ }^{l}$, Hui Gong ${ }^{3}$, Zuhong Lu ${ }^{1,2},{ }^{1}$ Key Laboratory of Child Development and Learning Science (Southeast University), Ministry of Education, Nanjing, China, ${ }^{2}$ School of Biological Science and Medical Engineering, Southeast University, Nanjing, China, ${ }^{3}$ Key Laboratory of Biomedical Photonics of Ministry of Education, Huazhong University of Science and Technology, Wuhan, China, ${ }^{4}$ Dept. of Psychiatry, University of Florida, Gainesville, USA

Voxel selection for fMRI data based on sparse representation, Yuanqing $\mathrm{i}^{1}$, Praneeth Namburi ${ }^{1,2}$, Cuntai Guan ${ }^{1}$, Jianfeng Feng, ${ }^{3},{ }^{1}$ Institute for Infocomm Research, Singapore, ${ }^{2}$ Nanyang Technological University, Singapore, ${ }^{3}$ Warwick University, United Kingdom

Source Based Morphometry: Using Independent Component Analysis to Identify Gray and White Matter Differences with Application to Schizophrenia, Lai Xu ${ }^{1,2}$, Godfrey Pearlson ${ }^{1,2}$, Vince Calhoun ${ }^{3,4},{ }^{1}$ The MIND Institute, Albuquerque, USA, ${ }^{2}$ Dept. of ECE, University of New Mexico, Albuquerque, USA, ${ }^{3}$ Olin Neuropsychiatry Research Center, Institute of Living, Hartford, USA, ${ }^{4}$ Dept. of Psychiatry, Yale University School of Medicine, New Haven, USA
592 W-PM

596 W-PM

$600 \mathrm{~W}-\mathrm{PM}$

604 W-PM

608 W-PM

612 W-PM
584 W-PM 
MOTOR BEHAVIOR

Basal Ganglia/Brainstem/Spinal Cord

Neural synchronization of distributed spinal activity as a sign of motor binding, Tjeerd Boonstra ${ }^{1,2}$, Andreas Daffertshofer ${ }^{2}$, Peter Beek, ${ }^{2}$ University of New South Wales, Randwick, Australia, ${ }^{2} V U$ University, Amsterdam, Netherlands

MOTOR BEHAVIOR

Eye Movements/Visuomotor Processing

Pure observational vs. imitation practice of hand actions: Correlation between behavioural outcome and neural activations., Satomi Higuchi ${ }^{1,2}$, Neil Roberts ${ }^{2}$, Simon B. Eickhoff ${ }^{3}$, Stefan Vogt ${ }^{1,2}$, ${ }^{I}$ Department of Psychology, University of Lancaster, Lancaster, United Kingdom, ${ }^{2}$ Magnetic Resonance and Image Analysis Research Centre, University of Liverpool, Liverpool, United Kingdom, ${ }^{3}$ Institut for Medicine, Research Center, Jülich, Germany

Secondary sensory area SII is crucially involved in the preparation of familiar movements compared to movements never made before., Martijn Beudel ${ }^{1,4}$, Sjouke Zijlstra ${ }^{2,4}$, Theo Mulder ${ }^{2}$, Inge Zijdewind ${ }^{3,4}$, Bauke de Jong ${ }^{1,4}$, ' dept. Neurology, University Medical Center Groningen, Groningen, Netherlands, ${ }^{2}$ dept. Human Movement Sciences, University of Groningen, Groningen, Netherlands, ${ }^{3}$ dept. Medical Physiology, University of Groningen, Groningen, Netherlands, ${ }^{4}$ BCN Neuroimaging Center, University of Groningen, Groningen, Netherlands

Influence of sensory and motor properties on the Parietal Cortex, Karin Nadig ${ }^{l}$, Lutz Jäncke ${ }^{1}$, Roger Lüchinger ${ }^{2}$, Kai Lutz ${ }^{1},{ }^{1}$ Department of Neuropsychology, University of Zurich, Zurich, Switzerland, ${ }^{2}$ Institute for Biomedical Engineering, Swiss Federal Institute of Technology (ETH) Zurich, Zurich, Switzerland

\section{NEUROANATOMY \\ DTI Studies, Application}

Effects of regular alcohol use during adolescence on white matter integrity, Francesca Filbey, Arvind Caprihan, Kent Hutchison, The MIND Research Network, Albuquerque, USA

Asymmetry of the arcuate fasciculus of the human brain studied by in- and ex-vivo DTI, as well as post-mortem microdissection, Kovacs Silvia ${ }^{l}$, Sage Caroline ${ }^{l}$, De Jong Lars ${ }^{2}$, Van Loon Johannes ${ }^{2}$, Sunaert Stefan ${ }^{I},{ }^{1}$ University Hospitals of the Catholic University Leuven, department of Radiology, Herestraat 49, Leuven, Belgium, ${ }^{2}$ University Hospitals of the Catholic University Leuven, Department of Neurosurgery, Herestraat 49, Leuven, Belgium

Probabilistic maps and reproducibility of the pyramidal tract by diffusion tensor imaging, mingguo qiu', Qiyu Li ${ }^{1}$, Guangjiu Liu', Bing Xie ${ }^{2}$, Jian Wang ${ }^{2}$, Shaoxiang Zhang ${ }^{I},{ }^{l}$ Department of Anatomy, Third Military Medical University, Chongqing, China, ${ }^{2}$ Department of Anatomy, Third Military Medical University, Chongqing, China, ${ }^{3}$ Department of Anatomy, Third Military Medical University, Chongqing, China, ${ }^{4}$ Department of Radiology, Southwest Hospital, Third Military Medical University, Chongqing, China, ${ }^{5}$ Department of Radiology, Southwest Hospital, Third Military Medical University, Chongqing, China, ${ }^{6}$ Department of Anatomy, Third Military Medical University, Chongqing, China

DTI Spatial Unbiased Infratentorial Template based on MPRAGE SUIT, Goran Vucurevic ${ }^{l}$, Paulo Dellani ${ }^{2}$, Andrea Kronfeld ${ }^{l}$, Andreas Konrad ${ }^{3}$, Peter Stoeter ${ }^{l},{ }^{1}$ Institute of Neuroradiology, Mainz, Germany, ${ }^{2}$ University Clinic, Department of Neurology, Mainz, Germany, ${ }^{3}$ University Clinic, Department of Psychiatry, Mainz, Germany

Evaluation of DTI fiber tracking strategy for clinical use, Perrine Clarisse ${ }^{1,2,3}$, Jean-Albert Lotterie $^{1,2,3}$, Matthieu Delion ${ }^{1}$, Kader Boulanouar ${ }^{1,2,3}$, Florent Aubry ${ }^{1,2,3}$, Pierre Celsis ${ }^{1,2,3}$, Isabelle Berry ${ }^{1,2,3},{ }^{1}$ INSERM U825, Toulouse, France, ${ }^{2}$ Université Toulouse III Paul Sabatier, Toulouse, France, ${ }^{3} \mathrm{CHU}$ de Toulouse, Toulouse, France

Kernohan's Notch Phenomenon demonstrated by Diffusion Tensor Imaging and Transcranial Magnetic Stimulation, Ji heon Hong ${ }^{1}$, Sung Ho Jang ${ }^{2}$, Sang Ho Ahn' ${ }^{2}$, Dong Seok Yang ${ }^{2},{ }^{1}$ Department of Physical Therapy, Graduate School of Rehabilitation Science Daegu University, Daegu, South Korea, ${ }^{2}$ Department of Physical Medicine and Rehabilitation, School of Medicine, Yeungnam University, Deagu, South Korea 
In vivo tract tracing of cortico-cortical connections in humans: a combined study of CCEP and Probabilistic Diffusion Tractography, Riki Matsumoto ${ }^{1}$, Nobukatsu Sawamoto ${ }^{2}$, Shin-ichi Urayama ${ }^{2}$, Nobuhiro Mikuni ${ }^{3}$, Takashi Hanakawa ${ }^{4}$, Timothy Behrens ${ }^{5}$, Akio Ikeda ${ }^{1}$, Ryosuke Takahashi ${ }^{1}$, Hidenao Fukuyama ${ }^{2}{ }^{1}$ Department of Neurology, Kyoto University Graduate School of Medicine, Kyoto, Japan,

${ }^{2}$ Human Brain Research Center, Kyoto University Graduate School of Medicine, Kyoto, Japan,

${ }^{3}$ Department of Neurosurgery, Kyoto University Graduate School of Medicine, Kyoto, Japan, ${ }^{4}$ Dept. Cortical Functional Disorder, National Institute of Neuroscience, National Center of Neurology,

Kodaira, Japan, ${ }^{5}$ FMRIB, Oxford University, Oxford, United Kingdom

Connectivity characteristics of eloquent cortical language sites, Stephen Dreyer ${ }^{l}$, Timothy Ellmore ${ }^{l}$, Thomas $O^{\prime}$ Neill $^{1}$, Giridhar Kalamangalam ${ }^{2}$, Nitin Tandon ${ }^{I},{ }^{1}$ Department of Neurosurgery, The University of Texas Medical School, Houston, USA, ${ }^{2}$ Department of Neurology, The University of Texas Medical School, Houston, USA

\section{SENSORY SYSTEMS Auditory/Vestibular}

The ability of absolute pitch and the cortical structure., Nobuko Hara ${ }^{l}$, Kimihiro Nakamura ${ }^{l}$, Chihiro Kuroki ${ }^{2}$, Yoshihiro Takayama ${ }^{1}$, Seiji Ogawa ${ }^{2},{ }^{I}$ Department of Speech Physiology, The University of Tokyo, Tokyo, Japan, ${ }^{2}$ Ogawa Laboratories for Brain Function Research, Tokyo, Japan

Regional specialization for processing auditory complexity: ALE meta-analysis and fMRI validation, Fabienne Samson ${ }^{1}$, Pascal Belin ${ }^{2}$, Alain Toussaint ${ }^{1}$, Laurent Mottron ${ }^{1}$, Thomas A. Zeffiro ${ }^{3}$, ${ }^{1}$ Hôpital Rivière-des-Prairies, University of Montréal, Montréal, Canada, ${ }^{2}$ Centre for Cognitive Neuroimaging \& Department of Psychology, University of Glasgow, Glasgow, United Kingdom, ${ }^{3}$ Neural Systems Group, Massachussetts General Hospital, Boston, USA

Involvement of Limbic Brain Centers in Sound Perception in Humans, Dave Langers ${ }^{1,2}$, Jennifer Melcher ${ }^{1,3},{ }^{1}$ Eaton-Peabody Laboratory, Massachusetts Eye and Ear Infirmary, Boston, USA, ${ }^{2}$ University Medical Center Groningen, Groningen, Netherlands, ${ }^{3}$ Tinnitus Research Initiative, Regensburg, Germany

Absolute pitch perception depends on morphology of the right Heschl's gyrus, Peter Schneider ${ }^{1,2}$, Martina Wengenroth ${ }^{2}$, Maria Blatow ${ }^{2}$, Konstantin Bodamer $^{3}$, Christoph Stippich ${ }^{1}$, Doris Geller ${ }^{3}$, Andre Rupp ${ }^{2}$, ${ }^{I}$ Dept. of Neurology, University Hospital Heidelberg, Heidelberg, Germany, ${ }^{2} 2$ Div. of Neuroradiology, University Hospital Heidelberg, Heidelberg, Germany, ${ }^{3}$ University of Music and Performing Arts, Mannheim, Germany

\section{SENSORY SYSTEMS} Tactile/Somatosensory

Neurophysiological basis of localization and delocalization of fMRI activation patterns, Natasja J.G. Maandag ${ }^{1,2}$, Daniel Coman ${ }^{l}$, Basavaraju G. Sanganahalli ${ }^{1}$, Peter Herman ${ }^{l}$, Arien J. Smith ${ }^{1,3}$, Hal Blumenfeld ${ }^{4}$, Robert G. Shulman ${ }^{1}$, Fahmeed Hyder ${ }^{1,5}$, IDiagnostic Radiology, Yale University, New Haven, USA, ${ }^{2}$ Anaesthesiology, University Medical Centre, Nijmegen, Netherlands, ${ }^{3}$ Neurosurgery, Mount Sinai Hospital, New York, USA, ${ }^{4}$ Neurology, Yale University, New Haven, USA, ${ }^{5}$ Biomedical Engineering, Yale University, New Haven, USA

Neural correlates of phantom limb perception in lower limb amputee patients during a sensation task., Erick H Pasaye ${ }^{1,5}$, Sarael Alcauter ${ }^{4,5}$, Maria del Refugio Pacheco ${ }^{3}$, Jorge Paz ${ }^{1}$, Roberto Mercadillo $^{2}$, Erika Aguilar ${ }^{1}$, Maria De Iturbe ${ }^{1}$, Perla M. Salgado ${ }^{1}$, Fernando A. Barrios $^{2},{ }^{\prime}$ Instituto Nacional de Neurología y Neurocirugía MVS, Mexico DF, Mexico, ${ }^{2}$ Instituto de Neurobiología, Universidad Nacional Autónoma de México, Queretaro, Mexico, ${ }^{3}$ Instituto Nacional de la Rehabilitación, Mexico DF, Mexico, ${ }^{4}$ Instituto Nacional de Psiquiatría Ramón de la Fuente, Mexico DF, Mexico, ${ }^{5}$ Posgrado en Ciecias Biomedicas UNAM, Mexico DF, Mexico

Dynamic texture perception for dominant and non-dominant hands within individuals: an fMRI study in adult healthy volunteers., Leeanne Carey $^{1,2}$, David Abbott ${ }^{3}$, Matt Harvey ${ }^{1,3}$, Aina Puce ${ }^{1,4}$, Rudiger Seitz ${ }^{1,5},{ }^{1}$ National Stroke Research Institute, Melbourne, Australia, ${ }^{2}$ LaTrobe University, Melbourne, Australia, ${ }^{3}$ Brain Research Institute, Melbourne, Australia, ${ }^{4}$ Center for Advanced Imaging, Morgantown, USA, ${ }^{5}$ University Hospital, Duesseldorf, Germany

Variability of somatosensory cortex localization over different fMRI centers - a multicenter patient study, Roland Beisteiner ${ }^{l}$, Nicolaus Klinger ${ }^{1}$, Markus Aichhorn ${ }^{2}$, Thomas Foki ${ }^{l}$, Alexander Geißler $^{1}$, Martin Kronbichler ${ }^{2}$, Janpeter Nickel ${ }^{4}$, Jakob Rath ${ }^{1}$, Christian Siedentopf ${ }^{3}$, Wolfgang Staffen ${ }^{2}$, 
Thomas Steinkellner ${ }^{1}$, Michael Verius ${ }^{3}$, Stephan Felber ${ }^{5}$, Stefan Golaszewski ${ }^{2}$, Florian Koppelstaetter ${ }^{3}$, Rüdiger Seitz ${ }^{4},{ }^{I}$ Study Group Clinical fMRI, MR Center of Excellence, Department of Neurology, Medical University of Vienna, Vienna, Austria, ${ }^{2}$ Department of Neurology, Christian Doppler Clinic and Center for Neurocognitive Research, Paracelsus Private Medical University, Salzburg, Austria, ${ }^{3}$ Department of Radiology, Subdivision Neuroradiology, Medical University of Innsbruck, Innsbruck, Austria, ${ }^{4}$ Department of Neurology, University Hospital Düsseldorf, Düsseldorf, Germany, ${ }^{5}$ Institute for Diagnostic Radiology, Stiftungsklinikum Mittelrhein, Koblenz, Koblenz, Germany

EEG source imaging and single-trial statistical analysis of distributed inverse solutions reveals late activation of insular cortex during light mechanical stimulation of the human hairy skin., Johan Wessberg, Goteborg University, Dept. of Physiology, Goteborg, Sweden

\section{SENSORY SYSTEMS Vision}

Functional MRI and DTI tractography in visual pathology, Christine Boucard ${ }^{l}$, Masahiro Ida ${ }^{2}$, Masaki Yoshida ${ }^{l}$, Takehiko Nagao ${ }^{3}$, Takaaki Haral, Thien Huong Nguyen ${ }^{4}$, Jean Louis Stievenart ${ }^{4}$, Chistophe Habas ${ }^{4}$, Takuya Shiba ${ }^{l}$, Kenji Kitaharal, Marie Therese Iba-Zizen ${ }^{4}$, Emmanuel Alain Cabanis ${ }^{4}$, Tohru Noda ${ }^{5}$, Hiroshi Tsuneoka ${ }^{l},{ }^{1}$ Department of Ophthalmology, The Jikei University School of Medicine, Tokyo, Japan, ${ }^{2}$ Department of Radiology, Tokyo Metropolitan Health and Medical Treatment Corporation Ebara Hospital, Tokyo, Japan, ${ }^{3}$ Department of Neurology, Tokyo Metropolitan Health and Medical Treatment Corporation Ebara Hospital, Tokyo, Japan, ${ }^{4}$ Service de Neuro-Imagerie, Centre Hospitalier National d'Ophtalmologie des XV-XX, Paris, France, ${ }^{5}$ Department of Ophthalmology, National Tokyo Medical Center, Tokyo, Japan

Topography of responses to colour and luminance in human subcortical visual pathways as revealed by high-resolution fMRI at 7T, Marcus Grueschow ${ }^{1,2,3}$, Jörg Stadler ${ }^{4}$, Claus Tempelmann ${ }^{3}$, Hans-Jochen Heinze, Jochem Rieger ${ }^{3}$, Oliver Speck ${ }^{5}$, John-Dylan Haynes ${ }^{2},{ }^{1}$ Max Planck Institute for Cognitive and Brain Sciences, Leipzig, Germany, ${ }^{2}$ Bernstein Center for Computational Neuroscience, Berlin, Germany, ${ }^{3} 3$ Dept. of Neurology II, Otto-von-Guericke University, Magdeburg, Germany, ${ }^{4}$ NonInvasive Imaging Lab, Leibniz-Institut für Neurobiologie, Magdeburg, Germany, ${ }^{5}$ Dept. of Biomedical Magnetic Resonance, Institute for Experimental Physics, Magdeburg, Germany

Fixation Based Event Related (FIBER) fMRI; Using individual fixations as events to reveal cortical processing, Jan Bernard C. Marsman ${ }^{1,2}$, Remco Renken ${ }^{2}$, Frans W. Cornelissen ${ }^{1,2}$, ${ }^{1}$ Laboratory of Experimental Ophthalmology, University Medical Center Groningen/University of Groningen, Groningen, Netherlands, ${ }^{2} B C N$ NeuroImaging Center, School of Behavioural and Cognitive

Neurosciences, University Medical Center Groningen/ University of Groningen, Groningen, Netherlands

The coding of colour, motion and their conjunction: revisited using pattern classifier analysis., Kiley Seymour ${ }^{l}$, Colin Clifford ${ }^{l}$, Nikos Logothetis ${ }^{2}$, Andreas Bartels ${ }^{2},{ }^{1}$ University of Sydney, Sydney, Australia, ${ }^{2}$ MPI for Biological Cybernetics, Tuebingen, Germany

Electrical Stimulation, Recording and BOLD fMRI of the Human Anterior Color Center, Michael Beauchamp ${ }^{1}$, Dona Murphey ${ }^{2}$, Daniel Yoshor ${ }^{2},{ }^{1}$ Univ of Texas Med School at Houston, Houston, USA, ${ }^{2}$ Baylor College of Medicine, Houston, USA

Positive and negative changes in motion coherence from adapted state always elicit positive BOLD responses in hV4., Mauro Costagli ${ }^{1,2}$, Kenichi Ueno ${ }^{1}$, Pei Sun ${ }^{l}$, Xiaohong Wan ${ }^{1}$, Emiliano Ricciardi ${ }^{2}$, Pietro Pietrini ${ }^{2}$, Keiji Tanaka ${ }^{1}$, Kang Cheng ${ }^{l},{ }^{l}$ RIKEN Brain Science Institute, Wako-shi, Japan,

${ }^{2}$ University of Pisa, Pisa, Italy

Inhibition of single word identification with TMS over dorsal area V5/MT+, Robin Laycock ${ }^{l}$, Sheila Crewther ${ }^{1}$, Paul Fitzgerald ${ }^{2}$, David Crewther ${ }^{3},{ }^{1}$ La Trobe University, Melbourne, Australia, ${ }^{2}$ Monash University and The Alfred Hospital, Melbourne, Australia, ${ }^{3}$ Swinburne University, Melbourne, Australia

Resonance properties of human occipital, parietal and frontal cortical areas studied by Transcranial Magnetic Stimulation (TMS) combined with high density EEG (hd-EEG)., Mario Rosanova ${ }^{I}$, Adenauer Casali, Valentina Bellina ${ }^{I}$, Federico Resta ${ }^{2}$, Maurizio Mariotti ${ }^{l}$, Marcello Massimini ${ }^{l},{ }^{l}$ Department of Clinical Science, University of Milan, Ospedale Luigi Sacco, Milan, Italy, ${ }^{2}$ Division of Radiology, Ospedale Luigi Sacco, Milan, Italy

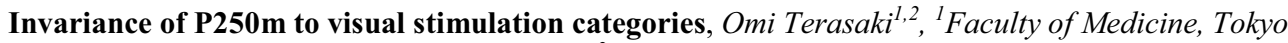
Medical and Dental University, Tokyo, Japan, ${ }^{2}$ Kurita Hospital, Kanagawa, Japan 
11:30 - 12:30 You Yangs Hall (Level 3)

\section{COGNITION \& ATTENTION}

Executive Function

Neural correlates of response inhibition deficits in schizophrenia - an fMRI and ERP study., Matthew Hughes ${ }^{1,2}$, William Fulham ${ }^{1,2}$, Janette Smith ${ }^{1}$, Johanna Badcock ${ }^{2,3,4}$, Patricia Michie ${ }^{1,2}$,

${ }^{1}$ University of Newcastle, Callaghan, Australia, ${ }^{2}$ Schizophrenia Research Institute, Sydney, Australia,

3 TH-AM

${ }^{3}$ Centre for Clinical Research in Neuropsychiatry, Perth, Australia, ${ }^{4}$ University of Western Australia,

Perth, Australia

Differential Prefrontal and Parietal Function in Spatial Working Memory, Tim Silk ${ }^{1,2,3}$, Cattram Nguyen ${ }^{3}$, Maree Farrow ${ }^{3}$, Alasdair Vance ${ }^{2}$, Ross Cunnington ${ }^{1,3},{ }^{1}$ School of Psychology and Queensland Brain Institute, University of Queensland, Brisbane, Australia, Australia, ${ }^{2}$ Academic Child Psychiatry Unit, Department of Paediatrics, University of Melbourne, Royal Children's Hospital, Murdoch Children's Research Institute, Melbourne, Australia, Australia, ${ }^{3}$ Howard Florey Institute, University of Melbourne, Melbourne, Australia, Australia

cTBS impairs dorsolateral prefrontal cortex function during sorting task and affects striatal dopamine: a TMS-PET study, $J i$ Hyun $\mathrm{Ko}^{l}$, Oury Monchi ${ }^{2}$, Alain Ptito ${ }^{1}$, Antonio P. Strafella ${ }^{3,4}$, ${ }^{1}$ Montreal Neurological Institute, McGill University, Montréal, Canada., Montreal, ${ }^{2}$ Functional Neuroimaging Unit, Geriatric's Institute, University of Montréal, Canada., Montreal, ${ }^{3}$ Toronto Western Research Institute and Hospital, University of Toronto, Canada., Toronto, ${ }^{4}$ PET Imaging Centre, Centre for Addiction and Mental Health, University of Toronto, Canada, Toronto,

Activity in anterior cingulate and parietal cortex predicts activity in prefrontal cortex, Justin Vincent $^{1,2}$, Abraham Snyder ${ }^{2}$, Lawrence Cabusora ${ }^{2}$, Michael Fox ${ }^{2}$, Randy Buckner ${ }^{1,3}$, Marcus Raichle ${ }^{2}$, ${ }^{1}$ Harvard University, Cambridge, USA, ${ }^{2}$ Washington University, St. Louis, USA, ${ }^{3}$ Howard Hughes Medical Institute, Cambridge, USA

Extracting Consistent Activated Patterns of Eyes Open and Eyes Closed Resting State fMRI Data by Independent Component Analysis, Mohammad Ali Oghabian ${ }^{l}$, Ameneh Boroumand ${ }^{l}$, Hajir Sikaroodi ${ }^{2}$, Ali Reza Ahmadian ${ }^{1},{ }^{1}$ Research Center for Sciences and Technology in Medicine, Tehran University/Medical Sciences, Tehran, Iran, ${ }^{2}$ Neurology Group, Shariaty Hospital, Tehran University /Medical Sciences, Tehran, Iran

\section{COGNITION \& ATTENTION Cognitive Development}

Neural encoding of perceptual decision making without awareness: Challenges for signal detection models of perception, Stefan Bode ${ }^{l}$, John-Dylan Haynes ${ }^{1,2},{ }^{l}$ Max Planck Institute for Human Cognitive and Brain Sciences, Leipzig, Germany, ${ }^{2}$ Bernstein Center for Computational Neuroscience Berlin, Charité - Universitätsmedizin, Berlin, Germany

\section{COGNITION \& ATTENTION}

\section{Executive Function}

Post-error performance optimization by modulation of goal-relevant sensory processing, Joseph $\mathrm{A}$. King $^{2}$, D. Yves von Cramon ${ }^{1,2}$, Markus Ullsperger ${ }^{1,2},{ }^{1}$ MPI for neurological research, Cologne, Germany, ${ }^{2}$ MPI for Human Cognitive and Brain Sciences, Leipzig, Germany

Effective Connectivity during Task Set Reconfiguration, Rei Akaishi, Yosuke Morishima, Vivian Rajeswaren, Katsuyuki Sakai, Grad. Sch. of Medicine, Univ. of Tokyo, Tokyo, Japan

Segregation of Posterior Inferior Frontal Gyrus and Inferior Frontal Junction Revealed by Modified Go/No-Go Task, Junichi Chikazoe, Koji Jimura, Tomoki Asari, Ken-ichiro Yamashita, Hiroki Morimoto, Satoshi Hirose, Yasushi Miyashita, Seiki Konishi, The Univ of Tokyo Sch of Med, Tokyo, 
A functional magnetic resonance imaging study in the patients with obsessive-compulsive disorder during task-switching paradigm before and after 4-month treatment, $\mathrm{Ji}$ Yeon Han ${ }^{\mathrm{I}}$, Do-Hyung $\mathrm{Kang}^{2}$, Bon-Mi Gul, Wi Hoon Jung ${ }^{l}$, Ji-Young Park ${ }^{l}$, Jung-Seok Choi ${ }^{2}$, Chi-Hoon Choi ${ }^{3}$, Jong-Min Lee ${ }^{3}$, Jun Soo Kwon ${ }^{1,2},{ }^{1}$ Interdisciplinary Program in Brain Science and in Cognitive Science, Seoul National University, Seoul, South Korea, ${ }^{2}$ Department of Psychiatry, Seoul National University College of Medicine, Seoul, South Korea, ${ }^{3}$ Department of Biomedical Engineering, Hanyang University, Seoul, South Korea

Optimizing anticipatory task-set reconfiguration., Frini Karayanidis ${ }^{1,2,3}$, Dearne Sanday ${ }^{l}$, Sharna Jamadar ${ }^{1,2}$, Robyn Loder ${ }^{I},{ }^{I}$ Functional Neuroimaging Laboratory, Newcastle, Australia, ${ }^{2}$ Schizophrenia Research Institute, Sydney, Australia, ${ }^{3}$ Hunter Medical Research Institute, Newcastle, Australia

Context determines neural correlates of deviant detection, Andreja Bubic ${ }^{1,3}$, D. Yves von Cramon ${ }^{1,4}$, Thomas Jacobsen ${ }^{2}$, Erich Schröger ${ }^{2}$, Ricarda I. Schubotz ${ }^{1,4}$, ' Max Planck Institute for Human Cognitive and Brain Sciences, Leipzig, Germany, ${ }^{2}$ BioCog-Cognitive and Biological Psychology, Institute of Psychology I, University of Leipzig, Leipzig, Germany, ${ }^{3}$ University of Leipzig, Leipzig, Germany, ${ }^{4}$ Max Planck Institute for Neurological Research, Cologne, Germany

The great mistake: brain responses to own and observed errors during cooperation and competition, Ellen R.A. de Bruijn ${ }^{l, 2}$, D. Yves von Cramon', Markus Ullsperger ${ }^{1},{ }^{l}$ Max Planck Institute for Human Cognitive and Brain Sciences, Leipzig, Germany, ${ }^{2}$ Nijmegen Institute for Cognition and Information (NICI), Radboud University, Nijmegen, Netherlands

Rule-Selection and action-selection have a shared neuroanatomical basis in the human prefrontal and parietal cortex., James Rowe ${ }^{1,2,3}$, Laura Hughes ${ }^{1,2}$, Doris Eckstein ${ }^{1,2}$, Adrian Owen ${ }^{2,3}$, ${ }^{1}$ Department of Clinical Neurosciences, Cambridge University, Cambridge, United Kingdom, ${ }^{2}$ MRC Cognition and Brain Sciences Unit, Cambridge, United Kingdom, ${ }^{3}$ MRC Behavioural and Clinical Neurosciences Institute, Cambridge, United Kingdom

Strategic changes in cognitive control across the adult lifespan., Lisa Whitson ${ }^{1}$, Frini Karayanidis ${ }^{1,2,3}$ Pat Michie ${ }^{1,2,3},{ }^{1}$ Functional Neuroimaging Laboratory, Newcastle, Australia, ${ }^{2}$ Schizophrenia Research Institute, Sydney, Australia, ${ }^{3}$ Hunter Medical Research Institute, Newcastle, Australia

\section{COGNITION \& ATTENTION}

\section{Perception, Imagery, Awareness}

Face representation in the categorical level, Yulwan Sung, Seiji Ogawa, Yoshiaki Someya, Masayuki Kamba, Ogawa Laboratories For Brain Function Research, Tokyo, Japan

The distinct neural network involved in pitch labelling of absolute pitch musicians, Carolyn $W u$, Ian Kirk, Jeff Hamm, Vanessa Lim, Research Centre for Cognitive Neuroscience, Department of Psychology, University of Auckland, Auckland, New Zealand

FMRI study on risk perception for driving task presented as video images, Makoto Takahashi ${ }^{l}$, Tomomi Aboshi ${ }^{1}$, Naoki Miura ${ }^{2}$, Hiroshi Ota ${ }^{3}$, Ryuta Kawashima ${ }^{4}$, Toshio Wakabayashi,

${ }^{I}$ Department of Quantum Science and Energy Engineering, Tohoku University, Sendai, Japan,

${ }^{2}$ Department of Intelligence Mechanical Systems Engineering, Kochi University of Technology, Kochi, Japan, ${ }^{3}$ Faculty of Engineering, Tohoku Institute of Technology, Sendai, Japan, ${ }^{4}$ Department of Functional Brain Imaging, Institute of Development, Aging and Cancer (IDAC), Tohoku University, Sendai, Japan

Cortical mechanism of reality monitoring (monitoring of perceptual knowledge congruency $\boldsymbol{\&}$ Agency), Yukihito Yomogidal, Motoaki Sugiura ${ }^{1,2}$, Yuko Sassa ${ }^{1,3}$, Keisuke Wakusawa ${ }^{1,4}$, Atsushi Sekiguchi $^{l}$, Ai Fukushimal, Hikaru Takeuchi ${ }^{1}$, Kaoru Horie ${ }^{5}$, Shigeru Sato ${ }^{5}$, Ryuta Kawashima ${ }^{1,3}$, ${ }^{I}$ Department of Functional Brain Imaging, Institute of Development, Aging and Cancer, Tohoku University, Sendai, Japan, ${ }^{2}$ Department of Cerebral Research, National Institute for Physiological Sciences, Okazaki, Japan, ${ }^{3}$ Research Institute of Science and Technology for Society, Japan Science and Technology Corporation, Kawaguchi, Japan, ${ }^{4}$ Department of Pediatrics, Tohoku University Graduate School of Medicine, Sendai, Japan, ${ }^{5}$ GSIGS, Tohoku Univercity, Sendai, Japan

Activation of visual cortex using crossmodal retinotopic mapping, Lotfi Merabet ${ }^{l}$, Dorothe Poggel ${ }^{2}$, William Stern ${ }^{l}$, Ela Bhatt ${ }^{l}$, Christopher Hemond ${ }^{l}$, Sara Maguire ${ }^{l}$, Peter Meijer ${ }^{3}$, Alvaro PascualLeone ${ }^{l},{ }^{I}$ Berenson-Allen Center for Noninvasive Brain Stimulation, Dept. of Neurology, BIDMC, Harvard Medical School,, Boston, USA, ${ }^{2}$ Center for Biomedical Imaging, Boston University Medical Center, Boston, USA, ${ }^{3}$ Developer of The vOICe,, Netherlands
39 TH-AM

43 TH-AM

47 TH-AM

51 TH-AM*

55 TH-AM

59 TH-AM

63 TH-AM

67 TH-AM

71 TH-AM

75 TH-AM

79 TH-AM 
The prefrontal cortex accumulates object evidence through differential connectivity to the visual and auditory cortices, Uta Noppeney, Dirk Ostwald, Mario Kleiner, Sebastian Werner, Max Planck Institute for biological Cybernetics, Tuebingen, Germany

Large scale neural synchrony correlates with visibility, local gamma oscillation with top-down representations during perceptual hysteresis, Lucia Melloni ${ }^{1,2}$, Notger Müller ${ }^{1,2}$, Wolf Singer ${ }^{3,4}$, Eugenio Rodriguez, ${ }^{3,5},{ }^{1}$ Cognitive Neurology Unit, Johann Wolfgang Goethe-University, Frankfurt am Main, Germany, ${ }^{2}$ Brain Imaging Center, Frankfurt am Main, Germany, ${ }^{3}$ Department of Neurophysiology, Max Planck Institute for Brain Research, Frankfurt am Main, Germany, ${ }^{4}$ Frankfurt Institute for Advanced Studies, Johann Wolfgang Goethe University, Frankfurt am Main, Germany, ${ }^{5}$ Laboratorio de Neurociencias, Escuela de Psicología, Pontificia Universidad Católica de Chile, Santiago, Chile

Self-Identification and empathy modulate error related brain activity during the observation of penalty shots between friend and foe, Roger Newman-Norlund ${ }^{l, 2}$, Shanti Ganesh ${ }^{l}$, Hein van Schie ${ }^{l}$, Ellen de Bruijn', Harold Bekkering ${ }^{1,2}$, ' Nijmegen Institute for Cognition and Information, Nijmegen, Netherlands, ${ }^{2}$ F.C. Donders Center for Cognitive Neuroimaging, Nijmegen, Netherlands

\section{DISORDERS OF THE NERVOUS SYSTEM Addiction}

Subdivisions of Corpus Callosum by Cortico-Cortical Connectivity with Diffusion Spectrum Imaging (DSI) in Alcoholism, Chih-Jui Chen ${ }^{I}$, I-Chao Liu ${ }^{2}$, Hsiao-Lan Wang ${ }^{l}$, Wen-Yang Chiang ${ }^{l}$, Wen-Yih Isaac Tseng ${ }^{1,3},{ }^{l}$ Center for Optoelectronic Biomedicine, National Taiwan University Hospital, Taipei, Taiwan, ${ }^{2}$ School of Medicine, Fu Jen Catholic University, Taipei, Taiwan, ${ }^{3}$ Department of Medical Imaging, National Taiwan University Hospital, Taipei, Taiwan

\section{DISORDERS OF THE NERVOUS SYSTEM Autism}

Visuo-motor integration in autism: implication of mirror and canonical neurons, Joëlle MARTINEAU, Nadia HERNANDEZ, Jean-Philippe COTTIER, Christophe DESTRIEUX, Inserm U 619, TOURS, France

\section{DISORDERS OF THE NERVOUS SYSTEM Brain \& Spinal Cord Trauma}

Evolution of Diffusion Tensor Imaging Findings After Mild Traumatic Brain Injury: Implications for Treatment of a Major Public Health Problem, Michael Lipton ${ }^{1,2,3}$, Erik Gellella ${ }^{l}$, Tamar Gold ${ }^{l}$, Sophia Rodriguez ${ }^{l}$, Keivan Shifteh ${ }^{l},{ }^{I}$ Department of Radiology, Albert Einstein College of Medicine and Montefiore Medical Center, Bronx, USA, ${ }^{2}$ Department of Psychiatry and Behavioral Sciences, Albert Einstein College of Medicine, Bronx, USA, ${ }^{3}$ The Center fro Advanced Brain Imaging, The Nathan $S$. Kline Institute for Psychiatric Research, Orangeburg, USA

Diffusion Tensor Tractography-Based Quantification in Detecting Traumatic Axonal Injury and Predicting Long-term Outcome, Jun Wang ${ }^{l}$, Hervé Abdi ${ }^{l}$, Khamid Bakhadirov ${ }^{l}$, Michael Devous ${ }^{2}$, Roddy McColl ${ }^{2}$, Carlos Marquez de la Plata ${ }^{2}$,Carol Moore ${ }^{2}$, Ramon Diaz-Arrastia ${ }^{2},{ }^{1}$ University of Texas at Dallas, Richardson, USA, ${ }^{2}$ University of Texas Southwestern Medical Center, Dallas, USA

Longer tracts may be preferentially damaged in traumatic brain injury., Virginia Newcombe ${ }^{l}$, Doris Chatfield ${ }^{l}$, Joanne Outtrim ${ }^{l}$, Jonathan Coles ${ }^{l}$, M.Giulia Abate ${ }^{l}$, Sally Harding ${ }^{2}$, John Pickard $^{2,3}$, Peter Hutchinson ${ }^{3}$, T.Adrian Carpenter ${ }^{2}$, Guy Williams ${ }^{2}$, David Menon ${ }^{1,2}$, ${ }^{1}$ Division of Anaesthesia, Cambridge University, Cambridge, United Kingdom, ${ }^{2}$ Wolfson Brain Imaging Centre, Cambridge University, Cambridge, United Kingdom, ${ }^{3}$ Academic Department of Neurosurgery, Cambridge University, Cambridge, United Kingdom

\section{DISORDERS OF THE NERVOUS SYSTEM Developmental Disorders}

Graded Degeneration of White Matter Fiber Tracts in Hereditary Spastic Paraplegia with Thin Corpus Callosum: A voxel-wise comparison based on diffusion spectrum imaging, Su-Chun Huang ${ }^{1}$, Wen-Yang Chiang ${ }^{1}$, Ming-Kai Pan ${ }^{2}$, Yu-Chun Lo ${ }^{1}$, Li-Wei Kuo ${ }^{3}$, Ming-Jen Lee ${ }^{2}$, Wen-Yih Isaac Tseng ${ }^{1,4},{ }^{I}$ Center for Optoelectronic Biomedicine, National Taiwan University College of Medicine, Taipei, Taiwan, ${ }^{2}$ Department of Neurology, National Taiwan University Hospital, Taipei, Taiwan, 
${ }^{3}$ Interdisciplinary MRI/MRS Lab, Department of Electrical Engineering, National Taiwan University,

Taipei, Taiwan, ${ }^{4}$ Department of Medical Imaging, National Taiwan University Hospital, Taipei, Taiwan

BOLD Response to Visual Stimulus in Pediatric Medulloblastoma Patients during Treatment and Follow-up, Ping Zou, Thomas E. Merchant, Amar Gajjar, Robert J. Ogg, St. Jude Children's Research Hospital, Memphis, USA

Cingulate-Fronto-Insular Cortical Thinning and Decreased Gray Matter Density in 8 Year-old Children with Disruptive Behavior Disorders, Cherine Fahim ${ }^{1,4,5}$, Uicheul Yoon ${ }^{1,2}$, Alan Evans ${ }^{1,2,3}$, Daniel Perusse ${ }^{4,5},{ }^{1}$ Department of Neurology and Neurosurgery, Mc Gill University, Montreal, Canada, ${ }^{2}$ Department of Biomedical Engineering, McGill University, Montreal, Canada, ${ }^{3}$ Department of Medical Physics, McGill University, Montreal, Canada, ${ }^{4}$ Sainte Justine Hospital Research Centre, Montreal, Canada, ${ }^{5}$ Department of Psychiatry, University of Montreal, Montreal, Canada

Decreased Corpus Callosum Thickness in Attention Deficit / Hyperactivity Disorder (ADHD), Eileen Luders ${ }^{1}$, Katherine L. Narr ${ }^{1}$, Liberty S. Hamilton ${ }^{1}$, Owen R. Phillips ${ }^{1}$, Paul M. Thompson ${ }^{1}$, Jessica S. Valle, Melissa Del'Homme ${ }^{3}$, Tony Strickland ${ }^{4}$, Arthur W. Toga', James T. McCracken ${ }^{3}$, Jennifer G. Levitt ${ }^{3},{ }^{1}$ Laboratory of Neuro Imaging, Department of Neurology, UCLA School of Medicine, Los Angeles, USA, ${ }^{2}$ Argosy University, Orange County, USA, ${ }^{3}$ Departments of Psychiatry and Neurology, UCLA Semel Institute for Neuroscience and Human Behavior, Los Angeles, USA, ${ }^{4}$ David Geffen School of Medicine at UCLA, Los Angeles, USA

Longitudinal fMRI Study of Neural Systems for Reading in Pediatric Medulloblastoma Patients, Ping Zou ${ }^{l}$, Gayatri Patel ${ }^{l, 2}$, Fred Laningham ${ }^{l}$, Heather Conklin ${ }^{l}$, Thomas Merchant ${ }^{l}$, Amar Gajjarl, Robert Ogg ${ }^{1},{ }^{l}$ St. Jude Children's Research Hospital, Memphis, USA, ${ }^{2}$ Rhodes College, Memphis, USA

\section{DISORDERS OF THE NERVOUS SYSTEM Epilepsy}

Functional Asymmetry Based on Spatial Correspondence: Application to Presurgical Memory Lateralization in Epilepsy, Sandhitsu Das ${ }^{1}$, Dawn Mechanic-Hamilton ${ }^{2}$, Marc Korczykowski ${ }^{2}$, Brian Avants ${ }^{1}$, John Detre ${ }^{2}$, James Gee ${ }^{1}$, Paul Yushkevich ${ }^{1},{ }^{1}$ Penn Image Computing and Science Laboratory (PICSL), Department of Radiology, University of Pennsylvania, Philadelphia, USA, ${ }^{2}$ Center for Functional Neuroimaging, Department of Neurology, University of Pennsylvania, Philadelphia, USA

Analysis of synchrony for seizure prediction, Levin Kuhlmann ${ }^{l}$, Anthony Burkitt ${ }^{1,3}$, Mark Cook ${ }^{2,3}$, Karen Fuller ${ }^{2}$, David Grayden ${ }^{1,3}$, Iven Mareels ${ }^{I},{ }^{I}$ Department of Electrical and Electronic Engineering, The University of Melbourne, Melbourne, Australia, ${ }^{2}$ St. Vincent's Hospital of Melbourne, Melbourne, Australia, ${ }^{3}$ The Bionic Ear Institute, Melbourne, Australia

Functional epilepsy networks: EEG-fMRI in secondary generalized epilepsy with tonic seizures, Neelan Pillay ${ }^{1,2,3}$, Danny Flanagan ${ }^{1,2,3}$, David Abbott ${ }^{1,2.3}$, Graeme Jackson ${ }^{1,2,3}$, ${ }^{\text {B Brain Research Institute }}$ Melbourne, Australia, ${ }^{2}$ University of Melbourne, Melbourne, Australia, ${ }^{3}$ Austin Health, Melbourne, Australia

\section{TEMPORAL DYNAMICS OF THALAMIC ACTIVITY IN BILATERAL SYNCRONOUS}

POLYSPIKES DISCHARGES, Francesca Benuzzi ${ }^{l}$, Stefano Meletti ${ }^{l}$, Francesca Antonelli ${ }^{1}$, Valentina Farinelli ${ }^{l}$, Matteo Pugnaghi ${ }^{I}$, Fausta Lui ${ }^{2}$, Paolo Nichelli ${ }^{1},{ }^{I}$ Dip. Neuroscienze, Università di Modena e Reggio Emilia, Modena, Italy, ${ }^{2}$ Dip. Scienze Biomediche, Università di Modena e Reggio Emilia, Modena, Italy

Spatiotemporal patterns of high frequency oscillation from intracranial electroencephalography before and during seizure., Karen Fuller ${ }^{1}$, Dean Freestone ${ }^{1,2,3}$, Simon Vogrin $^{1}$, Alan Lai ${ }^{1,3}$, Levin Kuhlmann ${ }^{2}$, David Grayden ${ }^{2,3}$, Anthony Burkitt ${ }^{2,3}$, Iven Mareels ${ }^{2}$, Mark Cook ${ }^{l},{ }^{1}$ Department of Neurology, St Vincents Hospital, Melbourne, Australia, ${ }^{2}$ Department of Electrical and Electronic Engineering, The University of Melbourne, Melbourne, Australia, ${ }^{3}$ The Bionic Ear Institute, Melbourne, Australia

Regional Increase of the adenosine $A_{1}$ receptor binding in patients with intractable temporal lobe epilepsy. -A positron emission tomography study-, Tadashi Nariai ${ }^{1}$, Kiichi Ishiwata ${ }^{2}$, Yuichi Kimura ${ }^{2}$, Kenji Ishii ${ }^{2}$, Chihiro Hosoda ${ }^{1}$, Motoki Inaji ${ }^{1}$, Taketoshi Maehara ${ }^{I}$, Kikuo Ohno ${ }^{1},{ }^{I}$ Department of Neurosurgery, Tokyo Medical and Dental University, Tokyo, Japan, ${ }^{2}$ Positron Medical Center, Tokyo Metropolitan Institute of Gerontology, Tokyo, Japan 
EEG-fMRI of temporal lobe epilepsy: correspondence between BOLD responses and EEG source localization using ICA, Maurício Sercheli ${ }^{1}$, Elizabeth Bilevicius ${ }^{2}$, Helka Ozelo ${ }^{1}$, Andrea Alessio ${ }^{2}$, Fabrício Pereira', Jane Rondina ${ }^{2}$, Fernando Cendes ${ }^{2}$, Roberto Covolan ${ }^{1},{ }^{1}$ Neurophysics Group, Instituto de Física "Gleb Wataghin", Unicamp, Campinas, Brazil, ${ }^{2}$ Neuroimaging Laboratory, Faculdade de Ciências Médicas, Unicamp, Campinas, Brazil

\section{DISORDERS OF THE NERVOUS SYSTEM Stroke \& Recovery of Function}

Alien hand syndrome: fMRI characteristics of a single case, Michael Dreyer, Gerald McInerney, Royal Hobart Hospital, Hobart, Australia

Using DTI to map the pathoanatomical basis in diagnostic dyspraxia, Mareike M. Menz, Kathrin Reetz, Rolf Verleger, Christian Erdmann, Detlef Kömpf, Ferdinand Binkofski, Department of Neurology and NeuroImage Nord, University of Luebeck, Luebeck, Germany

A functional MRI study of working memory in Obstructive-Sleep-Apnea (OSA) patients before and after PAP treatment, Stefano Cappa ${ }^{1,2,3,4}$, Nicola Canessa ${ }^{2,1}$, Vincenza Castronovo ${ }^{5}$, Daniela Perani $^{1,3,4}$, Andrea Falini ${ }^{6,4}$, Monica Consonni ${ }^{1,3}$, Sara Marelli ${ }^{5}$, Alice Bruschi ${ }^{5}$, Alessandro Oldani ${ }^{5}$, Antonella Iadanza ${ }^{6}$, Mark Aloia ${ }^{7}$, Luigi Ferini-Strambi $i^{5,3},{ }^{1}$ Center for Cognitive Neuroscience, San Raffaele Scientific Institute, Milan, Italy, ${ }^{2}$ CRESA, Vita-Salute San Raffaele, Milan, ${ }^{3}$ Faculty of Psychology, Vita-Salute San Raffaele, Milan, Italy, ${ }^{4}$ CERMAC, Vita-Salute San Raffaele, Milan, Italy, ${ }^{5}$ Sleep-Disorders Center, San Raffaele Scientific Institute, Milan, Italy, ${ }^{6}$ Neuroradiology Unit, Milan, Italy, ${ }^{7}$ Department of Medicine, National Jewish Medical and Research Center, Denver, USA

EFFECTS OF TRANSCRANIAL ANODAL DIRECT CURRENT BRAIN POLARIZATION OF PRIMARY MOTOR CORTEX ON HAND FUNCTION IN STROKE PATIENTS, Myoung-Hwan $\mathrm{Ko}^{l}$, Sang-Hyoung Han ${ }^{l}$, Sung-Hee Park ${ }^{l}$, Jeong-Hwan Seo ${ }^{l}$, Yun-Hee Kim ${ }^{2},{ }^{l}$ Chonbuk National University Medical School \& Hospital, Jeonju, South Korea, ${ }^{2}$ Sungkyunkwan University School of Medicine \& Samsung Medical Center, Seoul, South Korea

Mapping activated microglia along the corticospinal tract in subcortial stroke, Basia Radlinska ${ }^{2}$ Sasan Ghinani ${ }^{1,2}$, Ilana Leppert ${ }^{1,3}$, Michael Sidel ${ }^{1,2}$, Dean Jolly, Jean-Paul Soucy ${ }^{1,3}$, Alexander Thiel ${ }^{1,2}$, ${ }^{I}$ Mc Gill University, Montreal, Canada, ${ }^{2}$ Lady Davis Institute for medical research, Montreal, Canada,

${ }^{3}$ Montreal Neurological Institute, Montreal, Canada

\section{EMOTION \& MOTIVATION Decision Making}

Dissociable neural mechanisms underlying delay discounting of financial gain and loss, Hansem Sohn ${ }^{1}$, Jaeseung Jeong ${ }^{1,2},{ }^{1}$ Department of Bio and Brain Engineering, Korea Advanced Institute of Science and Technology (KAIST), Daejeon, South Korea, ${ }^{2}$ Department of Psychiatry, College of Physicians and Surgeons, Columbia University and New York State Psychiatric Institute, New York, USA

Put Your Money Where Your Heart Is: Affective Influences on Investment Behavior, Julie L. Hall ${ }^{l}$, Oliver C. Schultheiss ${ }^{1,2},{ }^{1}$ University of Michigan, Ann Arbor, USA, ${ }^{2}$ Friedrich-Alexander University, Erlangen, Germany

Identifying emotional prosody while ignoring emotional semantic content: an fMRI study, Matthias Wittfoth ${ }^{1}$, Sonja A. Kotz ${ }^{2}$, Hans-Jochen Heinze ${ }^{3}$, Reinhard Dengler ${ }^{1}$, Christine Schroeder ${ }^{l},{ }^{1}$ Department of Neurology, Medical School Hannover, Hannover, Germany, ${ }^{2}$ Max Planck Institute for Human Cognitive and Brain Sciences, Leipzig, Germany, ${ }^{3}$ Department of Neurology II, Magdeburg, Germany

\section{EMOTION \& MOTIVATION \\ Emotional Learning}

How is the medial prefrontal cortex involved in advanced emotion learning, Satoshi Umeda ${ }^{1,2}$, Chihiro Kuroki ${ }^{3}$, Motoichiro Kato ${ }^{1,2}$, Yu-ri Terasawa ${ }^{1}$, Seiji Ogawa ${ }^{2},{ }_{1}$ Keio University, Tokyo, Japan, ${ }^{2}$ Ogawa Laboratories for Brain Function Research, Hamano Life Science Research Foundation, Tokyo, Japan, ${ }^{3}$ Oita University Faculty of Medicine, Oita, Japan

The effects of positive and negative emotions on insight problem solving, Kazuhisa $\mathrm{NIKI}^{\mathrm{l}}$, Michiko Sakaki ${ }^{1,2},{ }^{1}$ National Institute of Advanced Industrial Science and Technology, Tsukuba, Japan, ${ }^{2}$ Japan Society for the Promotion of Science, Tokyo, Japan
167 TH-AM

163 TH-AM

171 TH-AM

175 TH-AM

179 TH-AM

183 TH-AM

187 TH-AM

191 TH-AM

195 TH-AM

199 TH-AM

203 TH-AM 


\section{EMOTION \& MOTIVATION \\ Emotional Perception}

Subregional Investigation of Brain Responses During Music Perception using Functional MRI

Combined with Probabilistic Anatomical Maps, Isabella Mutschler ${ }^{1,2,3,4}$, Andreas SchulzeBonhage $^{3,4,5}$, Jürgen Hennig ${ }^{4,6}$, Oliver Speck ${ }^{7}$, Tonio Ball ${ }^{3,4,5},{ }^{1}$ Department of Psychology, University of Basel, Basel, Switzerland, ${ }^{2}$ Department of Psychiatry, University Hospital Basel, Basel, Switzerland, ${ }^{3}$ Epilepsy Center, University Hospital Freiburg, Freiburg, Germany, ${ }^{4}$ Freiburg Brain Imaging, University Hospital Freiburg, Freiburg, Germany, ${ }^{5}$ Bernstein Center for Computational Neuroscience, Freiburg, Germany, ${ }^{6}$ MR-Physics, University Hospital Freiburg, Freiburg, Germany, ${ }^{7}$ MR-Physics, University Hospital Magdeburg, Magdeburg, Germany

Neural Correlates of Emotion regulation in MDMA users, Gloria Roberts ${ }^{1}$, Hugh Garavan ${ }^{2},{ }^{1}$ TCIN, TCD, Dublin, Ireland, ${ }^{2}$ TCIN, TCD, Dublin, Ireland

Habituation of Brain Responses During Music Perception in an Amygdalo-Cortical Network, Birgit Wieckhorst ${ }^{1,2}$, Isabella Mutschler ${ }^{1,2,3,4}$, Juergen Hennig ${ }^{4,5}$, Oliver Speck ${ }^{6}$, Andreas SchulzeBonhage $^{3,4,7}$, Erich Seifritz ${ }^{8}$, Tonio Ball ${ }^{3,4,7}{ }^{1}$ Department of Psychiatry, University, Basel, Switzerland, ${ }^{2}$ Department of Psychology, University, Basel, Switzerland, ${ }^{3}$ Epilepsy-Center, University Hospital, Freiburg, Germany, ${ }^{4}$ Freiburg Brain Imaging, University Hospital, Freiburg, Germany, ${ }^{5}$ MR-Physics, University Hospital, Freiburg, Germany, ${ }^{6}$ MR-Physics, University Hospital, Magdeburg, Germany, ${ }^{7}$ Bernstein Center for Computational Neuroscience, University, Freiburg, Germany, ${ }^{8}$ University Hospital of Psychiatry, Bern, Switzerland

Neural activation to harsh faces among patients with Borderline Personality Disorder as a function of suicide history, Michael McCloskey ${ }^{I}$, K. Luan Phan ${ }^{2}$, Rose McCarron ${ }^{l}$, Eunice Chen ${ }^{l}$, Emil Coccaro ${ }^{1},{ }^{1}$ University of Chicago, Chicago, USA, ${ }^{2}$ University of Michigan, Ann Arbor, USA

Maturational changes in facial emotion ERPs from 6 to 30 years: conscious versus nonconscious perception., Donna M Palmer ${ }^{1,2}$, Evian Gordon ${ }^{1,3,4}$, Leanne M Williams ${ }^{1,3}$, ${ }^{I}$ The Brain Dynamics Centre, Westmead Millennium Institute, Westmead Hospital, Westmead, Sydney, Australia, ${ }^{2}$ School of Psychology, University of Sydney, Camperdown, Sydney, Australia, ${ }^{3}$ Psychological Medicine, Western Clinical School, University of Sydney, Westmead, Sydney, Australia, ${ }^{4}$ Brain Resource International Database, Brain Resource Company, Ultimo, Sydney, Australia

Cognitive emotion regulation and the serotonin transporter, Dina Schardt ${ }^{1}$, Susanne Erk ${ }^{1}$, Corinna Nuesser ${ }^{I}$, Markus Noethen ${ }^{2,3}$, Marcella Rietschel $^{4}$, Per Hoffmann ${ }^{2,3}$, Markus Skowronek ${ }^{4}$, Sven Cichon $^{2,3}$, Kerstin Ludwig, ${ }^{2,3}$, Thomas Goschke, Henrik Walter ${ }^{1},{ }^{1}$ Division of Medical Psychology, Department of Psychiatry, University Bonn, Bonn, Germany, ${ }^{2}$ Department of Genomics, Life \& Brain Center, University Bonn, Bonn, Germany, Institute of Human Genetics, University Bonn, Bonn, Germany, ${ }^{4}$ Central Institute for Mental Health, Division of Genetic Epidemiology in Psychiatry, Mannheim, Germany, ${ }^{5}$ Institute of Psychology II, Technische Universitaet Dresden, Dresden, Germany

The role of emotional arousal in the automatic processing of emotional stimuli under unattended condition: an ERP study, Renlai $\mathrm{Zhou}{ }^{l, 2}$, Xin $\mathrm{Li}^{l}{ }^{\mathrm{I}}{ }^{1}$ State Key Laboratory of Cognitive Neurosciences and Learning, Beijing Normal University, Beijing, China, ${ }^{2}$ Research Center for Learning Science, Southeast China University, Nanjing, China

EEG Default Mode Network: Music Modulation from Post Painful Stress, Weijia Feng, Andrew CN Chen*, Center for Higher Brain Functions, Capital Medical University, Beijing, China

Ghrelin has stress hormone-like effects on brain function., Alain Dagher ${ }^{l}$, Diane Bedrossian ${ }^{l}$, Saima Malik ${ }^{1}$, Francis McGlone ${ }^{2},{ }^{1}$ Montreal Neurological Institute, Montreal, Canada, ${ }^{2}$ Unilever R\&D, Wirral, United Kingdom

Emotion processing in adolescent anorexia nervosa: An Event Related Potential Study, Ainslie Hatch $^{1,2}$, Sloane Madden ${ }^{5}$, Michael Kohn ${ }^{3,5}$, Simon Clarke ${ }^{3}$, Touyz Stephen ${ }^{2}$, Lea Williams ${ }^{1},{ }^{1}$ The $^{2}$ Brain Dynamics Centre, Westmead Millennium Institute, Westmead Hospital, Sydney, Australia, ${ }^{2}$ School of Psychology, University of Sydney, Camperdown, Sydney, Australia, ${ }^{3}$ Centre for Research into Adolescent S' Health (CRASH), Adolescent Medicine, Children's Hospital at Westmead \& Westmead Hospital, Sydney, Australia, ${ }^{4}$ Psychological Medicine, University of Sydney, Westmead 
Hospital, Sydney, Australia, ${ }^{5}$ Psychological Medicine, Children's Hospital at Westmead, Westmead, Sydney, Australia

Using fMRI to differentiate neural activity in depressed adolescents in response to personallyrelevant emotional phrases, Nancy Adleman ${ }^{1,2}$, Kiki Chang ${ }^{1,3}$, Amy Garrett ${ }^{I}$, Naama Barnea-Goraly ${ }^{I}$, Meghan Howe ${ }^{3}$, Allan Reiss ${ }^{1},{ }^{1}$ Center for Interdisciplinary Brain Sciences Research, Stanford University School of Medicine, Stanford, USA, ${ }^{2}$ Interdisciplinary Program in Neurosciences, Stanford University School of Medicine, Stanford, USA, ${ }^{3}$ Pediatric Bipolar Disorders Program, Stanford University School of Medicine, Stanford, USA

EEG Default Mode Network: Gamma Activity enhanced from Reversed Perception, Weijia Feng, Andrew CN Chen*, Center for Higher Brain Functions, Capital Medical University,, Beijing, China

Does valence of emotional pictures affect cortico-limbic functional connectivity in healthy subjects? A feasibility fcMRI study at 3T, Naranjargal Dashdorj, Dorothee Auer, Academic Radiology, School of Medical and Surgical Sciences, University of Nottingham, Nottingham, United Kingdom

Dissociable Neural Responses to Tasting and Swallowing of Pleasant and Disgusting Beverages during fMRI., Mbemba Jabbi ${ }^{1}$, Christian Keysers ${ }^{2},{ }^{1}$ Section on integrative Neuroimaging, Cognitive Brain Disorders Branch, Genes Cognition and Psychosis Lab, National Institutes of Mental Health, 9000 Rockville Pike, Bethesda, 20892 MD, USA, ${ }^{2}$ Social Brain Lab, BCN Neuroimaging Center, University Medical Center Groningen, Antonius Deusinglaan 2, 9713 AW, Groningen, Netherlands

\section{IMAGING TECHNIQUES \& CONTRAST MECHANISM Anatomical MRI}

Optimization of Accuracy and Efficiency in Measuring T1 in the brain using Simulated Inversion Recovery MRI, Maryam Abaei ${ }^{1}$, Paul Morgan ${ }^{1}$, Dorothee Auer ${ }^{1}$, Christopher Tench ${ }^{2},{ }^{1}$ Division of Academic Radiology, School of Medical and Surgical Sciences, University of Nottingham, Nottingham, United Kingdom, ${ }^{2}$ Division of Clinical Neurology, School of Medical and Surgical Sciences, University of Nottingham, Nottingham, United Kingdom

Optimal image contrast to noise ratio and SPM5 parameters for Voxel-Based Morphometry, Herve Lemaitre, Alan Barnett, Fabio Sambataro, Heike Tost, Beth Verchinski, Vankata Mattay, CBDB/NIMH, Bethesda, USA

Multi-parameter mapping of the human brain at $1 \mathrm{~mm}$ resolution in less than 20 minutes, Nikolaus Weiskopf ${ }^{1}$, Gunther Helms ${ }^{2},{ }^{1}$ Wellcome Trust Centre for Neuroimaging, University College London, London, United Kingdom, ${ }^{2}$ MR-Research in Neurology and Psychiatry, Goettingen University, Goettingen, Germany

A phantom based method for the outer cortical surface reconstruction of pediatric brain, Junki Lee, Alan C. Evans, McConnell Brain Imaging Centre, Montreal Neurological Institute, McGill University, Montreal, Canada

Combined Brain Morphometry and Skull Imaging with FLUSTER, André van der Kouwe, Thomas Benner, Athinoula A. Martinos Center for Biomedical Imaging, Department of Radiology, Massachusetts General Hospital, Charlestown, USA

\section{IMAGING TECHNIQUES \& CONTRAST MECHANISM} Diffusion MRI

A Comparative Study Between Constrained Spherical Deconvolution and Deconvolution Sharpening Transformation on High Angular-Resolution Diffusion Imaging, Shiou-Ping Lee ${ }^{l}$, Jacques-Donald Tournier ${ }^{2}$, Christopher P Hess ${ }^{3}$, Li-Wei Kuo ${ }^{4}$, Chung-Ming Chen ${ }^{1}$, Wen-Yih Tseng, ${ }^{1}$ Institute of Biomedical Engineering, National Taiwan University, Taipei, Taiwan, ${ }^{2}$ Brain Research Institute, Melbourne, Austria, ${ }^{3}$ Department of Radiology, University of California-San Francisco, San Francisco, USA, ${ }^{4}$ Interdisciplinary MRI/MRS Lab, Department of Electrical Engineering, National Taiwan University, Taipei, Taiwan, ${ }^{5}$ Center for Optoelectronic Biomedicine, National Taiwan University Medical College, Taipei, Taiwan

Simulated diffusion dataset for multi-tensor fiber tractography, Arish Qazi ${ }^{1,2}$, Gordon Kindlmann ${ }^{2}$, Carl-Fredrik Westin ${ }^{2},{ }^{1}$ University of Copenhagen, Copenhagen, Denmark, ${ }^{2}$ Laboratory of Mathematics in Imaging, Harvard Medical School, Boston, USA 
DIFFUSION KURTOSIS IMAGING USING TURBOPROP DWI, Chu-Yu Lee ${ }^{l}$, Donglai Hou ${ }^{2}$, Lina Karam ${ }^{l}$, Josef Debbins ${ }^{2},{ }^{1}$ Arizona State University, Tempe, USA, ${ }^{2}$ St. Joseph's Hospital and Medical Center, Phoenix, USA

High Resolution DTI in Whole, Fixed, Human Brain Reveals Cortical Fibre Patterns That Correspond Well with Histological Stains, Jennifer $\mathrm{McNab}^{l}$, Natalie Voets ${ }^{l}$, Steven Chance ${ }^{l}$, Gwenaelle Douaud ${ }^{1}$, Ned Jenkinson ${ }^{2}$, Tipu Aziz ${ }^{2,3}$, Karla Miller ${ }^{l},{ }^{1}$ Department of Clinical Neurology, Oxford University, Oxford, United Kingdom, ${ }^{2}$ Department of Physiology Anatomy and Genetics, Oxford University, Oxford, United Kingdom, ${ }^{3}$ Department of Neurosurgery, Oxford University, Oxford, United Kingdom

In vivo localisation of fibre tracts: Optimisation of fibre tracking to reduce voxel misclassification, Jacques-Donald Tournier ${ }^{1,2}$, Fernando Calamante ${ }^{1,2}$, Alan Connelly ${ }^{1,2},{ }^{1}$ Brain Research Institute, Melbourne, Australia, ${ }^{2}$ Department of Medicine, University of Melbourne, Melbourne, Australia

\section{IMAGING TECHNIQUES \& CONTRAST MECHANISM Multi-modal Integration}

Transient and Steady-State Components of fMRI BOLD and MEG Signals from Somatosensory Cortex, Michael Marxen ${ }^{1,2}$, Tara L. Dawson ${ }^{1}$, Tim Bardouille ${ }^{1,3}$, Bernhard Ross ${ }^{1,3}$, Fred Tam ${ }^{1}$, Simon J. Graham ${ }^{1,2,3,4},{ }^{1}$ Rotman Research Institute, Baycrest Centre for Geriatric Care, Toronto, Canada, ${ }^{2}$ Heart \& Stroke Foundation Centre for Stroke Recovery, Toronto, Canada, ${ }^{3}$ Department of Medical Biophysics, University of Toronto, Toronto, Canada, ${ }^{4}$ Sunnybrook Health Sciences Centre, Toronto, Canada

Low-frequency artifacts in concurrent transcranial magnetic stimulation (TMS) and fMRI caused by leakage currents, Nikolaus Weiskopf ${ }^{l}$, Oliver Josephs ${ }^{1}$, Christian Ruff ${ }^{1,2}$, Felix Blankenburg ${ }^{l}$, Eric Featherstone ${ }^{l}$, Anthony Thomas ${ }^{3}$, Sven Bestmann ${ }^{l}$, Jon Driver ${ }^{1,2}$, Ralf Deichmann ${ }^{1,4}$, ${ }^{1}$ Wellcome Trust Centre for Neuroimaging, University College London, London, United Kingdom, ${ }^{2}$ Institute of Cognitive Neuroscience, University College London, London, United Kingdom, ${ }^{3}$ The Magstim Company Limited, Whitland, United Kingdom, ${ }^{4}$ University Hospital, Brain Imaging Center, Frankfurt, Germany

Validation of calibrated MRI using continuous-wave and time-domain near-infrared spectroscopic imaging, Claudine Gauthier ${ }^{1,2}$, Louis Gagnon ${ }^{2,3}$, Juliette Selb ${ }^{4}$, David Boas ${ }^{4}$, Frédéric Lesage ${ }^{2,3}$, Richard Hoge ${ }^{1,2},{ }^{1}$ Université de Montréal, Montreal, Canada, ${ }^{2}$ Institut de gériatrie de Montréal, Montreal, Canada, ${ }^{3}$ École Polytechnique de, Montreal, Canada, ${ }^{4}$ Martinos Center for Biomedical Imaging, Massachusetts General Hospital, Charlestown, USA

Realignment parameter informed artifact correction for simultaneous EEG-fMRI recordings, Matthias Moosmann ${ }^{l}$, Vinzenz Schönfelder ${ }^{l}$, Tom Eichele ${ }^{I}$, Helge Nordby ${ }^{I}$, Kenneth Hugdahl, ${ }^{I}$ Department of Biological and Medical Psychology, University of Bergen, Bergen, Norway, ${ }^{2}$ Division of Psychiatry, Haukeland University Hospital, Bergen, Norway

\section{IMAGING TECHNIQUES \& CONTRAST MECHANISM Optical Imaging/NIRS/MRS (magnetic resonance spectroscopy)}

Measurement of brain activation using near-infrared spectroscopy: comparison of principal components for signal changes between short and long source-detector spacings, Makoto Kato ${ }^{1,3}$, Sachiko Takahama ${ }^{2,3},{ }^{1}$ Biol. ICT Grp., Kobe Adv. ICT Res. Ctr., NICT, Kobe, Japan, ${ }^{2}$ Sch. of Frontier Biosci., Osaka Univ., Osaka, Japan, ${ }^{3}$ CREST, JST, Kawaguchi, Japan

Functional connectivity in adult humans revealed with diffuse optical tomography of oxy-, deoxy-, and total hemoglobin, Brian White ${ }^{1}$. Joseph Culver ${ }^{2},{ }^{I}$ Department of Physics and School of Medicine, Washington University, St. Louis, USA, ${ }^{2}$ Department of Radiology, Washington University School of Medicine, St. Louis, USA

\section{IMAGING TECHNIQUES \& CONTRAST MECHANISM} Perfusion MRI

Simulation of Adaptive Sequential Design for Optimal Scheduling of Continuous ASL Samples, Jingyi Xie , Daniel Gallichan ${ }^{I}$, Roger Gunn ${ }^{2}$, Peter Jezzard ${ }^{I},{ }^{I}$ FMRIB Centre, University of Oxford, Oxford, United Kingdom, ${ }^{2}$ Clinical Imaging Centre, GlaxoSmithKline, London, United Kingdom 


\section{IMAGING TECHNIQUES \& CONTRAST MECHANISM PET/SPECT}

Assessment of ${ }^{18}$ F-FET PET uptake kinetics using Independent Component Analysis and SVM (Support Vector Machine) signal approximation., Kader BOULANOUAR ${ }^{1,2}$, Pierre PAYOUX ${ }^{1,2,3}$, Alexandra BENOUAICH-AMIEL ${ }^{4}$, Mathieu TAFANI ${ }^{2,3}$,Emmanuel GRAS ${ }^{2,3}$, Jean-Paul ESQUERRE ${ }^{2,3}$ Pierre CELSIS ${ }^{1,2},{ }^{1}$ INSERM Unit825, Toulouse, France, ${ }^{2}$ University of Toulouse, Toulouse, France, ${ }^{3}$ Nuclear Medecine Dept, CHU Purpan, Toulouse, France, ${ }^{4}$ Neurology Dept, CHU Pitie-Salpetriere, Paris, France

A Comparison of Visual Assessment and NeuroStat analysis of PiB and FDG in the Differential Diagnosis of Alzheimerís disease., Gareth Jones ${ }^{l}$, Victor L Villemagne ${ }^{1,2}$, Graeme O'Keefe ${ }^{l}$, Sze-Ting Lee ${ }^{I}$, Colin Masters ${ }^{3}$, Chris Rowe ${ }^{I},{ }^{I}$ Dept of Nuclear Medicine and Centre for PET, Austin Health, Melbourne, Australia, ${ }^{2}$ Dept of Medicine and Pathology, Melbourne, Australia, ${ }^{3}$ The Mental Health Research Institute of Victoria, Melbourne, Australia

\section{LANGUAGE \\ Comprehension}

The components of a Theory-of-Mind cortical network during narrative comprehension, Robert Mason, Chantel Prat, Marcel Just, Carnegie Mellon University, Pittsburgh, USA

Semantic processing in Hindi-English bilinguals using functional neuroimaging, Rajani Sebastian ${ }^{l}$, Swathi Kiran ${ }^{1,2},{ }^{\prime}$ Department of Communication Sciences and Disorders, University of Texas at Austin, Austin, USA, ${ }^{2}$ Institute of Neuroscience, University of Texas at Austin, Austin, USA

Processing negative polarity items in the absence of directed attention: Evidence from

Magnetoencephalography, Graciela Tesan, Stephen Crain, Macquarie University, Sydney, Australia

Differential Brain Activation during Language Processing in Children Prenatally Exposed to Methamphetamine, S. Christopher Nuñez ${ }^{I}$, Mirella Dapretto ${ }^{2,5}$, Elizabeth O'Hare ${ }^{1,2}$, Lisa H. Lu ${ }^{3}$, Lorna Quandt ${ }^{1}$, Lynne Smith ${ }^{4}$, Mary O'Connor ${ }^{5}$, Susan Bookhemer ${ }^{5}$, Elizabeth Sowell ${ }^{1,2},{ }^{1}$ UCLA Laboratory of Neuro Imaging, Department of Neurology, Los Angeles, USA, ${ }^{2}$ UCLA Interdepartmental Program for Neuroscience, Los Angeles, USA, ${ }^{3}$ Roosevelt University, Department of Psychology, Chicago, USA, ${ }^{4}$ Harbor-UCLA Medical Center, Department of Pediatrics, Torrance, USA, ${ }^{5}$ UCLA Department of Psychiatry and Biobehavioral Sciences, Los Angeles, USA

Embedding at the sentence and verb levels: An fMRI study, Einat Shetreet ${ }^{l}$, Naama Friedmann ${ }^{2}$, Uri Hadar ${ }^{I},{ }^{I}$ Department of Psychology, Tel Aviv University, Tel Aviv, Israel, ${ }^{2}$ Language and Brain Lab, School of Education, Tel Aviv University, Tel Aviv, Israel

How priming enables us to understand speech in an impoverished context, Johannes Tuennerhoff, Uta Noppeney, Cognitive Neuroimaging Group, Max Planck Institute for Biological Cybernetics, Tuebingen, Germany

An Investigation of the Effects of Syntactic Complexity, Task Demand, and Rate of Speech Input on the Neural Correlates of Sentence Comprehension, Kathleen Brumm ${ }^{l}$, David Swinney ${ }^{2}$, Frank Haist ${ }^{2}$, Tracy Love ${ }^{1,2,3}$, ISDSU/ UCSD Joint Doctoral Program in Language and Communicative Disorders, San Diego, USA, ${ }^{2}$ University of California, San Diego, La Jolla, USA, ${ }^{3}$ San Diego State University, San Diego, USA

The influence of colour and shape modifiers on the semantic processing of noun phrase in the congenital blind, Ji-Won Chun ${ }^{1,2,3}$, Jae-Jin Kim ${ }^{1,2,3,4}$, Joongil Kim ${ }^{2,3}$, ByungSik Seo ${ }^{2,3}$, Hae-Jeong Park ${ }^{1,2,3}$, 'Institute of Behavioral Science in Medicine, Yonsei University College of Medicine, Seoul, South Korea, ${ }^{2}$ Department of Diagnostic Radiology and Research Institute of Radiological Science, Nuclear Medicine, Yonsei University College of Medicine, Seoul, South Korea, ${ }^{3}$ Brain Korea 21 Project for Medical Science, Yonsei University College of Medicine, Seoul, South Korea, ${ }^{4}$ Department of Psychiatry, Yonsei University College of Medicine, Seoul, South Korea

An fMRI Study of Word Category on Word Recognition, Toshimune Kambara ${ }^{l}$, Satoru Yokoyama ${ }^{l}$, Kei Takahashi ${ }^{1,2}$, Naoki Miura ${ }^{1,3}$, Tadao Miyamoto ${ }^{2}$, Daiko Takahashi ${ }^{2}$, Shigeru Sato ${ }^{2}$, Ryuta Kawashima , ' Department of Functional Brain Imaging, IDAC, Tohoku University, Sendai, Japan, 363 TH-AM 355 TH-AM ${ }^{2}$ Graduate School of International Cultural Studies, Tohoku University, Sendai, Japan, ${ }^{3}$ Department of Intelligent Mechanical Systems Engineering, Kochi University of Technology, Kami, Japan 
Dynamic ERP Mapping Denoting Percept to Concept: Chinese Olympic Sport Symbols, Andrew CN Chen*, Peipei Wang, Center for Higher Brain Functions, Capital Medical University, Beijing, China

387 TH-AM

The role of the posterior superior temporal sulci in understanding linguistic and extralinguistic communicative intentions, Ivan Enrici , Mauro Adenzato ${ }^{1,2}$, Bruno G. Bara ${ }^{1,2}$, Stefano Cappa ${ }^{3,4}$, Marco Tettamanti ${ }^{4,5}$, ${ }^{1}$ Center for Cognitive Science, University of Torino, Torino, Italy, ${ }^{2}$ Neuroscience Institute of Turin, Torino, Italy, ${ }^{3}$ Vita-Salute San Raffaele University, Milano, Italy, ${ }^{4}$ CERMAC-HSR, Milano, Italy, ${ }^{5}$ Department of Nuclear Medicine, Scientific Institute HSR, Milano, Italy

\section{LANGUAGE \\ Reading/Writing}

Implicit and Explicit Morphologically Related Activation, Atira Bick ${ }^{1,2}$, Gadi Goelman ${ }^{2}$, Ram Frost ${ }^{3}$, ${ }^{I}$ ICNC, Hebrew University, Jerusalem, Israel, ${ }^{2}$ Medical Biophysics, Hadassah Hebrew University Hospital, Jerusalem, Israel, ${ }^{3}$ Psychology Department, Hebrew University, Jerusalem, Israel

The Different Function of the Dorsal and Ventral Pathways in the Spatial Processing of Chinese Characters: A fMRI Study, Yanlin Luo ${ }^{1}$, Andrew CN Chen ${ }^{l}$, xiujun $\mathrm{Li}^{2}$, Danlin Pen ${ }^{2},{ }^{I}$ Center for Higher Brain Functions, Capital Medical University, Beijing, China, ${ }^{2}$ Beijing normal University, Beijing, China

Using fMRI to Explore the Neural Underpinnings of Individual Differences in Reading Skill, Chantel Prat, Robert Mason, Marcel Just, Carnegie Mellon University, Pittsburgh, USA

Differential Associations with Socioeconomic Status and Brain Activation in Dyslexic versus Typical Adolescent Readers, Jessica M. Black ${ }^{1,2}$, Candy S. Ho ${ }^{I}$, Joshua Heitzmann ${ }^{l}$, Nahal Zakerani ${ }^{l}$, Allan L. Reiss ${ }^{l}$, Fumiko Hoeft ${ }^{l},{ }^{l}$ Center for Interdisciplinary Brain Sciences Research, Department of Psychiatry and Behavioral Sciences, Stanford University School of Medicine, Stanford, USA, ${ }^{2}$ School of Education, Stanford University, Stanford, USA

Neuronal processes in Kanji and Kana reading by Dyslexic children: An MEG study, Ryusaku Hashimoto ${ }^{1}$, Sunao Iwaki ${ }^{2}$, Mitsuru Kashiwagi ${ }^{l}$, Shuhei Suzuki ${ }^{I},{ }^{I}$ Osaka Medical College, Takatsuki, Japan, ${ }^{2}$ National Institutes of Advanced Industrial Science and Technology, Ikeda, Japan

411 TH-AM

Changes in neural microstructure associated with spelling and reading impairment in adolescents and young adults, Nenad Vasic ${ }^{I}$, Christian Robert Wolf ${ }^{1}$, Christina Lohr ${ }^{2}$, Claudia Steinbrink ${ }^{2}$, Manfred Spitzer ${ }^{1,2}$, Bärbel Herrnberger ${ }^{l},{ }^{1}$ University CLinic of Ulm, Deprtment of Psychiatry III, Ulm, Germany, ${ }^{2}$ Transfer Center for Neuroscience and Learning, University of Ulm, Ulm, Germany

\section{MEMORY \& LEARNING Learning (explicit \& implicit)}

Visuospatial Working Memory in Children with Attention Deficit Hyperactivity Disorder, Combined Type (ADHD-CT): A Functional Magnetic Resonance Imaging (fMRI) Study., Melissa Casey, ${ }^{1}$, Maree Farrow ${ }^{3}$, Ross Cunnington', Alasdair Vance ${ }^{1},{ }^{1}$ Academic Child Psychiatry Unit, Royal Children's Hospital, Murdoch Childrens Research Institute, Melbourne, Australia, ${ }^{2}$ Queensland Brain Institute, Brisbane, Australia, ${ }^{3}$ Howard Florey Institute, Melbourne, Australia

Boredom Susceptibility and Experience Seeking Predict Brain Responses to Repeated Visual Experience, Yang Jiang ${ }^{1}$, Joann Lianekhammy ${ }^{1}$, Adam Lawson ${ }^{1}$, Chunyan Guo ${ }^{1,2}$, Donald Lynam ${ }^{3}$, Jane Joseph $^{4}$, Brain Gold ${ }^{4}$, Thomas Kelly ${ }^{1},{ }^{1}$ Department of Behavioral Science, Lexington, USA, ${ }^{2}$ Department of Psychology, Beijing, China, ${ }^{3}$ Department of Psychological Sciences, Purdue University, West Lafayette, USA, ${ }^{4}$ Department of Anatomy \& Neurobiology, Lexington, USA

Comparable and dissociable neural correlates of spontaneous sensory-specific imagery versus perception of cue-unique sensory-perceptual outcome events, Leh Woon Mok ${ }^{l}$, Kathleen Thomas ${ }^{2}$, Ovidiu Lungu ${ }^{3},{ }^{1}$ Nanyang Technological University, Singapore, Singapore, ${ }^{2}$ University of Minnesota, Minneapolis, USA, ${ }^{3}$ Université de Montréal, Montreal, Canada

AVERSIVE UNCONDITIONED STIMULI CAN INHIBIT THE DEFENSIVE SYSTEM., Marta Andreatta, Andreas Muehlberger, Paul Pauli, University of Wuerzburg, Wuerzburg, Germany

Effects of implicit learning on repetitive recognition performance, Teruo Hashimoto ${ }^{l}$, Nobuo Usui ${ }^{2}$, Masato Taira ${ }^{2}$, Shozo Kojima ${ }^{1},{ }^{1}$ Dept of Psychology Keio Univ., Tokyo, Japan, ${ }^{2}$ Nihon University Advanced Research Institue for the Sciences and Humanities, Tokyo, Japan 
Guidance and Learning of Circular Eye Movements, Raimund Kleiser ${ }^{1}$, Thomas Matyas ${ }^{2}$, Hans-Jörg Wittsack, Rüdiger Seitz ${ }^{1,4}$, 'Department of Neurology, University Hospital, Duesseldorf, Germany, ${ }^{2}$ School of Psychology, LaTrobe University, Bundoora, Victoria, Australia, ${ }^{3}$ Department of Diagnostic

Radiology, University Hospital, Duesseldorf, Germany, ${ }^{4}$ Brain Imaging Centre West, Juehlich, Germany

Hippocampal Subregional Involvement in Encoding and Retrieval of Spatial Information, Nanthia Suthana ${ }^{1,2}$, Arne Ekstrom ${ }^{1,2}$, Saba Moshirvaziri ${ }^{1,2}$, Barbara Knowlton ${ }^{3}$, Susan Bookheimer ${ }^{1,2,3}$, ${ }^{1}$ Center for Cognitive Neurosciences, Semel Institute, UCLA, Los Angeles, USA, ${ }^{2}$ Dept. of Psychiatry and Biobehavioral Sciences, UCLA, Los Angeles, USA, ${ }^{3}$ Department of Psychology, UCLA, Los Angeles, USA

11:30 - 12:30 Corryong Hall (Level 2)
439 TH-AM

443 TH-AM*

453 TH-AM

KIBRA alleles modulate medial temporal lobe activity during episodic memory, $M$. R. Emery, $V . S$ Clinical Brain Disorders Branch, National Institute of Mental Health, NIH, Bethesda, USA

Semantic Knowledge Alters Functional Connectivity Recorded with MEG During Transverse Patterning Performance, Sandra Moses, Natasa Kovacevic, Christina Villate, Timothy Bardouille, Anthony Randal McIntosh, Jennifer Ryan, Rotman Research Institute, Baycrest Centre, Toronto, Canada

Functional connectivity of the hippocampi in healthy subjects, Kathrin Wagner ${ }^{1}$, Lars Frings ${ }^{1,2}$, Anne Buller ${ }^{I}$, Joachim Spreer ${ }^{3}$, Andreas Schulze-Bonhage ${ }^{I},{ }^{I}$ Epilepsy Center, University Hospital Freiburg, Freiburg, Germany, ${ }^{2}$ Gerontopsychiatry and Neuropsychology Section, Department of Psychiatry and Psychotherapy, University Hospital Freiburg, Freiburg, Germany, ${ }^{3}$ Department of Neuroradiology, University Hospital Freiburg, Freiburg, Germany

Stressed memories: effects of acute stress on medial temporal lobe activation during memory formation, Erno Hermans ${ }^{1,2}$, Marloes Henckens ${ }^{l}$, Zhenwei Pu ${ }^{3,1}$, Marian Joëls ${ }^{3}$, Guillen Fernández ${ }^{1,2}$, ${ }^{l}$ F.C. Donders Centre at the Radboud University Nijmegen, Nijmegen, Netherlands, ${ }^{2}$ Department of Neurology at the Radboud University Nijmegen Medical Centre, Nijmegen, Netherlands, ${ }^{3}$ SILS-CNS, University of Amsterdam, Amsterdam, Netherlands

Activity in the medial temporal lobes predicts realization of intentions for future actions, Jiro Okudal, Nobuhito Abe ${ }^{2}$, Maki Suzuki, ${ }^{3,4}$, Toshikatsu Fujii ${ }^{2},{ }^{1}$ Tamagawa University Brain Science Institute, Machida, Tokyo, Japan, ${ }^{2}$ Department of Behavioral Neurology and Cognitive Neuroscience, Tohoku University Graduate School of Medicine, Sendai, Japan, ${ }^{3}$ Division of Cyclotron Nuclear Medicine, Cyclotron and Radioisotope Center, Tohoku University, Sendai, Japan, ${ }^{4}$ The Japan Society for the Promotion of Science, Tokyo, Japan

Retrieval of associations between color and achromatic features activates two distinct areas in the ventral occipitotemporal cortex, Yan Wang ${ }^{1,2}$, JinHui Zhao ${ }^{2}$, FuCang Jia ${ }^{2}$, Sheng $\mathrm{He}^{3}$, Lin $\mathrm{Ma}^{4}$, DeJun $\mathrm{Li}^{4}, \mathrm{XuChu} \mathrm{Weng}^{2},{ }^{1}$ Department of Psychology, Laboratory for Cognition and Learning, Capital Normal University, BeiJing, China, ${ }^{2}$ Institute of Psychology, the Chinese Academy of Sciences, BeiJing, China, ${ }^{3}$ Department of Psychology, University of Minnesota, Minneapolis, USA, ${ }^{4}$ Department of Radiology, PLA General Hospital, BeiJing, China

MODELING \& ANALYSIS Exploratory Methods, Artifact Removal

Measurement of gamma band effects in MEG and concurrent EEG/fMRI at 7T, Matthew Brookes, Karen Mullinger, Claire Stevenson, Gerda Geirsdottir, Peter Morris, Richard Bowtell, University of Nottingham, Nottingham, United Kingdom

A Framework for Analyzing and Visualizing Multi-Modality Cross-Correlation, Satoru Hayasaka ${ }^{1,2}$, Paul Laurienti ${ }^{2}$, Joseph Maldjian ${ }^{2},{ }^{\prime}$ Biostatistical Sciences, Wake Forest University, Winston-Salem, USA, ${ }^{2}$ Radiology, Wake Forest University, Winston-Salem, USA

Average Gradient Artefact Subtraction: the effect on neuronal signals, Karen J. Mullinger, Matthew J. Brookes, Gerda B. Geirsdottir, Richard W. Bowtell, University of Nottingham, Nottingham, United Kingdom
461 TH-AM

465 TH-AM*

469 TH-AM

473 TH-AM

477 TH-AM

481 TH-AM

485 TH-AM 
Why sparse bump models?, Francois-B. Vialatte, Monique Maurice, Andrzej Cichocki, Riken BSI, Lab. ABSP, Wako-Shi, Japan

489 TH-AM

Hemodynamic response latency correction for improved fMRI functional connectivity, Catie Chang ${ }^{1}$, Moriah E. Thomason ${ }^{2}$, Gary H. Glover ${ }^{1,2,3},{ }^{1}$ Dept. of Electrical Engineering, Stanford University, Stanford, USA, ${ }^{2}$ Dept. of Psychology, Stanford University, Stanford, USA, ${ }^{3}$ Dept. of Radiology, Stanford, USA

Conquer and Divide: A novel approach to spatiotemporal significance testing that accounts for alpha error inflation, Sven P. Heinrich, Michael Bach, Jürgen Kornmeier, University of Freiburg, Freiburg, Germany

Assessing fiber similarity in probabilistic diffusion tractography, Luca Nanetti, Leonardo Cerliani, Valeria Gazzola, Christian Keysers, University Medical Center Groningen, Groningen, Netherlands

Regional Distribution of Outliers Across a Population of Diffusion MRI in Human Brain, Lindsay Walker ${ }^{1}$, Jinzhong Yang ${ }^{2}$, Xiaoying $\mathrm{Wu}^{2}$, Kristina Simonyan ${ }^{3}$, Ragini Verma ${ }^{2}$, Carlo Pierpaoli ${ }^{1},{ }^{1}$ NICHD, NIH, Bethesda, USA, ${ }^{2}$ Dept. of Radiology, University of Pennsylvania, Philadelphia, USA, ${ }^{3}$ NINDS, NIH, Bethesda, USA

\section{MODELING \& ANALYSIS Flattening, Segmentation}

Age and gender effect on Cerebral Spinal Fluid thickness, Anna Custo ${ }^{I}$, William M. Wells III ${ }^{1,2}$, W. Eric L. Grimson ${ }^{l},{ }^{1}$ Massachusetts Institute of Technology, CSAIL, Cambridge, USA, ${ }^{2}$ Brigham and Women's Hospital, HMS, Boston, USA

Semi-automated delineation of the tentorium cerebelli from MRI scans, Neeraja Penumetcha ${ }^{l}$, Suraj Kabadi $^{l}$, Bruno Jedynak ${ }^{l}$, Charles Walcutt ${ }^{2}$, Mokhtar H. Gado ${ }^{3}$, Lei Wang ${ }^{2}$, J. Tilak Ratnanather ${ }^{l}$, ${ }^{I}$ Center for Imaging Science, Johns Hopkins University, Baltimore, USA, ${ }^{2}$ Dept of Psychiatry,

Washington University School of Medicine, St. Louis, USA, ${ }^{3}$ Dept. of Radiology, Washington University School of Medicine, St. Louis, USA

MAPPING NEURODEGENERATION USING MULTI-ATLAS FLUID IMAGE ALIGNMENT, Yi-Yu Chou, Natasha Lepore, Xue Hua, Arthur Toga, Paul Thomposn, Laboratory of Neuro Imagimg, Department of Neurology, UCLA, Los Angeles, USA

Mapping Hippocampal Degeneration in 400 Subjects with a Novel Automated Segmentation Approach, Jonathan Morra ${ }^{I}$, Zhuowen Tu ${ }^{l}$, Liana Apostolova, ${ }^{1,2}$, Amity Green ${ }^{1,2}$, Christina Avedissian ${ }^{l}$, Sarah Madsen ${ }^{l}$, Neelroop Parikshak ${ }^{l}$, Xue Hua ${ }^{l}$, Arthur Toga ${ }^{l}$, Clifford Jack ${ }^{3}$, Norbert Schuff, Michael Weiner ${ }^{4,5}$, Paul Thompson ${ }^{1},{ }^{1}$ Laboratory of Neuro Imaging, UCLA, Los Angeles, USA, ${ }^{2}$ Dept. of Neurology, UCLA, Los Angeles, USA, ${ }^{3}$ Mayo Clinic College of Medicine, Rochester, USA, ${ }^{4}$ Dept. of Radiology, UCSF, San Francisco, USA, ${ }^{5}$ Dept. of Medicine and Psychiatry, UCSF, San Francisco, USA

\section{MODELING \& ANALYSIS}

Functional Connectivity and Structural Equation Modeling

Population dynamics under the Laplace assumption, Andre Marreiros, Jean Daunizeau, Stefan Kiebel, Lee Harrison, Karl Friston, Wellcome Trust Centre for Neuroimaging, University College London, London, United Kingdom

Principal Frequency of Resting State Networks, Rami Niazy ${ }^{1,2,3}$, Stephen Smith ${ }^{2}$, Christian Beckmann ${ }^{4},{ }^{1}$ Cardiff University Brain Research Imaging Centre (CUBRIC), School of Psychology, Cardiff University, Cardiff, United Kingdom, ${ }^{2}$ Centre for Functional MRI of the Brain (FMRIB), Department of Clinical Neurology, University of Oxford, Oxford, United Kingdom, ${ }^{3}$ Department of Engineering Science, University of Oxford, Oxford, United Kingdom, ${ }^{4}$ Clinical Neuroscience Department, Division of Neuroscience and Mental Health, Imperial College London, London, United Kingdom

Effect of alcohol on the resting state correlations., Pawel Skudlarskil, ${ }^{1,2}$ Sashwath Meda ${ }^{1}$, Vince Calhoune ${ }^{3}$, Godfrey Pearlson ${ }^{1,2},{ }^{1}$ Olin Neuropsychiatry Research Center, Hartford, USA, ${ }^{2}$ Department of Psychiatry Yale University School of Medicine, New Haven, USA, ${ }^{3}$ The Mind Institute, Albuquerue, NM, University of New Mexico, Albuquerque, USA

521 TH-AM

525 TH-AM 529 TH-AM 
Increasing specificity of resting-state fMRI-data using multiple regression analysis, Andreas Weissenbacher ${ }^{1,2}$, Rupert Lanzenberger ${ }^{3}$, Ewald Moser ${ }^{1,2}$, Christian Windischberger ${ }^{1,2},{ }^{1}$ MR Center of Excellence, Medical University, Vienna, Austria, ${ }^{2}$ Center for Biomedical Engineering and Physics, 537 TH-AM Medical University, Vienna, Austria, ${ }^{3}$ Department of Psychiatry and Psychotherapy, Medical University, Vienna, Austria

A Method for Improved Sensitivity and Flexibility of Psychophysiological Interactions in EventRelated FMRI Experiments, Donald McLaren ${ }^{1,2}$, Michele Ries ${ }^{1,2}$, Guofan Xu ${ }^{1,2}$, Michele Fitzgerald ${ }^{1,2}$, Erik Kastman $^{1,2}$, Gemma Gliori ${ }^{1,2}$, Britta Jabbar ${ }^{1,2}$, Sterling Johnson ${ }^{1,2},{ }^{1}$ William S. Middleton Memorial Veterans Hospital, Madison, USA, ${ }^{2}$ University of Wisconsin, Madison, USA

Discovering brain's functional connectivity through joint analysis of MEG and fMRI data by Dynamic Bayesian Network, Sergey Plis ${ }^{1}$, Michael P Weisend ${ }^{2}$, Mark Scully ${ }^{2}$, Vincent P Clark ${ }^{2}$, Terran Lane ${ }^{I},{ }^{1}$ Department of Computer Science, University of New Mexico, Albuquerque, USA, ${ }^{2}$ The Mind Research Network, Albuquerque, USA

Dynamical Consequences of Lesions in Cortical Networks, Christopher Honey, Olaf Sporns, Department of Psychological and Brain Sciences, Indiana University, Bloomington, USA

541 TH-AM

Asymmetry analysis of anterior cingulate cortex: functional connectivity using resting state fMRI, Xi-Nian ZUO ${ }^{1,2}$, Chao-Zhe ZHU ${ }^{l}$, Qi-Hong ZOU ${ }^{l}$, Yu-Feng ZANG ${ }^{l, *}$, ' State Key Laboratory of Cognitive Neuroscience and Learning, Beijing Normal University, Beijing, China, ${ }^{2}$ National Laboratory of Pattern Recognition, Institute of Automation, Chinese Academy of Sciences, Beijing, China

Modular Architecture of Weighted Human Brain Structural Network Revealed by Cortical Thickness from MRI, Zhang Chen, Yong He, Alan Evans, McConnell Brain Imaging Centre, Montréal Neurological Institute (MNI), McGill University, Montreal, Canada

Investigating reproducibility of effective connectivity using Dynamic Causal Modelling in a working memory task, Nia Goulden, Shane McKie, John Francis William Deakin, Rebecca Elliott, University of Manchester, Manchester, United Kingdom

Visual cues from mouth movements change the effective connectivity between V5/MT and Broca's area in the right hemisphere, Heejung Kim ${ }^{1,2}$, Yoon-Kyoung Yim ${ }^{1,2}$, Hyejin Kang ${ }^{1,3}$, Dong Soo Lee , Eunjoo Kang ${ }^{4},{ }^{1}$ Dept. of Nuclear Medicine, Seoul National University School of medicine, Seoul, South Korea, ${ }^{2}$ Interdisciplinary program in cognitive science, Seoul National University, Seoul, South Korea, ${ }^{3}$ Programs in Brain and Neuroscience, Seoul National University, Seoul, South Korea, ${ }^{4}$ Department of Psychology, Kangwon National University, Chuncheon, South Korea

Inferring neural signals' processing time: beyond the balloon model, Claudinei Eduardo Biazoli Jr, João Ricardo Sato, Edson Amaro Jr, NIF/LIM-44 Instituto de Radiologia do Hospital das Clínicas da Faculdade de Medicina da Universidade de São Paulo, São Paulo, Brazil

Dynamic causal modelling of distributed electromagnetic responses, Jean Daunizeau, Stefan Kiebel, Karl Friston, Welcome Trust Centre for Neuroimaging, London, United Kingdom

Reliability of Functional Connectivity in the Motor Cortex, Rao Gullapalli ${ }^{1}$, Neha Shah ${ }^{2}$, Steve Roys ${ }^{l}$, Jiachen Zhuo ${ }^{I},{ }^{I}$ Department of Radiology, University of Maryland School of Medicine, Baltimore, USA, ${ }^{2}$ Department of Computer Science and Electrical Engineering, University of Maryland Baltimore County, Baltimore, USA

Feedback Connections within Low-Level Emotion Processing Network Revealed by Dynamic Causal Modeling, Christian Kasess ${ }^{1,2}$, Rupert Lanzenberger ${ }^{3}$, Lukas Pezawas ${ }^{3}$, Ewald Moser ${ }^{1,2}$, Christian Windischberger ${ }^{1,2},{ }^{1}$ MR Center of Excellence, Medical University Vienna, Vienna, Austria, ${ }^{2}$ Center for Biomedical Engineering and Physics, Medical University Vienna, Vienna, Austria,

${ }^{3}$ Department of Psychiatry and Psychotherapy, Medical University Vienna, Vienna, Austria

MODELING \& ANALYSIS Multivariate Modeling, PCA, \& ICA

The effect of respiration variations on independent component analysis of resting state functional connectivity, Rasmus Birn, Kevin Murphy, Peter Bandettini, Laboratory of Brain and Cognition, 
Multivariate functional connectivity between fine-grained cortical activation patterns, Jakob Heinzle ${ }^{I}$, John-Dylan Haynes ${ }^{1,2},{ }^{I}$ Bernstein Center for Computational Neuroscience, Charité Universitätsmedizin, Berlin, Germany, ${ }^{2}$ Max Planck Institute for Cognitive and Brain Sciences, Leipzig, 589 TH-AM Germany

ICA of Brain Imaging Data - Validation by Resampling and Hierarchical Clustering, Radu Mutihac, University of Bucharest, Bucharest, Rumania

593 TH-AM

Frequency-wise inverse solutions to EEG recordings by state space modeling decomposition and dynamical LORETA, and its application to changes in slow delta activity during induction of anesthesia, Kin Foon Kevin Wong ${ }^{1,2}$, Andreas Galka ${ }^{2,3,4}$, Tohru Ozaki ${ }^{1,2,5}$, IJST RISTEX, Tokyo, Japan, ${ }^{2}$ Institute of Statistical Mathematics, Tokyo, Japan, ${ }^{3}$ Department of Neurology, University of Kiel, Kiel, Germany, ${ }^{4}$ Institute of Applied Physics, University of Kiel, Kiel, Germany, ${ }^{5}$ Graduate University for Advanced Studies, Kanagawa, Japan

A New Data-driven Analysis Method Based on the Temporal Structure of BOLD Response, Carlos Estombelo-Montesco ${ }^{1}$, Marcio Sturzbecher ${ }^{1}$, Oswaldo Baffa ${ }^{1}$, Allan Kardec ${ }^{2}$, Draulio de Araujo ${ }^{1}$,

${ }^{I}$ Department of Physics and Mathematics, FFCLRP, University of Sao Paulo, Ribeirao Preto, SP, Brazil,

${ }^{2}$ Department of Electrical Engineering, Federal University of Maranhao, Sao Luis, MA, Brazil

Independent Component Analysis of FMRI Wavelet Coefficients, Robert Johnson ${ }^{1,2}$, Jonathan Marchini ${ }^{I}$, Stephen Smith ${ }^{2}$, Christian Beckmann ${ }^{2,3},{ }^{1}$ Department of Statistics, University of Oxford, Oxford, United Kingdom, ${ }^{2} F M R I B$, University of Oxford, Oxford, United Kingdom, Imperial College, London, United Kingdom

Longitudinal Multivariate Tensor- and Searchlight-Based Morphometry Using Permutation Testing, Gerard Ridgway ${ }^{1}$, Brandon Whitcher ${ }^{2}$, Derek Hill ${ }^{1}$, Nick Fox ${ }^{3},{ }^{1}$ Centre for Medical Image Computing, UCL, London, United Kingdom, ${ }^{2}$ GSK Clinical Imaging Centre, London, United Kingdom, ${ }^{3}$ Dementia Research Centre, UCL, London, United Kingdom

The Impact of Dimensionality Estimation On Spatial Signal Detection In Multivariate Gaussian Image Data, Grigori Yourganov ${ }^{1,2}$, Stephen Strother ${ }^{2,}$, Institute of Medical Science, University of Toronto, Toronto, Canada, ${ }^{2}$ Rotman Research Institute of Baycrest Centre, University of Toronto, Toronto, Canada, ${ }^{3}$ Department of Medical Biophysics, University of Toronto, Toronto, Canada

\section{MOTOR BEHAVIOR \\ Basal Ganglia/Brainstem/Spinal Cord}

Putamen functional connectivity demonstrates a mechanism for the integration of motor and cognitive symptoms as well as cerebellar-basal ganglia communication, William Marchand ${ }^{l, 2}$, James Lee ${ }^{l}$, John Thatcher ${ }^{l}$, Edward Hsu ${ }^{l}$, Esther Rashkin', Yana Suchy', Gordon Chelune ${ }^{I}$, Jennifer Starr ${ }^{l}$, Sharon Barbera ${ }^{l},{ }^{1}$ University of Utah, Salt Lake City, USA, ${ }^{2}$ Department of Veterans Affairs VISN 19 MIRECC, Salt Lake City, USA

\section{MOTOR BEHAVIOR \\ Eye Movements/Visuomotor Processing}

Modulations of gamma and beta band activity during decision and preparation of saccades revealed by simultaneous intracranial recordings in human parietal and prefrontal cortex, Karim Jerbi $^{l, 2}$, Samson Freyermuth ${ }^{l}$, Olivier Bertrand ${ }^{2}$, Lorella Minotti ${ }^{3}$, Philippe Kahane ${ }^{3}$, Jean-Philippe Lachaux ${ }^{2}$, Alain Berhoz ${ }^{l},{ }^{1}$ Physiology of Perception and Action Lab, CNRS, Collège de France, Paris, France, ${ }^{2}$ INSERM, U821, Brain Dynamics and Cognition \& University Lyon 1, Lyon, France, ${ }^{3}$ Department of Neurology and INSERM U704, Grenoble Hospital, Grenoble, France

Neurons in the frontal eye fields projecting to the superior colliculus are crucial in making antisaccades: an fMRI-DTI study, AD de Weijer, RCW Mandl, IEC Sommer, SFW Neggers, Rudolf Magnus Institute of Neuroscience, Department of Psychiatry, University Medical Centre Utrecht, Utrecht, Netherlands

617 TH-AM

597 TH-AM

601 TH-AM

605 TH-AM

609 TH-AM

613 TH-AM

\section{NEUROANATOMY}

DTI Studies, Application

Exploring the Large-Scale Connectivity of the Human Visual System using Diffusion Tensor Tractography, Michael Capalbo, Alard Roebroeck, Rainer Goebel, University of Maastricht, Department of Cognitive Neuroscience, Maastricht, Netherlands 
Investigating the Biomechanisms of Cerebral Cortical Folding, Guangqiang Geng ${ }^{1,2}$, Leigh Johnston ${ }^{1,3}$, Edwin Yan ${ }^{4}$, David Walker ${ }^{5}$, Gary Egan ${ }^{1,6},{ }^{1}$ Howard Florey Institute, Florey Neuroscience Institutes, Melbourne, Australia, ${ }^{2}$ Graduate School of Biomedical Engineering, University of New South Wales, Sydney, Australia, ${ }^{3}$ Dept. of Electrical \& Electronic Engineering, University of Melbourne, Melbourne, Australia, ${ }^{4}$ National Trauma Research Institute, Alfred Hospital, Melbourne, Australia, ${ }^{5}$ Dept. of Physiology, Monash University, Melbourne, Australia, ${ }^{6}$ Centre for Neuroscience, University of Melbourne, Melbourne, Australia

The nigro-striatal pathway in the monkey brain using diffusion tensor imaging fiber tracking at 7T, Stephane Lehericy ${ }^{1,2}$, Essa Yacoub ${ }^{3}$, Eric Bardinet ${ }^{1,4}$, Romain Valabregue ${ }^{1,2}$, Chantal Francois ${ }^{2}$, Geoff Ghose ${ }^{3}$, Noam Harel ${ }^{3},{ }^{1}$ University Pierre and Marie Curie, Paris, France, ${ }^{2}$ INSERM, Paris, France, ${ }^{3}$ University of Minnesota, Minneapolis, USA, ${ }^{4}$ CNRS, Paris, France

Diffusion tensor MRI can anatomically segment human amygdaloid subregions in vivo, Eugenia Solano-Castiella, Alfred Anwander, Carol Docherty, Enrico Reimer, Marcel Weiss, Angela Friederici, Robert Turner, Max Planck Institute for Human Cognitive and Brain Sciences, Leipzig, Germany

A DTI tractography study on the functional relevance of inter-individual differences in callosal connectivity, René Westerhausen ${ }^{1}$, Renate Grüner ${ }^{2}$, Karsten Specht ${ }^{1,3}$, Kenneth Hugdahl, ${ }^{1,4}$, Dept of Biological and Medical Psychology, University of Bergen, Bergen, Norway, ${ }^{2}$ Dept of Radiology, Haukeland University Hospital, Bergen, Norway, ${ }^{3}$ Clinical Engineering Department, Haukeland University Hospital, Bergen, Norway, ${ }^{4}$ Division of Psychiatry, Haukeland University Hospital, Bergen, Norway

An omnibus test for case-control studies utilizing Tract-Based Spatial Statistics (TBSS), Matthew Cykowski $^{I}$, Jack Lancaster ${ }^{1}$, Roger Ingham ${ }^{1,2}$, Janis Ingham ${ }^{1,2}$, Anderson Winkler ${ }^{1}$, Peter Kochunov ${ }^{1}$, Peter Fox ${ }^{1,3},{ }^{1}$ IResearch Imaging Center, University of Texas Health Science Center at San Antonio, San Antonio, USA, ${ }^{2}$ University of California, Santa Barbara, Santa Barbara, USA, ${ }^{3}$ VA Medical Center, San Antonio, USA

Connectivity-Based Parcellations of the Human Lateral Premotor Cortex and its Relationship to Functional Activation Patterns, Thomas R. Knösche ${ }^{l}$, Alfred Anwander ${ }^{1}$, Ricarda I. Schubotz ${ }^{2}$, Marc Tittgemeyer ${ }^{2},{ }^{\prime}$ Max-Planck-Institute for Human Cognitive and Brain Sciences, Leipzig, Germany, ${ }^{2}$ MaxPlanck-Institute for Neurological Research, Cologne, Germany

Quantitative analysis of the registration errors in the combining voxel-based morphometry and diffusion tensor imaging (DTI-VBM)., Jun-Sung Park, Bang-Bon Koo, Chi-Hoon Choi, Jong-Min Lee, Department of Biomedical Engineering, Hanyang University, Seoul, South Korea

Non-Invasive Mapping of Human Trigeminal Brainstem Pathways, Jaymin Upadhyay ${ }^{l}$, Jamie Knudsen ${ }^{I}$, Julie Anderson ${ }^{1}$, Lino Becerral, David Borsook ${ }^{1,2},{ }_{1}^{1}$ P.A.I.N. Group, Brain Imaging Center, McLean Hospital, Belmont, USA, ${ }^{2}$ Athinoula A. Martinos Center for Biomedical Imaging Massachusetts General Hospital Harvard Medical School, Charlestown, USA

\section{SENSORY SYSTEMS Auditory/Vestibular}

Cortical representation of auditory objects, Amber Leaver, Josef Rauschecker, Georgetown University, Washington, USA

Neural correlates of auditory categorical perception revealed by magnetoencephalography, Hanna Renvall $^{l}$, Noël Staeren ${ }^{l}$, Nicolette Siep ${ }^{I}$, Ole Jensen ${ }^{2}$, Elia Formisano ${ }^{l}$, ${ }^{I}$ Department of Cognitive Neuroscience, Faculty of Pyschology, Maastricht, Netherlands, ${ }^{2}$ F.C. Donders Centre for Cognitive Neuroimaging, Nijmegen, Netherlands

3D pattern of brain changes in deaf subjects mapped using Tensor-Based Morphometry, Natasha Lepore $^{l}$, Patrick Vachon ${ }^{2}$, Franco Lepore ${ }^{2}, Y i-Y u$ Chou $^{l}$, Patrice Voss ${ }^{2}$, Caroline Brun ${ }^{l}$, Agatha D. Lee ${ }^{l}$, Arthur W. Toga ${ }^{l}$, Paul M. Thompson ${ }^{1},{ }^{1}$ Laboratory of Neuro Imaging, David Geffen School of Medicine at UCLA, Los Angeles, USA, ${ }^{2}$ Centre de Recherche en Neuropsychologie et Cognition, Universite de Montreal, Montreal, Canada

Post-lingual deaf potentiate the pre-existing normal speechreading network, but a different form of speechreading network is created in pre-lingual deaf: a Magnetoencephalographic Study, Myung-Whan Suh ${ }^{1}$, Hyo-Jeong Lee ${ }^{2}$, Chun Kee Jung ${ }^{3}$, June Sic Kim ${ }^{3}$, Min Hyun Park ${ }^{4}$, Ja Hyun Kim ${ }^{5}$, Seung $\mathrm{Ha} \mathrm{Oh}{ }^{1},{ }^{l}$ Department of Otorhinolaryngology, College of Medicine and Research Center for

633 TH-AM*

637 TH-AM

641 TH-AM

645 TH-AM

649 TH-AM

653 TH-AM

657 TH-AM

661 TH-AM*

665 TH-AM*

669 TH-AM

673 TH-AM

677 TH-AM 
Sensory Organs, Medical Research Center, Seoul National University, Seoul, Korea, ${ }^{2}$ Department of Otolaryngology-Head and Neck Surgery, Hallym University Sacred Heart Hospital, Seoul, Korea, ${ }^{3}$ Department of Neurosurgery, Seoul National University College of Medicine, MEG Center, Seoul, Korea, ${ }^{4}$ Department of Otorhinolaryngology, Seoul Municipal Boramae Hospital, Seoul, Korea,

${ }^{5}$ Department of Biomedical Engineering, College of Health Science, Yonsei University, Seoul, Korea

\section{SENSORY SYSTEMS}

Tactile/Somatosensory

High Resolution fMRI Mapping of the Primary Somatosensory Cortex and Thalamus in Humans at 7T, Feng Wang, Li Min Chen, Robert Friedman, Elizabeth Stringer, John Gore, Malcolm Avison, Christopher Gatenby, Vanderbilt University, Nashville, USA

Responsiveness of the sensorimotor cortex in fMRI to variable foot vibration using a controllable vibrating probe, Christian Siedentopf ${ }^{1,2}$, Karsten Heubach ${ }^{2,3}$, Anja Ischebeck ${ }^{2,4}$, Florian Koppelstaetter $^{1,2}$, Eugen Gallasch ${ }^{5}$, Martin Fend ${ }^{5}$, Ilka Haala ${ }^{1,2}$, Stephan Felber ${ }^{1,6}$, Franz Gerstenbrand ${ }^{7}$, Stefan Golaszewski ${ }^{1,2,8},{ }^{1}$ Department of Radiology, Medical University Innsbruck, Innsbruck, Austria, ${ }^{2}$ fMRI-Lab, Department of Psychiatry, Medical University Innsbruck, Innsbruck, Austria, ${ }^{3}$ Department of Surgery, St. Nepomuk Hospital, Erfurt, Germany, ${ }^{4}$ Department of Neurology, Medical University Innsbruck, Innsbruck, Austria, ${ }^{5}$ Department of Physiology, Medical University Graz, Graz, Austria, ${ }^{6}$ Stiftungsklinikum Mittelrhein St.Martin, Koblenz, Germany, ${ }^{7}$ Ludwig Boltzmann Institute for Restorative Neurology and Neuromodulation, Vienna, Austria, ${ }^{8}$ Department of Neurology, Paracelsus Medical University, Salzburg, Austria

Distinct Presentations of Heat pain and Touch in SI, SII and Insula in Humans Revealed by High Resolution fMRI at 7T, E.A. Stringer, R.M. Friedman, J.C. Gatenby, F. Wang, M.J. Avison, J.C. Gore, L.M. Chen, Department of Radiology and Radiological Science and Institute of Imaging Sciences, Vanderbilt University, Nashville, USA

Acupuncture Modulates Resting State Connectivity in Default and Sensorimotor Brain Networks, Polly Dhond ${ }^{l, 2}$, Calvin Yeh ${ }^{1}$, Kyungmo Park ${ }^{1,3}$, Norman Kettner ${ }^{2}$, Vitaly Napadow ${ }^{1,2}{ }^{1}{ }^{1}$ Martinos Center for Biomedical Imaging, Charlestown, USA, ${ }^{2}$ Logan College of Chiropractic, Chesterfield, USA,

${ }^{3}$ Kyunghee University, Yongin, South Korea

Transient phase-locking in somatosensory cortex during vibrotactile stimuli, Angela Langdon ${ }^{l, 2}$, Tjeerd Boonstra ${ }^{1,2}$, Stuart Knock ${ }^{1,2}$, Michael Breakspear ${ }^{1,2},{ }^{I}$ The School of Psychiatry, University of New South Wales, Sydney, Australia, ${ }^{2}$ The Black Dog Institute, Sydney, Australia

\section{SENSORY SYSTEMS} Vision

When apparent motion and real stimuli meet in primary visual cortex, Arjen Alink ${ }^{1,3}$, Caspar Schwiedrzik ${ }^{1,3}$, Axel Kohler ${ }^{1,3}$, Wolf Singer ${ }^{1}$, Lars Muckli ${ }^{2},{ }^{1}$ MPI for Brain Research, Neurophysiology, Frankfurt, Germany, ${ }^{2}$ University of Glasgow, dep. of Psychology, Glasgow, Scotland, ${ }^{3}$ Brain Imaging Centre, Frankfurt, Germany

MEG and EEG correlates of visual awareness and suppression of a face., Olivia Carter ${ }^{1,2,3}$, Ken Nakayama ${ }^{1}$, Dahlia Sharon ${ }^{3}$, Matti Hämäläinen ${ }^{3}$, Seppo Ahlfors ${ }^{3},{ }^{1}$ Vision Sciences Lab, Harvard University, Cambridge, USA, ${ }^{2}$ Brain Research Institute, Heidelberg West, Australia, ${ }^{3}$ Martinos Center, Massachusetts General Hospital, Charlestown, USA

Spatial scale tuning maps in human visual cortex, Jonathan Polimeni ${ }^{l}$, Oliver Hinds ${ }^{2}$, Christina Triantafyllou ${ }^{1,2},{ }^{I}$ Athinoula A. Martinos Center, Massachusetts General Hospital, Harvard Medical School, Chalestown, USA, ${ }^{2}$ McGovern Institute for Brain Research, Massachusetts Institute of 685 TH-AM

689 TH-AM

Technology, Cambridge, USA

Spatiotemporal frequency tuning of BOLD and Gamma band MEG responses compared in primary visual cortex, Suresh Muthukumaraswamy, Krish Singh, CUBRIC, Cardiff University, Cardiff, United Kingdom

Neural basis of modal and amodal completion: an fMRI investigation, Branka Spehar ${ }^{\text {, Scott }}$ McDonald ${ }^{2}$, Kiley Seymour ${ }^{2}$, Mark Schira ${ }^{1}$, Zoe Kourtzi ${ }^{3}$, Colin Clifford ${ }^{2},{ }^{I}$ The University of New South Wales, Sydney, Australia, ${ }^{2}$ University of Sydney, Sydney, Australia, ${ }^{3}$ University of Birmingham, Birmingham, United Kingdom 
Superposition of evoked and spontaneous activity in the visual cortex: a 7T study, Marta Bianciardi, Masaki Fukunaga, Peter van Gelderen, Silvina G. Horovitz, Jacco A. de Zwart, Jeff H. Duyn, Advanced MRI Section, LFMI, NINDS, NIH, Bethesda, USA

Multimodal Imaging combining fMRI and PET for the definition of early visual areas in humans, Florian Gerstl ${ }^{1,2}$, Christian Windischberger ${ }^{1,2}$, Rupert Lanzenberger ${ }^{3}$, Ewald Moser $^{1,2}$, Kurt Kletter ${ }^{4}$, Siegfried Kasper ${ }^{3},{ }^{1}$ MRCE, Medical University of Vienna, Vienna, Austria, ${ }^{2}$ Center for Biomedical Engineering and Physics, Medical University of Vienna, Vienna, Austria, ${ }^{3}$ Department of Psychiatry and Psychotherapy, Clinical Division of Biological Psychiatry, Medical University of Vienna, Vienna, Austria, ${ }^{4}$ Department of Nuclear Medicine, PET Centre, Medical University of Vienna, Austria, Vienna, Austria

Visual and auditory development: the use of entropy., Sarah Lippe ${ }^{1,2}$, Maryse Lassonde ${ }^{1,2}$, Natasa Kovacev ${ }^{3}$, Randy McIntosh ${ }^{3},{ }^{1}$ Hôpital Ste-Justine, Monreal, Canada, ${ }^{2}$ University of Montreal, Montreal, Canada, ${ }^{3}$ Rotman-Baycrest Center, Toronto, Canada

High resolution fMRI protocols are feasible for standard fMRI procedures demonstrated using retinotopy., Mark Schira ${ }^{1}$, Branka Spehar ${ }^{1}$, Michael Breakspear ${ }^{1}$, Christopher Tyler ${ }^{2},{ }^{1}$ University of New South Wales, Sydney, Australia, ${ }^{2}$ Smith Kettlewell Eye Research Institute, San Francisco, USA

Spontaneous activity associated with primary visual cortex in early blind, Kun $\mathrm{Wang}^{\mathrm{l}}$, Chunshui $Y u^{2}$, Lijuan $X u^{l}$, Wen Qin ${ }^{2}$, Kuncheng Li ${ }^{2}$, Tianzi Jiang ${ }^{l},{ }^{l}$ National Laboratory of Pattern Recognition, Institute of Automation, Chinese Academy of Sciences, Beijing, China, ${ }^{2}$ Department of Radiology, Xuanwu Hospital of Capital Medical University, Beijing, China

\section{COGNITION \& ATTENTION} Executive Function

The activaion of prefrontal area, basal ganglia, and paralimbic system involved in maintaining of goal-directed action without rewards, Masahiko Nishimura ${ }^{1,2}$, Jobu Watanabe ${ }^{3}$, Yoshihiko Yoshii ${ }^{1},{ }_{1}$ Department of Clinical Neuroscience Faculty of Medicine, University of the Ryukyus, Okinawa, Japan, ${ }^{2}$ Department of Occupational Therapy, Okinawa College of Rehabilitation and Welfare, Okinawa, Japan, ${ }^{3}$ Waseda Institute for Advanced Study Waseda University, Tokyo, Japan

Brain Substrates Associated with Strategic Mode in Executive Function: Comparison between the Tower of London Task and 2-Back Task Using fMRI, Ji-Eun Park ${ }^{l}$, Jin-Sup Eom ${ }^{1}$, Ik-Hyun Kim ${ }^{2}$, Mying-Ae Chung ${ }^{2}$, Hajime Nagai ${ }^{3}$, Jin-Hun Sohn ${ }^{1}$, I Dept. of Psychology, Institute for Brain Research, Chungnam Nat'l University, Daejeon, South Korea, ${ }^{2}$ Medical Information Convergence Service Research Team, ETRI, Daejeon, South Korea, ${ }^{3}$ BRAND'S Health Science Center, Cerebos Pacific Limited, China square central, Singapore

Freedom and Predictability of Choice Visualised by fMRI, Markus Thimm ${ }^{1,2}$, Ralph Weidner ${ }^{2}$, Gereon Fink $^{2,3}$, Walter Sturm ${ }^{I},{ }^{I}$ Department of Neurology, Section Clinical Neuropsychology, University Hospital RWTH Aachen, Aachen, Germany, ${ }^{2}$ Institute of Neurosciences and Biophysics - Medicine, Research Centre Jülich, Jülich, Germany, ${ }^{3}$ Department of Neurology, University Hospital Cologne, Cologne, Germany

Representation of situational context during preparation in task switching as mediated by task specific and behaviorally significant functional connectivity., A. Lenartowicz, L. E. Nystrom, J. D. Cohen, Neuroscience of Cognitive Control Laboratory, Princeton University, Princeton, USA

The neural substrate of task-switching behavior in major depressive disorder and obsessivecompulsive disorder, Peter Remijnse ${ }^{1,4}$, Marjan Nielen ${ }^{l}$, Harry Uylings ${ }^{2,3,4}$, Dick Veltman ${ }^{1,4}$, ${ }^{1}$ Department of Psychiatry, VU Medical Center, Amsterdam, Netherlands, ${ }^{2}$ Department of Anatomy and Neurosciences, VU Medical Center, Amsterdam, Netherlands, ${ }^{3}$ School for Mental Health and Neuroscience, division Neuropsychology, and Brain \& Behaviour Institute, University of Maastricht, Maastricht, Netherlands, ${ }^{4}$ Graduate School Neurosciences, Amsterdam, Netherlands

Exploring a common executive attention network in the brain across stimulus modalities using visual and auditory sorting tasks, Helene Hjelmervik ${ }^{l}$, Kenneth Hugdahl ${ }^{1,2}$, Karsten Specht ${ }^{1,3}$, ${ }^{1}$ Department of Biological and Medical Psychology, University of Bergen, Bergen, Norway, ${ }^{2}$ of Psychiatry and Bergen Mental Health Center, Haukeland University Hospital, Bergen, Norway,

${ }^{3}$ Clinical Engineering Department, Haukeland University Hospital, Bergen, Norway 
Errare humanum est, avoiding the error even more: fMRI evidence of brain networks involved in response suppression., Antonino Vallesi ${ }^{1}$, Anthony R. McIntosh ${ }^{1,2}$, Donald T. Stuss ${ }^{1,2},{ }^{1}$ Rotman Research Institute - Baycrest Centre, Toronto, Canada, ${ }^{2}$ University of Toronto, Toronto, Canada

The motivation-cognition interface: Effects of incentive valence, type, and magnitude on brain activity during working memory task performance, Todd Braver, Hannah Locke, Washington University, Saint Louis, USA

Gender difference in anticipation of monetary gain and loss on brain activation : An fMRI study, Yoonkyung Chung, Eunsoo Cho, Soonkoo Kwon, Hun Jeon, Eun Mo Yeon, Sung-il Kim, Korea University, Seoul, South Korea

Learning from errors: Error-related neural activity predicts improvements in future inhibitory control performance., Robert Hester, Janelle Madeley, Jason B. Mattingley, Queensland Brain Institute and School of Psychology, University of Queensland, St Lucia, Australia

A Dual-Process Model of Anticipatory Task Set Reconfiguration, Sharna Jamadar ${ }^{1,2}$, Frini Karayanidis, ${ }^{1,2,3}$, Pat Michie ${ }^{1,2,3}$, I Functional Neuroimaging Laboratory, Newcastle, Australia, ${ }^{2}$ Schizophrenia Research Institute, Sydney, Australia, ${ }^{3}$ Hunter Medical Research Institute, Newcastle, Australia

Executive functioning after Traumatic Brain Injury depends on difficulty., Fabienne Cazalis, Talin Babikian, Sarah Copeland, Claudia Kernan, Nina Newman, David Hovda, Christopher Giza, Robert Asarnow, UCLA - Brain Injury Research Center, Los Angeles, USA

Activation and Deactivation of the Default Mode, Omer Grigg ${ }^{1,2}$, Cheryl Grady ${ }^{1,2},{ }^{1}$ Rotman Research Institute, Toronto, Canada, ${ }^{2}$ University of Toronto, Toronto, Canada

Free selection of action: effects of ageing on behaviour and neural activity, James Rowe $e^{1,2,3}$, Laura Hughes ${ }^{1,2}$, Doris Eckstein ${ }^{1,2}$, Adrian Owen ${ }^{2,3},{ }^{I}$ Department of Clinical Neurosciences, Cambridge University, Cambridge, United Kingdom, ${ }^{2}$ MRC Cognition and Brain Sciences Unit, Cambridge, United Kingdom, ${ }^{3}$ MRC Behavioural and Clinical Neurosciences Institute, Cambridge, United Kingdom

\section{COGNITION \& ATTENTION}

Perception, Imagery, Awareness

The contralateral effect of auditory and visual stimuli on the event-related potential, Yoshimi Ohgami $^{l}$, Yasunori Kotani ${ }^{1}$, Tatsuya Yoshihiro ${ }^{1}$, Tetsuji Tsukamoto ${ }^{2}$, Junichiro Arai ${ }^{3}$, Yusuke Inoue ${ }^{4}$ ${ }^{I}$ Tokyo Institute of Technology, Tokyo, Japan, ${ }^{2}$ GE-Yokogawa Medical Systems, Tokyo, Japan, ${ }^{3}$ Daikin Industries, Osaka, Japan, ${ }^{4}$ The University of Tokyo, Tokyo, Japan

Top-down facilitation of visual object recognition, Tomoya Taminato ${ }^{l}$, Naoki Miura ${ }^{2,3}$, Motoaki Sugiura, ${ }^{4,6}$, Ryuta Kawashima ${ }^{5,6},{ }^{1}$ Tohoku University School of Medicine, Sendai, Japan, ${ }^{2}$ Department of Intelligent Mechanical Systems Engineering, kochi, Japan, ${ }^{3}$ CREST, Japan Science and Technology Agency, Kawaguchi, Japan, ${ }^{4}$ National Institute for Physiological Science, Department of Cerebral, Okazaki, Japan, ${ }^{5}$ RISTEX, Japan Science and Technology Agency, Kawaguchi, Japan, ${ }^{6}$ Department of Functional Brain Imaging, IDAC, Tohoku University, Sendai, Japan

Neural correlates of visual extinction or awareness revealed by fMRI in a series of righthemisphere stroke patients, Margarita Sarri, Christian Ruff, Geraint Rees, Jon Driver, University College London, London, United Kingdom

Changes of Low Frequency Fluctuation in Anterior Cingulate Cortex during Qigong Meditation, Weijun Tang ${ }^{1}$, Weilin Y ${ }^{2}$, Linbao $\mathrm{Ge}^{2}$, Xiaoyuan Feng ${ }^{1}, \mathrm{Ke} \mathrm{Li}^{1}$, Yizhang Cheng ${ }^{3},{ }^{1}$ Department of Radiology, Huashan Hospital, Fudan University, Shanghai, China, ${ }^{2}$ Shanghai qigong institute, Shanghai 72 TH-PM University of Traditional Chinese Medicine, Shanghai, China, ${ }^{3}$ Second Military Medical University, Shanghai, China

Dynamic switching of thalamocortical network with transition of human states between NREM and REM sleep, Takahiko Koike ${ }^{1}$, Shigeyuki Kan ${ }^{2,1}$, Masaya Misaki ${ }^{3,1}$, Satoru Miyauchi, ${ }^{1,2}$, ${ }^{I}$ National Institute of Information and Communications Technology, Kobe, Japan, ${ }^{2}$ Kyushu Institute of

Technology, Kitakyushu, Japan, ${ }^{3}$ Japan Society for the Promotion of Science, Tokyo, Japan 
Investigating the processing of chimaeric speech with MEG and DTI, Rebecca Millman ${ }^{I}$, Philip Quinlan', ${ }^{1}$ York Neuroimaging Centre, University of York, York, United Kingdom, ${ }^{2}$ Department of Psychology, University of York, United Kingdom

80 TH-PM

Imagery of a moving object affects activation patterns and directed influences of hMT/V5+, posterior parietal and early visual regions, Amanda Kaas ${ }^{1,2}$, Sarah Weigelt ${ }^{1}$, Alard Roebroeck ${ }^{2}$, Axel Kohler ${ }^{l}$, Wolf Singer ${ }^{l}$, Lars Muckli ${ }^{3},{ }^{\prime}$ Department of Neurophysiology, Max Planck Institute for Brain Research, Frankfurt am Main, Germany, ${ }^{2}$ Department of Cognitive Neuroscience, Faculty of Psychology, Maastricht University, Maastricht, Netherlands, ${ }^{3}$ Department of Psychology, UNiversity of Glasgow, Glasgow, United Kingdom

Is mental rotation a right parietal function? Investigation using ERPs and fMRI, Branka Milivojevic, Michael Corballis, Jeff Hamm, University of Auckland, Auckland, New Zealand

\section{DISORDERS OF THE NERVOUS SYSTEM Addiction}

Why we drink alcohol: Striatal activation in response to intravenous alcohol infusion in social drinkers, Jodi Gilman, Vijay Ramchandani, Megan Davis, James Bjork, Daniel Hommer, National Institutes of Alcohol Abuse and Alcoholism, Section of Brain Electrophysiology and Imaging,

Bethesda, USA

Differential effects of cognitive set on brain response to emotionally salient images in Alcoholdependent Patients and Healthy Controls, Daniel Hommer, Megan Davis, Jodi Gilman, NIH/NIAAA, Bethesda, USA

\section{DISORDERS OF THE NERVOUS SYSTEM} Autism

Neural substrates underlying Theory-of-Mind processing in children with autism: a functional MRI study, Rajesh Kana ${ }^{1,2}$, Timothy Keller ${ }^{2}$, Diane Williams ${ }^{3}$, Vladimir Cherkassky ${ }^{2}$, Nancy Minshew ${ }^{4}$, Marcel Just ${ }^{2},{ }^{1}$ University of Alabama, Birmingham, USA, ${ }^{2}$ Carnegie Mellon University, Pittsburgh, USA, ${ }^{3}$ Duquesne University, Pittsburgh, USA, ${ }^{4}$ University of Pittsburgh, Pittsburgh, USA

Alterations in Regional Homogeneity of Baseline Brain Activity in Autism Spectrum Disorder., Paakki Jyri-Johan ${ }^{1}$, Rahko Jukka ${ }^{2}$, Ebeling Hanna ${ }^{2}$, Jussila Katja ${ }^{2}$, Jansson-Verkasalo Eira ${ }^{3}$, Kuusikko Sanna ${ }^{2}$, Mattila Marja-Leena ${ }^{2}$, Moilanen Irma ${ }^{2}$, Nikkinen Juha ${ }^{1}$, Remes Jukka ${ }^{1}$, Starck Tuomo ${ }^{1}$, Tervonen Osmo ${ }^{1}$, Zang Yu-Feng ${ }^{4}$, Kiviniemi Vesa ${ }^{1},{ }^{1}$ Department of Diagnostic Radiology, Oulu University Hospital, Oulu, Finland, ${ }^{2}$ Department of Child Psychiatry, Oulu University Hospital, Oulu, Finland, ${ }^{3}$ Faculty of Humanities, Speech and Language Pathology, University of Oulu, Oulu, Finland, ${ }^{4}$ State Key Laboratory of Cognitive Neuroscience and Learning, Beijing Normal University, Beijing, China

\section{DISORDERS OF THE NERVOUS SYSTEM Brain \& Spinal Cord Trauma}

Sensory processing in patients with ALS: An fMRI study, Dorothee Lule ${ }^{1,2}$, Volker Diekmann ${ }^{l}$, Jan Kassubek ${ }^{l}$, Niels Birbaumer ${ }^{2}$, Albert Ludolph ${ }^{1},{ }^{l}$ Department of Neurology, University of Ulm, Ulm, Germany, ${ }^{2}$ Medical Psychology and Behavioural Neurobiology, University of Tuebingen, Tuebingen, Germany

fMRI reveals cognitive and emotional processing in a long-term comatose patient, Simon $B$ Eickhoff ${ }^{1}$, Manuel Dafotakis ${ }^{1}$, Christian Grefkes ${ }^{1,2}$, Tony Stöcker ${ }^{1,4}$, Jon N. Shah ${ }^{1,4}$, Karl Zilles ${ }^{1,3,4}$, Mario Siebler ${ }^{5},{ }^{1}$ Institute of Neuroscience and Biophysics, INB-3 Medicine, Research Centre Jülich, Jülich, Germany, ${ }^{2}$ Max-Planck-Institut for Neurological Research, Cologne, Germany, ${ }^{3}$ C\&O. Vogt Institute of Brain Research, University of Düsseldorf, Düsseldorf, Germany, ${ }^{4}$ Brain Imaging Center West (BICW), Jülich, Germany, ${ }^{5}$ Department of Neurology, Heinrich-Heine-University Düsseldorf, Düsseldorf, Germany

Assessing diffuse axonal injury in the corpus callosum using multimodal imaging, And Turken ${ }^{l}$, Timothy Herron ${ }^{I}$, Xiaojiang Kang ${ }^{1,2}$, David Woods ${ }^{I, 2}$, IVeterans Affairs Northern California Health Care 116 TH-PM System, Martinez, USA, ${ }^{2}$ University of California, Davis, Davis, USA

\author{
84 TH-PM
}

88 TH-PM

92 TH-PM

96 TH-PM

100 TH-PM

104 TH-PM

108 TH-PM

112 TH-PM

116 TH-PM 


\section{DISORDERS OF THE NERVOUS SYSTEM Developmental Disorders}

From genotype to phenotype: Diffusion imaging discovers disruption of optic radiation in patient with genetically-linked anophthalmia, Johannes $C$ Klein ${ }^{l}$, Heidi Johansen-Berg ${ }^{1}$, Timothy EJ Behrens ${ }^{1}$, Preeti Bakrania ${ }^{2}$, Nicola K Ragge ${ }^{2,3,4},{ }^{I}$ FMRIB Centre, University of Oxford, Oxford, United Kingdom, ${ }^{2}$ Department of Physiology, University of Oxford, Oxford, United Kingdom, ${ }^{3}$ Moorfields Eye Hospital, London, United Kingdom, ${ }^{4}$ Dept of Ophthalmology, Birmingham Children's Hospital, Steelhouse Lane, Birmingham, United Kingdom

Corpus Callosum development in the preterm infant: an MRI study, Deanne Thompson ${ }^{1,2,3}$, Terrie Inder ${ }^{2}$, Leigh Johnston ${ }^{1}$, Scott Kolbe ${ }^{1}$, Lex Doyle ${ }^{3,4}$, Gary Egan ${ }^{l},{ }^{1}$ Howard Florey Institute, Melbourne, Australia, ${ }^{2}$ St Louis Children's Hospital, St Louis, USA, ${ }^{3}$ Murdoch Childrens Research Institute, Melbourne, Australia, ${ }^{4}$ Royal Women's Hospital, Melbourne, Australia

Fractional anisotropy in the corticospinal tract, motor projection patterns, and hand motor outcome in children with unilateral cerebral palsy (CP) - Preliminary report., Linda Holmstrom ${ }^{l}$, Finn Lennartsson ${ }^{2}$, Kristina Tedroff ${ }^{\prime}$, Mominol Islam ${ }^{1}$, Chris Clark ${ }^{3}$, Jonas KE Persson ${ }^{4}$, Ann-Christin Eliasson ${ }^{I}$, Brigitte Vollmer ${ }^{I},{ }^{I}$ Department of Women and Child health, Karolinska Institute,, Stockholm, Sweden, ${ }^{2}$ MR-Center, Karolinska University Hospital, Stockholm, Sweden, ${ }^{3}$ Radiology and Physics unit, UCL, Institute of Child Health, London, United Kingdom, ${ }^{4}$ Neurophysiology unit, Karolinska University Hospital, Stockholm, Sweden

Abnormal Microstructure of the Cingulum Bundle in Agenesis of the Corpus Callosum: A 3T DTI Study, Michael Wahl ${ }^{1,2}$, Rita Jeremy ${ }^{3}$, James Barkovich ${ }^{1,2}$, Mari Wakahiro ${ }^{2}$, Steven Hetts ${ }^{1}$, Elliott Sherr ${ }^{2}$, Pratik Mukherjee ${ }^{1},{ }^{1}$ Dept. of Radiology, UCSF, San Francisco, USA, ${ }^{2}$ Dept. of Neurology, UCSF, San Francisco, USA, ${ }^{3}$ Dept. of Pediatrics, UCSF, San Francisco, USA

\section{DISORDERS OF THE NERVOUS SYSTEM Epilepsy}

Brain plasticity for verbal memory processing in patients with temporal lobe epilepsy and left hippocampal atrophy, Andrea Alessio ${ }^{I}$, Fabricio Pereira ${ }^{I}$, Mauricio Sercheli ${ }^{2}$, Jane Rondina ${ }^{I}$, Helka Ozelo ${ }^{2}$, Elisabeth Bilevicius ${ }^{1}$, Tatiane Pedro ${ }^{1}$, Marcelo Zibetti ${ }^{3}$, Roberto Covolan ${ }^{2}$, Benito Damasceno ${ }^{I}$, Fernando Cendes ${ }^{I},{ }^{1}$ Neuroimaging Laboratory, Campinas, Brazil, ${ }^{2}$ Institute of Physics Gleb Wataghin, Campinas, Brazil, ${ }^{3}$ Institute of Mathematics, Statistics and Computer Science, Campinas, Brazil

Function Cortical Mapping using High Frequency Intracranial Electroencephalography, Dean Freestone $^{1,2,3}$, Anthony Burkitt ${ }^{1,3}$, David Grayden ${ }^{1,3}$, Levin Kuhlmann ${ }^{1}$, Mark Cook ${ }^{2}$, Karen Fuller ${ }^{2}$, Simon Vogrin ${ }^{2}$, Iven Mareel ${ }^{1}$, Alan Lai ${ }^{2,3},{ }^{1}$ Deptartment of Electrical and Electronic Engineering, The University of Melbourne, Melbourne, Australia, ${ }^{2}$ Deptartment of Clinical Neurosciences, St. Vincent's Hospital, Melbourne, Australia, ${ }^{3}$ The Bionic Ear Institute, Melbourne, Australia

Event-related ICA of EEG/fMRI: BOLD changes before epileptiform events, Richard Masterton ${ }^{1,2}$, David Abbott ${ }^{1,2}$, Graeme Jackson ${ }^{1,2},{ }^{1}$ Brain Research Institute, Melbourne, Australia, ${ }^{2}$ The University of Melbourne, Melbourne, Australia

fMRI region of interest analysis of verbal memory task in controls and patients with left temporal lobe epilepsy, Jane Rondina ${ }^{1}$, Andréa Aléssio ${ }^{1}$, Fabrício Pereira', Sercheli Maurício ${ }^{2}$, Helka Ozelo ${ }^{2}$, Elizabeth Bilevicius ${ }^{1}$, Tatiane Pedro ${ }^{l}$, Marcelo Zibetti ${ }^{3}$, Roberto Covolan ${ }^{2}$, Benito Damasceno ${ }^{I}$, Fernando Cendes ${ }^{I},{ }^{1}$ Neuroimaging Laboratory, Campinas, Brazil, ${ }^{2}$ Institute of Physics Gleb Wataghin, Campinas, Brazil, ${ }^{3}$ Institute of Mathematics, Statistics and Computer Science, Campinas, Brazil

Correlation study of optimized voxel-based morphometry and ${ }^{1} \mathrm{H}$ MRS in patients with mesial temporal lobe epilepsy and hippocampal sclerosis (MTLE/HS), Brazdil Milan ${ }^{1}$, Marecek Radek', Fojtikova Dagmar', Mikl Michal ${ }^{1.2}$, Kuba Robert ${ }^{I}$, Krupa Petr ${ }^{3}$, Rektor Ivan ${ }^{I},{ }^{l}$ Brno Epilepsy Centre, Department of Neurology, St. Anne's Hospital, Masaryk University, Brno, Czech Republic, ${ }^{2}$ Faculty of Electrical Engineering and Communication, Brno University of Technology, Brno, Czech Republic, ${ }^{3}$ Department of Neuroimaging, St. Anne's Hospital, Masaryk University, Brno, Czech Republic 
Hemodynamic changes preceding the interictal spike in patients with different types of epilepsies investigated using simultaneous EEG-fMRI., Julia Jacobs ${ }^{1,2}$, Pierre LeVan ${ }^{2}$, Friederike Moeller ${ }^{1}$, Rainer Boor ${ }^{l}$, Ulrich Stephani ${ }^{1}$, Jean Gotman ${ }^{2}$, Michael Siniatchkin ${ }^{l},{ }^{1}$ Department of Neuropediatrics, University Clinic of Kiel,, Germany, ${ }^{2}$ Montreal Neurological Institute,, Canada

\section{HIPOCAMPAL FUNCTIONAL CONNECTIVITY MRI IN PATIENTS WITH LEFT MESIAL} TEMPORAL LOBE EPILEPSY AND CONTROL SUBJECTS DURING RESTING STATE, Fabricio Pereira ${ }^{l}$, Andrea Alessio ${ }^{I}$, Mauricio Sercheli ${ }^{2}$, Elisabeth Bilevicius ${ }^{1}$, Helka Ozelo ${ }^{2}$, Jane Rondina $^{l}$, Tatiane Pedro ${ }^{1}$, Marcelo Zibetti ${ }^{3}$, Gabriela Castellano ${ }^{2}$, Roberto Covolan ${ }^{2}$, Benito Damasceno ${ }^{1}$, Fernando Cendes ${ }^{1}$, ${ }^{1}$ Laboratory of Neuroimage, Campinas, Brazil, ${ }^{2}$ Institute of Physics Gleb Wataghin, Campinas, Brazil, ${ }^{3}$ Institute of Mathematics, Statistics and Scientific Computation, Campinas, Brazil

Dynamics of inter-ictal brain activity using correlation matrices from MEG signals, Maribel Pulgarin, Will Woods, Aziz Asghar, Gary Green, University of York, York, United Kingdom

\section{DISORDERS OF THE NERVOUS SYSTEM Stroke \& Recovery of Function}

Expensive toys or useful tools? FMRI and DTI in a patient with perinatal ischemia, Gunther Fesl ${ }^{l}$, Rainer Kopietz', Yvonne Mewald'2, Hartmut Brueckmann ${ }^{I},{ }^{I}$ Neuroradiology, University of Munich, Grosshadern, Munich, Germany, ${ }^{2}$ Neurology, University of Munich, Grosshadern, Munich, Germany

Brain Activation Patterns during a Category Fluency Task in Children with Neonatal Stroke, Anjali C. Raja ${ }^{1}$, Anthony R. McIntosh ${ }^{1}$, Mary Pat McAndrews ${ }^{2}$, Steven L. Small ${ }^{3,4}$, ${ }^{1}$ Rotman Research Institute of Baycrest Centre, University of Toronto, Toronto, Canada, ${ }^{2}$ Toronto Western Research Institute, University of Toronto, Toronto, Canada, ${ }^{3}$ University of Chicago, Department of Neurology, Chicago, USA, ${ }^{4}$ University of Chicago, Department of Psychology, Chicago, USA

Variable Resolution Electric-Magnetic Tomography (VARETA) in patients with High Blood Pressure., Maria Esther de Quesada ${ }^{I}$, Carolina Franco ${ }^{1}$, Monica Reyes ${ }^{1}$, Guido Diaz ${ }^{2}$, Department of Physiopathology, School of Medicin "J.M. Vargas", Central University of Venezuela, Caracas, Venezuela, ${ }^{2}$ Unit for Electrodiagnostic in Neuropsychiatry NPD, Caracas, Venezuela

Structural integrity of the corticospinal tract is related to motor function of the affected lower extremity in persons with stroke, Zheng-An Luo ${ }^{1}$, Wen-Yih Issac Tseng ${ }^{2,3}$, Yi-Hsin Ko ${ }^{l}$, Su-Chun Huang $^{2}$, Pei-Fang Tang ${ }^{1},{ }^{I}$ School and Graduate Institute of Physical Therapy, College of Medicine, National Taiwan University, Taipei, Taiwan, ${ }^{2}$ Center for Optoelectronic Biomedicine, College of Medicine, National Taiwan University, Taipei, Taiwan, ${ }^{3}$ Department of Medical Imaging, National Taiwan University Hospital, Taipei, Taiwan

\section{EMOTION \& MOTIVATION Decision Making}

"Your regret is my regret": empathy in post-decisional outcome evaluation, Nicola Canessa, Matteo Motterlini ${ }^{1}$, Cinzia Di Dio ${ }^{3}$, Stefano Cappa ${ }^{1,2,4,5}$, Daniela Perani $^{2,4,5}$, Vittorio Girotto ${ }^{6}$, Paola Scifo ${ }^{5}$, Giovanni Buccino ${ }^{3}$, Giacomo Rizzolatti ${ }^{3},{ }^{1}$ CRESA, Vita-Salute san Raffaele University, Milan, Italy, ${ }^{2}$ Center for Cognitive Neuroscience, San Raffaele Scientific Institute, Milan, Italy, ${ }^{3}$ Department of Neuroscience, University of Parma, Parma, Italy, ${ }^{4}$ Faculty of Psychology, Vita-Salute san Raffaele University, Milan, Italy, ${ }^{5}$ CERMAC, Vita-Salute San Raffaele University, Milan, Italy, ${ }^{6} I U A V$ University, Venice, Italy

Ventral striatum activity correlates with decision risk in a novel gambling paradigm, Jon $S$ Wegener $^{1,2,3}$, Julian Macoveanu ${ }^{1,2}$, Arnold Skimminge ${ }^{1,4}$, David Erritzoe ${ }^{2,5}$, Olaf B Paulson ${ }^{1,2,5}$, James B Rowe, ${ }^{2,},{ }^{1}$ Danish Research Centre for MR, Copenhagen University Hospital, Hvidovre, Denmark, ${ }^{2}$ Center for Integrated Molecular Brain Imaging, Copenhagen University Hospital, Copenhagen, Denmark, ${ }^{3}$ Learning Lab Denmark, Danish University of Education, Emdrup, Denmark, ${ }^{4}$ Informatics and Mathematical Modeling, Technical University of Denmark, Lyngby, Denmark, ${ }^{5}$ Neurobiology Research Unit, Copenhagen University Hospital, Rigshospitalet, Copenhagen, Denmark, ${ }^{6}$ Cambridge University Department of Clinical Neurosciences, Cambridge, United Kingdom

Correlation between delay discounting and mesial frontal gray matter volume in alcoholdependent patients and controls, Reza Momenan, James Bjork, Michael Kerich, Daniel Hommer, NIAAA, NIH, Bethesda, USA 


\section{COGNITION \& ATTENTION \\ Attention (visual)}

Investigating Attentional Networks in School Children using fMRI, Sina Wehrum ${ }^{l}$, Rudolf Stark ${ }^{l}$, Ulrich Ott ${ }^{2}$, Franziska Degé $e^{3}$, Gudrun Schwarzer ${ }^{3}$, Dieter Vaitt ${ }^{2},{ }^{1}$ Clinical and Physiological Psychology, JustusLiebig-University, Gießen, Germany, ${ }^{2}$ Bender Institute of Neuroimaging, Justus-Liebig-University, Gießen, Germany, ${ }^{3}$ Department of Developmental Psychology, Justus-Liebig-University, Gießen, Germany

\section{EMOTION \& MOTIVATION Emotional Learning}

Neural basis of reinforcement learning and dynamic decision adjustment in alcoholism, Jana Wrase ${ }^{l}$, Anne Beck ${ }^{l}$, Soyoung Park ${ }^{l}$, Thorsten Kahnt ${ }^{l}$, Mike X. Cohen ${ }^{2}$, Andreas Heinz ${ }^{l},{ }^{l}$ Charité, Psychiatry CCM, Berlin, Germany, ${ }^{2}$ Department of Epileptology, Bonn, Germany

Neural and electrodermal activity during fear conditioning with continuous and intermittent pairing rates, Katharina Tabbert ${ }^{l}$, Jan Schweckendiek ${ }^{l}$, Rudolf Stark ${ }^{l}$, Peter Kirsch ${ }^{2}$, Dieter Vaitl ${ }^{l}$, ${ }^{1}$ Bender Institut of Neuroimaging, University of Giessen, Giessen, Germany, ${ }^{2}$ Central Institute for Mental Health, Mannheim, Germany

\section{EMOTION \& MOTIVATION Emotional Perception}

Neural Correlates of Static and Dynamic Emotional Face Processing, Angela Mayes, Andrew Pipingas, Richard Silberstein, Patrick Johnston, Brain Sciences Institute, Hawthorn, Australia

Serotonergic and Noradrenergic Antidepressants Increase Attentional Bias to Positive Facial Emotional Stimuli during Emotional Expression Decoding. An Event Related Potential (ERP) Study, Pradeep Nathan ${ }^{1,2}$, Rebecca Kerestes ${ }^{2}$, Izelle Labuschagne ${ }^{2}$, K. Luan Phan ${ }^{3}$, Rodney Croft ${ }^{4}$, ${ }^{1}$ University of Cambridge, Cambridge, United Kingdom, ${ }^{2}$ Monash University, Melbourne, Australia,

${ }^{3}$ University of Michigan, Ann Arbor, USA, ${ }^{4}$ Swinburne University, Melbourne, Australia

\section{AMYGDALA VOLUME PREDICTS REACTIVITY TO POSITIVE BUT RECOVERY FROM NEGATIVE STIMULI AS INDEXED BY CORRUGATOR FACIAL EMG, Stacey Schaefer ${ }^{\prime}$, Matthew Sutterer ${ }^{l}$, Carien van Reekum ${ }^{1,2}$, Brendon Nacewicz ${ }^{l}$, Catherine Norris ${ }^{1,3}$, Regina Lapate ${ }^{l}$, David Bachhuber', Nicole Rute ${ }^{l}$, Richard Davidson ${ }^{l},{ }^{1}$ Waisman Laboratory for Brain Imaging \& Behavior, University of Wisconsin-Madison, Madison, USA, ${ }^{2}$ School of Psychology and CLS, University of Reading, Reading, United Kingdom, ${ }^{3}$ Psychological and Brain Sciences, Dartmouth College, Hanover, USA}

Inhibition-related activity in subgenual anterior cingulate is associated with harm avoidance and self directedness in adolescents, Tony Yang ${ }^{l}$, Scott Matthews ${ }^{l}$, Alan Simmons ${ }^{l}$, Susan Tapert ${ }^{l}$, Guido Frank $^{2}$, Martin Paulus ${ }^{1},{ }^{1}$ UC San Diego, San Diego, USA, ${ }^{2}$ University of Colorado at Denver and Health Sciences Center, Aurora, USA

Reading of facial expression with complex emotions: An fMRI study, Hyosun Jung ${ }^{l}$, Minjung Kim ${ }^{l}$, Woorim Jeong', Min Park', Seungbok Lee ${ }^{1}$, Hyo-Woon Yoon ${ }^{2}$, Hei-Rhee Ghim ${ }^{1}$, 'Department of Psychology, Chungbuk National University, Cheongju, South Korea, ${ }^{2}$ Neuroscience Research Institute, Gachon University of Medicine and Science, Incheon, South Korea

TMS disrupts the perception and embodiment of facial expressions, David Pitcher, Lucia Garrido, Vincent Walsh, Brad Duchaine, University College London, London, United Kingdom

Neural Circuits for Regulating Pleasant and Unpleasant Emotion: Beyond Reappraisal, Heather L. Urry ${ }^{l}$, Robert W. Roeser ${ }^{l}$, Sara W. Lazar ${ }^{2}$, Alan P. Poey ${ }^{l}$, Erin Phelps ${ }^{l}$, Richard M. Lerner ${ }^{l},{ }^{1}$ Tufts University, Medford, USA, ${ }^{2}$ Massachusetts General Hospital, Charlestown, USA

Decoding affective states from sustained large-scale patterns of brain activity, Silke Anders ${ }^{1,2}$, Thomas Ethofer ${ }^{3}$, John-Dylan Haynes ${ }^{2},{ }^{1}$ Neuroimage Nord, University of Luebeck, Department of Neurology, Luebeck, Germany, ${ }^{2}$ Bernstein Center for Computational Neuroscience, Berlin, Germany, ${ }^{3}$ Laboratory for Behavioral Neurology \& Imaging of Cognition, Geneva, Switzerland

EEG Default Mode Network: Mood Modulation (Happy-Sad) in Chinese Music (Butterfly Lovers, violin concerto), Huixuan Zhao, Andrew CN Chen*, Center for Higher Brain Functions, Capital Medical University, Beijing, China
196 TH-PM

204 TH-PM

212 TH-PM

216 TH-PM

220 TH-PM

224 TH-PM

228 TH-PM

232 TH-PM

236 TH-PM

240 TH-PM 
Emotion regulation in patients with major depression, Susanne Erk ${ }^{l}$, Alexandra Mikschl ${ }^{2}$, Sabine Stier ${ }^{2}$, Angela Ciaramidaro ${ }^{3}$, Volker Gapp ${ }^{2}$, Bernhard Weber ${ }^{2}$, Henrik Walter ${ }^{I},{ }^{\prime}$ Dept. of Psychiatry, Div. of Medical Psychology, University of Bonn, Bonn, Germany, ${ }^{2}$ Dept. of Psychiatry, Joh.-Wolfgang-Goethe University, Frankfurt/Main, Germany, ${ }^{3}$ Dept. of Cognitive Science, University of Turin, Turin, Italy

Prefrontal regulation of the emotional brain: Findings in depressed and healthy subjects from neuroimaging and psychophysiology, Tom Johnstone ${ }^{1,2}$, Gregory Kolden ${ }^{1}$, Sara Polis ${ }^{l}$, Michael Peterson ${ }^{I}$, Sandy Tierney ${ }^{I}$, Ned Kalin ${ }^{1}$, Richard Davidson ${ }^{l},{ }^{1}$ University of Wisconsin-Madison, Madison, USA, ${ }^{2}$ University of Reading, Reading, United Kingdom

Brain response to emotional anticipation is related to respiratory rate, Jennifer L. Aron ${ }^{1}$, Scott C. Matthews $^{1,2}$, Alan N. Simmons ${ }^{1,2,3}$, Irina A. Strigo ${ }^{1}$, Martin P. Paulus ${ }^{1,2,3}{ }^{1}{ }^{1}$ University of California, San Diego, La Jolla, USA, ${ }^{2}$ San Diego Veterans Administration, La Jolla, USA, ${ }^{3}$ Center of Excellence in Stress and Mental Health (CESAMH), San Diego, USA

Magnetoencephalographic evidence of right frontal impairment of negative emotion processing in bipolar disorder, Li-Fen Chen ${ }^{1,2}$, Ying-Chia Lin ${ }^{2}$, Yong-Sheng Chen ${ }^{3}$, Jen-Chuen Hsieh ${ }^{1,2}$, Tung-Ping $S u^{4,5},{ }^{1}$ Institute of Brain Science, National Yang-Ming University, Taipei, Taiwan, ${ }^{2}$ Integrated Brain Research Laboratory, Taipei Veterans General Hospital, Taipei, Taiwan, ${ }^{3}$ Department of Computer Science, Hsinchu, Taiwan, ${ }^{4}$ Division of Psychiatry, School of Medicine, National Yang-Ming University, Taipei, Taiwan, ${ }^{5}$ Psychiatric Department, Taipei Veterans General Hospital, Taipei, Taiwan

Repetition suppression in orbitofrontal cortex is modulated by anger in the voice, Thomas Ethofer ${ }^{1,2,3}$, Benjamin Kreifelts ${ }^{1}$, Sarah Wiethoff ${ }^{2}$, Jonathan Wolf ${ }^{4}$, Wolfgang Grodd ${ }^{2}$, Patrik

Vuilleumier ${ }^{3}$, Dirk Wildgruber ${ }^{1,2}$, ' Department of General Psychiatry, University of Tuebingen,

Tuebingen, Germany, ${ }^{2}$ Section on Experimental MR of the CNS, University of Tuebingen, Tuebingen,

Germany, ${ }^{3}$ Laboratory for Behavioral Neurology \& Imaging of Cognition, Department of Neurosciences $\&$ Clinic of Neurology, University Medical Center of Geneva, Geneva, Switzerland, ${ }^{4}$ Department of Child Psychiatry, University of Tuebingen, Tuebingen, Germany

Automatic and Controlled Emotion Processing: Preliminary Data, Nicole Joshua ${ }^{1,2}$, Susan Rossell ${ }^{1,3}$ ${ }^{I}$ MHRI, Melbourne, Australia, ${ }^{2}$ University of Melbourne, Melbourne, Australia, ${ }^{3}$ Monash University, Melbourne, Australia

\section{IMAGING TECHNIQUES \& CONTRAST MECHANISM} Anatomical MRI

MRI of Postmortem Human Brain Hemispheres: Changes in $\mathbf{T}_{\mathbf{2}}$ Relaxation during Formaldehyde Fixation, Robert Dawe ${ }^{1}$, David Bennett ${ }^{2}$, Julie Schneider ${ }^{2}$, Sunil Vasireddi ${ }^{1}$, Konstantinos Arfanakis ${ }^{1}$, ${ }^{I}$ Department of Biomedical Engineering, Illinois Institute of Technology, Chicago, USA, ${ }^{2}$ Rush Alzheimer's Disease Center, Rush University Medical Center, Chicago, USA

Evaluating Faster Structural MRI Acquisitions based on Automated Measures of Classified Local Brain Volumes, Michael Marxen ${ }^{l, 2}$, Tara L. Dawson ${ }^{I}$, M. Kate Hanratty, Gwenn S. Smith ${ }^{1,3}$, Simon J. Graham ${ }^{1,2,4,5},{ }^{1}$ Rotman Research Institute, Baycrest Centre for Geriatric Care, Toronto, Canada, ${ }^{2}$ Heart $\&$ Stroke Foundation Centre for Stroke Recovery, Toronto, Canada, ${ }^{3}$ Centre for Addiction and Mental Health, Toronto, Canada, ${ }^{4}$ Department of Medical Biophysics, University of Toronto, Toronto, Canada, ${ }^{5}$ Sunnybrook Health Sciences Centre, Toronto, Canada

Comparison of MMSE Scores with Postmortem Hippocampal Volumes, Robert Dawe ${ }^{l}$, David Bennett ${ }^{2}$, Julie Schneider ${ }^{2}$, Sunil Vasireddi ${ }^{I}$, Konstantinos Arfanakis ${ }^{I},{ }^{I}$ Department of Biomedical Engineering, Illinois Institute of Technology, Chicago, USA, ${ }^{2}$ Rush Alzheimer's Disease Center, Rush University Medical Center, Chicago, USA

Automatic Segmentation of White Matter Hyperintensities in FLAIR images at 3T, Erin Gibson ${ }^{l}$, Fuqiang Gaol, Sandra E. Black, ${ }^{1,2}$, Nancy J. Lobaugh ${ }^{1,2},{ }^{1}$ Sunnybrook Health Sciences Centre, Toronto, Canada, ${ }^{2}$ University of Toronto, Toronto, Canada

\section{IMAGING TECHNIQUES \& CONTRAST MECHANISM Diffusion MRI}

Simulated Framework for Fibre Tracking Validation, Thomas Close $e^{1,2,3}$, Jacques-Donald Tournier ${ }^{1,4}$, Leigh Johnston ${ }^{2,3,5}$, Fernando Calamante ${ }^{1,4}$, Iven Mareels ${ }^{2,3}$, Alan Connelly ${ }^{1,4}$, ${ }^{1}$ Brain Research Institute, Melbourne, Australia, ${ }^{2}$ National ICT Australia, Melbourne, Australia, ${ }^{3}$ Department of Electrical 
Engineering, University of Melbourne, Melbourne, Australia, ${ }^{4}$ Department of Medicine, University of Melbourne, Melbourne, Australia, ${ }^{5}$ Howard Florey Institute, Melbourne, Australia

Diffusion Tensor Imaging (DTI) at 3T and 7 T, Ralf Luetzkendorf, Tobias Moench, Maurice Hollmann, Sebastian Baecke, Johannes Bernarding, Institute for Biometry and Medical Informatics, Medical Faculty, University of Magde-burg, Magdeburg, Germany

Resolving crossing fibres: validation studies using DWI phantom data, Jacques-Donald Tournier ${ }^{1,2}$, Chun-Hung Yeh ${ }^{3}$, Fernando Calamante, ${ }^{1,2}$, Kuan-Hung Cho ${ }^{4}$, Alan Connelly ${ }^{1,2}$, Ching-Po Lin ${ }^{3,5},{ }^{1}$ Brain Research Institute, Melbourne, Australia, ${ }^{2}$ Department of Medicine, University of Melbourne, Melbourne, Australia, ${ }^{3}$ Department of Biomedical Imaging and Radiological Sciences, National YangMing University, Taipei, Taiwan, ${ }^{4}$ Interdisciplinary MRI/MRS Lab, Department of Electrical Engineering, National Taiwan University, Taipei, Taiwan, ${ }^{5}$ Lab for Brain Connectivity, Institute of Neuroscience, National Yang-Ming University, Taipei, Taiwan

Prediction of Motor Outcome using Diffusion Tensor Tractography in Pontine Infarct, min cheol Jang, sung ho Jang, sang ho Ahn, dong kyu Kim, Department of Physical Medicine \& Rehabilitation, Yeungnam University College of Medicine, Taegu, Korea

Measuring and correcting errors that occur in diffusion weighted images due to non-linear gradients, Zoltan Nagy, Chloe Hutton, Nikolaus Weiskopf, Wellcome Trust Centre for Neuriomaging, University College London, London, United Kingdom

Combining DTI with Partial-brain Q-Ball Imaging to Improve the Efficiency of Fiber Detection, Jiancheng Zhuang ${ }^{l}$, Nicolas Lori ${ }^{l, 2}$, ' University of Southern California, Los Angeles, USA, ${ }^{2}$ Coimbra University, Coimbra, Portugal

\section{IMAGING TECHNIQUES \& CONTRAST MECHANISM} Multi-modal Integration

Evaluating quality of ultrafast EEG signatures in a synchronized EEG-fMRI approach, Frank Freyer ${ }^{I}$, Petra Ritter ${ }^{1}$, Robert Becker ${ }^{1}$, Kimitaka Anami ${ }^{3}$, Gabriel Curio ${ }^{1}$, Arno Villringer ${ }^{1,2},{ }^{1}$ Berlin Neuroimaging Center, Charité Universitaetsmedizin, Berlin, Germany, ${ }^{2}$ Max Planck Institute for Brain and Cognitive Sciences, Leipzig, Germany, ${ }^{3}$ National Center Hospital for Mental, Nervous, and Muscular Disorders, Tokyo, Japan

Integration of MEG and EEG data in minimum L2 norm estimation, Antonio Molins ${ }^{3,2}$, Steven Stufflebeam ${ }^{2,4,3,5}$, Emery Brown ${ }^{3,2,6,1}$, Matti Hämäläinen ${ }^{2,5,3},{ }^{1}$ Brain and Cognitive Sciences, MIT, Cambridge, USA, ${ }^{2}$ MGH-MIT-HMS Athinoula A. Martinos Ctr. for Biomed. Imaging, Charlestown, USA, ${ }^{3}$ Harvard-MIT division for Hlth. Sci. and Technology, Cambridge, USA, ${ }^{4}$ Harvard Medical School, Cambridge, USA, ${ }^{5}$ Radiology, MGH, Boston, USA, ${ }^{6}$ Anesthesiology, MGH, Boston, USA

The MIND Clinical Imaging Consortium as an application for novel comprehensive quality assurance procedures in a multi-site heterogeneous clinical research study, H Jeremy Bockholt ${ }^{1}$, Sumner Williams ${ }^{1}$, Mark Scully ${ }^{1}$, Vincent Magnotta ${ }^{2}$, Randy Gollub ${ }^{3}$, John Lauriello ${ }^{4}$, Kelvin Lim ${ }^{5}$, Tonya White $^{5}$, Rex Jung ${ }^{1}$, Charles Schulz ${ }^{5}$, Nancy Andreasen ${ }^{2}$, Vince Calhoun ${ }^{1,4},{ }^{1}$ The MIND Institute, Albuquerque, USA, ${ }^{2}$ The University of Iowa, Iowa City, USA, ${ }^{3}$ Massachusetts, Charlestown, USA, ${ }^{4}$ The University of New Mexico, Albuquerque, USA, ${ }^{5}$ The University of Minnesota, Minneapolis, USA

Comparison of CBV changes with MRI and laser-Doppler: Implications on $\mathbf{C M R}_{\mathrm{O} 2}$ calculation, Peter Herman ${ }^{I}$, Basavaraju G. Sanganahalli, Fahmeed Hyder ${ }^{1,2},{ }^{1}$ Diagnostic Radiology, Yale University, New Haven, USA, ${ }^{2}$ Biomedical Engineering, Yale University, New Haven, USA

Relation between spatially and spectrally confined EEG rhythms and fMRI resting state networks, Petra Ritter ${ }^{1}$, Michael D. Greicius ${ }^{2}$, Robert Becker ${ }^{I}$, Arno Villringer ${ }^{I, 3}$, IBerlin Neuroimaging Center and Dept. Neurology, Charité, Universitätsmedizin Berlin, Berlin, Germany, ${ }^{2}$ Departments of Neurology and Psychiatry, Stanford University School of Medicine, Stanford, USA, ${ }^{3}$ Max Planck Institute for Brain and Cognitive Sciences, Leipzig, Germany

IMAGING TECHNIQUES \& CONTRAST MECHANISM Optical Imaging/NIRS/MRS (magnetic resonance spectroscopy)

Removal of skin blood flow artifact in fNIRS signal induced by an excessive finger tapping task though ICA, Satoru Kohno ${ }^{1,2}$, Akihiro Ishikawal, Shin-ichi Shiomi ${ }^{3}$, Shoichi Tsuneishi ${ }^{l}$, Haruhide 
Udagawa ${ }^{l}$, Takashi Amital, Yoshihiro Mukuta ${ }^{l},{ }^{I} R \& D$ Department Medical Systems Division, Shimadzu Corporation, Kyoto, Japan, ${ }^{2}$ Human Brain Research Center, Kyoto University Graduate School of Medicine, Kyoto, Japan, ${ }^{3} R \& D$ Department, Shimadzu System Development Corporation, Kyoto, Japan

Phase Imaging System of Oxygen Transport usingOxyhemoglobin and Deoxyhemoglobin - new index and phenomenon of brain function-, TOSHINORI KATO, Department of Brain Environmental Research, KATOBRAIN Co., Ltd.(http://www.nonogakko.com), Tokyo, Japan

\section{IMAGING TECHNIQUES \& CONTRAST MECHANISM Perfusion MRI}

A Bayesian Approach to Perfusion Quantification of Arterial Spin Labelling Data by Deconvolution, Michael Chappell, Salima Makni, Saad Jbabdi, Mark Woolrich, FMRIB Centre, Univeristy of Oxford, Oxford, United Kingdom

Using CASL Versus BOLD fMRI Techniques to Study Linguistic and Visuospatial Tasks: a comparison of findings, Georg Deutsch ${ }^{l}$, Amol Pednekar ${ }^{2}$, Omur Sen ${ }^{l}$, Beverly Corbitt ${ }^{1}$, William Evanochko ${ }^{I}$, Jan den Hollander ${ }^{l}$, Donald Twieg ${ }^{l},{ }^{I}$ University of Alabama Medical Center, Birmingham, USA, ${ }^{2}$ Philips Medical Systems NA, Bothell, USA

\section{IMAGING TECHNIQUES \& CONTRAST MECHANISM PET/SPECT}

Effect of transmission protocol on statistical analysis of brain ${ }^{18}$ F-FDG PET; Comparison between pre- and post-injection transmission scans, Masato Kobayashi, Takashi Kudo, Tetsuya Tsujikawa, Yasushi Kiyono, Yasuhisa Fujibayashi, Hidehiko Okazawa, Biomedical Imaging Research Center, University of Fukui, Fukui, Japan

Ictal SPECT Perfusion patterns in pathologically verified Mesial Temporal Sclerosis. Correlation with Surgical outcome, Pushpalatha Sudhakar Lakkuntal, Sita Jayalakshmi $S^{2}$, Prabhakar Rao V.V.S ${ }^{3}$, Manas Panigrahi ${ }^{4}$, Sundaram Challa ${ }^{5}$, Bhushan S. Murari ${ }^{6},{ }^{1}$ Department of Nuclear Medicine, Nizam's Institute of Medical Sciences, Hyderabad, India, ${ }^{2}$ Department of Neurology, Nizam's Institute of Medical Sciences, Hyderabad, India, ${ }^{3}$ Department of Nuclear Medicine, Nizam's Institute of Medical Sciences, Hyderabad, India, ${ }^{4}$ Department of Neuro Surgery, Nizam's Institute of Medical Sciences, Hyderabad, India, ${ }^{5}$ Department of Pathology, Nizam's Institute of Medical Sciences, Hyderabad, India, ${ }^{6}$ Department of Nuclear Medicine, Nizam's Institute of Medical Sciences, Hyderabad, India

\section{LANGUAGE \\ Comprehension}

Auditory-visual integration in speech perception: A pattern-analytic fMRI study of the McGurk effect, Kachina Allen ${ }^{1,2}$, Francisco Pereira ${ }^{l, 2}$, Matthew Botvinick ${ }^{1,2},{ }_{1}^{1}$ Princeton Neuroscience Institute, Princeton, USA, ${ }^{2}$ Psychology Department, Princeton University, Princeton, USA

fMRI in the service of linguistic theory: The case of optional complements, Einat Shetreet ${ }^{l}$, Naama Friedmann ${ }^{2}$, Uri Hadar ${ }^{I},{ }^{I}$ Department of Psychology, Tel Aviv University, Tel Aviv, Israel, ${ }^{2}$ Language and Brain Lab, School of Education, Tel Aviv University, Tel Aviv, Israel

Sex Hormones Affect Interhemispheric Connectivity during the Menstrual Cycle: an fMRI study, Susanne Weis ${ }^{I}$, Barbara Stoffers ${ }^{I}$, Markus Hausmann ${ }^{2}$, Walter Sturm ${ }^{l},{ }^{l}$ Clinical Neuropsychology, Department of Neurology, Aachen, Germany, ${ }^{2}$ Department of Psychology, Durham University, Durham, United Kingdom

The relation between auditory processing and prosodic perception in speech and music: An ERP study, Varghese Peter, Genevieve McArthur, Macquarie Centre for Cognitive Sciences, Macquarie University, Sydney, Australia

Neural correlates of metaphor comprehension: the role of the right hemisphere, Midori Shibata ${ }^{l}$, Atsushi Terao ${ }^{3}$, Tamaki Miyamoto ${ }^{2}$, Jun-ichi Abe ${ }^{I}$, ${ }^{I}$ Department of Psychology, Hokkaido University Graduate School of Letters, Sapporo, Japan, ${ }^{2}$ Brain Function Research Laboratory, Hokkaido University Graduate School of Medicine, Sapporo, Japan, ${ }^{3}$ Information Science Research Center, Aoyama Gakuin University, Tokyo, Japan

344 TH-PM

348 TH-PM

352 TH-PM

356 TH-PM

360 TH-PM

364 TH-PM

368 TH-PM 
Neural substrate for integrating semantic and orthographic processing in Chinese children, Mei-Yao Wu, Tai-Li Chou, Chih-Wei Chen, Shu-Hui Lee, Li-Ying Fan, Mei-En Hsieh, Department of Psychology, National Taiwan University, Taipei, Taiwan

372 TH-PM

Auditory Language Processing in Chinese: a functional MRI Study, Mea-Yuan $\mathrm{Lin}^{l}$, Chiao-Yi Wu ${ }^{l}$, Shuo-En Huangl, Wen-Yih Isaac Tseng ${ }^{2}$, S.H. Annabel Chen ${ }^{l},{ }^{l}$ Department of Psychology, National Taiwan University, Taipei, Taiwan, ${ }^{2}$ Department of Radiology, National Taiwan University College of Medicine, Taipei, Taiwan

When logical connectives modulate priming: An electrophysiological study of coordinate structures, Magda Dumitru, MACCS, Macquarie University, Sydney, Australia

380 TH-PM

Does the processing of words and pictures involving body parts recruit the motor cortex?, Analia Arevalo ${ }^{l}$, Nina Dronkers ${ }^{1,2,3},{ }^{1}$ Center for Aphasia and Related Disorders, VA Northern California Health Care System, Martinez, USA, ${ }^{2}$ University of California, Davis, Davis, USA, ${ }^{3}$ University of California, San Diego, La Jolla, USA

Dynamic ERP Mapping in Perception of International Phonetic Vowels, Andrew CN Chen*, Peipei Wang, Yanling Yin, Weijia Feng, Center for Higher Brain Functions, Capital Medical University, Beijing, China

Integration of speech and coverbal iconic gestures: Meaning matters, Antonia Green ${ }^{l}$, Benjamin Straube ${ }^{I}$, Susanne Weis ${ }^{2}$, Klaus Willmes ${ }^{2}$, Kerstin Konrad', Tilo Kircher ${ }^{l},{ }^{\prime}$ Department of Psychiatry and Psychotherapy, RWTH Aachen University, Aachen, Germany, ${ }^{2}$ Department of Neurology, RWTH Aachen University, Aachen, Germany, ${ }^{3}$ Department of Child and Adolescent Psychiatry and Psychotherapy, RWTH Aachen University, Aachen, Germany

\section{LANGUAGE \\ Reading/Writing}

Girls show more top-down influence on Fusiform during reading: an effective connectivity, fMRI study, Tali Bitan ${ }^{1}$, Jimmy Cheon ${ }^{2}$, Dong Lu ${ }^{2}$, Douglas Burman ${ }^{2,3}$, James Booth ${ }^{2,3}$, 'Department of Communication Disorders, Haifa University, Haifa, Israel, ${ }^{2}$ Department of Communication Sciences and Disorders, Northwestern University, Evanston, USA, ${ }^{3} 3$ Department of Radiology, Evanston Northwestern Healthcare,, Evanston, USA

Common and Unique Mechanisms for Phonological Decoding Real-words and Non-words., Richard Frye ${ }^{I}$, Jacqueline Liederman ${ }^{2}$, Benjamin Malmberg ${ }^{l}$, David Strickland ${ }^{l}$, Andrew Papanicolaou ${ }^{l},{ }^{1}$ University of Texas, Houston, USA, ${ }^{2}$ Boston University, Boston, USA

376 TH-PM

384 TH-PM

388 TH-PM

392 TH-PM 396 TH-PM

Hemodynamic response observation during typing tasks using NIRS-imaging, Nao Tatsumi ${ }^{l}$, Kayoko Yoshino ${ }^{l}$, Shun Ishizaki ${ }^{2},{ }^{l}$ Graduate School of Media and Governance, Keio University, Fujisawa, Japan, ${ }^{2}$ Faculty of Environmental Information, Keio University, Fujisawa, Japan

Investigation of the orthographic/phonological interaction and the L2 factor in the ERP rhyming effect, Yuchun Chen ${ }^{1,3}$, Jun-Ren Lee ${ }^{2,3}$, Shih-Kuen Cheng ${ }^{3,4}$, Daisy Hung ${ }^{3,4}$, Ovid Tseng ${ }^{3,4}$, ${ }^{1}$ Dept. of Special Education, National Taiwan Normal University, Taipei, Taiwan, ${ }^{2}$ Dept. of Educational Psychology and Counseling, National Taiwan Normal University, Taipei, Taiwan, ${ }^{3}$ Laboratory for Cognitive Neuroscience, National Yang-Ming University, Taipei, Taiwan, ${ }^{4}$ Institute of Cognitive Neuroscience, National Central University, Chung-Li, Taiwan

Neuroanatomical Correlates of Reading Development in Adolescents with Dyslexia: A Longitudinal Study, Candy Ho, Alexander Gantman, Black Jessica, Heitzmann Joshua, Zakerani Nahal, Reiss Allan, Hoeft Fumiko, Stanford University, Palo Alto, USA

\section{MEMORY \& LEARNING Learning (explicit \& implicit)}

The neural organization of individual voice categories, Attila Andics ${ }^{1,2}$, James M. McQueen ${ }^{2}$, Karl Magnus Petersson ${ }^{1,2},{ }^{1}$ FC Donders Centre for Cognitive Neuroimaging, Nijmegen, Netherlands, ${ }^{2}$ Max Planck Institute for Psycholinguistics, Nijmegen, Netherlands

Investigating cortical mechanisms related to enhancing memory by intellectual excitement., $A i$ Fukushima ${ }^{l}$, Motoaki Sugiura ${ }^{2,1}$, Yuko Sassa ${ }^{1,3}$, Ryuta Kawashima ${ }^{1,3}$, ${ }^{I}$ Department of functional brain
416 TH-PM 
imaging of IDAC, Tohoku University, Sendai, Japan, ${ }^{2}$ Department of Cortical research, National Institute for Physiological Sciences, Okazaki, Japan, ${ }^{3}$ RISTEX, JST, Kawaguchi, Japan

A MEG study of recognition memory, Sun-Kyoung Kim ${ }^{1}$, Myung-Sun Kim ${ }^{1}$, June Sic Kim ${ }^{2}$, Chun Kee Chung ${ }^{2},{ }^{I}$ Sungshin Women's University, Department of Psychology, Seoul, South Korea, ${ }^{2}$ Seoul National University Hospital, Department of Neurosurgery, Seoul, South Korea

424 TH-PM SPOKEN WORD MEMORY TRACES WITHIN THE HUMAN AUDITORY CORTEX, Pierre Gagnepain, Gael Chételat, Brigitte Landeau, Jacques Dayan, Francis Eustache, Karine Lebreton, Inserm - EPHE - Université de Caen Basse/Normandie, Unité U923, GIP Cyceron, CHU Côte de Nacre, Caen, France

Visuospatial Memory (VSM) in Children and Adolescents with Obsessive Compulsive Disorder (OCD): A Functional Magnetic Resonance Imaging (fMRI) Study, Eve Gu ${ }^{l}$, Hannah Shoemaker ${ }^{3}$, Melissa Casey ${ }^{I}$, Tim Silk', Michael Farrell', Alasdair Vance ${ }^{I},{ }^{2}$ Academic Child Psychiatry Unit, Royal Children's Hospital, Murdoch Childrens Research Institute, Melbourne, Australia, ${ }^{2}$ Queensland Brain Institute, Brisbane, Australia, ${ }^{3}$ Howard Florey Institute, Melbourne, Australia

"Does Size Matter? The relationship between hippocampal volume and memory ability in patients with treatment resistant MDD" , Kate Hoy, Alfred Psychiatry Research Centre, Prahran, Australia

The effects of prenatal methamphetamine exposure on brain activation during verbal learning, Lisa $H$ Lu ${ }^{l, 2}$, Lynne MSmith ${ }^{3}$, Mary J O'Connor ${ }^{4}$, Arianne Johnson ${ }^{l}$, Elizabeth D O'Hare ${ }^{1,5}$, Suzanne Houston ${ }^{I}$, Susan Y Bookheimer ${ }^{4,5}$, Elizabeth R Sowell ${ }^{1,5},{ }^{1}$ UCLA Laboratory of Neuro Imaging, David Geffen School of Medicine, Los Angeles, USA, ${ }^{2}$ Roosevelt University Dept of Psychology, Chicago, USA, ${ }^{3}$ Harbor-UCLA Medica Center Dept of Pediatrics, Torrance, USA, ${ }^{4}$ UCLA Dept of Psychiatry \& Biobehavioral Sciences, Los Angeles, USA, ${ }^{5}$ UCLA Interdepartmental PhD Program for Neuroscience, Los Angeles, USA

The neural substrate of Shogi pattern recognition shaped by long-term training in professional players, Xiaohong Wan, Hironori Nakatani, Kenichi Ueno, Takeshi Asamizuya, Kang Cheng, Keiji Tanaka, RIKEN Brain Science Institute, Wako_shi, Japan

11:30 - 12:30 Corryong Hall (Level 2)

MEMORY \& LEARNING

Long-term Memory (episodic, semantic, autobiographical)

Effective connectivity during recollection- and familiarity-based memory decisions, Thomas Lemmin, Alumit Ishai, Institute of Neuroradiology, University of Zurich, Zurich, Switzerland

Impact of Valence and Concreteness on Word List Learning in Young Adults: Differential Effects on Performance and Brain Activation, Olivier Piguet ${ }^{1,2}$, Paymon Varnamkhasti ${ }^{l}$, Keyma Prince ${ }^{l}$, Emily Connally ${ }^{I}$, Suzanne Corkin ${ }^{1,3},{ }^{I}$ Massachusetts Institute of Technology, Cambridge, USA, ${ }^{2}$ Prince of Wales Medical Research Institute, Sydney, Australia, ${ }^{3}$ MGH/MIT/HMS Athinoula A. Martinos Center for Biomedical Imaging, Charlestown, USA

An fMRI Study of Episodic Memory Retrieval at 7T, Bing Yao ${ }^{l}$, Tie-Qiang Li ${ }^{1}$, James Kroger ${ }^{2}$, Peter van Gelderen', Jacco de Zwart ${ }^{l}$, Jeff Duyn ${ }^{l},{ }^{l}$ NINDS, National Institutes of Health, Bethesda, USA,

${ }^{2}$ Department of Psychology, University of New Mexico, Albuquerque, USA

The role of facial expressions in animated characters during word encoding - an fMRI study, Henk Jansma ${ }^{I}$, Jan Ole Schumann ${ }^{I}$, Claus Tempelmann' ${ }^{2}$, Thomas Münte ${ }^{I},{ }^{I}$ Dept. of Neuropsychology, Otto von Guericke University, Magdeburg, Germany, ${ }^{2} 2$ Dept. of Neurology II and CAI, University of Magdeburg, Magdeburg, Germany

Differential Connectivity During Memory Encoding for Patients with MCI versus Controls: A Partial Least Squares Account of Encoding Success, Andrea B. Protzner ${ }^{l}$, Mary Pat McAndrews ${ }^{1}$, Jennifer L. Mandzia ${ }^{2}$, Sandra E. Black ${ }^{2},{ }^{1}$ Krembil Neuroscience Program, Toronto Western Hospital, Toronto, Canada, ${ }^{2}$ Cognitive Neurology Unit, Sunnybrook Health Sciences Centre, Toronto, Canada

Predicting Successful Memory Formations using fMRI and Discriminant Analyses, Julie Yoo ${ }^{l}$, Noa Ofen $^{2}$, Susan Gabrieli ${ }^{1,2}$, Oliver Hinds ${ }^{l}$, Christina Triantafyllou ${ }^{1,3}$, John Gabrieli ${ }^{1,2},{ }^{1}$ McGovern Institute $^{2}$ for Brain Research, Massachusetts Institute of Technology, Cambridge, USA, ${ }^{2}$ Department of Brain and
428 TH-PM

432 TH-PM

436 TH-PM

440 TH-PM

444 TH-PM

454 TH-PM

458 TH-PM

462 TH-PM

466 TH-PM

470 TH-PM*

474 TH-PM* 
Cognitive Sciences, Massachusetts Institute of Technology, Cambridge, USA, ${ }^{3}$ Athinoula A. Martinos Center, Department of Radiology, MGH, Harvard Medical School, Boston, USA

MODELING \& ANALYSIS

Exploratory Methods, Artifact Removal

Evaluating the sensitivity of a peak fit analysis to speech-associated manual gestures during naturalistic audiovisual language comprehension, E Elinor Chen, Michael Andric, Steven Small, The University of Chicago, Chicago, USA

Volume Acquisition Noise-Induced Activation, Shuowen $\mathrm{Hu}^{l}$, Olumide Olulade ${ }^{l}$, Joseph Santos ${ }^{2}$, Gregory Tamer ${ }^{2}$, Wen-ming Luh ${ }^{3}$, Thomas Talavage ${ }^{1,2}$, 'School of Electrical and Computer Engineering, Purdue University, West Lafayette, USA, ${ }^{2}$ Weldon School of Biomedical Engineering, Purdue University, West Lafayette, USA, ${ }^{3}$ National Institute of Mental Health, Bethesda, USA

In Vivo Simulation of Arbitrary Activation Waveforms for Exploring phMRI Pre-Processing and Statistical Analysis Streams, Lisa Nickerson ${ }^{1,2}$, Sarabeth Fox ${ }^{3}$, Blaise Frederick ${ }^{1,2},{ }^{1}$ McLean Hospital, Belmont, USA, ${ }^{2}$ Harvard Medical School, Boston, USA, ${ }^{3}$ University of Texas, San Antonio, USA

Investigation of analyzing process on voxel-based analysis using diffusion tensor imaging data sets., Haruyasu Yamada, ${ }^{1,}$, Osamu Abe ${ }^{2}$, Hidenori Yamasue ${ }^{3}$, Kiyoto Kasai ${ }^{3}$, Shigeki Aoki ${ }^{2}$, Yusuke Inoue ${ }^{2}$, Atsuya Watanabe ${ }^{1}$, Toshiyuki Okubo ${ }^{1}$, Kuni Ohtomo ${ }^{2},{ }^{1}$ Department of Radiology, Teikyo University Chiba Medical Center, Ichihara, Japan, ${ }^{2}$ Department of Radiology, University of Tokyo, Tokyo, Japan, ${ }^{3}$ Department of Psychiatry, University of Tokyo, Tokyo, Japan

Is Cardiac Gating in Clinical DTI Studies with Single-Shot EPI Acquisition a Good Strategy?, SungWon Chung ${ }^{1,2}$, Blandine Courcot ${ }^{4}$, Michael Sdika ${ }^{3}$, Kirsten Moffat ${ }^{5}$, Caroline Rae ${ }^{4}$, Roland G. Henry ${ }^{1,2},{ }^{1}$ UCSF / UC Berkeley Joint Graduate Group in Bioengineering,, USA, ${ }^{2}$ Department of Radiology, University of California, San Francisco, USA, ${ }^{3}$ Department of Neurology, University of California, San Francisco, USA, ${ }^{4}$ Prince of Wales Medical Research Institute, Sydney, Australia, ${ }^{5}$ Symbion Clinical Research Imaging Centre, Sydney, Australia

Spatial characterisation of cardiac- and respiratory-related phase fluctuations in EPI, Chloe Hutton, Eric Featherstone, Nikolaus Weiskopf, Wellcome Trust Centre for Neuroimaging, University College London, London, United Kingdom

Effect of ventilation variations on attention system activation during a scrutiny perception task in Social Anxiety Disorder, Hector Ortiz ${ }^{1,2}$, Jesus Pujol ${ }^{1}$, Benjamin Harrison ${ }^{1,3}$, Carles Soriano-Mas ${ }^{1}$, Marina Lopez-Solà ${ }^{1,4}$, Monica Gimenez-Navarro ${ }^{l}$, Joan Deus ${ }^{1,5}$, Narcis Cardoner ${ }^{1,6}$, Javier Rosell ${ }^{2}$, Emilio Merlo-Pich ${ }^{7},{ }^{1}$ Institut d'Alta Tecnologia (IAT) - CRC Corporació Sanitària, Barcelona, Spain, ${ }^{2}$ Electronic Engineering Department, Technical University of Catalonia (UPC), Barcelona, Spain, ${ }^{3}$ Melbourne Neuropsychiatry Centre, Department of Psychiatry, The University of Melbourne, Melbourne, Australia, ${ }^{4}$ Clinical Sciences Departament. Faculty of Medicine. University of Barcelona, Barcelona, Spain, ${ }^{5}$ Department of Clinical and Health Psychology, Autonomous University of Barcelona, Barcelona, Spain, ${ }^{6}$ Department of Psychiatry, Bellvitge University Hospital, Barcelona, Spain, ${ }^{7}$ Psychiatry Centre for Excellence in Drug Discovery, Clinical Pharmacology and Discovery Medicine, GlaxoSmithKline SpA, Verona, Italy

Fractional Amplitude of Low Frequency Fluctuation: An Improved Approach for Detecting the Resting-State Functional MRI Signal, Qi-Hong Zou ${ }^{l}$, Chao-Zhe Zhu ${ }^{l}$, Yihong Yang ${ }^{2}, \mathrm{Xi-Nian} \mathrm{Zuo}$, Xiang-Yu Long ${ }^{1,4}$, Qing-Jiu Cao ${ }^{3}$, Yu-Feng Wang ${ }^{3}$, Yu-Feng Zang ${ }^{1}$, I'State Key Laboratory of Cognitive Neuroscience and Learning, Beijing Normal University, Beijing, China, ${ }^{2}$ Neuroimaging Research Branch, National Institute on Drug Abuse, National Institutes of Health, Baltimore, USA, ${ }^{3}$ Institute of Mental Health, Peking University, Beijing, China, ${ }^{4}$ National Laboratory of Pattern Recognition, Institute of Automation, Chinese Academy of Sciences, Beijing, China

\section{MODELING \& ANALYSIS \\ Flattening, Segmentation}

An improved tissue atrophy simulation based on delaunay triangulation, Meng Li, Huiguang He, Bin Lv, Mingchang Zhao, Institute of Automation, Chinese Academy of Sciences, Beijing, China 
Restoration of the sphere-cortex homeomorphism for coarse cortical triangle meshes, Michael Wagner ${ }^{l}$, Andreas Mang ${ }^{2}$, Manfred Fuchs ${ }^{l}$, Jörn Kastner ${ }^{1}$, Jan Müller ${ }^{2},{ }^{l}$ Compumedics Neuroscan, Hamburg, Germany, ${ }^{2}$ University of Lübeck, Lübeck, Germany

GIfTI: A geometry data format for interoperable exchange of surface-based brain mapping data., John Harwell ${ }^{1}$, Hester Bremen ${ }^{2}$, Olivier Coulon ${ }^{3}$, Donna Dierker ${ }^{l}$, Richard C. Reynolds ${ }^{4}$, Claudio Silva ${ }^{5}$ Kevin Teich ${ }^{6}$, David C. Van Essen ${ }^{1}$, Simon K. Warfield ${ }^{7}$,Ziad S. Saad ${ }^{4},{ }^{1}$ Department of Anatomy and Neurobiology, Washington University School of Medicine, Saint Louis, USA, ${ }^{2}$ Brain Innovation B.V.,, Netherlands, ${ }^{3}$ Laboratoire LSIS, UMR 6168, CNRS, Marseille, France, ${ }^{4}$ Scientific and Statistical Computing Core, National Institute of Mental Health, NIH, Department of Health and Human Services, Bethesda, USA, ${ }^{5}$ Scientific Computing and Imaging Institute and School of Computing, University of Utah, Salt Lake City, USA, ${ }^{6}$ Department of Radiology, Massachusetts General Hospital, Charlestown, USA, ${ }^{7}$ Computational Radiology Laboratory, Department of Radiology, Children's Hospital Boston, Boston, USA

Brain MRI Segmentation Based on Local Markov Random Fields and Sub Volume Probabilistic Atlases, Jussi Tohkal, Ivo Dinov ${ }^{2}$, David Shattuck ${ }^{2}$, Arthur Toga ${ }^{2},{ }^{1}$ Tampere University of Technology, Tampere, Finland, ${ }^{2}$ University of California, Los Angeles, Los Angeles, USA

\section{MODELING \& ANALYSIS}

Functional Connectivity and Structural Equation Modeling

Spontaneous Activity is Modulated by Task Independently of the Evoked BOLD Response, Mark McAvoy $^{l}$, Linda Larson-Prior ${ }^{1}$, Abraham Snyder ${ }^{I}$, Debra Gusnard ${ }^{l}$, Marcus Raichle ${ }^{I}$, Giovanni d'Avossa, ${ }^{2},{ }^{1}$ Washington University School of Medicine, Saint Louis, USA, ${ }^{2}$ Bangor University, Bangor, United Kingdom

Limbic-cortical networks in an affective shift task, Allison Nugent ${ }^{l}$, Julie Frost-Bellgowan ${ }^{1}$, Gang Chen $^{2}$, Wayne Drevets ${ }^{I}$, Maura Furey ${ }^{I},{ }^{I}$ Section on Neuroimaging in Mood and Anxiety Disorders, NIMH, Bethesda, USA, ${ }^{2}$ Scientific and Statistical Computing Core, NIMH, Bethesda, USA

Predicting Resting-State Functional Connectivity from Structural Connectivity, Christopher Honey ${ }^{1}$, Olaf Sporns ${ }^{1}$, Leila Cammoun ${ }^{2}$, Xavier Gigandet ${ }^{2}$, Reto Meuli ${ }^{3}$, Patric Hagmann ${ }^{3},{ }^{I}$ Department of Psychological and Brain Sciences, Indiana University, Bloomington, USA, ${ }^{2}$ Signal Processing Institute, Ecole Polytechnique Federale de Lausanne, Lausanne, Switzerland, ${ }^{3}$ Department of Radiology, University Hospital Center and University of Lausanne, Lausanne, Switzerland

Brain effective connectivity study based on conditional Granger causality, Zhenyu Zhou ${ }^{1,2}$, Yonghong Chen ${ }^{3}$, Guojun He ${ }^{I}$, Paul Wright ${ }^{1}$, Mingzhou Ding ${ }^{3}$, Yijun Liu ${ }^{I}$, 'Dept. of Psychiatry, University of Florida, Gainesville, USA, ${ }^{2}$ Key Laboratory of Child Development and Learning Science (Southeast University), Ministry of Education, Nanjing, China, ${ }^{3}$ Dept. of Biomedical Engineering, University of Florida, Gainesville, USA

Characterize the Resting State fMRI of the Brain, aviv mezer, yaniv assaf, Tel Aviv University, Tel Aviv, Israel

Modeling Functional Connectivity in the Amygdala: A Meta-Analytic Approach, Jennifer Robinson ${ }^{1}$, Angela Laird ${ }^{2}$, David Glahn ${ }^{1,2}$, Peter Fox ${ }^{2},{ }_{1}^{1}$ Department of Psychiatry, University of Texas Health Science Center, San Antonio, USA, ${ }^{2}$ Research Imaging Center, University of Texas Health Science Center, San Antonio, USA

Estimating mental chronometry from fMRI signals via solving the hemodynamic inverse problem, Vasily Vakorin ${ }^{1}$, Ron Borowsky ${ }^{2,3}$, Olga Krakovska ${ }^{4}$, Gordon Sarty ${ }^{2,5}$, Antony McIntosh ${ }^{1,6}$, ${ }^{1}$ Rotman Research Institute of Baycrest, Canada, , ${ }^{2}$ Department of Psychology, University of Saskatchewan, Canada, , ${ }^{3}$ Division of Neurosurgery, University of Saskatchewan, Canada,, ${ }^{4}$ Department of Applied 550 TH-PM Mathematics, University of Western Ontario, Canada,, ${ }^{5}$ Division of Biomedical Engineering, University of Saskatchewan, Canada, " Department of Psychology, University of Toronto, Canada,,

Resting State Functional Connectivity of the Dorsolateral Prefrontal Cortex: Laterality effects, Nick Bradfield, David Reutens, Amanda Wood, Department of Medicine (Neurosciences), Southern Clinical School, Monash University, Melbourne, Australia

538 TH-PM

542 TH-PM

546 TH-PM

Granger causality analysis of fMRI data reveals true neuronal connectivity despite HRF variability, Gopikrishna Deshpande ${ }^{I}$, Krish Sathian ${ }^{2,3}$, Xiaoping Hu ${ }^{l},{ }^{l}$ Coulter Department of 
Biomedical Engineering, Georgia Institute of Technology and Emory University, Atlanta, USA,

${ }^{2}$ Departments of Neurology, Psychology and Rehabilitation Medicine, Emory University, Atlanta, USA,

${ }^{3}$ Atlanta VAMC Rehabilitation R\&D Center of Excellence, Atlanta, USA

Granger Causality analysis of the default network, Luis Hernandez-Garcia, Scott Peltier, Mostafa Rezaie, University of Michigan, Ann Arbor, USA

562 TH-PM

Diffusion tensor imaging analysis methods for comparisons at group level: tractwise fractional anisotropy statistics and intersubject fiber tracking, Jan Kassubek, Anne-Dorte Sperfeld, Axel Riecker, Albert C. Ludolph, Alexander Unrath, Hans-Peter Müller, Dept. of Neurology, University of Ulm, Ulm, Germany

Cortical interactions, masses and clouds: The geometry of system and measurement noise., Stuart Knock ${ }^{l, 2}$, Michael Breakspear ${ }^{1,2}$, 'S Chool of Psychiatry, University of New South Wales, Australia, Sydney, Australia, ${ }^{2}$ The Black Dog Institute, Randwick, NSW, Australia, Sydney, Australia

A multistart procedure to recover functional networks in MEG/EEG based on anatomical and functional K-means with spatial limitation constraints., Anael Dossevi ${ }^{l, 2}$, Line Garnero ${ }^{l}$, Habib Ammari ${ }^{2},{ }^{1}$ Cognitive Neuroscience \& Brain Imaging Lab CNRS UPR 640, Paris, France, ${ }^{2}$ Center of Applied Mathematics, Ecole Polytechnique/CNRS UMR 7641, Palaiseau, France

Resting state brain functional connectivity is associated with EEG beta activity, Jaroslav Hlinka ${ }^{l}$, Charilaos Alexakis ${ }^{1}$, Ana Diukova, ${ }^{1,2}$, Peter F. Liddle ${ }^{2}$, Paul S. Morgan', Dorothee P. Auer ${ }^{l},{ }^{1}$ Division of Academic Radiology, School of Medical and Surgical Sciences, University of Nottingham, Nottingham, United Kingdom, ${ }^{2}$ Division of Psychiatry, School of Community Health Sciences, University of Nottingham, Nottingham, United Kingdom

Meta-analysis of the default mode network: Connectivity patterns for activations and deactivations, Angela Laird, Peter Fox, Research Imaging Center, University of Texas Health Science Center, San Antonio, USA

MODELING \& ANALYSIS

Multivariate Modeling, PCA, \& ICA

Detecting time-varying connectivity in EEG/MEG imaging, Felix Carbonell ${ }^{1}$, Keith Worsley ${ }^{l, 2}$, Nelson Trujillo-Barreto ${ }^{3}$, Roberto Sotero ${ }^{3},{ }^{1}$ Department of Mathematics and Statistics, McGill University, Montreal, Canada, ${ }^{2}$ McConnell Brain Imaging Centre, Montreal Neurologic Institute, Montreal, Canada, ${ }^{3}$ Cuban Neuroscience Centre, Havana, Cuba

Constrained Canonical Correlation Analysis using a Local Region Growing Algorithm, Mingwu Jin, Dietmar Cordes, University of Colorado Denver, Denver, USA

Clinical utility of distributed source modeling of scalp EEG in focal epilepsy, Chris Plummer ${ }^{1,3}$, Michael Wagner $^{2}$, Manfred Fuchs ${ }^{2}$, Simon Vogrin ${ }^{1}$, Lucas Litewka ${ }^{1}$, Steve Farish ${ }^{3}$, A.Simon Harvey ${ }^{3,4}$, Mark Cook ${ }^{1,3}$, ${ }^{I}$ St Vincent's Hospital, Melbourne, Australia, ${ }^{2}$ Compumedics Neuroscan, Hamburg, Germany, ${ }^{3}$ University of Melbourne, Melbourne, Australia, ${ }^{4}$ Royal Children's Hospital, Melbourne, Australia

594 TH-PM

Reproducibility Based Group-level Independent Component Analysis, Zhi Yang ${ }^{l}$, Stephen LaConte ${ }^{2}$, Xuchu Weng ${ }^{l}$, Xiaoping $\mathrm{Hu}^{3}$, ${ }^{I}$ Lab. for Higher Brain Function, Institute of Psychology, the Chinese Academy of Sciences, Beijing, China, ${ }^{2}$ Department of Bioengineering, Rice University, Houston, USA, ${ }^{3}$ Department of Biomedical Engineering, Emory University, Atlanta, USA

Algorithm for automated identification of intrinsic brain networks in group studies by clustering independent components across subjects, Sridharan Devarajan, Elena Rykhlevskaia, Kaustubh Supekar, Catherine Chang, Michael Greicius, Vinod Menon, Stanford University, Stanford, USA

602 TH-PM

Directed Partial Correlation to assess functional interactions in fMRI time series, David Feess ${ }^{1,3}$, Wolfgang Mader ${ }^{1,3}$, Rüdiger Lange ${ }^{3}$, Dorothee Saur ${ }^{3}$, Volkmar Glauche, ${ }^{3}$, Cornelius Weiller ${ }^{2,3}$, Jens Timmer ${ }^{1,2}$, Björn Schelter ${ }^{1,2},{ }^{I}$ FDM, Center for Data Analysis and Modeling, University of Freiburg, Freiburg, Germany, ${ }^{2}$ BCCN, Bernstein Center for Computational Neuroscience, University of Freiburg, Freiburg, Germany, ${ }^{3}$ Department of Neurology, University Hospital Freiburg, Freiburg, Germany

Neuronal dynamics in Stop-signal paradigm: EEG/MEG source localization, Alexander Savostyanov $^{1,2}$, Arthur Tsay ${ }^{2}$, Michelle Liou ${ }^{2}$, Juin-Der Lee $^{2}$, Evgeny Levin $^{1}$, Alexey Yurganov ${ }^{1}$, 
Gennadiy Knyazev ${ }^{I},{ }^{I}$ Institute of Physiology of SB RAMS, Novosibirsk, Russia, ${ }^{2}$ Institute of Statistical Science of Academia Sinica, Taipei, Taiwan

MOTOR BEHAVIOR

Basal Ganglia/Brainstem/Spinal Cord

Gender differences in voluntary micturition control - An fMRI study., Jürgen Baudewig ${ }^{1}$, Sandra Seseke $^{2}$, Kai Kallenberg ${ }^{1}$, Rolf H Ringert ${ }^{2}$, Florian Seseke ${ }^{3}$, Peter Dechent ${ }^{1},{ }^{I}$ MR-Research in Neurology and Psychiatry, University Medical Center, Göttingen, Germany, ${ }^{2}$ Department of Urology, University Medical Center, Göttingen, Germany, ${ }^{3}$ Department of Urology, Martha-Maria Hospital, Halle, Germany

614 TH-PM

\section{MOTOR BEHAVIOR}

Eye Movements/Visuomotor Processing

Interhemispheric Transfer Visualized by fMRI: Are there BOLD Signal Changes in White Matter?, Jürgen Baudewigl', Julia Böhm², Peter Dechent ${ }^{1}$, Aribert Rothenberger ${ }^{2}$, Veit Roessner ${ }^{2}$, ${ }^{1}$ MR-Research in Neurology and Psychiatry, University Medical Center, Göttingen, Germany,

${ }^{2}$ Department of Child and Adolescent Psychiatry, University Medical Center, Göttingen, Germany

618 TH-PM

Eye hand coordination task by Children with Developmental Coordination Disorder: An fMRI study, Mitsuru Kashiwagi $i^{1}$, Sunao Iwaki ${ }^{2}$, Ryusaku Hashimoto ${ }^{1}$, Shuhei Suzuki ${ }^{2},{ }^{1}$ Osaka Medical College, Takatsuki, Japan, ${ }^{2}$ National Institutes of Advanced Industrial Science and Technology, Ikeda, Japan

COMPARISON OF OBSERVING AN ACTION AS IF IT WERE PERFORMED BY ONESELF OR THE OTHER PERSON USING EVENT RELATED fMRI, Satomi Higuchi ${ }^{l, 3}$, Stefan Vogt $t^{l, 3}$, Francis McGlone ${ }^{2}$, Neil Roberts ${ }^{3},{ }^{1}$ Department of Psychology, Lancaster University, Lancaster, United Kingdom, ${ }^{2}$ Cognitive Neuroscience, Unilever R\&D, Port Sunlight Laboratories, Wirral, United Kingdom, ${ }^{3}$ Magnetic Resonance and Image Analysis Research Centre, University of Liverpool, Liverpool, United Kingdom

\section{NEUROANATOMY \\ DTI Studies, Application}

MAPPING GENETIC INFLUENCES ON BRAIN FIBER ARCHITECTURE WITH HIGH
ANGULAR RESOLUTION DIFFUSION IMAGING (HARDI), Ming-Chang Chiang ${ }^{1}$, Marina ANGULAR RESOLUTION DIFFUSION IMAGING (HARDI), Ming-Chang Chiang, Marina
Barysheva $^{1}$, Agatha D. Lee ${ }^{I}$, Sarah Madsen ${ }^{1}$, Andrea D. Klunder ${ }^{1}$, Arthur W. Toga ${ }^{1}$, Katie L. McMahon ${ }^{2}$, Greig I. de Zubicaray ${ }^{2}$, Matthew Meredith ${ }^{2}$, Margaret J. Wright ${ }^{3}$, Anuj Srivastava ${ }^{4}$, Nikolay Balov $^{4}$, Paul M. Thompson ${ }^{1},{ }^{1}$ Laboratory of Neuro Imaging, Department of Neurology, UCLA School of Medicine, Los Angeles, USA, ${ }^{2}$ Functional MRI Laboratory, Centre for Magnetic Resonance, University of Queensland, Brisbane, Australia, ${ }^{3}$ Queensland Institute of Medical Research, Brisbane, Australia, ${ }^{4}$ Department of Statistics, Florida State University, Tallahassee, USA

Connectivity-based parcellation of the cortical surface using q-ball diffusion imaging, Pamela Guevara $^{1,2}$, Muriel Perrin ${ }^{1,2,3}$, Pascal Cathier ${ }^{1,2}$, Yann Cointepas ${ }^{1,2}$, Denis Rivière ${ }^{1,2}$, Cyril Poupon ${ }^{1,2}$, Jean-François Mangin ${ }^{1,2},{ }^{l}$ CEA, Neurospin, Gif-sur-Yvette, France, ${ }^{2}$ Institut Fédératif de Recherche 49, Gif-sur-Yvette, France, ${ }^{3}$ GE Healthcare, Buc, France

Disparate Gender Effects on White Matter Tracts in Fronto-striato-thalamic Circuit: A Diffusion Spectrum Imaging Study, Y.C. Lo ${ }^{1}$, S.C. Huang ${ }^{2}$, W.Y. Chiang ${ }^{2}$, L.W. Kuo ${ }^{4}$, F.C. Yeh ${ }^{2}$, V.J. Wedeen ${ }^{5}$, W.Y.I. Tseng ${ }^{1,2,3}$, Institute of Biomedical Engineering, National Taiwan University, Taipei, Taiwan, ${ }^{2}$ Center for Optoelectronic Biomedicine, National Taiwan University College of Medicine, Taipei, Taiwan, ${ }^{3}$ Department of Medical Imaging, National Taiwan University Hospital, Taipei, Taiwan, ${ }^{4}$ Department of Electrical Engineering, National Taiwan University, Taipei, Taiwan, ${ }^{5}$ MGH Martinos Center for Biomedical Imaging, Harvard Medical School, Charlestown, USA

Comparison of white matter indices in healthy ageing, Emmanuel A Stamatakis ${ }^{1}$, Meredith A Shafto ${ }^{2}$, Lorraine K Tyler ${ }^{2},{ }^{1}$ School of Psychological Sciences and Division of Imaging Science and Biomedical Engineering, University of Manchester, Manchester, United Kingdom, ${ }^{2}$ Centre for Speech, Language and the Brain, Department of Experimental Psychology, University of Cambridge, Cambridge, United Kingdom

Gender difference in gray/white matter volume and diffusion tensor data during normal aging, Osamu Abe ${ }^{l}$, Hiddenori Yamasue ${ }^{l}$, Haruyasu Yamada ${ }^{2}$, Yoshitaka Masutani $^{l}$, Hideyuki Inoue ${ }^{l}$, Kunio 
Takei $^{I}$, Motomu Suga ${ }^{1}$, Hiroyuki Kabasawa ${ }^{I}$, Kiyoto Kasai ${ }^{l}$, Shigeki Aoki ${ }^{l}$, Kuni Ohtomo ${ }^{l}$, ${ }^{1}$ University of Tokyo, Tokyo, Japan, ${ }^{2}$ Teikyo University, Chiba, Japan

Uncertainty of apparent white matter fiber tract size in DTI fiber tracking and region of interest analyses: A multi-resolution study, Daniel Franc ${ }^{1}$, Christophe Lenglet ${ }^{2}$, Gloria Haro ${ }^{3}$, Paul Thompson ${ }^{4}$, Bryon Mueller ${ }^{1}$, Guillermo Sapiro ${ }^{1}$, Kelvin Lim ${ }^{l},{ }^{1}$ University of Minnesota, Minneapolis, USA, ${ }^{2}$ Siemens Corporate Research, Princeton, USA, ${ }^{3}$ UPC, Barcelona, Spain, ${ }^{4}$ UCLA Medical School, Los Angeles, USA

Gender Differences in White Matter Asymmetry in Relation with Cortical Thickness Asymmetry, Chi-Hoon Choi ${ }^{1,2}$, Jong-Min Lee ${ }^{2}$, Bang-Bon Koo ${ }^{2}$, Jun Sung Park ${ }^{2}$, Jun Soo Kwon ${ }^{3}$, Sun I. Kim ${ }^{2}$, ${ }^{I}$ Department of Diagnostic Radiology, National Medical Center, Seoul, South Korea, ${ }^{2}$ Department of Biomedical Engineering, Hanyang University, Seoul, South Korea, ${ }^{3}$ Department of Psychiatry, Seoul National University College of Medicine, Seoul, South Korea

Comparative SPM and ROI analyses of fractional anisotropy maps in preterm and normal newborns, Paola Scifo, Cristina Baldoli, Silvia Pontesilli, Valeria Blasi, Roberta Scotti, Giuseppe Scotti, Ferruccio Fazio, Scientific Institute H San Raffaele, Milan, Italy

DTI Fiber Tractography Reveals Precentral-Postcentral Gyral Connectivity, John Bogovic, Aaron Carass, Jing Wan, Bennett Landman, Jerry Prince, Image Analysis and Communications Laboratory, Electrical and Computer Engineering, the Johns Hopkins University, Baltimore, USA

\section{SENSORY SYSTEMS Auditory/Vestibular}

Diffusion tensor imaging study on congenitally deaf, Yonghui $L i^{l}$, Yuan Zhou ${ }^{l}$, Jun $\mathrm{Li}^{l}$, Chunshui Yu ${ }^{2}$, Wen Qin ${ }^{2}$, Kuncheng Li ${ }^{2}$, Yong Liu ${ }^{1}$, Ni Shu ${ }^{1}$, Tianzi Jiang ${ }^{1},{ }^{l}$ National Laboratory of Pattern Recognition, Institute of Automation, Chinese Academy of Sciences, Beijing, China, ${ }^{2}$ Department of Radiology, Xuanwu Hospital of Capital Medical University, Beijing, China

rTMS over medial posterior parietal cortex impairs fine auditory spatial discrimination, Santani Teng, David Whitney, Center for Mind and Brain/Dept. of Psychology, UC Davis, Davis, USA

Electrophysiological mapping of the human auditory cortex using click-train stimulation, Kirill Nourski ${ }^{1}$, Hiroyuki Oya ${ }^{1}$, Hiroto Kawasaki ${ }^{1}$, Richard Reale ${ }^{1,2}$, Albert Fenoy ${ }^{1}$, Paul Poon ${ }^{3}$, Matthew Howard ${ }^{l}$, John Brugge ${ }^{1,2}$, 'The University of Iowa, Iowa City, USA, ${ }^{2}$ University of Wisconsin-Madison, Madison, USA, ${ }^{3}$ National Cheng Kung University, Tainan, Taiwan

Plastic Functional Connectivity in Musicians' Brain: a Resting State fMRI Study, Han Zhang ${ }^{l}$, Ying Han $^{l}$, Hong Yang ${ }^{2}$, He-Han Tang ${ }^{2}$, Qi-Yong Gong ${ }^{2}$, Yu-Feng Zang ${ }^{1}$, Chao-Zhe Zhu ${ }^{l}$, , ${ }^{l}$ State Key Laboratory of Cognitive Neuroscience and Learning, Beijing Normal University, Beijing, China, ${ }^{2}$ Huaxi MR Research Center (HMRRC), Department of Radiology, West China Hospital of Sichuan University, Chengdu, China

\section{SENSORY SYSTEMS}

Tactile/Somatosensory

MEG Event-Related Desynchronization and Synchronization Differences During Basic Somatosensory Processing in Individuals with ADHD, Colleen Dockstader ${ }^{1}$, William Gaetz ${ }^{1,2}$, Douglas Cheyne ${ }^{1,2}$, Christina M. Popovich ${ }^{1,3}$, Frank Wang ${ }^{1,3}$, F. Xavier Castellanos ${ }^{4}$, Rosemary Tannock ${ }^{1,5},{ }^{1}$ Neurosciences and Mental Health Program, The Hospital for Sick Children, Toronto, Canada, ${ }^{2}$ Department of Diagnostic Imaging, The Hospital for Sick Children, Toronto, Canada, ${ }^{3}$ Institute of Medical Science, University of Toronto, Toronto, Canada, ${ }^{4}$ Child Study Center, New York University, New York, USA, ${ }^{5}$ Human Development \& Applied Psychology, Ontario Institute for Studies in Education, Toronto, Canada

Perceptive limits linked to differential $600 \mathrm{~Hz}$ activity in the somatosensory system, Ulrike Jaros ${ }^{l}$, Bernd Hilgenfeld ${ }^{l}$, Stephan Lau ${ }^{1,2}$, Gabriel Curio ${ }^{3}$, Jens Haueisen ${ }^{1,2}$, ${ }^{I}$ Biomagnetic Center, Department of Neurology, University Hospital Jena, Jena, Germany, ${ }^{2}$ Institute of Biomedical Engineering and Informatics, Technical University Ilmenau, Ilmenau, Germany, ${ }^{3}$ Neurophysics Group, Department of Neurology, Charité - University Medicine Berlin, Berlin, Germany 
Top-down control of cortical ongoing mu rhythm $(7-13 \mathrm{~Hz})$ in sensory awareness of a weak stimulus, Yan Zhang, Mingzhou Ding, J. Crayton Pruitt Family Department of Biomedical Engineering, University of Florida, Gainesville, USA

Cuff-type pneumatic stimulator for somatosensory mapping of finger afferences with fMRI, Eugen Gallasch $^{1}$, Martin Fend ${ }^{l}$, Dietmar Rafolt ${ }^{2}$, Christian Siedentopf ${ }^{5,6}$, Stefan Golaszewski ${ }^{3,5}$, Roland Beisteiner ${ }^{4},{ }^{1}$ Dept. of Physiology, Medical University of Graz, Graz, Austria, ${ }^{2}$ Center for Biomedical Engineering and Physics, Medical University of Vienna, Vienna, Austria, ${ }^{3}$ Dept. of Neurology, 694 TH-PM Paracelsus Medical University Salzburg, Salzburg, Austria, ${ }^{4}$ Dept. of Neurology, Medical University of Vienna, Vienna, Austria, ${ }^{5}$ fMRI Lab, Dept. of Psychiatry, Medical University Innsbruck, Innsbruck, Austria, ${ }^{6}$ Dept. of Radiology, Medical University Innsbruck, Innsbruck, Austria

A fMRI Study of Acupuncture: Human Brain Activity in the Manipulation of Needle Rotation., Hiroaki Mano ${ }^{1}$, Masahiro Umeda ${ }^{2}$, Masaki Fukunaga ${ }^{2}$, Toshihiro Higuchi ${ }^{l}$, Chuzo Tanaka ${ }^{l}$,

${ }^{l}$ Department of Brain Surgery, Meiji Unisersity of Integrated Medicine, Nantan, Japan, ${ }^{2}$ Department of Medical Informatics, Meiji Unisersity of Integrated Medicine, Nantan, Japan

\section{SENSORY SYSTEMS} Vision

Multivoxel fMRI analysis reveals the representation of spatial frequency information in the human primary visual cortex, Bahador Bahrami ${ }^{1,2}$, Geraint Rees ${ }^{1,2}$, 'Institute of Cognitive Neuroscience, London, United Kingdom, ${ }^{2}$ Wellcome Department of Imaging Neuroscience, London, United Kingdom

Brain Mechanisms of Vision in Human Amblyopia: A Magnetoencephalography (MEG) Study., Filomeno Cortese ${ }^{I}$, Herbert C. Goltz ${ }^{I}$, Zahra Hirji ${ }^{1}$, Douglas O. Cheyne ${ }^{2}$, Agnes F.M. Wong ${ }^{1}$, ${ }^{l}$ Department of Ophthalmology \& Vision Sciences, The Hospital for Sick Children, Toronto, Canada, ${ }^{2}$ Diagnostic Imaging, The Hospital for Sick Children, Toronto, Canada

The transformation of representational similarity along human ventral-stream stages of visualobject processing, Nikolaus Kriegeskorte, Marieke Mur, Jerzy Bodurka, Peter Bandettini, NIMH, Bethesda, USA

N170 amplitude reflects the seen number of faces irrespective of low-level stimulus variables, Aina Puce $^{I}$, Marie McNeely ${ }^{I}$, Olivia Carrick ${ }^{l}$, Michael Berrebi ${ }^{I}$, James Epling ${ }^{l}$, James Thompson ${ }^{I, 2}$, Jillian Hardee ${ }^{1}$, Leor Zellner ${ }^{1}$, Julie Brefcynski-Lewis ${ }^{l},{ }^{1}$ Center for Advanced Imaging, West Virginia University, Morgantown, USA, ${ }^{2}$ Psychology Department, George Mason University, Fairfax, USA

MEG demonstrates a shift to higher gamma frequencies in primary visual cortex for moving versus stationary stimuli., Jennifer B. Swettenham, Krish D. Singh, CUBRIC, School of Psychology, Cardiff University, Cardiff, United Kingdom

Increasing the measured BOLD signal in human lateral geniculate nucleus and superior colliculus using cardiac gating, Martin Hebart ${ }^{l}$, Ignacio Vallines ${ }^{1,2}$, I Department of Experimental Psychology, Ludwig Maximilian University, Munich, Germany, ${ }^{2}$ Department of Experimental Psychology, University of Regensburg, Regensburg, Germany

Exploring the relationship between natural fluctuations in electrical measures of brain activity and the BOLD response, during visual stimulation., Karen J. Mullinger, Gerda B. Geirsdottir, Matthew J. Brookes, Peter F. Liddle, Richard W. Bowtell, University of Nottingham, Nottingham, United Kingdom

Using inter-session repeatability to improve the results of phase-encoded retinotopic mapping, Krish D. Singh ${ }^{1}$, Simon K. Rushton ${ }^{1}$, Tom C.A. Freeman ${ }^{1}$, Petroc Sumner ${ }^{l}$, Paul A. Warren ${ }^{l}$, Andy T. Smith ${ }^{2},{ }^{1}$ CUBRIC, School of Psychology, Cardiff University, Cardiff, United Kingdom, ${ }^{2}$ Dept. of Psychology, Royal Holloway, University of London, Egham, United Kingdom

\section{SENSORY SYSTEMS}

Tactile/Somatosensory

Proprioceptive perception, an fMRI study of brain lateralization and its relationship with behavioral measures, Ettie Ben-Shabat ${ }^{1,2}$, Gaby S Pell ${ }^{3}$, Amy Brodtmann ${ }^{2}$, Thomas A Matyas ${ }^{1,2}$, Leeanne M Carey ${ }^{1,2},{ }^{1}$ La Trobe University, Melbourne, Australia, ${ }^{2}$ National Stroke Research Institute, 